\title{
BRUNO CHRCANOVIC ON FAILURE OF ORAL IMPLANTS
}
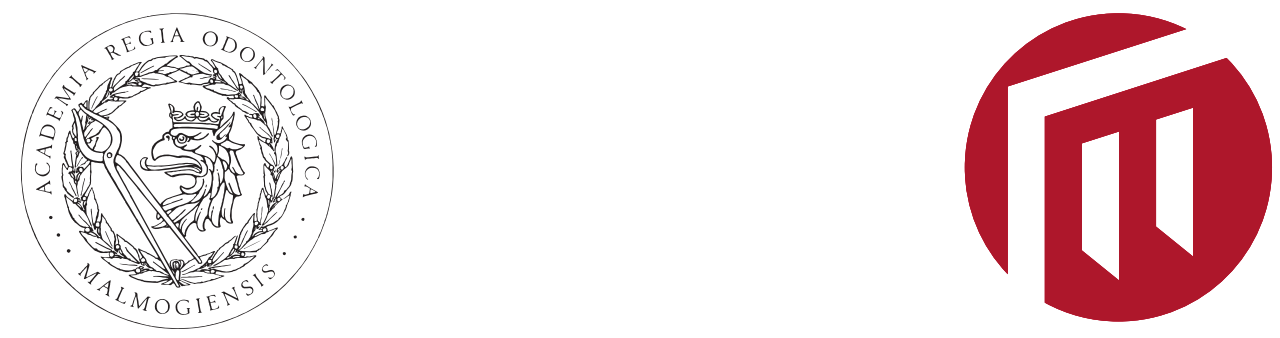

ON FAILURE OF ORAL IMPLANTS 
Malmö University, Faculty of Odontology Doctoral Dissertations 2017

(C) Bruno Chrcanovic 2017 ISBN 978-9I-7I04-766-3 (print)

ISBN 978-9I-7 I04-767-0 (pdf)

Holmbergs, Malmö 20I7 


\section{BRUNO CHRCANOVIC ON FAILURE OF ORAL IMPLANTS}

Malmö University, 2017

Faculty of Odontology Department of Prosthodontics Malmö, Sweden 
This publication is also available at: www.mah.se/muep 
To my beloved wife Aleksandra, my precious son Nathan, and my mother and my father 

This thesis is number 50 in a series of investigations on implants, hard tissues and the locomotor apparatus originating from the Department of Biomaterials, University of Gothenburg and the Department of Prosthodontics/Material Sciences, Malmö University, Sweden.

1. Anders R Eriksson DDS, 1984. Heat-induced Bone Tissue Injury. An in vivo investigation of heat tolerance of bone tissue and temperature rise in the drilling of cortical bone. Thesis defended 21.2.1984. External examiner: Docent K-G. Thorngren.

2. Magnus Jacobsson MD, 1985. On Bone Behaviour after Irradiation.

Thesis defended 29.4.1985. External examiner: Docent A.

Nathanson.

3. Fredric Buch MD, 1985. On Electrical Stimulation of Bone Tissue.

Thesis defended 28.5.1985. External examiner: Docent T. Ejsing-Jörgensen.

4. Peter Kälebo MD, 1987. On Experimental Bone Regeneration in Titanium Implants. A quantitative microradiographic and histologic investigation using the Bone Harvest Chamber. Thesis defended 1.10.1987. External examiner: Docent N. Egund.

5. Lars Carlsson MD, 1989. On the Development of a new Concept for Orthopaedic Implant Fixation.

Thesis defended 2.12.1989. External examiner: Docent L-Å Broström.

6. Tord Röstlund MD, 1990. On the Development of a New Arthroplasty.

Thesis defended 19.1.1990. External examiner: Docent Å. Carlsson. 
7. Carina Johansson Res Tech, 1991. On Tissue Reaction to Metal Implants.

Thesis defended 12.4.1991. External examiner: Professor K.

Nilner.

8. Lars Sennerby DDS, 1991. On the Bone Tissue Response to Titanium Implants.

Thesis defended 24.9.1991. External examiner: Dr J.E.

Davies.

9. Per Morberg MD, 1991. On Bone Tissue Reactions to Acrylic Cement.

Thesis defended 19.12.1991. External examiner: Docent K. Obrant.

10. Ulla Myhr PT, 1994. On factors of Importance for Sitting in Children with Cerebral Palsy.

Thesis defended 15.4.1994. External examiner: Docent K. Harms-Ringdahl.

11. Magnus Gottlander MD, 1994. On Hard Tissue Reactions to Hydroxyapatite- Coated Titanium Implants.

Thesis defended 25.11.1994. External examiner: Docent P. Aspenberg.

12. Edward Ebramzadeh MScEng, 1995. On Factors Affecting Long-Term Outcome of Total Hip Replacements. Thesis defended 6.2.1995. External examiner: Docent L. Linder.

13. Patricia Campbell BA, 1995. On Aseptic Loosening in Total Hip Replacement: the Role of UHMWPE Wear Particles. Thesis defended 7.2.1995. External examiner: Professor D. Howie.

14. Ann Wennerberg, DDS, 1996. On Surface Roughness and Implant Incorporation.

Thesis defended 19.4.1996. External examiner: Professor P-O. Glantz. 
15. Neil Meredith BDS MSc FDS RCSm, 1997. On the Clinical Measurement of Implant Stability Osseointegration. Thesis defended 3.6.1997. External examiner: Professor J. Brunski.

16. Lars Rasmusson DDS, 1998. On Implant Integration in Membrane-Induced and Grafter Bone.

Thesis defended 4.12.1998. External examiner: Professor R. Haanaes.

17. Thay Q Lee MSc, 1999. On the Biomechanics of the Patellfemoral Joint and Patellar Resurfacing in Total Knee Arthroplasty.

Thesis defended 19.4.1999. External examiner: Docent G. Nemeth.

18. Anna Karin Lundgren DDS, 1999. On Factors Influencing Guided Regeneration and Augmentation of Intramembraneous Bone.

Thesis defended 7.5.1999. External examiner: Professor B. Klinge.

19. Carl-Johan Ivanoff DDS, 1999. On Surgical and Implant Related Factors Influencing Integration and Function of Titanium Implants. Experimental and Clinical Aspects. Thesis defended 12.5.1999. External examiner: Professor B. Rosenquist.

20. Bertil Friberg DDS MDS, 1999. On Bone Quality and Implant Stability Measurements. Thesis defended 12.11.1999. External examiner: Docent P. Åstrand.

21. Åse Allansdotter Johansson MD, 1999. On Implant Integration in Irradiated Bone. An Experimental Study of the Effects of Hyperbaric Oxygeneration and Delayed Implant Placement.

Thesis defended 8.12.1999. External examiner: Docent K. Arvidsson-Fyrberg. 
22. Börje Svensson FFS, 2000. On Costochondral Grafts Replacing Mandibular Condyles in Juvenile Chronic Arthritis. A Clinical, Histologic and Experimental Study. Thesis defended 22.5.2000. External examiner: Professor Ch. Lindqvist.

23. Warren Macdonald BEng, MPhil, 2000. On Component Integration on Total Hip Arthroplasties: Pre-Clinical Evaluations.

Thesis defended 1.9.2000. External examiner: Dr A.J.C. Lee.

24. Magne Røkkum MD, 2001. On Late Complications with HA Coated Hip Arthroplasties.

Thesis defended 12.10.2001. External examiner: Professor P. Benum.

25. Carin Hallgren Höstner DDS, 2001. On the Bone Response to Different Implant Textures. A 3D analysis of roughness, wavelength and surface pattern of experimental implants. Thesis defended 19.11.2001. External examiner: Professor S. Lundgren.

26. Young-Taeg Sul DDS, 2002. On the Bone Response to Oxidised Titanium Implants: The role of microporous structure and chemical composition of the surface oxide in enhanced osseointegration.

Thesis defended 7.6.2002. External examiner: Professor J.E. Ellingsen.

27. Victoria Franke Stenport DDS, 2002. On Growth Factors and Titanium Implant Integration in Bone.

Thesis defended 11.6.2002. External examiner: Associate Professor E. Solheim.

28. Mikael Sundfeldt MD, 2002. On the Aetiology of Aseptic Loosening in Joint Arthroplasties and Routes to Improved cemented Fixation.

Thesis defended 14.6.2002. External examiner: Professor N. Dahlén. 
29. Christer Slotte CCS, 2003. On Surgical Techniques to Increase Bone Density and Volume. Studies in Rat and Rabbit.

Thesis defended 13.6.2003. External examiner: Professor C.H.F. Hämmerle.

30. Anna Arvidsson MSc, 2003. On Surface Mediated Interactions Related to Chemomechanical Caries Removal. Effects on surrounding tissues and materials.

Thesis defended 28.11.2003. External examiner: Professor P. Tengvall.

31. Pia Bolind DDS, 2004. On 606 retrieved oral and craniofacial implants. An analysis of consequently received human specimens.

Thesis defended 17.12.2004. External examiner: Professor A. Piattelli.

32. Patricia Miranda Burgos DDS, 2006. On the influence of micro- and macroscopic surface modifications on bone integration of titanium implants.

Thesis defended 1.9.2006. External examiner: Professor A. Piattelli.

33. Jonas P. Becktor DDS, 2006. On factors influencing the outcome of various techniques using endosseous implants for reconstruction of the atrophic edentulous and partially dentate maxilla.

Thesis defended 17.11.2006. External examiner: Professor K.F. Moos.

34. Anna Göransson DDS, 2006. On Possibly Bioactive CP Titanium Surfaces.

Thesis defended 8.12.2006. External examiner: Professor B. Melsen.

35. Andreas Thor DDS, 2006. On platelet-rich plasma in reconstructive dental implant surgery. Thesis defended 8.12.2006. External examiner: Professor E.M. Pinholt. 
36. Luiz Meirelles DDS MSc, 2007. On Nano Size Structures for Enhanced Early Bone Formation.

Thesis defended 13.6.2007. External examiner: Professor Lyndon F. Cooper.

37. Pär-Olov Östman DDS, 2007. On various protocols for direct loading of implant-supported fixed prostheses. Thesis defended 21.12.2007. External examiner: Professor B. Klinge.

38. Kerstin Fischer DDS, 2008. On immediate/early loading of implant supported prostheses in the maxilla.

Thesis defended 8.2.2008. External examiner: Professor K. Arvidsson Fyrberg.

39. Alf Eliasson 2008. On the role of number of fixtures, surgical technique and timing of loading.

Thesis defended 23.5.2008. External examiner: Professor K. Arvidsson Fyrberg.

40. Victoria Fröjd DDS, 2010. On Ca2+ incorporation and nanoporosity of titanium surfaces and the effect on implant performance.

Thesis defended 26.11.2010. External examiner: Professor J.E. Ellingsen.

41. Lory Melin Svanborg DDS, 2011. On the importance of nanometer structures for implant incorporation in bone tissue.

Thesis defended 01.06.2011. External examiner: Associate professor C. Dahlin.

42. Byung-Soo Kang MSc, 2011. On the bone tissue response to surface chemistry modifications of titanium implants. Thesis defended 30.09.2011. External examiner: Professor J. Pan.

43. Kostas Bougas DDS, 2012. On the influence of biochemical coating on implant bone incorporation. Thesis defended 12.12.2012. External examiner: Professor T. Berglundh. 
44. Arne Mordenfeld DDS, 2013. On tissue reaction to and adsorption of bone substitutes.

Thesis defended 29.5.2013. External examiner: Professor C. Dahlin.

45. Ramesh Chowdhary DDS, 2014. On efficacy of implant thread design for bone stimulation.

Thesis defended 21.05.2014. External examiner: Professor Flemming Isidor.

46. Anders Halldin MSc, 2015. On a biomechanical approach to analysis of stability and load bearing capacity of oral implants.

Thesis defended 28.05.2015. External examiner: Professor J. Brunski.

47. Francesca Cecchinato MSc, 2015. On magnesium-modified titanium coatings and magnesium alloys for oral and orthopaedic applications: in vitro investigation.

Thesis defended 20.11.2015. External examiner: Professor C. Stanford.

48. Jonas Anderud DDS, 2016. On guided bone regeneration using ceramic membranes.

Thesis defended 27.05.2016. External examiner: Professor S. Lundgren

49. Silvia Galli DDS, 2016. On magnesium-containing implants for bone applications.

Thesis defended 08.12.2016. External examiner: Professor J.E. Ellingsen.

50. Bruno Chrcanovic DDS MSc, 2017. On Failure of Oral Implants.

Thesis to be defended 08.06.2017. External examiner:

Associate Professor B. Friberg.

see www.mah.se/muep 


\section{TABLE OF CONTENTS}

THESIS AT A GLANCE ........................................ 19

ABSTRACT ................................................. 23

POPULÄRVETENSKAPLIG SAMMANFATTNING .............. 26

LIST OF PAPERS .............................................. 29

ABBREVIATIONS AND ACRONYMS.......................... 31

INTRODUCTION ............................................ 34

OSSEOINTEGRATION ........................................ 37

REPORTED CLINICAL OUTCOME OF

OSSEOINTEGRATED IMPLANTS ................................ 44

RESEARCH METHODOLOGIES

- ADVANTAGES AND DISADVANTAGES AND

IMPACT ON IMPLANT SURVIVAL RESULTS .................... 46

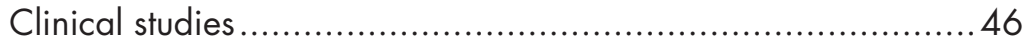

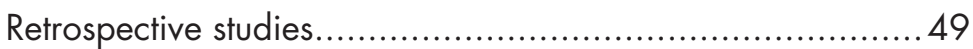

Prospective studies ..................................................... 50

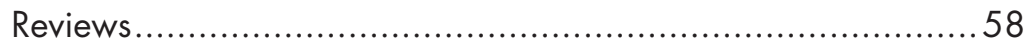

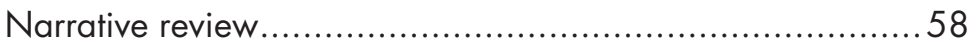

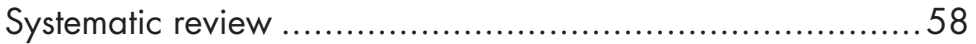

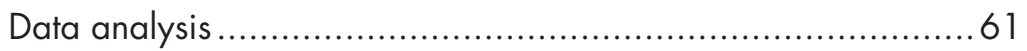

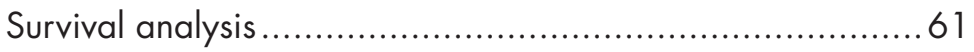

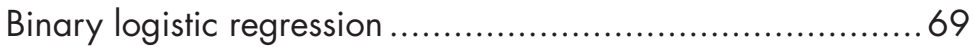

Generalized estimating equation .................................. 71

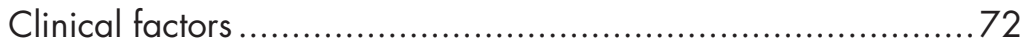

Time of follow-up ..................................................... 72

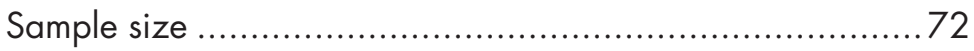

Unaccounted for implants in drop out patients ...................74 
AIMS ......................................................... 77

MATERIALS AND METHODS ................................... 79

RESULTS AND DISCUSSION ................................ 83

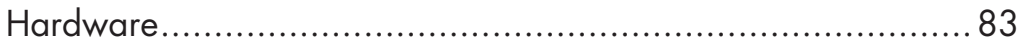

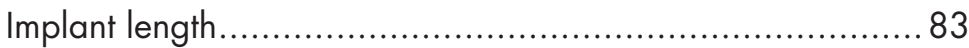

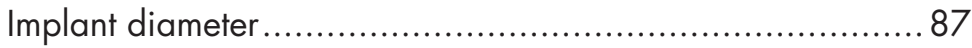

Implant design (non-threaded versus threaded,

cylindrical versus tapered) .......................................... 91

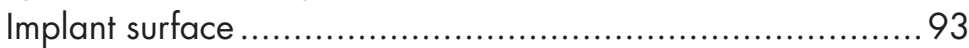

"Copycat" implants and implant systems that were withdrawn due to clinical problems ..................................96 96

Surface contamination .............................................. 99

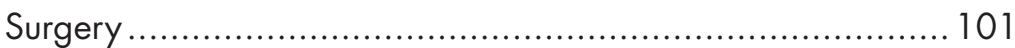

Use of prophylactic antibiotics..................................... 101

Insertion torque......................................................... 102

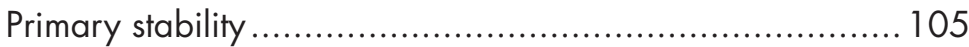

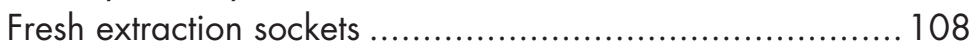

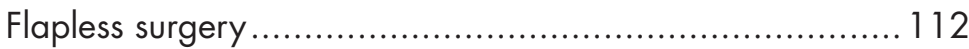

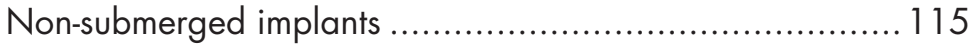

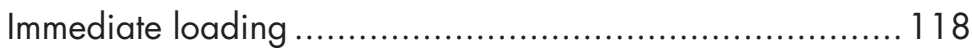

Tilted implants .................................................... 120

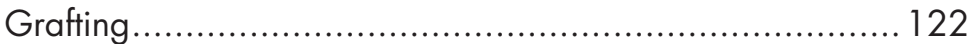

Reoperation ........................................................... 124

Anatomy .............................................................. 126

Location - maxilla and mandible, anterior

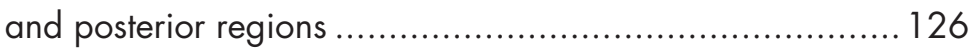

Bone quantity and quality ........................................ 127

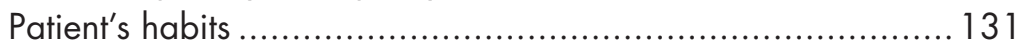

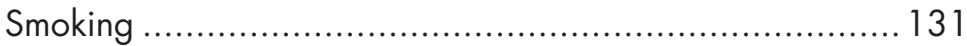

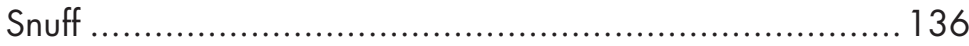

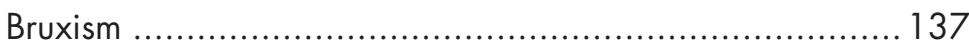

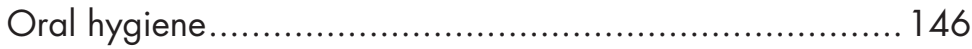

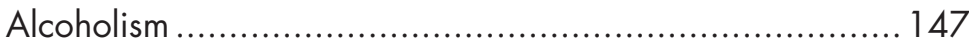

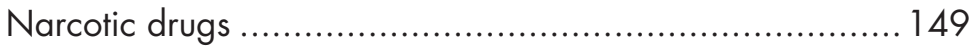

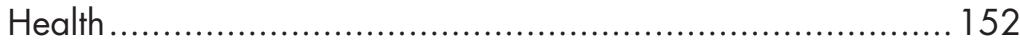

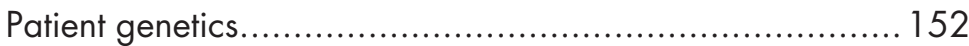

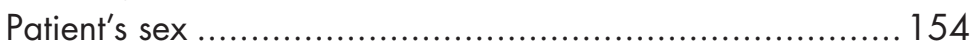

History of periodontitis ............................................ 158 


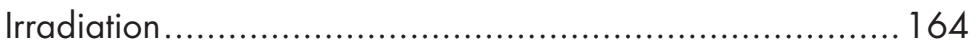

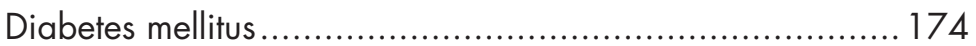

Pharmaceutical reasons for implant problems .................. 178

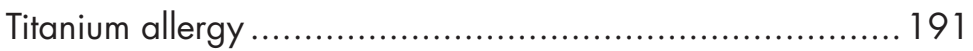

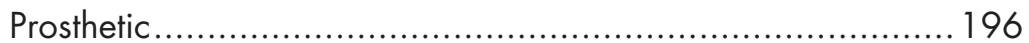

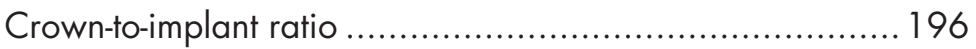

Number of implants per patient .................................. 198

Prosthetic rehabilitation type.......................................... 199

Occlusal versus non-occlusal loading...............................200

Prosthodontist experience/skill/judgment........................201

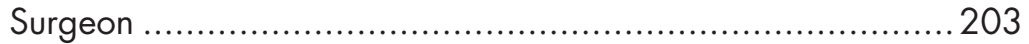

The surgeon's experience ............................................ 203

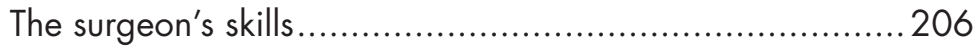

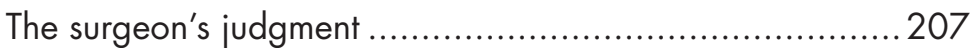

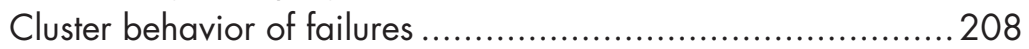

ON REASONS FOR MARGINAL BONE LOSS

AROUND ORAL IMPLANTS ..................................21 1

The traditional theory of reasons for marginal bone loss..........21 1

The immunologically based theory behind marginal bone loss ... 213

FRACTURE OF IMPLANTS ...................................214

CONTRAINDICATIONS FOR IMPLANTS .....................220

CONCLUSIONS ......................................................

ACKNOWLEDGEMENTS .................................23

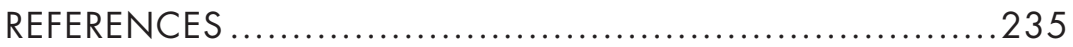

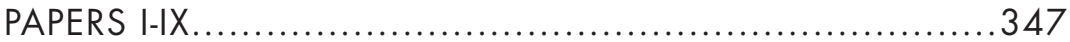





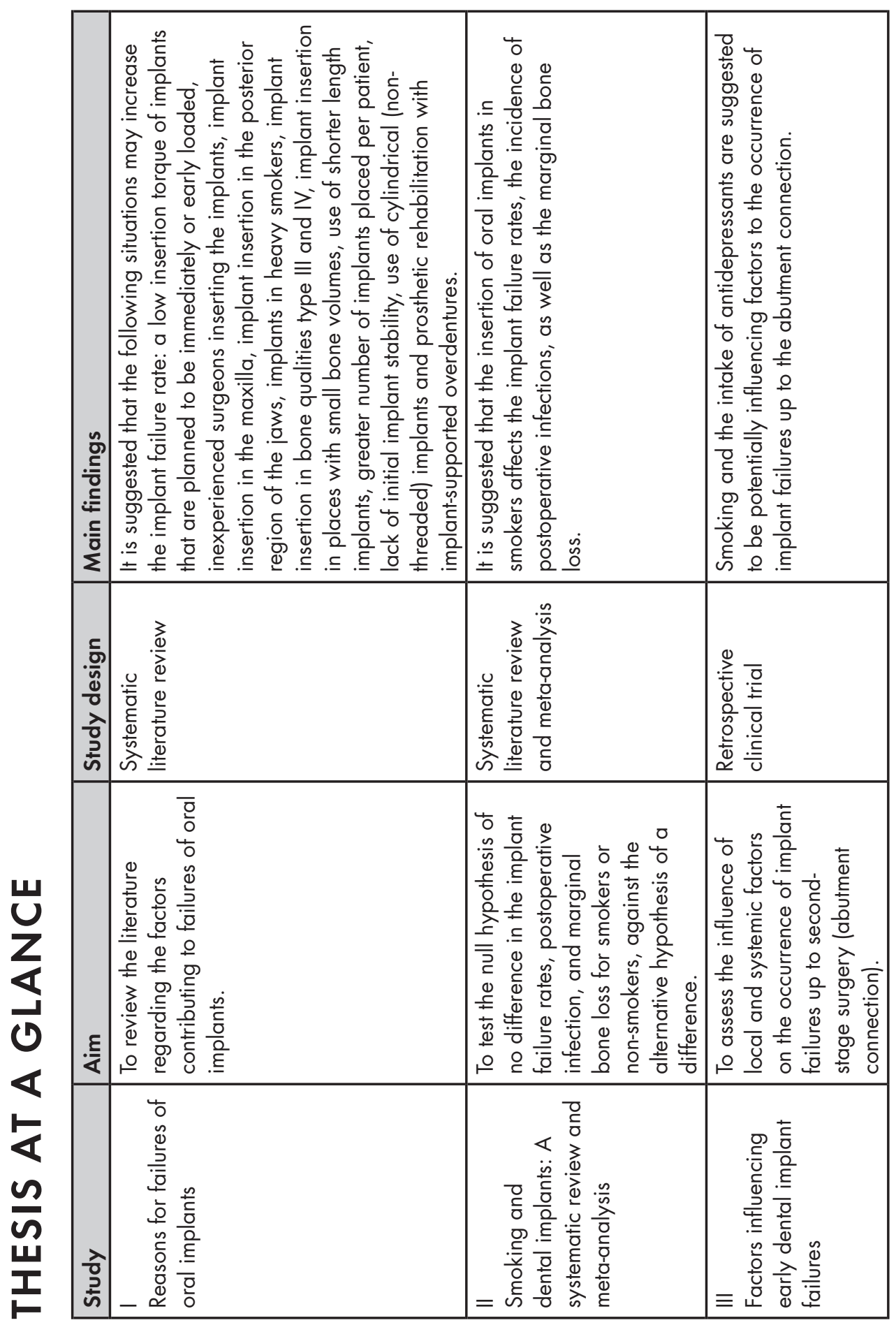




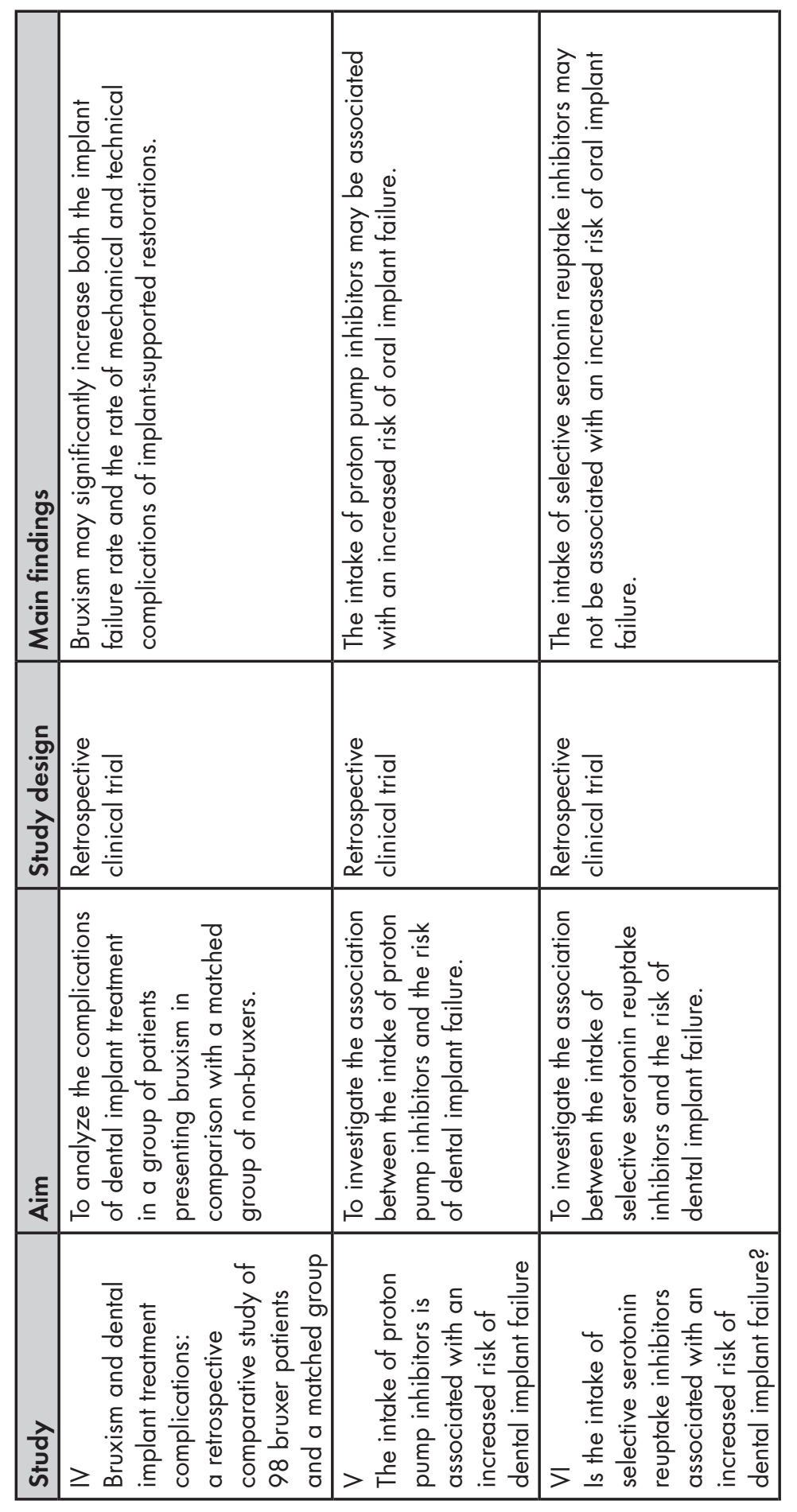




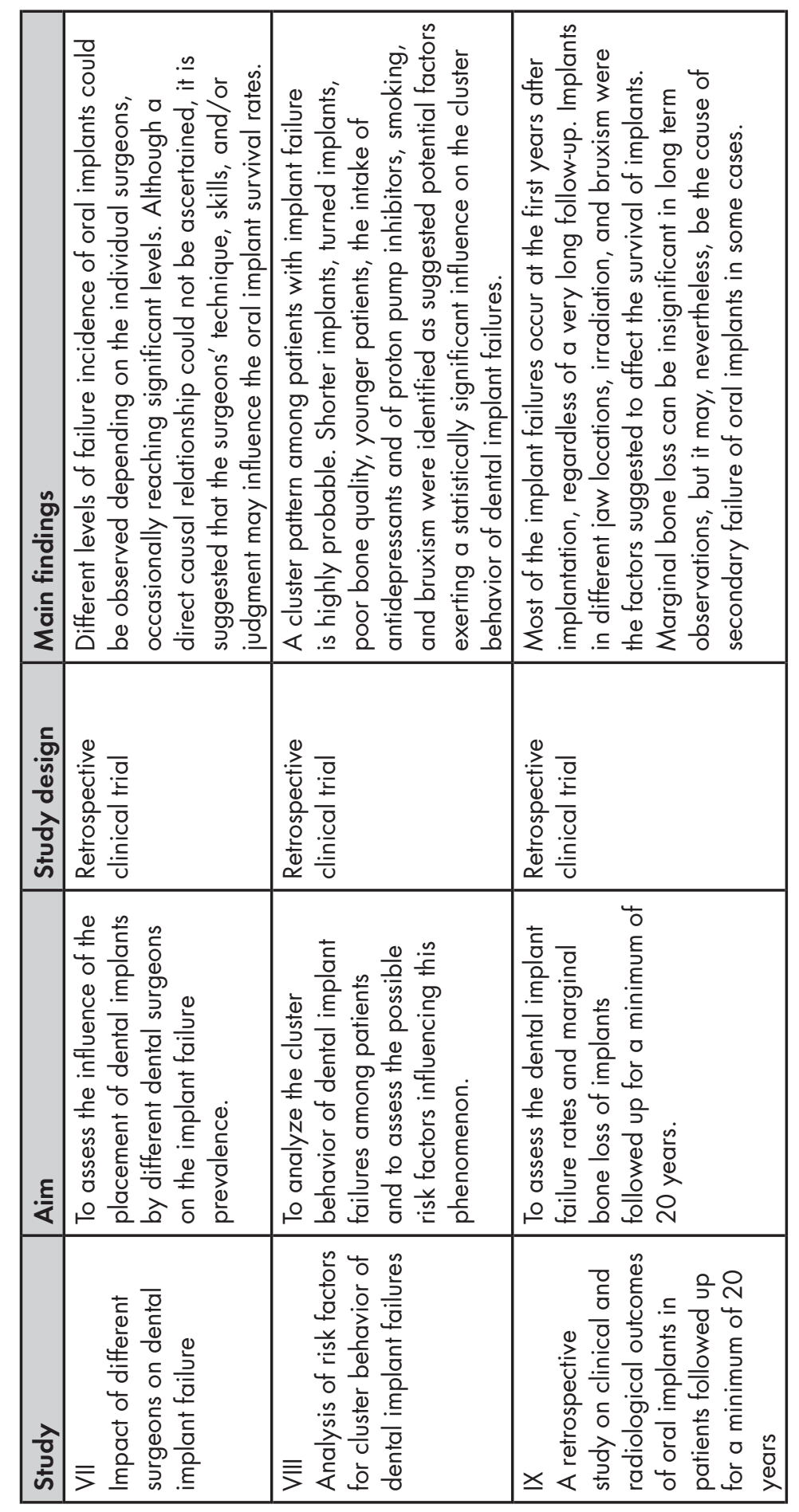





\section{ABSTRACT}

\section{Introduction}

Nowadays oral implant placement is an effective and predictable treatment modality for replacing missing teeth in both fully and partially edentulous patients. Despite the high implant survival and success rates, there is a general appreciation that some risk factors predispose individuals to more complications and implant failures and may result in lower implant survival and success rates. Determining the exact elements that are critical for osseointegration would be extremely useful. A better understanding of the factors associated with implant failure provide data for the planning of future studies, facilitate clinical decision-making, and may enhance implant success. Once identified, the risks can then be avoided, or an alternative intervention can be applied, implying in the least cost to produce a given level of effectiveness in oral rehabilitation. The general aim of the present thesis was to assess the impact of several factors on the failure of oral implants.

\section{Materials and Methods}

The articles included in the present thesis consist of three types of studies. First, a general overview was performed in order to identify risk factors associated with the failure of oral implants (Study I). Then, a systematic review of the literature with meta-analyses was performed to analyze the influence of one risk factor (smoking) on the failure of oral implants, marginal bone loss (MBL), and postoperative infection around implants (Study II). The seven retrospective studies (Study III-IX) were based on all 2,670 patients provided with 10,096 implants, consecutively treated on a routine basis at one specialist 
clinic (Clinic for Prosthodontics, Centre of Dental Specialist Care, Malmö, Sweden - Folktandvården Skåne AB, Specialisttandvård Malmö) during the period from 1980 to 2014. The dental records of all patients ever treated with implants in this clinic were read in order to collect the data. The data were directly entered into a SPSS file (SPSS software, version 23, SPSS Inc., Chicago, USA) as the files were being read. Several anatomical-, patient-, health-, and implant-related factors were collected. The clinical studies included in the thesis focused on the association between implant failure and bruxism, the intake of proton pump inhibitors and of selective serotonin reuptake inhibitors and their relation to failure, the impact of different surgeons, and the number of early failures, cluster behavior of failures, and clinical and radiological outcomes of implants followed up for a minimum of 20 years.

Besides descriptive statistics and tests for the comparison of 2 or of 3 or more independent and dependent groups of continuous and categorical data, survival analyses, logistic regression models, generalized estimating equation method, and multilevel mixed effects parametric survival analysis were performed, depending on the study.

\section{Results and Conclusions}

After a systematic review of the literature, it may be suggested that the following situations may increase the implant failure rate: a low insertion torque of implants that are planned to be immediately or early loaded, inexperienced surgeons inserting the implants, implant insertion in the maxilla, implant insertion in the posterior region of the jaws, implants in heavy smokers, implant insertion in bone qualities type III and IV, implant insertion in places with small bone volumes, use of shorter length implants, greater number of implants placed per patient, lack of initial implant stability, use of cylindrical (non-threaded) implants and prosthetic rehabilitation with implantsupported overdentures. Moreover, it may be suggested that the following situations may be correlated with an increase in the implant failure rate but with a weaker association than the factors listed above: use of the non-submerged technique, immediate loading, implant insertion in fresh extraction sockets, smaller diameter implants. Some recently published studies suggest that modern, moderately rough implants may present with similar results irrespective if placed in 
maxillae, in smoking patients or using only short implants (Study I). The systematic review of the literature with meta-analyses suggested that the insertion of oral implants in smokers affects the implant failure rates, the incidence of postoperative infections, as well as the marginal bone loss (Study II).

Smoking and the intake of antidepressants are suggested to be potentially influencing factors with respect to the occurrence of implant failures up to the second-stage surgery - abutment connection (Study III). Bruxism may significantly increase both the implant failure rate and the rate of mechanical and technical complications of implant-supported restorations (Study IV). The intake of proton pump inhibitors may be associated with an increased risk of oral implant failure (Study V), but not so the intake of selective serotonin reuptake inhibitors (Study VI). Different levels of failure incidence of oral implants could be observed depending on the individual surgeons, occasionally reaching significant levels. Although a direct causal relationship could not be ascertained, it is suggested that the surgeons' technique, skills, and/or judgment may influence the oral implant survival rates (Study VII). A cluster pattern among patients with implant failure is highly probable. Shorter implants, turned implants, poor bone quality, younger patients, the intake of antidepressants and of proton pump inhibitors, smoking, and bruxism were identified as suggested potential factors exerting a statistically significant influence on the cluster behavior of dental implant failures (Study VIII). Most of the implant failures occur at the first years after implantation, regardless of a very long follow-up. Implants in different jaw locations, irradiation, and bruxism were the factors suggested to affect the survival of implants. Marginal bone loss can be insignificant in long term observations, but it may, nevertheless, be the cause of secondary failure of oral implants in some cases (Study IX). 


\section{POPULÄRVETENSKAPLIG SAMMANFATTNING}

\section{Introduktion}

Behandling med orala implantat är idag en effektiv och fungerande metod att ersätta bettfunktion vid partiell eller hel tandlöshet. Trots höga överlevnads- och lyckandesiffror finns ändå riskfaktorer som för vissa patienter kan resultera i implantatförlust. Man behöver noggrant utreda olika faktorer som kan påverka benförankringen, osseointegrationen, av implantaten för att ytterligare förbättra lyckandesiffrorna. Om faktorer som leder till ökad implantatförlust kan identifieras, skulle det underlätta för såväl tandläkare som för patienten genom att man då får möjlighet att undvika eller kompensera för individuella risker. Den här avhandlingen har haft som syfte att presentera kända riskfaktorer bakom implantatförluster.

\section{Material och metodik}

Artiklarna som ingår i denna avhandling kan indelas i tre olika sorters studier. Först gjordes en allmän översikt om kända faktorer bakom implantatproblem (arbete I). Därefter utfördes en metaanalys innebärande en genomgång av relevant litteratur för att mer djupgående kunna analysera en selekterad riskfaktor, nämligen att patienter som röker kan påverka såväl benförlust runt implantatet som postoperativ infektionsrisk och implantatförlust.(Arbete II). Därefter utfördes sju retrospektiva (tillbakablickande) studier(Arbeten III-IX) baserat på 10.096 implantat, konsekutivt insatta på tillsammans 2670 patienter på en klinik - folktandvården i Skåne, specialisttandvården 
Malmö under tiden 1980 till 2014. Efter godkännande från etisk nämnd har det blivit möjligt att datorbehandla tandläkarjournaler från detta patientmaterial. Data införs på en SPSS fil (SPSS software, version 23, SPSS Inc, Chicago, USA). Anatomiska-, patient-, hälsooch implantatrelaterade faktorer registrerades. De kliniska studier som ingår $\mathrm{i}$ avhandlingen har fokuserats kring frågeställningar som implantatförlust och bruxism(tandgnissling), medikamentell behandling med protonpumpsinhibitorer(magsårsmedicin) eller serotonin(antidepressivt medel), lyckandedata för implantat insatta av olika kirurger, tidiga förluster och $\mathrm{s} k$ klusterbeteende. Klusterbeteende avser att implantatförluster inte är jämnt fördelade bland patienterna, utan att det rör sig främst om ett fåtal patienter som har många misslyckanden. Därtill utfördes röntgenologiska beräkningar av benförlust runt implantat som varit insatta under mer än 20 års tid. Olika statistiska modeller användes för att testa om erhållna data var signifikant skilda åt i jämförelse med relevanta kontroller.

\section{Resultat och Diskussion}

Den systematiskta genomgången av litteraturen antydde att det fanns en serie faktorer som kunde påverka implantatutgången; $t$ ex lågt vridmoment vid implantatinsättningen om man samtidigt avsåg belasta implantaten snabbt; att oerfarna eller dåligt skolade kirurger har sämre kliniskt resultat än mer skolade kollegor; implantatinsättning i överkäken; implantatinsättning i bakre delen av käkarna eller i ben av dålig kvalitet/kvantitet; rökande patienter; användning av korta implantat eller för många implantat; insättning av icke gängade implantat eller användande av s k täckprotes. Därtill fanns antydningar om andra faktorer som möjligen skulle kunna påverka implantatframgången negativt, som att låta implantaten ha direkt genomgång i slemhinnan utan tidigare vilofas, som direktbelastning av implantaten, som insättning av implantat direkt efter tandutdragning, som implantat med liten diameter. En del senare studier antydde att moderna moderat råa implantatytor kunde kompensera för risker med en del faktorer som insättning i överkäken, användande av korta implantat eller vid direktbelastning (Studie I). Den mer systematiska analysen av rökande patienter fann en ökning av implantatförlusterna hos rökare och därtill påverkades 
infektionsrisken efter implantatinsättningen och man fick ökad benförlust runt implantaten när patienten rökte (Studie II).

Rökning kunde verifieras som riskfaktor i den kliniska analysen och även om patienten åt antidepressiva tabletter av serotonintyp, så ökade i vart fall de tidiga implantatförlusterna (Studie III). Patienter som gnisslade tänder hade signifikant ökning av implantatförluster och fick därtill ökning av implantatfrakturer och brott på tandbroar (Studie IV). Resultaten visade också att en vanlig typ av magsårsmedicin; s k proton pumphämmare, resulterade i ökning av antalet implantatförluster (Studie V). Man fann däremot inga säkra tecken på ökad implantatförlust vad gällde sena resultat för de som åt antidepressiva mediciner av serotonintyp (Studie VI). Vidare fanns klara antydningar att vissa kirurger hade fler implantatförluster än andra, något som kan ha att göra med vald kirurgisk teknik, kirurgisk förmåga, kirurgiskt omdöme(vissa tänkta implantatpatienter skall kanske inte operas utan få annan behandling) (Studie VII). Vissa patienter med specifika karaktäristika som pekats på i studie I förlorade klart fler implantat än medelpatienten (Studie VIII). Slutligen noterades en mycket klar övervikt för tidiga implantatförluster under de första två åren efter implantatinsättningen, varefter ytterliga implantatförluster förekom men var långt mer ovanliga. En särskild faktor som antytts ställa till stora kliniska problem, som benförlust runt implantaten, visade sig i verkligheten sällan leda till implantatförlust. De flesta implantat som uppvisade benförlust fungerade trots detta utmärkt kliniskt (Studie IX).

Den övergripande målsättningen med denna avhandling har varit att öka kunskapen om olika faktorer bakom implantatförlust för att på så sätt kunna bidra till ytterligare förbättring av kliniska resultat $i$ framtiden. 


\section{LIST OF PAPERS}

This dissertation is based on the following papers, which will be referred to in the main text by their Roman numerals, except for the Study I, which is a general review. Reference to the papers in the text will not exacly appear in the numerical order shown below. The papers are appended at the end of the thesis.

I. Chrcanovic BR, Albrektsson T, Wennerberg A. Reasons for failures of oral implants. Journal of Oral Rehabilitation 2014 Jun;41(6):443-476.

II. Chrcanovic BR, Albrektsson T, Wennerberg A. Smoking and dental implants: A systematic review and meta-analysis. Journal of Dentistry 2015 May;43(5):487-498.

III. Chrcanovic BR, Kisch J, Albrektsson T, Wennerberg A. Factors influencing early dental implant failures. Journal of Dental Research. 2016 Aug;95(9):995-1002.

IV. Chrcanovic BR, Kisch J, Albrektsson T, Wennerberg A. Bruxism and dental implant treatment complications: a retrospective comparative study of 98 bruxer patients and a matched group. Clinical Oral Implants Research. 2016. In press.

V. Chrcanovic BR, Kisch J, Albrektsson T, Wennerberg A. The intake of proton pump inhibitors is associated with an increased risk of dental implant failure. International Journal of Oral and Maxillofacial Implants 2016. Accepted for publication. 
VI. Chrcanovic BR, Kisch J, Albrektsson T, Wennerberg A. Is the intake of selective serotonin reuptake inhibitors associated with an increased risk of dental implant failure? International Journal of Oral and Maxillofacial Surgery. 2017 Jun;46(6):782-788.

VII. Chrcanovic BR, Kisch J, Albrektsson T, Wennerberg A. Impact of different surgeons on dental implant failure. International Journal of Prosthodontics. 2017. Accepted for publication.

VIII. Chrcanovic BR, Kisch J, Albrektsson T, Wennerberg A. Analysis of risk factors for cluster behavior of dental implant failures. Clinical Implant Dentistry and Related Research. 2017. In press.

IX. Chrcanovic BR, Kisch J, Albrektsson T, Wennerberg A. A retrospective study on clinical and radiological outcomes of oral implants in patients followed up for a minimum of 20 years. 2017. Submitted.

All published and accepted papers were reprinted with permission from the copyright holders.

Contribution by the respondent

The respondent performed most of the work of planning, and he performed data collection and analysis of the data. The respondent was also the main contributor to writing the manuscripts. 


\title{
ABBREVIATIONS AND ACRONYMS
}

\author{
${ }^{\circ} \mathrm{C} \quad$ degree Celsius \\ $95 \% \mathrm{Cl} \quad 95 \%$ confidence interval \\ AB Aktiebolag (Swedish) \\ AG Aktiengesellschaft (German) \\ AIDS Acquired immune deficiency syndrome \\ AMSTAR Assessing the Methodological Quality of Systematic Reviews \\ ANOVA Analysis of variance \\ BIC Bone-to-implant contact \\ BMD Bone mineral density \\ BMI Body mass index \\ BMP-2 Bone morphogenetic protein 2 \\ BP Bisphosphonate \\ BRONJ Bisphosphonate-related osteonecrosis of the jaw \\ c.p. $\mathrm{Ti}$ Commercially pure titanium \\ $C / I$ ratio Crown-to-implant ratio \\ CCB Calcium-channel blocker \\ CCT Controlled clinical trial \\ CNC Computer Numerical Control \\ CsA Cyclosporin A \\ CSR Cumulative survival rate \\ CT Computed tomography \\ e.g. exempli gratia (Latin) \\ EU European Union \\ FEA Finite element analysis \\ g gram
}




\begin{tabular}{|c|c|}
\hline GEE & Generalized estimating equation \\
\hline Gy & Gray \\
\hline HA-coated & Hydroxyapatite-coated \\
\hline $\mathrm{HbAlc}$ & Glycosylated hemoglobin \\
\hline $\mathrm{HBO}$ & Hyperbaric oxygen \\
\hline HIF-1 $\alpha$ & Hypoxia-inducible factor 1-alpha \\
\hline HIV & Human immunodeficiency virus \\
\hline$H R$ & Hazard ratio \\
\hline i.e. & id est (Latin) \\
\hline |L-1 & Interleukin 1 \\
\hline $\mid \mathrm{L}-1 \alpha$ & Interleukin 1 alpha \\
\hline IL-1 $\beta$ & Interleukin 1 beta \\
\hline IL-6 & Interleukin 6 \\
\hline |L-8 & Interleukin 8 \\
\hline ISQ & Implant stability quotient \\
\hline KM & Kaplan-Meier \\
\hline LTT & Lymphocyte transformation test \\
\hline MBF & Maximal bite force \\
\hline MBL & Marginal bone loss \\
\hline MD & Mean difference \\
\hline MDMA & 3,4-methylenedioxymetamphetamine \\
\hline MELISA & Memory Lymphocyte Immunostimulation Assay \\
\hline $\mathrm{mg}$ & milligram \\
\hline MIP-1 $1 \alpha$ & Macrophage inflammatory protein-1 alpha \\
\hline MIT & Maximum insertion torque \\
\hline $\mathrm{mm}$ & millimeter \\
\hline MMP & Matrix metalloprotenase \\
\hline MRT & Maximum removal torque \\
\hline MSC & Mesenchymal stem cell \\
\hline $\mathrm{NCm}$ & Newton centimeter \\
\hline $\mathrm{nm}$ & nanometer \\
\hline $\mathrm{O}_{2}$ & Oxygen (dioxygen) \\
\hline OR & Odds ratio \\
\hline PCP & Periodontally compromised patient \\
\hline PDGF-AA & Platelet-derived growth factor-AA \\
\hline
\end{tabular}




$\begin{array}{ll}\text { PEEK } & \text { Polyether ether ketone } \\ \text { PGE2 } & \text { Prostaglandin E2 } \\ \text { PHP } & \text { Periodontally healthy patient } \\ \text { PICF } & \text { Peri-implant crevicular fluid } \\ \text { ppb } & \text { parts per billion } \\ \text { PPI } & \text { Proton pump inhibitor } \\ \text { Ppm } & \text { parts per million } \\ \text { PRISMA } & \text { Preferred Reporting ltems for Systematic Reviews } \\ \text { PTH } & \text { and Meta-Analyses } \\ \text { QCT } & \text { Parathyroid hormone } \\ \text { RCT } & \text { Quantitative computed tomography } \\ \text { RFA } & \text { Randomized clinical trial } \\ \text { RR } & \text { Resonance frequency analysis } \\ \text { SD } & \text { Risk ratio } \\ \text { SEM } & \text { Standard deviation } \\ \text { SPSS } & \text { Scanning electron microscopy } \\ \text { SPT } & \text { Statistical Package for the Social Sciences } \\ \text { SSRI } & \text { Supportive periodontal treatment } \\ \text { TGF- } \beta 1 & \text { Selective serotonin reuptake inhibitor } \\ \text { TiO } 2 & \text { Transforming growth factor-beta 1 } \\ \text { TNF- } \alpha & \text { Titanium dioxide } \\ \text { VEGF } & \text { Tumor necrosis factor alpha } \\ \mu m & \text { Micrometer } \\ & \end{array}$




\section{INTRODUCTION}

Failures of oral implants are much less commonly described in the literature than are surviving and successful cases. One reason for this may certainly be that implants fare very well, provided documented oral implant systems are placed by trained individuals. Since the great majority of published papers indeed are authored by implant scholars, this means that failures are few, typically in the range of $<5 \%$ over 10 years with modern, documented implants. ${ }^{1}$ Another reason why failures are being discussed much less often than are successful cases may relate to our inborn wish to remain positive. Indeed, no clinic is particularly promoted if mainly failed implants are being discussed.

But implant failures nevertheless occur. One way of categorizing failures would be to separate early (primary) failures from secondary ones. Some implants simply never osseointegrate; those are the primary failures. They are not easy to explain. Palma-Carrió et al. ${ }^{2}$ presented a review over early failures with few possible conclusions, even if factors such as patient smoking, implant posterior location or poor bone quality or quantity seemed to be of some relevance. Sakka et al. ${ }^{3}$ listed a lack of primary stability, surgical trauma and infection as reasons for early failures. An odd reason for early oral implant failure in form of low serum levels of vitamin D was analyzed by Mangano et al., ${ }^{4}$ however without finding clear statistics incriminating low vitamin D levels. Secondary failures, i.e. implants that have lost a previously established osseointegration, were explained by the sum of the trauma due to patient smoking, patient genetic factors, poor surgery, adverse loading, excess cement or intake of certain medicines all leading to an immunologically derived shifted balance between 
bone formation and bone resorption in favor of the latter. ${ }^{5}$ Sakka et al. ${ }^{3}$ believed in occlusal overload, peri-implantitis, suboptimal implant designs and improper prosthetic constructions to explain later implant failure.

A lot has been written about an alleged disease entitled periimplantitis that supposedly hit implants in a similar way as teeth suffer from periodontitis. Clearly inflated definitions of such a disease have resulted in claims that up to $50 \%$ of all placed implants suffer from it (for review see Qian et al. ${ }^{6}$ ). These theories are seemingly impossible to couple to the clinical reality and, indeed, Jemt et al. ${ }^{7}$ who followed up a group of allegedly sick implants 9 years after their preliminary diagnosis reported that clearly more than $90 \%$ of them seemed to function quite well with no indications at all of an imminent failure.

Chee and Jivray ${ }^{8}$ said that most implant failures can be classified into four categories: (1) loss of osseointegration, (2) positional failures, (3) soft tissue defects, and (4) biomechanical failures. Hadi and co-workers ${ }^{9}$ listed a greater number of factors contributing to implant failure, namely the medical status of the patient, patient smoking, bone quality, bone grafting, irradiation, bacterial contamination, lack of preoperative antibiotics, degree of surgical trauma and operator experience. In addition, the authors listed implant surface properties, roughness and premature loading to be associated with failing patterns.

Another way of categorizing implant failure, but from the patient perspective, was suggested by Penchas. ${ }^{10}$ Patient pain, swelling or inflammation or a general feeling that the implant is loose were summarized as patient factors.

This wide spectrum of factors believed to be associated with oral implant failure in the literature was the background to the present study. Although there were many viewpoints on reasons for failure that had been suggested in the literature, a major study devoted only to reasons for oral implant failures seemed to be missing in the literature. In a series of meta-analyses or reviews the author behind this thesis started by summing up the literature. ${ }^{11-31}$ However, summarizing the literature only, of which two papers ${ }^{11,20}$ have been inserted in this thesis, is far from satisfactory in an attempt to cover the important topic of implant failure. The applicant, therefore, 
decided to go through a consecutive series of 10,096 implants placed at one Malmö clinic since 1980. Every clinical record available from no fewer than 2,670 patients was carefully read and data were computerized. To the knowledge of the present author, thanks to the computerization of all data in dozens and dozens of independent variables for each and every implant, this may be the largest implant material ever possible to analyze in great detail, even if I am certainly aware that the Brånemark clinic in Gothenburg has access to about 40,000 implants in their clinical patient records. My major motivation behind this thesis is to present an attempt to learn more about oral implant failures to encompass even further increase of implant success rates for the future. 


\section{OSSEOINTEGRATION}

Oral implants of the past were anchored in soft tissues and had one thing in common; they showed an intolerably poor clinical outcome. No decent University had oral implants on their curriculum. Early attempts to place implant with a direct bone anchorage had failed. ${ }^{32}$

Per-Ingvar Brånemark (Figure 1) of the Gothenburg University in Sweden discovered in 1962 what was later referred to as osseointegration, a direct bone anchorage of titanium implants (Figure 2 ), whilst working with experimental implants in rabbits. Brånemark intended to use his implants for visualization of the microcirculation, but noticed at the termination of his experiment that the implants were most difficult to remove from the bone (Figure 3). Only 3 years later, in 1965, Brånemark operated his first patient with oral implants (Figure 4). His ideas were initially not very well received by dentistry, but this was to change in Scandinavia in 1977 and internationally in 1982. There were different definitions offered of osseointegration; one of them claimed "a structural and functional connection between ordered, living bone and the surface of a load carrying implants". ${ }^{33}$ Another early definition suggested that osseointegration was "a process whereby clinically asymptomatic rigid fixation of alloplastic material is achieved and maintained in bone during functional loading". ${ }^{34}$ Six factors were found important to control to achieve reliable osseointegration; implant biocompatibility, design and surface features, status of the host bed, surgical technique and what was referred to as "loading conditions". ${ }^{35}$ In case one or several of those parameters were not controlled, implantation resulted in spit out of the implant, i.e. a primary failure (Figure 5). 


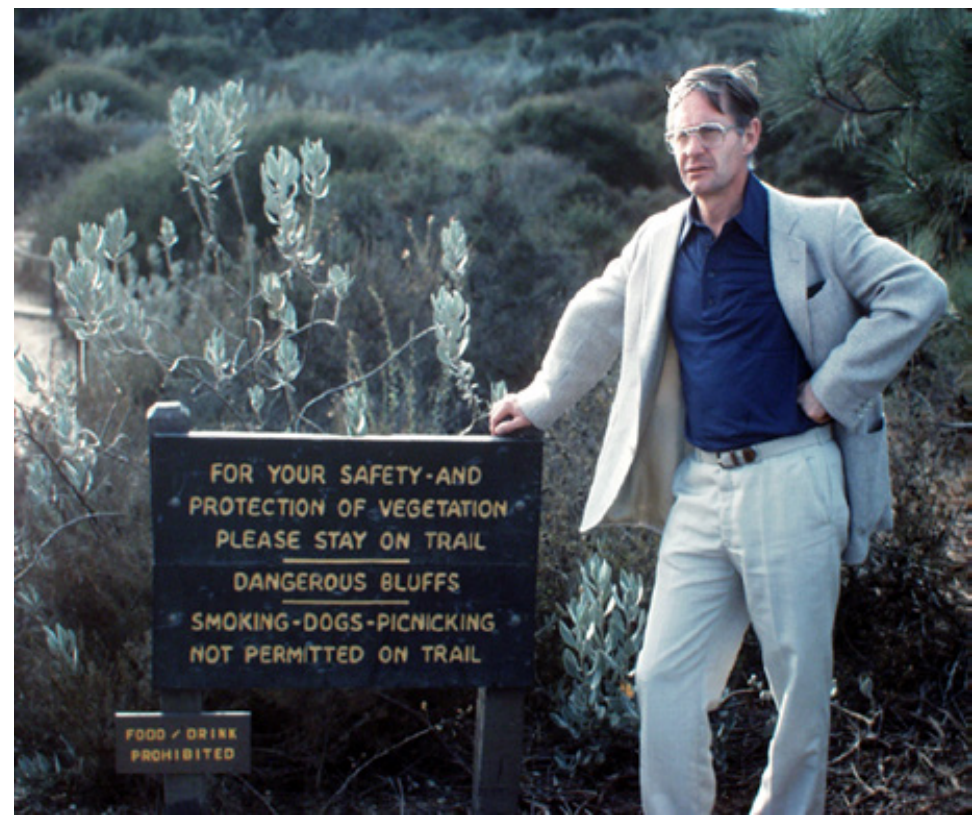

Figure 1. Per-Ingvar Brånemark. La Jolla, California, 1981.

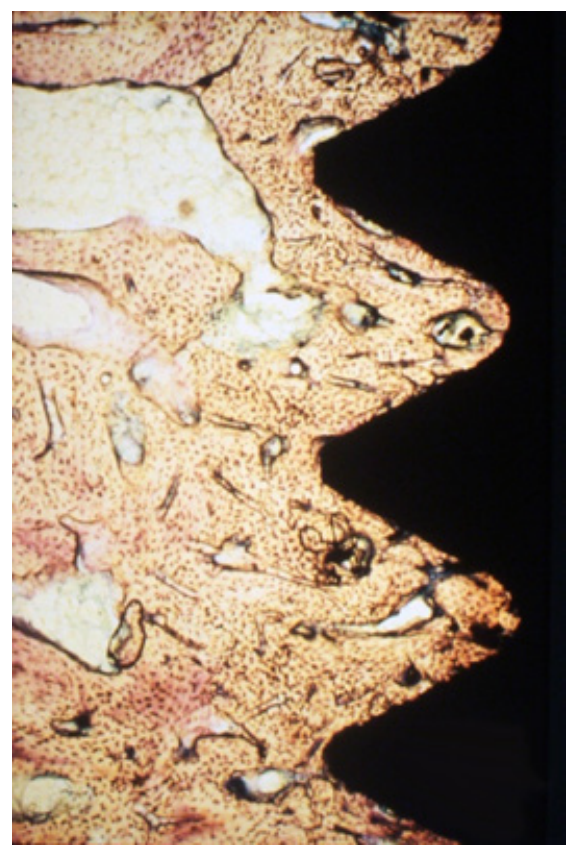

Figure 2. Osseointegration represents a direct contact (at the light microscopic level of resolution) between bone and implant. 


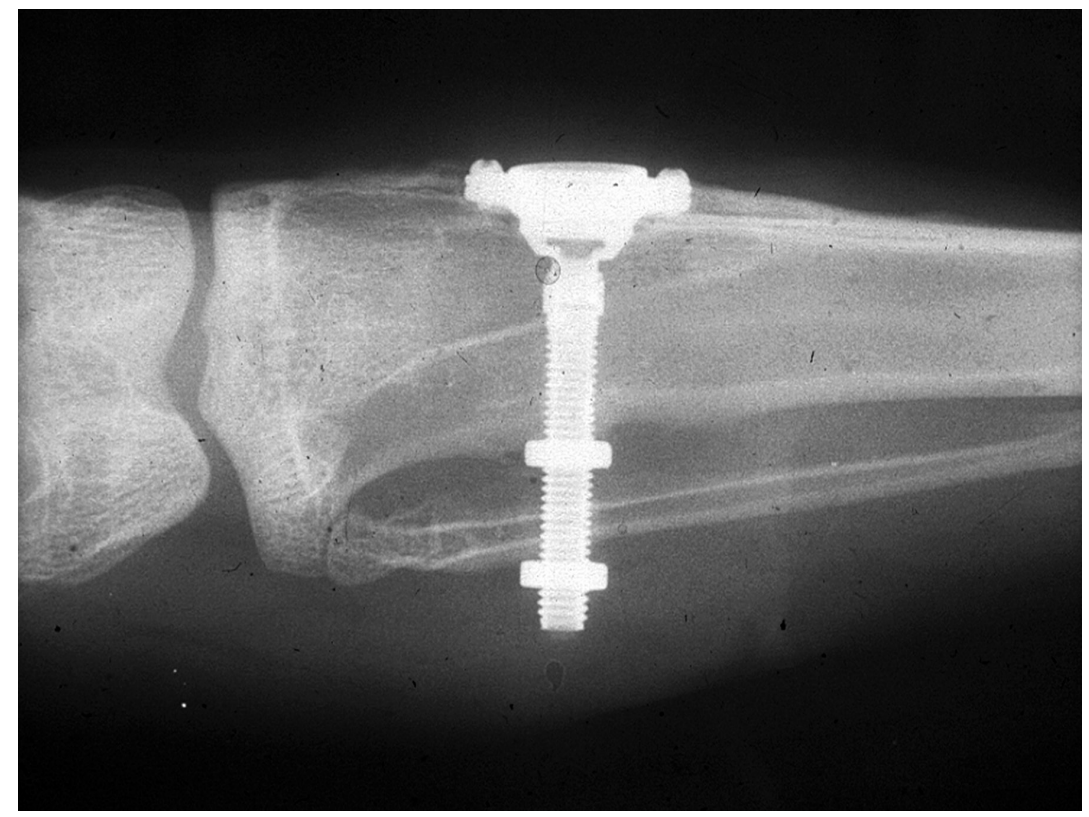

Figure 3. This is a radiogram of the original chamber, placed in rabbit bone, that led to the discovery of osseointegration in 1962.
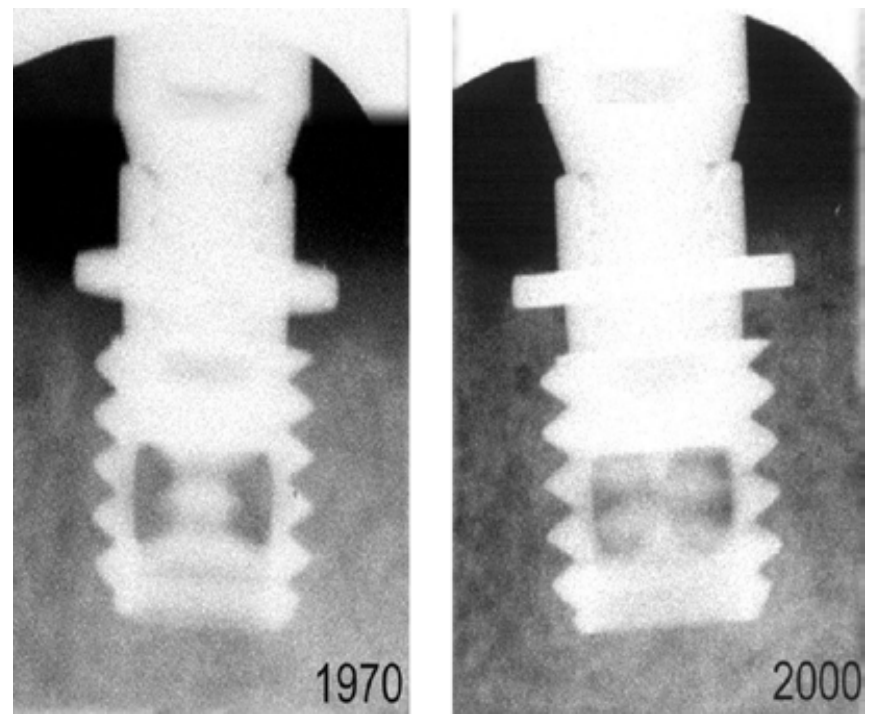

Figure 4. One very early implant design with flanges used by P-I Brånemark and his team between 1965 and 1970. The radiogram to the right is a 30 -year control. 


\section{Implant Placement}

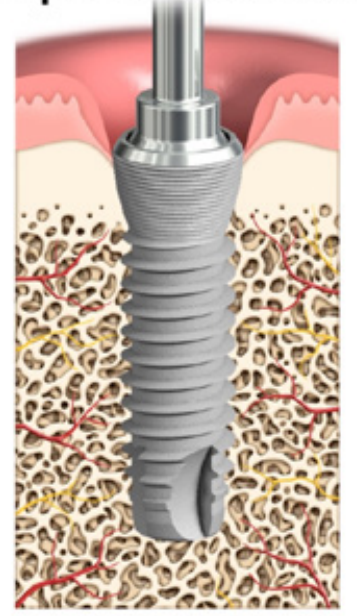

Rejection

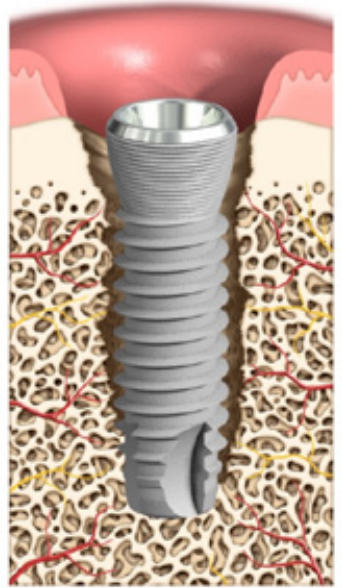

Figure 5. Primary failure of an implant (from Albrektsson et al. ${ }^{44}$ Published with permission Periodontology 2000).

Osseointegration meant an enormous breakthrough for the discipline of jaw reconstruction. Today it is estimated that somewhat between 15 and 20 million oral implants are placed annually in the world. Long term clinical success rates of more than $90 \%$ have been reported in the literature. ${ }^{36}$ The present thesis is mainly built on a consecutive series of 10,096 osseointegrated implants placed at one Malmö clinic since 1980. This controlled material of oral implants is probably the second largest in the world, second only to the implants placed at the Brånemark clinic of Gothenburg where 39,077 oral implants have been followed up and, to part, have had clinical results analyzed under varying conditions. ${ }^{37}$ Clinical records of the entire Malmö material have been computerized in several independent variables, which is an advantage that enables simple long-term analysis of the patient material.

With time the definition of and outlook on osseointegrated implants changed; whereas in the early days titanium implants were regarded to only result in a wound healing phenomenon ${ }^{38,39}$ due to their superb biocompatibility, the general viewpoint today is that titanium does represent a foreign body and is handled so by the host tissues. ${ }^{40-42}$ The scenario is such that titanium is regarded as a 
foreign body by the defense mechanisms and the response is to form bone around the titanium to protect the body tissues from it. This is indeed splendid for clinical dentistry since the newly formed bone will improve loading of the implant. Our suggestion for a new definition of osseointegration would be: "Osseointegration is a foreign body reaction where interfacial bone is formed as a defense reaction to shield off the implant from the tissues" (Figures 6 and 7). ${ }^{43}$

\section{Foreign body Encapsulated reaction \\ Foreign body}

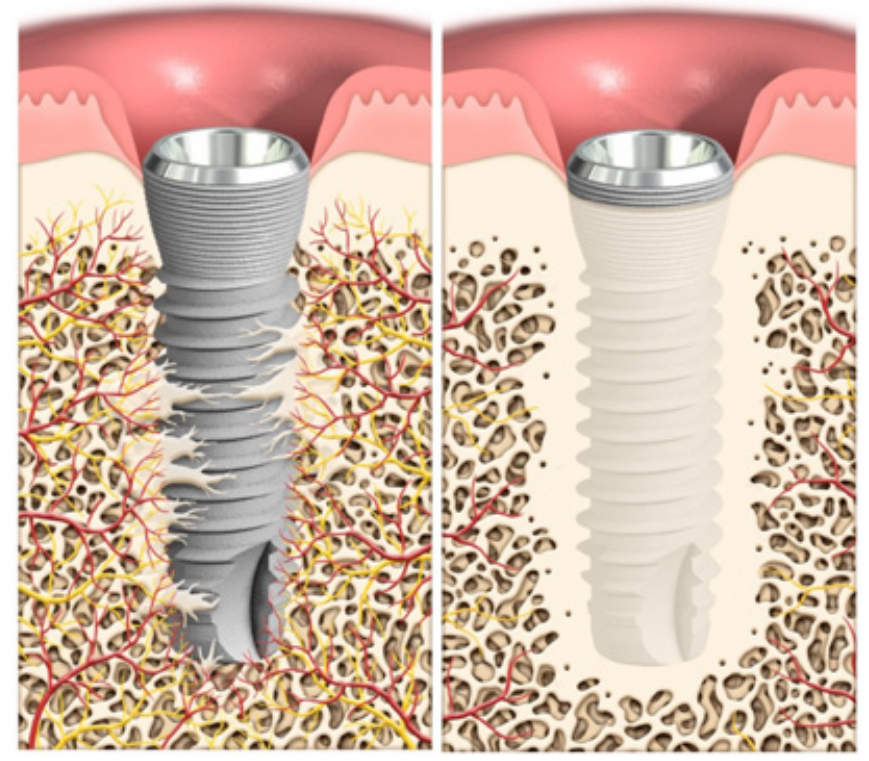

Figure 6. C.p. titanium is seen as a foreign body and the organism protects itself by forming bone around titanium, a fact we can use clinically to load our implants (from Albrektsson et al. ${ }^{44}$ Published with permission Periodontology 2000). 


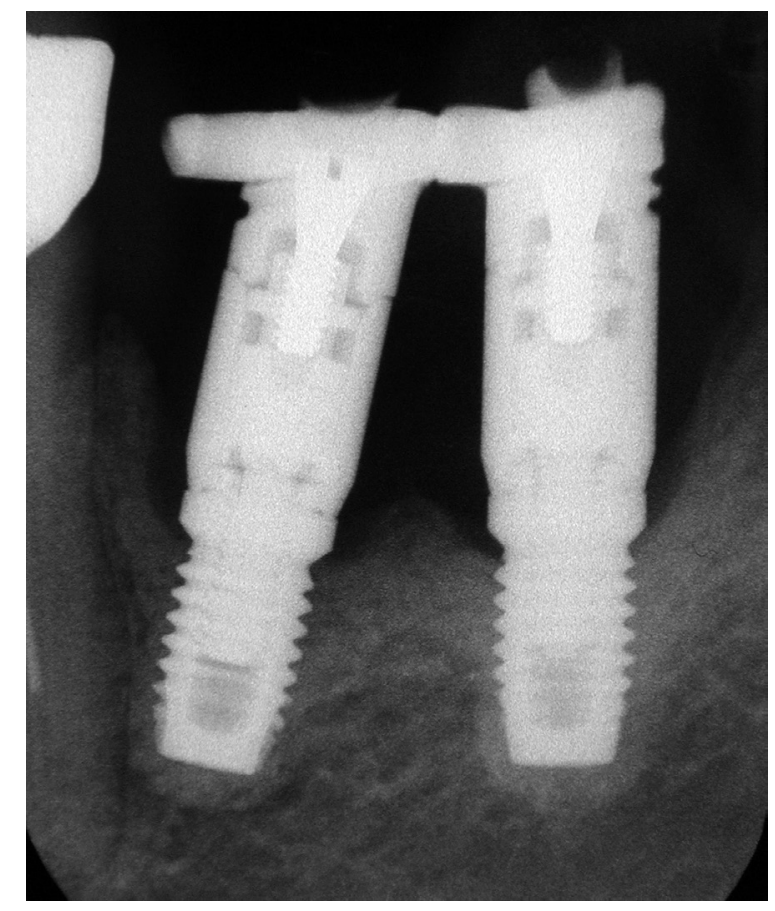

Figure 7. Two clinical implants where bone condensation representing foreign body bone is visualized.

The great majority of oral implants today are manufactured from commercially pure titanium (c.p. Ti). Despite this material presenting a foreign body reaction, it works very well with case reports of clinical function of up to 50 years. ${ }^{44}$ Alternative materials for oral implants have been hydroxyapatite coated titanium, titanium alloys and zirconia. All these materials are biocompatible despite handled as foreign elements by the defense mechanisms of the body. However, hydroxyapatite-coated (HA-coated) implants of the first generation failed and caused bone resorption, possibly due to the thick coats of hydroxyl-apatite that were applied. ${ }^{45}$ Titanium-6Aluminum4Vanadium alloys have been used in relatively small numbers in oral implantology with one clinical report claiming $83.3 \%$ success and $93.4 \%$ survival over 5 years of follow-up. ${ }^{46}$ Other titanium alloy implants containing zirconia have, to the knowledge of the authors, not yet been followed up for 5 years. The same is true for zirconia implants ${ }^{47}$ and zirconia has the additional problem of increasing 
brittleness due to ageing in the human body, even if it is unknown whether we will see an increasing number of fractures of the implant over time. In the present thesis, all 10,096 implants investigated were manufactured from c.p. Ti. 


\section{REPORTED CLINICAL OUTCOME OF OSSEOINTEGRATED IMPLANTS}

Brånemark discovered the process of osseointegration in the 1960s and placed the first dental implant in a patient about 50 years ago. ${ }^{48}$ Since then dental implants became an increasingly viable alternative to conventional prosthodontic treatment options, supported by evidenced-based data. Thousands of clinical studies on dental implants were performed and published. Most studies present data on implant and prosthesis survival, as well as on bone response adjacent to the implants. However, many of the studies are on a relatively short- or medium-term basis. ${ }^{49}$ There is an increase in the number of dental implants being placed in the world annually and follow-up is essential to determine and predict a future clinical course. The patients receiving implants expect to keep them for years and years, and therefore it is more reliable to have a basis for dental implants prognosis with long follow-up studies.

One of the first studies to report at least 10 years of follow-up of dental implants was the one published by Brånemark et al. ${ }^{50}$ At the time, the Brånemark implant (Nobel Biocare $\mathrm{AB}$, Göteborg, Sweden), the so-called turned or machined original Brånemark implant, was one of few available ones in the market. The study ${ }^{50}$ reported a survival rate of $78.6 \%$ for implants placed in the maxilla and $92.6 \%$ for implants installed in the mandible. Several studies of at least 10 years of follow-up assessing the survival rates of the turned Brånemark implants followed, with almost invariably showing higher survival rates for implants placed in the mandible (usually around 95\%) than for implants placed in the maxilla (usually around 90\%). ${ }^{49,51-62}$ More 
recently, Chrcanovic et al. ${ }^{63}$ (Study IX) assessed the implant failure rates of 1045 implants (98\% of them Nobel turned implants) and marginal bone loss (MBL) of 300 of these implants, all followed up for a mean \pm SD of $24.25 \pm 2.8$ years (min-max, 20.3-36.5). It was observed a failure rate of $18.4 \%$ for implants placed in maxillae and $7.1 \%$ for implants placed in mandibles. The study observed that $59.3 \%$ the implant failures occurred at the three first years after implantation, regardless of a very long follow-up.

Later, the Nobel Biocare launched implants with an oxidized surface, the so-called TiUnite. Studies of at least 10 years of follow-up with TiUnite implants ${ }^{64-66}$ showed improved survival rates than the long-term studies with the turned Brånemark implants, sometimes reaching $100 \%$ of survival. Other modern implants presented similar positive long-term results, as the case of the SLA implants (Straumann AG, Basel, Switzerland), ${ }^{67-75}$ TiOblast implants (Astra Tech AB, Mölndal, Sweden), ${ }^{76-83}$ Osseotite implants (Zimmer Biomet Dental, Warsaw, USA), ${ }^{84,85}$ and XiVE implants (Dentsply, York, USA). ${ }^{86}$

In general, modern oral implants have displayed better clinical results than previously used devices, at least in maxillary cases or when challenging factors apply such as patient smoking, direct or rapid loading, the use of short implants, irradiation to tissues, or bone grafting. ${ }^{1}$ After all, a clinical outcome of $95 \%$ or more of modern implants when followed up for ten years or more ${ }^{44}$ suggests most good a clinical outcome. However, as has been pointed out in the past, ${ }^{1}$ this good an outcome may be limited to properly trained clinicians placing controlled implant types and cannot automatically be considered valid in all cases. 


\section{RESEARCH METHODOLOGIES \\ - ADVANTAGES AND \\ DISADVANTAGES AND IMPACT ON IMPLANT SURVIVAL RESULTS}

Research plays a vital role within biomedicine. Scientifically appropriate research provides a basis for appropriate medical decisions; conversely, inappropriate research may lead to flawed 'best medical practices' which, when followed, contribute to avoidable morbidity and mortality. ${ }^{87}$ Informed decision making requires health professionals to combine their own clinical expertise and training with high quality scientific evidence. New scientific knowledge is emerging all the time and all health workers need to update their knowledge continuously. ${ }^{88}$ Despite the advent of evidence-based medicine, many research methods have become 'standard' even though there are legitimate scientific reasons to question the conclusions reached by such methods. ${ }^{87}$

In the present chapter some research methodologies are discussed, pointing out their possible advantages and limitations, as well as a discussion on some already established and other more recent statistical methods used to evaluate dental implant survival and/or failure. Moreover, the impact of some clinical factors on the study results is examined.

\section{Clinical studies}

Study designs can be categorized as observational or interventional. Observational studies, in which the investigator observes events 
without attempting to influence them, are done to establish the frequency of a disease or characteristic and/or to compare two groups. Following a population over a period of time, and then analyzing the differences between those who do or do not get a specific disease, or the differences between patients who lose or did not lose dental implants, are examples of observational studies. Observational studies can identify an association between two variables. However, they cannot establish a causal link. ${ }^{89}$

Types of observational studies include case-control, cohort, and cross-sectional investifations. ${ }^{89}$ A case-control study is superior to a case series because of the presence of a control group. Cases with and without the condition of interest are identified. The degree of exposure to a possible risk factor is then compared between the 2 groups. The case-control study design assumes that (1) cases differ from controls only in having the disease, (2) exposure should be equally distributed between cases and controls if the exposure does not cause the disease, and (3) greater exposure among cases would indicate that exposure increases the risk of the disease. The exposure is determined retrospectively. Ideally, the data collectors are unaware of whether a subject is a case or a control, and the data collectors should be unaware of the study hypothesis. The cases and the controls must be assessed for exposure in the same way. ${ }^{90}$ There are both strengths and weaknesses in case-control studies. The strengths include: a retrospective study has fewer constraints by the frequency of the disease and it is easier to assess conditions where there is a long latency between exposure and disease, and thus allows study of rare occurrences, it has shorter waiting time than a prospective cohort study, it is sometimes feasible when an randomized clinical trial (RCT) is not and, as it uses existing records, it costs less and has fewer practical restrictions. The weaknesses include: a less well defined target population; risk of selection bias; and it is difficult or impossible to ascertain cause-and-effect, because of confounding factors. ${ }^{90}$ With a matched case-control study design, the control subjects are selected so they resemble (match) the cases with regard to certain characteristics (e.g., age, comorbidity, severity of disease). The goal is to compare case and control patients who have similar characteristics and thereby to adjust for potential confounders and increase the precision of the comparison..$^{90}$ 
In a cross-sectional study, the study sample is selected (e.g., the population treated in a clinic), and the outcome variable (e.g., dental implant failure) and the predictor variable (smoking) are measured simultaneously. Because the data are collected at one point in time, the investigator has no control over extraneous factors. In contrast to a cohort study, there is no follow-up period in a cross-sectional study. Cross-sectional studies are effective in determining the prevalence of a disease (the proportion of the population that has a disease at a particular point in time) and the coexistence of associated variables. They do not give us information about disease/outcome incidence (the proportion of the population that gets an illness or a failed dental implant over a specified period of time). ${ }^{89}$

Cohort studies can also be used to establish the frequency of a disease or event. A cohort is a group of patients with a specific characteristic (e.g., patients being rehabilitated with dental implants). An investigator may want to answer the question, "Do patients who smoke present dental implant losses more often than patients who do not smoke?" Using a retrospective cohort study design, the investigator might look at all dental records of the cohort of patients who lost an implant (the outcome variable), and match these records with the cohort of patients without any dental implant lost. The investigator can then review the records to see how often the presence of smoking habits (the predictor variable) was documented in these two cohorts. The retrospective nature of this study design means the investigator is dependent on documentation of a dental implant failure to properly classify patients. Cohort studies are particularly effective at describing the incidence of disease and analyzing associations between risk factors and outcomes, although it cannot prove that the observed factor was the cause of the outcome. ${ }^{89}$ For example, the result of an observational study may be that heavy smoking and dental implant failure are associated with each other, but the statement cannot be accurately made that heavy smoking causes dental failure, based on the result of an observational study alone.

Interventional studies, also known as clinical or experimental trials, are used to address the question of causation. In this study type the investigator intervenes in the events being studied by manipulating a specific variable, and observing the effect of this manipulation on a 
specified outcome ${ }^{89}$ For example, a clinical trial might be designed to investigate the effects of a drug agent added to a poor bone implant site immediately before implantation on the implant survival. In such a trial, the investigators would intervene in the management of the implant site by the delivery of the drug or an appropriate placebo in order to establish whether the use of the drug would result in an improved osseointegration or clinical outcome.

Observational studies can be subdivided into retrospective and prospective studies. The primary difference between these subdivisions is the timing of the measurement of the outcome variable. In a prospective study, the outcome variable has not yet occurred; the investigator will measure it in the future. The investigator follows the population over time, looking for the presence of the outcome variable. All interventional studies are prospective. ${ }^{89}$

\section{Retrospective studies}

A retrospective study uses existing data that have been recorded for reasons other than research. In health care these are often called "chart reviews" because the data source is the medical record. ${ }^{90}$ A retrospective cohort study is based on a group of individuals who are known to have been subject to a particular exposure or intervention in the past, e.g. work environment, dental implants, and about whom additional information has been collected from existing sources, often medical records, concerning the consequences of the intervention or exposure. Data for a retrospective study thus suffer the limitation that they were originally collected and reported in records but not standardized for a specific research purpose. Retrospective cohort studies are usually less costly than prospective cohort studies and also take less time to complete. However, they are limited to studies of outcomes which have been documented insofar as data are retrievable and reliable. Retrospective cohort studies are especially suitable for studies of rare exposures, or where the latent period between exposure and disease is long.

As advantages of this research design, the retrospective study can help to focus the study question, clarify the hypothesis, determine an appropriate sample size, and identify feasibility issues for a prospective study. There are, however, several important disadvantages of a retrospective study. First, the investigator depends on the availability 
and accuracy of the medical record..$^{90}$ There usually are gaps in information and incomplete records. All data rely on the accuracy of the original examination and documentation. Items may have been excluded in the initial examination or not recorded in the medical chart. Secondly, the retrospective study is subject to selection bias because the investigator self-selects the cases..$^{90}$ Thirdly, retrospective studies are dependent upon the quality of information recorded by the clinicians involved in the study. Since different clinicians may practice differently, the retrospective study may demonstrate a larger variability of techniques or materials. ${ }^{91}$ Fourthly, retrospective studies tend to be conducted over longer periods of time. During those time periods there is a greater likelihood that minor changes in materials, techniques or designs can alter the therapy enough to create distinctly different treatment groups. With these changes, survival data may also change. If this is recognized, it is possible that the authors may describe specific "before and after" data, but if it is not recognized, significant improvements in design may not be apparent to the reader. ${ }^{91} \mathrm{An}$ actual example from many retrospective studies is implants followed up over long time, without recognizing that machined implants were used until one time period and modern, moderately rougher implants from another time period. A potential benefit of the latter group may "drown" in the information from the former group. Comparison of pooled data in such a study could create erroneous impressions when the reader compares the data to the results of other studies. For these reasons, the use of time-dependent statistical analysis may be even more critical to the establishment of meaningful conclusions with retrospective studies. ${ }^{91}$

\section{Prospective studies}

In a prospective cohort study, the investigator is in the position to plan and decide in beforehand what data to collect, when, and by which method the data will be analyzed. Usually this type of study must continue over a long period of time in order to observe a sufficient number of cases, and thereby obtain a fairly reliable estimate of incidence. The length of follow-up time required is dependent on the incidence rate and the size of the population at risk.

Prospective studies generally have inclusion and exclusion criteria. In some instances, prospective studies may be designed to eliminate 
known risk factors that could contribute to higher failure rates. Welldesigned prospective studies consider the anticipated differences in treatment outcomes when the number of subjects for enrollment is considered; this is generally described as a power analysis. ${ }^{91}$

Prospective studies are generally more expensive to complete since the study must enroll specific patients and must exclude others. Data must be maintained. The dependence on personnel to complete these tasks is greater with prospective studies. These studies are generally performed over shorter time periods and often have closer patient follow-up. The ability to discover late complications or compliance related to complications with prospective studies is thereby compromised..$^{91}$

Clinicians and policymakers often distinguish between the efficacy and the effectiveness of an intervention. Efficacy trials (explanatory trials) determine whether an intervention produces the expected result under ideal circumstances. Effectiveness trials (pragmatic trials) measure the degree of beneficial effect under "real world" clinical settings. ${ }^{92}$ Hence, hypotheses and study designs of an effectiveness trial are formulated based on conditions of routine clinical practice and on outcomes essential for clinical decisions. ${ }^{93}$ Of the principal methods available to measure effectiveness in a prospective way, one important distinction is between nonrandomized studies and RCTs.

\section{Controlled (non-randomized) clinical trial}

A controlled clinical trial (CCT) is a clinical study that includesa comparison (control) group. The comparison group receives a placebo, another treatment, or no treatment at all. When the study is not randomized, it is called non-randomized controlled trial or simply CCT. The principal difference in design compared with an RCT investigation is that participants in the trial are not allocated at random to treatment. ${ }^{94}$ This kind of trial can detect associations between an intervention and an outcome.

Because the trial participants are not randomly allocated to treatment, the trial is liable to allocation bias. Allocation bias is a systematic difference between participants in how they are allocated to treatment. ${ }^{95}$ Because participants are not randomly allocated to treatment and in case the treatment groups are not similar in the 
number of participants, the two treatment groups are unlikely to be similar in baseline characteristics. Therefore, the association between treatment and outcome is prone to be confounding, ${ }^{94}$ in other words, one cannot rule out the possibility that the association was caused by a third factor linked to both intervention and outcome. ${ }^{96}$ Hence, the non-randomized controlled trial study design may not promote internal validity. ${ }^{94}$ Internal validity is the extent to which the observed results can be ascribed to differences in treatment and not be confounding, thereby allowing the inference of causality to be inferred. ${ }^{97}$

The potential contribution that non-randomized studies can make to the evaluation of the effectiveness of healthcare interventions has generated considerable debate. In particular, it has been suggested that the lack of randomization in a non-randomized controlled trial, which potentially leads to differences between treatment groups in outcome owing to differences between treatment groups in baseline characteristics, results in the study design having limited value. However, in some trials random allocation may not be appropriate. ${ }^{94}$

Although not intrinsic to the design of non-randomized controlled trials, when the trial is community based (an entire community within the population is included in the trial), such an approach to sampling of study participants promotes external validity, i.e. the extent to which the study results can be generalized to the population that the sample is meant to represent. ${ }^{97}$ This is in contrast to RCTs, which often have stringent inclusion criteria and can recruit only those patients who are willing to be randomized. This may introduce biases that make the trial participants not representative of the population. ${ }^{94}$

\section{Randomized clinical trials}

RCTs are quantitative, comparative, controlled experiments in which groups are randomly assigned to receive 1 of 2 or more educational interventions. A large, well-designed and conducted RCT, in which randomization is undertaken in a way that ensures that allocation is actually random, will provide groups that can be expected to be comparable in every way except for the intervention..$^{98-100}$ Consequently, it is reasonable to attribute any significant observed difference in outcome between the two groups to differences in the effect of the interventions, provided both the practitioner and 
patient are blinded and the outcome is assessed in a way that does not introduce bias. ${ }^{98}$

The results of an RCT study can be analyzed and presented in two ways: according to the treatment to which the patients were randomized or according to the treatment they actually received. ${ }^{101}$ The planned treatment of the former demonstrates the efficacy of a treatment, while the pragmatic latter alternative establishes its effectiveness. The explanatory analysis is based on the principle that avoidance of bias is a prime goal in the RCT study, that randomization is the prime mechanism for avoiding bias, and that bias can arise from any post-randomization made by patients or by investigators. ${ }^{102}$ More simply, the effectiveness study may be more prone to bias. The disadvantage is that anything that happens to a patient after a treatment has been randomly assigned is thereafter included in the events ascribed to that treatment, regardless of why and how the events occurred. ${ }^{103}$ Most RCTs adopt the explanatory analysis. Therefore, clinicians should check the method of data analysis used in the literature when they read the results of an RCT. ${ }^{91}$

Although randomization is considered the most powerful experimental design in clinical trials, the method is not without limitations. RCTs are more likely than observational studies to be free of bias, ${ }^{104}$ but, because they usually enroll selected participants, external validity may suffer. This problem of unsuitable participants is also termed distorted assembly. ${ }^{102}$ Participants in RCTs tend to be different (including being healthier ${ }^{105,106}$ ) than those who choose not to take part, a function of the restricted entry criteria. Moreover, the process of randomization can be compromised such that the allocation results in biased groups of patients. A trial which has had its randomization compromised may apparently show a treatment effect that is entirely due to biased allocation. The results of such a study are more damaging than results from an explicitly non-randomized study, as bias in the latter is acknowledged and the statistical analysis and subsequent interpretation takes this into account. ${ }^{107}$ In the past attempts were not generally made to conceal randomization schedules from investigators who recruited patients. However, unconcealed randomization can lead to clinicians scheduling patients such that patients with particular characteristics would receive a certain allocation, thereby biasing the allocation. ${ }^{108}$ While much of the 
evidence on subverting randomization is anecdotal, a review found that randomization had been compromised in several controlled trials. ${ }^{108}$ This review showed that trials which did not adequately conceal randomization from the investigators demonstrated, on average, a $41 \%$ increase in effect for the active treatment compared with an adequately concealed trial. ${ }^{108}$ To avoid bias it is important that randomization is well concealed. Recent evidence has questioned the rigor of using local randomization (use on envelopes). Randomization should be distant and separate from clinicians (telephone or internet randomization) conducting the trial. ${ }^{107}$

RCTs are certainly not the most appropriate research design for all questions. ${ }^{109}$ For example, for etiological questions, it would neither be possible or ethical to randomize subjects to many harmful exposures. ${ }^{88}$ Exposing patients to an intervention believed to be inferior to current treatment is often thought unethical. ${ }^{96}$ In other circumstances an RCT study may be ethical but infeasible - for example, because of difficulties with randomization or recruitment. ${ }^{96}$ Randomization is unnecessary when the effect of an intervention is so dramatic that the contribution of unknown confounding factors can plausibly be ignored. Examples include penicillin for streptococcal infection and defibrillation for ventricular fibrillation. ${ }^{98}$ Randomization may be inappropriate where a trial would have to be of disproportionate size and duration, and thus expense, if it were to detect very rare long-term adverse effects or the impact of policies designed to prevent rare events. ${ }^{98}$ Randomization may be misleading where the process of random allocation may affect the effectiveness of the intervention. This can arise when the subjects cannot be blinded to the intervention because the intervention requires their active participation, which in turn will be affected by their underlying beliefs and preferences. ${ }^{98}$ Strong patient preferences may also limit recruitment and bias outcomes if not accommodated within the study design. ${ }^{110}$

If a population contains many individuals with little ability to benefit from the intervention in question and a few with a high ability to benefit, even though the overall sample size is large, the latter group may be very small and randomization may not ensure that they are evenly distributed between the two arms. In this case, the disproportionate effect exerted may lead to serious bias. ${ }^{11}$ 
Most evaluative studies fail to document adequately the characteristics of eligible patients who do not participate. The effect of nonparticipation differs between RCTs that evaluate interventions designed to treat an existing condition and those directed at preventing disease. ${ }^{112}$ Participants in the former tend to be less affluent, less educated, and more severely ill than eligible patients who do not participate. ${ }^{113}$ In contrast, participants in RCTs evaluating preventive interventions tend to be more affluent, better educated, and more likely to have adopted a healthy lifestyle than patients who decline. ${ }^{114}$ It is plausible that low participation in RCTs of treatment may exaggerate treatment effects by including more skillful practitioners and subjects with a greater capacity to benefit, while RCTs of prevention may underestimate effects as participants have selectively less capacity to benefit. ${ }^{115}$ Studies of RCTs with varying quality of randomization have shown that inadequate or unclear methods of treatment allocation exaggerate effect sizes. ${ }^{108}$

A large, inclusive, fully blinded RCT incorporating appropriate subgroup analysis is likely to provide the best possible evidence of effectiveness, but there will always be circumstances in which randomization, especially on an inclusive basis, is unethical or impractical. ${ }^{116}$ In circumstances where there are genuine reasons for not randomizing, ${ }^{117}$ non-randomized studies can provide useful evidence. In such studies, adjustment for baseline imbalances should always be attempted, as rigorously and extensively as possible, and the procedures should be reported explicitly to help readers' evaluations. $^{115}$

\section{Randomized versus non-randomized studies}

Evaluations of healthcare interventions can either randomize subjects to comparison groups, or not. In both designs there are potential threats to validity, which can be external (the extent to which they are generalizable to all potential recipients) or internal (whether differences in observed effects can be attributed to differences in the intervention). ${ }^{115}$ Non-randomized studies face greatest risk to their internal validity through allocation bias, though it may be possible to overcome this during analysis, by either risk adjustment or examination of comparable subgroups. The internal validity of unblinded RCTs may also be threatened by the consequences 
of patient preference, which may result in misleading estimations of treatment effect. ${ }^{98}$ Threats to external validity have been seen as of greater importance for RCTs, though the issue also arises in non-randomized studies. This can be due to restricted eligibility criteria, which can be addressed by expanding the criteria, or limited participation by centers or by patients and practitioners, which can be addressed by ensuring the design is pragmatic with less demanding consent requirements. Potential subjects may not be invited to participate in the research because of practitioner preferences for one of the treatments or simply due to administrative oversight. ${ }^{98}$ Some investigators have argued that RCTs tend to exclude, consciously or otherwise, some types of patient to whom results will subsequently be applied. Furthermore, in unblinded trials the outcome of treatment may be influenced by practitioners' and patients' preferences for one or other intervention. Though non-randomized studies are less selective in terms of recruitment, they are subject to selection bias in allocation if treatment is related to initial prognosis. ${ }^{115}$ Various factors act through their effect on the distribution of the potential to benefit among different groups. As patients are excluded or do not participate, the study population becomes a progressively smaller subset of the reference population, in principle increasing the scope for selection bias and raising the question of whether it is valid to apply the results obtained to the reference population. ${ }^{115}$ It is argued that non-randomized studies, which often have more inclusive entry criteria and procedures, may include subjects that are more representative of the population to whom the results will be applied. The process of recruitment, in which only individuals willing to be randomized will be recruited, may introduce hidden biases that make subjects unrepresentative of the reference population. ${ }^{98}$

An RCT study may produce a greater effect if the patients enrolled in it receive higher quality care or are selected so that they have greater capacity to benefit than patients in non-randomized studies. However, the RCT study may produce a lower estimate of treatment effect for several reasons: (1) in non-randomized studies, patients tend to be allocated to treatments that are correctly considered most appropriate for their individual circumstances; (2) exclusions from an RCT create a sample with less capacity to benefit than in a nonrandomized study; (3) an unblinded RCT fails to capture patients with 
strong preferences for a particular treatment who show an enhanced response to treatment; (4) non-randomized studies of preventive interventions include disproportionate numbers of individuals who, by virtue of their health related behavior, have greater capacity to benefit; (5) publication bias leads to negative results being less likely to be published from non-randomized studies than from RCTs. ${ }^{115}$

The limited evidence indicates that the results of non-randomized studies best approximate to results of RCTs when both use the same exclusion criteria and when potential prognostic factors are well understood, measured, and appropriately controlled in nonrandomized studies. ${ }^{118}$ The results of RCTs and non-randomized studies of similar patients may not, after adjustment, be substantially different in relative effect size. Any variations are no greater than those between different RCTs or among non-randomized studies. Differences in effect sizes could be due to chance or differences in populations studied, timing, or nature of the intervention. ${ }^{115}$ Britton et al. ${ }^{98}$ observed that within the limits of statistical significance, the results obtained by the randomized and non- randomized studies are frequently similar and that any differences were most often, but not always, of similar magnitude of estimated treatment effect rather than in the same direction, and only rarely did the two approaches favor different interventions.

Evaluative research is undertaken predominantly in university or teaching centers, but nonrandomized studies are more likely than RCTs to include nonteaching centers, and criteria for participation in RCTs may include the achievement of a specified level of clinical outcome. ${ }^{115}$ The available evidence suggests that this may exaggerate the measured treatment effect. ${ }^{119}$

When both randomized and nonrandomized studies have been conducted it is important to ascertain whether estimates of treatment effect are consistent for patients at similar risk across studies. If so, it may be reasonable to accept the results of the less exclusive nonrandomized studies. Differences in results cannot be assumed to be solely due to the presence or lack of randomization - differences in study populations, characteristics of the intervention, and the effects of patients' preferences may also affect the results. ${ }^{115}$ 


\section{Reviews}

\section{Narrative review}

Narrative reviews are the traditional approach and usually do not include a section describing the methods used in the review. These reviews are generally on a very broad topic, they use informal, unsystematic and subjective methods to search for, collect and interpret information, which is often summarized with a specific hypothesis in mind, and without critical appraisal, and summarized with an accompanying convenient narrative. Though these narrative reviews are often conducted by people with expert knowledge of their field, it may be the case that this expertise and experience may bias the authors. The absence of a clear and objective method section leads to a number of methodological flaws, which can bias the author's conclusions. ${ }^{120}$ Thus, it can be said that narrative reviews are highly subjected to publication selection bias, and it can favor one treatment in comparison of another one based on the author's biased preferences to choose the studies. Authors tend to choose papers that agree with their point of view and also tend to effusively quote themselves.

\section{Systematic review}

Systematic reviews of interventions require a thorough, objective and reproducible search of a range of sources to identify as many relevant studies as possible (within resource limits). This is a major factor in distinguishing systematic reviews from traditional narrative reviews and helps to minimize bias and therefore assist in achieving reliable estimates of effects. ${ }^{121}$

By addressing the methods of the identified primary studies, systematic reviews and meta-analyses can identify common methodological weaknesses and errors that might materially affect the results - these may be missed using a less systematic approach. ${ }^{88}$ By weighting the trials and producing a pooled overall estimate, which is a quantitative, rather than a qualitative assessment of the apparent strength of the results, systematic reviews may often conclude that the effect of a treatment is less impressive than a traditional narrative review would conclude. ${ }^{88}$

There is evidence that systematic reviews improve the reliability and accuracy of the conclusions, however the results are rarely 
unequivocal and require careful appraisal and interpretation: ${ }^{122}$ clinicians therefore need to integrate the results with clinical expertise and the patient's preferences.

There are several forms of systematic bias that need to be considered in reviews. One of the most important is selection bias, in other words, a systematic bias in the selection of primary research studies that are included in the review. For example, a reviewer may only select those studies that support his prior beliefs. Selection bias, however, may also be due to "publication bias" (the tendency of investigators, reviewers and editors to differentially submit or accept manuscripts for publication based on the direction or strength of the study findings), ${ }^{123}$ "language of publication bias" (studies finding a treatment effect are more likely to be published in English-language journals, whilst opposing studies may be published in non-Englishlanguage journals ${ }^{124}$ and biases introduced by an over reliance on electronic databases (electronic databases do not offer comprehensive or unbiased coverage of the relevant primary literature).${ }^{88}$

Unfortunately, there is considerable evidence that key information is often poorly reported in systematic reviews, thus diminishing their potential usefulness. ${ }^{125}$ Systematic reviews may differ in quality, and yield different answers to the same question. ${ }^{126}$ As a result, users of systematic reviews should be critical and look carefully at the methodological quality of the available reviews. ${ }^{127}$ Therefore, when performing systematic reviews, it is important to make use of instruments in order to attain certain methodological quality, such as the AMSTAR ${ }^{128}$ or guidelines such as PRISMA, ${ }^{129}$ an evidencebased minimum set of items for reporting in systematic reviews and meta-analyses.

\section{The use of meta-analysis}

Systematic reviews usually, but not necessarily, apply meta-analysis to examine a research question. ${ }^{130}$ Meta-analysis is the statistical combination of the results of several studies into one pooled value and can be a useful way of reducing random error and increasing precision. ${ }^{88}$ Meta-analyses generally provide a greater degree of precision when compared with other study types because the estimate from the combined studies is based on a larger sample than any of the individual studies. This will provide narrower $95 \%$ confidence intervals than any study conducted on its own. ${ }^{131}$ 
A meta-analysis can be misleading unless it is performed in the context of a systematic review of the literature to avoid systematic biases. Whereas a systematic review can be applied to any form of research question, a meta-analysis should not be used indiscriminately, especially when the primary data are inadequate. ${ }^{88}$ The first stage in conducting a systematic review is the formulation of a clear question. The nature of the clinical question determines the optimal primary study design and hence the a priori inclusion and exclusion criteria. ${ }^{88}$ The most reliable study design would be an RCT. Systematic reviews of studies other than RCTs have their own methodological problems: guidelines exist for undertaking reviews (and meta-analyses) of diagnostic tests ${ }^{132}$ and the observational epidemiological designs used in etiological research. ${ }^{133} \mathrm{~A}$ different approach would be to select specific studies, but this has its problems as well and opens the door to biased choices. The studies selected for meta-analyses are usually all of high quality - often it is an inclusion criterion that they are randomized and double blind. ${ }^{130}$

Meta-analysis may not always be appropriate. First of all, it is necessary to ensure that the individual studies are really looking at the same question: the criteria used to select including studies and to assess the quality of the studies included should be explicit and consistent with the focus of the review. Results from poor quality studies can result in misleading conclusions and so inclusion of these studies in a meta-analysis may bias the pooled result towards an overestimate of the effectiveness of the intervention being evaluated. ${ }^{134}$ The methodological quality of the included studies is important even if the results or quality of the included studies do not vary: if the evidence is consistent but all the studies are flawed, the conclusions of the review would not be nearly as strong as if consistent results were obtained from a series of high quality studies. ${ }^{88}$

Potential biases are likely to be greater for non-randomized studies compared with RCTs, so results should always be interpreted with caution when they are included in reviews and meta-analyses. ${ }^{121}$ So what was the reason to include non-randomized studies in the present meta-analysis? The issue is important because meta-analyses are frequently conducted on a limited number of RCTs. ${ }^{135}$ Shrier ${ }^{136}$ gave fundament to this observation by reviewing a random $1 \%$ sample of meta-analyses published by the Cochrane Collaboration 
in 2003 and found that 6 of 16 reviews included two studies or fewer. Furthermore, 158 of 183 analyses conducted in 7 additional studies were limited to two or fewer studies. Given the limited number of RCTs included in 13/16 reviews, the inclusion of other types of evidence - for example, cohort, case-control, basic science would probably have provided a stronger foundation for a rational decision of treatment. ${ }^{136}$ In meta-analyses such as these, adding more information from observational studies may aid in clinical reasoning and establish a more solid foundation for causal inferences. ${ }^{135}$ In a meta-analysis, homogeneity implies a mathematical compatibility between the results of each individual trial. Narrowing the inclusion criteria increases homogeneity but also excludes the results of more trials and thus risks the exclusion of significant data. In the Cochrane Review approach, if one RCT is available then all "weaker" data are ignored. However, it is not clear how much stronger the data needs to be to ignore "weaker" data. ${ }^{137}$ Further, it ignores the fact that other types of studies could help the reviewer discuss treatment effectiveness in other populations - for example, different age groups - or similar but not identical conditions. ${ }^{136}$ Cochrane Reviews currently enjoy a credibility level that may not be warranted. Reviews that are based on zero, one, or two RCTs without reference to other types of evidence may be omitting important information that would be useful to a clinician who needs to treat actual patients. ${ }^{136}$

A drawback associated with meta-analyses includes lack of accepted or consistent methodology in a scientific study(s), which can bias the results. This can lead to inaccurate estimates of the effectiveness of clinical procedures. Important caveats to consider in a meta-analysis include pooling of data and conflicting results of studies, which can influence outcomes. In addition, the consistency in clinical and research methodology that has been used, the sample sizes, number of variables in a study design, and the probability for error or bias in the results of clinical studies need to be considered when performing a meta-analysis. ${ }^{131}$

\section{Data analysis}

Survival analysis

With any study there is a need for data analysis. Whether data are followed forward (prospective study) or backward (retrospective 
study), they must be collated and evaluated in such a way as to make them meaningful. Raw data can be easily distorted to demonstrate results that do not adequately describe clinical performance. Statistical analysis of data, particularly time-dependent analysis, is more valuable if there is a risk of time-dependent deterioration, complication, or failure. ${ }^{91}$ Studies of how patients respond to treatment over time are fundamentally important to understanding how therapies influence quality of life and progression of disease during survivorship. ${ }^{138}$ Survival analyses are statistical methods used to examine changes over time to a specified event. It follows comments on some of the used methods of survival analysis.

\section{Life table}

The most straightforward way to describe the survival in a sample is to compute the life table, or the Cutler-Ederer method. ${ }^{139}$ The life table technique is one of the oldest methods for analyzing survival data. The distribution of survival times is divided into a certain number of intervals. For each interval we can then compute the number and proportion of cases or objects that entered the respective interval 'alive,' the number and proportion of cases that failed in the respective interval (number of terminal events, or number of cases that died, or number of dental implants that failed), and the number of cases that were lost or censored in the respective interval. Based on those numbers and proportions, several additional statistics can be computed, such as, the number of cases at risk, proportion failing, proportion surviving, survival function, hazard rate, and median survival time. This procedure is used for larger samples where the time intervals are large enough to be broken down into smaller units. $^{140}$

The advantages of the life-table analysis were listed by Ferguson: ${ }^{141}$ (1) the method uses the data of all patients; (2) calculations are simple enough to be performed by a non-statistician and by hand; (3) it does not require knowledge of exact follow-up times; (4) hypothesis testing is based on distribution-free methods. That is to say, no assumption is required regarding the distribution of survival times and, therefore, the form of the survival distribution need not be known. This is particularly important, since in general it is not known; (5) results can be represented graphically, promoting comprehension and 
simplifying communication of results. This is particularly important when the target audience consists of non-statisticians; (6) life tables can suggest which factors are important for establishing a prognosis, for example, by comparing life tables in separate patient categories based on treatment, gender, etc; (7) the log-rank test is optimal for assessing the equality of life tables when the event rate in one group consistently exceeds that of another; (8) the log-rank tests can be used to compare more than two groups; (9) it is possible to adjust survival comparisons for the confounding effects of prognostic variables by means of a stratified log-rank test. However, the potential to do so is limited compared with other techniques, for example, regression based on the proportional hazards model.

As disadvantages of the life table, it can be said that ${ }^{141}$ (1) the lifetable analysis shows cumulative event-free survival and, therefore, does not directly show changes in the event rate; (2) it does not take into account the exact ordering of dental implant losses within each interval; (3) it is an approximation to the Kaplan-Meier product-limit survival estimate and owes its popularity, in some measure, to the relative ease of calculations performed in large data sets. However, this is no longer an important consideration given the ubiquity of computers; (4) survival estimates are somewhat arbitrary in that they depend on the time intervals chosen. This is not a problem with the Kaplan-Meier product-limit method of survival analysis; (5) large steps and long flat regions in the survival curve can result from small numbers of patients at risk. These distortions are not biologically significant; (6) the most recent observations are the least reliable because of the decreasing number of patients at risk for the event of interest; (7) the log-rank test is relatively insensitive to early differences in event-free survival. Consequently, it is less likely than the Gehan test to detect these.

More specifically to the implant dentistry, it is possible to report 5 -year cumulative survival rate (CSR) results before all implants have been followed up for a full of 5 years, i.e. a considerable advantage for those who wish to report results as rapidly as possible. Hence, the CSR technique involving the use of so called life tables presents a quite good way of reporting clinical outcome with one major exception; the treatment of so called drop-out patients that are assumed to have the same results as those remaining in the study. This may be true for 
patients who have not, as yet, had the chance of being followed up for the decided time period, it may further be true for those patients who die with their implants in situ, but it is a great risk that it is untrue for many other patient categories where patient drop outs may coincide with more actual implant problems compared to those who remain in a study. In the drop-out category, it is possible that a number of high risk patients may be lost to follow-up. Similarly dissatisfied patients are unlikely to return for follow-up care which further underestimates the number of failures experienced in the patients who do not return. If high risk and dissatisfied patients are lost their higher incidence of implant failure would not be recorded thereby providing the reader with the impression that a higher number of implants succeed than is actually the case. The solution to this potential misrepresentation is to count all "dropouts" as clinical failures and let data so handled present the lower confidence interval and then to conversely count all "dropouts" as successes and record this as the upper confidence interval. The way that most articles are reported today is to ignore the dropouts entirely and simply report the performance of the known or followed patients. When reporting this, the authors are making a concerted effort to describe in the life table all the known data but may not recognize that this method likely inflates the results. We are likely to have an overrepresentation of drug abuse and other risk patients and, furthermore, dissatisfied patients who do not wish to go back to the treating dentist that well may represent double the failure rate compared to those who remained in the study.

One more disadvantage of life tables as used in implant dentistry is that too few implants have been included that are actually followed up for the full study period, hence a study that is titled as a "five-year cumulative survival analysis" frequently presents results in which only a very small percentage of patients treated in the study have reached the described five-year time frame. A more appropriate title might describe a mean survival with up to a maximum of five years. The presented 5 year CSR-outcome has a substantial uncertainty in that it is assumed that these very few implants reflect the reality for each and every implant of the study. Another substantial shortcoming of commonly used simple CSR tables in most dental implant reports is that too little data is being presented in the tables that, at best, present a survival rate. 
It is also important to stress that life-table analyses are based mainly on criteria that are easy to evaluate (e.g. implant function and immobility, no pain) instead of criteria that are more difficult to evaluate (e.g. peri-implant pocket depth, bleeding disposition, and bone loss), but which would provide more information about peri-implant soft and hard tissue health. ${ }^{142}$ Life-table analyses that consider multiple implants placed in one patient produces data that are not independent (i.e., they have some common variables that may predispose to failure). Thus, statistics based on each implant as the index of analysis may lead to bias. Others have suggested selecting one implant at random for each patient providing independent data, ${ }^{143}$ but this method discards much of the data collected ${ }^{144}$ and has the drawback of falsifying the result depending on false positive or negative choices. ${ }^{142}$

\section{Kaplan-Meier analysis}

The Kaplan-Meier (KM) method is maybe the most prevalent in the dental literature. ${ }^{91}$ Kaplan and Meier ${ }^{145}$ first described the approach and formulas for the statistical procedure that took their name. The method permits estimation of subject survival over time, even when subjects are lost to follow-up or followed for different lengths of time. ${ }^{91}$ Incomplete observations often occur because contact with some sample members is lost before the event, some other intervening variable affects the event, or insufficient time has passed to observe the event in all sample members. Any of these cases would result in a participant being censored. ${ }^{138}$ At each time point, the KM method typically involves computing the number of subjects that had died at each time point (numerator) divided by the total number of subjects that were still in the study at that time point (denominator). The censored cases, i.e. the subjects who are lost to follow-up, are no longer included in the denominator from the first time point at which they were lost. ${ }^{91}$ The KM method allows the practitioner to estimate the probability of a subject surviving at a given time point, e.g., 5 years, from the cumulative probability of a subject surviving each preceding time interval (years 1, 2, 3, and 4). Therefore, although a probability calculated in a given interval may not be accurate because of a relatively small number of subject deaths in that interval, the overall probability of surviving to each time point is more accurate. ${ }^{91}$ 
The KM method is not without limitations. Missing data is a problem that can potentially bias data analyses and statistics. An important assumption is that censored patients have the same likelihood of survival as those continuing in the study (an assumption not easily testable), and that survival probabilities are the same whether individuals enter a study early or late. ${ }^{146}$ One important way to deal with this is to track the numbers of patients in each arm who withdraw and reasons for withdrawal and to include this information in research reports. ${ }^{138}$ In most studies some subjects drop out for reasons unrelated to the condition under study (for example, emigration). If, however, for some patients in this study censoring was related to failure to conceive this would have biased the estimated survival probabilities downwards. ${ }^{147}$ Completeness of follow-up is important, especially in clinical trials, since unequal follow-up in the treatment groups can bias the analysis of results. In survival studies, information on participants who do not complete the study is often omitted because their data can be included up to the time at which they were lost to follow-up. ${ }^{148}$

A second assumption would be that the survival probabilities are the same for subjects recruited early and late in the study. ${ }^{147}$ In a long term observational study of patients with dental implants, the cohort may change over the period of recruitment, or there may be an innovation in treatment, for example, turned implants were installed at the beginning years of the study and then enlarged-surface implants were adopted. A third assumption is that the event happens at the time specified. This is not a problem for the conception data, but could be, for example, if a dental implant failure was detected at a regular examination. All we would know is that the event happened between two examinations. This imprecision would bias the survival probabilities upwards. ${ }^{147}$

Most clinical trials have a minimum follow-up time, at which point the status of each patient (implant) is known. The survival rate at this point becomes the most accurate reflection of the survival rate of the group. Survivor function at the far right of a Kaplan-Meier survival curve should be interpreted cautiously, since there are fewer patients remaining in the study group and the survival estimates are not as accurate. ${ }^{149}$ After the first patient is censored the survival curve becomes an estimate, since we do not know if censored patients would 
have experienced an event at some point later in their life. Thus, the more patients censored in a study (especially early in the study), the less reliable is the survival curve. Ludbrook and Royse ${ }^{150}$ showed that the KM approach underestimates the cumulative survival probability. The KM method for estimating survival or incidence is unsuitable for use when there are two competing risks. It occurs because if relapse is the event of interest, then according to the KM method, both those who are dead and those alive should be censored. But unlike the case in which there is only one event and observations are censored because the subject is alive or is inaccessible to further follow up, death precludes the possibility of relapse. ${ }^{150}$ However, the presence of competing risks is more common in cancer studies, not in dental implant studies. As examples of competing risks, ${ }^{148}$ there would be death from a cause "not tumor related", precluding the observation of death from cancer, death precluding the observation of time to diagnosis of a second cancer in lymphoma patients after high-dose chemotherapy and stem-cell transplants, and analysis of local and distant recurrence.

Likewise, it is helpful to know why patients were censored. If many patients were censored in a given group(s), one must question how the study was carried out or how the type of treatment affected the patients. This stresses the importance of showing censored patients as tick marks in survival curves. ${ }^{149}$

Moreover, the shape of the KM survival curve is important to evaluate. Curves that have many small steps usually have a higher number of participating subjects, whereas curves with large steps usually have a limited number of subjects and are thus not as accurate. Concerning the amount of censored subjects and the distribution of censored subjects, if there is a large number of censored subjects one must question how the study was carried out or if the treatment was ineffective resulting in subjects leaving the study to pursue different therapies. A curve that does not demonstrate censored patients should be interpreted with caution. ${ }^{149}$

\section{Cox Proportional Hazards Regression}

Cox proportional hazards regression is widely used to assess the influence of multiple factors on time to event. ${ }^{151}$ It is one of the most popular regression models in medical research. The model enables 
one to assess a relationship between patients' (or dental implants') survival time and one (univariable) or multiple (multivariable) explanatory variables. Additionally, it allows an estimated risk of an event occurrence for an individual to take into account statistically significant prognostic variables. ${ }^{152}$ Similarly to other regression analyses, Cox regression produces coefficients which provide an estimate of size of effect due to the variable of interest as well as confidence intervals. These estimates are called hazard ratios (HR) and represent the chance of the event (e.g. dental implant loss) occurring in those with the variable of interest (e.g. smoker) as a ratio of the hazard of the event occurring in those without (e.g. non-smoker). Therefore a HR of 1 represents no difference while a HR of 3 implies that at any time point the event will occur 3 times more commonly in those with the variable of interest compared to control participants. In the case of continuous predictor variables the HR represents the ratio of hazards for each unit of change in the predictor variable. The HR does not provide information about speed of time to event (or recovery) so it is incorrect to interpret a HR of 3 as saying people with the treatment will recover 3 times faster than those without the treatment. ${ }^{153}$ In other words, the HRs of two people are independent of time, and are valid only for timeindependent covariates. This means that the hazard functions for any two individuals at any point in time are proportional. ${ }^{140}$ If an individual has a risk of having a dental implant failure at some initial point in time that is twice as high as that of another individual, then at all later times the risk of implant failure remains twice as high. Then, a key assumption when performing Cox regression is that the HR for the explanatory variable is relatively constant across time. ${ }^{154}$

When the HR for the explanatory variable is not constant across time (the curves cross in the plot of survival curves, and the HR clearly changes over time), then there is strong evidence the proportional hazards assumption is violated. ${ }^{154}$ An example would be a trial comparing steroid injection to exercise therapy in people with sub acromial impingement as the HR would likely change over time. The event of interest could be time to $50 \%$ reduction in pain. In the short term (e.g. day 1) you would expect more people to have the event if they received steroid injection, however, at a later time point (e.g. 6 weeks) it is likely a larger number of people receiving 
exercise therapy will have the event. This is a clear violation of the proportional hazards assumption. Cox regression cannot be used in this case without modifications. ${ }^{154}$ One strategy to deal with situations where the proportional hazards assumption is violated is to divide the time interval. ${ }^{155}$ In other words the HRs are generated for smaller intervals of time where the HR is more constant.

\section{Multilevel mixed effects parametric survival approach}

The multilevel mixed effects parametric survival model is a very recent survival statistical analysis. The model is used in the analysis of clustered survival data, such as repeated events, multicenter clinical trials, and individual participant data meta-analyses, to investigate heterogeneity in baseline risk and covariate effects. ${ }^{156}$ It takes into consideration that the occurrence of clustered survival data is commonplace in medical research, where event times are clustered within groups of the same or similar individuals, which means event times from the same group are likely to be correlated. It considers situations where a researcher wants to make an average inference across all clusters while accounting for any heterogeneity between clusters. ${ }^{156}$

The method is very recent, and as the developers of this statistical model stated, further work is needed to evaluate the estimation methods in clinically realistic scenarios, to investigate how well it performs for varying effect sizes, heterogeneity standard deviation sizes, number of clusters, and number of participants per cluster. ${ }^{156} \mathrm{~A}$ deeper discussion about the advantages and limitations of the method would be, statistically speaking, too complex, which goes beyond the scope of the present text.

Only few studies have used the method in the dental literature for the analysis of implant failures. ${ }^{157-161}$

\section{Binary logistic regression}

Logistic regression models are used to study effects of predictor variables on categorical outcomes and normally the outcome is binary, such as presence or absence of disease, in which case the model is called a binary logistic model. ${ }^{162}$ An event is a binary variable that can only have a yes or no value (e.g., dental implant failure, death, 
hospital discharge or readmission, heart attack, recovery from an infection, relapse from smoking cessation, etc.). ${ }^{138}$

Logistic regression may include only one or multiple independent variables, although examining multiple variables is generally more informative because it reveals the unique contribution of each variable after adjusting for the others. ${ }^{163}$ And this is important in implant dentistry, since implant failure frequently involves many different factors. For a logistic regression model with only one independent variable, the odds ratio $(\mathrm{OR})$ is considered "unadjusted" because there are no other variables whose influence must be adjusted for or subtracted out. In contrast, if the logistic regression model includes multiple independent variables, the ORs are now "adjusted" because they represent the unique contribution of the independent variable after adjusting for (or subtracting out) the effects of the other variables in the model. As a result, adjusted odds ratios are often lower than their unadjusted counterparts. ${ }^{163}$

Carefully selecting one's independent variables is an essential step. While logistic regression is quite flexible in that it accommodates different variable types, including continuous (e.g., age, implant length), ordinal (e.g., local bone classification in quantity or quality), and categorical (e.g., implant surface, implant system, reason for tooth extraction before implantation), one must always justify variable selection using well-established theory, past research, clinical observations, preliminary statistical analysis, or some sensible combination of these different options. ${ }^{163}$ One must always keep in mind that too many independent variables in the model may lead to a mathematically unstable outcome, with decreased generalizability beyond the current study sample. ${ }^{164,165}$ Thus, it is suggested that first a univariate effect of each factor on implant failure is evaluated and then only the factors that shown to have a statistically significant effect size in the univariate logistic regression would be included in the multiple/multivariate analysis.

Concerning the selection of the independent variables, the challenge is to select the smallest number of independent variables that best explains the outcome while being mindful of sample size constraints. ${ }^{164,165}$ For instance, if one selects 50 people for the study sample and includes 50 independent variables in the logistic regression analysis, the result is an overfit (and therefore unstable) model. 
An overfit model has estimated beta coefficients for independent variables that are much larger than they should be, as well as higher than-expected standard errors. ${ }^{164}$

What, then, is the correct number of outcomes for avoiding an overfit model? While there is no universally accepted standard, there are some common "rules of thumb" based in part on simulation studies. One such rule states that for every independent variable, there should be no fewer than 10 outcomes for each binary category (e.g., lost implant/survived implant), with the least common outcome determining the maximum number of independent variables. ${ }^{166,167}$ For example, in a dental implant failure study, assume that 30 patients lost dental implants and 600 patients did not lose any implant. The logistic regression model could reasonably accommodate, at most, three independent variables (since 30 is the smallest outcome). However, the issue has not been definitively settled, and some would argue that fewer than 10 outcomes per independent variable may be appropriate in certain research contexts. ${ }^{164}$

\section{Generalized estimating equation}

In clinical dental research, each participant often contributes multiple, potentially clustered observations to a database. For example, in dental implants, multiple implants placed within the same individual will be clustered or correlated. It is common practice for those conducting dental research to assume independent outcome observations in survival analyses. ${ }^{168,169}$ These approaches tend to underestimate the variability of the estimated covariate effects or survival probabilities, because the possible within-cluster correlations are overlooked. To account for correlation, many authors have recommended randomly selecting 1 implant per person, and the use of only the subset for analysis. ${ }^{53,170}$ However, this approach does not fully utilize all the available information, and thus may be inefficient. This gives rise to the need for the application of other methods that can efficiently analyze clustered dental survival data. ${ }^{171}$

Responses measured on the same subject are usually assumed to be correlated and, therefore, a proper modeling approach is needed that accounts for within subject correlation. ${ }^{172}$ One of the most important approaches is the generalized estimating equation (GEE) proposed by Liang and Zeger. ${ }^{173}$ Particularly in implant dentistry, 
the method accounts for the fact that repeated observations (several implants) can be available for a single patient. Because the outcome relating to implants within a single patient must be more closely correlated to each other than implants in separate patients, ignoring these correlations could result in a bias in $p$-value computations. ${ }^{174}$ A deeper discussion about the advantages and limitations of the method goes beyond the scope of the present text, being very complex and specific to advanced statistics. Literature about it has been published. ${ }^{175,176}$

\section{Clinical factors}

\section{Time of follow-up}

When a reader compares studies, it is critical to compare similar time periods and time intervals. If a study begins many years prior to a second study, it is distinctly possible that results of the two studies may differ simply because of changes in the standards of practice that occurred during the time period covered by the two studies. When two studies are conducted in two distinctly different time periods, even if the duration of the studies is similar, distortion of results is possible. It is distinctly possible that the earlier study may represent a different generation or version of the device being tested and therefore may have little relevance to the more current study. Materials, devices, and techniques used in the 1980s study may not even be available for use a decade and a half later. Therefore, comparisons that lead to conclusions about the two different approaches should not be made. ${ }^{91}$

Moreover, when studies differ in follow-up periods, a longer follow-up period can lead to an increase in the failure rate, especially if it extended beyond functional loading, because other prosthetic factors can influence implant failure from that point onward. This might lead to an underestimation of actual failures in some studies. Long-term survival rates of implants are critical for clinicians to consider before they can make decisions to place them in their patients.

\section{Sample size}

A research can be conducted for various objectives. It may be done to establish a difference between two treatment regimens in terms 
of predefined parameters like beneficial effects, side effects, and risk factors of these regimens. It may also be carried out to prove similarity between groups. Sometimes, the purpose may be to achieve certain estimation in the population, such as the prevalence of a disease. Whatever the aim, one can draw a precise and accurate conclusion only with an appropriate sample size. A smaller sample will give a result which may not be sufficiently powered to detect a difference between the groups and the study may turn out to be falsely negative leading to a type II error. A study on a small sample is quite tempting for obvious reasons, but it is a waste of time and money as the result will be invariably inconclusive. Very often, a small sample size is decided arbitrarily based on the researchers' convenience, available time, and resources, resulting in a null trial due to insufficient number of subjects studied. The underpowered studies should be interpreted cautiously and the 'absence of evidence' in these studies should not be taken as 'evidence of absence'. Only for a rare disease or indication is an underpowered study justified, due to logistics as the data from such a study is helpful in meta-analysis. ${ }^{177}$

Concerning implant dentistry, because implant survival rates are generally high, sample sizes need to be large to demonstrate statistically significant differences for meaningful clinical differences in implant survival performance. ${ }^{91}$ When a new dental implant is evaluated for its clinical efficacy, there might be conflicting results from the published literature. For instance, two different studies might have reported opposite conclusions about the dental implants being good for their patients. However, one of the studies might have placed the implants in a smaller group of patients (e.g., 20 patients) by a single surgeon, while the second study might have carried out a larger RCT (e.g., 2000 patients). The conclusions from the second study might carry more validity in terms of being more acceptable to clinicians, while the first study's conclusions might constitute a mere opinion from a clinician's experience. When appraising the literature, the dentist needs to consider these previously mentioned factors so that the more valid study results can be used clinically. ${ }^{131} \mathrm{~A}$ power analysis would permit a clinician researcher to predict the number of implants that need to be included in a study for it to provide data that can be analyzed in a statistically meaningful manner. ${ }^{91}$ 


\section{Unaccounted for implants in drop out patients}

The probability of missing data is particularly high in longitudinal epidemiological studies. ${ }^{178}$ Subject loss to follow-up is a form of selection bias that is particular to the cohort design. ${ }^{179,180}$ It occurs when subjects are permanently lost to follow-up. ${ }^{181}$ Loss to follow-up is inevitable in most cohort studies and commonly leads to bias and loss of statistical power. ${ }^{182,183}$ Loss to follow-up can severely compromise a study's validity. Incomplete follow-up biases the results when either the dropout rates are different between study groups or the patients who drop out are different from those who do not drop out. These situations make a difference because in each situation, those lost to follow-up often have a different prognosis than those who complete the study. ${ }^{184}$ For example, patients with dental implants may not return for follow-up because they might think that there is no problem and felt no need to return to see the surgeon. Conversely, some patients may not return because they had a particularly bad outcome or complication and had chosen to go to another dentist instead, or because they died. In either case, bias can affect the validity of the inferences drawn from the study. Some have suggested that $<5 \%$ loss of follow-up leads to little bias, while $>20 \%$ poses serious threats to validity. ${ }^{185}$ This may be a good rule of thumb, but keep in mind that even small proportions of patients lost to follow-up can cause significant bias. ${ }^{186}$ In the presence of missing data, the choice of analytical method has important implications on the estimates of the outcome response and of the relationship of the outcome to predictors. That is, the effect of missing data on inference depends on the underlying missingness process. ${ }^{178}$

When it comes to the implant dentistry, Albrektsson and Zarb ${ }^{187}$ suggested that each and every implant should be evaluated as part of a four-grade scale representing success, survival, unaccounted for, and failure. The success category includes implants that meet (and have been tested for) all of the success criteria, including stability tests and individual radiograms. The survival category encompasses those unattached implants that were not checked for mobility, where periapical films were not used, or where the prosthesis was not removed at the evaluation for other reasons. Such implants can never be regarded as successful because there are not enough data to evaluate them as such. Implants in the unaccounted for category include all those in patients who died or dropped out of the study, or who were 
not available at a specific recall appointment. Naturally, the larger the number of unaccounted for implants, the more uncertain will be the estimates of implant success or survival. The failure category includes all removed implants irrespective of the cause of their failure or removal. Even an individual implant that has been lost (e.g., in a motor vehicle accident) must be categorized as a failure. Correctly applied, it is believed this new type of evaluation will encompass an improved diagnosis of the outcome of oral implants.

In their article, the authors ${ }^{187}$ describe an example of how to apply the categories (Figure 8): 100 consecutively placed implants are to be evaluated with respect to their outcome for 1, 3, and 5 years. At the 1-year recall, 78 of those implants were diagnosed as successful based on the listed criteria. Four implants were impossible to evaluate radiographically because of poor radiograms, 4 were placed in a patient who subsequently died, 4 others were placed in a patient who suddenly dropped out of the study, 6 were reported to be painful, 2 were mobile, and the remaining 2 had been removed. This would result in a 1 -year outcome of $78 \%$ success, $10 \%$ survival, $8 \%$ unaccounted for, and $4 \%$ failure.

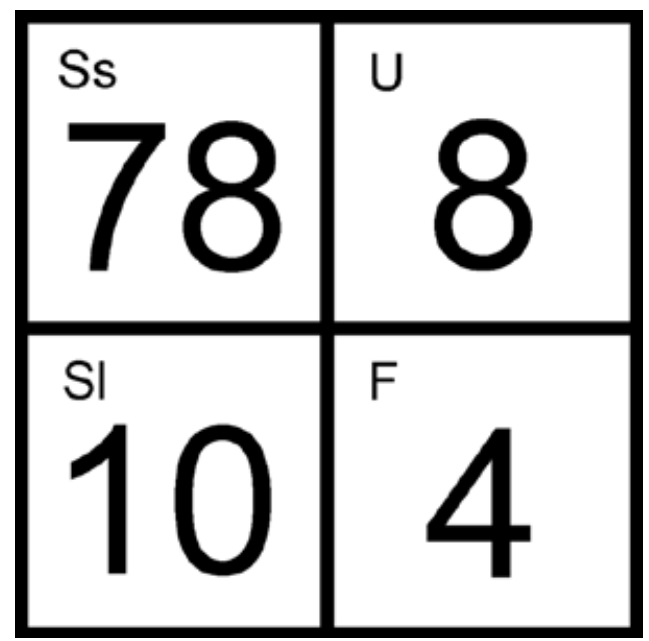

Figure 8. Four-field diagram for evaluating the outcome of an implant procedure (from Albrektsson and Zarb ${ }^{187}$ ). An example of 100 consecutively placed implants where 78 implants passed the success criteria. $\mathrm{Ss}=$ success, $\mathrm{Sl}=$ other survivals, $\mathrm{U}=$ unaccounted for, $\mathrm{F}=$ failure. All figures in percent. 
As a final remark, it can be said that a well-constructed statistical model is not the sole determinant of high quality research—developing a clinically relevant and objectively measurable hypothesis, implementing an appropriate study design and statistical analysis plan, and accurately reporting both outcomes and conclusions are all important considerations. Therefore, readers who pay close attention to the parameters of their statistical analysis within the context of a well-designed and soundly executed study will make the most meaningful contribution to evidence-based dentistry. ${ }^{163}$ 


\section{AIMS}

The general aim of the present thesis was to investigate and identify factors associated with the failure of oral implants, by performing systematic reviews and clinical trials including the population rehabilitated with dental implants at the Clinic for Prosthodontics, Centre of Dental Specialist Care, Malmö, Sweden (Folktandvården Skåne $\mathrm{AB}$, Specialisttandvård Malmö).

The specific aims were:

1. To identify risk factors that may contribute to failures of oral implants, in a systematic review of the literature (Study I);

2. To analyze the influence of one risk factor (smoking) on the failure of oral implants, marginal bone loss, and postoperative infection, in a systematic review of the literature with metaanalysis (Study II);

3. To assess the influence of local and systemic factors on the occurrence of oral implant failures up to second-stage surgery abutment connection, in a retrospective clinical trial (Study III);

4. To analyze the complications of oral implant treatment in a group of patients presenting bruxism in comparison with a matched group of non-bruxers, in a retrospective clinical trial (Study IV);

5. To investigate the association between the intake of two types of medicaments, i.e. proton pump inhibitors and selective serotonin reuptake inhibitors, and the risk of oral implant failure, in retrospective clinical trials (Studies V and VI); 
6. To assess, among other factors, the influence of the placement of oral implants by different dental surgeons on the implant failure prevalence, in a retrospective clinical trial (Study VII);

7. To analyze cluster behavior of oral implant failures among patients, to assess the possible risk factors influencing this phenomenon, and to describe and compare this group of patients with one not presenting this behavior, in a retrospective clinical trial (Study VIII);

8. To assess the dental implant failure rates and marginal bone loss of implants followed up for a minimum of 20 years, in a retrospective clinical trial (Study IX). 


\section{MATERIALS AND METHODS}

The articles included in the present thesis consist of three types of studies. First, a general overview was performed in order to identify risk factors associated with the failure of oral implants (Study I). Then, a systematic review of the literature with meta-analyses was performed to analyze the influence of one risk factor (smoking) on the failure of oral implants, MBL, and postoperative infection around implants (Study II). Details about the procedures performed in these two reviews are described in the articles included at the end of this thesis.

The other studies consisted of retrospective clinical observations (Studies III-IX). In general, these are the materials and methods for these seven clinical trials:

Materials. The retrospective studies were based on all 2,670 patients provided with implants, consecutively treated on a routine basis at one specialist clinic (Clinic for Prosthodontics, Centre of Dental Specialist Care, Malmö, Sweden - Folktandvården Skåne AB, Specialisttandvård Malmö) during the period from 1980 to 2014. The study protocol was approved by the regional Ethical Committee, Lund, Sweden (Dnr 2014/598; Dnr 2015/72).

Definitions. An implant was considered a failure if presenting signs and symptoms that led to implant removal. Thus, a failed implant in our study is equal to a lost implant. The failures were classified into two types: (1) implants lost due to lack/loss of osseointegration and (2) fractured implants. Primary failures were those occurring until/at the day of the $2^{\circ}$ stage surgery (abutment connection). 
Inclusion and exclusion criteria. Patients with all modern types of implants with cylindrical or conical design were included. Zygomatic implants were not included in the study, as well as implants detected in radiographies, but without basic information about them in the patients' files.

Data collection. The dental records of all patients ever treated with implants in the aforementioned clinic were read in order to collect the data. The data were directly entered into a SPSS file (SPSS software, version 23, SPSS Inc., Chicago, USA) as the files were being read. The following data were collected:

(a) Implant-related factors: implant system, implant surface (turned/machined or enhanced surfaces, the latter including sandblasted, acid-etched, sandblasted + acid-etched, anodized, hydroxyapatite-coated surfaces), implant diameter and length, and implant design (cylindrical or conical), number of implants in maxilla and mandible;

(b) Site-related factors: implant jaw location (maxilla/ mandible), anterior or posterior location of the implant (sites from right canine to left canine teeth were considered anterior location), bone quantity and quality of the implant sites, which were classified at the time of surgery according to the Lekholm and Zarb ${ }^{188}$ classification, implant sites with previous implant failures (reoperation), elements adjacent to the implant (both sides edentulous, one tooth + edentulous, two teeth, implant + edentulous, two implants, one tooth + one implant);

(c) Patient-related factors: patient's sex, age of the patient at the implant insertion surgery, general health, and behavioral history. The presence of a medicament list in the patients' records was also used to correlate the use of certain drugs to specific health conditions. Health factors assessed: diabetes types I and II, hypertension, hypercholesterolemia, hypothyroidism, asthma, psoriasis, chemotherapy, and irradiation of the head and neck region. The patients were also classified according to the intake of the following medication types: antidepressants, immunosuppressives, bisphosphonates, antithrombotic agents (antiplatelet, anticoagulant, thrombolytic drugs), hormone replacement therapy in women, and medicaments to reduce 
the acid gastric production (proton pump inhibitors - PPIs). The behavioral factors assessed were smoking habits, use of snuff, and bruxism;

(d) Other factors: surgeon who performed the implant surgery, prescription of antibiotics (the prophylactic antibiotic regimen was usually starting 1-2 hours before surgery and going from 5-7 days postoperatively), bone graft procedures, reason for tooth extraction (periodontal disease, tooth fracture, trauma, advanced caries, tooth agenesis, other), type of implantsupported prosthetic restoration (single crown, partial bridge with 2-6 prosthetic elements, partial bridge with 7-10 prosthetic elements, full-arch, overdenture), number of days until failure, time between loss of an implant and replacement by another one, and follow-up time.

Statistical analyses. The SPSS software version 23 (SPSS Inc., Chicago, USA) was used for the statistical analyses. In general, the mean, standard deviation, and percentages were presented as descriptive statistics. The Kolmogorov-Smirnov test was performed to evaluate the normal distribution of the variables, and Levene's test evaluated homoscedasticity. The performed tests for the comparison of continuous data of two independent groups were Student's t-test or Mann-Whitney test, depending on the normality. The performed tests for the comparison of continuous data of two dependent groups were paired-samples $t$-test or Wilcoxon signed-rank test, depending on the normality. The performed tests for the comparison of three or more independent groups were one-way analysis of variance (one-way ANOVA) or Kruskal-Wallis test for continuous variables, depending on the normality. Pearson's chi-squared test or Fisher's exact test was used in the analysis of contingency tables of categorical data of independent groups, and McNemar's test for dependent groups. The degree of statistical significance was considered $p<0.05$.

- The use of other statistical analyses varied among the articles included in the thesis:

- Survival analyses: life table and Kaplan-Meier analysis;

- Logistic regression models: used at the patient-level as well as at the implant-level; 
- Generalized estimating equation (GEE) method: used at the implant-level to account for the fact that repeated observations (several implants) were available for a single patient;

Multilevel mixed effects parametric survival analysis: with a binomial distribution and a logit link function, while assuming an exchangeable working correlation structure.

Details specific to each of the seven retrospective clinical trials are described in the articles included at the end of this thesis. 


\section{RESULTS AND DISCUSSION}

In my work about oral implant failure I have decided to include 9 different scientific papers. However, since the topic or oral implant failure is so huge, this means that I have had to select 9 papers from the 33 papers $^{11-31,44,63,158,160,161,189-195}$ I have actually authored and published on this topic. I have, therefore, decided to present the results and discussion by thematic headlines going through my own results and those of others and discussing them in the same part of the thesis. In this chapter, the influence of several factors on implant survival/failure will be discussed. The aim is not to cover every possible factor affecting osseointegration, but the main issues. The papers included in the thesis will be referred to in the following text by their Roman numerals (see 'List of Papers'). Study I is not referred to in the following text, since it is a general review.

\section{Hardware}

Implant length

Many implant models are available for specific clinical applications, with varying lengths and diameters. The choice of implants depends on the type of edentulism, the volume of residual bone, the amount of space available for the prosthetic reconstruction, the emergence profile, and the type of occlusion. ${ }^{196}$ The use of implants may be restricted where there are limitations imposed by the geometry and volume of the alveolar bone. These restrictions are more common in the posterior regions of the maxilla and the mandible. ${ }^{197}$ Surgical treatment options to overcome these limitations include either supplementary bone augmentation procedures or the use of implants 
reduced in length. ${ }^{198}$ The advantages of using short implants include the avoidance of invasive bone augmentation surgery associated with donor site morbidity, additional treatment duration and financial burden. ${ }^{199}$

Some articles showed clearly that short implants failed more often than longer implants. ${ }^{200-205}$ Telleman et al. ${ }^{206}$ observed in their review that there is a tendency towards an increasing survival rate per implant length. The meta-analysis of Pommer et al. ${ }^{207}$ observed increased failure rates of short implants compared to longer implants with significant differences observed in the anterior and posterior maxilla. Another review observed that the failure rate appeared to be higher in shorter small diameter implants than in longer ones. ${ }^{208}$ However, others reported that implant length did not appear to significantly influence the survival rate. ${ }^{169,209-211}$ Some factors have been suggested to explain the difference found in some studies, such as the quality of the patient's bone, amount of bone-to-implant contact (BIC), crown-to-implant ratio, and stress on bone.

In sites associated with poor bone density and jaw bone resorption, a prevalence of short implants and/or wide-diameter implants might be used. In these particular situations, failure rates may be increased. Jaw shape and bone density must be considered as one of the most influential factors in implant survival. It should be understood that the length of the implant, in most of the studies reflects the state of jaw bone resorption. ${ }^{212}$ When the relationship between implant length and available jaw bone were examined, Herrmann et al. ${ }^{205}$ found that $29.4 \%$ of the $7-\mathrm{mm}$ implants were placed in jaws with jaw shape $\mathrm{E}$ and $25.5 \%$ were placed in jaw with jaw shape $\mathrm{D}$, according to the Lekholm and Zarb ${ }^{188}$ classification.

Compared with longer implants, there is less BIC when short implants are used, because of the smaller implant surface. ${ }^{211}$ However, as some studies show comparable survival rates for short and long implants observed, ${ }^{169,209-211}$ the difference in the total implant surface might not be crucial for implant survival.

Concerning crown to implant ratio, because of the extensive resorption in the posterior region, a higher crown to implant ratio is created over short dental implants, ${ }^{211}$ which might in theory contribute to an increase in implant failure rate. ${ }^{206}$ 
Several researchers ${ }^{213-218}$ have stated that short implants produce higher stress and strain over the bone because of the smaller contact area at the implant-bone interface in combination with a less homogenous stress distribution. ${ }^{219}$ Rubo and Capello Souza ${ }^{220}$ showed that the stress decreased $\approx 14 \%$ when the implant length increased from $10 \mathrm{~mm}$ to $13 \mathrm{~mm}$. Petrie and Williams ${ }^{221}$ reported that an increase in the implant length might facilitate a decrease in bone deformation and maximum stress compared with short implants. ${ }^{217}$ However, a finite element analysis (FEA) study conducted by Pierrisnard et al. ${ }^{222}$ found that the shear stress during oblique loads in implants is concentrated on the first $7 \mathrm{~mm}$ and observed no differences related to the implant length. It appears that, whatever the implant length is in the cancellous bone, stress is always located at the implant neck level and has a slight influence on the peri-implant bone stress values. ${ }^{222} \mathrm{~A}$ study of fixed single-unit restorations showed that a relationship between implant length and success may not exist, especially over $13 \mathrm{~mm}$ in length. ${ }^{223}$ No relationship between initial mobility and implant length has been established, and mechanical analyses have supported the view that increasing the implant length may only increase the success rate to a certain extent. ${ }^{224}$ Moreover, according to Geng et al., ${ }^{225}$ if the bone is of good quality (bone type I), the implant length does not influence the treatment success because the degree of inequality in the stress distribution over the interface depends on the difference in the elastic modulus of both structures; with better bone quality, there is less difference in the stiffness of these two structures. Some have stated that in the long term, the length of the implant could be more important than the diameter, because before oral cavity exposure and loading, vertical osseous loss is present, and it can be close to $0.2 \mathrm{~mm}$ per year; in the future, the implant may lose important contact between bone and implant surface. ${ }^{226,227}$

For immediate implant cases, the literature reports contradictory statements about the influence of implant length on bone biomechanics based in finite element analyses. In a clinical follow-up study of Schnitman et al., ${ }^{228}$ the authors conclude that $50 \%$ failures of immediately loaded external hexagon implants during 10 years were related with utilization of implants shorter than $10 \mathrm{~mm}$. Miyamoto et al. ${ }^{229}$ stated that length is a weak factor for primary 
stability in immediate implants cases. However implants longer than $10 \mathrm{~mm}$ may result in a considerable strain reduction. ${ }^{230}$ Kong et al. ${ }^{231}$ concluded that greater implant length can reduce the cortical and cancellous bone stress and the implant displacement during axial loads in immediate implant cases.

Surface treatment may have some influence on the results of short implants. The review of Renouard and Nisand ${ }^{197}$ demonstrated a trend toward an increased failure rate with short- and wide-diameter implants. However, they identified that out of the 12 studies which have documented an increased failure rate with short implants, 11 used of machined-surfaced implants. . $^{53,200,202,204,205,212,232-236}$ In the other hand, out of the nine studies which have indicated that implant length did not influence the survival rate, six $^{168,169,209,237-239}$ were performed with textured-surfaced implants. As an example, Feldman et al. ${ }^{209}$ demonstrated survival rates of $91.6 \%$ and $97.7 \%$, respectively, in a the 5-year survival rate of short machined-surfaced and short dualacid-etched surfaced implants. In this study a statistically significant difference in CSR was found between short machined-surfaced implants and standard machined-surfaced implants. It is noteworthy that this difference increased dramatically in the posterior maxilla. For the dual-acid-etched implants, no statistically significant differences were demonstrated between short and standard-length implants. When comparing, the CSRs in poor bone density, Feldman et al. ${ }^{209}$ demonstrated that short dual-acid-etched implants provided better outcomes than machined-surfaced implants $(96 \%$ and $86.5 \%$, respectively). Kotsovilis et al. ${ }^{240}$ concluded from their systematic review that the placement of short rough-surface implants is not a less efficacious treatment modality compared with the placement of conventional rough surface implants. In the meta-analysis of Pommer et al. ${ }^{207}$ turned implants demonstrated a significant impact of implant length overall as well as in the anterior and posterior maxilla. However, no significant overall effect could be substantiated for rough-surfaced implants.

It is worth mentioning that short implants should not be compared with long implants placed in good bone density, but should then be compared with the failure rates and morbidity of advanced surgical procedures such as bone grafting, sinus lifting, and alveolar nerve lateralization. Thus both survival rates and morbidity must 
be considered when comparing the outcomes of short implants and advanced surgical procedures to allow adequate comparisons. ${ }^{197} \mathrm{~A}$ RCT aimed to evaluate whether short dental implants could be an alternative to bone augmentation together with placement of longer implants in posterior atrophic jaws; results revealed that, 1 year after loading, short implants achieved similar results compared with longer implants placed in augmented bone. ${ }^{241}$ The authors concluded that short implants might be a preferable choice to bone augmentation because the treatment is faster, less expensive, and associated with less morbidity.

A review ${ }^{197}$ analyzing 53 human studies observed that studies which have used surgical preparation adapted to the bone density, texturedsurfaced implants, and modified case selection have reported survival rates for short implants and for wide-diameter implants which were comparable with those obtained with long-implants and standarddiameter implants.

It is important to stress that there is still no consensus in the literature on the definition of a short implant. ${ }^{211}$ Some authors consider $10 \mathrm{~mm}$ the minimal length for predictable success; thus, they consider any implant $<10 \mathrm{~mm}$ in length as short. ${ }^{242}$ Others defined an implant length of $10 \mathrm{~mm}$ also as a short Implant. ${ }^{198}$

\section{Implant diameter}

The original standard Brånemark System implant was $3.75 \mathrm{~mm}$ in diameter, but owing to treatment needs, i.e. the adaptation of the implant to the existing anatomy, implants of several other diameters were introduced as well. Today, many types of implants are available, which have different external designs, surfaces, platforms, connections, diameters and lengths.

It has been said that the diameter of the implant could be considered the most effective factor when compared with the length. ${ }^{243-245}$ It has been suggested that increasing the implant diameter appears to compensate for the implant length, ${ }^{246,247}$ even though the results of a meta-analysis ${ }^{207}$ found no influence of implant diameter on the failure rate of short implants.

Availability of bone in the edentulous ridge determines the implant dimensions that can be used in that site. ${ }^{248,249}$ The main 2 advantages of narrow implants are the ability to apply less invasive surgical 
procedures when there are circumferential bone deficiencies and the ability to place narrow implants in reduced interradicular spaces, such as the edentulous ridge of the mandibular incisors. ${ }^{250,251}$ Thus, narrow implants are indicated in areas with reduced horizontal ridge width or mesio-distal prosthetic space. ${ }^{252}$ Conceptually narrow implants are often placed in compromised clinical scenarios or subjected to higher risks of increased implant body fracture possibility or prosthetic complications..$^{253,254}$

In some cases, surgeons try to use narrow implants, which are advantageous in treating bone defects and avoiding anatomical risk areas. ${ }^{255}$ In the use of narrow diameter implants, the similarity of the survival rates to those of regular implants does not extend to their mechanical behavior. ${ }^{248,256}$ Biomechanically, the implant diameter appears to influence the stress concentration at the implant as well as at the surrounding bone and, hence, affects the success rate. ${ }^{213,257}$ In vitro studies and finite element analyses have illustrated that stress values affecting the crestal cortical bone are reciprocal to the dental implant diameter, which means that especially small diameters result in disadvantageous stress peaks at the implant-bone interface. ${ }^{258}$ Ding et al. ${ }^{259}$ showed that the stress values at the implant-bone interface rise more significantly by reducing the diameter from $4.1 \mathrm{~mm}$ to 3.3 $\mathrm{mm}$, compared to reducing the diameter from $4.8 \mathrm{~mm}$ to $4.1 \mathrm{~mm}$. As a biological implication, inadequate overloading of narrow-diameter implants might possibly lead to disadvantageous peri-implant crestal bone resorption resulting in clinical complications. The implant itself is also more prone to fatigue fracture as a result of a reduced implant diameter. ${ }^{253}$ An increase of the implant diameter, comparing the same load, produces a decrease in stress in the implant and the peri-implant bone. ${ }^{244,258-262}$ Lower stress values in the bone and the implant have been observed in implants with larger diameter, which is justified as a better distribution by the largest contact area between the implant and peri-implant bone. ${ }^{215,262,263}$ The usefulness of small-diameter implants has to be discussed with an awareness of their potential limitations. It has been estimated that a 3.3-mm-diameter, screw-shaped, c.p. Ti implant possesses $25 \%$ less resistance to fracture when compared with a similar regular-diameter implant. ${ }^{264}$ Decreasing the diameter also means increasing the risk for implant fracture because of reduced mechanical stability and increasing the risk for overload. ${ }^{265}$ 
An increase in the implant diameter produces significant stress reduction, particularly in the cortical bone, ${ }^{262}$ whereas the length of the implant has a certain influence on the stress patterns in the trabecular bone implant interface. ${ }^{258,266}$ Iplikcioglu and Akça ${ }^{267}$ demonstrated that the implant length does not influence stress reduction over the peri-implant bone, in contrast to the implant diameter. When the implant diameter increases by $0.25 \mathrm{~mm}$, the contact surface is augmented by $\approx 10 \% .{ }^{268}$ In a direct comparison, the use of a 5.0 $\mathrm{mm}$ diameter implant that is $6 \mathrm{~mm}$ long increases the surface area available to contact the bone similar to that of a $3.75 \mathrm{~mm}$ diameter implant that is $10 \mathrm{~mm}$ in length. ${ }^{201}$ An increased implant surface area of wider implants can engage more cortical bone. It has also been shown in a study in rabbit tibia that wider implant diameters resulted in increased removal torque values. ${ }^{269}$ In 1994, Lazzara ${ }^{270}$ recommended the immediate placement of a wide-diameter implant after extraction. The volume of the alveolus is best filled by increasing the diameter of the implant to provide adequate primary stability.

Finite element studies suggest that implants with a wider diameter are more favorable in reducing the stress distribution in bone surrounding the implants compared to more narrow implants. ${ }^{271,272}$ The use of wider diameter implants allows engagement of a maximal amount of bone and improved distribution of stress in the surrounding bone, ${ }^{254}$ providing more BIC, bicortical engagement, and immediate placement in failure sites. ${ }^{211}$ The use of wider components also allows for the application of higher torque in the placement of prosthetic components. The use of wide implants, however, is limited by the width of the residual ridge and esthetic requirements for a natural emergence profile. ${ }^{273}$

Implants of 5- and 6-mm diameter are, respectively, three and six times more resistant to fracture than standard implants. The supporting surface of the top area of 5- and 6-mm implants is increased by $122 \%$ and $281 \%$, respectively, compared with standard implants. ${ }^{274}$ This feature allows better distribution of occlusal forces. Petrie and Williams ${ }^{221}$ concluded that the increase of the implant diameter decreases the stress in the alveolar crest 3.5-times, with a better effect for short and tapered implants because the diameter predominates over the length and taper. Kong et al. ${ }^{275}$ reported that the narrowing of the implant neck also induces a more favorable 
stress distribution in the bone. It is hard to say whether all the biomechanical advantages of wider implants may result in more advantageous clinical conditions when it comes to MBL. A review ${ }^{197}$ analyzing 53 human studies observed that there was no relationship between MBL and implant diameter in most of the studies, which reported rather low changes in crestal bone levels.

Wide-diameter implants are indicated in cases of poor bone quality and inadequate height of bone and to replace nonintegrated or fractured implants. Wide-diameter implants can be used in special situations in which they can increase the surface area available for implant anchorage and improve their primary stabilization. Such wide implants provide a means of managing difficult bone situations, such as inadequate quality and/or height of bone. ${ }^{226,270,274} \mathrm{~A}$ biological impediment to the use of wide-diameter implants can be a lower blood supply because of minimum existing cancellous bone. ${ }^{276}$ Wider implants are used when bone is scarce and the influence of diameter on BIC may not translate into a clinical advantage. ${ }^{277}$

Some studies involving implants of various diameters reach the conclusion that failure is more common for the $5.0-\mathrm{mm}$ implant as compared to the $3.75-\mathrm{mm}$ and $4.0-\mathrm{mm}$ standard design. ${ }^{277,278}$ As possible causes for the outcome, the $5.0-\mathrm{mm}$ implant was frequently placed as a rescue implant in poor quantities of low-quality bone without utilizing an adapted surgical technique and without extended healing time, ${ }^{277,279}$ and the use of this implant diameter when primary stability could not be achieved with a standard-diameter implant. ${ }^{277}$ This view was supported by the study of Mordenfeld et al. ${ }^{280}$ in which wide-diameter implants were placed in unfavorable situations such as poor bone density, and compromised bone volume. As such, in some studies a trend could be drawn with a prevalence of early failures. ${ }^{223,277,281}$

Moreover, implants of wider diameter are usually placed in the posterior areas of the jaws. Due to the anatomical limitations (maxillary sinus, inferior alveolar canal), these implants are also shorter. Some studies have observed that shorter implants have a higher failure rate than longer implants. ${ }^{200-205}$ And in posterior partially edentulous arches it is often difficult to achieve implant stability because of both compromised bone quality and/or quantity. Initial implant stability has been pointed out as another parameter of importance for establishing osseointegration. ${ }^{35}$ 
Furthermore, the 5.0-mm-diameter implant usually lacks a neck and has a differently threaded profile, compared to standard and 4.0-mm-diameter implants. Certainly, this might also have had an impact on the treatment results, both with regard to implant survival and MBL. The implant neck may play an important role in stabilizing the implant during its final tightening when placed, especially in poor quality bone and in combination with a thin marginal compact bone layer. ${ }^{277}$

There are no significant differences in failures rates when narrow-diameter implants are compared to their regular-diameter counterparts, according to two literature reviews on the subject. ${ }^{207,208}$

It is worth mentioning again that the review of Renouard and Nisand ${ }^{197}$ including 53 human studies observed that studies which have used surgical preparation adapted to the bone density, texturedsurfaced implants, and modified case selection have reported survival rates for short implants and for wide-diameter implants which were comparable with those obtained with long-implants and standarddiameter implants.

\section{Implant design (non-threaded versus threaded, cylindrical versus tapered)}

When considering only studies comparing the survival rates between cylindrical non-threaded and threaded implants, four studies showed that there is a greater risk of implant failures with insertion of nonthreaded implants when compared to threaded implants. ${ }^{168,227,238,282}$ The statistical analysis of four other studies indicated that the type of implant did not influence the failure rate of the implants. ${ }^{210,283-285}$ It is worth to mention the case of the German IMZ implant, a solid non-threaded cylindrical implant with a plasma sprayed titanium surface. Life table analysis revealed an extremely low CSR for maxillary implants (37.9\% at 100 months of follow-up) in a clinical study. ${ }^{286}$ According to Karoussis et al., ${ }^{282}$ the possible reasons for inferior survival rates of non-threaded implants in comparison to threaded implants are largely unknown and may only be speculated upon.

However, others have hypothesized that the differences could be related to accumulation of stress around the implant neck. Implant design has been proven to have an effect on peri-implant bone when loaded. ${ }^{287}$ Implants with threaded designs are preferred because they 
have a greater ability to reduce shear force than cylindrical nonthreaded implants. ${ }^{287,288}$ In general, stresses developing in the neck region of threaded implants are lower than those around cylindrical implants. ${ }^{289}$ Moreover, studies ${ }^{288,290,291}$ have suggested that the use of threaded implants decreases implant mobility at the time of placement, disperses interfacial stresses, and increases the contact area between the implant and bone.

Another possible explanation for the extremely poor clinical results of the cylindrical implants relates to the most common surface modification used at the time for this implant design, the plasma sprayed surface. This surface modification presented extremely high values of roughness, which was a short-term disadvantage for bone tissue. ${ }^{292}$

Concerning cylindrical and tapered implants, it has been proposed that tapered implants favor load distribution to surrounding bone by mimicking the natural root form. ${ }^{293}$ The theory behind the use of tapered implants is to provide for a degree of compression of the cortical bone in a poor bone-implant site. ${ }^{294}$ Some studies have investigated the difference between these two different implant designs. O'Sullivan et al. ${ }^{294}$ analyzed the mechanical performance and the primary and secondary stability characteristics of tapered implants when compared with the standard Brånemark parallel-walled design placed in rabbits, and observed that the tapered implants resulted in a better primary stability. Sakoh et al. ${ }^{295}$ investigated the differences with respect to primary stability between a conical implant and a cylindrical screw-type implant, in an in vitro study. The conical implant showed significantly higher primary stability than the cylindrical hybrid implant using the insertion torque, Periotest, and push-out tests. In an experimental study on dogs, Kim et al. ${ }^{296}$ compared the mechanical properties of tapered and parallel-walled implants in terms of the success rate. Maximum insertion torque (MIT) and maximum removal torque (MRT) were assessed. The results showed significantly high values of MIT and MRT for tapered implants than parallel-walled implants. ${ }^{296}$ Romanos et al. ${ }^{297}$ placed 90 parallel-walled and 90 tapered implants in freshly slaughtered cow ribs and noticed that tapered implants achieved greater stability than parallel-walled implants, measured by resonance frequency analysis (RFA). ${ }^{297}$ Similar results were reported in another experimental study 
performed by the same group. ${ }^{298}$ Toyoshima et al. ${ }^{299}$ observed that the mean MIT and implant stability quotient (ISQ) were significantly higher for tapered implants than for cylindrical ones in an ex vivo model. The general results that tapered implants may present higher primary stability than cylindrical implants might have an impact on the implant survival rates, as it has been stated that primary stability is a crucial prerequisite for achieving osseointegration. ${ }^{299}$

\section{Implant surface}

To achieve successful BIC (i.e. osseointegration), oral implants placed according to a two-stage surgical protocol have been advocated to remain unloaded for a healing period of 3-6 months. ${ }^{300}$ The biological rationale supporting this approach resides predominantly in the fact that implant micro-movements caused by premature loading during wound healing may promote connective tissue encapsulation of the implant rather than healing by direct BIC. ${ }^{300}$ Numerous efforts have been made to make implant therapy more appealing for potential patients by simplifying clinical procedures. One of these efforts has been a general reduction of the healing period using new titanium surfaces that shorten and improve the osseointegration process. ${ }^{301}$ Nevertheless, this reduction of the initial osseointegration period should not result in a higher failure rate of the dental implants inserted with the chosen loading protocol. ${ }^{302}$

To improve its functional properties, the titanium implant surface is generally modified by increasing the surface roughness on the titanium surface or by altering the crystal structure and chemical composition (Figure 9). Dental implant surfaces are modified in an attempt to optimize the cell and tissue interactions. ${ }^{303}$ When a biomaterial is inserted into living body, it absorbs proteins before cells adhere to its surface. ${ }^{304}$ These proteins significantly affect the attachment, adhesion, and spreading of osteoblasts, the cells that form bony tissues. ${ }^{305}$ For such cells, the implant's surface-charge influences their reactions to the implant, by affecting the type and amount of proteins attached on its surface. ${ }^{306}$ The moderately enlarged surfaces provide better possibilities for micro-biomechanical retention due to larger tissue contacts and thus more retention for proteins (on the nanoscale level) to attach and new bone formation. ${ }^{307-311}$ 

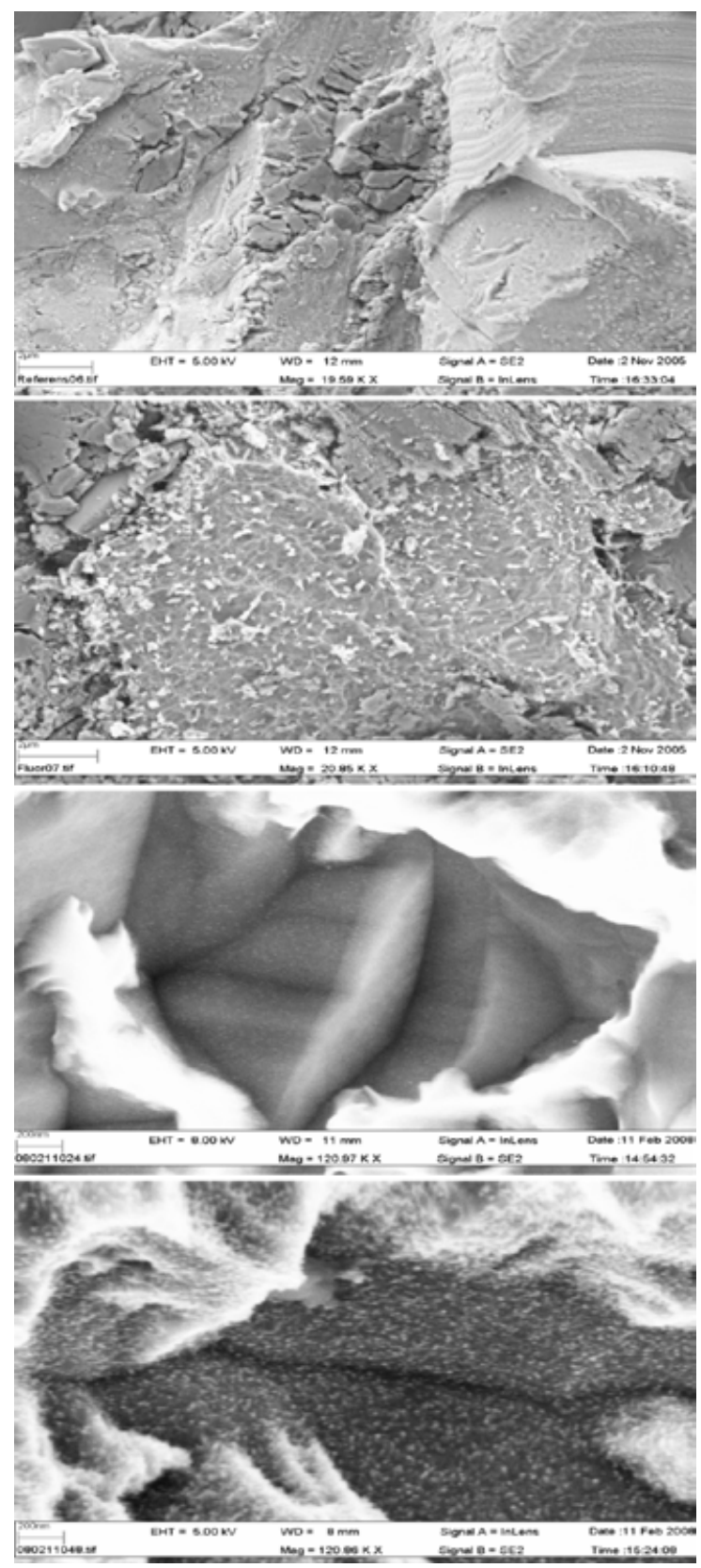

Figure 9. High resolution SEM images of TiOblast (top image) and Osseospeed (middle upper image) implant surfaces (Scale bar $2 \mu \mathrm{m}$ ). High power SEM images of SLA (middle lower image) and SLActive (bottom image) implant surfaces (Scale bar $200 \mathrm{~nm}$ ). There are clear nanopatterns on Osseospeed and SLActive implant surfaces. 
In addition to surface roughness, the surface physics is another factor important for peri-implant bone apposition, because it influences surface charge and surface wettability. Surface wettability is largely dependent on surface energy, and influences the degree of contact with the physiologic environment. Increased wettability enhances interaction between the implant surface and the biologic environment. ${ }^{312}$ Textor et al. ${ }^{313}$ concluded that a certain similarity of the clean hydrophilic titanium oxide surface to water can be assumed as a consequence of extensive hydroxylation/ hydration of the oxide layer. This leads to high wettability by water ${ }^{314}$ and to an interaction of the surface with the water shell around biomolecules such as proteins. Such an interaction might accelerate and enhance bone deposition and therefore osseointegration. ${ }^{315}$ In addition, this also increases removal torque values during this early healing phase. ${ }^{316}$ This in turn might allow for further reductions in the time needed for healing around the implant or might provide more stability early in the course of healing, when the stability of the implant is generally decreased. ${ }^{301}$

In the late 1980s and 1990s, several research groups started to examine altered titanium surfaces, and focused on subtractive surface techniques such as sandblasting and/or acid-etching procedures. ${ }^{292,317-325}$ Various techniques have been used over the years, such as sandblasting, ${ }^{292,320-323}$ acid-etching, ${ }^{326-329}$ a combination of acidetching and sandblasting, ${ }^{318,330,331}$ oxidation methods, ${ }^{332,333}$ anodic plasma-chemical treatments, ${ }^{334}$ calcium phosphate and hydroxyapatite coatings on the titanium surface, ${ }^{335}$ the fabrication of nanotubular structures on a titanium surface by anodic oxidation, ${ }^{336}$ fluoride modification of the titanium surface, ${ }^{337-339}$ the addition of calcium on the surface,,$^{340,341}$ and the incorporation of magnesium..$^{342-345}$ Several of these experimental studies demonstrated that the new titanium surfaces had better bone integration than machined titanium surfaces. It was also showed a significantly higher removal torque values and a greater percentage of BIC adjacent to moderately roughened titanium surfaces, compared with plasma sprayed, machined or polished titanium surfaces. Clinical studies evaluating implants with rougher surfaces usually show short- and long-term higher survival rates when compared to turned implants, $28,37,191,346,347$ (Study III included) but this was not always the case. ${ }^{76,210,348-351}$ An extremely rough surface may 
not be ideal for osseointegration. It was shown that a highly increased surface roughness compared to a moderately increased one is a shortterm disadvantage for bone tissue. ${ }^{292,320-322}$ So the correct development was not to produce "rougher surfaces" but to achieve a moderately rough surface. Plasma-sprayed rough implants did seem to give rise to more MBL than did smoother implants. ${ }^{352,353}$

When it comes to MBL, it was reported that a surface roughness of more than $2 \mu \mathrm{m}\left(\mathrm{S}_{\mathrm{a}}\right)$ is associated with a higher risk of periimplantitis. ${ }^{354}$ Rougher implant surfaces are more susceptible to accumulation of bacteria on hard surfaces. ${ }^{355,356}$ Bacterial infection, characterized as bacterial colonization and biofilm formation on dental implants, is an important risk factor for peri-implantitis. A roughened surface does not only increase the susceptibility for periimplantitis, but also reduces the treatment efficacy of the bacteria biofilm. ${ }^{357}$ The progression of ligature induced bone resorption in dogs is more pronounced at implants with a moderately rough surface than at implants with a turned surface. ${ }^{358}$ These results must be carefully interpreted due to the animal model. However, this could mean that with rough surface, the prevalence of peri-implantitis is not higher but once there is a peri-implantitis the progression is increased compare to the turned surface. ${ }^{359}$ Hence, moderate surface modifications may improve implant therapy in terms of speeding up the treatment, but may be disadvantageous for the patients prone to peri-implantitis. ${ }^{360}$ However, it must be pointed out that these viewpoints so far are speculative in nature. There is no clinical evidence available that moderately rough surfaces see more clinical problems with peri-implantitis of other surfaces. ${ }^{361}$

\section{"Copycat" implants and implant systems that were withdrawn due to clinical problems}

Some dental implant companies have been researching the science of dental implants for years and years. There are copycat companies that are manufacturing cheap, low-quality knock-off implants that are marketed to dentists as low-cost dental implants. Often these copycat implants look very similar but unfortunately do not carry guarantee of high standards and research based quality, i.e. they are not all subject to the same rigor of material quality control and continuing quality verification. Therefore, they might not ensure an identical outcome. 
Many implant companies try to take advantage of research undertaken by the more established providers, stating "we use the same materials" or "the principles are the same for all dental implant systems", when clearly it is not acceptable to make such claims about systems that are not supported by their own research. ${ }^{362}$ Good two-way communication can be an excellent way to find out more about the products available, and the choice should be based on known and extensive research with comprehensive documentation. It's unlikely that cheaper copycat products will have this scope of evidence. ${ }^{362}$ Although is supposedly assumed that the copycat implants might not perform as well as the dental implants that have been on the market for years, clinical studies on the performance of copycat implants are lacking.

Some implant systems with designs of their own have failed in the long run and been withdrawn from the market. In some cases the precise reason for the system failing was easy to pinpoint, but in the majority of cases it must be regarded as uncertain whether one implant characteristic or a combination of characteristics was responsible for the poor clinical outcome. The crystalline bone screw of aluminum oxide had poor strength and the estimated 5 year success rate was only $25 \% .{ }^{363}$ The Frialit implant of aluminum oxide was introduced in the 1980s with, for its time, very good clinical research behind it. ${ }^{364,365}$ However, a $41 / 2$ year follow-up (average) reported only $87 \%$ survival rate. ${ }^{366}$ Other authors confirmed the poor rate of long term success with Frialit 1 and the aluminum oxides did not meet the expectations in terms of long term stability and was finally withdrawn from marketing. ${ }^{367}$ Single crystal aluminum oxide implants marketed by Kyocera of Japan was allegedly most popular, but when clinical results were scrutinized implant fractures resulted in quite poor 5- and 10-year outcomes of, respectively, $75.7 \%$ and $64.7 \%^{368}$ and the system disappeared.

The Core Vent implant, allegedly the most commonly used brand in the US around 1990, was a hollow cylinder of titanium 6 aluminum 4 vanadium material, with some threads in the construction. However, when tested in a clinical study ${ }^{369}$ analyzing 47 consecutively placed core vent cylinders, the survival of 1-4 years was only $71 \%$ and the success rate over the same time was estimated to $9 \%$. The implants lost marginal bone at a most rapid rate and were unable to maintain the osseointegrated state and the system was withdrawn in 1991. It is 
possible that the problems with Core Vent implants were associated with the hollow cylinder design, but precise data documenting this hypothesis are lacking.

IMZ was a German solid cylinder implant with a plasma sprayed titanium surface. When analyzed clinically without a radiographic documentation, the system seemed to function quite well, ${ }^{370}$ but this was not the case at all over longer times of follow up when a severe resorption of marginal bone was documented. ${ }^{352,353}$ The IMZ implants never displayed even close to a steady state situation with respect to MBL that was documented to $0.5 \mathrm{~mm}$ annually (average) between year 1 and year 5 and accelerated thereafter. ${ }^{371} \mathrm{~A}$ clinical report finding only $37.9 \%$ survival of maxillary IMZ implants 100 months of follow-up ${ }^{286}$ was followed by withdrawal of this oral implant system in 1997. Reasons for MBL may have been the cylindrical shape without threads of the implants in combination with the plasma sprayed surface. Another solid cylindrical implant with a plasma sprayed HA-coated surface was the Calcitek implant. It likewise failed to display maintained marginal bone levels ${ }^{45,372}$ and were withdrawn from sales due to this, even if short term reports without bone loss evaluations were quite positive. ${ }^{373}$ The type of HA-coatings used in those days was plasma sprayed with a minimal thickness of some 50-75 $\mu \mathrm{m}$. In animal studies it was demonstrated that such thick HA-coatings were attacked by macrophages/foreign body. Giant cells that initiated resorption of the bone as well ${ }^{335}$ which may have been an additional problem with the Calcitek cylinders.

The Bicortical screw implant caused major problems in form of MBL possibly due its design in form of a needle-like device with threads. Attempts to place this implant in patients ended up with major problems due to the loss of harboring bone and most implants were removed at nearby University clinics. This implant design with due clinical problems resulted in Scandinavian court cases with banning of its further usage. ${ }^{374}$

The clinical problems observed with Nobel Direct implants ${ }^{375,376}$ was probably due to strange clinical recommendations to grind the implant down in situ and, thereafter, loading it directly. Such actions resulted in large bone loss and implant failure in one third of cases at 18 months of follow-up, ${ }^{377}$ presumably due to vibrations from the rotating instruments in combination with later direct loading of the 
implants. The Swedish control body; Läkemedelsverket, forbade the sales of this implant until instructions for its use were re-written. ${ }^{378}$ Quite acceptable clinical results with the same Nobel Direct implant was reported for devices not ground down in situ. ${ }^{377}$

\section{Surface contamination}

In order to discuss the factor of surface contamination, it would be impossible to not mention the work that Dr. Dirk Duddeck has been conducting. His work focuses in investigating the quality of dental implant surfaces, which depends on a number of different factors. Surface contamination is one of them.

Once the titanium implant blank has been computer numerical control (CNC)-machined, it is further processed using different techniques that ultimately result in the product's specific surface structure. ${ }^{379}$ There must be a distinction between the manufacturing process, from the CNC-machined blank with product-specific surface processing, and the handling of the sterile-packaged implant. Various production processes ultimately contribute to product quality: the production itself, the cleaning steps, post-production handling (i.e., quality control), packaging and sterilization processes and the packaging itself. ${ }^{379}$ The packaging itself may also be a source of organic contamination of the implant surface. ${ }^{380}$

Several implants in the study that did not feature contact-free packaging but were delivered in soft sealed polyethylene bags exhibited various amounts of organic contaminants or plastic residue, depending on their surface roughness. ${ }^{380}$ The manufacturers and users of implants which in the study of Duddeck and Neugebauer ${ }^{380}$ exhibited some organic contaminants report clinical success rates that do not differ from those of implants by other manufacturers. Statements such as "Our success rates are high, so what is the problem?" may be fully justified when discussing statistical average. But the question remains: What happens to those organic contaminants once in the bone? It is hard to imagine that those organic contaminants should have a positive influence on osseointegration. At best, there will be areas with a lack of osseointegration, i.e. smaller areas or entire outer thread edges with less bone contact, because the osteoblasts will have better things to do than to settle on polyethylene residue. Or macrophages cause phagocytosis of these 
materials during the first remodeling phase, which would amount to biological purification of industrially manufactured surfaces. What then becomes of the phagocytized materials is another question. ${ }^{380}$ Particles with a diameter of less than $10 \mu \mathrm{m}$ are susceptible to uptake by macrophages through phagocytosis. ${ }^{381}$ Particle-induced macrophage activation is associated with an increased osteoclastogenesis and may therefore cause increased bone resorption. ${ }^{382}$ Implant debris activates macrophages to secrete TNF- $\alpha$, IL-1 $\beta$, IL-6, and PGE2, which stimulates differentiation of osteoclast precursors into mature osteoclasts and increases peri-prosthetic bone resorption, which is not replaced by new bone. ${ }^{383,384}$

It is possible that residue on implants is tolerated in healthy patients. But can we be sure that this is also the case in immunocompromised high-risk patients? How about extended augmentation sites? Therefore, surfaces ought to be properly cleaned to avoid increasing the clinical risks with implants. These questions, however, should actually not arise in the first place, because impurities are preventable, as this study clearly shows. ${ }^{380}$

There is also the concern of the inorganic impurities. There is continuing concern regarding the release of soluble metal ions [aluminum $(\mathrm{Al})$, chromium $(\mathrm{Cr})$, vanadium $(\mathrm{V})$, cobalt $(\mathrm{Co})$, and titanium (Ti), among others], which bind to proteins, remain in solution, and disseminate into the surrounding tissues, bloodstream, and remote organs. ${ }^{381}$ At least when it comes to orthopedic implants, patients with titanium-base alloy implants have demonstrated elevated titanium, aluminum, and vanadium levels in joint pseudocapsules (with up to $200 \mathrm{ppm}$ of titanium, six orders of magnitude greater than that of controls, $880 \mathrm{ppb}$ of aluminum, and $250 \mathrm{ppb}$ of vanadium). ${ }^{385}$ Implant debris can elicit inflammation, osteolysis, hypersensitivity, and neuropathy. Concerns about implant debris becoming carcinogenic and toxic, or either, persist. ${ }^{381}$

The clinical relevance of minuscule particles and contaminants on dental implants remains speculative. Parameters such as total surface area and particle size do matter, and the most important determinant of inflammation is probably particle load, i.e. concentration of phagocytosable particles per tissue volume, assuming particles are under $10 \mu \mathrm{m}$ and able to be phagocytized by cells. However, it is important to note that all particles are not equally bioreactive. ${ }^{381}$ 


\section{Surgery}

\section{Use of prophylactic antibiotics}

The risk of infection in any dental implant surgical procedure depends on several patient factors, management procedures during implant placement, and the care of the surgical team in maintaining the basic principles of surgery and asepsis. ${ }^{386,387}$ Although many surgeons believe that antibiotic coverage for implant placement is not necessary, they continue to use them to protect against claims of malpractice. ${ }^{388}$ However, the benefits of antibiotic prophylaxis in relatively normal patients undergoing routine surgical procedures, such as the placement of endosseous dental implants, remain a controversial issue, ${ }^{389}$ even for the placement of implants into infected sites. ${ }^{189}$ Moreover, there is a general trend of providing inappropriate antibiotic prescriptions in dental practice, usually in excess, therapeutically as well as prophylactically. ${ }^{390}$

Among the possible disadvantages of routine antibiotic prophylaxis are the risk of drug-related complications (such as hypersensitivity and anaphylaxis), which may be greater than the risk of postoperative infection occurring with that particular operation, and the possibility that its use may lead to lax surgical techniques and actual increase of complications. ${ }^{389}$ In addition, in view of the growing concerns that health care professionals have regarding the potential for an increase in new antibiotic-resistant bacteria, antibiotic prophylaxis guidelines are being reviewed and modified by health care professionals. ${ }^{388}$

The indiscriminate use of antibiotics may produce unwanted adverse effects in patients, cause unnecessary costs, and may add to the increasing emergence of resistant bacterial strains. The recent increases in resistant bacteria observed in the health care environments represents a major concern that requires all health care professionals to closely examine each procedure in which prophylactic antibiotics are frequently used in order to determine their true effectiveness. ${ }^{391}$ Even though oral surgeons often prescribe antibiotics routinely following implant surgery, the usefulness of pre- and post-operative antibiotics in reducing the failure rates of endosseous implants remain unclear. ${ }^{11}$ Most of the conclusions about the use of prophylactic antibiotic regimen in implant dentistry originate from retrospective analyses, with multiple operators, different antibiotic prescriptions, and different regimens. Despite the widespread use of oral implants 
in dentistry, we are still lacking a clear guideline on if, when, and how to prescribe antibiotics for this type of surgery. ${ }^{392}$

A recent systematic review and meta-analysis on subject ${ }^{14}$ suggested that the difference between the use versus nonuse of antibiotics significantly affected the implant failure rates $(p=0.0002)$, with a risk ratio (RR) of 0.55 (95\% CI 0.41-0.75). This means that healthy patients taking prophylactic antibiotics for implant placement surgery may experience a $45 \%$ lesser risk of having an implant loss in comparison to healthy patients not taking prophylactic antibiotics for the same procedure. Moreover, there were no apparent significant effects of prophylactic antibiotics on the occurrence of postoperative infections in healthy patients receiving implants $(p=0.520)$.

It is also important to mention another variable, the surgeons' surgical experience, which was evaluated together with the use of prophylactic antibiotics in one study. ${ }^{389}$ When implant survival rates were compared according to surgeons' previous implant surgery experience and use or non-use of preoperative antibiotics, those surgeons with greater than 50 implant placements prior to the study had a slightly higher implant survival rate $(2.9 \%)$ when preoperative antibiotics were used. There was an even greater increase in survival rate $(7.3 \%)$ when less experienced surgeons $(<50$ previous implant placements) used preoperative antibiotic coverage, which would suggest that as surgical skill increases with experience, there is less reliance on antibiotics for survival of implants. However, this should not be interpreted that antibiotics will make up for lax and poor surgical techniques.

Although the exact mechanism by which prophylactic antibiotics has a significant effect on implant survival is not known, it may be that a more aseptic local environment during the time of implant placement and in the immediate perioperative period results in improved healing and, ultimately, a better state of osseointegration. ${ }^{389}$

\section{Insertion torque}

The goal of achieving primary stability at the time of implant placement is to limit excessive micromotion at the bone-implant interface, which could prevent osseointegration..$^{393,394}$ The use of a slightly narrower final drill with a tapered implant design has been often associated with elevated insertion torque ${ }^{395-399}$ and localized 
bone compression, ${ }^{400}$ and these factors may help to increase primary implant stability. ${ }^{401}$ The achievement of high insertion torque is likely related to the achievement of higher primary fixation ${ }^{402}$ and to the amount of micromotion. ${ }^{403}$ The measurement of insertion torque values to quantify primary implant stability has been reported. Some examples follow.

Maló et al. ${ }^{404}$ assessed implants placed in the esthetic zone, of which only those reaching a minimum installation torque of 32 $\mathrm{NCm}$ were immediately loaded. With the CSR of $96 \%$ after only 2 years, the authors stated that the minimum installation torque used in the study was safe for immediate function. Hui et al. ${ }^{405}$ studied immediate placement of single-tooth implants in the esthetic zone with a minimum installation torque of $40 \mathrm{NCm}$, with an immediate provisional protocol. No failures were reported, however, the implants were observed between 1-15 months. Lorenzoni et al. ${ }^{406}$ evaluated the immediate loading of single implants placed in fresh extraction sockets in the anterior maxilla, and recommended immediate provisionalization for single implants inserted into fresh extraction sites only when a minimum torque value of $32 \mathrm{Ncm}$ is reached. Nikellis et al. ${ }^{399}$ observed that the immediate loading of all 190 dental implants in their study with a minimum of $32 \mathrm{NCm}$ resulted in no failures after a follow-up up to 2 years. It is important to mention that these studies did not attempt to insert implants with less than $32 \mathrm{NCm}$, which would be more appropriate for direct comparisons.

Ottoni et al. ${ }^{402}$ compared implants restored immediately (test group) with implants restored after a healing period (the control group), and primary stability was standardized with a minimum insertion torque of $20 \mathrm{Ncm}$. The test group included 10 failed implants, of which 9 had been placed with an insertion torque of $20 \mathrm{Ncm}$. Only one implant from the control group failed during the 24-month follow-up period. The authors concluded that immediate loading in singletooth indications should only be considered if the implant can be placed with an insertion torque greater than $32 \mathrm{Ncm}$. In the animal study of Neugebauer et al., ${ }^{407}$ four to six immediately loaded and unloaded dental implants with a microstructured surface were placed in the mandible and the maxilla in seven minipigs. Implants showed osseointegration if the average insertion torque of the implants within 
one bridge was $>35 \mathrm{Ncm}$. If the primary stability of the bridge was $<35 \mathrm{Ncm}$, all implants in the quadrant were lost after 4 months. Grandi et al. ${ }^{408}$ conducted a study very similar to Hui et al..$^{405}$ and Lorenzoni et al. ${ }^{406}$ One implant failed and was removed 3 weeks after placement because of progressive mobility. The failed implant had a final insertion torque of $35 \mathrm{Ncm}$, whereas all the successful implants had a final insertion torque ranging between 50 and $80 \mathrm{Ncm}$. It was reported that the implant was inserted in D3 quality bone and the authors hypothesized that the presence of micromovements at the implant-bone interface probably led to the formation of a soft-tissue encapsulation around the fixture, causing its failure. ${ }^{408}$ Khayat et al..$^{401}$ assessed the clinical outcome of 42 implants placed with an insertion torque $\geq 70 \mathrm{Ncm}$. All implants were clinically stable after 2-3 months of non-submerged healing. The use of high insertion torques (up to $176 \mathrm{Ncm}$ ) did not prevent osseointegration. The implants were followed for 1 year, and no signs of pressure necrosis, crestal bone change, or untoward healing were noted compared with the control group. An increased static strain in the bone not only creates higher implant stability at the time of insertion, but also generates increased implant stability throughout the observation period of 3-24 days. ${ }^{409}$

It seems there is no consensus on the minimal insertion torque required for some kinds of treatments with dental implants. When it comes to the maximum insertion torque, traditionally, implants were preferably placed with a torque not exceeding the empirically set limit of $45 \mathrm{Ncm}$ because excessive strain on the surrounding bone was thought to negatively affect osseointegration. ${ }^{396}$ Excessive tightening creates important compression forces in the surrounding bone. This has been theorized to disturb microcirculation and lead to bone resorption, but the theory has never been scientifically investigated. However, there seems to be no limits concerning the maximal insertion torque allowed provided of course that the bone does not fracture, according to the results of the study of Khayat et al. ${ }^{401}$ 


\section{Primary stability}

Implant stability, an indirect indication of osseointegration, is a measure of the clinical immobility of an implant. ${ }^{325,393}$ It can occur at two different stages: primary and secondary. ${ }^{410}$ Primary stability of an implant is attained at implant placement and is determined by numerous factors, including density and mechanical properties of the bone, the implant design, site complications, and the surgical technique. ${ }^{411-415}$ It has been stated that primary stability is a crucial prerequisite for achieving osseointegration. ${ }^{299}$ Secondary stability depends on the further reaction of surrounding tissue to the implantation and offers biological stability through bone regeneration and remodeling. ${ }^{325,393,415,416}$ In case of premature loading of an implant, lack of primary stability may result in micromotion at the tissue-implant interface, which may lead to fibrous encapsulation rather than bone formation. Therefore, if good primary stability can be obtained in small amounts of dense bone, for instance, by using an adapted surgical technique with narrower drills and self-tapping implants, it is possible that the implant can remain stable during functional loading. ${ }^{417}$ The conventional method is to insert the implant into a hole of a smaller diameter than usual. This leads to the generation of compressive forces along the implant-bone interface and results in enhanced implant stability. ${ }^{299}$ The results of Toyoshima et al. ${ }^{299}$ showed that the mean MIT, ISQ, and push-out tests for implants with standard drilling in corticocancellous bone were significantly higher than those with undersized or standard drilling in cancellous bone. Another approach in cases with insufficient bone is to choose a tapered implant for insertion into a standard parallel-sided hole. ${ }^{298,418}$ The idea behind this approach is to induce controlled compressive forces in the cortical layer of bone when the implant is inserted. ${ }^{297}$ These forces would be expected to increase the primary stability of the implant. ${ }^{294,419}$ Still, the implants could be fabricated with a shorter thread pitch in order to increase the available surface area. ${ }^{420}$ As pitch distance is inversely related to the number of threads, implants with a shorter pitch should carry an increased surface area. This, in turn, should lead to a higher degree of BIC, improved mechanical anchorage, and improved primary stability, which are vital in cases with insufficient bone. ${ }^{421} \mathrm{~A}$ three-dimensional FEA study showed that 
implants with threads of $0.8 \mathrm{~mm}$ pitch offered stronger resistance to vertical loads than those with 1.6 or $2.4 \mathrm{~mm}$ pitch. ${ }^{422}$

Overloading may also play a role and result in complete loss of osseointegration and replacement of bony by fibrous tissue, which has been demonstrated. ${ }^{423-428}$ Then it may be assumed that immediate or early loading of implants, especially in situations with very loose trabecular bone, may also result in primary instability of the implants, thus jeopardizing the establishment of a direct BIC. ${ }^{429}$ The results of Balshi et al. ${ }^{415}$ suggest that initial bone quality at implantation can affect the rate of bone remodeling and thus secondary stability. There are some studies in literature supporting these statements.

For instance, Friberg et al. ${ }^{212}$ reported that implants placed in extremely soft bone and/or lacking initial stability, as evidenced by "lack of resistance during final tightening of the cover screw or mobility of the fixture mount when still on the implant," constituted $32 \%$ of the implant failures recorded in their study. However, they did not report the integration rate of implants that were mobile at placement or their long-term survival rates.

In the study of Orenstein et al. ${ }^{430}$ with Core Vent Spectra System implants, $93.8 \%$ of the implants that were mobile at placement were clinically integrated at uncovering, compared with $97.5 \%$ of the implants not mobile at placement. The 3-year postplacement survival for the implants that were mobile at placement, however, was significantly lower $(79.8 \%)$ than for implants that exhibited primary stability $(93.4 \%)$ at the time of placement. In the study of Morris et al. ${ }^{414}$ with Ankylos implants, of the implants mobile at placement, $97.7 \%$ were stable at uncovering. The 3 -year postplacement survival of initially mobile implants was $84.1 \%$ compared with $96.8 \%$ for implants not mobile at placement.

Glauser et al..$^{431}$ observed that only $66 \%$ of implants placed in poorly mineralized trabecular bone in the posterior maxilla were successfully integrated after 1 year following immediate loading in comparison with $91 \%$ successful implants placed in mineralized bone. Glauser et al. ${ }^{431}$ studied machined implants.

In the study of Lioubavina-Hack et al. ${ }^{429}$ titanium implants were placed in rats in a secluded space created for the formation of new bone, either in contact with the bone surface of the mandibular ramus, being stable, or not, could easily be moved. All primarily 
stable implants presented increasing percentages of direct BIC during the experimental period, reaching about $80 \%$ of the entire implant length after 9 months. Primarily unstable implants, on the other hand, failed to present osseointegration at any observation time, despite the formation of considerable amounts of new peri-implant bone inside the capsules. The authors stated that this finding would indicate that primary implant stability is a prerequisite for obtaining osseointegration of titanium oral implants.

In addition, it has been stated that secure primary stability leads to predictable secondary stability. ${ }^{432}$ However, primary implant stability, while desirable, is not a prerequisite for achieving osseointegration..$^{414,430}$ Implants that are mobile at placement may benefit significantly if given more time to integrate without loads than those implants that are stable at placement. ${ }^{414}$ Progressive loading of implants has been shown to improve Periotest values ${ }^{433}$ and may also promote more complete osseointegration of initially mobile implants. Moreover, the low values of RFA obtained from implants placed in softer bone seem to increase over time and match the RFA values of the implants placed in more dense bone sites. ${ }^{434}$ Thus, the most important factors to affect osseointegration could be a matter of how unstable the implant is right after placement and how soon, strong, and frequent is the load to which the implant is subjected to.

Based on the findings of several studies, ${ }^{398,415,434-441}$ a table of RFA ISQ ranges was developed based on bone type, implant location, and patient gender that can be used as a guideline for determining the specifics of immediate loading. ${ }^{442}$ An ISQ value below the predictor range may indicate that the implant does not have sufficient primary stability to undergo immediate functional loading. An ISQ value above the predictor range may represent either an inaccurate identification of bone quality at the implant site or an incorrect resonance frequency measurement technique. ${ }^{415}$ However, these guidelines should be used with caution. There is a need to maintain a consistent protocol in the measurements, as the ISQ level was often influenced by orientation of the transducer to the axis of the alveolar ridge. It was observed that measurements in one orientation (for instance, perpendicular or parallel) may have ISQs equal to or higher than the measurements made in different orientations. ${ }^{415}$ The distance from the transducer to the first bone contact ${ }^{435,443}$ is another factor of potential importance, 
such as the abutment length and possible marginal bone resorption as well. ${ }^{434}$ The application of the Periotest as a clinical diagnostic aid to measure implant stability has been contested by some, ${ }^{444}$ due to the sensitivity of the device to clinical variables including striking heights and hand-piece angulation.

\section{Fresh extraction sockets}

The insertion of dental implants immediately after teeth extractions has become a routine clinical procedure in implant dentistry. Tooth extraction results in a reduction of the bone quantity, which may prevent placement of an implant because of the decreased bone volume. Therefore, some authors advocate immediate placement. Several studies mainly of moderately rough-surfaces implants have reported that successful osseointegration is possible when implants are inserted immediately after tooth extraction, with similar survival rates when compared to implants inserted in healed sites, with or without the help of guided bone regeneration procedures. Placing an implant immediately after tooth extraction offers several advantages, including a decrease in rehabilitation treatment time, fewer surgical sessions, the ability to place the fixture in an ideal axial position, positive psychological impact on the patient, and enhanced hard and soft tissue maintenance. ${ }^{445,446}$ However, some other studies have shown that implants placed in fresh extraction sockets shows higher failure rates than when inserted in mature bone. ${ }^{404,447-452} \mathrm{~A}$ systematic review and meta-analysis was conducted ${ }^{18}$ in order to address the conflicting clinical data, and observed that the insertion of dental implants in fresh extraction sockets increased the implant failure rates $(p<0.0001)$, with a RR of 1.58 (95\% CI 1.27-1.95) when compared to placement in healed sites.

A suggested explanation for this is the fact that implant placement in fresh extraction sockets is technique-sensitive because primary implant stability is critical; these implants are usually not in direct contact with the alveolar bone because the extraction socket is broader than is the implant. The anatomic characteristics of the socket after tooth extraction are different from the socket environment after 1 year of healing. Implants placed immediately into fresh extraction sites engage precisely prepared bony walls only in their apex, whereas the coronal space is filled by the end of the healing phase. ${ }^{453}$ It has 
been stated that the procedure should be limited to alveoli with sufficient bone for primary stability, which is generally achieved by exceeding the apex by 3 to $5 \mathrm{~mm}$ or by using implants that are wider than the alveolus. ${ }^{454}$ To achieve these conditions, a minimum of 4 to $5 \mathrm{~mm}$ of alveolar crest width and a residual bone length no less than $10 \mathrm{~mm}$ are recommended. ${ }^{455}$ Simultaneous bone regeneration may be required. ${ }^{456}$

It is also suggested that periodontitis-affected tissues may have a negative local influence on the failure rates due to the presence of infrabony defects, which could increase the gap between bone and implant, ${ }^{457}$ or jeopardize the achievement of primary stability ${ }^{458}$ at immediate implant placement.

The aforementioned review ${ }^{18}$ observed a higher failure rate of immediate implants in relation to non-immediate implants in the maxilla in comparison to the mandible, which may be attributed to the low density of medullary bone and thin cortical plates, ${ }^{448}$ which may have resulted in significant reduction in insertion torque for implants in the maxilla and less implants with primary stability, and further resulted in a lack of resistance to mechanical stresses. ${ }^{450}$

Concerning the influence of the prosthetic rehabilitation on the failure rates, a statistically significant difference between the placement of implants in fresh extraction sockets and healed sites was found when studies only evaluating patients with implant-supported single crowns were pooled, the same not happening when full-arch prostheses were the only prosthetic rehabilitation performed. ${ }^{18}$ From a biomechanical point of view, single implants may be at higher risk of overloading, ${ }^{459}$ because of the lack of splinting. The splinting of the implants in full-arch prostheses allows a more even distribution of the occlusal forces, thereby reducing stresses at the bone-implant interface ${ }^{460}$ as well as micromotion. ${ }^{461}$

Moreover, it is suggested that the healing/loading period may also influence the implant failure rates and immediate loading may appear to be at a higher risk of failure than conventionally loaded ones. It was believed that too-early loading of an implant leads to an interfacial formation of fibrous tissue instead of bone. ${ }^{462}$ Presently, it appears that premature loading per se does not lead to fibrous tissue encapsulation. Rather, it is due to an excessive amount of micromotion at the bone-implant interface, during the healing 
phase. ${ }^{440}$ Most of the included studies exclusively evaluating implants being rehabilitated with single crowns or with full-arch prostheses applied immediate loading to the implants. The splinting of the implants in the full-arch prostheses might have influenced the results.

A study ${ }^{450}$ comparing the survival rates of implants subjected to immediate or delayed restoration in periodontally susceptible patients observed that the stratification of the implants between extraction and non-extraction sites revealed no statistically significant difference between the groups in either insertion torque, insertion ISQ (Implant Stability Torque), or change in ISQ from insertion to 12 months. Based on these data, primary stability did not contribute to implant failure in extraction sites.

Some studies specifically addressed this issue of MBL when implants were placed immediately in extraction sockets. In a prospective clinical study, Schropp et al. ${ }^{463}$ observed that both delayed and immediate approach resulted in statistically significant reduction in bony defects. Another study found that the amount of resorption has been reduced in immediate implants compared to the delayed implants. ${ }^{464}$ Raes et al. ${ }^{465}$ observed that a trend toward bone gain was found following insertion in fresh extraction sockets, which may be explained by the fact that the gap between the original bone and implant diminishes during healing, and the BIC increases in coronal direction during the healing phase. These findings can be related to a coronal bone remodeling around immediate implants and a healing pattern with new bone apposition around the neck of the implants. ${ }^{466}$ These studies did not lead to a clear conclusion about the subject, and the possible influence of several factors ${ }^{42}$ makes it difficult to estimate the real effect of the insertion of implants in fresh extraction sockets on the marginal bone level. The meta-analysis of Chrcanovic et al. ${ }^{18}$ found no apparent significant effect of implants inserted in fresh extraction sockets on the magnitude of MBL in comparison with implants placed in mature bone.

One important factor to consider is the implant position in relation to the extraction socket crestal bone level. A study ${ }^{467}$ observed that there are reasons to suggest that either crestal bone loss or its preservation may be partly due to the crestal or subcrestal implant position. The study also showed that there is a marked hard tissue 
alteration during the 8-week healing period following tooth extraction and immediate implant placement, which affected both buccal and lingual bone plates. The experiment of Caneva et al. ${ }^{468}$ installed immediate implants into extraction sockets in the mandibles of six dogs and concluded that implants should be positioned approximately $1 \mathrm{~mm}$ below the alveolar crest and in a lingual position in relation to the center of the alveolus to reduce or eliminate the exposure above the alveolar crest of the endosseous rough portion of the implant. In a clinical trial using a multivariate model to analyze factors that may affect bone alterations during healing after immediate implant placement, Tomasi et al. ${ }^{469}$ observed that the position of the implant opposite the alveolar crest of the buccal ridge and its bucco-lingual implant position influenced the amount of buccal crest resorption. Furthermore, the thickness of the buccal bony wall in the extraction site and the vertical as well as the horizontal positioning of the implant in the socket must be considered because these factors will influence hard tissue changes during healing.

Another factor that may determinate the amount of bone loss that will occur at crestal bone level is the implant-abutment design. ${ }^{470}$ The influence of different microgap configurations can cause different amount of bone loss, even before prosthetic loading. Subcrestal placement of a butt-joint microgap design may lead to more pronounced bone loss at least at early times of follow up. ${ }^{471,472}$

It is suggested that grafting procedures may also exert some influence on the marginal bone levels. The marginal gap that may exist following implant placement in an extraction socket may be resolved by hard tissue filling during healing, which can possibly change the results, but the literature shows conflicting results. A preclinical study $y^{473}$ on beagle dogs demonstrated that socket grafting modified the process of hard tissue healing, provided additional amounts of hard tissue at the entrance of the previous socket and improved the level of marginal BIC. In contrast, an animal study ${ }^{474}$ on mongrel dogs demonstrated that such procedure resulted in significant buccal bone loss with low osseointegration. Last but not least, the use of different radiological techniques to assess changes in crestal bone level is an additional influencing factor on the results. 


\section{Flapless surgery}

When placing dental implants, a flap is traditionally elevated to better visualize the implant recipient site, providing that some anatomical landmarks are clearly identified and protected. When a limited amount of bone is available, a flap elevation can help implant placement to reduce the risk of bone fenestrations or perforations. ${ }^{475}$ More recently, the concept of flapless implant surgery has been introduced for patients with sufficient keratinized gingival tissue and bone volume in the implant recipient site. In a flapless procedure, a dental implant is installed through the mucosal tissues without reflecting a flap. The alleged reasons to choose the flapless technique are to minimize the possibility of postoperative peri-implant tissue loss and to overcome the challenge of soft tissue management during or after surgery. ${ }^{476}$ Other alleged advantages of the flapless implant surgery include less traumatic surgery, decreased operative time, rapid postsurgical healing, fewer postoperative complications and increased patient comfort. ${ }^{477,478}$ A disadvantage of this technique is that the true topography of the underlying available bone cannot be observed because the mucogingival tissues are not raised, which may increase the risk for unwanted perforations which in turn could lead to esthetical problems or implant losses. ${ }^{479}$ Moreover, there is the potential for thermal damage secondary to reduced access for external irrigation during osteotomy preparations. ${ }^{478}$

A systematic review and meta-analysis on the subject ${ }^{12}$ suggested that there is a statistically significant difference in implant failure rates when implants are inserted through the flapless approach in comparison to an open flap surgery (RR 1.75, 95\% CI 1.07-2.86; $p=0.03$.

Currently, some software systems using computed tomography (CT) scans have been proposed to aid in planning surgery and to produce surgical drilling guides to transfer the planned position to the surgical field. These guides are manufactured in such a way that they match the location, trajectory, and depth of the planned implant with a high degree of precision. As the dental practitioner places the implants, the guides stabilize the drilling by restricting the degrees of freedom of the drill trajectory and depth. ${ }^{480,481}$ It was stated that by using computer-assisted surgery predictability, precision and safety in flapless dental implantology are ensured. ${ }^{482}$ 
However, the precision of the whole procedure depends largely on the ability to position accurately the drill guide, and to maintain that stable position during the whole procedure. ${ }^{480}$ In the case of the placement of implants in completely edentulous jaws, there must be a way to assure the stability of the drill guide, and this is done by fixing the surgical guide onto the bone using osteosyntheses screws. Asymmetric distribution of the screws or uneven tightening of the screws could bring the drilling template out of balance. Furthermore, a certain error is induced as the diameter of the steel tubes is slightly larger than the drill diameter. ${ }^{400}$ Finally, the largest error is probably due to the fact that the final step in the procedure is carried out manually, depending on the surgical guide used. In these cases, implant placement cannot be done through the surgical drill guide because of present mechanical limitations. The drill guide, therefore, has to be removed before the implant is actually inserted, leaving the possibility of additional deviation. ${ }^{480}$ Because of these reasons, the surgical drill guide may provide a false security in decreasing the risk of bone fenestrations or perforations. This may be one of the reasons why the review of Chrcanovic et al. ${ }^{12}$ observed a higher percentage of implant failures with the flapless technique when compared with the open flap surgery.

Still concerning the precision of the implant insertion, it is worth commenting about the technique used by the study of Sennerby et al. ${ }^{376}$ They made use of a slide-over guide sleeve to evaluate and determine the position of the implant. This system is based on the surgeons' imprecise opinion of what is the exact direction of the implants to be placed and it is subjected to flaws, which may have led to an increasing incidence of implant bone plate fenestrations or perforations, and consequently higher implant failure in this group ( $7.9 \%$ versus no failure in the open flap surgery). Correct bur angulation is critical in the procedure. ${ }^{480} \mathrm{With}$ the CT-guide surgery, it is possible to verify in advance the presence of concavities of the vestibular and lingual/palatal bone plates surrounding the planned implant surgical site, thus planning the correct bur angulation and decreasing the chance of implant bone fenestrations or perforations.

Moreover, one in vitro study ${ }^{483}$ analyzed deviations in position and inclination of implants placed with flapless surgery compared with the ideally planned position and examined whether the outcome was 
affected by the experience level. The authors observed that the threedimensional location of implants installed with flapless approach differed significantly from the ideal, although neighboring teeth were present and maximal radiographical information was available. The outcome was not influenced by the level of experience with implant surgery. It was suggested that these deviations would in a clinical situation lead to complications such as loss of implant stability, as well as aesthetical and phonetical consequences. The authors recommended the performance of more precise measurements of soft tissue in situ or additional use of guiding systems.

Since flapless implant placement generally is a "blind" surgical technique, care must be taken when placing implants. Angulation of the implants affected by drilling is critical so as to avoid perforation of the cortical plates, both lingually and buccally, but with presumed more problems with the lingual side in the mandibular molar area and the anterior maxilla. ${ }^{48}$ Therefore, the surgeon must weigh the benefits of the flapless technique against the increasing risk of implant bone fenestrations or perforations, which allegedly may impair implant success or increase the implant failure rates. ${ }^{485}$ Violation of the dental implant beyond the alveolar housing may result in infection and ultimate loss of the implant. ${ }^{486}$ There should be no problem if the patient has been appropriately selected and an appropriate width of bone is available for implant placement. ${ }^{484}$ Some authors ${ }^{484}$ suggested a minimum of $7 \mathrm{~mm}$ of bone width and substantial training to use the appropriate technique.

Another hypothetical drawback of the flapless procedure is that it could interfere with osseointegration because of implant surface contamination and the deposition of epithelial and connective cells from the oral mucosa in the bone during surgical preparation. ${ }^{487}$

On the other hand, a flapless procedure could have a positive effect on the early bone remodeling process, because during the surgical procedure, the bone remains covered by the periosteum. However, the strongly tightened surgical template used to insert implants in totally edentulous jaws may hinder access of saline water and proper cooling during the drilling procedure, which could negatively influence the implant surrounding the bone and the remodeling process during healing. ${ }^{488}$ 
Concerning the MBL, one may expect that the open flap surgery may cause higher MBL due to decreased supraperiosteal blood supply because of the raising the tissue flap during the surgical procedure. Studies have demonstrated that flap reflection often results in bone resorption around natural teeth. ${ }^{489}$ However, it was showed in five studies that the flapless technique generated more MBL around the implants. ${ }^{479,490-493}$ The authors of some of the articles here reviewed provided some reasonable explanations for this. De Bruyn et al. ${ }^{479}$ suggested that this was probably caused in their study due to overdoing of the countersinking procedure. More extensive widening of the crestal bone was necessary to remove enough bone as to allow proper placement of the healing abutment. By countersinking wider and deeper, the coronal portion of the implant is not always in intimate contact with the bone. In the flapped sites, the countersinking procedure was more controlled according to the guidelines of the manufacturer because visual inspection in situ was possible. Rousseau ${ }^{492}$ discussed that this is due to implants being installed blindly, and thus implants are installed more deeply with the flapless technique than with the open flap technique. Therefore, a portion of the transmucosal (supracrestal) part of the implant is slightly below the crestal bone level. Because the coronal part of the implant is smooth titanium, rearrangement of bone around the neck of the implant is normal. When an open flap technique is used, the implant is installed under visual control directly at the right crestal bone position, which results in less bone rearrangement around the implant neck. ${ }^{492}$ The results found in the study of Van de Velde et al. ${ }^{493}$ may be related to the fact that the implants inserted through the flapless technique were immediately loaded, whereas the implants inserted through open flap surgery were loaded only after 6 weeks.

\section{Non-submerged implants}

Historically, the original Brånemark protocol for placing implants prescribed a two-stage surgery with a submerged healing period of at least 3 months in the mandible and 6 months in the maxilla, ${ }^{35}$ allowing the implant to osseointegrate without being exposed to external forces. After bone healing, a second surgery is performed to connect a healing abutment. One of the main reasons for implant insertion in the two-stage procedure was to minimize the risk of 
infection, since the peri-implant tissue is allowed to heal separately from the oral microbial environment. ${ }^{494}$ The predictable outcome of this two-stage installation technique was verified in several clinical trials that reported high success and survival rates for implants that were initially submerged. Extended treatment times, two surgical interventions, and the need for interim prostheses during healing are disadvantages of conventional implant treatment. ${ }^{495}$

With time, the concepts of implant placement in fresh extraction sockets, ${ }^{189,496}$ immediate loading, and non-submerged implants were introduced, focusing on shorter and less invasive procedures. To reduce the treatment time and offer the patient early function and esthetics, it is necessary to use a one-stage surgical procedure and to load the implants as soon as possible. In the one-stage surgical approach (non-submerged implant), the coronal part of the implant is positioned above the gingiva level in case of one-part implants, or transmucosal healing abutments are placed in case of two-part implants. In the one-stage surgical approach the implant can be immediately loaded or not. The encouraging early experiences of immediate loading in the mandible and the development of new implant designs and surfaces have inspired researchers to further explore applications of immediate loading. ${ }^{497}$

Inserting implants in one stage has several advantages. Only one surgical intervention is required, which is convenient for the patient, especially for the medically compromised patient. In addition, there is a considerable cost-benefit advantage. The prosthetic phase can start earlier because there is no wound-healing period involved related to a second surgical procedure. Furthermore, the implants are accessible for clinical monitoring during the osseointegration period. ${ }^{498}$ It allows for a healed peri-implant mucosa at the time of prosthetic rehabilitation. Although immediate loading of implants shortens treatment duration and also provides patients with an acceptable esthetic appearance, there is concern that immediate loading may increase the risk of implant failure. However, reports have shown that immediate loading can lead to clinical and histological osseointegration. ${ }^{499,500}$

In a meta-analysis, Chrcanovic et al. ${ }^{21}$ compared dental implant failures in patients being rehabilitated with immediately loaded nonsubmerged dental implants versus delayed loaded submerged implants. It was observed that the difference between the procedures significantly 
affected the outcome $(p=0.02)$, with a higher implant failure rate for the immediately loaded non-submerged implants (RR 1.78, 95\% CI 1.12, 2.83). However, the results of the analysis may have been influenced by the fact that all implants in the non-submerged group were immediately loaded.

A possible explanation for the possibility of a difference in MBL between submerged and non-submerged implants could be that the trauma of the second operation is avoided by preservation of the biologic width by means of a more superficial placing of implants. ${ }^{501}$ A less extensive countersinking in the non-submerged implants may also contribute to less bone resorption. ${ }^{501}$ The presence of a microgap between the implant and the prosthetic abutment when two-part implants are used is another hypothesis. It has been observed that bacteria colonize the inner region of two-part implants following abutment connection and that this, in turn, may result in MBL..$^{502,503}$ Weber et al. ${ }^{504}$ demonstrated that in one-stage implants a large percentage of initial bone loss occurred during the first months, whereas in two-stage implants $40 \%$ of initial bone resorption was found after re-entry. The authors explain their findings with bacterial colonization of one-stage implants and the additional surgical trauma in two-stage protocols. However, a comparison of MBL between one- and two-piece implants was performed in the study of Engquist et al. ${ }^{501}$ and the results suggest that the microgap has no obvious effect on the clinical outcome. Putative periodontal pathogens have been implicated in the onset and progression of peri-implantitis, but it remains unclear whether these bacteria always constitute a risk factor for the maintenance of dental implants. ${ }^{505}$ In fact, in some studies ${ }^{506,507}$ no correlation was established between the frequency of any group of microorganisms and the clinical parameters of peri-implantitis. Although suspected periodontal pathogens were identified at implant sites in these latter studies, the clinical parameters were not indicative for deteriorating support, suggesting that the presence of potential periodontal pathogens around implants may not always be associated with future attachment loss or implant failure. As it was observed in a review, there are many original reasons for MBL around oral implants, reasons not associated with any primary infection or overloading alone, but instead coupled with the used hardware, clinical handling, and different patient factors or dependent on foreign body reactions. ${ }^{6}$ 


\section{Immediate loading}

A healing period of 4 to 6 months was initially proposed to ensure osseointegration of endosseous dental implants. ${ }^{38}$ The desire for fewer surgical interventions and shorter implant treatment times has led to the development of revised placement and loading protocols. With the improvements in oral implantology resulting in improved prognosis and outcomes, the traditional protocol for implant dentistry has been constantly reevaluated. Recent steps were the reduction of the treatment time by immediate placement of implants into fresh extraction sockets ${ }^{18,189}$ and by loading the implants immediately. ${ }^{508}$ In 1990, Schnitman et al. ${ }^{509}$ reported on the fabrication of immediate (immediately following stage I surgery) fixed mandibular interim prostheses supported by 2 -stage threaded implants. These authors felt that the use of threaded implants in these cases was important in order to achieve "immediate stabilization within cortical bone." Immediate loading protocols have since been extensively discussed in the literature and found to be a viable treatment approach in selected cases. $^{500}$

In a meta-analysis, Chrcanovic et al. ${ }^{21}$ compared dental implant failures in patients being rehabilitated with immediately loaded nonsubmerged dentalimplants versus delayed loaded submerged implants. It was observed that the difference between the procedures significantly affected the outcome $(p=0.02)$, with a higher implant failure rate for the immediately loaded non-submerged implants (RR 1.78, 95\% CI 1.12, 2.83). However, things are not as black and white as they might see, as the authors discussed in their article.

Concerning the immediate loading, that might have influenced the results, Pilliar et al. ${ }^{510}$ observed that an amount of $150 \mu \mathrm{m}$ of micromovement may be a critical level above which healing would undergo fibrous repair rather than the desired osseous regeneration. Micromotion or motion of the implant surface relative to the bone can result from functional overloading immediately after implantation. It has been for long believed that micromotion can also disturb the early remodeling phase, and a critical degree of micromotion caused by overload can result in fibrous repair at the interface rather than osseous regeneration and osseointegration. ${ }^{511}$ However, reports have shown that immediate loading can lead to clinical and histological osseointegration. ${ }^{499,500}$ It is therefore justifiable to 
question whether this healing period is an absolute prerequisite to obtaining osseointegration, or if under certain circumstances this period can be shortened without jeopardizing osseointegration and long-term results. ${ }^{512}$ Moreover, several implant features, like geometry of the implant body specially designed for critical bone conditions and implant surfaces combined with high insertion torques during bone healing may have minimized the risk of early failure of immediately loaded implants. The immediate loading of implants installed according to the non-submerged procedure might inhibit osseointegration, although implants inserted in the submerged way sometimes fail to osseointegrate as well. Thus, the true reason for the failure to osseointegrate largely remains obscure. ${ }^{494}$

Speculatively, the immediate loading may cause a greater MBL in comparison with delayed loading. However, early functional loading to a determined, controlled extent during the healing phase may instead have a positive effect on marginal bone levels. ${ }^{513}$ Early loading stimuli at the bone-implant interface leads to functional adaptation of the bone to the loading situation (remodeling) and to an improved differentiation of the bone structures, resulting in a higher marginal bone level. ${ }^{393,514}$

Furthermore, the peri-implant bone reactions around delayed and immediately loaded implants have been evaluated in an animal study. Histologic and histomorphometric observations and data have already been published ${ }^{515}$ and showed no histologic differences in the two loading groups. It should be noted that the lack of statistically significant differences between the two loading groups does not mean that the peri-implant conditions are the same, ${ }^{516}$ because the sample size in most of the studies with available information on MBL were relatively small.

It has been suggested that it is not the absence of loading per se that is critical for osseointegration, but rather the absence of excessive micromotion at the interface. ${ }^{517}$ Micromotion consists of relative movement between the implant surface and surrounding bone during functional loading and above a certain threshold excessive interfacial micromotion early after the implantation interferes with local bone healing, predisposes to a fibrous tissue interface, and may prevent the fibrin clot from adhering to the implant surface during healing. ${ }^{440}$ Splinting of the provisional restoration might have protected these implants from micromotion. ${ }^{461}$ 
The biological differences in peri-implant tissue responses between immediate nonfunctional loading and immediate functional loading implants were already analyzed in animal models, and no difference was observed between the ultrastructural morphology of the cells at the interface of implants from both groups in the early phases of the osseointegration in minipigs, ${ }^{518}$ and no statistically significant differences in the BIC percentages between the groups, in a study performed in dogs. ${ }^{519}$

\section{Tilted implants}

The loss of posterior teeth, particularly at an early age, leads to the loss of alveolar bone with a relative surfacing of the inferior alveolar nerve in the mandible, thus often prohibiting placement of implants in the posterior regions. ${ }^{520}$ Bone grafting and the use of short implants have been proposed to overcome these anatomic limitations. An alternative could be the inferior alveolar nerve lateral transposition ${ }^{521,522}$ or the use of tilted implants, which allows for maximum use of the existing bone and placement of posterior fixed teeth with minimum cantilevers, in a region where bone height and nerve proximity does not allow for the placement of axial implants. ${ }^{520}$ Concerning the upper jaw, implant anchorage in the totally edentulous maxilla is often restricted owing to bone resorption, which is especially frequent in the posterior region of the maxillary arch, where bone grafting is often indicated. ${ }^{523}$ There is also the problem of the pneumatization, an inferior expansion of the maxillary sinus in relation to fixed anatomic landmarks which develops with time after the extraction of the posterior maxillary teeth. Pterygomaxillary and zygomatic implants could be used, but these techniques present considerable surgical complexity. ${ }^{27,480,524,525}$ The use of tilted implants in the anterior or posterior maxillary sinus walls leads to the exclusion of maxillary sinus elevation or bone grafts, resulting in a simpler and less time-consuming treatment and significantly less morbidity, in decreased financial costs associated with those procedures, and in a more comfortable postsurgical period for the patients. ${ }^{526,527}$

A recent systematic review ${ }^{17}$ focused on comparing the effect of tilted versus axially placed implants on the implant failure rates. A meta-analysis observed that the difference between the procedures 
did not significantly affect the implant failure rates (RR 1.14, 95\% CI $0.84-1.56 ; p=0.40)$. This suggests that tilted implants may achieve the same outcome as implants placed in an upright position. This result is associated with biomechanical advantages in the case of fixed full-arch prostheses with splinted implants, the most common rehabilitation observed in the studies here included, since in this protocol implants are placed in strategic positions from a load-sharing point of view. Placement of two or more well-anchored posterior tilted implants together with anterior axially oriented implants can provide a predictable foundation for implant-supported full-arch prostheses. ${ }^{527}$ A FEA study concluded that there is a biomechanical advantage in using splinted tilted distal implants rather than axial implants supporting a higher number of cantilever teeth. ${ }^{528}$ Tilting of the implants may allow using longer implants that may engage greater quantity of residual bone, which is beneficial to implant stability. ${ }^{529,530}$ Moreover, a more even distribution of stress around implants is achieved when implants with longer length are used. ${ }^{221}$

It is also important to make some consideration about the splinting of the implants. It has been suggested that immediate loading would not be as critical for osseointegration as the presence of excessive micromotion at the interface. Micromotion consists of relative movement between the implant surface and surrounding bone during functional loading and above a certain threshold excessive interfacial micromotion early after the implantation interferes with local bone healing, predisposes to a fibrous tissue interface, and may prevent the fibrin clot from adhering to the implant surface during healing. ${ }^{440}$ Splinting of the implants in the case of the immediateloaded fixed full-arch prostheses might have protected these implants from micromotion. ${ }^{461}$ Splinting allows a more even distribution of the occlusal forces, thereby reducing stresses at the bone-implant interface. ${ }^{460}$ It was also suggested that the reason for the high survival of tilted implants may be the increased contact between cortical bone and tilted implants, increasing the initial stability, ${ }^{531}$ which may be true for the maxilla, but not necessarily for the mandible. However, when a sensitivity analysis was performed pooling the studies evaluating implants inserted in maxillae only, a statistically significant difference was observed, favoring axially placed implants. This might be associated with the lower bone density encountered 
at the posterior regions of the edentulous maxilla, where the tilted implants were inserted.

Concerning MBL, it was suggested by FEA studies which reported accentuated stresses around non-axially placed implant necks ${ }^{532-534}$ that unfavorable loading direction could in theory induce greater bone resorption around tilted implants as compared to axially placed ones. Tilted implants may be also subjected to bending, possibly increasing marginal bone stress. ${ }^{535}$ On the other side, it was shown in FEA studies for full-arch prosthesis that the reduction of the cantilever length achieved by tilting of the distal implants allows for a more widespread distribution of the occlusal forces under loading, and consequently for a reduction of the stresses at the implant neck..$^{528,536,537}$ It is interesting to note that photo elastic and FEA studies analyzing single angulated implants ${ }^{532-534}$ showed increase of stress in the surrounding bone, whereas FEA studies ${ }^{528,536,537}$ analyzing tilted implants in splinted full-arch prostheses observed more favorable results for tilted implants concerning MBL, due to the splinting effect. The cantilever length of the prosthesis also has some influence, as shorter cantilevers have been correlated to a reduced peri-implant bone loss. ${ }^{538}$ The meta-analysis of Chrcanovic et al. ${ }^{17}$ did not find an apparent significant effect of tilted dental implants on the occurrence of greater MBL in comparison with axially placed implants. The fact that fixed full-arch prostheses with splinted implants were the most common rehabilitation observed in several studies might have collaborated to these findings. However, these results should be interpreted with caution due to the lack of use of a standardized technique aiming to obtain a precise and reproducible bone loss measurement, and further due to the variability of the follow-up period among the studies.

\section{Grafting}

The insertion of dental implants requires sufficient bone volume to achieve primary stability ${ }^{539}$ and ensure good long-term results. The reconstruction of defective mandibles using non-vascularized bone grafts and dental implant insertion was originally described by Brånemark et al. ${ }^{540}$ and has become a well-established procedure for prosthetic rehabilitation. ${ }^{539,541,542}$ Interpositional bone grafting, ${ }^{543}$ vascularized bone grafting, ${ }^{544,545}$ and nerve repositioning, including lateralization and transposition techniques, ${ }^{522}$ represent valid 
alternatives to non-vascularized bone grafting procedures. In cases of non-vascularized procedures, and depending on clinical conditions, endosseous implants can be inserted at the time of reconstruction as long as there is sufficient residual bone to ensure primary stability. ${ }^{546,547}$ Alternatively, implants may be inserted later, in a delayed procedure, after the graft has been left to consolidate for 3 to 6 months. ${ }^{542}$

Many authors have advocated the delayed protocol, ${ }^{548-557}$ stating that the immediate placement of implants exposes the patient to some risks, such as partial or total loss of the graft in the case of wound dehiscence, membrane or onlay graft exposure and/or infection, and non-integration of implants related to the immediate placement into avascular bone. As it would be possible to place implants in a revascularized graft when a delayed protocol is performed, and since the regenerative capacity of bone is determined by the presence of vessels, bone marrow, and vital bone surfaces, a delayed approach would permit a better integration of implants (higher values of BIC) and stability of implants as compared with immediate implant placement. ${ }^{549,558,559}$ Although it is difficult to determine a clear indication for immediate or delayed implant placement, the majority of authors suggest immediate implant placement when the residual alveolar bone presents adequate quality and quantity. ${ }^{560}$ Thus, primary stability of dental implants would be closely related to these parameters. ${ }^{561}$

A systematic review ${ }^{561}$ comparing success rates in immediate and delayed dental implant placement following guided bone regeneration or onlay bone block ridge augmentation observed that these two procedures are reliable techniques, providing sufficient bone volume to allow implant placement in the case of vertical and/ or horizontal defects of partially or totally edentulous patients. Most studies demonstrated that success rates of implants placed in the augmented areas by means of GBR or onlay graft techniques are similar to those reported for implants placed in pristine bone. Another review ${ }^{562}$ observed that implants placed in onlay or inlay graft procedures presented a very low failure rates, and that the marginal bone conditions around implants in grafted sites were comparable to what has generally been reported for non-grafted sites.

Specifically to the superior jaw, maxillary sinus floor augmentation is a technique that is widely used to enable the placement of dental implants in the severely resorbed posterior region. The success of the 
maxillary sinus lift procedure is determined by the amount of vital bone formation after maturation of the graft and the long-term survival rate of the implants placed in that region. ${ }^{563} \mathrm{~A}$ recent systematic review on the subject ${ }^{564}$ observed that the most frequently reported reasons for the loss of implants were instability during implant insertion ${ }^{565-569}$ and the placement of implants after dental extraction in alveolar regions with less than $2 \mathrm{~mm}$ of height, ${ }^{570}$ demonstrating the importance of primary stability in the rehabilitation of regions by sinus lift.

\section{Reoperation}

Replacement of implants in sites where previous implants have failed has often been the main treatment alternative in most cases. ${ }^{571}$ It was suggested more than 30 years ago that when a dental implant is lost, a flap should primarily cover the entrance to the site, and after 9 to 12 months a new implant could be replaced. ${ }^{300}$ More recently, it was suggested that a 1-year healing period may not be necessary, provided that the socket can be prepared to eliminate thread grooves and invasive soft tissue, the replacement implant is larger in diameter than the original implant, and sufficient available bone remains for the procedures. ${ }^{572}$

Failed implant sites present a challenging therapeutic dilemma to the clinician because the alveolar bone in these sites is usually further reduced due to marginal bone resorption, resulting in greater difficulties for integration of yet another implant. Implant therapy is, however needed for a fixed prosthetic reconstruction in most cases. ${ }^{573}$

Chrcanovic et al. ${ }^{192}$ assessed the survival of dental implants placed in sites of previously failed implants, and observed that the survival of implants placed at the same location after implant failure was $73 \%$ compared to the $94 \%$ survival rate of traditionally placed implants in the original cohort. These results are in agreement with other studies. Grossmann and Levin ${ }^{574}$ observed that 9 of 31 (29\%) re-operated implants failed in their study. Machtei et al. ${ }^{573}$ reported an overall survival rate of $83.5 \%$ for re-operated dental implants. Kim et al. ${ }^{575}$ found a lower failure rate $(11.7 \%)$ for reimplantation, similar to the results of Alsaadi et al. ${ }^{576}$ with $12.1 \%$ failure rates ( 7 of 58 implants), and Wagenberg and Froum ${ }^{577}$ of only $3.92 \%$ failures ( 2 of 51 ). Kim et al. ${ }^{575}$ reported that few implants failed the second time and were replaced with a third implant, this time with good results. However, 
these were only a few implants, which were only followed up for a short period of time. The lower survival rate observed in these studies represents three- to fourfold increase in the proportions of lost fixtures compared with the rate often reported for non-affected first-time implant sites. ${ }^{577}$

The lower survival rate for implants placed in previously failed sites suggests site- or patient-specific risk factors. ${ }^{571,573}$ The sitespecific theory is mainly supported by the finding that failed implants are often found in patients where other implants were successfully osseointegrated and in function. ${ }^{229}$ Machtei et al. ${ }^{573}$ concluded that the lower failure rate observed for re-operated implants in their study was not related to any of the common implant- or patient-related factors. Thus, they have suggested a possible site-specific negative effect that might be associated with this phenomenon. A greater percentage of lost implants in the study of Chrcanovic et al. ${ }^{192}$ were placed in bone sites having been classified as quantities D and $\mathrm{E}$ and qualities 3 and 4, according to the Lekholm and Zarb ${ }^{188}$ classification. Moreover, bone quality in the area with failed implants is usually further reduced and is poor compared with other areas without implants. ${ }^{575}$ There was a statistically significant higher failure rate for turned (machined) implants versus surface enlarged implants in cases of replaced failed implants. ${ }^{192}$ The same was observed in the study of Alsaadi et al. ${ }^{576}$ The mean Implant diameter was rather similar in the original operation and the reoperations. Evaluating the length of the original and replacement implants, it could be observed that the mean length of the replacement implants were actually shorter than the original ones. Furthermore, some implants were replaced a short period after the explantation. ${ }^{192}$ These two factors may have also contributed to the higher failure rates. It has been suggested that the success of replacement may be increased by improving the implantation site with bone augmentation and the use of larger implants with improved surfaces, ${ }^{576}$ although recent evidence has criticized bone augmentation. ${ }^{578}$ It has been reported that implants with an enlarged surface experience faster bone apposition in relation to turned (machined) implants, which allows the former to achieve a proper fixation even if a remaining endosseous lesion tends to compromise the osseointegration process. ${ }^{579}$ This difference in bone apposition may be relevant for replacing implants..$^{576}$ 
On the other hand, the cluster phenomenon supports the patientspecific factor as a possible explanation for failure of implants. ${ }^{580}$ Concerning this issue, there is a general appreciation that risk factors predispose individuals to more complications and implant failures and may result in lower implant survival rates. ${ }^{11}$ Chrcanovic et al. ${ }^{192}$ found no clear relationship between the patients' habits and systemic conditions and failures of the re-operated implants. However, this does not necessarily mean that these variables are not risk factors for dental implants, but it is suggested that the substantial negative effect of these sites with previous failures outweighs these patients' and environmental variables, thus masking their effect. ${ }^{573}$ It is important to stress that, once an implant has failed, replacement of that implant is subjected to at least all the initial factors that led to the failure. ${ }^{574}$

\section{Anatomy}

\section{Location - maxilla and mandible, anterior and posterior regions}

According to its structure, jawbone can be classified as cortical or trabecular. With respect to osseointegration, cortical bone is beneficial to implant stability, whereas trabecular bone is beneficial to blood supply. ${ }^{581}$ Their relative proportion strongly affects implant survival. ${ }^{582}$ In general, there is a tendency to have more bone tissue in anterior than in posterior regions. ${ }^{583}$ In the posterior maxilla, there is commonly thinner cortical bone combined with thicker trabecular bone compared to the mandible. ${ }^{583-585}$ This higher failure rate in this region may be attributable to a combination of the demanding preconditions often present in the posterior maxilla, such as barely sufficient bone volume, poor bone quality, and high functional forces. ${ }^{431}$ The cortical layer of both jaws tends to become thinner and more porous posteriorly. Moreover, posterior implants have to withstand the heaviest loading and are in general short due to insufficient quantity of available bone (the maxillary sinus and the inferior alveolar nerve are the main anatomical limitations). The high survival rates presented in the canine and/or premolar maxillary area in some studies ${ }^{212,577,586,587}$ could be attributed to favorable bone density and adequate bone quantity due to relatively rare anatomic limitations. A study ${ }^{577}$ found high failure rates in the mandibular anterior region, which was suggested to be caused by overheating of 
the bone when long implants $(15-18 \mathrm{~mm})$ were placed. ${ }^{577}$ The cortical bone that is very hard with a low blood supply and the trabecular bone with a low density do not provide favorable host conditions for good prognosis of dental implants. ${ }^{588}$ Implant survival rates can be significantly associated with implant location. Clinical studies have reported dental implants in the mandible to have higher survival rates compared to those in the maxilla, especially for the posterior maxilla. ${ }^{346,589}$ Bone quality has been considered as the basic cause of this difference.

\section{Bone quantity and quality}

Bone quality is often referred to as the amount and their topographic relationship of cortical and cancellous bone in which the recipient site is drilled. ${ }^{590}$ There are two main reasons for assessing jawbone tissue in dental implant treatment: first, as a diagnostic tool to assess whether the jawbone tissue is sufficient for implant treatment, and second, as a prognostic tool to predict the probability of success or failure, as the bone tissue characteristics of quality, quantity and density are considered important with regard to treatment outcomes. ${ }^{212}$

A poor bone quantity and quality have been indicated as the main risk factors for implant failure as it may be associated with excessive bone resorption and impairment in the healing process compared with higher density bone. ${ }^{205,590,591}$ Therefore, methods used prior to dental implant placement are valuable as diagnostic tests for the planning of implant placement in bone tissue. ${ }^{592}$

Chrcanovic et al. ${ }^{30}$ performed the so far only meta-analysis comparing the survival rate of dental implants, when these were inserted in bone sites of different qualities and quantities, according to the Lekholm and Zarb ${ }^{188}$ classification. The results suggest that dental implants inserted in bone quality 1 have a higher failure rate in comparison with implants inserted in bone qualities 2 and 3, the insertion of implants in bone quality 3 have a higher failure rate in comparison with implants inserted in bone quality 2 , and the insertion of implants in bone quality 4 have a higher failure rate in comparison with implants inserted in bone qualities 1, 2 and 3. These results are hypothesized to be related to some factors. It has been shown that achieving optimum primary stability in bones with poor densities is difficult, and this is related to a higher implant 
failure rate. ${ }^{590,593}$ There is a strong correlation between bone density and dental implant stability. ${ }^{594}$ Dental implant stability is positively associated with the thickness of cortical bone. ${ }^{229}$ Bone quality is not the only factor, and stability is also influenced by the surgical technique, the surface implant morphology, or its diameter, bone compaction techniques and cortical anchor for implant placement. Wide diameters that increase the contact area between the treated bone and implant surface treated will increase primary stability.

The results of the review ${ }^{30}$ in relation to the bone quantity suggest that dental implants inserted in bone quantity A have a higher failure rate in comparison to implants inserted in bone quantities B and $\mathrm{C}$, but failure less when compared to implants placed in bone quantity D. Implants in bone quantity B have a lower failure rate in comparison with implants inserted in bone quantities $\mathrm{C}$ and $\mathrm{D}$. Implants in bone quantity $\mathrm{C}$ have a lower failure rate in comparison with implants inserted in bone quantity D. Implants in bone quantity $\mathrm{E}$ have a higher failure rate in comparison with implants inserted in all other bone quantities. The higher failure rate of implants inserted in bone quantity $\mathrm{A}$ in comparison with implants inserted in bone quantities B and C may be related to the heat generation during implant site preparation. One in vitro study ${ }^{595}$ measured in blocks of bovine cortical femur bone the thermal changes elicited during drilling and tapping procedures used in site preparation for implants. Significantly greater temperature increase was noted at the 8-mm depth versus the 4-mm depth with drills. Dentists tend to use longer implants for sites with more available bone. This increases the probability of a thermal injury at the implant site. Frictional heat generated during uncontrolled surgical preparation of hard tissues at implant sites produces a zone of devitalized bone around the bur holes. The extent of this necrotic zone varies exponentially with the magnitude of temperature reached and the duration of thermal injury. ${ }^{596}$ Eriksson et al. ${ }^{597}$ performed histological, histochemical and vital microscopic study in the rabbit and established a threshold of $47^{\circ} \mathrm{C}$ for 1 minute to cause thermal necrosis of the cortical bone. Also they evaluated the effects of defined temperature rise on bone regeneration and found that heating of the test implants (titanium) to $47^{\circ} \mathrm{C}$ or $50^{\circ} \mathrm{C}$ for 1 minute caused a significant reduction in the bone formation in the implants while no significant effects were observed 
when the heating was done to $44{ }^{\circ} \mathrm{C}$ for $1 \mathrm{~min}$. These results reflected the importance of controlling the heat generated during the surgical process that hampers the proper bone regeneration. Concerning the case of bone quantities $\mathrm{D}$ and $\mathrm{E}$, the poor bone volume does not allow the placement of longer implants with presumably high degrees of BIC, which may lead to a difficulty to attain primary stability.

Some things must be said about the classification system presented by Lekholm and Zarb. ${ }^{188}$ This classification was chosen for several reasons: (1) it is well known; (2) it describes jawbone tissue from both qualitative and quantitative aspects; and (3) results indicate a good correlation with bone mineral content. ${ }^{598}$ However, the classification is not perfect. It does not provide quantitative data about bone quality and density. ${ }^{599,600}$ The original publication by Lekholm and Zarb ${ }^{188}$ contains no definitions of bone characteristics. The suggestion that anatomical features of bone tissue be considered before the preparation of implant sites was based on experience. The foundation for five groups of jaw shapes or four groups of bone quality was not discussed. ${ }^{601}$ In a systematic review to evaluate the evidence for the diagnostic accuracy of clinical methods to assess bone density, quantity, or quality prior to and during dental implant placement, ${ }^{592}$ the authors observed that no study examined the accuracy of the method originally described by Lekholm and Zarb. ${ }^{188}$ The authors concluded that the evidence for the efficacy of clinical methods to assess jawbone tissue prior to and during endosseous dental implant placement is sparse. This emphasizes the need for studies that incorporate accepted methodological criteria for diagnostic efficacy. ${ }^{592}$ In a later review, ${ }^{601}$ the same authors observed that though it had become routine to include the reference by Lekholm and Zarb, ${ }^{188}$ apparently there was no knowledge of the original description of either bone characteristics or the recommended assessment methods. This is an inadequate approach to research as inaccuracy of measurements can affect the reported results of any intervention. ${ }^{601}$ Authors should describe how these outcomes have been measured and whether any particular steps have been taken to increase the reliability of the measurements. ${ }^{602,603}$

The reliability of surgeons' perceptions of bone quality during surgery is difficult to investigate. ${ }^{601}$ Shapurian et al. ${ }^{604}$ found the observer agreement of two examiners to be low when assessing 
bone quality as correlated with Hounsfield units values $(\mathrm{r}=0.65$, $p<0.001$ ), concluding that their finding underscored the subjective nature of the Lekholm and Zarb ${ }^{188}$ classification system. The fact that there are no guarantees against operator errors, coupled with the notion that there are no clear-cut boundaries assigned between poor, moderate, and good bone quality, opens the door to the possibility of variability. ${ }^{605}$

Besides being a subjective nonspecific technique to determine bone density, ${ }^{606}$ there is a lot of overlap between its various categories. ${ }^{599,600}$ For example, the results obtained from the study of Rokn et al. ${ }^{607}$ show that the Lekholm and Zarb ${ }^{188}$ classification as a preoperative predictor and the tactile sense of the surgeon as an indicator at the time of surgery significantly distinguish quality bones 1,2 , and 3 from each other but fail to differentiate quality bones 3 from 4 . Other studies ${ }^{608-611}$ demonstrated a similar overlap between quality 2 and 3 in bone classification, making it difficult to distinguish these 2 bone types by subjective evaluations. In this regard, Lindh et al. ${ }^{608}$ demonstrated a higher interobserver accuracy when the clinicians classified the bone density according to trabecular bone pattern and in 3 categories.

On the other hand, the applicability of the Lekholm and Zarb ${ }^{188}$ classification of bone quality was supported by the results of four studies. In the first study, ${ }^{612}$ panoramic radiographic appearances of bone quality assessed according to Lekholm and Zarb ${ }^{188}$ were found to be correlated with bone mineral density of the body of the mandible as measured by dual energy X-ray absorptiometry. In two other studies, ${ }^{610,613}$ implant sites were assessed by quantitative computed tomography (QCT) where the QCT bone density values were compared to the Lekholm and Zarb ${ }^{188}$ classification system. An overall correlation between the QCT values and the subjective density scores was observed. In the fourth study, ${ }^{598}$ bone mineral density measurements from CT and RFA were used to assess bone quality. Bone classification significantly correlated with mean bone mineral density and mean RFA values, and bone mineral density was higher at implant sites where torque was $\geq 35 \mathrm{Ncm}$ at implant placement. ${ }^{598}$ However, none of the included studies in the review of Chrcanovic et al. ${ }^{30}$ had performed dual energy X-ray absorptiometry, $\mathrm{CT}$, or RFA in order to validate their findings. 
Although subjective assessments of the implant recipient area are relatively accepted methods for determining bone quality clinically, ${ }^{600}$ a more accurate evaluation should be used to determine bone quality, especially in patients in whom bone quality is more important in the treatment plan, such as patients needing early or immediate loading. ${ }^{607} \mathrm{CT}$ has been regarded as the best radiographic method for analyzing the morphological and qualitative analysis of the residual bone. ${ }^{600,614,615} \mathrm{CT}$ is also a valuable means for evaluating the relative distribution of cortical and cancellous bone. ${ }^{613}$ Hounsfield units can be determined on CT scan images and is an objective and reliable technique for the evaluation of bone density. ${ }^{606,613}$

Bone quality as a term is a potentially ambiguous one. Bone quality recordings of the osteotomy site at the time of implant placement are subjective and based upon a surgeon's assessment usually accomplished by visual and tactile inspection/palpation at the time the osteotomy site is prepared. Clinicians would benefit from an objective measure to reliably predict osseointegration. ${ }^{605}$ Accurate analysis of the bone content and architecture would facilitate clinical decision-making regarding patient selection, implant type and surface, and the surgical technique used. ${ }^{613}$ Classification systems are needed in order to provide a framework for the orderly, scientific study of treatment and treatment outcomes. ${ }^{601}$ Similar assessment methods, classification systems and measurement units are essential prerequisites for comparing the results of different studies and for improving our understanding of treatment outcomes in relation to different bone characteristics. If there is no distinct and generally accepted definition of bone tissue characteristics, comparisons of results cannot be trusted. ${ }^{601}$

\section{Patient's habits}

\section{Smoking}

Nicotine is the most important constituent among more than 4,000 potentially toxic substances in tobacco products. It is the main chemical component responsible for tobacco addiction, appears to mediate the hemodynamic effects of smoking, ${ }^{616}$ and has been implicated in the pathogenesis of numerous diseases. ${ }^{617}$

When it comes to the negative effects in bone, smoking, especially nicotine, impairs new bone formation, reduces calcium absorption, 
and decreases bone mineral density transiently. ${ }^{618}$ Chen et al. ${ }^{619}$ observed that mean union rates were 7.1 months in smokers and 4.1 months in non-smokers, in a study of healing after orthopedic surgery to the arm. Moreover, six smokers $(30 \%)$ and no non-smokers experienced delayed union or nonunion. Osteoporosis is usually observed in long-term smokers who routinely show lower bone density than non-smoking cohorts. ${ }^{620-622}$ One study showed that smokers and ex-smokers had lower trabecular bone mineral content when compared to never-smokers. ${ }^{623}$ In a study of 41 pairs of twins, those who smoked more heavily had bone mineral density values $5 \%$ and $10 \%$ lower at the femoral neck and lumbar spine, respectively, for each 20 pack-year difference (the lifetime tobacco use was calculated as the total number of years of smoking multiplied by the average number of cigarettes smoked per day, divided by 20 , and expressed as packyears of smoking).${ }^{624}$ Glassman et al. ${ }^{625}$ studied the effect of cigarette smoking and smoking cessation on spinal fusion in a retrospective review of 357 patients, and found that the nonunion rate was $14.2 \%$ for non-smokers and $26.5 \%$ for patients who continued to smoke after surgery $(p<0.05)$. Patients who quit smoking after surgery for longer than 6 months had a nonunion rate of $17.1 \%$. Bain ${ }^{626}$ found that patients following a smoking cessation protocol (complete cessation of smoking for 1 week before and 8 weeks after initial implant placement) presented statistically significant less failure rates than smokers who continued to smoke. These results may help to validate the hypothetical assumption that postoperative smoking cessation helps to reverse the impact of cigarette smoking on bone healing. However, it is important to notice that there is much more evidence of the detrimental effect of smoking on implant outcomes than there is on the potential benefit of stopping smoking. ${ }^{627}$

Besides the implication in several systemic diseases, various clinical studies have also demonstrated the detrimental effect of smoking on oral health. Tobacco smoke is associated with the poorer healing of the socket post tooth extraction, ${ }^{628}$ tooth loss, ${ }^{629}$ marginal and alveolar bone loss, ${ }^{630}$ and periodontal diseases. ${ }^{631}$ Concerning the dental implant interface, the deleterious effects of tobacco smoke reflects a series of direct and indirect systemic and local effects on bone metabolism. ${ }^{632}$ It has been strongly suggested that local exposure 
of the peri-implant tissues to tobacco products is the main factor leading to an overall increase in implant failure rate in smokers. ${ }^{631}$

A systematic review and meta-analysis on the effect of smoking on dental implants ${ }^{20}$ (Study II) suggested that the insertion of dental implants in smokers affects the implant failure rates. Sensitivity analyses suggested that smoking also significantly affects the survival of implants inserted only in the maxilla or in the posterior regions of the jaws, implants receiving single-crown restorations, implants in full-arch implant-supported fixed prostheses, implants that received immediate loading, and of implants submitted to any surface modification here reviewed (turned, acid-etched, sandblasted and acid-etched, sandblasted and fluoride-modified, and oxidized). The lack of statistical significance for the mandible was surprising but is most likely explained by the limited number of studies ${ }^{633-637}$ reporting implant survival for smokers and non-smokers exclusively in the inferior jaw. Moreover, a previous review ${ }^{638}$ on the subject suggested that smoking may be a significant risk factor with an adverse effect on implant survival and success in areas of loose trabecular bone, but may not be as significant for good bone sites. A recent clinical study ${ }^{191}$ (Study III) assessed the influence of local and systemic factors on the occurrence of dental implant failures up to the second-stage surgery (abutment connection). As the study of failures only before the abutment connection limits the observation to the stage before the prosthetic treatment, some confounding factors are eliminated. Smoking was one of the two variables to exert some significant effect on the failures during this pre-prosthetic period.

The increase of implant failure rates due to smoking is hypothesized to be related mainly to the effect of smoking in osteogenesis and angiogenesis. Concerning the effects on osteogenesis, Ma et al. ${ }^{639}$ showed that nicotine inhibited the gene expression of BMP-2, TGF- $\beta 1$, PDGF-AA and VEGF in osteoblasts. BMP-2 is the most potent osteogenic induction factor regulating osteoblast differentiation, ALP expression and subsequent mineralization. ${ }^{640}$ TGF- $\beta 1$ is produced by osteoblasts and incorporated into the bone matrix. During bone remodeling, TGF- $\beta 1$ plays an important role in the regulation of osteoblast proliferation, differentiation and apoptosis, with subsequent important effects on bone formation and remodeling. ${ }^{641}$ PDGF and VEGF have angiogenic effects during bone healing. ${ }^{642}$ 
Whereas their expression can be detected in osteoblasts, they are considered to be able to regulate osteoblast activity as well. VEGF can interact synergistically with bone morphogenetic protein (BMP) to promote skeletal development and bone healing by enhancing cell recruitment, prolonging cell survival, and increasing angiogenesis. ${ }^{643}$ BMP acts as an important regulator that stimulates production of VEGF in osteoblasts. ${ }^{64}$ Therefore, the inhibitive effect of nicotine on osteoblastic activity may contribute to the failure of dental implant osseointegration. ${ }^{639}$

In addition, some studies showed that osteogenesis and angiogenesis are tightly coupled during bone formation, and angiogenesis plays a pivotal role in skeletal development and bone repair. ${ }^{645-647}$ Besides carrying oxygen and nutrients to bone tissue, blood flow play an active role in bone formation and remodeling by mediating the interactions among osteoblasts, osteocytes, osteoclasts, and vascular cells at a variety of levels. ${ }^{648}$ The deleterious effects of smoking have not only been shown on osteoblasts, but also on the microcirculation, including morphologic aspects, particularly vessel wall injury and capillary loss and functional aspects, predominantly changes in tissue perfusion and its regulatory mechanisms, notable reactive hyperemia, and sequestration of blood cells in the microcirculation. ${ }^{649}$ Studies ${ }^{650,651}$ demonstrated that nicotine exposure has direct effects on blood vessels, producing vasoconstriction and systemic venoconstriction, which decreases blood perfusion and causes low oxygen and ischemia, which is the major stimulus for initiating the angiogenic cascade. ${ }^{652}$ Hypoxia and ischemia owing to nicotine exposure could stimulate HIF- $1 \alpha$ expression, leading to an increased expression of VEGF, which, in turn, stimulates angiogenesis. However, the enhanced vessel formation is incapable of compensating for the adverse effect of the reduced blood flow possibly caused by nicotine-induced vasoconstriction. ${ }^{61}$ And even though the increased expression of VEGF caused by hypoxia and ischemia may stimulate angiogenesis, it may also contribute to the compromised bone healing, due to the fact that excessive VEGF may lead to impairment in bone formation, possibly by promoting mesenchymal stem cell differentiation toward an endothelial lineage, ${ }^{653}$ consequently reducing the availability of mesenchymal stem cells (MSCs) for osteogenic differentiation. ${ }^{654}$ Alternatively, excessive VEGF may increase recruitment of osteoclasts into the 
bone-regeneration sites and lead to an excessive bone resorption. ${ }^{654}$ Another fact that should also be taken into consideration is that the inflammatory response to bone trauma plays an important role in initiating the repair cascade. The trauma activates downstream factors such as cytokines and growth factors that recruit osteoprogenitor and mesenchymal cells to the injury site. ${ }^{653,655}$ The problem is that nicotine is an anti-inflammatory agent. ${ }^{656}$

Moreover, it is known that the surface properties of dental implants such as topography and chemistry are relevant for the osseointegration process influencing ionic interaction, protein adsorption and cellular activity at the surface. ${ }^{311}$ In a meta-analysis performed more than 10 years ago, Bain et al. ${ }^{627}$ examined the outcomes of clinical studies that monitored the performance of turned and acid-etched surface implants, also isolating the effect of smoking, and it was observed that there was no significant difference between smokers and non-smokers with either type of surface, but there was a significant difference between turned and acid-etched surface in both smokers and nonsmokers. Balshe et al. ${ }^{657}$ observed that smoking was not identified as significantly associated with implant failure among the roughsurface implants, while it was associated with implant failure among the group with smooth-surface implants. More recently, Sayardoust et al. ${ }^{658}$ showed that turned implants failed more frequently and lost more marginal bone in smokers, and that oxidized implants showed similar failure rates and bone loss in smokers and never-smokers. In the review, ${ }^{20}$ however, a higher RR was observed for implants with roughened surfaces in comparison with turned implants in smokers. These contrasting results indicate that there is a need for more studies to evaluate the long-term outcome of implants with altered surface characteristics in smokers. ${ }^{638}$

It is important to stress that some studies on the subject have a short-term follow-up period, of up to 3 years. In 12-month follow-up study, Kan et al. ${ }^{659}$ reported a $93.04 \%$ success for non-smokers and an $82.82 \%$ success for smokers. In a second study by the authors, ${ }^{660}$ but now with a 60 -month follow-up, the success rate for the nonsmokers was $82.7 \%$ and for smokers was $65.3 \%$. Thus, if one considers the difference in success rates for smokers and non-smokers with implants placed in loose trabecular bone sites that are followed over a longer period of time, the adverse effect of smoking may be more evident. 
The fact that the insertion of dental implants in smokers statistically affected the $\mathrm{MBL}^{20}$ may be related to the deleterious effects of smoking in osteogenesis and angiogenesis. Lindquist et al. ${ }^{633}$ correlated the amount of cigarette consumption to the greater MBL in smokers than in non-smokers. The multivariate analyses performed in their study observed that the smoking habit was the most important factor among those analyzed for association with MBL. Cigar and cigarette smokers have significantly greater loss of bone height than matched non-smokers. ${ }^{661}$ Moreover, a higher MBL in smokers in comparison to non-smokers could be partially explained by the elevated levels of pyridinoline (a molecule specific to the collagen matrix of bone and cartilage) in the crevicular fluid associated with dental implants of smokers ${ }^{662}$ and suggest that smoking may affect implant success in part through alterations in the levels of bone resorption. A strong correlation between pyridinoline levels and radiographic and histologic signs of tissue destruction has been demonstrated. ${ }^{663}$

\section{Snuff}

Whereas the effect of smoking on periodontal health is abundantly documented, little is known about the possible effects of non-smoked tobacco products, which are mainly used as chewing tobacco and snuff. ${ }^{664}$ Like other smokeless tobacco products, snuff is rapidly delivering high doses of nicotine, which leads to dependence. ${ }^{665,666}$ The nicotine is readily absorbed via the mucosal membrane by passive diffusion. ${ }^{667,668}$

Localized oral manifestations, such as gingival recessions and mucosal lesions (snuff dipper's lesion) at the site of snuff placement, are common in users of moist snuff. ${ }^{669-673}$ After cessation of use, however, mucosal lesions seem to heal clinically as well as histologically. ${ }^{672,674,675}$ However, the use of snuff was not associated with the presence of periodontal bone loss. ${ }^{664,676,677}$ Cigarette smokers were found to have a statistically significant higher risk of severe periodontitis than nontobacco users and users of snuff. Using snuff did not seem to be a risk factor for periodontitis. ${ }^{676} \mathrm{It}$ is hard to tell whether the effect of snuff on teeth would reflect the same periodontal results on implants, because studies on the effect of snuff on dental implants are rare. Chrcanovic et al. ${ }^{191}$ (Study III) and Chrcanovic et al. ${ }^{193}$ (Study VIII) found no influence of snuff on dental implant failures in multiple 
regression analyses assessing factors that could influence dental implant failures up to the abutment connection and cluster behavior of dental implant failures among patients, respectively.

\section{Bruxism}

Occlusal parafunction includes bruxism (clenching, grinding), lip biting, thumb sucking, and abnormal posturing of the jaw. In contrast to functional behaviors such as mastication, deglutition, or speaking, activities classified as "parafunction" appear to have no functional purpose. ${ }^{678}$ Concerning the overloading related to parafunction, it can cause various complications, such as occlusal surface wear (Figures 10 and 11), fracture, loosened screws, or abutment and implant fracture. ${ }^{679}$ As the frequency of parafunction is very common, ${ }^{678}$ the usage of implants in patients with parafunctional habits is unavoidable. Moreover, a significantly high percentage of newly gained parafunction was reported in patients with implant-supported superstructures. ${ }^{680}$ Some authors have suggested that overloading of implants or abnormal occlusal stress, as seen in patients with bruxism habits, may contribute to failure. ${ }^{681}$ As the possible occurrence of parafunctional habits is evident in any stage of dental treatment, the risks for implant therapy must be considered. ${ }^{679}$ Therefore, bruxism is often considered a contraindication for implant treatment, although the evidence for this is usually based on clinical experience only. ${ }^{62}$

Bruxism has been suggested to cause excessive occlusal load of dental implants and their suprastructures, ultimately resulting in bone loss around the implants or even in implant failure, ${ }^{682}$ even though some studies on the subject have not provided clear conclusions on the issue, ${ }^{199,452,683}$ or have not supported bruxism as a causative effect of dental implant failures. ${ }^{514}$ The only meta-analysis on the subject ${ }^{23}$ found a statistically significant difference when comparing dental implant failures in bruxers and non-bruxers. However, the included studies have a lot of limitations, thus being not possible to suggest that the insertion of dental implants in bruxers affects the implant failure rates.

Two clinical studies ${ }^{158,190}$ evaluating the effect of bruxism on dental implants and implant-supported prosthetic restorations were recently published. The first one ${ }^{190}$ (Study IV) analyzed the complications of dental implant treatment in a group of 98 patients with bruxism 
in comparison with a matched group of 98 non-bruxers. The odds ratio of implant failure in bruxers in relation to non-bruxers was $2.71(95 \%$ CI $1.25,5.88 ; p=0.01)$. Considering the same number of patients with the same total number of implants equally distributed between groups, the bruxers group had a higher prevalence of mechanical complications (Figures 12,13, 14, and 15) in comparison to the non-bruxers group. The second study ${ }^{158}$ used multilevel mixed effects parametric survival analysis to test the association between bruxism and risk of implant failure adjusting for several potential confounders. The statistical model showed that bruxism (among other factors) was a statistically significantly risk factor to implant failure (HR 3.396; 95\% CI 1.314, 8.777; $p=0.012$ ). 

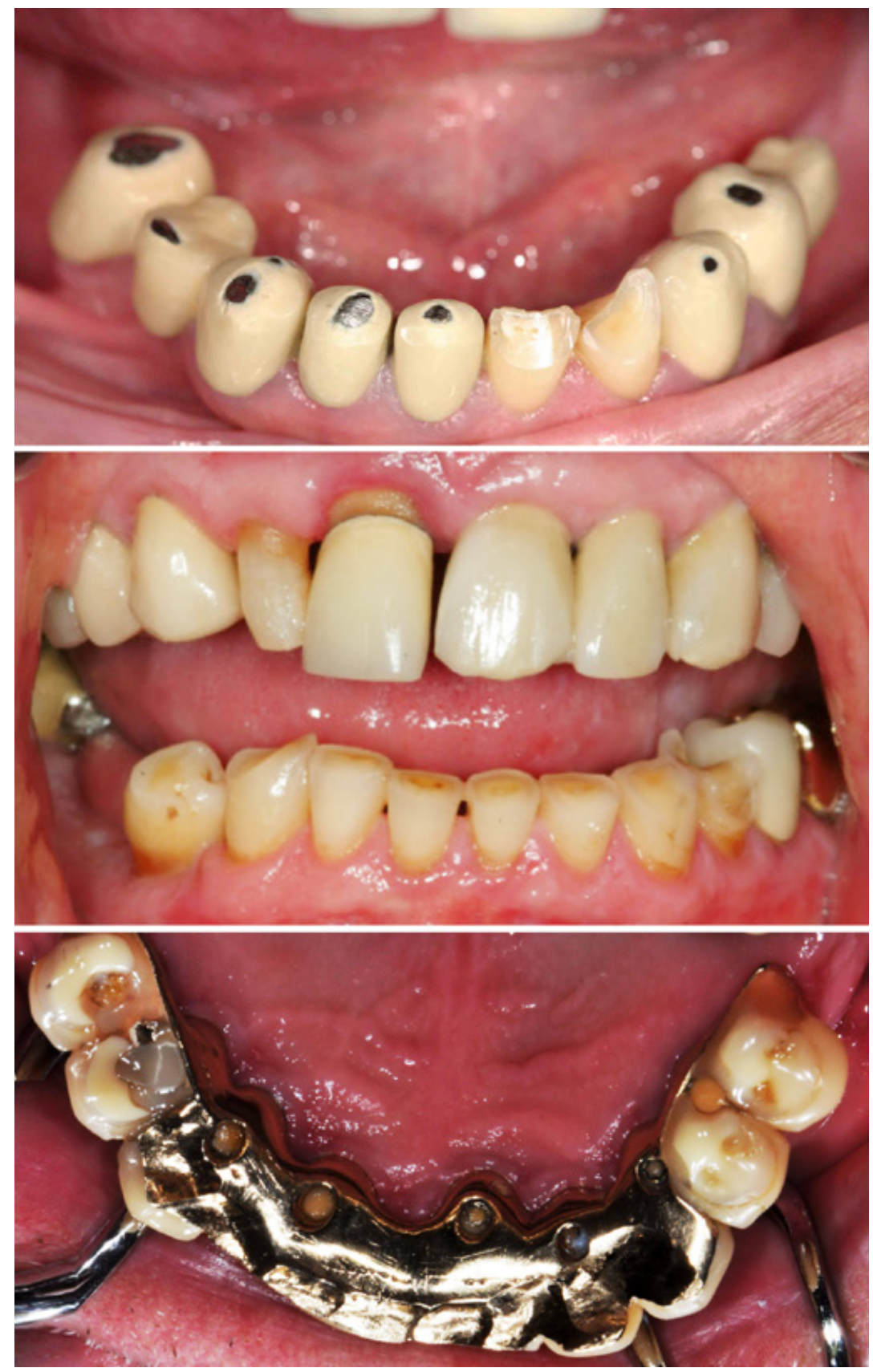

Figure 10. Severe occlusal surface wear in bruxers (middle and bottom images: Courtesy of Dr. Jenö Kisch). 

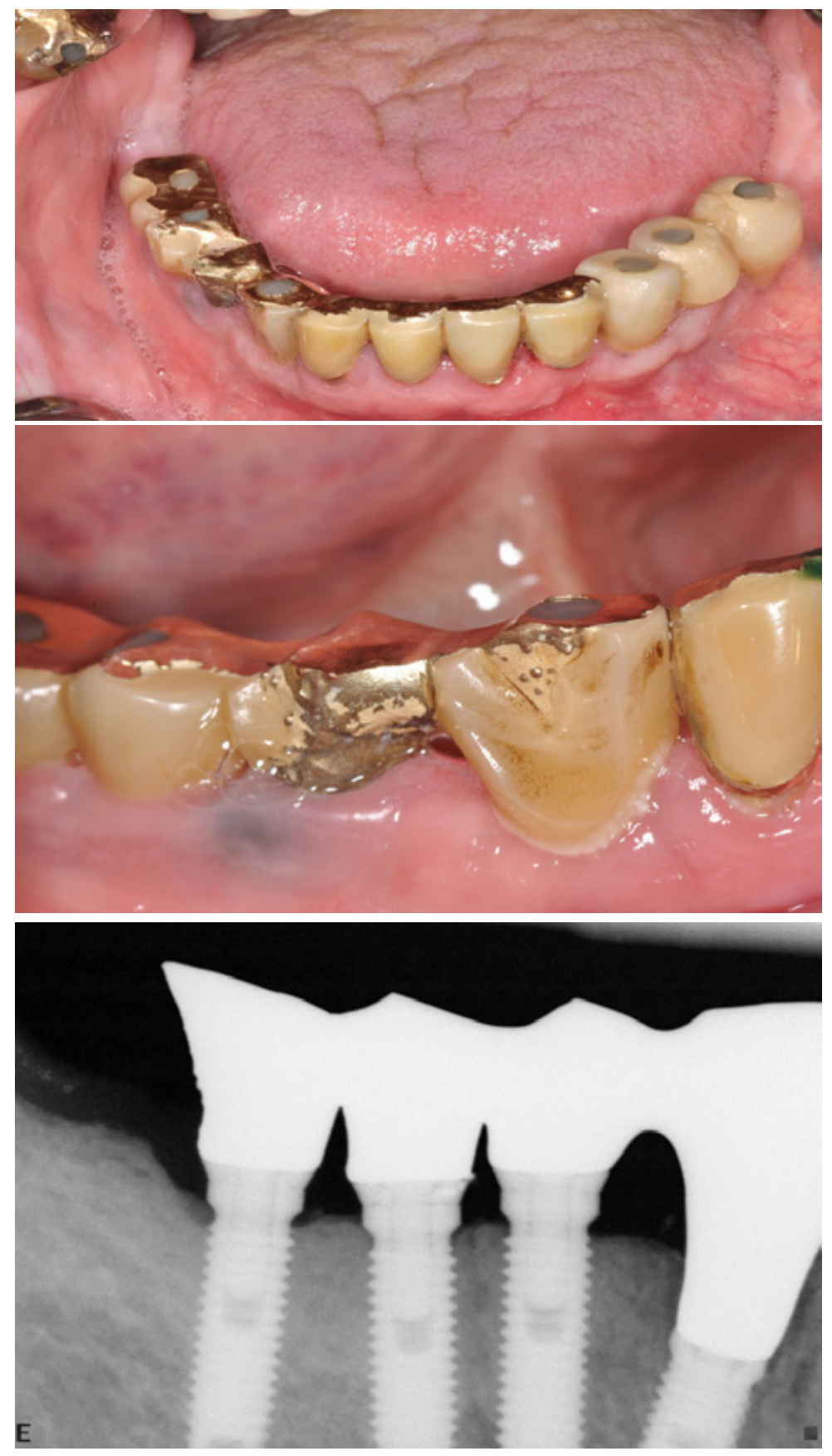

Figure 11. Severe occlusal surface wear in a bruxer patient (Courtesy of Dr. Jenö Kisch). 


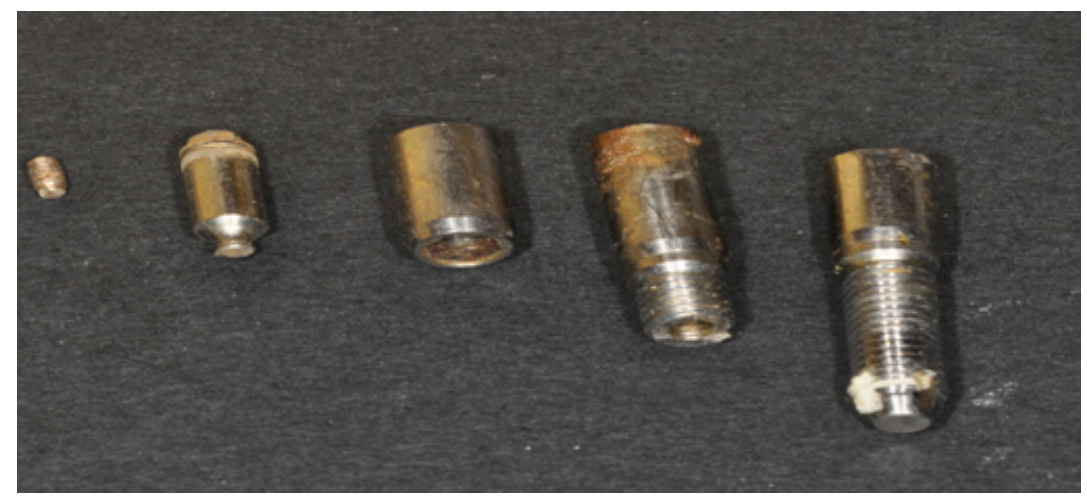

Figure 12. Mechanical complications observed in the same bruxer patient: screw fracture, abutment fracture, implant fracture, and implant exfoliation (Courtesy of Dr. Jenö Kisch).
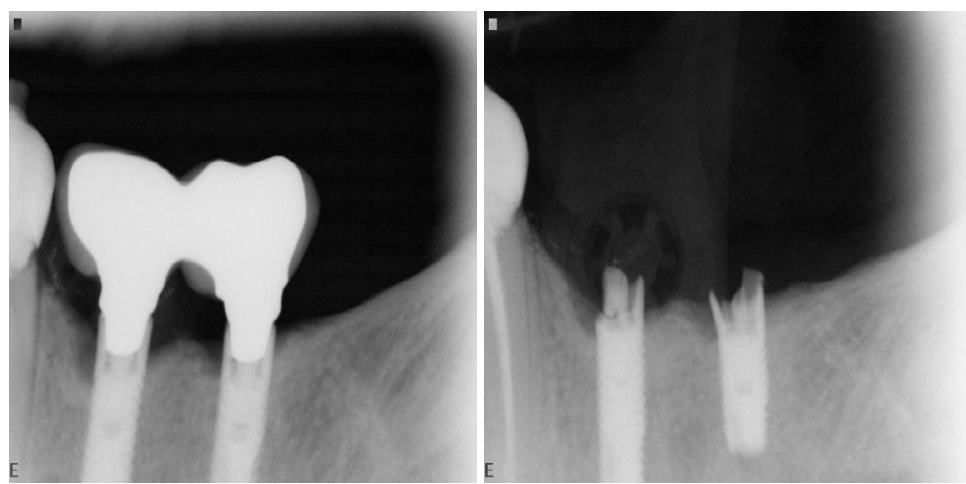

Figure 13. Fracture of implants in a bruxer patient (Courtesy of Dr. Jenö Kisch).

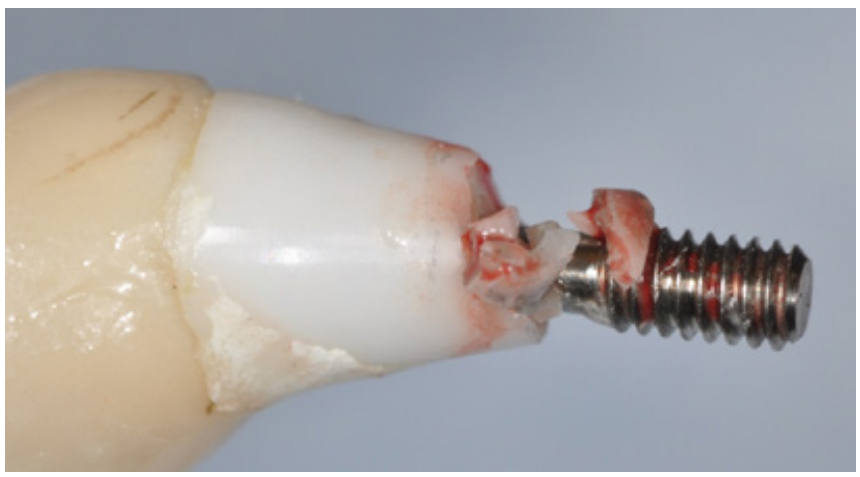

Figure 14. Fracture of ceramic abutment in a bruxer patient (Courtesy of Dr. Jenö Kisch). 

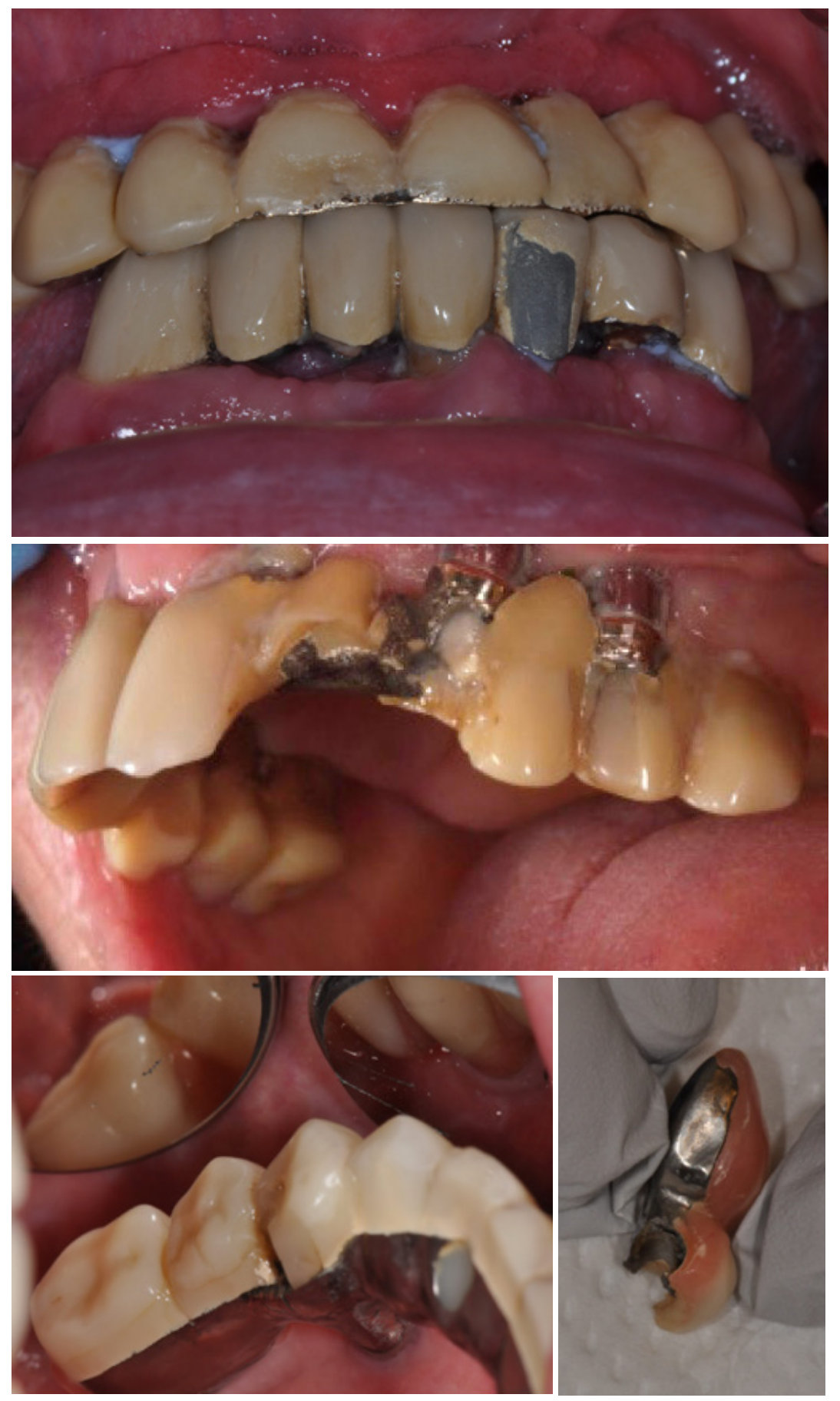

Figure 15. Fracture of prosthetic work in bruxer patients (Courtesy of Dr. Jenö Kisch). 
Even though the cause-effect relationship still needs to be confirmed by more studies, it worth reminding that a high and unpredictable or uncontrolled loading of the implant could lead to micromotions above the critical limit, resulting in fibrous encapsulation of the implant instead of osseointegration. ${ }^{513}$ It is also important to stress that the periodontal ligament of natural teeth provides the central nerve system with feedback for sensory perception and motor control. ${ }^{684}$ Proprioception around dental implants is limited because of the absence of a periodontal ligament, causing lower tactile sensitivity. Consequently, the proprioceptive feedback mechanisms to the jaw closing muscles are limited as well. In addition, the perception of forces is limited in implant patients. ${ }^{685}$ It is, therefore, not unlikely that forces that are applied to implants during bruxism are even larger than those exerted during mastication, ${ }^{682}$ making them more prone to occlusal overload and possible subsequent failure. ${ }^{64}$ Chewing is supposed to be a physiological load for dental implants; bruxism, an overload. ${ }^{682}$

When it comes to MBL, contrary to early failures, late biological failures are characterized by pathological bone loss after full osseointegration was obtained at an earlier stage. Late biological implant failures are, among other reasons, associated with overload. ${ }^{682}$ Occlusal overloading has been suggested to cause periimplant MBL and constitutes a high risk for early implant failure. ${ }^{686}$ Natural teeth have a lower detection threshold of minimal pressure compared to implants, but stresses are distributed evenly around them, whereas stresses around implants tend to concentrate at the crestal bone region instead of distributing themselves evenly ${ }^{687}$ In case of overload, equilibrium between bone resorption and deposition is being disturbed, thereby causing fatigue-related micro-fractures at, and around, the bone-implant interface. ${ }^{688}$

In a clinical human study, Lindquist et al. ${ }^{538}$ showed that parafunctional activity such as bruxism, reported as tooth clenching and occlusal wear on the prosthesis, led to increased bone loss around Brånemark implants. Animal studies also evaluated the relationship between implant occlusal overload and MBL. Miyata et al. ${ }^{689}$ investigated the relationship between occlusal overload and peri-implant tissue and suggested that there is a possibility of bone resorption around the implants caused by excess occlusal trauma, 
even when there is no inflammation in the peri-implant tissue. Duyck et al. ${ }^{690}$ showed that dynamic overload generated by the grinding of teeth resulted in severe angular bone loss. In a recent review, ${ }^{691}$ the authors pointed out that animal experimental studies indeed suggested the potential detrimental effect of excessive mechanical load on peri-implant bone, although RCTs or CCTs of treatment interventions of oral implants designed to study overload are lacking. The authors also observed that the level of evidence of the studies on bone response to implant loading is weak and does not indicate that overload can lead to peri-implant bone loss, except in case of inflammation. Thus, the subject is still controversial.

It is important to say that there is still a lack of agreement about the definition of bruxism, which makes it sometimes difficult to unequivocally interpret the available evidence. ${ }^{62}$ The clinical features for diagnosis of bruxism include complaint of jaw muscle discomfort, fatigue, stiffness, and/or occasional headaches, the presence of tooth wear, tooth sensitivity, muscle hypertrophy, TMJ clicking or jaw lock, and tongue indentation. The clinical diagnosis of bruxism is based on orofacial examination and is usually supported by patient history, self-reports, or parental/partner reports. Considering that many sleep bruxism patients are not aware of grinding if they sleep alone or with a partner who sleeps deeply, ${ }^{692}$ and that the overall prevalence of daytime clenching awareness has been reported by approximately $20 \%$ of the adult population, ${ }^{693}$ this may misguide the clinician to the correct clinical diagnosis. ${ }^{692}$ Polysomnographic analysis has been proposed for a more accurate diagnosis, ${ }^{679}$ even though the process of diagnosing sleep bruxism by means of polysomnography is considered to be complicated by some authors. ${ }^{694}$ Thus, there appears to be a need to establish more accurate and objective methodology for detecting bruxism. ${ }^{679}$ Many clinical studies ${ }^{199,431,452,514,634,695-697}$ did not even report the adopted criteria to classify a patient as having bruxing habits, i.e. the mode of bruxism determination is not given at all.

In the study of Chrcanovic et al. (Study IV), ${ }^{190}$ the authors followed the definition of bruxism proposed by Lobbezoo et al. ${ }^{698}$ : "Bruxism is a repetitive jaw-muscle activity characterized by clenching or grinding of the teeth and/or by bracing or thrusting of the mandible. Bruxism has two distinct circadian manifestations: it can occur during sleep (indicated as sleep bruxism) or during wakefulness (indicated as 
awake bruxism)." The self-conscience of the condition was evaluated with some questions, according to suggestions of a previous study, ${ }^{699}$ questions like the following were asked: (a) Are you aware of the fact that you grind your teeth during sleep? (b) Did anyone tell you that you grind your teeth during sleep? (c) On morning awakening or on awakenings during the night, do you have your jaws thrust or braced? (d) Do you clench your teeth whilst awake? (e) Do you grind your teeth whilst awake? All questions could be answered with either 'yes' or 'no'. The patients were instructed to answer 'yes' if considered their habit to be frequent enough to be clinically relevant (e.g. frequency of more than thrice a week and/or several hours per day). ${ }^{699}$ The clinical condition was re-evaluated to assess possible bruxism-related signs and symptoms. The sign and symptoms of bruxism were listed according to the International Classification of Sleep Disorders ${ }^{700}$ : (a) presence of regular or frequent tooth grinding sounds occurring during sleep, (b) abnormal tooth wear consistent with above reports of tooth grinding during sleep, (c) transient morning jaw muscle pain or fatigue; and/or temporal headache; and/or jaw locking on awakening consistent with above reports of tooth grinding during sleep. Moreover, clenching or grinding of the teeth and/or by bracing or thrusting of the mandible during wakefulness was also considered, according to a recent international consensus. ${ }^{698}$ As reliable and valid diagnostic tools for bruxism are scarce, ${ }^{698}$ a diagnostic grading system of 'possible', 'probable' and 'definite' sleep or awake bruxism was used, as suggested for clinical and research purposes. ${ }^{701}$ According to an international consensus, ${ }^{698}$ 'possible' sleep or awake bruxism should be based on self-report, by means of questionnaires and/or the anamnestic part of a clinical examination. 'Probable' sleep or awake bruxism should be based on self-report plus the inspection part of a clinical examination. 'Definite' sleep bruxism should be based on selfreport, a clinical examination, and a polysomnographic recording, preferably along with audio/video recordings. As electromyography and/or polysomnography were not used due to high cost and limited availability, the patients of the study of Chrcanovic et al. ${ }^{190}$ (Study IV) would only fall into the categories 'possible' or 'probable'. Thus, a patient was considered as presenting bruxism based on self-report of clenching/grinding during sleep or during wakefulness, plus the inspection part of a clinical examination. 
Without a definitive diagnosis of bruxism having been established, it is acknowledged that some of the outcomes illustrated in some of the clinical cases may be due to such load-increasing or materialsrelated factors, rather than to bruxism per se. ${ }^{694}$ Therefore, the possible cause-and-effect relationship between bruxism and implant failure do not yield consistent and specific outcomes. This is partly because of the large variation in the literature in terms of both the technical aspects and the biological aspects of the study material. ${ }^{682}$

It is also important to comment that when overload occurs, the level of stress concentration at the implant-bone interface depends on several factors related to load transfer, such as the direction of the functional loads, the resiliency properties of the implant and alveolar bone, the implant macrogeometry and microgeometry, and the quality of the bone support, ${ }^{702}$ and it is impossible to control these all variables.

Although a hard stabilization splint for nightly use (night guard) will not prevent a parafunctional habit from occurring, it contributes to optimally distributing, and vertically redirecting, the forces that go with nocturnal teeth grinding and clenching. ${ }^{679,703}$

\section{Oral hygiene}

A proportion of implants are unsuccessful due to various inflammatory pathoses in peri-implant tissues. These include two main disease entities: peri-implant mucositis, which is a condition limited to the mucosa surrounding the implant, and peri-implantitis, characterized by a loss of peri-implant bone. ${ }^{704}$ Some studies showed that experimentally induced mucositis was reversible at the clinical and biomarker level once the biofilm was disrupted and oral hygiene procedures were reinforced. ${ }^{705,706}$ On the other side, mechanical debridement alone has shown limited efficacy for periimplantitis. ${ }^{707,708}$ Gingivitis of natural teeth and peri-implant mucositis of implants would be the precursors of periodontitis and periimplantitis, respectively. ${ }^{709}$ Therefore, prevention and management of peri-implant mucositis are critical in long-term maintenance of implants. ${ }^{710}$ In contrast, some researchers see mucositis as a possible indication of an immunological reaction, not in itself dependent on oral hygiene. ${ }^{5}$ 
A clinical study showed that the absence of preventive maintenance in individuals with pre-existing peri-implant mucositis was associated with a high incidence of peri-implantitis. Clinical parameters, such as bleeding on peri-implant probing, periodontal probing depth and the presence of periodontitis were associated with a higher risk of developing peri-implantitis. ${ }^{711}$ Others have reported that bleeding on probing and probing depth are meaningless tools for diagnosis of disease. ${ }^{712}$ Aguirre-Zorzano et al. ${ }^{713}$ observed that the MBL around implants in patients with treated chronic periodontitis is minimal if they are in a controlled supportive periodontal therapy program and there is individual control of plaque index. Rinke et al. ${ }^{714}$ observed an association between regular prophylaxis/ supportive periodontal therapy and the reduction of the risk for peri-implantitis by more than 11 times.

When it comes to peri-implantitis, adjunctive measures (e.g. local antibiotics/antiseptics, laser application) may be effective in arresting disease progression at initial sites, however, moderate to advanced peri-implantitis lesions commonly require a more demanding surgical intervention (e.g. resection of the bony wall, filling of the defect or surface modifications of the implant) or even removal of the implant. ${ }^{708,715}$ In contrast, there is currently published evidence claiming that the reported frequency of peri-implantitis is grossly exaggerated. ${ }^{1}$

There are several studies showing that maintenance therapy decrease the risk of tooth loss. ${ }^{716-719}$ However, the same cannot be said about studies on implants. In the field of implant dentistry there is still a lack of long-term longitudinal studies to determine the actual significance of the effect of maintenance therapy to minimize implant loss.

\section{Alcoholism}

Alcohol abusers are defined by consumption of at least five drinks (more than $60 \mathrm{~g}$ of ethanol) per day for several months or years. ${ }^{720}$ After consumption, alcohol is readily distributed throughout the body in the blood stream and crosses biological membranes, affecting virtually all biological processes inside the cell. ${ }^{721}$ Excessive alcohol consumption substantially promotes adipogenesis and inhibits osteogenesis. ${ }^{722-724}$ Moreover, both chronic and binge consumptions of ethanol are 
known to cause suppression of bone formation and enhancement of bone resorption. Chronic alcoholism is associated with increased risk of fracture and incidence of osteoporosis. ${ }^{725,726}$ Bone metabolism is affected by alcohol consumption because alcohol inhibits osteoblast proliferation. ${ }^{727,728}$ Several in vitro studies investigating the effects of alcohol on bone have demonstrated diminished osteoblast numbers and function. ${ }^{721,727,729-736}$ Other in vitro studies indicated a possible increase in osteoclast numbers resulting in increased resorption. ${ }^{737,738}$ One of these studies ${ }^{738}$ showed that ethanol, even at blood concentrations experienced by the social drinker, has an immediate direct effect on bone cells in vitro, resulting in increased resorption by osteoclasts. According to the results of their rat model study, Trevisiol et al. ${ }^{739}$ concluded that reduced osteoinduction may contribute to impaired bone healing in alcoholics.

Moreover, the consumption of alcohol was found to be associated with suppression of activation and proliferation of $\mathrm{T}$ lymphocytes, as well as with adherence, mobility, and phagocytic activity of monocytes, macrophages, and neutrophils. ${ }^{740,741}$ As these cells populations are crucial in the initiation, regulation, and propagation of cellular immunity, alcoholism may as well be associated to an increased risk of postoperative infection. Although most of these immunological changes have been demonstrated to be related to alcohol, other factors, such as malnutrition and cigarette smoking, may contribute to the increased susceptibility to infection in alcohol abusers. ${ }^{720}$

Few studies have studied the effect of high consumption of alcohol on the osseointegration and peri-implant tissues of oral implants. Bombonato-Prado et al. ${ }^{742}$ evaluated the effect of alcoholic beverage administration on reparative bone formation around hydroxyapatite/ tricalcium phosphate implants inside the alveolar sockets of rats. A significant delay in reparative bone formation was detected in the alveolus of alcoholic rats by a histometric differential point counting method. Koo et al. ${ }^{743}$ evaluated bone formation in alcohol-fed rabbits following the insertion of dental titanium implants and compared the results with a control group. Their results showed that the alcoholic rabbits demonstrated significantly less bone density and direct BIC. Galindo-Moreno et al. ${ }^{74}$ conducted a prospective clinical study to explore the possible link between peri-implant bone loss and the 
widespread habits of tobacco smoking and alcohol consumption. Their results showed that peri-implant MBL was significantly related to a daily consumption of $>10 \mathrm{~g}$ of alcohol, tobacco use and increased plaque levels and gingival inflammation. Torricelli et al. ${ }^{736}$ investigated the proliferation and synthetic activity of osteoblasts isolated from the trabecular bone of rats previously exposed to 7-week intermittent exposure to ethanol vapor when cultured on standard c.p. Ti. Their results suggest that alcohol abuse affects bone repair and decreases the ability to form bone around standard c.p. Ti. Alissa and Oliver ${ }^{745}$ assessed whether alcohol consumption, among other risk indicators, was associated with patients with at least one implant failure using a case-control study design. Heavy drinkers ( $>5$ units/day) had a statistically higher percentage of implant failure compared with nondrinkers and patients who reported consumption of a lesser amount of alcohol ( $<5$ units/day). de Deco et al. ${ }^{746}$ evaluated osseointegration of implants in femurs of rats fed with alcohol and presenting induced estrogen deficiency. Their results showed that the combination of alcohol intake and estrogen deficiency, and the combination of estrogen deficiency and reduced ingestion of food can negatively affect osseointegration in rats.

\section{Narcotic drugs}

Narcotics are chemical agents that induce stupor, coma or, as in the case of morphine, methadone, and codeine, insensibility to pain. These drugs are also used to affect mood or behavior and some are sold illegally for nonmedical purposes, such as cannabis, heroin, and cocaine. Legally speaking, the term "narcotic" is imprecisely defined and typically has negative connotations. ${ }^{747}$ In the medical community, however, the term is more precisely defined and generally does not carry the same negative connotations. Any substance that can affect bone metabolism could, in theory, have some influence on the osseointegration of oral implants. Several studies evaluated the effect of narcotics on bone status and metabolism.

Analgesic therapy with opioids and the use of opioid drugs seem to induce the inhibition of gonadotropin-releasing hormone production, and chronic hypogonadism is an important cause of osteoporosis in both sexes. ${ }^{78}$ Hypogonadism in young women can subsequently 
cause amenorrhea. This endocrinological misbalance is a well-known negative factor for bone mineral density (BMD) metabolism. ${ }^{749-754} \mathrm{In}$ addition, opioids may contribute to increased fracture risk by directly interfering with bone formation. ${ }^{748,755-757}$ Prolonged heroin addiction was associated with accelerated bone turnover and osteopenia in cortical bone without evidence of metabolic bone disease. ${ }^{758}$ Opioids were associated with significantly reduced BMD. ${ }^{759}$ One study ${ }^{760}$ observed that the chronic abuse of opioid drugs may be associated with altered bone metabolism and reduced trabecular bone mass, attributable, at least in part, to gonadal deficiency. However, these alterations seem reversible after drug discontinuation. Another study showed that $83 \%$ of the patients enrolled in a methadone maintenance treatment had low BMD. ${ }^{761}$

A role for the endocannabinoid system (membrane receptors for the psychoactive principle in Cannabis) in the regulation of bone mass has been demonstrated, because mice lacking either of the cannabinoid receptors $\mathrm{CB}_{1}$ or $\mathrm{CB}_{2}$ have abnormal bone phenotypes. ${ }^{762}$ Furthermore, cannabinoid receptor agonists and inverse agonists reduce bone loss in mice following ovariectomy and have direct effects on both osteoclasts and osteoblasts in vitro. ${ }^{763-}$ ${ }^{766}$ Bourne et al. ${ }^{767}$ found no association between cannabis and bone mineral density of the hip or spine in adults, for any level of use. On the other hand, the study of Sophocleous et al. ${ }^{768}$ found that heavy cannabis use is associated with low bone mineral density, low body mass index (BMI), high bone turnover, and an increased risk of fracture. Heavy cannabis use negatively impacts on bone health both directly and indirectly through an effect on BMI.

Balabanova et al. ${ }^{769}$ observed that daily administration of cocaine in sheep caused elevations in plasma parathyroid hormone (PTH) and calcitonin. However, these effects could only be maintained for 10 days of treatment before lack of a response was noted. These authors acknowledged that a physical tolerance may have been reached and that graded increase in cocaine been administered further release of these calciotropic hormones. Given the osteolytic response to high circulating levels of PTH, the consequences of long term cocaine use may include reduced bone mass.

Rico et al. ${ }^{770}$ evaluated osteocalcin by radioimmunoassay at the time of delivery in mothers and in the umbilical arteries of newborns 
in a group of pregnant drug users (eight heroin users and seven cocaine users) and compared with findings from a group of 18 normal mothers and their newborns. Osteocalcin, a vitamin K-dependent protein synthesized by osteoblasts, ${ }^{771,772}$ is a major noncollagenous protein found in bone matrix. ${ }^{773,774}$ Serum concentrations of osteocalcin have been shown to correlate well with histologic parameters of bone formation. ${ }^{774}$ The results of the study of Rico et al. ${ }^{770}$ suggest a toxic effect of these drugs on the osteoblast, which could account for the lower birth weights and skeletal alterations reported in the infants of drug users.

Seifert and Church ${ }^{775}$ examined the long term effects of prenatal cocaine exposure on femoral growth and mineralization in male rats. Femur dry weight, ash weight, organic matrix weight and density were significantly reduced in these animals compared to normal or pair-fed controls. The authors stated that their results suggested that prenatal exposure to cocaine may have long term postnatal impact on bone cell or mineral metabolism.

Only one clinical study ${ }^{776}$ observed a possible association between narcotic abuse and oral implant outcome, showing addiction to narcotics in a group of patients presenting multiple implant failures. An in vivo study investigated whether marijuana smoke influences bone healing around titanium implants in rats. ${ }^{777}$ A group of marijuana smoke inhalation rats was compared to a control group. A negative effect of marijuana smoke was observed in cancellous bone for BIC and bone area. The authors of the study suggested that the deleterious impact of cannabis sativa smoke on bone healing may represent a new concern for implant success/ failure. Other studies focused on alveolar bone loss and periodontitis. Nogueira-Filho et al. ${ }^{778}$ noticed that cannabis smoke may impact alveolar bone by increasing bone loss resulting from ligature-induced periodontitis in rats. Breivik et al. ${ }^{779}$ investigated the effects of longterm exposure (4 weeks) to the widely used narcotic drug and putative neurotoxicant "ecstasy" (3,4-methylenedioxymetamphetamine - MDMA) on neuronal transmitter transport and progression of experimental periodontitis in rats. Their results showed that longterm exposure to MDMA affects the serotonergic and dopaminergic transport systems in the rat brain and increased the susceptibility to the psychosomatic ailment periodontitis following disturbances of 
brain immune-regulatory systems. It is a matter of debate whether similar results could occur around implants, in an experimental peri-implantitis.

We know very little, if anything, on narcotic drug abuse and its possible effects on oral implants, but that we cannot ignore the possibility that some drugs may cause implant problems.

\section{Health}

\section{Patient genetics}

The fact that implant losses tend to cluster in a specific group of individuals ${ }^{193}$ (Study VIII) could indicate specific host characteristics that disturb the osseointegration process and are influenced by genetic factors. ${ }^{780}$ Most of the genetic research in the oral disease has focused on gene polymorphisms that play a role in the immune response, tissue destructive process, or metabolic mechanism. ${ }^{781}$ A combination of the Greek words poly (meaning multiple) and morph (meaning form), polymorphism is a term used in genetics to describe multiple forms of a single gene that exist in an individual or among a group of individuals. A gene is said to be polymorphic if more than one allele occupies that gene's locus within a population. ${ }^{782}$ In some situations, genetic polymorphisms could cause a change in the protein or its expression, possibly resulting in alterations in innate and adaptative immunity and may thus be deterministic in disease progression. ${ }^{781}$ On the other hand, genetic polymorphisms may also act like a protector or a destructive factor for a disease. ${ }^{783-786}$

The genetic variants in the interleukin-1 (IL-1) gene region can be listed as an example of the suggestive influence of genetics on implant dentistry. IL-1 is a potent proinflammatory mediator that is mainly released by monocytes, macrophages, and dendritic cells. Immunological reactions are believed to cause abnormal tissue destruction leading to periodontitis. Inflammatory cytokines are involved in the initiation and continuation of such immune reactions. ${ }^{787,788}$ Involvement of IL-1 in the pathogenesis of periodontal disease has long been suspected because of similarities between its known biological effects and the manifestations of the disease, as well its presence in the lesions. ${ }^{789}$ Feloutzis et al. ${ }^{790}$ investigated the relation between specific IL-1 gene polymorphisms and peri-implant bone loss at dental implants. The results of the study suggested that in heavy 
cigarette smokers, carriage of a functionally significant IL-1 gene complex polymorphism is associated with an increased risk for periimplant bone loss following prosthetic reconstruction and during the supportive periodontal care phase of the treatment. Gruica et al. ${ }^{791}$ reported a follow-up study using the increased number of patients in the same institution as Feloutzis et al. ${ }^{790}$ They observed that there is a synergistic effect between a positive IL-1 genotype and smoking that puts dental implants at a significantly higher risk of developing biologic complications during function. Laine et al. ${ }^{792}$ results provide evidence that IL-1RN gene polymorphism is associated with periimplantitis and may represent a risk factor for this disease. Petkovic et al. ${ }^{793}$ examined the peri-implant crevicular fluid (PICF) levels of IL-1 $\beta$, tumor necrosis factor $\alpha$ (TNF- $\alpha$ ), interleukin-8 (IL-8) and macrophage inflammatory protein- $1 \alpha$ (MIP- $1 \alpha)$ in patients with nonmanifesting inflammation, early and late stages of mucositis. Their results suggest that cytokines could be prognostic markers of implant failure. Vaz et al. ${ }^{781}$ analyzed the association between polymorphisms in the IL-1 gene cluster and failure of dental implants, and showed that the alleles 1 and 2 of IL- $1 \alpha$ gene and the alleles 1 and 2 of IL-1 $\beta$ gene were statistically associated with the success or no success of the dental implants. The study of Shimpuku et al. ${ }^{794}$ suggested that the IL-1 $\beta-5112 / 2$ genotype has a significant association with the incidence of early MBL around implants.

Studies suggesting a lack of association between IL-1 gene polymorphisms and dental implant biological problems have also been published. Wilson and Nunn ${ }^{795}$ could not find statistical association with positive genotype for IL-1 in their group of failed implants (27 patients who had lost implants or had implants with at least $50 \%$ of bone loss on radiographs). In addition, Rogers et al. ${ }^{796}$ could not find any statistical association with combined positive genotype of IL-1 in the group of failed implants (19 patients with failed implants).

It is suggested that peri-implantitis implant success and survival may also be influenced by other gene polymorphisms. Nosaka et al. ${ }^{797}$ observed that patients with a type calcitonin receptor genotype were 20 times more likely to suffer buccal MBL around implants in the mandible than patients with another calcitonin receptor genotype. Shimpuku et al. ${ }^{798}$ showed that BMP-4 genetic polymorphism 
influences early MBL around implants. The results of Santos et al. ${ }^{799}$ suggested a possible relationship between polymorphisms in the promoter region of human matrix metalloprotenase 1 (MMP-1) or a C-1562T substitution in the MMP-9 gene and early implant failure. Later, the same group of researchers ${ }^{800}$ observed similar results with polymorphism in the same gene. Casado et al. ${ }^{801}$ investigated the association between BRINP3 genetic variation and expression and susceptibility to chronic periodontitis and peri-implantitis. The study showed that a BRINP3 polymorphic variant and the low level of BRINP3 expression are associated with peri-implantitis, independently from the presence of chronic periodontitis.

However, not every gene polymorphism seems to be associated with dental implant problems. For example, Dos Santos et al ${ }^{802}$ observed that polymorphisms in the TGF- $\beta 1$ gene are not associated separately or in haplotype combinations with early implant failure, suggesting that the presence of those single nucleotide polymorphisms alone do not constitute a genetic risk factor for early implant failure. Cury et al. ${ }^{803}$ did not find an association between a specific polymorphism in the tumor necrosis factor TNF- $\alpha$ gene and peri-implant bone loss following prosthetic reconstruction.

Although a review on the association between genetic predisposition and dental implant biological complications ${ }^{804}$ pointed out that a small number of properly designed studies have been performed to address the genetic susceptibility to implant failure in terms of biological complications and that methodological and study design issues restrict the possibility to draw robust conclusions, the matter of a possible genetic influence on dental implants cannot be ignored. It seems that we are still incipient on this issue, and further research is needed.

\section{Patient's sex}

In humans, biological sex is determined by some factors present at birth, such as the presence or absence of a Y chromosome, the type of gonads, the internal reproductive anatomy, the external genitalia, and the proportion of the different sexual hormones. ${ }^{805}$ The biological sex is important, as the function of cells and organs depends on their sex, determined by the interplay among the genome and biological and social environments. Sex has profound effects on physiology and 
the susceptibility to disease. ${ }^{806}$ Even though success and survival rates for osseointegrated dental implants are well documented, it is still unknown whether there is influence of sex on the clinical outcomes of the therapy with endosseous dental implants.

The only systematic review and meta-analysis on the subject ${ }^{22}$ with more than 52000 implants suggested that when implants are inserted in men, failures are 1.21 times likely to happen than failures when implants are inserted in women (RR 1.21, 95\% CI 1.07, $1.37 ; p=0.002)$. The difference in failure rates between the sexes is hypothesized to be related to some factors.

Osteoporosis is one of them. After the early to mid-20s, a slow but steady decline in bone mass takes place in both men and women. This is because less than $100 \%$ of the bone that is resorbed every 180 days is replaced by newly forming bone. Over the years, this slight shortfall in bone renewal adds up to a significant loss of bone mass. ${ }^{807}$ It is heightened in women because after menopause natural levels of estrogen are greatly reduced, and estrogen is one of the requirements for osteoblast differentiation from bone marrow osteoprogenitor cells. ${ }^{808}$ Therefore, postmenopausal women undergo a more rapid and significant decline in bone mass. ${ }^{809}$ Furthermore, more women stay with less strong bone than men. Therefore, it takes longer time for males to suffer the consequences of biological bone loss with increasing age. Compromise of systemic bone metabolism may be a risk factor affecting osseointegration and maintenance of osseointegration. One study retrospectively evaluated 13 patients with osteoporosis of the axial or appendicular skeleton, including the jaw bone, that were subjected to oral implant treatment. ${ }^{810}$ The results of the study showed that implant placement in patients in whom the average bone density showed osteoporosis in both lumbar spine and hip as well as poor local bone texture may be successful over a period of many years. The study did not compare the result between sexes.

If a higher degree of osteoporosis may be more prevalent in women than in men, the same does not happen with periodontitis. Epidemiologic studies provide broad-based evidence that men are at greater risk of developing severe periodontal disease than women. ${ }^{811,812}$ A study estimated the prevalence, severity and extent of periodontitis in the adult US population with data from the a national survey and 
observed that after adjustment for the effect of age, total periodontitis was significantly higher in men than in women aged 30 years and older (males $=57 \%$; females $=39 \%$ ), with males showing a 3\%, $7 \%$ and $8 \%$ higher prevalence of mild, moderate and severe periodontal disease, respectively, compared with women. ${ }^{813}$ Freitag-Wolf et al. ${ }^{814}$ investigated interactions of single-nucleotide polymorphism genotypes with male and female sex with respect to the risk for aggressive periodontitis on a genome-wide scale, and observed an increased risk of aggressive periodontitis in men and a decreased risk in women. Moreover, the results of a systematic review and metaanalysis ${ }^{15}$ suggest that an increased susceptibility for periodontitis may translate to increased susceptibility for peri-implantitis. We do not know the mechanisms that connect periodontitis with increased rates of peri-implantitis and loss of implants. Periodontitis is a complex genetic disorder that shows clear differences to the genetics of periimplantitis. ${ }^{815}$ Hence, the connection between a disease of teeth and an immunological reaction affecting foreign body implants ${ }^{787,788}$ may depend on quite different gene patterns and not necessarily being indicative of a similarity between these two conditions. Whatever the reason, the increased rate of peri-implantitis could possibly explain the higher failure rates we found in men.

Concerning the association between gender and recall compliance to supportive periodontal treatment (SPT), there is no common consensus on this issue, and the variance in the studies outcomes may be explained by differences in the study populations, variations in the observation periods, levels of maintenance measures and the use of different criteria to define periodontitis/peri-implantitis. ${ }^{816}$ Even though many studies ${ }^{817-821}$ demonstrated that no significant relationship was found between degree of compliance and gender, several others ${ }^{822-827}$ revealed a predominance of females in compliance with SPT. After the incorporation of implant-supported restorations, special oral hygiene measures are known to be necessary for preventing inflammation, peri-implant diseases, and even implant loss. This suggests that the difference of compliance between implant patients of different sexes to SPT may also play a role in the different implant failure rates.

A survey conducted in the United States reported that, in 2005, the current cigarette smoking prevalence of the population aged $\geq 18$ 
years was higher among males $(23.9 \%)$ than females $(18.1 \%)$. These numbers were $20.5 \%$ and $18.1 \%$ for males and females, respectively, in the survey conducted in 2013 including 34,557 respondents. ${ }^{828} \mathrm{~A}$ study tried to identify predictors of implant failure and peri-implant bone loss, and observed that only smoking and recall compliance had a significant influence on implant failure, based on multivariate analysis. ${ }^{829}$ The results of a recent meta-analysis analyzing 107 clinical trials suggest that the insertion of dental implants in smokers statistically affects the implant failure rates. ${ }^{20}$ These findings suggest that a higher prevalence of smokers among men may, to some degree, be associated with the higher risk of implant failure in men than in women. However, in some societies such as Sweden, females today smoke more than men and it will be interesting to follow up whether this fact will influence future failures or not. ${ }^{830}$

Another explanation behind the noticed difference in implant failure between the sexes may be related to the fact that men are prone to load their implants more strongly than females. Under normal conditions the extra load may not be problematic, but in case of complicating factors or rapid onset of extra load such as the case of suddenly failed teeth around an implant, then overloading may be a significant factor behind the greater implant loss reported in men. According to a recent review on the subject, ${ }^{831}$ total loss of osseointegration appears possible with an already osseointegrated dental implant, when the applied force exceeds the biological adaptable limit, but this has been very rarely documented, and this threshold is currently unknown. The fact is that bone response to functional load depends on a complicated interaction of strain magnitude and time. ${ }^{832}$ Strain (deformation of the bone) is defined as the relative change in the length of the bone and the amount of strain is directly correlated to the stress applied to the bone; for example, occlusal loading, but it is also dependent on the mechanical properties of the bone. ${ }^{831}$ It is reasonable to assume that with different bone types and composition, the thresholds of destructive strain values will be different for each individual, while age and sex might play a significant role in this diversity. ${ }^{831}$

When it comes to sex differences of bite forces, a study observed that the voluntary maximal bite force $(\mathrm{MBF})$ values for men were one third higher than for women. ${ }^{833}$ This sex-related difference in 
MBF may be a result of anatomic differences. Tuxen et al. ${ }^{834}$ used electromyography and biopsy to further explore why MBF is higher in men than in women. The men's masseter muscles had type II fibers with larger diameter and sectional area than those of the women, and the authors suggested that hormonal differences might contribute to the composition of the muscle fibers. The results of Cosme et al. ${ }^{833}$ showed that voluntary MBF was no different between bruxers and non-bruxers when controlling for some potential confounders. One possible explanation may be that the voluntary MBF is different from the MBF exerted during bruxism.

Concerning bruxism, this parafunctional habit has been for many years considered a reason for implant failure or MBL after successful bone-to-implant osseointegration. A recent systematic review on epidemiology of bruxism in adults ${ }^{835}$ observed that bruxism was not found to be a disorder related to sex, since sex differences were not relevant for any of the bruxism activities, even though a female-tomale ratio was reported only in a few studies.

\section{History of periodontitis}

The question if patients with a history of periodontitis are more at risk for peri-implant disease has received increasing attention in the last years. ${ }^{836}$ There is some evidence that patients treated for periodontitis may experience more implant loss and complications around implants including higher bone loss and peri-implantitis than non-periodontitis patients. ${ }^{837} \mathrm{~A}$ history of treated periodontitis does not seem to adversely affect implant survival rates but it could have a negative influence on implant survival/success rates, particularly over longer periods. ${ }^{638}$

Some clinicians assume that periodontally compromised patients (PCPs) present a potentially higher risk for implant failure. The reason for this assumption is that a similar pathogenous bacterial flora forms around diseased teeth and diseased implants, though with some differences in partially and completely edentulous patients. ${ }^{838}$ Implants are rapidly colonized by indigenous periodontal pathogens in partially dentate patients harboring periodontal lesions. ${ }^{838}$ An association between periodontal and peri-implant conditions over 10 years in partially edentulous patients was showed. Marginal bone level at 10 years was significantly associated with smoking, implant 
location, full-mouth probing attachment levels, and change, over time, in full-mouth probing pocket depths. ${ }^{839}$ However, Cecchinato et al. ${ }^{840}$ who evaluated the progressive MBL that had occurred at implants and teeth in the same segment of the dentition following implant placement in partially dentate subjects, reported that when implants lost bone teeth bone was stable and, vice-versa, when teeth lost bone implant bone seemed stable.

A systematic review and meta-analysis ${ }^{15}$ comparing PCPs and periodontally healthy patients (PHPs) suggested that an increased susceptibility for periodontitis may also translate to an increased susceptibility for implant loss, loss of supporting bone, and postoperative infection. The difference between the patients significantly affected the implant failure rates (RR 1.78, 95\% CI 1.50-2.11; $p<0.00001)$, also observed when only the CCTs were pooled (RR 1.97, 95\% CI 1.38-2.80; $p=0.0002$ ). There was significant effects of dental implants inserted in PCPs on the occurrence of postoperative infections (RR 3.24, 95\% CI 1.69-6.21; $p=0.0004)$ and in MBL (MD 0.60, 95\% CI 0.33-0.87; $p<0.0001$ ) when compared to PHPs.

This observation is not surprising if one assumes that periodontal disease not only decreases available bone volume, but also affects the capacity of osseous tissue to remodel ${ }^{841}$ In patients in whom teeth were lost for periodontal reasons, the disease may have decreased the available bone following tooth extraction or resulted in the necessity to place the implant with a more exposed surface to achieve ideal prosthetic position. Both of these situations could result in a greater implant failure rate. ${ }^{577}$ Other factors may also exert influence on the fact that implant failure is more frequent in patients classified in the aggressive periodontitis group. De Boever et al ${ }^{842}$ noticed that it is apparent that in these patients a combination of this form of periodontitis, current smoking habits and general (systemic) diseases was often observed. It should be stressed that the classification used by De Boever et al ${ }^{842}$ to classify periodontitis as 'aggressive' may not be the same as used in other studies.

Here is important to mention the possible high influence of smoking habits on the implants failure rates. Tobacco smoking is considered the principal environmental risk factor affecting the pathogenesis of periodontitis. Tobacco smokers were shown to 
be more prone to developing periodontitis compared with nonsmokers ${ }^{843}$ Furthermore, the results after periodontal therapies are less predictable in smokers compared with non- or former smokers ${ }^{844}$ and the risk of periodontitis recurrence appears to be higher as well..$^{845}$ Karoussis et al. ${ }^{846}$ showed in their study that there was a tendency for a poorer survival rate of implants in smokers versus non-smokers in patients with a history of periodontitis, indicating that the smoking patient susceptible to periodontitis yields a documented higher risk for implant loss than the non-smoking periodontitis patient or the patient not susceptible to periodontitis at all. In the study of Koldsland et al., ${ }^{847}$ the combination of tobacco smoking and a history of treated periodontitis was significantly associated with implant loss after a mean observation time of 8.4 years. Aglietta et al. ${ }^{848}$ showed that tobacco smokers with a history of treated periodontitis displayed lower implant survival rates and higher MBL rates compared with smokers PHPs, independent of other factors such as implant type, healing modality and operator, and despite the fact that smokers PCPs were treated for their periodontal conditions before implant placement and were regularly enrolled in a SPT.

A relationship between marginal implant bone loss and smoking habits was also observed. In the study of Feloutzis et al., ${ }^{790}$ IL-1 gene polymorphisms were determined and compared to the annual rate of bone loss in periodontally susceptible patients. It was revealed that IL-1 genotype positive smoking patients yield a higher risk for peri-implant bone loss than IL-1 negative smokers. Heavy smoking patients, which in this study were the patients smoking 20 cigarettes per day, also significantly demonstrated higher rates of peri-implant alveolar bone loss than non-smokers. Nitzan et al. ${ }^{849}$ showed a higher incidence of marginal implant bone loss in their study in the smoking group, which was more pronounced in the maxilla. Hardt et al. ${ }^{850}$ observed that the amount of longitudinal peri-implant bone loss is related to pretreatment experience of loss of periodontal bone support. The exact relationship between periodontitis and periimplantitis remains unknown; we really do not know whether these two types of disease are similar in origin at all since a demonstrated positive correlation between PCP and implant failure in itself does not prove such a connection. In some studies PCPs demonstrated higher implant failure rates but this difference did not reach statistical 
significance. Obviously, treatment of periodontal infections before implant placement would seem important to avoid placing an implant in an infected bed.

No difference was found for implant failures and the MBL around implants between the PHPs and patients with chronic periodontal disease in the aforementioned meta-analysis. ${ }^{15}$ This is likely due to the thorough supportive maintenance program in which patients from almost all studies were enrolled, and also due to the more mild nature of the chronic periodontitis in comparison with the aggressive ones. In some studies PCPs demonstrated higher implant failure rates but this difference did not reach statistical significance, which could be attributed to the fact that the patients were treated in a periodontal clinic and their periodontal condition was "controlled." Obviously, treatment of periodontal infections before implant placement, achievement of high levels of oral hygiene and enrollment of patients in a regular supportive therapy program contributed to the positive outcomes (or less difference) in some studies. The lack of adhesion to SPT is associated with a higher incidence of bone loss and implant loss, as it has proven to be a key factor in enhancing long-term outcomes of implant therapy by controlling re-infection. ${ }^{836}$ These results may underestimate the value of the SPT in enhancing longterm outcomes of implant therapy, particularly in subjects affected by severe periodontitis. ${ }^{836}$

However, the compliance to SPT cannot always be counted as a factor to decrease or avoid possible periodontal complications. A small number of periodontal maintenance patients seem to be refractory to treatment and go on to experience continued and significant tooth loss. These subjects also have a high level of implant complications and failure. ${ }^{851}$ Moreover, long-term outcomes demonstrated that implants in non-smoking PCPs previously treated for periodontitis were more prone to developing MBL compared with those in PHPs. ${ }^{852}$ These results were obtained despite the fact that all patients were regularly enrolled in and compliant with a SPT program over 10 years. ${ }^{852}$ Furthermore, Fardal and Linden ${ }^{851}$ observed that smoking, stress and a family history of periodontal disease were identified as factors associated with a refractory outcome, and these variables remained significant after multivariate analysis. 
Analysis of the data disclosed different patterns regarding the distribution of the implant losses over time in the two categories of patients. In the study of Hardt et al., ${ }^{850}$ while the number of implants lost during the first year were similar in the two groups, there was subsequently only one implant lost in PHPs compared to five implants (four patients) in PCPs, all with a length of $\geq 10$ $\mathrm{mm}$. Rosenberg et al. ${ }^{853}$ observed that failure up to 1 year of loading occurred relatively frequently in both the PCP group and the PHP group, with no significant differences, indicating that a history of periodontitis in a particular patient does not affect the healing process of osseointegration. On the other side, failure significantly occurred more frequently in the PCP group than in the PHP group after 1 year of loading. Gianserra et al ${ }^{854}$ observed in a retrospective study that patients with a previous history of periodontitis did not lose significantly more implants after 5 years in function than PHPs. However, Roccuzzo et al. ${ }^{836}$ showed that the difference between PHP and PCP is negligible during the first 5 years, but becomes more pronounced later on, being in accordance with the findings of Karoussis et al. ${ }^{846}$ who first demonstrated that a 5-year follow-up is usually not sufficient to evaluate the differences in the clinical outcomes of the various groups of patients. In particular, the difference in the survival rates was not obvious during the first 6 years of follow-up, whereas during the later 4 years, a higher implant loss was observed in PCP. ${ }^{846}$ In the study of Levin et al., ${ }^{855}$ the extended Cox model revealed that until around 50 months, periodontal status is not a significant factor but after 50 months, the hazard for implant failure is eight times greater for the severe PCP. This means that a higher susceptibility for the loss of implants in patients with a history of periodontitis compared to patients with no such history may first become evident after prolonged observation periods of 5-10 years. Rosenberg et al. ${ }^{853}$ hypothesized that one possible explanation for the difference between the 2 groups in this pattern of failure is the influence of the host, which plays an important role in the variable inflammatory process and may be significant in patients with a history of periodontal disease. Another possible explanation could be related to local factors. A reduced quantity of hard tissue in the PCP group may be related to periodontal loss prior to tooth extraction. ${ }^{853}$ 
A problem in comparing these studies is the fact that the studies used different definitions for the presence of periodontal disease, depending on the threshold chosen for the definition of periodontitis, or which conjunct of characteristics may be considered a periodontal disease, i.e., the diagnostic criteria are less clear. Periodontitis comprises a variety of pathologic conditions that affect the health of the periodontium, with patients usually exhibiting gingival inflammation and loss of the connective tissue attachment to teeth, but sometimes also loss of the periodontal ligament, disruption of its attachment to the cementum, and resorption of the alveolar bone can also occur. ${ }^{856}$ Thus, a clear classification system needs to be implemented with clinical evaluation related to a more specific pathology. Moreover, the outcome measures were not related to the type of periodontitis in every study. When it was reported, there was a statistically significant difference concerning the implant failure rates, favoring the less aggressive type of periodontitis in comparison with the more aggressive one.

It has been stated that endodontic problems associated with teeth to be extracted may affect the long-term survival of implants immediately placed into the same sites. ${ }^{857}$ However, a systematic review ${ }^{189}$ on the subject got to the conclusion that the high survival rate and the normal marginal bone changes obtained in several studies support the hypothesis that implants may be successfully osseointegrated when placed immediately after extraction of teeth presenting endodontic and periodontal lesions, provided that appropriate clinical procedures are performed before the implant surgical procedure. Moreover, primary closure over immediately placed implants is another variable that could influence the outcome. Although gingival grafts may be placed over the socket if primary closure is not achieved, in numerous cases the socket may have had exposure to the oral environment during healing. ${ }^{858}$ Numerous implants in some studies ${ }^{857}$ were placed at the same time as periodontal surgical procedures were being carried out. The influence of this co-therapy on implant contamination during the procedure has not been investigated. At the immediate and early implant placement it can be speculated that periodontitis-affected tissues might have had a negative local influence due to the presence of infrabony defects; this could increase the gap between bone and implant ${ }^{457}$ or jeopardize achievement of primary stability. ${ }^{458}$ 
It has been reported that in partially edentulous patients, periodontal pathogens may be transmitted from teeth to implants, implying that periodontal pockets may serve as reservoirs for bacterial colonization around implants. ${ }^{838,859}$ At sites of implants having been in function for 3-4 years, deeper probing depths and higher detection frequencies of periodontal pathogens were observed compared to sites of implants having been in function for only 1-2 years, thus providing evidence for the spread of pathogens with time. ${ }^{860}$ The similarity in microbial flora responsible for periodontitis and peri-implantitis supports the concept that periodontal pathogens may be associated with periimplant infections and failing implants. ${ }^{861}$ However, more recent studies show different results. Carcuac and Berglundh ${ }^{862}$ performed histologic and immunohistochemical analysis of soft tissue biopsies sampled from patients with severe forms of periodontitis or periimplantitis. They observed that the peri-implantitis lesions were larger and comprised greater proportions of plasma cells, macrophages and polymorphonuclears than the periodontitis lesions. They suggested that peri-implantitis and periodontitis lesions exhibit critical histopathological differences, which contribute to the understanding of dissimilarities in onset and progression between the two diseases. Moreover, the study of Cecchinato et al. ${ }^{840}$ evaluated the MBL that occurred at implants and teeth in the same segment of the dentition following implant placement in partially dentate subjects and showed that MBL at implants and teeth in many partially dentate subjects might be independent phenomena.

\section{Irradiation}

Following radiation and surgical resection of oral cancer most patients suffer from defects of soft and hard tissue resulting in functional disabilities and esthetic deformity. These maxillofacial defects are usually reconstructed with vascularized and non-vascularized flaps. This treatment modality alters hard and soft tissues, and the oral cavity in particular is influenced in its integrity by the surgical reconstruction and the adverse effects of the radiotherapy. Dental rehabilitation by conventional prosthesis may be hampered because of the fact that reconstructive surgery changes oral anatomy and radiotherapy results in vulnerable mucosa, xerostomia and bone healing disturbances. Therefore, in most patients a conventional 
prosthetic rehabilitation is unsatisfactory or may not even be a possibility. In this situation dental implants are potentially a more effective oral rehabilitation with regard to mastication, esthetics and speech function. ${ }^{863}$ As dental implants have been increasingly used in oral cancer patients, it is important to know how much irradiated patients in the head and neck region are more at risk of losing dental implants.

Radiotherapy is largely used for treatment of head and neck cancer, as primary therapy, adjuvant to surgery, in conjunction with concurrent chemotherapy or as palliative treatment for late stage and unresectable head and neck malignancies. Although the radiotherapy can increase cure rates, the irradiated patient is susceptible to secondary effects and a series of potential orofacial complications. Radiotherapy may result in progressive fibrosis of blood vessels and soft tissues, in xerostomia, in osteoradionecrosis, and in reduction of bone-healing capacity, among others. ${ }^{864-866}$ Because of the cumulative effects of radiation on bone vascularity, the regenerative capacity of these tissues is limited, which may have a deleterious impact on subsequent implant osseointegration. ${ }^{867}$

A recent systematic review and meta-analysis ${ }^{24}$ assessed the influence of radiotherapy on dental implants. The meta-analyses performed suggest that irradiation negatively affects the survival rates of dental implants, and that implants placed in irradiated maxillae perform much worse than implants placed in irradiated mandibles. The study has failed to support the effectiveness of hyperbaric oxygen (HBO) therapy in irradiated patients requiring dental implants. It also suggests that there is no statistically significant difference in survival when implants are inserted before or after 12 months after the radiotherapy. Moreover, it was observed a tendency to a lower survival rate of implants inserted in the patients submitted to higher irradiation doses.

The significantly higher failure rates in irradiated compared to non-irradiated patients might be caused by the long-term effects of reduced vascularization compromising the implantation site. ${ }^{863} \mathrm{In}$ general, radiation therapy has two antagonistic effects with regard to recovery of irradiated tissue: a short-term positive cellular effect resulting in the improvement of reduced bone-healing capacity, ${ }^{868}$ and a long-term negative effect resulting in permanent damage of 
osteoprogenitor cells ${ }^{869}$ and a gradual, progressive endarteritis obliterans, with thrombosis of small blood vessels, fibrosis of the periosteum and mucosa and damage to osteocytes, osteoblasts and fibroblasts. ${ }^{870}$ Moreover, irradiation of tissues that contain integrated implants increases the risk of soft tissue dehiscences around the implants, and osteoradionecrosis may lead to loss of the implants. ${ }^{871}$ This failure difference after radiation therapy was observed in several studies, although the radiation dosage and observation period varied in the majority of these cases.

Another aspect to consider is the use of HBO. There is no strict consensus about the use of adjunctive $\mathrm{HBO}$ therapy but many studies stressed the advantages of $\mathrm{HBO}$ treatment for wound healing in the irradiated soft and hard tissue. ${ }^{863}$ As a result, some authors use $\mathrm{HBO}$ as an additive therapy when implant therapy in irradiated bone is planned. It is stated in the literature that $\mathrm{HBO}$ results in an increased oxygen tension in the irradiated ischemic bone and provokes capillary neoangiogenesis ${ }^{869}$ and bone formation. ${ }^{872}$ The exact correlation between HBO dose and duration of antifibrotic response is not yet settled. It has been convincingly demonstrated that such treatment dramatically decreases the risk of trauma-induced osteoradionecrosis by promoting vascular proliferation, collagen synthesis, bone remodeling activities, and healing of bone wounds in irradiated tissues. ${ }^{873}$ Moreover, $\mathrm{HBO}$ therapy increases the amount of force necessary to unscrew integrated titanium implants in irradiated bone, ${ }^{874}$ which suggests that promoted integration has occurred. Larsen et al. ${ }^{872}$ showed a difference of $13.9 \%$ in mean percent of integration after 4 months in the osseointegration surface of irradiated and non-irradiated animals. This difference dropped to $6.38 \%$ when animals received $\mathrm{HBO}$ before and after implantation. The difference was shown to be significant, though without establishing whether this reduction is of significance in relation to implant support. As long as the direct bone-implant interface is sufficiently large to support the suprastructure, it can be argued that additional support to the implant surface is unnecessary, particularly in light of patient inconvenience and the cost of $\mathrm{HBO}$ treatment. ${ }^{875} \mathrm{HBO}$ therapy needs expensive equipment, requires significant patient compliance and involves financial costs per patient treatment. In addition, HBO therapy is not without risks and adverse effects like barotrauma, 
particularly of the middle ear, $\mathrm{O}_{2}$ seizures or a change in the refractive power of the lens. ${ }^{876}$ Moreover, results from animal experiments may only partly be extrapolated to clinically relevant conditions for endosseous implants in irradiated bone. Apart from considerable methodological limitations in the simulation of the therapeutic reality and temporal restrictions, events during the follow-up (induction of neoplasms, loss of implants, osteoradionecrosis, etc), differences related to cell cycles, structural peculiarities of the bone matrix, the periosteal situation, and the enzyme system of the species have to be taken into account. ${ }^{877}$ It is no less important to mention that several studies $^{875,878-898}$ demonstrated a high survival rate for the implants placed in irradiated jaws without the use of adjunctive HBO therapy. It might have been expected a significantly higher failure for patients submitted to HBO, since most of the patients selected for $\mathrm{HBO}$ could have been at a higher risk for osteoradionecrosis due to a higher irradiation dose, and consequently at a higher risk of implant loss. For example, the difference in implant survival between the $\mathrm{HBO}$ and non-HBO treated patients observed in the study of Schoen et al. ${ }^{894}$ was remarkable, but was mainly caused by one $\mathrm{HBO}$ treated patient who developed osteoradionecrosis and subsequently lost all four implants. However, the meta-analysis of Chrcanovic et al. ${ }^{24}$ found no statistically significant difference when comparing implant failures in irradiated patients receiving $\mathrm{HBO}$ or not; this is most likely the result of the small sample size in many studies and selection bias. There are still no randomized, controlled, double-blind studies conducted to prove that $\mathrm{HBO}$ really has a significant osseointegration stimulating effect in irradiated patients. However, there are certain technical difficulties related to designing such a study, such as blinding a chamber treatment and the design of the placebo treatment. ${ }^{899}$

The time span between the irradiation and the implant surgery may influence the survival of the dental implants, and recommendations for an optimal time interval are inconsistent. Dental implants can be inserted during the ablative surgery or after completed radiotherapy. When the implants are inserted during the ablative surgical session, a large part of the integration will occur in the period between surgery and radiotherapy, i.e. within 4-6 weeks. ${ }^{900}$ However, a large majority of studies reviewed by Chrcanovic et al. ${ }^{24}$ report on implants installed after radiotherapy. The advantage of placement after radiotherapy 
is that the anatomical situation, residual function and prognosis can be taken into account in the decision of whether to use implants or not. Moreover, tumor recurrence has been reported to occur most often between 8 and 12 months after surgery according to one study, ${ }^{901}$ or within 2 years according to another one; ${ }^{879}$ thus oral rehabilitation may be delayed until this period of highest risk has passed. The disadvantage is that patients are often psychologically and physically weakened by the therapy, resulting in postponement or even cancellation of prosthetic rehabilitation. ${ }^{902}$ Moreover, after radiotherapy the vascularization and regenerative ability of the irradiated tissues can be decreased, which may lessen the prospect of successful osseointegration of the dental implants. ${ }^{903}$ The subject is controversial. King et al. ${ }^{904}$ indicated that blood vessels that were destroyed radiologically show partial recovery after 3 to 6 months. Wächter and Stoll ${ }^{905}$ conducted histomorphometric studies, the results of which state that implantation can be performed, at the earliest, 12 to 18 months after the conclusion of irradiation. Jacobsson ${ }^{868}$ reported an improvement in the bone healing capacity by a factor of almost 2.5 during a 12-month period following irradiation. According to the author, functional self-regeneration of the damaged tissue would provide sufficient implant healing and osseointegration capacities of the bone 1 year after radiation therapy. On the other side, Marx and Johnson ${ }^{869}$ stated that after 6 months, fibrosis is expected to start in the irradiated tissues as a result of reduced cell reproducibility and progressive ischemia. This process will increase with time, especially when curative doses of radiotherapy have been given. The meta-analysis of Chrcanovic et al. ${ }^{24}$ found no statistically significant difference when failure of implants was compared for the implants installed before and after a period of 12 months from the radiotherapy, and this is most likely dependent on the fact that only three studies ${ }^{863,906,907}$ performed this comparison. Two other studies did not report defined time points to enable the comparison, but did not find any influence of time of implantation on the implant failure rates. ${ }^{883,891}$

Named advantages of implant installation during ablative tumor surgery include ${ }^{900}$ : (1) Implant surgery in an area compromised by radiotherapy can be avoided, thus reducing the risk of late complications, such as development of osteoradionecrosis; (2) initial 
implant healing (osseointegration) will take place before irradiation; (3) the patient can benefit from the support of the implants at an earlier stage after treatment, e.g. concerning the rehabilitation of speech and swallowing. Prosthetic rehabilitation can start early providing the patient with a more timely improvement in oral function; (4) there is no need for adjunctive prophylaxis such as the long-term use of antibiotics or hyperbaric oxygen therapy. The risks of installing implants during ablative surgery have to be named as well: (1) improper implant positioning, especially when ablative surgery will result in gross alterations in the anatomical situation and/or intermaxillary relationship, e.g. after mandibular continuity resections or if the anatomical situation is changed due to reconstructive surgery; (2) interference with or delay of oncological therapy including radiotherapy; (3) development of post-treatment complications caused by installation of implants during ablative surgery; and (4) lack of use of implants, due to early tumor recurrence or patients refusing abutment connection surgery. ${ }^{900}$ The advantages of placement of implants sometime after ablative and reconstructive surgery include the ability to make detailed assessment of disease status, oral function, motivation, and rehabilitation requirements of the patient before commencing radiotherapy treatment. ${ }^{144}$

Concerning the radiation dose, the implants inserted into locations irradiated with $\geq 50$ Gy in four studies ${ }^{879,906-908}$ had a lower survival rate than implants in locations that are irradiated with $<50 \mathrm{~Gy}$. The same happened in another study, ${ }^{909}$ but comparing different dose thresholds ( $>54$ versus < 54 Gy). Generally, high radiation doses $>50$ Gy have been reported to result in chronic complications like radioxerostomia and impaired wound healing. ${ }^{910}$ Also, irradiation doses above 65 Gy may significantly increase the risk of development of osteoradionecrosis, ${ }^{911}$ which may also be a reason for implant failures. Moreover, it was shown that there is a distinct dose-dependent relationship between the duration and extent of irradiation and the resulting reduced osteogenesis. ${ }^{912}$ Bone healing was delayed at lower doses of radiation with the formation of more impaired fibrous tissue and non-lamellar bone, but severely disturbed at radiation doses used to treat head and neck cancer. ${ }^{913}$ However, the meta-analysis of Chrcanovic et al. ${ }^{24}$ did not find a significant higher risk of losing an implant in patients receiving higher doses of radiotherapy. Once 
again, this result may have been influenced by the limited number of studies performing this comparison. The comparable survival rates of both groups in the study of Niimi et al. ${ }^{886}$ and even a higher survival rate of implants inserted into locations of higher doses in the study of Shaw et al. ${ }^{144}$ might be caused by the effects of reduced vascularization that compromises both irradiated and non-irradiated locations. ${ }^{869}$

Another circumstance that may increase implant failure is the anatomy of the implantation site. Oral anatomy may change after ablative surgery and eventually following reconstruction by grafts and flaps, and the resulting defects and bulky areas or the presence of insufficient soft tissues for coverage may compromise prosthetic rehabilitation. ${ }^{914,915}$ One could speculate that the bony section of the graft may have been non-vital from the time of placement or that placement of implants in the grafted block of bone may have compromised the vitality of the block. ${ }^{916}$ Some studies on this topic address the increased implant loss in augmented bone to the reduced primary stability of dental implants due to impaired mechanical bone quality as well as to increased bone resorption kinetics. ${ }^{544,558,917}$ The lower survival of implants in the grafted bone may be the result of differences in bone quality, bone volume and revascularization compared with the original residual bone. There is also a difference when it comes to the graft type. Vascularized bone flaps maintain their viability even following prolonged periods of ischemia provided that the medullary nutrient blood supply is later restored. ${ }^{918}$ This is unlike nonvascularized cortical bone grafts, where implant placement must await a lengthy delay to allow for 'creeping substitution' ${ }^{645}$ to occur. Even with all these disadvantages, Chrcanovic et al. ${ }^{24}$ found no statistically significant difference concerning failures of dental implants when inserted in irradiated grafted bone or in irradiated native bone. This could be related to the assumption that the clinical courses of implants may not be affected by the presence of grafted bone once the graft has succeeded. ${ }^{915}$ However, this is difficult to ascertain, as not every study included in the review of Chrcanovic et al. ${ }^{24}$ provided detailed information about grafting procedures. On the other side, when the failure rates were compared in irradiated versus non-irradiated patients, ${ }^{24}$ there was a statistically significant difference for implants inserted in grafted bone and in native bone. 
Once again, when the term 'irradiation' is inserted into the equation, there are significant differences.

Concerning the different jaws, it was observed in one study ${ }^{906}$ that the most dominant variable influencing implant survival in irradiated bone is the implant's location in the maxilla or mandible. The side effects of radiotherapy appear to be more serious in the mandible than in the maxilla because of its inferior blood supply. 883 However, it appears that implant failure in the maxilla follows a similar course in irradiated and non-irradiated patients. ${ }^{24}$ The results comparing implant failures in irradiated and non-irradiated maxilla and in irradiated and non-irradiated mandible must be interpreted with caution, due to the limited number of studies reporting this information ( 2 and 4 , respectively). It is also important to consider whether all implants had been inserted in the field of irradiation, even though this information was rarely provided by the studies. In the case of the mandible, for example, the external beam radiation therapy for oral malignancies does not always include the whole mandible and, hence, implants inserted anterior to the mental foramina might be inserted in a field of lower radiation dose. This will naturally affect the outcome for the implants. ${ }^{899}$

When it comes to postoperative infection, soft tissue may be affected by irradiation, and the causes might be the post-operative and post-irradiation xerostomia, different saliva quantity and quality and altered microflora as well as the presence of scar tissue after reconstruction and the loss of attached and keratinized gingiva adjacent to the implants. ${ }^{863,891}$ It may be suggested that stable soft tissue conditions are crucial for the possibility to perform oral hygiene and can influence the clinical performance of the implants in irradiated patients. ${ }^{896}$ This could influence the incidence of periimplantitis and consequently the MBL. Chrcanovic et al. ${ }^{24}$ observed a statistically significant difference between the irradiated versus non-irradiated patients concerning the MBL, favoring non-irradiated patients. However, this result must be interpreted with caution, due to the limited number of studies that evaluated the condition. The same can be said about the meta-analysis results for the outcome postoperative infection.

There are a considerable number of confounding factors that might also influence therapy with dental implants in irradiated patients. 
First, bone resections may result in unfavorable prosthetic circumstances by producing bulky and soft areas. In these situations, (removable) prosthetic appliances are often complicated and may cause overloading of the implants. ${ }^{914}$ This negative influence may be responsible for lower survival rate of implants inserted into jaws treated with bone resections, compared with that of implants inserted into jaws that did not undergo bone surgery. ${ }^{906}$

Second, the percentage of patients who had received postoperative radiotherapy may have decreased over time among the survivors, as it was observed in many studies, which could have contributed to the relatively low failure rate of implants in irradiated bone. Those patients selected to be submitted to radiotherapy may be the patients with cancers of worse prognosis.

Third, in the analysis of implant survival, it is important to ascertain whether the implants that were placed and considered osseointegrated were, in fact, used for the final implant-supported prosthesis. ${ }^{919}$ A longer follow-up period can lead to an increase in the failure rate, especially if it extended beyond functional loading, because other prosthetic factors can influence implant failure from that point onward. This might have led to an underestimation of actual failures in some studies. However, it is hard to define what it would be considered a short follow-up period to evaluate implant failures when comparing these techniques.

Fourth, most oral cancer patients have a history of smoking, drinking poor oral hygiene. ${ }^{920}$ The local environment is less favorable to dental implants than in healthy subjects. Saliva quantity and quality as well as oral microflora change considerably after salivary gland resection. Landes and Kovács ${ }^{891}$ observed that plaque and peri-implant bleeding indices remained typically high compared with non-cancer patients. Oral hygiene and compliance were limited although patients received individual oral hygiene instruction. On the other hand, a rapid plaque accumulation and bleeding tendency was concomitant with postoperative and post-irradiation xerostomia, altered microflora, saliva quantity and quality, smoking and drinking. Irradiation leaves a lessviable bone that is prone to infection; furthermore, oral xerostomia post-radiotherapy complicates the situation..$^{909}$

Fifth, as oral cancer represents around $3 \%$ of the total cancer incidence, ${ }^{921}$ most of the studies were not able to create a homogenous 
collective for more than the factors such as oral squamous cell carcinoma, edentulousness or severely reduced tooth number. By breaking down the study by each variable, the authors of the studies may had had serious doubts regarding the clinical value in a study that is totally homogenous in tumor staging, Gy of irradiation, etc. It can be said that in order to have sufficient group sizes and a clinically relevant conclusion, groups in the studies were therefore not supposedly broken down further. Homogenous or matched collectives somewhat have a higher significance but they tend to be inhibited by small case numbers. ${ }^{891}$

Sixth, it is known that the surface properties of dental implants such as topography and chemistry are relevant for the osseointegration process influencing ionic interaction, protein adsorption and cellular activity at the surface. ${ }^{311}$ The studies here included made use of implants with different brands and surface treatments. Titanium with different surface modifications shows a wide range of chemical, physical properties, and surface topographies or morphologies, depending on how they are prepared and handled, ${ }^{922}$ and it is not clear whether, in general, one surface modification is better than another. $^{311}$

Seventh, there was a great variation of the implant healing time before the prosthetic loading. In irradiated tissue, the local healing ability is impaired ${ }^{886,909}$ and the vulnerable time period following insertion becomes jeopardized with early loading. The overloading of the implants, induced by altered oral anatomy following ablative surgery or reconstruction by grafts and flaps may also increase implant failure in malignant cancer patients compared with healthy patients. ${ }^{913}$ The optimal head and neck oncology treatment-related healing time of implants before loading is still in need of further research and probably can be shortened significantly. ${ }^{895}$

Eighth, patients in some studies were subject to adjunctive chemotherapy. The effect of chemotherapy on the osseointegration and survival of endosteal implants is not well established, even though it was shown that the risk of irradiation-induced bone damage is increased by chemotherapy. ${ }^{923}$ It is suggested by animal model studies that chemotherapeutic agents have an adverse effect on normal physiological bone turnover, especially osteoblastic activity, and would also be expected to alter fracture-healing and bone- 
allograft incorporation by these same mechanisms..$^{924,925}$ However, chemotherapy did not have a detrimental effect on the survival and success of dental implants in some studies, ${ }^{142,896,926}$ even though implant insertion in these studies was performed at least 6 months after chemotherapy. Thus, it is unknown whether the time point of chemotherapy might be decisive.

Ninth, it is important that the patients are followed-up long enough before reporting implant success in irradiated bone. ${ }^{899}$ However, since the life-span prognosis for most patients with oral malignant tumors is rather poor and the 5-year survival rate is reached by only approximately $50 \%$ of the patients, it is difficult to collect long-term data on implants placed in these patients. ${ }^{903}$ Moreover, one study ${ }^{927}$ showed that the early dropout after the final prosthetic treatment is high and that some patients were hindered to attend the regular recall sessions due to serious health conditions, which makes it even more difficult to collect substantial data on the long-term situation. It is also important to stress that the patients with better oral and general health conditions are usually the ones who are more willing to pay a recall visit.

The failure of dental implants in irradiated patients is therefore subjected to many considerations and predictability is dependent upon issues like the use of $\mathrm{HBO}$ and chemotherapy, the timing of the implant placement in relation to radiation therapy, the radiation dosage, the insertion of implants in native of grafted bone, selection of anatomic site, the patients' oral hygiene conditions and habits (smoking, drinking), the implant surface treatment, the prosthetic loading conditions, the type of prosthetic restoration, the period of follow-up, and risk of osteoradionecrosis. ${ }^{24}$

\section{Diabetes mellitus}

Dental implant survival is initially dependent upon successful osseointegration following placement. Any alteration of this biological process by excessive surgical trauma, infection or metabolic upset may adversely affect treatment outcomes. ${ }^{928}$ Subsequently, as an implant is restored and placed into function, bone remodeling becomes a critical aspect of implant survival in responding to the functional demands placed on the implant restoration and supporting bone. The critical dependence on bone metabolism for implant survival may be 
heightened in patients with diabetes. ${ }^{929}$ Diabetes is a chronic disease that occurs when the pancreas does not produce enough insulin, or when the body cannot effectively use the insulin it produces. The number of people with diabetes increased from 153 (127-182) million in 1980, to 347 (314-382) million in 2008. ${ }^{930}$ These trends highlight the urgency for a better understanding of diabetes as well as for improving the care of patients with diabetes.

Diabetic patients have increased frequency of periodontitis and tooth loss, ${ }^{931}$ and together with the fact that diabetes is associated with delayed wound healing, ${ }^{932}$ prevalence of microvascular disease, ${ }^{933}$ and impaired response to infection, ${ }^{934}$ diabetes has been considered a risky condition for dental implants. Accordingly, it has been stated that diabetes remains a relative contraindication for implant therapy; ${ }^{935}$ well-controlled diabetic patients may be considered appropriate for implant therapy while diabetic patients lacking good glycemic control may be denied the benefits of implant therapy. ${ }^{929}$ Decreased levels of implant osseointegration have been demonstrated in hyperglycemic animals consistent with untreated type 1 diabetes. ${ }^{936,937}$ However, the subject is contradictory, since there are numerous studies that offer indirect evidence for diabetes patients to benefit from oral rehabilitation using dental implant therapy.

It has been suggested that the relative contraindication for implant surgery is related to the stability of the diabetic's blood sugar level. Unfortunately, the application of the finding from many studies to clinical practice is limited by the lack of specific information characterizing the subjects' diabetic status. While most of these studies describe the subjects' diabetes as being 'well-controlled', the authors do not report how they assessed glycemic control. ${ }^{938}$ A systematic review and meta-analysis assessing the influence of diabetes on dental implants therapy ${ }^{16}$ identified two ${ }^{938,939}$ of the 14 publications included ${ }^{855,928,938-949}$ in the study in which patients lacking acceptable glycemic control (through the estimation of glycosylated hemoglobin - HbA1c) were included. Five studies ${ }^{940,942,944-946}$ reported that the patients' diabetes was under control, but it was not mentioned the level of control, whereas the other studies included in the review did not provide any information about glycemic control. In the study of Moy et al., ${ }^{950}$ even patients with controlled diabetes were almost 3 times as likely to develop implant failure compared 
to other patients. Glycemic control is a primary consideration for patients with diabetes, and there is a clear correlation between glycemic control and the development of microvascular and macrovascular complications. ${ }^{951}$ Tissue hyperglycemia impacts every aspect of wound healing by adversely affecting the immune system, including neutrophil and lymphocyte function, chemotaxis, and phagocytosis. ${ }^{952}$ This also leads to a greater predisposition to infection of the wound. Moreover, animal studies showed negative effects of hyperglycemia, not only on bone formation, but also on bone strength and fracture healing. ${ }^{936,953,954}$ These effects are suggested to affect the osseointegration. However, a prospective evaluation of 58 patients with presumably well-controlled diabetes who received mandibular implants reported that glycemic control was not related significantly to implant success over five years. ${ }^{955}$ Dowell et al..$^{938}$ and Tawil et al. ${ }^{939}$ also observed that compromises in glycemic control may not affect implant success in human subjects.

Heterogeneity in eligibility criteria for implantation in different diabetic populations may explain the wide between-study variations. The true differences in metabolic effects between type 1 and type 2 diabetes remain unclear. ${ }^{929}$ It has been proposed that diabetes leads to decreased bone turnover, with reductions in both resorption and formation and that it is the difference in ages of onset of types 1 and 2 diabetes relative to bone growth patterns that lead to these distinctions in outcomes. ${ }^{956}$ Because type 1 has an earlier onset than type 2 diabetes, one can assume that implant loss is more frequent in patients with the former form of diabetes. One possible reflection in oral implantology was observed by Alsaadi et al., ${ }^{945}$ who detected a significant effect of diabetes type 1 on early implant failures ( $p$ $=0.02)$, the same not happening with diabetes type $2(p=0.39)$. However, it is important to observe that in the study of Alsaadi et al.,${ }^{945}$ only one implant was placed in the only patient with diabetes type 1 in the study, and this implant failed, whereas 25 implants were inserted in patients with diabetes type 2 , with only one failure (694 implants were placed in non-diabetic patients, with 13 failures). One of the possible problems in including patients with diabetes type 1 in dental implant clinical studies is its prevalence: over $90 \%$ of people with diabetes have type 2 . Since implant outcomes for patients with type 1 diabetes may differ from those for patients with type 2 
diabetes, it is important for studies that include both patients' types to report the outcome data separately for each group. ${ }^{638}$

In animal studies it was shown that uncontrolled diabetes hinders bone formation, bone remodeling, and wound healing ${ }^{957}$ and causes reduction in BIC and bone thickness, ${ }^{958}$ while insulin upregulates bone formation ${ }^{936}$ and maintains BIC. ${ }^{959}$ The effects of a hyperglycemic state have been shown to include inhibition of osteoblastic cell proliferation and collagen production during the early stages of callus development, resulting in reduced bone formation, as well as diminished mechanical properties of the newly formed bone. ${ }^{953}$ Reduced BIC may indicate a poorer healing response and may predict a reduced ability of the implant to withstand bacterial and load challenges. If the lack of BIC is carried to the extreme, osseointegration would be deemed to have failed and the implant would be found to be mobile at stagetwo surgery. ${ }^{928}$ Oates et al. ${ }^{960}$ demonstrated alterations in implant stability consistent with impaired implant integration for persons with type 2 diabetes mellitus in direct relation to hyperglycemic conditions. It was observed that persons with $\mathrm{HbA} 1 \mathrm{c} \geq 8.1 \%$ had a greater maximum decrease in stability from baseline and required a longer time for healing, which also suggests alterations in the biologic integration of the implants in direct relation to glycemic control. ${ }^{960}$ It seems reasonable to postulate that an implant demonstrating reduced BIC may be less able to withstand functional stresses placed upon it during the healing phase. Further breakdown of the peri-implant bone could therefore ensue and this could result in a loosening of the implant and its ultimate failure. ${ }^{928}$ However, this has not yet been clinically proved, since most of the studies reviewed by Chrcanovic et al. ${ }^{16}$ did not inform of the times which the implants placed in diabetics were loaded. The study of Tawil et al. ${ }^{939}$ was the one only in which some implants $(\mathrm{n}=58)$ inserted in diabetic patients were submitted to immediate loading, but no failure was observed after a mean follow-up of 42 months.

In some dental implant studies including diabetic patients, ${ }^{938,945}$ the patients were followed for a short period (up to 6 months). Thus, even though it is especially during the healing time, up to abutment surgery, that systemic factors can be most easily identified as other risk factors that occur after abutment surgery do not apply, ${ }^{961}$ only early failures could be assessed. A longer follow-up period can lead 
to an increase in the failure rate. Moreover, the results found in the studies differed from each other, and this difference could be due to factors such as differences in the patients included in the study or the clinicians placing and restoring the implants. For example, Olson et al. ${ }^{955}$ observed that implant failure had a statistically significant association with an increase in years of diabetic history. The authors hypothesized that, as duration of diabetes is associated with increased classic microvascular complications, this increase in microvascular disease may be postulated to have contributed to implant failure. However, Tawil et al. ${ }^{939}$ divided the patients with well-controlled diabetes into four groups (with reference to duration of diabetes), and the results showed no significant differences in implant survival rates between the groups.

The study of Morris et al. ${ }^{941}$ was the only one associating some variables to diabetes and implant failure rates. They reported improved implant survival for patients who were treated with antibiotics in comparison to those treated without prophylactic antibiotics, but the survival improvement was greater in diabetic patients $(97.1 \%$ versus $86.6 \%$ ) than in non-diabetic patients $(95.1 \%$ versus $90.6 \%)$. The same happened in diabetic and non-diabetic patients when the use or non-use of chlorhexidine rinses was evaluated.

In the meta-analysis of Chrcanovic et al., ${ }^{16}$ the difference between the insertion of dental implants in non-diabetic and diabetic patients did not statistically affect the implant failure rates. However, the authors suggested that is believed that this lack of difference applies only when well-controlled type 2 diabetes patients are considered. They also detected that studies which include both patient types with a larger sample size and report the outcome data separately for each group are lacking.

\section{Pharmaceutical reasons for implant problems}

\section{Effects of antidepressants and clinical depression}

Selective serotonin reuptake inhibitors (SSRIs) are a class of drugs that are typically used as antidepressants in the treatment of major depressive disorder and anxiety disorders. There is evidence suggesting a relationship between some neuroendocrine mechanisms related to the serotonin system and the impairment of bone metabolism. ${ }^{962-964}$ Therefore, the intake of SSRIs could in theory interfere with the 
osseointegration process. In the case of dental implants in particular, the findings of recent studies ${ }^{157,191}$ (Study III included) suggest that the treatment with antidepressants is associated with an increased failure risk of osseointegrated implants, while others ${ }^{944,945,965}$ did not find a relationship between these two factors. Thus, there is still no clear consensus on the influence of antidepressants on the risk of dental implant failure.

It is possible that neuroendocrine mechanisms related to the serotonin system could regulate osteoclast differentiation/activation, because osteoclasts derive from hematopoietic cell precursors and a relationship between bone and the immune system has been established. ${ }^{962-964}$ Studies have identified a functional serotonin system in osteoblasts and osteoclasts, ${ }^{966-968}$ in which the serotonin transporter and several receptors are expressed in osteoblasts as well as in osteoclasts. ${ }^{966,967}$ The presence of serotonin receptors and the serotonin transporter in bone raises the question whether medications that antagonize serotonin reuptake could influence bone metabolism. It has been shown in in vitro studies that activity of the serotonin transporter is required for osteoclast differentiation. While blockage of the serotonin transporter reduced osteoclast differentiation when fluoxetine, an antidepressant, was administered to produce $\mu \mathrm{M}$ concentrations, ${ }^{968,969}$ there was an increase in osteoclast differentiation for the same medicament in the $\mathrm{nM}$ concentrations. ${ }^{969}$ In vivo studies demonstrated detrimental effects of fluoxetine on trabecular architecture $^{970}$ and on bone mineral density ${ }^{970,971}$ in mice. Another in vivo study showed that serotonin acts on osteoblasts inhibiting their proliferation. ${ }^{972}$ These studies in animal models indicate a negative effect of SSRIs on bone mass and suggest that these antidepressants may possess direct anti-anabolic skeletal effects, through the pharmacological inhibition of the serotonin transporter.

In the field of dentistry, some studies have already assessed whether the intake of antidepressants might be associated with an increased risk of implant failures. Alsaadi et al ${ }^{945}$ and Alsaadi et al. ${ }^{965}$ evaluated the impact of local and systemic factors on the incidence of oral implant failures up to abutment connection, finding no relationship between these two factors. The same group of researchers ${ }^{944}$ evaluated the impact of the same factors on the incidence of late oral implant failures as well, also finding no relationship. However, 
these three studies did not specify whether this kind of medication was limited to the class of SSRIs. A more recent study ${ }^{191}$ (Study III) evaluated a cohort study on the same conditions as these two previous studies, i.e. failures up to abutment connection, having several implant- and health-related factors as confounders, and not limiting antidepressants to the SSRIs class. In contrast to Alsaadi et al., ${ }^{94,945,965}$ they observed an increased failure risk of implants with the use of these medicaments (in this study, ${ }^{191}$ an increased failure risk up to abutment connection was also associated with smoking). Wu et al. ${ }^{157}$ was the only study among these previous publications that evaluated only SSRIs, excluding other classes of antidepressants, and they found a statistically significant association. However, it is important to make a few more considerations about the study of Wu et al. ${ }^{157} \mathrm{~A}$ table in their publication included 9 factors except the intake of SSRIs, which was presented alone and in a different table. It is not entirely clear from the publication whether they included the intake of SSRIs in this final model or whether they analyzed the factor SSRIs alone in a crude model. If they didn't analyze the factor SSRIs in the model with the other 9 factors, this could generate a misspecified model, i.e. one that is not complete - it is missing key/important explanatory variables and so it does not adequately represent what one is trying to model or trying to predict. Supposing that the variable SSRI was not included in the model together with the other confounders, and if it were, the interaction of other confounders of strong effect on the outcome (implant diameter and smoking, in this case in particular) could have influenced the significance of the factor SSRIs. It may happen that when there is the inclusion of a new variable into a model, something that was previously statistically significant becomes not significant. ${ }^{973}$ Thus, the results of the study of Wu et al. ${ }^{157}$ must be analyzed carefully.

Chrcanovic et al. ${ }^{160}$ (Study VI) used several statistical methods to test the association between SSRIs exposure and risk of implant failure adjusting for several potential confounders. Patients were included in the study if there were only taking SSRIs and no other medicament, and did not present any other systemic condition. Thus, the authors tried to isolate the status 'taking SSRIs' as much as possible from the potential influence any other systemic condition or medication. Even though descriptive statistics had pointed out that 
the implant failure rates were statistically significant higher for SSRIs users in comparison to nonusers $(12.5 \%$ versus $3.3 \%, p=0.007)$, the same being observed by Kaplan Meier analysis $(p<0.001)$, the other two statistical methods used in the study (multivariate GEE method and a multilevel mixed effects parametric survival analysis) did not detect any statistically significant difference between the groups. This was due to differences in the statistical methods applied. The Fisher's exact test found a statistically significant association between the intake of SSRIs and an increased risk of implant failure. However, this test does not take into consideration the other confounders and the factor time. The Kaplan Meier analysis showed a statistically significant difference in the CSRs between SSRIs users and nonusers. Even though this test considers the factor time, it does not count the influence of the other variables. The multivariate GGE did not find a statistically significant association, but even analyzing the influence of the other confounders on the failures, the model only takes the factor time as a simple confounder. The multilevel mixed effects parametric survival analysis could be the best analysis, and it showed no statistically significant association between the intake of SSRIs and the risk of dental implant failure.

It is important to take into consideration that the alleged higher risk of dental implant failures could be associated with the patient's mental condition rather than by the intake of antidepressants per se. Some studies have demonstrated that psychosocial stress may induce neglect of oral hygiene ${ }^{974,975}$ and that people suffering from psychosocial stress may show resistance to periodontal therapies. ${ }^{976}$ Thus, it is suggested that psychosocial stress may be an important risk factor in defining the progression of periodontitis, even if the patient is undergoing therapy. ${ }^{977}$ Moreover, a study showed that depression was associated with more extensive periodontitis, with continuous periodontal breakdown, and with differences in serum antibody titers to pathogens associated with periodontitis. ${ }^{978} \mathrm{It}$ is a matter of debate whether the results relating depression to periodontitis (around teeth) could be similar around implants (peri-implantitis).

\section{Effects of proton pump inhibitors}

Proton pump inhibitors (PPIs) are in widespread use for the treatment of acid-related disorders, such as gastroesophageal reflux disease or 
gastric ulcer disease. They exert their curative effects by inhibiting the acid output of the stomach. The higher probability of losing an implant in patients taking medicaments to reduce the acid gastric production is suggested to be related to observations indicating that a reduction of gastric acidity may impair effective calcium uptake through the intestines. ${ }^{979}$ As the calcium balance is essential for the maintenance of bone health, among a multitude of other physiological processes, it seems reasonable to believe that the unbalance of calcium may to some degree affect osseointegration.

A recent clinical study ${ }^{161}$ (Study V) investigated the association between the intake of PPIs and the risk of dental implant failure. The implant failure rates were $12.0 \%(30 / 250)$ for PPIs users and $4.5 \%(148 / 3309)$ for nonusers $(p<0.001)$. The multilevel mixed effects parametric survival analysis performed in the study showed that the intake of PPIs significantly affected the implant survival rate (HR 2.811; 95\% CI 1.139, 6.937; $p=0.025$ ), among other factors.

Some reasons could theoretically account for the suggested association between PPIs intake and the increased likelihood of dental implant failures. The most prominent hypothesis assumes that the reduced acidity in the stomach impairs the intestinal absorption of dietary calcium. Thus, there can be a decreased calcium absorption under PPI therapy. ${ }^{980-982}$ Graziani et al. ${ }^{980}$ observed in eight healthy volunteers that postprandial calcium concentrations did not increase in subjects on a PPI regime (omeprazole $20 \mathrm{mg} 3 \mathrm{x}$ daily), whereas control subjects demonstrated a clear spike in serum calcium levels. They as well observed that the urine calcium excretion in the PPI group was also reduced in comparison to the control group. O'Connell et al. ${ }^{981}$ measured the intestinal calcium absorption and reported that 7 days of PPI (omeprazole $20 \mathrm{mg}$ once a day) intake significantly reduced calcium absorption in elderly women under fasting conditions compared with the placebo group. Schinke et al. ${ }^{982}$ showed that genetically manipulated mice to be achlorhydric (with absence of hydrochloric acid from gastric juice) presented decreased serum calcium levels and developed osteoporosis and secondary hyperparathyroidism in an effort to maintain calcium balance. As the calcium balance is essential for the maintenance of bone health, among a multitude of other physiological processes, it seems reasonable to believe that the unbalance of calcium may to some degree affect osseointegration. 


\section{Effects of bisphosphonates}

Bisphosphonates (BP) are a class of drugs that prevent the loss of bone mass, used to treat osteoporosis and malignant diseases involving bone resorption, such as bone lesions of multiple myeloma and metastatic bone diseases. BP inhibits osteoclast-mediated bone resorption and renewal, therefore retaining existing bone. This increases mineralization under normal function, resulting in an increase in bone mineral density and theoretically an increased resistance to osteoporosis-related fractures. ${ }^{983}$ However, more recent studies has been showing that preventing osteoclast-mediated bone resorption/renewal results only in the retention of old bone, which lives out its natural life span and becomes brittle and thus even more prone to fracture. ${ }^{984}$ Removing osteoclasts and their useful function in skeletal homeostasis by drugs can be expected to result in the same complications seen in the genetic loss of osteoclast function in the disease known as osteopetrosis, such as exposed bone in the maxilla and/or mandible and leg fractures. ${ }^{985}$ In the case of use of BP, it is not a matter of genetic expression but one of dose and accumulation in bone over time. ${ }^{807}$

BPs are incorporated into mineralized structures and released during mineral resorption. Upon release, BPs are internalized by osteoclasts. The non-nitrogen-containing BPs are metabolized by osteoclasts into cytotoxic analogs of adenosine triphosphate. ${ }^{986}$ Once concentrated within the osteoclast, the analogs induce osteoclast apoptosis. The final outcome from this process is a decreased quantity of osteoclasts, resulting in less bone resorption. ${ }^{987}$ Nitrogen-containing BPs work by a separate pathway. Once ingested, nitrogenous BPs bind to the enzyme farnesyl diphosphate synthase in the 3-hydroxy-3-methylglutaryl-CoA pathway and block the synthesis of farnesol and genanylgeraniol. The end result is osteoclast cellular dysfunction and death. ${ }^{988,989}$

The increase of implant failure rates to $\mathrm{BP}$ is hypothesized to be related mainly to the impairment and cellular death (apoptosis) of not only adult osteoclasts but osteoclast precursors in the bone marrow by BP. ${ }^{990,991}$ In the normal bone, in areas of greater stress, the osteoblasts/ osteocytes reduce their secretion of osteoprotegerin, which is a decoy receptor for the receptor activator of nuclear factor-kappaB ligand (RANKL). Osteoprotegerin is also known as osteoclastogenesis 
inhibitory factor and inhibits both differentiation and function of osteoclasts. The osteoblasts/osteocytes also increase their secretion of RANKL, so that the area can resorb and re-form to accommodate this greater stress; an adaptive response. ${ }^{992}$ If the now-stimulated osteoclast attempts to resorb this stressed bone that contains a BP, it begins to do so but dies off before completing the resorption. This disrupts the resorption-renewal cycle. The osteocytes then succumb to the stress and die before their usual 180-day life span, or they live out their life span but are not replaced. The bone may first become sclerotic (overmineralized), but then it often becomes necrotic and exposed. ${ }^{807}$ Moreover, an animal study demonstrated that alendronate (a type of oral BP) inhibited the repair of normal bone microdamage, leading to the accumulation of microdamage and possible osteonecrosis. ${ }^{993}$ It has been hypothesized that as bone remodeling is decreased by BPs, an infection into the bone exposure site or microcrack area might lead to a continuous and deeper progression of the inflammatory process and infection, which are not always observed in peri-implantitis, where bone resorption exists along with the bone-implant interface. Thus, when bisphosphonate-related osteonecrosis of the jaw (BRONJ) is not triggered by peri-implantitis or surgical trauma but developed at the well-functioning implant, bacterial infection to the microcrack area in peri-implant trabecular bone might contribute to the en bloc sequestration of a BRONJ lesion. ${ }^{994}$ All the above mentioned mechanisms could presumably interfere with the healing process after implant placement, and consequently increase the failure rates.

It is important to stress that the differences in the BP therapy also interfere with the occurrence of osteonecrosis, which may directly influence the implant survival rates. Considering the different ways of BP administration, for example, osteonecrosis caused by intravenous BPs is more prevalent and more severe than osteonecrosis induced by oral BPs, ${ }^{995}$ being a result of the immediate uptake and binding of BPs to bone via the intravenous route, as compared to the oral route, where less than $0.7 \%$ is absorbed through the gastrointestinal mucosa before it circulates and binds to bone. ${ }^{996}$ Thus, it can be said that intravenous BPs load and accumulate in bone at a rate 142.8 times higher than oral BP. ${ }^{807}$ As none of the studies included in a meta-analysis on the subject ${ }^{25}$ provided information about the evaluated patients taking intravenous BPs, an underestimation of the 
implant failure rates can exist. Still, the meta-analysis suggested that BPs affect the implant failure rates.

A second point concerning the BP therapy is that variations in the chemical molecular structure of the BPs confer different degrees of potency, with zolendronate being the most potent of the intravenous BPs and alendronate being the most potent of the oral BPs, therefore causing the majority of the cases of osteonecrosis of the jaws for intravenous ${ }^{997}$ and oral types, ${ }^{998}$ respectively. The action of a BP also depends on the drug's chemical structure. The two main categories are the non-nitrogen-containing $\mathrm{BP}$ and the nitrogen-containing BP. ${ }^{999}$ The addition of an amine group to the end of a side chain increases the drug's potency. ${ }^{1000}$

A third point is the fact that the effect of patient compliance and adherence to their medication regimens is unknown. Evidence indicates that adherence to BP therapy at 6 months may be less than $50 \%$ for both once-weekly and daily dosing regiments. ${ }^{1001}$ Fourth, studies involving a retrospective chart review do not usually provide information regarding the duration of BP usage. This would have been an important variable to examine, as the absorbed nitrogencontaining BPs remain bound to their bone targets until they are released when the bone is resorbed. ${ }^{1002}$ This limitation prevents one from stratifying the population of oral BPs users by duration to analyze the effect of increasing duration on implant failure rate. Fifth, much of the research in the field is limited by small cohort sizes and short follow-up periods. The occurrence of osteonecrosis can also exert some influence on the implant survival rates. ${ }^{994}$ Some studies show that effects of BP persist for extended periods, which could explain why osteonecrosis appears after long-term treatment and even in cases in which BP treatment was discontinued. ${ }^{1003,1004}$ This is some concern because oral $\mathrm{BP}$ are intended for ongoing use over several years in, for example, postmenopausal women. However, it is hard to define what would be considered a short follow-up period to evaluate implant failures in patients taking BP.

Concerning MBL, several studies showed that BPs may not only cause apoptosis of osteoclasts cells, but also inhibit angiogenesis. ${ }^{990,991,1005,1006}$ These processes may lead to increased amounts of avascular and acellular bone that may be less tolerant to oral bacterial insult and lead to increased MBL. ${ }^{1007}$ Moreover, 
the microdamage accumulation reduces the mechanical properties of bone, enhancing the effect of occlusal forces and leading to increased MBL. ${ }^{993}$ Although animal studies ${ }^{1008,1009}$ have shown that BPs may be useful in minimizing MBL around osseointegrated implants, those implants were placed in rat tibia. Thus, those findings may not be directly comparable to results of clinical trials since occlusal forces and bacterial flora are absent and may not be directly applicable to humans.

The only meta-analysis assessing the intake of BPs and dental implants failure rates ${ }^{25}$ suggested a statistically significant risk ratio for implant failure (RR 1.73, 95\% CI 1.21-2.48, $p=0.003$ ) in patients taking $\mathrm{BP}$ in comparison to patients not taking this class of medicaments. However, the authors stressed that conclusions are difficult due to a limited number of published studies, ${ }^{577,947,995,1007,1010-}$ 1020 all characterized by a low level of specificity, and most of them dealing with a limited number of cases without a proper control group. Therefore, the real effect of BPs on the osseointegration and survival of dental implants is still not well established. Still, there are recommendations for the discontinuation of oral BP therapy for three to six months prior to implant insertion, and also for several months following implant insertion, which may allow bone turnover to recover. ${ }^{1021}$ It must be pointed out that these results apply to oral consumption or parenteral administration of BPs and may not be relevant for BP-coated implants. ${ }^{1022}$ Abtahi et al. ${ }^{1022}$ compared the effects of the local delivery of BP from the implant surface and the effects of systemic BP on the fixation of dental implants in an animal study in rats, and observed that local BP treatment appears to improve implant fixation in a setting where systemic treatment caused osteonecrosis of the jaw.

It is believed that future controlled studies with a larger number of patients in the BP group (several studies included far fewer patients taking $\mathrm{BP}$ than patients not taking $\mathrm{BP}$ ) are required to determine the real effect of this medicament on the dental implant outcome. Given the large number of post-menopausal women, and an increasing number of elderly men, who are being treated for osteopenia or osteoporosis with BPs medications, whether dental implant therapy leads to lower survival rates is important information to discuss with patients during the treatment planning phase. ${ }^{1014}$ There is 
also a need to weigh the benefits of a "drug holiday" (a temporary discontinuation of a bisphosphonate to allow bone marrow recovery and the formation of new osteoclasts to repopulate the population that has been depleted by the bisphosphonate ${ }^{807}$ for implant therapy against the risk of progression of osteoporosis in the absence of oral BP therapy. ${ }^{1019}$

\section{Effects of chemotherapeutic agents}

Implant placement in patients after ablative surgery for malignant disease and benign tumors presents special difficulties. Resection results in an unfavorable environment for dental implants. Radiation therapy for treatment of oral cancer further endangers implant placement. ${ }^{142}$ Chemotherapy is the third standard treatment method for cancer patients. All the different antineoplastic agents cause severe acute side-effects in many tissues, e.g. bone marrow, kidneys, and oral mucosa (stomatitis/mucositis). As a late side-effect, vascular cells may be altered and thereby cause malnutrition of the bones. ${ }^{1023}$ It was also shown that the effect of chemotherapy with cisplatin is a diminished bone remodeling, especially at implant-host interfaces in orthopedic surgery. ${ }^{925}$ However, the real effect of chemotherapy on the osseointegration and survival of endosteal dental implants is still not well established, with most of the reports dealing with a limited number of cases without a control group.

The effect of chemotherapy on the osseointegration and survival of endosteal implants is not well established, even though it was shown that the risk of irradiation-induced bone damage is increased by chemotherapy. ${ }^{923}$ In the study of Friedlaender et al., ${ }^{924}$ the effects of the chemotherapeutic agents on trabecular bone were quantified with respect to changes in total bone mass, new-bone formation, and resorption, in a rat model. Both drugs used in the study significantly and profoundly diminished bone-formation rates by nearly $60 \%$. The toxic effect on osteoblasts was also reflected in reduced volume and thickness of osteoid, but the total numbers of osteoblasts and the percent of trabecular surface covered by bone-forming cells were not affected. Young et al. ${ }^{925}$ investigated the influence of cisplatin chemotherapy on the bone turnover analyzing bone markers in a dog model, and their results showed that significant effects of cisplatin were observed at the site of active cellular induction and 
proliferation, such as implant-host interface, and fewer effects were seen at the sites of normal bone turnover. Thus, it is suggestive that chemotherapeutic agents have an adverse effect on normal physiological bone turnover, especially osteoblastic activity, and would also be expected to alter fracture-healing and bone-allograft incorporation by the same mechanisms. ${ }^{924}$ In the second publication of the study, a biomechanical and histologic analysis on the effects of chemotherapy either pre- or postoperatively, Young et al. ${ }^{1024}$ observed that postoperative chemotherapy resulted in less bone formation, but extracortical capsule formation was not inhibited, while preoperative chemotherapy did not alter the formation of new bone. Animal experiments are difficult to translate into a clinical setting, but formation of peri-implant connective tissue would be detrimental to the osseointegration of dental implants. In the study of Karr et al., ${ }^{1025}$ many patients who have undergone chemotherapy with pre-existing implants have suffered serious complications and lost multiple implants. On the other hand, as claimed by other reports, implants have been successful with chemotherapy treatments before and after implant placement. ${ }^{1026-1028}$ However, in the study of Moy et al., ${ }^{950}$ data analysis revealed a success rate of $90 \%(R R=0.63)$ in patients undergoing chemotherapy treatment. Although the sample size for these patients was small, no significant increase in implant failure was seen when compared with the healthy population. Moreover, in three other studies ${ }^{142,896,926}$ chemotherapy did not have a detrimental effect on the survival and success of dental implants, even though implant insertion in these studies was performed at least 6 months after chemotherapy. It is still unknown whether the time point of chemotherapy might be decisive. The only meta-analysis on the subject $^{26}$ did not show that the implant failure rate was affected by the fact that the patients were submitted or not to chemotherapy. The authors concluded that, as there are a limited number of published clinical series with patients being rehabilitated with dental implants and submitted to chemotherapy, ${ }^{142,875,879,897,926,942,1029,1030}$ and most of them deal with a limited number of cases without a well-defined control group, the real effect of chemotherapy on the osseointegration and survival of endosteal dental implants is still not well established.

Considering gender of the patients, women with breast cancer have an increased risk for osteoporosis because of treatment- 
induced premature ovarian failure and the direct effects of cytotoxic chemotherapy. ${ }^{1031}$ Estrogens limit bone resorption, and decreases in available estradiol levels can accelerate bone resorption, resulting in overall bone loss. ${ }^{1032}$ Clinically significant bone loss may continue during 2-5 years following chemotherapy, in women who experienced permanent ovarian failure. ${ }^{1033}$ Therefore, the long term follow-up is also important. In the case of men, androgen deprivation-induced bone loss is a significant clinical concern in patients with hormone-sensitive prostate cancer receiving long-term androgen deprivation therapy, which decreases circulating levels of estrogen and testosterone, both of which maintain bone mass through suppression of bone resorption and promotion of bone formation. ${ }^{1032,1034}$ To which extent this may affect the osseointegration of dental implants is unknown, since a study assessing the chemotherapy effects on implants taking into account the patients' gender is lacking.

\section{Effects of immunosuppressives}

Immunosuppressive drugs/agents are drugs that inhibit or prevent activity of the immune system. They are mainly used to prevent or reduce the chance of rejection of transplanted organs and tissues, and to treat autoimmune diseases. Cyclosporin A (CsA) is the most commonly used immunosuppressant agent for preventing graft rejection. Its major effect on the immune system is inhibition of T-cell action and proliferation, thereby altering the T-cell-mediated immune response. It has also been shown to affect a subset of B cells. ${ }^{1035}$ CsA has also been associated with deleterious effects upon bone metabolism. ${ }^{1036}$ The precise mechanisms underlying the CsA effect on bone metabolism are still unknown. However, it may be suggested that CsA affects the immune system by selectively influencing the lymphokine-monokine cascade and it has been recognized that cytokines, such as IL-1, are among a number of products of the lymphokine-monokine cascade that affects bone metabolism. ${ }^{1037}$ Moreover, it is known that the immune system actively participates in bone mineral metabolism and that the $\mathrm{T}$ lymphocytes play a critical role in the development of CsA-induced osteopenia. ${ }^{1038}$ This is not surprising, as the $\mathrm{T}$ cell is the traditional target of CsA and naturally occurring T-lymphocyte perturbations are implicated in the development of primary osteoporosis in humans. ${ }^{1038} \mathrm{~T}$-lymphocyte 
suppression results in a high bone-metabolism state where the bone formation is supplanted by the bone resorption leading to a decrease in the trabecular bone volume. ${ }^{1038,1039}$

In vivo studies observed that CsA administration may result in a severe high turnover osteopenic state, ${ }^{1040-1042}$ which is dependent on both the dose and duration of the therapy. ${ }^{1039}$ In vitro studies indicate that CsA inhibits bone resorption and osteoclast formation. ${ }^{1043} \mathrm{Fu}$ et al. ${ }^{1044}$ observed an increased osteoclasia in periodontal sites and reduced bone formation in symphyseal sites in rats exposed to CsA compared to the controls. Two in vivo studies ${ }^{1045,1046}$ in a rabbit model suggested that the administration of immunosuppressive drugs may negatively influence bone healing around titanium implants. Moreover, it was observed that the administration of CsA may impair the mechanical retention of dental implants previously integrated to the bone. ${ }^{1047}$

The effects of immunosuppressives on bone metabolism may be different when there is concomitant use of calcium-channel blockers (CCB). CCBs have been successfully utilized for the control of CsAinduced toxicity. ${ }^{1048} \mathrm{~A}$ number of clinical and experimental studies have suggested that the effects of CCBs are not only limited to the cardiovascular system but might also involve skeletal calcium metabolism, due to the presence of calcium channels in bone cells. ${ }^{1049-}$ ${ }^{1051}$ The results of a study ${ }^{1052}$ suggested that short-term administration of CsA, associated with nifedipine, may not influence bone density in a lateral zone to the titanium implant surface inserted in rabbits.

$\mathrm{Gu}$ and $\mathrm{Yu}^{1053}$ published a case series of 13 liver transplant patients who received 45 implants and were followed up for 3 years, with no implant losses. They reported that MBL and Periotest values were within acceptable ranges without bleeding on probing or pathological probing depth. Montebugnoli et al. ${ }^{1054}$ published which was probably the first controlled clinical study in humans. The authors concluded that the bone response around submerged dental implants in immunocompromised organ transplant patients $(n=10)$ does not differ from that observed in control patients $(n=10)$. However, the study had a follow-up of only 3 months. A couple of years later the authors published further results of the study, now with 3 more patients in each group and 1 year after prosthetic loading. ${ }^{1055}$ The authors came to the same conclusions as in their previous publication. 
However, even though they had followed the patients for a longer period, actual follow-up time was limited.

It is a matter of debate whether the implications proposed by the results of these studies could have some significant clinical impact on dental implants in human beings in the long run, due to the lack of long-term controlled clinical studies with a considerable number of patients regarding the effect of immunosuppressive agents on the osseointegration process around titanium implants.

\section{Titanium allergy}

Various types of metallic and organic materials have been used for dental prostheses. Some of these materials have been reported to have pro-allergenic properties. ${ }^{1056}$ Mercury, ${ }^{1057-1059}$ nickel, ${ }^{1059,1060}$ chromium, ${ }^{1061}$ palladium and cobalt ${ }^{1060,1061}$ are classic allergens. When it comes to dental implants, the most commonly used systems nowadays are manufactured of c.p. Ti.

Titanium implants are covered by a protective adherent oxide layer, composed primarily of titanium dioxide $\left(\mathrm{TiO}_{2}\right)$, created upon exposure to air and biological fluids, which provides pure titanium and titanium-based alloys with outstanding resistance to corrosion. ${ }^{1062}$ Nevertheless some corrosion of titanium from oral implants has been demonstrated. ${ }^{1063}$ Release and in vivo studies have proven that the oxide layer can be compromised, and ions and particles are indeed released from c.p. Ti and titanium alloys into biological fluids and tissue (Figure 16). ${ }^{1064}$ Incorporation of other metals in the $\mathrm{TiO}_{2}$ passive layer might weaken the protective properties, and this might explain the poorer stability of titanium alloys than of $\mathrm{TiO}_{2}$ materials, and explain the differences in stability between different titanium alloys despite formation of the oxide layer. ${ }^{1064}$ 


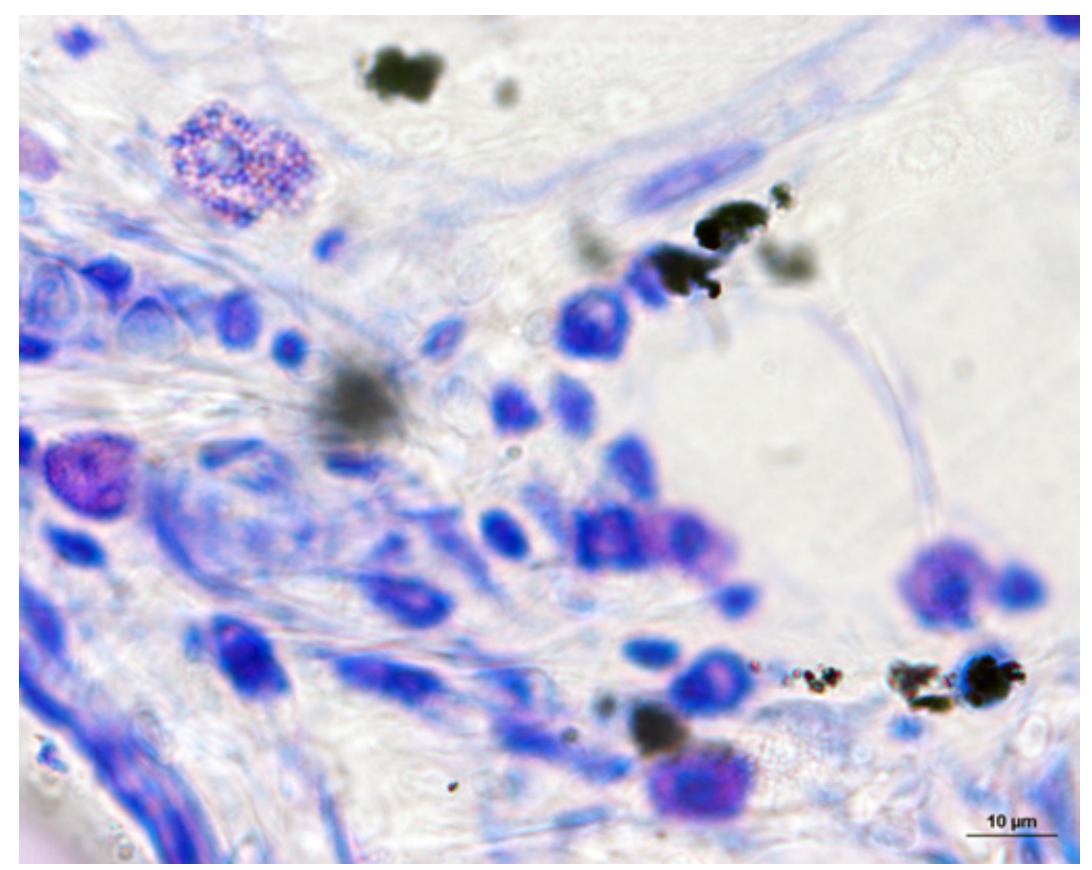

Figure 16. Leaked out titanium from a clinical implant. Black dots represent titanium in the implant-near tissues.

Cadosch et al. ${ }^{1065}$ showed that human osteoclast precursors are able to grow and differentiate to form mature osteoclasts on titanium and aluminum, and that the mature cells are able to directly corrode the metal surface and take up corresponding metal ions, which may subsequently be released and thereby induce the formation of osteolytic lesions in the peri-prosthetic bone, contributing to the loosening of the implant.

Several studies of humans and animals with inserted implants showed an increased presence of titanium ions in serum, ${ }^{1066-}$ 1069 in surrounding tissue, ${ }^{1070}$ urine, ${ }^{1067}$ peri-implant tissues, ${ }^{1070}$ regional lymph nodes, ${ }^{1071-1073}$ spleen, ${ }^{1071}$ adjacent muscle, ${ }^{1071}$ and lungs, ${ }^{1071,1073,1074}$ particularly in combination with loosened implants. On the other hand, there are studies that found no statistically significant difference on the increased release of titanium between test and control human ${ }^{1075,1076}$ or animal groups, ${ }^{1063,1077-1079}$ or in in vitro studies. ${ }^{1080,1081}$ 
Notwithstanding, whether dental materials comprising titanium are associated with allergic symptoms is controversial. Titanium is known to possess good biocompatibility. However, recent studies have reported cases of allergic symptoms caused by titanium-based dental materials. For example, Müller and Valentine-Thon ${ }^{1082}$ investigated the potential of titanium to induce hypersensitivity in patients chronically exposed to titanium-based dental or endoprosthetic implants. Fiftysix patients who had developed clinical symptoms after receiving titanium-based implants were tested. Although all patients that were patch-tested accused negative reaction to titanium, these same patients showed remarkable clinical improvement following removal of the implants. Moreover, 15 of these patients were retested in the optimized lymphocyte transformation test (LTT) MELISA and showed clinical improvement correlated with normalization in MELISA reactivity. Thomas et al. ${ }^{1083}$ reported a patient who developed eczema upon titanium-based osteosynthesis. Patch testing gave no reactions to titanium or to nickel, chromium, or cobalt. However, in the LTT, the patient's lymphocytes showed markedly enhanced proliferation in vitro to titanium. After removal of the titanium material, fracture healing was achieved and the eczema cleared. Parallel to this, in vitro hyperactivity to titanium disappeared. ${ }^{1083}$ Egusa et al. ${ }^{1084}$ reported facial eczema in association with a titanium dental implant placed for a mandibular overdenture supported by 2 implants. The patient showed positive LTTs index for titanium. LTTs have been recently validated for metal sensitivity testing, including testing for titanium hypersensitivity. ${ }^{1082,1085,1086}$ Complete remission was achieved by the removal of the titanium material. Sicilia et al. ${ }^{1087}$ evaluated the presence of titanium allergy by the anamnesis and examination of 1,500 patients, together with the selective use of cutaneous and epicutaneous testing, in patients treated with or intending to receive dental implants of such material. They observed that nine patients $(0.6 \%)$ displayed positive reactions to titanium allergy tests. Five of these nine patients proved to have suffered some form of acute reaction, post-implant surgery allergic reaction or short-term complications, or spontaneous rapid exfoliation of an implant. Hosoki et al. ${ }^{1056}$ reported a case that exhibited allergic symptoms (eczema) after orthopedic surgery. The titanium screws used in the orthopedic surgery that he underwent were removed 1 year later, but 
the eczema remained. The patient clearly exhibited positive reactions to many reagents (patch tests), including titanium. After removal of dental implants, the eczema disappeared completely. Degidi et al. ${ }^{1088}$ compared the inflammatory profiles of peri-implant soft tissues adjacent to either c.p. Ti or experimental zirconia healing abutments in five human subjects after 6 months of healing. They observed significant elevations in pro-inflammatory infiltrates (lymphocytes, plasma cells, and histiocytes), as well as increased expression of VEGF and nitric oxide synthase isoforms 1 and 3 adjacent to titanium healing abutments, as compared to zirconia abutments.

Contrastingly, there are studies showing no effect of titaniumbased dental materials on tissue reactions. Barwacz et al. ${ }^{1089}$ assessed differences in the pro-inflammatory cytokine and bone metabolism mediator protein expression in the PICF adjacent to transmucosal abutments, made of either c.p. Ti or zirconia. No significant differences in pro-inflammatory cytokine or bone metabolism mediator profiles were observed biochemically, with the exception of leptin (a protein that has been demonstrated to induce osteoblastogenesis and inhibit osteoclastogenesis ${ }^{1090,1091}$ ), for the abutment biomaterials of titanium or zirconia. The authors concluded that the molecular PICF findings of their study support the observed clinical biocompatibility of both titanium and zirconia abutments. Moreover, Flatebø et al. ${ }^{1092}$ histologically evaluated non-perforated mucosa covering submerged maxillary titanium implants with regard to induced tissue reactions, and tissue sensitivity reactions to titanium implants were not disclosed.

Of course that with data from non-experimental studies such as some of the aforementioned, cause-effect relations cannot be established, and so the conclusions must be interpreted as hypotheses to be confirmed later on. Still, the clinical evidence is suggestive, and some of these clinical studies were supported by in vitro and laboratorial tests.

It is also important to remember that one component containing titanium, the $\mathrm{TiO}_{2}$, is one of the most popular pigments in use today. ${ }^{1093} \mathrm{TiO}_{2}$ is authorized as a food color in the European Union (EU) as $\mathrm{E} 171^{1094}$ and is commonly used as a food additive. ${ }^{1095} \mathrm{TiO}_{2}$ is used as an additive in a plethora of food groups, such as confectionery (chocolate products, sweets, candy bars, chewing gum), fine bakery 
wares (cake, pies, pastries, cookies and biscuits), sauces (dressings and savoury sauces), milk products (cheeses (soft and hard), coffee creamer and milk powder, ices and desserts, cappuccino, dairy drinks), drinks (sports and soft drinks, fruit drinks), and cereal products (popcorn and rice, breakfast cereals). ${ }^{1095-1097}$ It was observed that there is a constant intake of nanoparticles $\mathrm{TiO}_{2}$ by humans, and the products contributing most this are toothpaste (in young children only), candy, coffee creamer, fine bakery wares and sauces. ${ }^{1098} \mathrm{TiO}_{2}$ is also applied in paints, coatings, plastics, papers, inks, drugs, cosmetics (e.g. sunscreens, creams, lip balm) and toothpaste. ${ }^{1099,1100}$ Recent oral studies with $\mathrm{TiO}_{2}$ nanoparticles indicate that these particles can have toxic effects ${ }^{1100-1102}$ and repeated oral exposure to these particles may result in tissue accumulation in the long run. ${ }^{1103}$ However, there is an enormous lack of epidemiological data regarding $\mathrm{TiO}_{2}$ nanoparticles in spite of its increased production and use. ${ }^{1100}$

Prevalence of allergy-positive reactions against titanium reagents is far lower than that for other materials such as chromium, mercury, palladium and nickel. No patient has exhibited an allergy-positive reaction only for a titanium reagent. In light of this, it can be suggested that titanium is a relatively safe material that causes allergic symptoms rarely. However, one must bear in mind that as hypersensitization can take months or even years to develop, ${ }^{1104}$ along with the fact of its infrequency and the uncertainty of its symptomatic expression, ${ }^{1105}$ it is difficult to perform deeper studies in this field. As titanium allergy is barely recognized in the field of implant dentistry, some authors have suggested that this could just be the very tip of the iceberg, and advocate further awareness. ${ }^{1106-1108}$ Possible restrictions deriving from the use of cutaneous and epicutaneous tests, ${ }^{1085}$ the fact that some hypersensitive reactions appear over the long-term and the differences between cutaneous reactivity and that of other organs, ${ }^{1109,1110}$ as well as the potential yet unlikely appearance of immunological tolerance or non-specific immunosuppression caused by implant degradation products, ${ }^{1105}$ may contribute to underestimate the actual prevalence rate of titanium allergy. Moreover, dental implants have special conditions that could prevent them from being affected by an allergic reaction despite developing a cutaneous reaction: the implant-host contact surface is very limited, ${ }^{1111,1112}$ the prosthetic structures and implants are partially isolated by a layer of glycoproteins ${ }^{1113}$ and the 
bone and oral mucosa have a low reactivity. ${ }^{1109,1110,1113}$ Last but not least, it is not entirely known whether the titanium alloys usually used to manufacture orthopedic implants may have the same reactivity as c.p. Ti and the titanium alloys usually used to manufacture dental implants and titanium-based dental materials, respectively.

If the allergy to titanium would be diagnosed in a patient, the surgeon must then determine whether it is suitable to place a titanium implant in patients with a positive reaction to it, given the potential medical and legal complications that could derive from such a procedure. ${ }^{1087}$ Some alternative materials could be considered, such as tantalum, ${ }^{1114,1115}$ hydroxyapatite, ${ }^{1116-1118}$ zirconium,,${ }^{1119,1120}$ or polyether ether ketone - PEEK. ${ }^{1121}$

\section{Prosthetic}

\section{Crown-to-implant ratio}

The crown-to-root ratio is defined as the physical relationship between that portion of the tooth within the alveolar bone and that portion not within alveolar bone, as determined by a radiograph. ${ }^{1122}$ The crown-to-root ratio is determined by dividing the length of the tooth coronal to the bone by the length of the root that resides in bone. ${ }^{1123}$

Dentists use the crown-to-root ratio as an important prognostic indicator to determine the suitability of a tooth to act as an abutment for a fixed or removable partial denture. Furthermore, the crownto-root ratio is used as a prime indicator of the long-term prognosis of a given tooth. ${ }^{1124,1125}$ It extrapolates the biomechanical concept of a class I lever for evaluating abutment teeth with the fulcrum lying in the middle portion of the root residing in alveolar bone. As progressive bone loss occurs, the fulcrum moves apically, and as a result, the tooth is more susceptible to harmful lateral occlusal forces. ${ }^{1126}$ Newman et al. ${ }^{1127}$ reinforce this by stating that because of disproportionate crown-to-root ratios and the reduced root surface available for periodontal support, the periodontium may be more susceptible to injury by occlusal forces.

The concept of crown-to-root ratio was then extrapolated to the dental implant field, coining the term crown-to-implant (C/I) ratio. The "anatomic" crown-to-implant ratio is measured from the bottom of the fixture to the implant-abutment connection and then from 
that point to the top of the crown. The "clinical" crown-to-implant ratio is measured from the top of the crestal bone in contact with the implant to the top of the crown; thus the implant length is measured from the bottom of the fixture to the top of the crestal bone in contact with the implant. This is an important difference because of the fact that an implant of conventional length $(>10 \mathrm{~mm})$ may exhibit a large $\mathrm{C} / \mathrm{I}$ ratio if the crestal bone has remodeled to a level far below the implant-abutment connection. ${ }^{1123}$

From the biomechanical viewpoint, long- and wide-diameter implants with low $\mathrm{C} / \mathrm{I}$ ratios provide better peri-implant tissue stability in the long-term because of favorable distribution of occlusal forces. ${ }^{218,224,1128}$ On the other hand, the limited surface area of short implants with high C/I ratios could be a biomechanical disadvantage in the long-term because such implants may be less resistant to detrimental nonaxial occlusal forces. ${ }^{1129-1131}$ Increased C/I ratio, higher bite forces, and bone density in posterior area are common factors that increase stress and may explain the reasons of the higher failure rates of short implants. ${ }^{1132}$ Excessive $\mathrm{C} / \mathrm{I}$ ratios have been reported as being detrimental to the long-term stability of peri-implant bone and implant survival, ${ }^{197,1133}$ and is generally associated with higher stress values in the peri-implant region. ${ }^{249}$

However, disproportionate $\mathrm{C} / \mathrm{I}$ ratios have also been associated with predictable long-term prognosis. Neither C/I ratio nor estimated implant surface area (small or large) affected steady-state crestal bone levels in the study of Rokni et al. ${ }^{1134}$ that evaluated partially edentulous patients with implant-supported fixed prostheses in a follow-up study of almost 4 years. Blanes et al. ${ }^{1135}$ suggested that implant restorations with $\mathrm{C} / \mathrm{I}$ ratios between 2 and 3 may be successfully used in the posterior areas of the jaw, a 10-year follow-up study. Contrary to what would be expected according to the biomechanical viewpoint, Blanes et al. ${ }^{1135}$ found a positive correlation between increasing crown-to-implant ratios and increasing first BIC levels over 1 year. Higher clinical crown-to-implant ratios showed lower average bone loss when compared with lower crown-toimplant ratios. This may be attributed to the design of the nonsubmerged implants, the clinical crown-to-implant calculations, or the fact that most of the restorations were splinted. ${ }^{1123}$ Schulte et al. ${ }^{1136}$ observed that the crown-to-implant ratios of those implants 
that were in function were similar to those implants that failed. The results of the study of Birdi et al. ${ }^{1123}$ suggest that a C/I ratio of 2.0:1 and even greater can produce a stable favorable outcome. However, this study had a mean follow-up of only 20.9 months. Urdaneta et al. ${ }^{1137}$ observed that larger C/I was associated with a significant increase in prosthetic complications but had no significant effect on crestal bone levels on single-tooth locking-taper implants. According to a review, ${ }^{1138}$ a higher $\mathrm{C} / \mathrm{I}$ ratio could be considered a risk factor for some mechanical complications of implant-supported rehabilitations as screw loosening, porcelain fractures loosening of maxillary anterior crowns, fractures of 2-mm-wide implant abutments supporting single crowns in posterior areas, ${ }^{697,1137}$ but an increased $\mathrm{C} / \mathrm{I}$ ratio cannot be considered a risk factor for biological complications around dental implants and implant failure. ${ }^{1138}$ Sun et al. ${ }^{1139}$ observed in patients who had been treated with single Astra implants for replacement of missing premolars and molars that the higher $\mathrm{C} / \mathrm{I}$ ratio and anatomical crown length did not increase the risk of peri-implant MBL during 6 years of functional loading. Instead, they noticed that higher anatomical crown lengths are associated with higher technical complications, the same as observed in other studies. ${ }^{1140,1141}$ It has been hypothesized that implant restorations with high anatomical crown length overload the prosthetic components, which increase the risk of technical complications such as screw fracture, screw looseness, loss of retention, and veneering fracture. ${ }^{1139}$ In a study with extra-short implants, ${ }^{1142}$ there was a significant positive correlation between bone loss and crown height space, but not between MBL and the crown-to-implant ratio. A possible explanation for this is that an increase in crown length from 10 to $20 \mathrm{~mm}$ will increase the occlusal forces on an implant by $100 \% .{ }^{1143}$ Moreover, a crown height space higher than $15 \mathrm{~mm}$ has been reported as unfavorable and to result in higher stress concentrations at the bone-implant interface. ${ }^{1140,1141}$

\section{Number of implants per patient}

One may hypothesize that placing multiple implants requires more mucoperiosteal stripping, compromising blood supply, more operating time, and more contamination of the wound, all of which may contribute to the increased complication rate. ${ }^{1144}$ Only few 
clinical studies corroborate this hypothesis. Smith et al. ${ }^{1144}$ studied 313 implants, and the univariate analysis of risk factors showed that the number of implants placed per patient did correlate with implant failure. The patients receiving from 4.1 implants had a greater probability of having failures than the patients receiving until 2.2 implants $(p=0.016)$. Naert et al. ${ }^{202}$ studied the outcome of 1,956 implants, and according to their results, the higher the number of implants a patient has, the higher the hazard rate $(p=0.005)$. Increasing the number of implants by one increases the hazard rate 0.14 times. However, there are also examples of studies in which the number of lost implants was independent of the number of implants placed per patient. ${ }^{53,1145}$

\section{Prosthetic rehabilitation type}

Several studies observed statistically significant difference in the implant failure rates between implants used to support different prosthetic configurations, being overdentures versus fixed full-arch prostheses, favoring fixed prostheses, ${ }^{235}$ overdenture versus fixed partial prostheses, favoring fixed prostheses, ${ }^{372}$ overdentures versus all types of fixed prostheses (single-tooth, partial, full-arch), favoring fixed prostheses, ${ }^{1146-1148}$ overdentures versus fixed full-arch bridges, favoring fixed prostheses (but only in the maxilla), ${ }^{1149}$ overdentures versus all types of fixed prostheses, favoring overdentures and full-arch fixed prostheses, ${ }^{1150}$ and single- versus multiple-implant supported fixed prostheses, favoring single-tooth prostheses. ${ }^{376}$ Many studies did not observe statistically significant differences in the implant failure rates between implants used to support different prosthetic configurations, being fixed prosthesis versus single-tooth restorations, ${ }^{52}$ single crowns versus fixed partial prosthesis, ${ }^{202}$ overdenture versus fixed full-arch prosthesis, ${ }^{1151}$ single- versus multiple-implant supported fixed prosthesis, ${ }^{577}$ and all-ceramic versus metal-ceramic implant-supported single-tooth restorations. ${ }^{1152}$ Generally speaking, it is difficult to say which type of prosthetic restoration type would be better than the other, since several influencing factors may play a role in the implant failure rates.

One of the important decisions in implant prosthodontics is the choice of the connection type of the final restoration to the implant via the screw-retained abutment. The restorative connection can 
be either screw- or cement-retained. Four controlled studies ${ }^{1153-1156}$ evaluated the differences in the clinical outcomes between cemented versus screw-retained implant restorations, and all 4 observed no statistically significant difference in implant failure rates between the two techniques. Concerning MBL, three of these four studies compared MBL between cemented and screw-retained implant restorations. Nissan et al. ${ }^{1153}$ observed that the mean MBL was statistically significantly higher $(p<0.001)$ for screw-retained $(1.4 \pm 0.6 \mathrm{~mm})$ than for cemented $(0.69 \pm 0.5 \mathrm{~mm})$ restorations. Crespi et al. ${ }^{1156}$ found no statistically significant differences in MBL between cemented versus screw-retained implants restorations, the same in the study of Vigolo et al. ${ }^{1155}$ at the 10-year follow-up appointment.

\section{Occlusal versus non-occlusal loading}

Within immediate loading, two types of have been described in the literature. One is the immediate functional loading, or immediate occlusal loading, which refers to the use of a temporary or definitive prosthesis seated the same day as the surgery in occlusal contact with the opposing arch. ${ }^{1157}$ The other one has been proposed to modify the immediate temporary restoration to avoid occlusal contact in centric and lateral excursions, in order to reduce the early risks of mechanical overload caused by functional or parafunctional forces, the immediate nonfunctional loading, or immediate non-occlusal loading. ${ }^{1158}$ Thus, the modified restoration would still be involved in the chewing process, but the mechanical loading stress is reduced. ${ }^{1159}$ Chrcanovic et al. ${ }^{13}$ tested the null hypothesis of no difference in the implant failure rates between these two procedures in a meta-analysis. The results showed that the procedure used (nonfunctional versus functional) did not significantly affect the implant failure rates (RR $0.87,95 \%$ CI $0.44,1.75 ; p=0.70)$.

The idea behind the concept of keeping temporary restoration out of occlusion is to control the load on the prosthesis in order to allow undisturbed healing. The role of tongue pressure and perioral musculature may be an underestimated factor in immediately provisionalized but non-loaded implants. Moreover, occlusion might not be the only determinant of implants survival ${ }^{1160}$ because there was no statistically significant difference between the immediate nonfunctional loading and immediate functional loading. The 
increase of load, applied to the prosthesis caused by the presence of the normal occlusal contact, seems to be unable to jeopardize or alter the healing process of the implant. ${ }^{1159}$ Some factors may have contributed to this outcome in some studies: the use of a resilient acrylic resin for the fabrication of the temporary restoration, the exclusion of parafunctional bruxist patents, and the splinting of the temporary prosthetic work.

\section{Prosthodontist experience/skill/judgment}

The experience of the surgeon may affect the survival rates of implants, and the same is true for the experience of the prosthodontist performing the restorative part of the dental implant rehabilitation.

The construction of an inadequate implant-supported restoration, which could be a result of an oral prosthetic rehabilitation conducted by a less experienced and less skilled prosthodontist, among other factors, might jeopardize the implant long-term survival. It has been suggested that the passiveness of the framework fit, for instance, may have an influence on implant failures and/or delayed component failure. ${ }^{233,300,1161-1163}$ Passive fit is supposedly one of the prerequisites for the maintenance of the bone-implant interface. To provide passive fit or a strain-free superstructure, a framework should, theoretically, induce absolute zero strain on the supporting implant components and the surrounding bone in the absence of an applied external load. ${ }^{1164}$ However, there is no longitudinal clinical study that reports implant failure specifically attributed to framework misfit. Moreover, first, it is hard to tell what level of misfit is clinically important, beyond which damage is likely to occur, and second, passiveness of a framework is not an easy task to measure it in a clinical situation. ${ }^{165}$ And yet, although more recent procedures such as laser welding, framework cemented on prepared abutments and other methods have minimized the misfit of structures on implants, ${ }^{1166,1167}$ an absolute passive fit cannot be obtained. ${ }^{1168}$

Another factor to consider is the establishment of a balanced occlusion, which is, in most part, verified, controlled and corrected by the prosthodontist. The application of an external load on an implant-supported prosthesis induces stresses within the entire loadbearing system and stress reactions in the supporting bone which are theoretically the same in magnitude, but in opposite directions. 
During clinical loading of an implant, the direction of forces almost never coincides along its central long axis, providing an absolute axial loading. On the contrary, the occlusal force is applied at different locations and frequently, in a direction that creates a lever-arm, which causes reacting forces and bending moments in the bone. ${ }^{1169,1170}$ This bending moment is the force times the orthogonal distance between the force direction line and the counter-acting support. The longer the distance, the greater will be the bending moment. ${ }^{1171}$ Accordingly, the fraction of force transmitted to implants and the induced stresses are dependent particularly on where the load is applied on the prosthesis. ${ }^{1172,1173}$ Hence, it is of paramount importance that the prosthodontist do not overlook a non-balanced occlusion, i.e. do not neglect to establish an equilibrium between acting and counteracting forces.

The aforementioned conditions are hypothetical situations in which the prosthodontist could negatively influence the implant-prosthetic rehabilitation work, and most of the studies assessing the influence of the experience of the practitioner on the implant survival concern the dentists who surgically place the implant, not the prosthodontist. The only one, perhaps, to somehow address the issue of the influence of the prosthodontist on implant clinical performance was Bryant. ${ }^{1174}$ He observed that the crestal bone loss during the first to fourth year of loading and after the first year of loading, and the estimated crestal bone level at 10 years after loading among prosthetic sites varied with differences in the dentist who placed the first prosthesis.

There are, however, some studies correlating the prevalence of restorative complication in implant-supported prostheses with the experience of the professional performing the restorative part of the treatment. Wang et al., ${ }^{175}$ for example, found a correlation between screw loosening in single-implant crowns and operator experience (measured by number of implants restored during the study period), with more experienced operators suffering fewer screw-loosening complications. They also observed that increased operator experience is associated with reduced food packing and contact point complications. 


\section{Surgeon}

Some studies have assumed that the operator skill and/or experience is of importance for achieving high implant success rates and that a learning curve does exist for dental implant therapy. ${ }^{452,1176-1178}$ However, this subject is not without controversy, and a significant association of 'experienced surgeon-less implant failures' was not verified by all studies. Melo et al. ${ }^{1179}$ found no statistically significant difference in implant survival rates among oral and maxillofacial residents of different levels of training. However, the authors stressed that this observation may be due to the fact that more complicated cases were assigned to surgeons with more experience in implant surgery. Another study ${ }^{456}$ observed that clinical experience had no significant impact on implant survival, at least not on the basis of multivariate analyses. The authors suggested that a significant difference in favor of inexperienced surgeons based on univariate methods could be explained by the fact that the more challenging cases with poor bone quality and/or quantity had been treated by the academic staff; whereas, standard cases had been usually treated by clinicians-in-training. Considering the assumption of no difference in the implant failure rates between beginners and experienced surgeons, what could possibly be influencing the difference in failure rates between different surgeons?

\section{The surgeon's experience}

Weyant ${ }^{1180}$ showed that the surgeon's experience was associated with implant health; as a provider's experience grows, the likelihood of peri-implant soft tissue health problems decreases. A study ${ }^{1176}$ comparing treatment outcomes in implant therapy between surgeons with a minimum of 2 years implant experience and surgeons just beginning involvement in implant techniques observed that the surgeon's experience had a major impact on the failure probability of unloaded implants. The authors suggested that the loading conditions and the design of the prosthesis may be the decisive determinants for the probability of success with loaded implants, but also pointed out that it is likely that implant positioning, angulation, and technique will affect the result for the entire lifespan of the restoration. ${ }^{1176}$ Moreover, the authors observed that the surgeons' learning curve showed that after 2 years of function, the probability of success 
of the prosthesis remained constant, which might be biased since increased experience might have caused the operator to adopt a more selective approach toward prosthodontic solutions. Lambert et al. ${ }^{1177}$ observed that implants placed by inexperienced surgeons $(<50$ implants) failed twice as often as those placed by experienced surgeons ( $>$ or $=50$ implants). Similar results were observed by Morris et al. ${ }^{1178} \mathrm{Ji}$ et al. ${ }^{452}$ reported in their study that the implant failure rate for 2 surgeons with $>5$ years of surgical experience was $2.4 \%$ ( 2 of 85 implants), whereas the remaining 18 surgeons, that is, those with $\leq 5$ years of surgical experience, incurred an implant failure rate of $12.2 \%$ (26 of 212 implants). On the other hand, Morris et al. ${ }^{941}$ noticed an only slightly greater survival for implants placed by the more experienced dentists in their study, evaluating more than 2,600 implants followed up for 3 years. Melo et al. ${ }^{1179}$ found no statistically significant difference in implant survival rates among oral and maxillofacial residents of different levels of training. However, the authors stressed that this observation may be due to the fact that more complicated cases were assigned to surgeons with more experience in implant surgery. Another study ${ }^{456}$ observed that clinical experience had no significant impact on implant survival, at least not on the basis of multivariate analyses. The authors suggested that a significant difference in favor of inexperienced surgeons based on univariate methods could be explained by the fact that the more challenging cases with poor bone quality and/or quantity had been treated by the academic staff; whereas, standard cases had been usually treated by clinicians-in-training.

There is also a relationship between procedure volume and clinical outcomes. Although surgical volume is less important for simple procedures, it might be for complex procedures. Procedure volume may be linked to other delivery factors associated with surgical outcomes, but it has been assumed that volume is important in large part because it serves as a proxy for operative proficiency. ${ }^{1181}$ A study ${ }^{1182}$ examined mortality rates for 12 surgical procedures of varying complexity in 1,498 hospitals to determine whether there is a relation between a hospital's surgical volume and its surgical mortality, and observed that hospitals in which 200 or more of these operations were done annually had death rates, adjusted for case mix, 25 to $41 \%$ lower than hospitals with lower volumes. The 
results may reflect the effect of volume or experience on mortality, or referrals to institutions with better outcomes, as well as a number of other factors, such as patient selection. Another study ${ }^{1183}$ examined the relations between operative mortality and surgeon volume and hospital volume (each in terms of total procedures performed per year), with adjustment for characteristics of the patients and other characteristics of the provider. The study results showed that the surgeon volume was inversely related to operative mortality for all eight procedures assessed.

A systematic review on the subject ${ }^{31}$ found only 8 studies reporting and comparing implant failure rates between groups of (relatively) more and less experienced surgeons. In the first meta-analysis performed in the study, including four retrospective comparative studies that defined experienced professionals as specialists, the experience of the surgeon did not significantly affect the implant failure rate (OR 1.24, 95\% CI 0.62, 2.48; $p=0.54$ ). In the second meta-analysis, including two retrospective studies that defined experienced surgeons as those professionals that had placed more than 50 implants, the expertise of the surgeon did significantly affect the implant failure rates (OR 2.18, 95\% CI 1.40, 3.39; $p=0.0005$ ). However, based only on the results on this study it is not possible to state that the insertion of dental implants by experienced surgeons would result in a lower risk of implant failures in comparison to nonexperienced surgeons, due to a limited number of published studies.

A recent clinical study ${ }^{194}$ (Study VII) assessed the influence of several factors on dental implant failure prevalence, with special consideration on the placement of implants by different dental surgeons. Only the data of patients and implants treated by surgeons who had inserted a minimum of 200 implants at one clinic were included. The differences between the survival curves of each of the 10 eligible surgeons were statistically significant. The surgeon with the highest absolute number of failures was also the one who inserted most implants in sites of poor bone and used turned implants in most cases, whereas the surgeon with the lowest absolute number of failures used mainly modern implants. There were statistically significant differences in cumulative survival when separate survival analyses of turned and modern implants were stratified for the individual surgeon. The study also identified the surgeon among the 
statistically significant factors affecting the implant survival rates when a binary logistic regression analysis was performed using a GEE method.

\section{The surgeon's skills}

Besides the procedure volume and experience of the surgeon, the skill is another important factor to take into consideration. A considerable body of research suggests that some surgeons have better results than others, with some studies showing wide variation in risk-adjusted patient mortality across surgeons. ${ }^{181,1184,1185}$ To quote an example, a study assessing the influence of the surgical skill on the complications rates after bariatric surgery ${ }^{1181}$ observed that the technical skill of practicing bariatric surgeons varied widely, and greater skill was associated with fewer postoperative complications and lower rates of reoperation, readmission, and visits to the emergency department. Surgical skill was strongly related to procedure volume, but not related to years in bariatric surgery practice, status with respect to completion of a fellowship in advanced laparoscopy or bariatric surgery, or practice at a teaching or nonteaching hospital.

In many procedures, the technical skill of the operating surgeon may be an important determinant of the outcomes. ${ }^{1186}$ A high level of surgical skill may be essential in preventing intraoperative problems, such as failure to properly maintain or use equipment and instruments that may compromise drilling precision. If an imprecise implant site is prepared or if tapping procedures are excessive, initial implant stability and subsequent osseointegration may be compromised. A bony dehiscence or defect may occur as a result of implant placement too facially or lingually. Moreover, an excessive countersinking may result in the loss of a superior cortical bone plate to stabilize the implant. The failure to preserve keratinized gingiva was even mentioned as a factor, as it may compromise maintenance of peri-implant tissue health in the long-term. ${ }^{1187}$ Because failure to osseointegrate can, in part, be related to imprecise surgical technique, it follows that these complications are more likely to occur after operations performed by less skilled surgeons.

The impact of stress on the surgeon's skill must also be considered. According to Renouard et al., ${ }^{1188}$ everyone will, at one time or another, experience the negative impact stress can have on their capacity to 
perform a procedure or deliver a treatment to the best of their ability. Moreover, in the event of acute stress, people may regress mentally and completely lose their ability for rational decision-making. ${ }^{1189-1191}$

\section{The surgeon's judgment}

It is important to take into consideration some facts concerning the observed statistically significant difference in implant failure rates between some surgeons in the study of Chrcanovic et al. (Study VII). ${ }^{194}$ The success of a technique depends on careful and scrupulous adherence to the prescribed clinical protocol by the combine surgical-prosthodontic team. The surgeon might be addicted to bad/bold habits and bad choices, as for example, risk too much in inserting implants in and/or choosing patients with sites with poor bone quantity/quality. The level of complexity of cases treated by different dentists may vary, and some professionals may be more willing to take more risky cases. The surgeons may overestimate their ability to execute a complex or high-risk procedure. ${ }^{347,1174,1188}$ There are some categories of behavior that are frequently cited to increase the likelihood of one or more complications occurring during the implementation of a procedure, and such behaviors are also relevant in the dental/medical field and may make the surgeon less careful. ${ }^{1192}$ In the study of Chrcanovic et al. ${ }^{194}$ (Study VII), a separate analysis showed a far unequal distribution of implants placed in sites of poor bone (bone quantity $\mathrm{D} / \mathrm{E}$, bone quality 4 ) among the surgeons. The surgeon with the highest absolute number of failures was also the one who mostly inserted implants in sites of poor bone. It was suggested that one of the most important factors responsible for implant failures is probably the local anatomic structure regarding bone quality and quantity, or rather the lack thereof. ${ }^{776}$ Clinicians may embark on a particular course of treatment to address a complex case despite less-than-ideal conditions from a patient safety point of view. ${ }^{1188}$ Some surgeons might have had considerable experience with some implant systems but not with other systems. Moreover, some surgeons used old implants more often than other surgeons, but this is also related to the fact that some surgeons inserted more implants on the 1980's and 1990's. Modern implants having an enlarged surface had a statistically significant lower probability to fail in comparison to turned implants. It has been shown that the 
osseointegration process is influenced by several factors, among them the surface texture. ${ }^{1193,1194}$ There is supportive evidence for a positive relationship between an improved bone healing around implants and its surface roughness, ${ }^{1195}$ which enhances the process of osseointegration. However, it is also important to note that the group of turned-surface implants had statistically significant longer mean follow-up time than the group of enlarged surface implants, which can lead to an increase in the failure rate.

Unfortunately, it was not possible for Chrcanovic et al. ${ }^{194}$ (Study VII) to assess implant survival based on dentist experience in implant surgery, as this information was not archived in the records. Dentists from the specialist clinic where the study was based on come with a diverse background; some enter the specialist training program after only 2 years of working in general practice after leaving the dental school (minimum requirement to enter a specialist program in the country), whereas others come from a long private dental practice. It is important to remember that Chrcanovic et al.'s ${ }^{194}$ study includes implants placed as long ago as 1980, and some of the surgeons are already retired. It was not possible to know for every surgeon whether the initial implants placed for each of them occurred at the clinic in particular, and how many implants were placed outside the clinic. Moreover, some surgeons with less experience might have had more opportunity to gain additional experience than those with more experience, i.e. those with less experience might have put more implants after a couple of years than the more experienced surgeons. Another important issue is the fact that the experience of the surgeon may be masked by that of the restorative dentist. With the existing data forms, the particular experience of each surgeon was difficult to ascertain, and any conclusions regarding experience drawn from these data must be considered with caution in mind. However, if one hypothetically assumes that the surgeons analyzed would have the level of experience, and assuming all other factors equal, the variations in the failure rates could be, in part, explained by the surgeons' skill and/or judgment. ${ }^{194}$

\section{Cluster behavior of failures}

Results of some studies indicated that implant failures are commonly concentrated in few patients, rather than to be evenly distributed 
among all treated patients. ${ }^{776,1196-1200}$ Implant failures are not randomly distributed in all patients and a cluster behavior can occur. ${ }^{1198}$ Cluster was once defined as more than one implant failure per patient, not necessarily in the same area or quadrant. ${ }^{1199}$ As such, these failing patients have been described as "cluster patients," and even though they have seemingly been observed in a randomized pattern, it is reasonable to assume that the patient with failing implant has certain individual characteristics that separate them from the more successful implant patients, ${ }^{1198}$ i.e. these implant loss clusters happen in specific high-risk groups and individuals. ${ }^{1201}$

One recent study ${ }^{193}$ (Study VIII) analyzed the cluster behavior of dental implant failures among patients and assessed the possible risk factors influencing this phenomenon. Only patients receiving at least three implants were included. There were 1,406 patients with three or more implants, totaling 8,337 implants, with 592 implant failures. Sixty-seven $(4.77 \%)$ of these patients were identified as presenting cluster behavior, who received 620 implants, of which 331 failed. The 67 cluster patients had a mean implant failure rate of $53.4 \%$ (range $20-100 \%$ ), and they presented $56 \%$ of all failures in the study. Twelve patients lost all their implants. The intake of antidepressants and bruxism were identified as potential negative factors exerting a statistically significant influence on a cluster behavior at the patientlevel. The negative factors at the implant-level were turned implants, short implants, poor bone quality, age of the patient, the intake of medicaments to reduce the acid gastric production, smoking, and bruxism. The authors concluded that a cluster pattern among patients with implant failure is highly probable. Factors of interest as predictors for implant failures could be a number of systemic and local factors, although a direct causal relationship cannot be ascertained.

It is important to stress that some patients should have been treated differently than what was done in the first place. Grafting procedures might have been advantageous in the cases with poor bone quantity and quality that displayed large number of failures. Parameters that can be available before the surgical and prosthodontics treatment would certainly be of importance to identify information to allow a careful discussion regarding risks and problems prior to surgery, and also to allow for measures to be taken to reduce the risk by, for 
example, choosing other surgical or prosthodontic techniques. ${ }^{1198}$ The indication for the use of oral implants should sometimes be reconsidered when alternative prosthetic treatments are available and when possibly multiple interfering systemic or local factors are identified. 


\section{ON REASONS FOR MARGINAL BONE LOSS AROUND ORAL IMPLANTS}

MBL around oral implants has been recognized as a potential problem for long time survival of the implant. Therefore, it was natural to include a MBL parameter in the criteria for success of oral implants presented in $1986 .{ }^{919}$ With respect to MBL as handled in those criteria, a successful implant must display less than $0.2 \mathrm{~mm}$ annually of MBL following the implant's first year of service. The reason why the authors did not include MBL during the first year in their criteria was the fact that knowledge from the first year was lacking in the then available clinical material due to the fact that Brånemark did not endorse radiograms during the initial phase of implant incorporation. In a publication from 1993, an acceptable MBL during the first year was considered to be $1 \mathrm{~mm}$ accumulated. ${ }^{187}$

\section{The traditional theory of reasons for marginal bone loss}

The success criteria described by Albrektsson et al. ${ }^{187,919}$ were behind what was regarded as a dangerous MBL by author such as RoosJansåker et al. ${ }^{1202}$ and Fransson et al. ${ }^{1203}$ These authors in their initial reports accepted $1.8 \mathrm{~mm}$ of $\mathrm{MBL}^{1202}$ or three threads of $\mathrm{MBL}^{1203}$ but deemed larger bone loss as pathological if coupled to bleeding on probing and pus on probing. With these definitions they regarded $6.6 \%^{1202}$ or $12.8 \%^{1203}$ of all placed Brånemark implants followed up over respectively $9-14$ years and 5 to 20 years to suffer from a disease entitled peri-implantitis. With time the same authors, then using more liberal criteria for pathological MBL, reported between 18 and $43.3 \%$ of all implants to suffer from peri-implantitis. A consensus report ${ }^{1204}$ 
reported that peri-implantitis is an infectious disease, which affects the supporting bone as well as the mucosa. Mucositis occurs as a response to bacteria, originating either from surgical contamination during implant placement or from a later occurring infection. The bacteria will secondarily lead to marginal bone resorption. In parallel to the clinical studies, experimental investigations with ligatures were conducted. ${ }^{1205}$ Albouy and co-workers ${ }^{1206-1208}$ performed a series of experimental studies with ligatures placed around commercially available implants. The ligatures provoked progressive bone resorption and were then removed. Whereas turned implants spontaneously saw no further bone resorption after ligature removal, the other commercially available implants nevertheless saw continued MBL. The investigators then mechanically cleaned the implants and were able to stop further bone resorption around two of the tested implants, but not around the third type of implant that had a porous surface structure allegedly more difficult to clean mechanically. One may certainly question the clinical relevance of such ligature studies, but they remain one mode of investigating MBL.

The studies by Roos-Jansåker et al. ${ }^{1202}$ and Fransson et al. ${ }^{1203}$ have been criticized for making pathology of MBL that in many cases may spontaneously stop without really resulting in risk of implant loosening. Jemt et al. ${ }^{7}$ reported of the long term fate of the allegedly sick implants in the Fransson et al. ${ }^{1203}$ study when analyzed a further 9.1 years after the completion of the original 5-20 year clinical follow up study. Jemt et al. ${ }^{7}$ reported that $91.3 \%$ of the allegedly sick implants had seen no significant further bone resorption and that $95.4 \%$ of the implants were still clinically functioning, thus questioning the actual clinical problems with the majority of the implants suffering from peri-implantitis. Coli et al. ${ }^{712}$ have questioned the use of Bleeding on probing and probing depth around implants in the diagnosis of MBL. Albrektsson et al. ${ }^{1}$ questioned the high rates of reported disease. Based on 10 different 10-year studies of modern implants, only $1-2 \%$ of the implants had problems with MBL and the authors concluded that peri-implantitis is not as common as previously reported, at least if properly controlled oral implant systems are used by trained individuals. 


\section{The immunologically based theory behind marginal bone loss}

During the first decades of using osseointegrated titanium implants, the general concept was that titanium implants worked very well and were incorporated into bone in the manner of a simple wound healing. However, this theory has not been supported by biomaterials research; instead the titanium implant is perceived by the bodies as a foreign element and osseointegration represents the body's defense mechanism in encapsulation of the foreign material. $5,40-42,44,1209,1210$ This means that when the osseointegration of an implant is threatened, immunological reactions are likely to participate in the process. In fact, a recent $\mathrm{PhD}$ thesis of orthopedic implants analyzed the reason for what orthopedic researchers have called aseptic loosening of implants. The aseptic loosening was found to be caused by immunological reactions, with evidence of both an innate and adaptive immune response as incriminating agents. ${ }^{1211}$ Landgraeber et al. ${ }^{1212}$ mainly believed in innate immune system reactions.

According to the immunologically based theory, the initial reason behind MBL is due to immunological reactions, but this does not necessarily prevent that bacteria play a role in the bone response as well. ${ }^{788}$ There are principally three different theories concerning the role of interfacial bacteria; that they merely represent a secondary opportunistic colonization, ${ }^{1213}$ that they recruit the same bone resorbing cells as those recruited by immunological agents ${ }^{788}$ or that the bacteria themselves are perceived as foreign elements leading to activation of the bone response. ${ }^{5}$

The immunologically based theory has been criticized by those who believe that Periodontitis around teeth and peri-implantitis around implants are similar forms of disease. However, recent evidence speaks strongly against this suggestion. ${ }^{815}$ Furthermore, Cecchinato et al. ${ }^{840}$ reported that teeth and implants in the same patient did not see bone resorption simultaneously; if teeth displayed bone resorption implants showed a steady state situation of the marginal bone and alternatively if implants lost bone, then teeth had a steady state marginal bone status. In only $3 \%$ of analyzed cases, did the investigators see simultaneous bone resorption around teeth and implants. 


\section{FRACTURE OF IMPLANTS}

Knowledge regarding the types of complications that can occur in dental implant oral rehabilitations is an important aspect of the post-treatment care. Among the mechanical complications, implant fractures offer a challenging situation because of their functional, restorative, surgical, and emotional implications. ${ }^{1214}$ Implant fracture is the most catastrophic failure of implant components because it usually causes the loss of the implant and prosthesis. ${ }^{1215}$ Fortunately, they do not occur as frequently as abutment screw or prosthesis gold screw fractures. ${ }^{190,1216}$ (Study IV included) Implant fractures were first described by Brånemark et al., ${ }^{38}$ who reported on 13 fractured of a total of 1,618 osseointegrated implants. Chrcanovic et al. ${ }^{195}$ observed a $0.40 \%$ implant fracture rate among more than 10,000 implants. The prevalence in several studies altogether is reported to be around $1.0-1.5 \%$ according to two review papers, ${ }^{1214,1216}$ mostly when older Brånemark implants were considered. However, one study of 157 ITI implants (Straumann AG, Waldenburg, Switzerland) that were followed up for a mean of 40 months reported a $1.9 \%$ fracture rate. ${ }^{1217}$

Several factors have been implicated as potential causes of implant fracture.

(a) Bending overload. Bending overload is defined as the load on an implant-supported prosthesis that exerts a bending moment on the fixture cross-section at the crestal bone level, leading to MBL and/or implant fatigue fracture. ${ }^{1218}$ The mechanism of fracture has a multi-factorial etiology; when number, position, dimension, design of implant and restoration are inadequate to the site needing 
rehabilitation, the situation of bending overload is present and an initial bone loss around the implant begins. ${ }^{1219}$ If no correction of the prosthesis is introduced, the coronal screws become soon exposed and a crater-like appearance of the surrounding bone is observable. At this time, the coronal portion of the implant represents a locus minoris resistensiae (i.e., a site of lesser resistance), being the implant internally filleted and consequently extremely thin, upon which overstress promotes the creation of numerous micro-fractures that can result, ${ }^{1220-1223}$ after a variable time range, in a complete fatigue fracture. Ideally, implants with internal abutment connection should be more suitable to this complication. ${ }^{1219}$

Previous case series and reports, involving investigations of fractured implant surfaces, revealed fatigue striations ${ }^{1214,1215,1219,1221,1224}$ consistent with those obtained in vitro when implants were cyclically loaded to fracture. ${ }^{1221}$ An interesting case is the one from Capodiferro et al. ${ }^{1219}$ who performed an accurate analysis of a removed fractured implant both by stereomicroscopy and making a three-dimensional reconstruction by confocal laser scanning microscopy. The histological evaluation of the peri-implant bone showed no signs of periimplantitis or fibrous integration of the implant. After removal of the bone, the fracture surface presented the typical features of the fatigue fracture observed by other studies using fractography with scanning electronic microscopy - main transversal fracture surface with a variable number of fatigue striations indicating the advancement of the crack front under cycling loading. ${ }^{53,1225,1226}$ Moreover, numerous surface irregularities were also detected on the roots of the screw thread of the well-osseointegrated apical portion of the implant, which the authors defined as microfractures or cracks of the apical portion of the implant despite adequate osseointegration. ${ }^{1219}$ This would suggest that bending overload and resultant fatigue failure to be the usual mode of fracture of an implant. ${ }^{1227}$

(b) Restoration design. Cantilever design bridges increase the stress upon an implant, and have been found to be associated with fractured implants, ${ }^{1221,1224,1225,1228,1229}$ even though fractured fixtures have also been reported to occur more frequently in single implant supported, single unit restorations. ${ }^{1224,1225,1229-1232}$ Implant fractures associated with a combined dento-implant supported restoration have also been reported. ${ }^{1214,1225,1233,1234}$ In addition, an implant supporting a bridge 
has a smaller tendency to fracture than an implant supporting a removable prosthesis. ${ }^{1196}$

(c) Accuracy of fit of restoration. Stress caused by screw joint connections to the implants from an ill-fitting prosthetic framework may result in constant shear load on the implant, predisposing it to fracture. Frequently, loosening of the screw(s) on the supraimplant component precedes implant fracture and may be a warning sign that the framework needs re-evaluation. ${ }^{1228}$ Some studies have shown implant fracture in partially edentulous fixed prostheses particularly with older, less passively fitting prostheses..$^{300,1235-1237}$

(d) Number of implants, positioning. A higher incidence of implant fractures has been reported in fixed partial dentures supported by only 2 implants. ${ }^{232,1238-1241}$ Theoretical models suggest the effects of loading on implant supported restorations to be significantly reduced by the placement of additional implants, the use of wider platform fixtures and the avoidance of implants being positioned in a straight line. ${ }^{1224,1225,1242}$ In a survey by Rangert et al., ${ }^{1225}$ most fractured implants occurred in single or double implant-supported restorations in posterior partially edentulous jaws where occlusal loads were higher. Thus, it was suggested that implant fixed partial dentures be supported by 3 implants placed in a tripod alignment to minimize stress and torque distribution. ${ }^{1225,1241}$ The proximity of the molar to the temporomandibular joint creates a mechanically unfavorable situation because of the high magnitude of force transmission. In a similar sense, the molar has a larger occlusal table than premolar or anterior teeth. The larger occlusal surface, supported by a single implant, will create forces that are not in line with the long axis of the implant. These tipping forces may lead to chronically high, complex force patterns that contribute to implant fracture. ${ }^{1224}$

(e) Marginal bone loss. Implant fractures have been associated with increased MBL. 1214,1221,1224,1225,1228,1230,1231 According to a review on clinical complications of implants, ${ }^{1216}$ most fractures of implants reported in the literature occurred between the third and fourth implant thread, which corresponds to the last thread of the abutment screw. ${ }^{1243,1244}$ Bone resorption exceeding three threads in the apical direction will expose the weaker portion of the implant apical to the abutment screw engagement, which may become the fulcrum for the 
loading, and thus contributes to an overload of the implant. ${ }^{1225,1245}$ This may particularly be the case in fixtures whose design features incorporate a significant void in this region. ${ }^{1230-1232}$ It has been shown in an animal study that craterlike bone loss will occur around implants with excessive dynamic loading ${ }^{690}$ and this loss of bone support will allow flexing of an implant under loading and possibly contribute to a fatigue stress fracture. Moreover, any significant bone loss at the crestal level increases the unsupported coronal length of the implant, increasing the crown to implant ratio, resulting in the fixture becoming more at risk to bending forces. ${ }^{1227}$ Morgan et al. ${ }^{1221}$ analyzed the surface of in vivo fractured implants and compared to new titanium implants fractured in the laboratory under monotonic and cyclic loads. The researchers concluded that the failure mechanism was fatigue and that the failures were associated with MBL, which was thought to have been induced by overload before the fracture occurred. ${ }^{1221}$ Implant fracture involves progressive fatigue failure until the implant lacks adequate strength to maintain integrity, culminating in a catastrophic failure. During the progression of the fracture process, it is possible that an infective process may be involved in the observed pattern of bone loss, i.e. when an implant begins to fracture, it becomes secondarily infected which causes the accelerated MBL. In the former situation, bone loss is an etiologic factor for the fracture, while the latter instance would have the fracture causing bone loss. ${ }^{1224}$ It is unclear whether MBL is a cause or an effect of implant fracture, or if they are both consequences of unfavorable loading. ${ }^{1224,1225}$

(f) Bruxism. Implant fracture has been associated with parafunction. A parafunctional occlusal habit can contribute to the potential overload, as load magnitude, duration, frequency, and direction are increased by such activity. Six of the eight fractured implants in the study of Balshi ${ }^{1228}$ supported posterior prostheses and all eight fractured implants occurred in patients with parafunctional habits. The results of the clinical study of Chrcanovic et al. ${ }^{190}$ (Study IV) suggest that bruxism may be an important contributor to the rate of mechanical complications in implant-supported restorations, as well as for a higher prevalence of implant fracture. The study compared groups of bruxers and non-bruxers having the same number of patients $(n=98)$ with the same total number of implants $(n=427)$ equally distributed between them. Bruxers presented 16 fractured 
implants, whereas non-bruxers did not present any case of implant fracture. In another study, specifically assessing implant fractures, Chrcanovic et al. ${ }^{195}$ observed that bruxism was one of the factors presenting a statistically significant influence on the prevalence of implant fractures, according to a multivariate GEE statistical model.

(g) Chemical factors. Titanium implant components adsorb hydrogen in the biological environment and it has been suggested that this makes them more brittle and prone to fatigue. ${ }^{1223}$ Hydrogen embrittlement of titanium is associated with the formation of a brittle hydride phase. ${ }^{1246}$ Amongst the factors that determine the service life of a titanium device for medical use may therefore be the rate of hydrogen absorption in the device and the rate of diffusion of hydrogen in the alloy. ${ }^{1247}$

(b) Implant composition. In the study of Eckert et al., ${ }^{1224}$ all of the fractured implants were made from c.p. Ti grade 1 . The use of a different implant material provides a higher threshold for fatigue failure, since fatigue appears to have some relationship with the tensile strength of the implant material. ${ }^{1248}$ Because c.p. Ti is ductile and the implant will thus bend, it is not possible to fracture a c.p. Ti implant in situ with just one loading cycle. With repeated load, however, the material may start to develop internal microcracks, which may increase in size with the number of load cycles. The fatigue strength determines the capacity for withstanding such repeated loads. ${ }^{1225}$ The fatigue strength of grade $1 \mathrm{c}$.p. Ti is practically independent of its tensile strength. ${ }^{1249}$ The tensile strength may be increased by work hardening to surpass the minimum strength specified for grade 3 , but this has limited influence on the long-term fatigue behavior. Such strength increases have practical consequences only over a small number of loading cycles, and thus have little clinical significance. The most effective way of increasing clinically relevant implant strength, based on a grade 1 c.p. Ti implant, is therefore to increase its diameter. ${ }^{1225}$

It may sound strange that different grades of c.p. Ti may be subject to different fracture percentages, bearing in mind that all c.p. Ti consists of about $99.7 \%$ of titanium. However, the fact is that the remaining $0.3 \%$ of constituents is of clear importance for the strength 
of the material, particularly if the iron contents increase which is the case for higher grades of titanium. One of the major commercial companies used only titanium grade 1 until the latter part of the 1990s when, finally, the change to grade 4 occurred with due higher strength of their implants.

MBL and mechanical failure of the restorative components (i.e., frequent loosening and/or fracturing of prosthetic screws) should be considered as warning signs. ${ }^{1214,1228}$ Several methods have been reported to try to decrease the risk of implant failure ${ }^{1225,1228}$ : reduction of cantilever use, narrowing of buccolingual width/mesiodistal length of the teeth, flattening the cuspal inclination, centering the occlusal contacts, using additional implants with larger diameters, occlusal guard treatment in patients with parafunctional habits, and insertion of implants not in a straight line.

Different treatment alternatives have been suggested for implant fractures. The least invasive approach is to leave the apical portion embedded in bone either following the patient's informed decision or when retrieval of the implant may compromise the integrity of adjacent vital structures (i.e., proximity to inferior alveolar canal). ${ }^{1214}$ Another option is to flatten the head of the implant and make it smooth to simulate the surface of the original implant face, retapping the internal abutment screw thread and placing a longer abutment connector, followed by the necessary modifications of the prosthesis. ${ }^{1220,1250}$ The latter option should be considered only as an emergency procedure in the transition to a more permanent solution, since the crown-to-implant body ratio can be considerably altered, offering a more complex biomechanical challenge; while creating a butt joint repair between the fractured portion and the newly connected abutment, microleakage and the lack of antirotational features may further compromise the surrounding bone integrity. The third and presumably the most common option would be the removal of the fractured implant, ${ }^{226}$ using a trephine drill to core out the bone to three quarters of the length of the implant. An elevator is then used to fracture the most apical integration. A long healing abutment is threaded in the remaining internal threads and lifted out with forceps. The site may be tapped to receive a wider implant, or following a suitable period of healing, usually 3 to 6 months, a new implant can be placed. 


\section{CONTRAINDICATIONS FOR IMPLANTS}

It has been suggested that some local and systemic factors could represent contraindications to dental implant treatment. ${ }^{1251-1254}$ Several factors have been implicated as contraindications of implant surgery. Sugerman and Barber ${ }^{1251}$ suggested that conventional dentures or fixed partial prostheses may be preferable to dental implants in growing and epileptic patients and patients at risk of oral carcinoma, anaphylaxis, severe hemorrhage, steroid crisis, endocarditis, osteoradionecrosis, myocardial infarction, or periimplantitis. Hwang and Wang ${ }^{1253}$ suggested that the absolute contraindications for dental implants include recent myocardial infarction and cerebrovascular accident, transplant or valvular prosthesis surgery, profound immunosuppression, severe bleeding issues, active treatment of malignancy, drug abuse, psychiatric illness, as well as intravenous bisphosphonate use. However, there is little or no evidence to support most of these contentions. ${ }^{1255}$ Here follows a discussion about some of these conditions that were suggested as contraindications for treatment with oral implants.

(a) Bleeding disorders. In patients with bleeding disorders, hemorrhage associated with implant surgery is more common and may be prolonged, ${ }^{1256}$ particularly if the patient is taking warfarin or acenocoumarol, both anticoagulants that function as vitamin $\mathrm{K}$ antagonists. The treatment of a patient suffering from classical hemophilia with repeated surgical session; for ankylosis of the right temporomandibular joint, for bilateral sagittal split procedures, for extraction of teeth, and for placement of implants ${ }^{1257}$ provides 
some evidence to support the placement of implants in patients with bleeding disorders. The important fact here to consider is that the patient was prepared medically on each of the surgical occasions with factor VIII replacement concentrate as well as oral antifibrinolytic therapy (tranexamic acid). However, Gornitsky et al. ${ }^{1257}$ did not provide information on the follow-up of the patients. Bacci et al. ${ }^{1258}$ evaluated the incidence of bleeding complications following surgical implant therapy in a group of 50 consecutive patients receiving oral anticoagulant therapy (warfarin) without interruption or modifications of their therapy. The authors observed no statistically significant difference in the bleeding risk between these patients and 109 other patients not taking anticoagulants. They concluded that local hemostasis in dental implant surgery is capable of preventing bleeding complications in patients on oral anticoagulants, allowing these surgical procedures to be performed on an outpatient basis. Again, the authors did not provide information on the follow-up of the patients after the placement of the implant-supported prosthesis. There is no evidence that bleeding disorders are an absolute contraindication to oral implant surgery, although these patients may be at risk of prolonged hemorrhage and blood loss. ${ }^{1255}$ In face of the low number of publications focusing on this issue, with no information on follow-up, the impact of bleeding disorders on the long-term implant survival may be considered unknown.

(b) Bone diseases. There are only few reported cases of oral implant placement in patients with osteogenesis imperfect, ${ }^{1259-1265}$ polyarthritis, ${ }^{1266-1268}$ or ankylosing spondylitis. ${ }^{1269}$ With only few cases described in the literature, there is little evidence to suggest whether these osseous diseases could affect implant survival or not. Thus, there is no sufficient material to say whether these conditions would be contraindication to oral implant surgery or not.

Two retrospective clinical trials ${ }^{1270,1271}$ assessed implant outcomes in patients suffering from autoimmune rheumatoid arthritis with or without concomitant connective tissue diseases. A high implant survival rate was noted with follow-ups of 3 and 3.5 years, respectively. Patients with isolated rheumatoid arthritis demonstrated acceptable marginal bone resorption and good soft tissue conditions, while patients with rheumatoid arthritis and concomitant connective tissue diseases showed increased bone 
resorption. Although two clinical trials were published on the subject, they are both from the same research group, and most of the patients included in the studies are very probably the same ones.

Another bone condition to consider is osteoporosis. According to a review, the evidence for an association between osteoporosis and implant failure was low. ${ }^{1272}$ Several clinical trials in humans have focused on the subject. ${ }^{810,1017,1273-1278}$ In general, results from the studies led the authors to suggest that oral implant therapy in patients suffering from osteoporosis is a reliable treatment option with comparable integration rates as in healthy patients. Thus, osteoporosis would not be considered as a contraindication for dental implants. For more details on osteoporosis and the possible effect on dental implants, see "Results and Discussion", section "Health", sub-item "Patient's sex" of the present thesis.

(c) Intravenous bisphosphonate (BP) use. The possible effects of the use of bisphosphonates on the oral implant survival were already discussed in the "Results and Discussion" of the present thesis. A few number of patients rehabilitated with oral implants and being under intravenous administration of BPs have been reported in the literature, ${ }^{1279,1280}$ with no implant failures recorded. However, as the cases consisted of only a couple of patients, there is no strong clinical evidence to allege complete security of these medicaments under parenteral administration. It has been observed that osteonecrosis caused by intravenous BPs is more prevalent and more severe than osteonecrosis induced by oral BPs. ${ }^{995}$ A higher prevalence of osteonecrosis with the use of intravenous BPs could presumably interfere with the healing process after implant placement, and consequently increase the failure rates. Therefore, it is suggested that the use of intravenous BPs might be a relative contraindication for the placement of oral implants. For a deeper discussion on the subject, see "Results and Discussion", section "Health", item "Pharmaceutical reasons for implant problems", sub-item "Effects of bisphosphonates" of the present thesis.

(d) Corticosteroid therapy. Steroids, also called corticosteroids, are anti-inflammatory medicines used to treat a range of conditions, such as asthma, rheumatoid arthritis, inflammatory bowel disease and autoimmune diseases. ${ }^{1281}$ Steroids do not tend to cause 
significant side effects if they are taken for a short time or at low doses. Long-term corticosteroid use may be associated with more serious sequelae, which includes osteoporosis, increased epithelial fragility and immunosuppression, among many other side effects. ${ }^{1282}$ As such, it has been suggested that the chronic use of systemic glucocorticoids might compromise oral implant osseointegration and peri-implant healing. ${ }^{1283}$ Therefore, corticosteroids have been cited as a contraindication for placement of implants in the jaws. ${ }^{1284}$ However, only few studies have actually evaluated the association between corticosteroid therapy and oral implants. Werner et al. ${ }^{1285}$ assessed the effect of dexamethasone on the first stages of the postimplantation reparative process in rats. The extension of osteogenic peri-implant response was greater in dexamethasone -treated animals than in controls. Fujimoto et al. ${ }^{1284}$ evaluated the effects of steroid administration on the osseointegration of c.p. Ti implants in an in vivo study in rabbits. They observed no significant difference in removal torque of the implants placed in the mandible between prednisolonetreated group of rabbits and a control group, besides no significant correlation between the bone density of the femur and the removal torque of the implants placed in the mandible. Moy et al. ${ }^{950}$ retrospectively analyzed risk factors associated with the outcome of 4,680 implants placed in 1,140 patients. A logistic regression analysis indicated that steroid therapy was not associated with a significant increase in implant failure. Bencharit et al. ${ }^{1283}$ reported a case of a patient with polymyalgia rheumatica, under chronic treatment with systemic glucocorticoids and treated with oral implants. There were no clinical problems after one year of follow-up. Shibuya et al. ${ }^{1286}$ retrospectively analyzed risk factors associated with the outcome of 619 TiUnite implants. A logistic regression analysis identified that a history of steroid treatment, among other factors, was significant predictors of implant failure. Due to the limited number of studies on the subject, there is no clear evidence that corticosteroid therapy is a contraindication to treatment with oral implants.

(e) Head and neck cancer patients. Radiotherapy can significantly affect the dental implant failure rates. ${ }^{24}$ It is suggestive that treatment with implants can be a contraindication in patients during radiotherapy, just before or in the few months after it, in patients who received or are planned to receive irradiation doses above $65 \mathrm{~Gy}$, 
and in patients showing sign and symptoms of osteoradionecrosis. For a deeper discussion on the subject, see Chapter "Results and Discussion", section "Health", sub-item "Irradiation" of the present thesis.

(f) Alcoholism. Bone metabolism is affected by alcohol consumption because alcohol inhibits osteoblast proliferation. However, there is no clear evidence in the literature that alcoholism might be a contraindication to the placement of oral implants, although the literature is suggestive that alcoholic patients may be at increased risk of complications. For a deeper discussion on the subject, see Chapter "Results and Discussion", section "Patient's Habits", subitem "Alcoholism" of the present thesis.

(g) Diabetes mellitus. Diabetes is a chronic disease that occurs when the pancreas does not produce enough insulin, or when the body cannot effectively use the insulin it produces. It has been suggested that the relative contraindication for implant surgery is related to the stability of the diabetic's blood sugar level. However, there is still no clear evidence that diabetes is a contraindication to treatment with oral implants. For a deeper discussion on the subject, see Chapter "Results and Discussion", section "Health", sub-item "Diabetes mellitus" of the present thesis.

(b) Cardiovascular disease. Some forms of cardiovascular disease (hypertension, atherosclerosis, vascular stenosis, coronary artery disease, and congestive heart failure) may impair the healing process, which depends on oxygen supply delivered by a normal blood flow. However, several studies suggest that cardiovascular diseases may not be a risk factor for dental implants. Khadivi et al. ${ }^{1287}$ conducted a retrospective study including 246 patients, of which 39 patients had some type of cardiovascular diseases, 109 patients had a history of other systemic disease, and 98 patients were healthy. The study found no statistically significant differences in implant failure rates between the groups, and the authors concluded that cardiovascular diseases may not be a risk factor for successful osseointegration. Several other studies observed that the presence of cardiac problems and/or the intake of antihypertensive drugs did not affect the implant failure rates (Studies III, V and VIII included). ${ }^{161,191,193,942,944,945,950,965,1288} \mathrm{Wu}$ et al. ${ }^{159}$ observed a higher survival rate of dental implants among 
users of antihypertensive drugs compared to nonusers. There is no clear evidence in the literature that cardiovascular diseases or the intake of antihypertensive drugs might be a contraindication to the placement of oral implants. Patients with cardiac problems should, however, receive careful supervision of bleeding time, as many of these patients usually take blood thinners or antithrombotic agents (antiplatelet drugs, anticoagulants, thrombolytic drugs). Moreover, cardiovascular events such as recent myocardial infarction, stroke, and cardiovascular surgery, might represent an absolute contraindication to implant therapy. ${ }^{1253}$ Due to the high risk of complications following a myocardial infarction or cerebrovascular accident, the surgeon must wait until preliminary stabilization before the performance of an elective oral implant surgery. ${ }^{1253}$

(i) Hyposalivation. Sjögren's syndrome is probably the most common cause of hyposalivation. Sjögren's syndrome is a chronic autoimmune disorder of the exocrine glands with associated lymphocytic infiltrates in the affected glands. Involvement of the salivary glands results in progressive dryness of the mouth, difficulties with chewing, swallowing and speech, reduced oral clearance, and a shift in oral flora. ${ }^{1289}$ As Sjögren's syndrome is associated with several oral problems ${ }^{1290}$ one has raised the question whether patients with this condition might be contraindicated to receive oral implants. Nevertheless, several case reports and small case series reported the use of dental implants in patients with the condition, ${ }^{1270,1291-1300}$ reporting excellent clinical results. Albrecht et al. ${ }^{1301}$ investigated prevalence and patient-reported outcomes of dental implants in patients with Sjögren's syndrome. A total of 5 of 104 (4.8\%) implants in the patients with the syndrome and none of the 14 implants in the controls had to be removed. A total of $75 \%$ of the patients were highly satisfied with the implants and $97 \%$ would recommend them to other patients with Sjögren's syndrome. Korfage et al. ${ }^{1302}$ performed a cohort study of well-classified patients with Sjögren's syndrome $(\mathrm{n}=50)$ and its possible association to oral implants clinical outcomes. The researchers observed that peri-implant health was reasonably good in syndromic patients with minor MBL and a peri-implantitis prevalence of $14 \%$, comparable with healthy controls. Despite the fact that a worse oral functioning was associated with more dryness complaints, lower patient satisfaction, and worse subjective chewing 
ability, the implant survival rate reached $97 \%$ (4 out of 142 implants failed) after a median follow-up 46 months. All the studies support the idea that the syndrome is no contraindication for the treatment with oral implants.

(j) Immunocompromised patients. It has been suggested that patients taking immunosuppressive drugs/agents might have some impairment of bone metabolism, which in theory could have some negative influence on osseointegration. For a deeper discussion on the subject, see "Results and Discussion", section "Health", item "Pharmaceutical reasons for implant problems",sub-item "Effects of immunosuppressives" of the present thesis.

There are few clinical studies evaluating oral implants outcomes in patients who are immunocompromised. Most of them deal with the acquired immune deficiency syndrome (AIDS), a spectrum of conditions caused by the infection of components of the human immune system such as $\mathrm{CD}^{+} \mathrm{T}$ cells, macrophages and dendritic cells by the human immunodeficiency virus (HIV). Stevenson et al. ${ }^{1303}$ investigated the short-term clinical outcome of implant placement in a group of HIV-positive and HIV-negative individuals who required complete dentures, and the success rate was $100 \%$ for both groups. There was also no difference in clinical outcome (presence of pain, mobility, soft tissue status, and radiographic bone level) between the groups. The limitation of this study is the short term follow-up, of only 6 months. Oliveira et al. ${ }^{1304}$ evaluated the 12-month after loading success rate of 59 dental implants placed in patients who were HIV-positive and were receiving different regimens of highly active anti-retroviral therapy, as the same time as comparing those patients with a control group of other HIV-negative patients. The study showed very similar results between the groups, regardless of $\mathrm{CD} 4{ }^{+}$cell count, viral load levels and type of antiretroviral therapy. Once again, the limitation of this study was its short term follow-up. Gay-Escoda et al. ${ }^{1305}$ retrospectively assessed 9 HIV-positive subjects treated with 57 dental implants, with a mean follow-up time of 77.5 months. The implant survival was $98.3 \%$, and the patients that attended regular periodontal maintenance visits had significantly less mean bone loss than non-compliant patients $(1.3 \mathrm{~mm}$ and 3.9 $\mathrm{mm}$ respectively). Gherlone et al. ${ }^{1306,1307}$ examined the associations between the success of implant-prosthetic treatment and systemic 
$\mathrm{CD}^{+}$level, smoking habits, and oral hygiene. The authors concluded that placement of dental implants in HIV-positive patients with stable disease seems a reasonable treatment option, regardless of $\mathrm{CD}^{+}$cell count, provided that they are in a normal range. The follow-up was of only 12 months. May et al. ${ }^{1308}$ investigated the 5 -year clinical outcome of 33 oral implants in 16 patients diagnosed with AIDS. The study found a slightly higher failure rate of $10 \%$ in patients with AIDS than the previous clinical trials on the same subject, probably due to a longer follow-up. There are in the literature other reports of oral implant treatment in HIV-positive subjects, mostly consisting of few or isolated case reports, ${ }^{1309-1317}$ all showing good clinical outcomes. Thus, HIV-positive patients do not apparently have any contraindication to receive oral implants, provided that the disease is controlled. However, longer follow-up studies including more patients are necessary to add more clinical evidence.

Another condition that causes immunodeficiency is Crohn's disease, a type of inflammatory bowel condition that may affect any part of the gastrointestinal tract from mouth to anus. Crohn's disease is characterized by the presence of many antibody-antigen complexes, leading to autoimmune inflammatory processes in several parts of the body, which in theory could as well occur at the interface with biocompatible implants, but which could in Crohn's patients be recognized as non-self, thus affecting the outcome of the implant treatment. ${ }^{942}$ The study of van Steenberghe et al., ${ }^{942}$ which had three patients with Crohn's disease, with two of them having implant failure ( 3 out of 10 implants) and one patient having no implant failure (0 out of 3 implants). However, both patients with early failures, suffered from other medical conditions. Alsaadi et al. ${ }^{944}$ observed 11 of 12 oral implants placed in patients with Crohn's disease in their retrospective study integrated successfully. There is low level of evidence to suggest any contraindication or not of the use of oral implants in patients with Crohn's disease.

There are some case reports of successful implant placement in patients with Papillon-Lefèvre syndrome, ${ }^{1318-1324}$ a rare autosomal recessive disorder, which oral manifestations include rapidly progressive periodontal disease resulting in premature exfoliation of primary and permanent dentitions. However, many of these patients presented in these publications were followed up for only one year. 
Only two cases ${ }^{1325}$ of oral rehabilitation using oral implants were reported in patients presenting Chediak-Higashi syndrome, a rare genetic immunodeficiency disorder that arises from a mutation of a lysosomal trafficking regulator protein, which leads to a decrease in phagocytosis. The decrease in phagocytosis results in recurrent pyogenic infections. Most patients present severe periodontal disease with an unfavorable prognosis. The two cases presented good clinical results, but with only one year of follow up.

(k) Neuro-psychiatric disorders and disabilities. Case reports and clinical series have shown good clinical outcomes with implantsupported oral rehabilitations in patients with cerebral palsy, ${ }^{1295,1326-}$ ${ }^{1328}$ Down syndrome, ${ }^{1295,1327-1329}$ autism, ${ }^{1329}$ psychiatric disorders, ${ }^{1330}$ dementia, ${ }^{1327}$ intellectual disability, ${ }^{1331}$ mental retardation, ${ }^{1295,1328,1329}$ bulimia, ${ }^{1332}$ Parkinson disease, ${ }^{1333,1334}$ epilepsy, ${ }^{1328,1329,1335}$ and multiple disabilities. ${ }^{1329,1335}$ However, it is important to stress that poor oral hygiene, oral parafunction habits such as bruxism, harmful habits such as repeated introduction of the fingers into the mouth and behavioral problems are not uncommon in patients with neuropsychiatric diseases, and oral implants in such patients may lead to complications. ${ }^{1255,1328}$ Therefore, patients with neuro-psychiatric diseases might be relatively contraindicated to receive oral implants. Medical advice is highly recommended in these cases, ${ }^{1330}$ and the success of the oral rehabilitation in patients with neuro-psychiatric diseases will depend fundamentally on the appropriate patient selection. ${ }^{1331}$ The course of the disease, patient condition, quality of life and life expectancy must be taken into consideration before adopting a treatment with oral implants in this kind of patients. ${ }^{1336}$ Implants can be successful if these patients are given continuous professional support. ${ }^{1295}$ There exist no apparent biological reasons for patients with most of the above disorders to lose implants. ${ }^{1253}$ However, one state where implant placement is not recommended is in a patient unable to comprehend and anticipate dental treatment logically. ${ }^{1253}$

(l) Titanium allergy. Titanium allergy is an absolute contraindication for implants manufactured with alloys containing this metal, provided that is properly proven that a patient is allergic. Titanium allergy is deeply discussed in the "Results and Discussion" of the present thesis, section "Health", sub-item "Titanium allergy". 
(m) Oral mucosal diseases. Several case reports and clinical series evaluated the outcomes of oral implant treatment with four mucosal diseases, namely ectodermal dysplasia, epidermolysis bullosa, oral lichen planus, and Sjögren's syndrome. They show that implant survival rates in patients with these conditions are comparable to those of patients without oral mucosal diseases. Sjögren's syndrome is discussed somewhere else in this chapter.

Ectodermal dysplasia is not a single disorder, but a group of syndromes all deriving from abnormalities of the ectodermal structures. It is a hereditary condition in which hypodontia is the second most frequently occurring sign. Hypodontia is associated with lack of development of the alveolar ridge and results in less volume of bone for support of conventional prostheses. Minimal development of the alveolar ridge can affect the bone volume available for the placement of dental implants. ${ }^{1337}$ Several cases reports and clinical series of oral rehabilitations with implants in patients with ectodermal dysplasia have been reported, ${ }^{1333,1337-1352}$ usually with high survival rates. The implant failure rates in children and adolescents, however, tend to be worse than in adults. ${ }^{1342,1345}$

Epidermolysis bullosa represents a group of mainly hereditary skin disorders, manifested by an exceptional tendency of the skin and mucosa to form bullae and vesicles after minor friction and trauma. Oral features include repeated blistering, scar formation, elimination of buccal and vestibular sulci, and alveolar bone resorption. ${ }^{1353}$ There are several case reports and clinical series of successful implant placement in patients with epidermolysis bullosa. ${ }^{1353-1362}$ The main reported complication during the implant surgical procedure was the formation of bleeding blisters by minimal trauma. During the follow-up period many patients also developed ulcers in the areas of prosthesis contact, but these complications did not affect the successful implant outcome.

Oral lichen planus is an inflammatory disease of the oral mucosa with pathognomonic changes seen both in the epithelium and the subepithelial layer of connective tissue. Implants penetrate the oral mucosa into the alveolar bone to which they osseointegrate. The epithelial attachment forms the barrier that separates the infected oral environment from the internal tissues. It has been postulated that oral lichen planus may directly alter the nature of the barrier affect, 
jeopardizing the long-term success of the implants. ${ }^{1363}$ Moreover, Langerhans cells and keratinocytes, in the oral lichen planus lesions up-regulate the pro-inflammatory response by increasing interleukin-2 and interferon- secretion. ${ }^{1364,1365}$ These cytokines have an important role in local bone resorption ${ }^{1366}$ and may lead to alveolar bone loss around the implants. The studies evaluating oral implant outcomes in patients with oral lichen planus ${ }^{1363,1367-1370}$ usually showed very high implant survival rates.

Final remarks on contraindications. The impact of many oral and systemic conditions on the outcome of implant therapy is still unclear. ${ }^{1251,1255}$ Dental management of the medically compromised patient requires acquisition of a complete health history of the patient. This should include documentation via questionnaire as well as a verbal history. ${ }^{1371}$ It seems like the medical control of the disease is more important than the disease itself. ${ }^{1255}$ It is important that the patient's surgical risks should be weighed against the potential benefits offered by the dental implant. ${ }^{1372}$ 


\section{CONCLUSIONS}

From the studies included in the present thesis, it can be concluded that:

1. After a systematic review of the literature, it may be suggested that the following situations may increase the implant failure rate: a low insertion torque of implants that are planned to be immediately or early loaded, inexperienced surgeons inserting the implants, implant insertion in the maxilla, implant insertion in the posterior region of the jaws, implants in heavy smokers, implant insertion in bone qualities type III and IV, implant insertion in places with small bone volumes, use of shorter length implants, greater number of implants placed per patient, lack of initial implant stability, use of cylindrical (non-threaded) implants and prosthetic rehabilitation with implant-supported overdentures. Moreover, it may be suggested that the following situations may be correlated with an increase in the implant failure rate but with a weaker association than the factors listed above: use of the non-submerged technique, immediate loading, implant insertion in fresh extraction sockets, smaller diameter implants. Some recently published studies suggest that modern, moderately rough implants may present with similar results irrespective if placed in maxillae, in smoking patients or using only short implants (Study I);

2. The systematic review of the literature with meta-analyses suggested that the insertion of oral implants in smokers affects the implant failure rates, the incidence of postoperative infections, as well as the marginal bone loss (Study II); 
3. Smoking and the intake of antidepressants are suggested to be potentially influencing factors to the occurrence of implant failures up to the second-stage surgery - abutment connection (Study III);

4. Bruxism may significantly increase both the implant failure rate and the rate of mechanical and technical complications of implant-supported restorations (Study IV);

5. The intake of proton pump inhibitors may be associated with an increased risk of oral implant failure (Study V), but not so the intake of selective serotonin reuptake inhibitors (Study VI);

6. Different levels of failure incidence of oral implants could be observed depending on the individual surgeons, occasionally reaching significant levels. Although a direct causal relationship could not be ascertained, it is suggested that the surgeons' technique, skills, and/or judgment may influence the oral implant survival rates (Study VII);

7. A cluster pattern among patients with implant failure is highly probable. Shorter implants, turned implants, poor bone quality, younger patients, the intake of antidepressants and of proton pump inhibitors, smoking, and bruxism were identified as suggested potential factors exerting a statistically significant influence on the cluster behavior of dental implant failures (Study VIII);

8. Most of the implant failures occur at the first years after implantation, regardless of a very long follow-up. Implants in different jaw locations, irradiation, and bruxism were the factors suggested to affect the survival of implants. Marginal bone loss can be insignificant in long term observations, but it may, nevertheless, be the cause of secondary failure of oral implants in some cases (Study IX). 


\section{ACKNOWLEDGEMENTS}

I would like to express my sincere gratitude to everyone who contributed in one way or another to the progress and completion of this work. This thesis would not have been possible without all the inspiring and generously supporting people around me. In particular I wish to thank:

Professor Tomas Albrektsson, my main supervisor. There are not enough words to express my deep and sincere gratitude for your guidance, all the great scientific discussions, encouragement and your invaluable endless support. Indeed, your commitment, supervision and passion towards my $\mathrm{PhD}$ training are untold. Thank you very much.

Professor Ann Wennerberg, my supervisor. Indeed there are not enough words to express my deep and sincere gratitude for your expertise guidance, remarkable and tireless support, encouragement throughout my entire time as a doctoral student, and for always believing in me. Thank you very much.

My profound appreciation goes to Övertandläkare Doctor Honoris Causa Jenö Kisch, who I had the opportunity and pleasure to meet, have some good laughs, and research with at the Specialisttandvård Malmö, Folktandvården Skåne AB. Without you this work would not have been accomplished. Thank you very much. 
Professor Björn Klinge for the friendly atmosphere and your support as a co-supervisor, as well as for the help in getting access to some databases.

My colleagues PhD students, dentists and nurses of the Department of Prosthodontics, Faculty of Odontology, Malmö University. And the administrative and service staff of the Faculty of Odontology, Malmö University. Thank you all for your invaluable support, comments, encouragement and friendship.

VD Marika Qvist, Vice VD och Forsknings- och utvecklingschef Ewa Ericsson, Enhetschef Kristoffer Alin, Övertandläkare Björn Gjelvold, the dentists and nurses, and the administrative and service staff of the Folktandvården Skåne $\mathrm{AB}$, for their generous support and friendship.

Finally

My beloved wife Aleksandra and my precious son Nathan for your love, understanding, support, encouragement and patience for my work. I treasure your presence in my life.

And my parents for your endless love, care and support.

This work was supported by research funds from the Oral Health Related Research by Region Skaine (Odontologisk Forskning i Region Skane, OFRS 414321), Sweden, and from the Scientific Research Council of Sweden (Vetenskapsradet, Dnr 2015-02971). This work was supported by Folktandvarden AB, Region Skane, Sweden and by CNPq, Conselho Nacional de Desenvolvimento Científico e Tecnológico, Brazil. 


\section{REFERENCES}

1. Albrektsson T, Buser D, Sennerby L. Crestal bone loss and oral implants. Clin Implant Dent Relat Res 2012;14(6):783-791.

2. Palma-Carrió C, Maestre-Ferrin L, Penarrocha-Oltra D, PenarrochaDiago MA, Penarrocha-Diago M. Risk factors associated with early failure of dental implants. A literature review. Med Oral Patol Oral Cir Bucal 2011;16(4):e514-517.

3. Sakka S, Baroudi K, Nassani MZ. Factors associated with early and late failure of dental implants. J Investig Clin Dent 2012;3(4):258-261.

4. Mangano F, Mortellaro C, Mangano N, Mangano C. Is Low Serum Vitamin D Associated with Early Dental Implant Failure? A Retrospective Evaluation on 1625 Implants Placed in 822 Patients. Mediators Inflamm 2016;2016:5319718.

5. Albrektsson T, Canullo L, Cochran D, De Bruyn H. "Peri-Implantitis": A Complication of a Foreign Body or a Man-Made "Disease". Facts and Fiction. Clin Implant Dent Relat Res 2016;18(4):840-849.

6. Qian J, Wennerberg A, Albrektsson T. Reasons for marginal bone loss around oral implants. Clin Implant Dent Relat Res 2012;14(6):792807.

7. Jemt T, Sundén Pikner S, Gröndahl K. Changes of Marginal Bone Level in Patients with "Progressive Bone Loss" at Branemark System(R) Implants: A Radiographic Follow-Up Study over an Average of 9 Years. Clin Implant Dent Relat Res 2015;17(4):619-628.

8. Chee W, Jivraj S. Failures in implant dentistry. Br Dent J 2007;202(3):123-129.

9. Hadi SA, Ashfaq N, Bey A, Khan S. Biological factors responsible for failure of osseointegration in oral implants. Biol Med 2011;3(2):164170.

10. Penchas J. Dental Implants Made Simple: eBookit.com; 2011. 
11. Chrcanovic BR, Albrektsson T, Wennerberg A. Reasons for failures of oral implants. J Oral Rehabil 2014;41(6):443-476.

12. Chrcanovic BR, Albrektsson T, Wennerberg A. Flapless versus conventional flapped dental implant surgery: a meta-analysis. PLoS One 2014;9(6):e100624.

13. Chrcanovic BR, Albrektsson T, Wennerberg A. Immediate nonfunctional versus immediate functional loading and dental implant failure rates: a systematic review and meta-analysis. J Dent 2014;42(9):1052-1059.

14. Chrcanovic BR, Albrektsson T, Wennerberg A. Prophylactic antibiotic regimen and dental implant failure: a meta-analysis. J Oral Rehabil 2014;41(12):941-956.

15. Chrcanovic BR, Albrektsson T, Wennerberg A. Periodontally compromised vs. periodontally healthy patients and dental implants: A systematic review and meta-analysis. J Dent 2014;42(12):1509-1527.

16. Chrcanovic BR, Albrektsson T, Wennerberg A. Diabetes and oral implant failure: a systematic review. J Dent Res 2014;93(9):859-867.

17. Chrcanovic BR, Albrektsson T, Wennerberg A. Tilted versus axially placed dental implants: A meta-analysis. J Dent 2015;43(2):149-170.

18. Chrcanovic BR, Albrektsson T, Wennerberg A. Dental implants inserted in fresh extraction sockets versus healed sites: A systematic review and meta-analysis. J Dent 2015;43(1):16-41.

19. Chrcanovic BR, Albrektsson T, Wennerberg A. Platform switch and dental implants: A meta-analysis. J Dent 2015;43(6):629-646.

20. Chrcanovic BR, Albrektsson T, Wennerberg A. Smoking and dental implants: A systematic review and meta-analysis. J Dent 2015;43(5):487-498.

21. Chrcanovic BR, Albrektsson T, Wennerberg A. Immediately loaded non-submerged versus delayed loaded submerged dental implants: A meta-analysis. Int J Oral Maxillofac Surg 2015;44(4):493-506.

22. Chrcanovic BR, Albrektsson T, Wennerberg A. Dental implants inserted in male versus female patients: a systematic review and metaanalysis. J Oral Rehabil 2015;42(9):709-722.

23. Chrcanovic BR, Albrektsson T, Wennerberg A. Bruxism and Dental Implants: A Meta-Analysis. Implant Dent 2015;24(5):505-516.

24. Chrcanovic BR, Albrektsson T, Wennerberg A. Dental implants in irradiated versus nonirradiated patients: A meta-analysis. Head Neck 2016;38(3):448-481.

25. Chrcanovic BR, Albrektsson T, Wennerberg A. Bisphosphonates and dental implants: A meta-analysis. Quintessence Int 2016;47(4):329342. 
26. Chrcanovic BR, Albrektsson T, Wennerberg A. Dental Implants in Patients Receiving Chemotherapy: A Meta-Analysis. Implant Dent 2016;25(2):261-271.

27. Chrcanovic BR, Albrektsson T, Wennerberg A. Survival and Complications of Zygomatic Implants: An Updated Systematic Review. J Oral Maxillofac Surg 2016;74(10):1949-1964.

28. Chrcanovic BR, Albrektsson T, Wennerberg A. Turned versus anodised dental implants: a meta-analysis. J Oral Rehabil 2016;43(9):716-728.

29. Chrcanovic BR, Nilsson J, Thor A. Survival and complications of implants to support craniofacial prosthesis: A systematic review. $J$ Craniomaxillofac Surg 2016;44(10):1536-1552.

30. Chrcanovic BR, Albrektsson T, Wennerberg A. Bone Quality and Quantity and Dental Implant Failure: A Systematic Review and Metaanalysis. Int J Prosthodont 2017.

31. Sendyk DI, Deboni MCZ, Chrcanovic BR, Wennerberg A, Albrektsson T. Does the surgical experience influence the implant survival rate? A systematic review and meta-analysis. Int J Prosthod 2017.

32. Southam JC, Selwyn P. Structural changes around screws used in the treatment of fractured human mandibles. Br J Oral Surg 1971;8(3):211-221.

33. Brånemark PI. Introduction to osseointegration. In: Brånemark PI, Zarb G, Albrektsson T, editors. Tissue Integrated prostheses. Chicago: Quintessence Publishing; 1985. pp. 11-76.

34. Zarb G, Albrektsson T. Osseointegration - a requiem for the periodontal ligament? - An editorial. Int J Periodontics Restorative Dent 1991;11:88-91.

35. Albrektsson T, Brånemark PI, Hansson HA, Lindström J.

Osseointegrated titanium implants. Requirements for ensuring a longlasting, direct bone-to-implant anchorage in man. Acta Orthop Scand 1981;52(2):155-170.

36. Buser D, Sennerby L, De Bruyn H. Modern implant dentistry based on osseointegration: 50 years of progress, current trends and open questions. Periodontol 2000 2017;73(1):7-21.

37. Jemt T, Olsson M, Franke Stenport V. Incidence of First Implant Failure: A Retroprospective Study of 27 Years of Implant Operations at One Specialist Clinic. Clin Implant Dent Relat Res 2015;17 Suppl 2:e501-510.

38. Brånemark PI, Hansson BO, Adell R, Breine U, Lindström J, Hallen O, Ohman A. Osseointegrated implants in the treatment of the edentulous jaw. Experience from a 10-year period. Scand J Plast Reconstr Surg Suppl 1977;16:1-132. 
39. Albrektsson T, Brånemark PI, Hansson HA, Kasemo B, Larsson K, Lundström I, McQueen DH, Skalak R. The interface zone of inorganic implantsIn vivo: Titanium implants in bone. Annals of Biomedical Engineering 1983;11(1):1-27.

40. Donath K, Laass M, Gunzl HJ. The histopathology of different foreignbody reactions in oral soft tissue and bone tissue. Virchows Arch A Pathol Anat Histopathol 1992;420(2):131-137.

41. Anderson JM, Rodriguez A, Chang DT. Foreign body reaction to biomaterials. Semin Immunol 2008;20(2):86-100.

42. Albrektsson T, Dahlin C, Jemt T, Sennerby L, Turri A, Wennerberg A. Is Marginal Bone Loss around Oral Implants the Result of a Provoked Foreign Body Reaction? Clin Implant Dent Relat Res 2014;16(2):155165.

43. Albrektsson T, Chrcanovic B, Jacobsson M, Wennerberg A. Osseointegration of implants - a biological and clinical overview. 2017.

44. Albrektsson T, Chrcanovic B, Östman PO, Sennerby L. Initial and long-term crestal bone responses to modern dental implants. Periodontol 2000 2017;73(1):41-50.

45. Albrektsson T. Hydroxyapatite-coated implants: a case against their use. J Oral Maxillofac Surg 1998;56(11):1312-1326.

46. Deporter D, Watson P, Pharoah M, Levy D, Todescan R. Five- to six-year results of a prospective clinical trial using the ENDOPORE dental implant and a mandibular overdenture. Clin Oral Implants Res 1999;10(2):95-102.

47. Apratim A, Eachempati P, Krishnappa Salian KK, Singh V, Chhabra S, Shah S. Zirconia in dental implantology: A review. J Int Soc Prev Community Dent 2015;5(3):147-156.

48. Brånemark PI, Adell R, Breine U, Hansson BO, Lindström J, Ohlsson A. Intra-osseous anchorage of dental prostheses. I. Experimental studies. Scand J Plast Reconstr Surg 1969;3(2):81-100.

49. Ekelund JA, Lindquist LW, Carlsson GE, Jemt T. Implant treatment in the edentulous mandible: a prospective study on Branemark system implants over more than 20 years. Int J Prosthodont 2003;16(6):602608.

50. Brånemark PI, Svensson B, van Steenberghe D. Ten-year survival rates of fixed prostheses on four or six implants ad modum Branemark in full edentulism. Clin Oral Implants Res 1995;6(4):227-231.

51. Lindquist LW, Carlsson GE, Jemt T. A prospective 15-year follow-up study of mandibular fixed prostheses supported by osseointegrated implants. Clinical results and marginal bone loss. Clin Oral Implants Res 1996;7(4):329-336. 
52. Parein AM, Eckert SE, Wollan PC, Keller EE. Implant reconstruction in the posterior mandible: a long-term retrospective study. J Prosthet Dent 1997;78(1):34-42.

53. Lekholm U, Gunne J, Henry P, Higuchi K, Linden U, Bergstrom C, van Steenberghe D. Survival of the Branemark implant in partially edentulous jaws: a 10-year prospective multicenter study. Int J Oral Maxillofac Implants 1999;14(5):639-645.

54. Carlsson GE, Lindquist LW, Jemt T. Long-term marginal periimplant bone loss in edentulous patients. Int J Prosthodont 2000;13(4):295302.

55. van Steenberghe D, Quirynen M, Naert I, Maffei G, Jacobs R. Marginal bone loss around implants retaining hinging mandibular overdentures, at 4-, 8- and 12-years follow-up. J Clin Periodontol 2001;28(7):628-633.

56. Leonhardt A, Grondahl K, Bergstrom C, Lekholm U. Long-term follow-up of osseointegrated titanium implants using clinical, radiographic and microbiological parameters. Clin Oral Implants Res 2002;13(2):127-132.

57. Jemt T, Johansson J. Implant treatment in the edentulous maxillae: a 15 -year follow-up study on 76 consecutive patients provided with fixed prostheses. Clin Implant Dent Relat Res 2006;8(2):61-69.

58. Jemt T. Single implants in the anterior maxilla after 15 years of followup: comparison with central implants in the edentulous maxilla. Int J Prosthodont 2008;21(5):400-408.

59. Rocci A, Rocci M, Scoccia A, Martignoni M, Gottlow J, Sennerby L. Immediate loading of maxillary prostheses using flapless surgery, implant placement in predetermined positions, and prefabricated provisional restorations. Part 2: a retrospective 10-year clinical study. Int J Oral Maxillofac Implants 2012;27(5):1199-1204.

60. Mordenfeld A, Albrektsson T, Hallman M. A 10-year clinical and radiographic study of implants placed after maxillary sinus floor augmentation with an 80:20 mixture of deproteinized bovine bone and autogenous bone. Clin Implant Dent Relat Res 2014;16(3):435-446.

61. Simion M, Gionso L, Grossi GB, Briguglio F, Fontana F. TwelveYear Retrospective Follow-Up of Machined Implants in the Posterior Maxilla: Radiographic and Peri-Implant Outcome. Clin Implant Dent Relat Res 2015;17 Suppl 2:e343-351.

62. Benic GI, Bernasconi M, Jung RE, Hammerle CH. Clinical and radiographic intra-subject comparison of implants placed with or without guided bone regeneration: 15 -year results. J Clin Periodontol 2017;44(3):315-325. 
63. Chrcanovic BR, Kisch J, Albrektsson T, Wennerberg A. A retrospective study on clinical and radiological outcomes of oral implants in patients followed up for a minimum of 20 years. 2017.

64. Degidi M, Nardi D, Piattelli A. 10-year follow-up of immediately loaded implants with TiUnite porous anodized surface. Clin Implant Dent Relat Res 2012;14(6):828-838.

65. Östman PO, Hellman M, Sennerby L. Ten years later. Results from a prospective single-centre clinical study on 121 oxidized (TiUnite) Branemark implants in 46 patients. Clin Implant Dent Relat Res 2012;14(6):852-860.

66. Glauser R. Implants with an Oxidized Surface Placed Predominately in Soft Bone Quality and Subjected to Immediate Occlusal Loading: Results from an 11-Year Clinical Follow-Up. Clin Implant Dent Relat Res 2016;18(3):429-438.

67. Buser D, Janner SF, Wittneben JG, Bragger U, Ramseier CA, Salvi GE. 10-year survival and success rates of 511 titanium implants with a sandblasted and acid-etched surface: a retrospective study in 303 partially edentulous patients. Clin Implant Dent Relat Res 2012;14(6):839-851.

68. Fischer K, Stenberg T. Prospective 10-year cohort study based on a randomized controlled trial (RCT) on implant-supported full-arch maxillary prostheses. Part 1: sandblasted and acid-etched implants and mucosal tissue. Clin Implant Dent Relat Res 2012;14(6):808-815.

69. Roccuzzo M, Bonino L, Dalmasso P, Aglietta M. Long-term results of a three arms prospective cohort study on implants in periodontally compromised patients: 10 -year data around sandblasted and acidetched (SLA) surface. Clin Oral Implants Res 2014;25(10):1105-1112.

70. Roccuzzo M, Gaudioso L, Bunino M, Dalmasso P. Long-term stability of soft tissues following alveolar ridge preservation: 10-year results of a prospective study around nonsubmerged implants. Int J Periodontics Restorative Dent 2014;34(6):795-804.

71. van Velzen FJ, Ofec R, Schulten EA, Ten Bruggenkate CM. 10-year survival rate and the incidence of peri-implant disease of 374 titanium dental implants with a SLA surface: a prospective cohort study in 177 fully and partially edentulous patients. Clin Oral Implants Res 2015;26(10):1121-1128.

72. Kuchler U, Chappuis V, Gruber R, Lang NP, Salvi GE. Immediate implant placement with simultaneous guided bone regeneration in the esthetic zone: 10-year clinical and radiographic outcomes. Clin Oral Implants Res 2016;27(2):253-257. 
73. Nedir R, Nurdin N, Vazquez L, Abi Najm S, Bischof M. Osteotome Sinus Floor Elevation without Grafting: A 10-Year Prospective Study. Clin Implant Dent Relat Res 2016;18(3):609-617.

74. Roccuzzo M, Grasso G, Dalmasso P. Keratinized mucosa around implants in partially edentulous posterior mandible: 10-year results of a prospective comparative study. Clin Oral Implants Res 2016;27(4):491-496.

75. Zhang XX, Shi JY, Gu YX, Lai HC. Long-Term Outcomes of Early Loading of Straumann Implant-Supported Fixed Segmented Bridgeworks in Edentulous Maxillae: A 10-Year Prospective Study. Clin Implant Dent Relat Res 2016;18(6):1227-1237.

76. Vroom MG, Sipos P, de Lange GL, Grundemann LJ, Timmerman MF, Loos BG, van der Velden U. Effect of surface topography of screwshaped titanium implants in humans on clinical and radiographic parameters: a 12-year prospective study. Clin Oral Implants Res 2009;20(11):1231-1239.

77. Jacobs R, Pittayapat P, van Steenberghe D, De Mars G, Gijbels F, Van Der Donck A, Li L, Liang X, Van Assche N, Quirynen M, Naert I. A split-mouth comparative study up to 16 years of two screw-shaped titanium implant systems. J Clin Periodontol 2010;37(12):1119-1127.

78. Al-Nawas B, Kammerer PW, Morbach T, Ladwein C, Wegener J, Wagner W. Ten-year retrospective follow-up study of the TiOblast dental implant. Clin Implant Dent Relat Res 2012;14(1):127-134.

79. Gotfredsen K. A 10-year prospective study of single tooth implants placed in the anterior maxilla. Clin Implant Dent Relat Res 2012;14(1):80-87.

80. Mertens C, Meyer-Baumer A, Kappel H, Hoffmann J, Steveling HG. Use of 8-mm and 9-mm implants in atrophic alveolar ridges: 10-year results. Int J Oral Maxillofac Implants 2012;27(6):1501-1508.

81. Ravald N, Dahlgren S, Teiwik A, Grondahl K. Long-term evaluation of Astra Tech and Branemark implants in patients treated with full-arch bridges. Results after 12-15 years. Clin Oral Implants Res 2013;24(10):1144-1151.

82. Cecchinato D, Parpaiola A, Lindhe J. Mucosal inflammation and incidence of crestal bone loss among implant patients: a 10-year study. Clin Oral Implants Res 2014;25(7):791-796.

83. Donati M, Ekestubbe A, Lindhe J, Wennstrom JL. Implant-supported single-tooth restorations. A 12-year prospective study. Clin Oral Implants Res 2016;27(10):1207-1211. 
84. Calvo-Guirado JL, Gomez-Moreno G, Delgado-Ruiz RA, Mate Sanchez de Val JE, Negri B, Ramirez Fernandez MP. Clinical and radiographic evaluation of osseotite-expanded platform implants related to crestal bone loss: a 10-year study. Clin Oral Implants Res 2014;25(3):352-358.

85. Schropp L, Wenzel A, Stavropoulos A. Early, delayed, or late single implant placement: 10-year results from a randomized controlled clinical trial. Clin Oral Implants Res 2014;25(12):1359-1365.

86. Degidi M, Nardi D, Piattelli A. 10-year prospective cohort followup of immediately restored XiVE implants. Clin Oral Implants Res 2016;27(6):694-700.

87. Berger VW, Matthews JR, Grosch EN. On improving research methodology in clinical trials. Stat Methods Med Res 2008;17(3):231242.

88. Cipriani A, Geddes J. Comparison of systematic and narrative reviews: the example of the atypical antipsychotics. Epidemiol Psichiatr Soc 2003;12(3):146-153.

89. Hall KN, Kothari RU. Research fundamentals: IV. Choosing a research design. Acad Emerg Med 1999;6(1):67-74.

90. Hess DR. Retrospective studies and chart reviews. Respir Care 2004;49(10):1171-1174.

91. Eckert SE, Choi YG, Koka S. Methods for comparing the results of different studies. Int J Oral Maxillofac Implants 2003;18(5):697-705.

92. Godwin M, Ruhland L, Casson I, MacDonald S, Delva D, Birtwhistle R, Lam M, Seguin R. Pragmatic controlled clinical trials in primary care: the struggle between external and internal validity. BMC Med Res Methodol 2003;3:28.

93. Gartlehner G, Hansen RA, Nissman D, Lohr KN, Carey TS. AHRQ Technical Reviews. Criteria for Distinguishing Effectiveness From Efficacy Trials in Systematic Reviews. Rockville (MD): Agency for Healthcare Research and Quality (US); 2006.

94. Sedgwick P. What is a non-randomised controlled trial? Bmj 2014;348:g4115.

95. Sedgwick P. Selection bias versus allocation bias. BMJ : British Medical Journal 2013;346:f3345.

96. Sibbald B, Roland M. Understanding controlled trials. Why are randomised controlled trials important? Bmj 1998;316(7126):201.

97. Sedgwick P. Randomised controlled trials: internal versus external validity. BMJ : British Medical Journal 2014;348:g1742. 
98. Britton A, McKee M, Black N, McPherson K, Sanderson C, Bain C. Choosing between randomised and non-randomised studies: a systematic review. Health Technol Assess 1998;2(13):i-iv, 1-124.

99. Roberts C, Torgerson D. Randomisation methods in controlled trials. Bmi 1998;317(7168):1301.

100. Altman DG, Bland JM. Statistics notes. Treatment allocation in controlled trials: why randomise? Bmj 1999;318(7192):1209.

101. Fletcher RH, Fletcher SW, Wagner EH. Clinical Epidemiology: The Essentials. 5th ed. Baltimore: Williams \& Wilkins; 1996.

102. Feinstein AR. Randomized clinical trial. In: Feinstein AR, editor. Clinical Epidemiology: The Architecture of Clinical Research. Philadelphia: Saunders; 1985. pp. 683-718.

103. Feinstein AR. Implementation of the outline: Outcome events. In: Feinstein AR, editor. Clinical Epidemiology: The Architecture of Clinical Research. Philadelphia: Saunders; 1985. pp. 311-351.

104. Chalmers TC, Celano P, Sacks HS, Smith H, Jr. Bias in treatment assignment in controlled clinical trials. N Engl J Med 1983;309(22):1358-1361.

105. Halbert JA, Silagy CA, Finucane P, Withers RT, Hamdorf PA. Recruitment of older adults for a randomized, controlled trial of exercise advice in a general practice setting. J Am Geriatr Soc 1999;47(4):477-481.

106. Moinpour CM, Lovato LC, Thompson IM, Jr., Ware JE, Jr., Ganz PA, Patrick DL, Shumaker SA, Donaldson GW, Ryan A, Coltman CA, Jr. Profile of men randomized to the prostate cancer prevention trial: baseline health-related quality of life, urinary and sexual functioning, and health behaviors. J Clin Oncol 2000;18(9):1942-1953.

107. Torgerson DJ, Roberts C. Understanding controlled trials. Randomisation methods: concealment. Bmj 1999;319(7206):375-376.

108. Schulz KF, Chalmers I, Hayes RJ, Altman DG. Empirical evidence of bias. Dimensions of methodological quality associated with estimates of treatment effects in controlled trials. Jama 1995;273(5):408-412.

109. Sackett DL, Wennberg JE. Choosing the best research design for each question. Bmj 1997;315(7123):1636.

110. Brewin CR, Bradley C. Patient preferences and randomised clinical trials. Bmj 1989;299(6694):313-315.

111. Ioannidis JP, Lau J. The impact of high-risk patients on the results of clinical trials. J Clin Epidemiol 1997;50(10):1089-1098.

112. Hunninghake DB, Darby CA, Probstfield JL. Recruitment experience in clinical trials: literature summary and annotated bibliography. Control Clin Trials 1987;8(4 Suppl):6s-30s. 
113. Barofsky I, Sugarbaker PH. Determinants of patients nonparticipation in randomised clinical trials for the treatment of sarcomas. Cancer Clin Trials 1979;2:237-249.

114. Davies G, Pyke S, Kinmonth AL. Effect of non-attenders on the potential of a primary care programme to reduce cardiovascular risk in the population. Family Heart Study Group. Bmj 1994;309(6968):1553-1556.

115. McKee M, Britton A, Black N, McPherson K, Sanderson C, Bain C. Methods in health services research. Interpreting the evidence: choosing between randomised and non-randomised studies. Bmj 1999;319(7205):312-315.

116. Black N. Why we need observational studies to evaluate the effectiveness of health care. Bmj 1996;312(7040):1215-1218.

117. McPherson K. The Cochrane Lecture. The best and the enemy of the good: randomised controlled trials, uncertainty, and assessing the role of patient choice in medical decision making. J Epidemiol Community Health 1994;48(1):6-15.

118. Horwitz RI, Viscoli CM, Clemens JD, Sadock RT. Developing improved observational methods for evaluating therapeutic effectiveness. Am J Med 1990;89(5):630-638.

119. Stukenborg GJ. Comparison of carotid endarterectomy outcomes from randomized controlled trials and Medicare administrative databases. Arch Neurol 1997;54(7):826-832.

120. Mulrow CD. The medical review article: state of the science. Ann Intern Med 1987;106(3):485-488.

121. Higgins JPT, Green S, Cochrane Collaboration. Cochrane handbook for systematic reviews of interventions. Chichester, England ; Hoboken, NJ: Wiley-Blackwell; 2008.

122. Hopayian $\mathrm{K}$. The need for caution in interpreting high quality systematic reviews. Bmj 2001;323(7314):681-684.

123. Gilbody SM, Song F. Publication bias and the integrity of psychiatry research. Psychol Med 2000;30(2):253-258.

124. Egger M, Smith GD. Bias in location and selection of studies. Bmj 1998;316(7124):61-66.

125. Moher D, Tetzlaff J, Tricco AC, Sampson M, Altman DG. Epidemiology and reporting characteristics of systematic reviews. PLoS Med 2007;4(3):e78.

126. Moher D, Soeken K, Sampson M, Ben-Porat L, Berman B. Assessing the quality of reports of systematic reviews in pediatric complementary and alternative medicine. BMC Pediatr 2002;2:3. 
127. Jadad AR, Moher M, Browman GP, Booker L, Sigouin C, Fuentes $\mathrm{M}$, Stevens R. Systematic reviews and meta-analyses on treatment of asthma: critical evaluation. Bmj 2000;320(7234):537-540.

128. Shea BJ, Grimshaw JM, Wells GA, Boers M, Andersson N, Hamel C, Porter AC, Tugwell P, Moher D, Bouter LM. Development of AMSTAR: a measurement tool to assess the methodological quality of systematic reviews. BMC Med Res Methodol 2007;7:10.

129. Liberati A, Altman DG, Tetzlaff J, Mulrow C, Gotzsche PC, Ioannidis JP, Clarke M, Devereaux PJ, Kleijnen J, Moher D. The PRISMA statement for reporting systematic reviews and meta-analyses of studies that evaluate health care interventions: explanation and elaboration. PLoS Med 2009;6(7):e1000100.

130. Leucht S, Kissling W, Davis JM. How to read and understand and use systematic reviews and meta-analyses. Acta Psychiatr Scand 2009;119(6):443-450.

131. Kashi A, Saha S. Evidence-based techniques to assess the performance of dental implants. J Oral Implantol 2013;39(6):655-661.

132. Irwig L, Tosteson AN, Gatsonis C, Lau J, Colditz G, Chalmers TC, Mosteller F. Guidelines for meta-analyses evaluating diagnostic tests. Ann Intern Med 1994;120(8):667-676.

133. Stroup DF, Berlin JA, Morton SC, Olkin I, Williamson GD, Rennie D, Moher D, Becker BJ, Sipe TA, Thacker SB. Meta-analysis of observational studies in epidemiology: a proposal for reporting. Metaanalysis Of Observational Studies in Epidemiology (MOOSE) group. Jama 2000;283(15):2008-2012.

134. Detsky AS, Naylor CD, O’Rourke K, McGeer AJ, L'Abbe KA. Incorporating variations in the quality of individual randomized trials into meta-analysis. J Clin Epidemiol 1992;45(3):255-265.

135. Shrier I, Boivin JF, Steele RJ, Platt RW, Furlan A, Kakuma R, Brophy J, Rossignol M. Should meta-analyses of interventions include observational studies in addition to randomized controlled trials? A critical examination of underlying principles. Am J Epidemiol 2007;166(10):1203-1209.

136. Shrier I. Cochrane Reviews: new blocks on the kids. Br J Sports Med 2003;37(6):473-474.

137. Slavin RE. Best evidence synthesis: an intelligent alternative to metaanalysis. J Clin Epidemiol 1995;48(1):9-18.

138. Dudley WN, Wickham R, Coombs N. An Introduction to Survival Statistics: Kaplan-Meier Analysis. J Adv Pract Oncol 2016;7(1):91100.

139. Cutler SJ, Ederer F. Maximum utilization of the life table method in analyzing survival. J Chronic Dis 1958;8(6):699-712. 
140. Singh R, Mukhopadhyay K. Survival analysis in clinical trials: Basics and must know areas. Perspect Clin Res 2011;2(4):145-148.

141. Ferguson JG. Life tables for clinical scientists. J Vasc Interv Radiol 1992;3(4):607-615.

142. Kovács AF. Influence of chemotherapy on endosteal implant survival and success in oral cancer patients. Int J Oral Maxillofac Surg 2001;30(2):144-147.

143. Schliephake H, Neukam FW, Schmelzeisen R, Wichmann M. Longterm results of endosteal implants used for restoration of oral function after oncologic surgery. Int J Oral Maxillofac Surg 1999;28(4):260265.

144. Shaw RJ, Sutton AF, Cawood JI, Howell RA, Lowe D, Brown JS, Rogers SN, Vaughan ED. Oral rehabilitation after treatment for head and neck malignancy. Head Neck 2005;27(6):459-470.

145. Kaplan EL, Meier P. Nonparametric Estimation from Incomplete Observations. Journal of the American Statistical Association 1958;53(282):457-481.

146. Jager KJ, van Dijk PC, Zoccali C, Dekker FW. The analysis of survival data: the Kaplan-Meier method. Kidney Int 2008;74(5):560-565.

147. Bland JM, Altman DG. Survival probabilities (the Kaplan-Meier method). Bmj 1998;317(7172):1572.

148. Bollschweiler E. Benefits and limitations of Kaplan-Meier calculations of survival chance in cancer surgery. Langenbecks Arch Surg 2003;388(4):239-244.

149. Rich JT, Neely JG, Paniello RC, Voelker CC, Nussenbaum B, Wang EW. A practical guide to understanding Kaplan-Meier curves. Otolaryngol Head Neck Surg 2010;143(3):331-336.

150. Ludbrook J, Royse AG. Analysing clinical studies: principles, practice and pitfalls of Kaplan-Meier plots. ANZ J Surg 2008;78(3):204-210.

151. Cox DR. Regression Models and Life-Tables. Journal of the Royal Statistical Society. Series B (Methodological) 1972;34(2):187-220.

152. Babi ska M, Chudek J, Chełmecka E, Janik M, Klimek K, Owczarek A. Limitations of Cox Proportional Hazards Analysis in Mortality Prediction of Patients with Acute Coronary Syndrome. Studies in Logic, Grammar and Rhetoric; 2015. p. 33.

153. Spruance SL, Reid JE, Grace M, Samore M. Hazard ratio in clinical trials. Antimicrob Agents Chemother 2004;48(8):2787-2792.

154. Hancock MJ, Maher CG, Costa Lda C, Williams CM. A guide to survival analysis for manual therapy clinicians and researchers. Man Ther 2014;19(6):511-516. 
155. Hosmer DW, Lemeshow S, May S. Applied Survival Analysis:

Regression Modeling of Time-to-Event Data. 2nd ed. Hoboken: WileyInterscience; 2008.

156. Crowther MJ, Look MP, Riley RD. Multilevel mixed effects parametric survival models using adaptive Gauss-Hermite quadrature with application to recurrent events and individual participant data metaanalysis. Stat Med 2014;33(22):3844-3858.

157. Wu X, Al-Abedalla K, Rastikerdar E, Abi Nader S, Daniel NG, Nicolau B, Tamimi F. Selective serotonin reuptake inhibitors and the risk of osseointegrated implant failure: a cohort study. J Dent Res 2014;93(11):1054-1061.

158. Chrcanovic BR, Kisch J, Albrektsson T, Wennerberg A. Bruxism and dental implant failures: a multilevel mixed effects parametric survival analysis approach. J Oral Rehabil 2016;43(11):813-823.

159. Wu X, Al-Abedalla K, Eimar H, Arekunnath Madathil S, Abi-Nader S, Daniel NG, Nicolau B, Tamimi F. Antihypertensive Medications and the Survival Rate of Osseointegrated Dental Implants: A Cohort Study. Clin Implant Dent Relat Res 2016;18(6):1171-1182.

160. Chrcanovic BR, Kisch J, Albrektsson T, Wennerberg A. Is the intake of selective serotonin reuptake inhibitors associated with an increased risk of dental implant failure? Int J Oral Maxillofac Surg 2017;46(6):782788.

161. Chrcanovic BR, Kisch J, Albrektsson T, Wennerberg A. The intake of proton pump inhibitors is associated with an increased risk of dental implant failure. Int J Oral Maxillofac Implants 2017.

162. Nick TG, Campbell KM. Logistic regression. Methods Mol Biol 2007;404:273-301.

163. Stoltzfus JC. Logistic regression: a brief primer. Acad Emerg Med 2011;18(10):1099-1104.

164. Hosmer DW, Lemeshow SL. Applied Logistic Regression. 2nd ed. Hoboken: Wiley-Interscience; 2000.

165. Tabachnick BG, Fidell LS. Using Multivariate Statistics. 5th ed. Boston: Pearson Education, Inc.; 2007.

166. Peduzzi P, Concato J, Kemper E, Holford TR, Feinstein AR. A simulation study of the number of events per variable in logistic regression analysis. J Clin Epidemiol 1996;49(12):1373-1379.

167. Agresti A. An Introduction to Categorical Data Analysis. Hoboken: Wiley; 2007.

168. Buser D, Mericske-Stern R, Bernard JP, Behneke A, Behneke N, Hirt HP, Belser UC, Lang NP. Long-term evaluation of non-submerged ITI implants. Part 1: 8-year life table analysis of a prospective multi-center study with 2359 implants. Clin Oral Implants Res 1997;8(3):161-172. 
169. Testori T, Wiseman L, Woolfe S, Porter SS. A prospective multicenter clinical study of the Osseotite implant: four-year interim report. Int J Oral Maxillofac Implants 2001;16(2):193-200.

170. Weibrich G, Buch RS, Wegener J, Wagner W. Five-year prospective follow-up report of the Astra tech standard dental implant in clinical treatment. Int J Oral Maxillofac Implants 2001;16(4):557-562.

171. Chuang SK, Cai T. Predicting clustered dental implant survival using frailty methods. J Dent Res 2006;85(12):1147-1151.

172. Jaman A, Latif MA, Bari W, Wahed AS. A determinant-based criterion for working correlation structure selection in generalized estimating equations. Stat Med 2016;35(11):1819-1833.

173. Liang K-Y, Zeger SL. Longitudinal data analysis using generalized linear models. Biometrika 1986;73(1):13-22.

174. Shintani A. Primer of statistics in dental research: Part II. J Prosthodont Res 2014;58(2):85-91.

175. Odueyungbo A, Browne D, Akhtar-Danesh N, Thabane L. Comparison of generalized estimating equations and quadratic inference functions using data from the National Longitudinal Survey of Children and Youth (NLSCY) database. BMC Med Res Methodol 2008;8:28.

176. Khajeh-Kazemi R, Golestan B, Mohammad K, Mahmoudi M, Nedjat S, Pakravan M. Comparison of Generalized Estimating Equations and Quadratic Inference Functions in superior versus inferior Ahmed Glaucoma Valve implantation. J Res Med Sci 2011;16(3):235-244.

177. Nayak BK. Understanding the relevance of sample size calculation. Indian J Ophthalmol 2010;58(6):469-470.

178. Touloumi G, Babiker AG, Pocock SJ, Darbyshire JH. Impact of missing data due to drop-outs on estimators for rates of change in longitudinal studies: a simulation study. Stat Med 2001;20(24):3715-3728.

179. Greenland S. Response and follow-up bias in cohort studies. Am J Epidemiol 1977;106(3):184-187.

180. Kleinbaum DG, Morgenstern H, Kupper LL. Selection bias in epidemiologic studies. Am J Epidemiol 1981;113(4):452-463.

181. Schafer JL, Graham JW. Missing data: our view of the state of the art. Psychol Methods 2002;7(2):147-177.

182. Rothman KJ, Greenland S. Modern Epidemiology. 2nd ed. Philadelphia: Lippincott-Raven; 1998.

183. Deeg DJH. Attrition in longitudinal population studies: Does it affect the generalizability of the findings? Journal of Clinical Epidemiology 2002;55(3):213-215.

184. Dettori JR. Loss to follow-up. Evid Based Spine Care J 2011;2(1):7-10. 
185. Sackett DL, Richardson WS, Rosenberg W, Haynes RB. EvidenceBased Medicine: How to Practice and Teach EBM. 1st ed. New York: Churchill Livingstone; 1997.

186. Bhandari M, Guyatt GH, Swiontkowski MF. User's guide to the orthopaedic literature: how to use an article about a surgical therapy. $J$ Bone Joint Surg Am 2001;83-a(6):916-926.

187. Albrektsson T, Zarb GA. Current interpretations of the osseointegrated response: clinical significance. Int J Prosthodont 1993;6(2):95-105.

188. Lekholm U, Zarb GA. Patient selection and preparation. In: Brånemark PI, Zarb GA, Albrektsson T, editors. Tissue integrated prostheses: osseointegration in clinical dentistry. Chicago: Quintessence Publishing Company; 1985. pp. 199-209.

189. Chrcanovic BR, Martins MD, Wennerberg A. Immediate placement of implants into infected sites: a systematic review. Clin Implant Dent Relat Res 2015;17 Suppl 1:e1-e16.

190. Chrcanovic BR, Kisch J, Albrektsson T, Wennerberg A. Bruxism and dental implant treatment complications: a retrospective comparative study of 98 bruxer patients and a matched group. Clin Oral Implants Res 2016.

191. Chrcanovic BR, Kisch J, Albrektsson T, Wennerberg A. Factors Influencing Early Dental Implant Failures. J Dent Res 2016;95(9):9951002.

192. Chrcanovic BR, Kisch J, Albrektsson T, Wennerberg A. Survival of dental implants placed in sites of previously failed implants. Clin Oral Implants Res 2016.

193. Chrcanovic BR, Kisch J, Albrektsson T, Wennerberg A. Analysis of risk factors for cluster behavior of dental implant failures. Clin Implant Dent Relat Res 2017.

194. Chrcanovic BR, Kisch J, Albrektsson T, Wennerberg A. Impact of different surgeons on dental implant failure. Int J Prosthod 2017.

195. Chrcanovic BR, Kisch J, Albrektsson T, Wennerberg A. Factors influencing the fracture of dental implants. 2017.

196. Degidi M, Piattelli A, Carinci F. Clinical outcome of narrow diameter implants: a retrospective study of 510 implants. J Periodontol 2008;79(1):49-54.

197. Renouard F, Nisand D. Impact of implant length and diameter on survival rates. Clin Oral Implants Res 2006;17 Suppl 2:35-51.

198. das Neves FD, Fones D, Bernardes SR, do Prado CJ, Neto AJ. Short implants--an analysis of longitudinal studies. Int J Oral Maxillofac Implants 2006;21(1):86-93. 
199. Nedir R, Bischof M, Briaux JM, Beyer S, Szmukler-Moncler S, Bernard JP. A 7-year life table analysis from a prospective study on ITI implants with special emphasis on the use of short implants. Results from a private practice. Clin Oral Implants Res 2004;15(2):150-157.

200. Bahat O. Branemark system implants in the posterior maxilla: clinical study of 660 implants followed for 5 to 12 years. Int J Oral Maxillofac Implants 2000;15(5):646-653.

201. Winkler S, Morris HF, Ochi S. Implant survival to 36 months as related to length and diameter. Ann Periodontol 2000;5(1):22-31.

202. Naert I, Koutsikakis G, Duyck J, Quirynen M, Jacobs R, van Steenberghe D. Biologic outcome of implant-supported restorations in the treatment of partial edentulism. part I: a longitudinal clinical evaluation. Clin Oral Implants Res 2002;13(4):381-389.

203. Attard NJ, Zarb GA. Implant prosthodontic management of partially edentulous patients missing posterior teeth: the Toronto experience. $J$ Prosthet Dent 2003;89(4):352-359.

204. Weng D, Jacobson Z, Tarnow D, Hurzeler MB, Faehn O, Sanavi F, Barkvoll P, Stach RM. A prospective multicenter clinical trial of $3 \mathrm{i}$ machined-surface implants: results after 6 years of follow-up. Int J Oral Maxillofac Implants 2003;18(3):417-423.

205. Herrmann I, Lekholm U, Holm S, Kultje C. Evaluation of patient and implant characteristics as potential prognostic factors for oral implant failures. Int J Oral Maxillofac Implants 2005;20(2):220-230.

206. Telleman G, Raghoebar GM, Vissink A, den Hartog L, Huddleston Slater JJ, Meijer HJ. A systematic review of the prognosis of short $(<10$ $\mathrm{mm}$ ) dental implants placed in the partially edentulous patient. J Clin Periodontol 2011;38(7):667-676.

207. Pommer B, Frantal S, Willer J, Posch M, Watzek G, Tepper G. Impact of dental implant length on early failure rates: a meta-analysis of observational studies. J Clin Periodontol 2011;38(9):856-863.

208. Sohrabi K, Mushantat A, Esfandiari S, Feine J. How successful are small-diameter implants? A literature review. Clin Oral Implants Res 2012;23(5):515-525.

209. Feldman S, Boitel N, Weng D, Kohles SS, Stach RM. Five-year survival distributions of short-length (10 mm or less) machined-surfaced and Osseotite implants. Clin Implant Dent Relat Res 2004;6(1):16-23.

210. Lemmerman KJ, Lemmerman NE. Osseointegrated dental implants in private practice: a long-term case series study. J Periodontol $2005 ; 76(2): 310-319$. 
211. Mijiritsky E, Mazor Z, Lorean A, Levin L. Implant diameter and length influence on survival: interim results during the first 2 years of function of implants by a single manufacturer. Implant Dent 2013;22(4):394398.

212. Friberg B, Jemt T, Lekholm U. Early failures in 4,641 consecutively placed Branemark dental implants: a study from stage 1 surgery to the connection of completed prostheses. Int J Oral Maxillofac Implants 1991;6(2):142-146.

213. Akca K, Iplikcioglu H. Finite element stress analysis of the influence of staggered versus straight placement of dental implants. Int J Oral Maxillofac Implants 2001;16(5):722-730.

214. Tada S, Stegaroiu R, Kitamura E, Miyakawa O, Kusakari H. Influence of implant design and bone quality on stress/strain distribution in bone around implants: a 3-dimensional finite element analysis. Int J Oral Maxillofac Implants 2003;18(3):357-368.

215. Qian L, Todo M, Matsushita Y, Koyano K. Effects of implant diameter, insertion depth, and loading angle on stress/strain fields in implant/ jawbone systems: finite element analysis. Int J Oral Maxillofac Implants 2009;24(5):877-886.

216. Hasan I, Heinemann F, Aitlahrach M, Bourauel C. Biomechanical finite element analysis of small diameter and short dental implant. Biomed Tech (Berl) 2010;55(6):341-350.

217. de Carvalho NA, de Almeida EO, Rocha EP, Freitas AC, Jr., Anchieta RB, Kina S. Short implant to support maxillary restorations: bone stress analysis using regular and switching platform. J Craniofac Surg 2012;23(3):678-681.

218. Pellizzer EP, Verri FR, de Moraes SL, Falcon-Antenucci RM, de Carvalho PS, Noritomi PY. Influence of the implant diameter with different sizes of hexagon: analysis by 3-dimensional finite element method. J Oral Implantol 2013;39(4):425-431.

219. Bourauel C, Aitlahrach M, Heinemann F, Hasan I. Biomechanical finite element analysis of small diameter and short dental implants: extensive study of commercial implants. Biomed Tech (Berl) 2012;57(1):21-32.

220. Rubo JH, Capello Souza EA. Finite-element analysis of stress on dental implant prosthesis. Clin Implant Dent Relat Res 2010;12(2):105-113.

221. Petrie CS, Williams JL. Comparative evaluation of implant designs: influence of diameter, length, and taper on strains in the alveolar crest. A three-dimensional finite-element analysis. Clin Oral Implants Res 2005;16(4):486-494.

222. Pierrisnard L, Renouard F, Renault P, Barquins M. Influence of implant length and bicortical anchorage on implant stress distribution. Clin Implant Dent Relat Res 2003;5(4):254-262. 
223. Eckert SE, Meraw SJ, Weaver AL, Lohse CM. Early experience with Wide-Platform Mk II implants. Part I: Implant survival. Part II: Evaluation of risk factors involving implant survival. Int J Oral Maxillofac Implants 2001;16(2):208-216.

224. Lum LB. A biomechanical rationale for the use of short implants. $J$ Oral Implantol 1991;17(2):126-131.

225. Geng JP, Xu DW, Tan KB, Liu GR. Finite element analysis of an osseointegrated stepped screw dental implant. J Oral Implantol 2004;30(4):223-233.

226. Langer B, Langer L, Herrmann I, Jorneus L. The wide fixture: a solution for special bone situations and a rescue for the compromised implant. Part 1. Int J Oral Maxillofac Implants 1993;8(4):400-408.

227. Spiekermann H, Jansen VK, Richter EJ. A 10-year follow-up study of IMZ and TPS implants in the edentulous mandible using bar-retained overdentures. Int J Oral Maxillofac Implants 1995;10(2):231-243.

228. Schnitman PA, Wöhrle PS, Rubenstein JE, DaSilva JD, Wang NH. Ten-year results for Branemark implants immediately loaded with fixed prostheses at implant placement. Int J Oral Maxillofac Implants 1997;12(4):495-503.

229. Miyamoto I, Tsuboi Y, Wada E, Suwa H, Iizuka T. Influence of cortical bone thickness and implant length on implant stability at the time of surgery--clinical, prospective, biomechanical, and imaging study. Bone 2005;37(6):776-780.

230. Georgiopoulos B, Kalioras K, Provatidis C, Manda M, Koidis P. The effects of implant length and diameter prior to and after osseointegration: a 2-D finite element analysis. J Oral Implantol 2007;33(5):243-256.

231. Kong L, Gu Z, Li T, Wu J, Hu K, Liu Y, Zhou H, Liu B. Biomechanical optimization of implant diameter and length for immediate loading: a nonlinear finite element analysis. Int J Prosthodont 2009;22(6):607615.

232. van Steenberghe D, Lekholm U, Bolender C, Folmer T, Henry P, Herrmann I, Higuchi K, Laney W, Linden U, Astrand P. Applicability of osseointegrated oral implants in the rehabilitation of partial edentulism: a prospective multicenter study on 558 fixtures. Int J Oral Maxillofac Implants 1990;5(3):272-281.

233. Jemt T. Failures and complications in 391 consecutively inserted fixed prostheses supported by Branemark implants in edentulous jaws: a study of treatment from the time of prosthesis placement to the first annual checkup. Int J Oral Maxillofac Implants 1991;6(3):270-276. 
234. Bahat O. Treatment planning and placement of implants in the posterior maxillae: report of 732 consecutive Nobelpharma implants. Int J Oral Maxillofac Implants 1993;8(2):151-161.

235. Jemt T, Lekholm U. Implant treatment in edentulous maxillae: a 5-year follow-up report on patients with different degrees of jaw resorption. Int J Oral Maxillofac Implants 1995;10(3):303-311.

236. Wyatt CC, Zarb GA. Treatment outcomes of patients with implantsupported fixed partial prostheses. Int J Oral Maxillofac Implants 1998;13(2):204-211.

237. Ellegaard B, Baelum V, Karring T. Implant therapy in periodontally compromised patients. Clin Oral Implants Res 1997;8(3):180-188.

238. Brocard D, Barthet P, Baysse E, Duffort JF, Eller P, Justumus P, Marin P, Oscaby F, Simonet T, Benque E, Brunel G. A multicenter report on 1,022 consecutively placed ITI implants: a 7-year longitudinal study. Int J Oral Maxillofac Implants 2000;15(5):691-700.

239. Romeo E, Lops D, Margutti E, Ghisolfi M, Chiapasco M, Vogel G. Long-term survival and success of oral implants in the treatment of full and partial arches: a 7-year prospective study with the ITI dental implant system. Int J Oral Maxillofac Implants 2004;19(2):247-259.

240. Kotsovilis S, Fourmousis I, Karoussis IK, Bamia C. A systematic review and meta-analysis on the effect of implant length on the survival of rough-surface dental implants. J Periodontol 2009;80(11):1700-1718.

241. Esposito M, Pellegrino G, Pistilli R, Felice P. Rehabilitation of postrior atrophic edentulous jaws: prostheses supported by $5 \mathrm{~mm}$ short implants or by longer implants in augmented bone? One-year results from a pilot randomised clinical trial. Eur J Oral Implantol 2011;4(1):21-30.

242. Morand M, Irinakis T. The challenge of implant therapy in the posterior maxilla: providing a rationale for the use of short implants. $J$ Oral Implantol 2007;33(5):257-266.

243. Lee JS, Kim HM, Kim CS, Choi SH, Chai JK, Jung UW. Long-term retrospective study of narrow implants for fixed dental prostheses. Clin Oral Implants Res 2013;24(8):847-852.

244. Santiago Junior JF, Pellizzer EP, Verri FR, de Carvalho PS. Stress analysis in bone tissue around single implants with different diameters and veneering materials: a 3-D finite element study. Mater Sci Eng C Mater Biol Appl 2013;33(8):4700-4714.

245. Vairo G, Sannino G. Comparative evaluation of osseointegrated dental implants based on platform-switching concept: influence of diameter, length, thread shape, and in-bone positioning depth on stress-based performance. Comput Math Methods Med 2013;2013:250929. 
246. Ding X, Liao SH, Zhu XH, Zhang XH, Zhang L. Effect of diameter and length on stress distribution of the alveolar crest around immediate loading implants. Clin Implant Dent Relat Res 2009;11(4):279-287.

247. Lee JS, Lim YJ. Three-dimensional numerical simulation of stress induced by different lengths of osseointegrated implants in the anterior maxilla. Comput Methods Biomech Biomed Engin 2013;16(11):11431149 .

248. Romeo E, Lops D, Amorfini L, Chiapasco M, Ghisolfi M, Vogel G. Clinical and radiographic evaluation of small-diameter (3.3- $\mathrm{mm})$ implants followed for 1-7 years: a longitudinal study. Clin Oral Implants Res 2006;17(2):139-148.

249. Bayraktar M, Gultekin BA, Yalcin S, Mijiritsky E. Effect of crown to implant ratio and implant dimensions on periimplant stress of splinted implant-supported crowns: a finite element analysis. Implant Dent 2013;22(4):406-413.

250. Olate S, Lyrio MC, de Moraes M, Mazzonetto R, Moreira RW. Influence of diameter and length of implant on early dental implant failure. J Oral Maxillofac Surg 2010;68(2):414-419.

251. Elsyad MA, Gebreel AA, Fouad MM, Elshoukouki AH. The clinical and radiographic outcome of immediately loaded mini implants supporting a mandibular overdenture. A 3-year prospective study. $J$ Oral Rehabil 2011;38(11):827-834.

252. Davarpanah M, Martinez H, Tecucianu JF, Celletti R, Lazzara R. Small-diameter implants: indications and contraindications. J Esthet Dent 2000;12(4):186-194.

253. Allum SR, Tomlinson RA, Joshi R. The impact of loads on standard diameter, small diameter and mini implants: a comparative laboratory study. Clin Oral Implants Res 2008;19(6):553-559.

254. Arisan V, Bolukbasi N, Ersanli S, Ozdemir T. Evaluation of 316 narrow diameter implants followed for 5-10 years: a clinical and radiographic retrospective study. Clin Oral Implants Res 2010;21(3):296-307.

255. Rodriguez AM, Rosenstiel SF. Esthetic considerations related to bone and soft tissue maintenance and development around dental implants: report of the Committee on Research in Fixed Prosthodontics of the American Academy of Fixed Prosthodontics. J Prosthet Dent 2012;108(4):259-267.

256. Andersen E, Saxegaard E, Knutsen BM, Haanaes HR. A prospective clinical study evaluating the safety and effectiveness of narrowdiameter threaded implants in the anterior region of the maxilla. Int $J$ Oral Maxillofac Implants 2001;16(2):217-224. 
257. Anitua E, Orive G. Finite element analysis of the influence of the offset placement of an implant-supported prosthesis on bone stress distribution. J Biomed Mater Res B Appl Biomater 2009;89(2):275281.

258. Baggi L, Cappelloni I, Di Girolamo M, Maceri F, Vairo G. The influence of implant diameter and length on stress distribution of osseointegrated implants related to crestal bone geometry: a threedimensional finite element analysis. J Prosthet Dent 2008;100(6):422431.

259. Ding X, Zhu XH, Liao SH, Zhang XH, Chen H. Implant-bone interface stress distribution in immediately loaded implants of different diameters: a three-dimensional finite element analysis. J Prosthodont 2009;18(5):393-402.

260. Lin D, Li Q, Li W, Swain M. Dental implant induced bone remodeling and associated algorithms. J Mech Behav Biomed Mater 2009;2(5):410-432.

261. Tsuge T, Hagiwara Y. Influence of lateral-oblique cyclic loading on abutment screw loosening of internal and external hexagon implants. Dent Mater J 2009;28(4):373-381.

262. Okumura N, Stegaroiu R, Kitamura E, Kurokawa K, Nomura S. Influence of maxillary cortical bone thickness, implant design and implant diameter on stress around implants: a three-dimensional finite element analysis. J Prosthodont Res 2010;54(3):133-142.

263. Chu CM, Huang HL, Hsu JT, Fuh LJ. Influences of internal tapered abutment designs on bone stresses around a dental implant: threedimensional finite element method with statistical evaluation. $J$ Periodontol 2012;83(1):111-118.

264. Jorneus H. Developing the narrow platform. Nobel Biocare Global Forum. 1996:3.

265. Vigolo P, Givani A. Clinical evaluation of single-tooth mini-implant restorations: a five-year retrospective study. J Prosthet Dent 2000;84(1):50-54.

266. Bozkaya D, Muftu S, Muftu A. Evaluation of load transfer characteristics of five different implants in compact bone at different load levels by finite elements analysis. J Prosthet Dent 2004;92(6):523530.

267. Iplikcioglu H, Akca K. Comparative evaluation of the effect of diameter, length and number of implants supporting three-unit fixed partial prostheses on stress distribution in the bone. J Dent 2002;30(1):41-46.

268. Oliveira EJ. Princípios de bioengenharia em implantes oseointegrados. 1st ed. Rio de Janeiro: Pedro Primeiro Ltda; 1997. 
269. Ivanoff CJ, Sennerby L, Johansson C, Rangert B, Lekholm U. Influence of implant diameters on the integration of screw implants. An experimental study in rabbits. Int J Oral Maxillofac Surg 1997;26(2):141-148.

270. Lazzara RJ. Criteria for implant selection: surgical and prosthetic considerations. Pract Periodontics Aesthet Dent 1994;6(9):55-62; quiz 64.

271. Himmlová L, Dostalova T, Kacovsky A, Konvickova S. Influence of implant length and diameter on stress distribution: a finite element analysis. J Prosthet Dent 2004;91(1):20-25.

272. Mohammed Ibrahim M, Thulasingam C, Nasser KS, Balaji V, Rajakumar M, Rupkumar P. Evaluation of design parameters of dental implant shape, diameter and length on stress distribution: a finite element analysis. J Indian Prosthodont Soc 2011;11(3):165-171.

273. Lee JH, Frias V, Lee KW, Wright RF. Effect of implant size and shape on implant success rates: a literature review. J Prosthet Dent 2005;94(4):377-381.

274. Graves SL, Jansen CE, Siddiqui AA, Beaty KD. Wide diameter implants: indications, considerations and preliminary results over a two-year period. Aust Prosthodont J 1994;8:31-37.

275. Kong L, Hu K, Li D, Song Y, Yang J, Wu Z, Liu B. Evaluation of the cylinder implant thread height and width: a 3-dimensional finite element analysis. Int J Oral Maxillofac Implants 2008;23(1):65-74.

276. Anner R, Better H, Chaushu G. The clinical effectiveness of $6 \mathrm{~mm}$ diameter implants. J Periodontol 2005;76(6):1013-1015.

277. Ivanoff CJ, Grondahl K, Sennerby L, Bergstrom C, Lekholm U. Influence of variations in implant diameters: a 3- to 5-year retrospective clinical report. Int J Oral Maxillofac Implants 1999;14(2):173-180.

278. Becker W, Becker BE, Alsuwyed A, Al-Mubarak S. Long-term evaluation of 282 implants in maxillary and mandibular molar positions: a prospective study. J Periodontol 1999;70(8):896-901.

279. Friberg B, Ekestubbe A, Sennerby L. Clinical outcome of Branemark System implants of various diameters: a retrospective study. Int J Oral Maxillofac Implants 2002;17(5):671-677.

280. Mordenfeld MH, Johansson A, Hedin M, Billstrom C, Fyrberg KA. A retrospective clinical study of wide-diameter implants used in posterior edentulous areas. Int J Oral Maxillofac Implants 2004;19(3):387-392.

281. Shin SW, Bryant SR, Zarb GA. A retrospective study on the treatment outcome of wide-bodied implants. Int J Prosthodont 2004;17(1):52-58. 
282. Karoussis IK, Brägger U, Salvi GE, Bürgin W, Lang NP. Effect of implant design on survival and success rates of titanium oral implants: a 10-year prospective cohort study of the ITI Dental Implant System. Clin Oral Implants Res 2004;15(1):8-17.

283. Lazzara R, Siddiqui AA, Binon P, Feldman SA, Weiner R, Phillips $\mathrm{R}$, Gonshor A. Retrospective multicenter analysis of $3 \mathrm{i}$ endosseous dental implants placed over a five-year period. Clin Oral Implants Res 1996;7(1):73-83.

284. Heydenrijk K, Raghoebar GM, Meijer HJ, van der Reijden WA, van Winkelhoff AJ, Stegenga B. Two-stage IMZ implants and ITI implants inserted in a single-stage procedure. A prospective comparative study. Clin Oral Implants Res 2002;13(4):371-380.

285. Meijer HJ, Raghoebar GM, Van’t Hof MA, Visser A. A controlled clinical trial of implant-retained mandibular overdentures: 10 years' results of clinical aspects and aftercare of IMZ implants and Branemark implants. Clin Oral Implants Res 2004;15(4):421-427.

286. Haas R, Mensdorff-Pouilly N, Mailath G, Watzek G. Survival of 1,920 IMZ implants followed for up to 100 months. Int J Oral Maxillofac Implants 1996;11(5):581-588.

287. Steigenga JT, al-Shammari KF, Nociti FH, Misch CE, Wang HL. Dental implant design and its relationship to long-term implant success. Implant Dent 2003;12(4):306-317.

288. Huang HL, Chang CH, Hsu JT, Fallgatter AM, Ko CC. Comparison of implant body designs and threaded designs of dental implants: a 3-dimensional finite element analysis. Int J Oral Maxillofac Implants 2007;22(4):551-562.

289. Abu-Hammad OA, Watkinson AC, Harrison A. A 3D finite element method study of stress distribution around dental implants supporting complete overdentures. In: Middleton J, Jones ML, Pande N, editors. Computer Methods in Biomechanics and Biomedical Engineering, Volume 1. Amsterdam: Gordon and Breach Publishers; 1996. pp. 472440.

290. Hansson S. The implant neck: smooth or provided with retention elements. A biomechanical approach. Clin Oral Implants Res 1999;10(5):394-405.

291. Sykaras N, Iacopino AM, Marker VA, Triplett RG, Woody RD. Implant materials, designs, and surface topographies: their effect on osseointegration. A literature review. Int J Oral Maxillofac Implants 2000;15(5):675-690. 
292. Wennerberg A, Albrektsson T, Andersson B. Bone tissue response to commercially pure titanium implants blasted with fine and coarse particles of aluminum oxide. Int J Oral Maxillofac Implants 1996;11(1):38-45.

293. Maiorana C, Santoro F. Maxillary and mandibular bone reconstruction with hip grafts and implants using Frialit-2 implants. Int J Periodontics Restorative Dent 2002;22(3):221-229.

294. O’Sullivan D, Sennerby L, Meredith N. Influence of implant taper on the primary and secondary stability of osseointegrated titanium implants. Clin Oral Implants Res 2004;15(4):474-480.

295. Sakoh J, Wahlmann U, Stender E, Nat R, Al-Nawas B, Wagner W. Primary stability of a conical implant and a hybrid, cylindric screwtype implant in vitro. Int J Oral Maxillofac Implants 2006;21(4):560566.

296. Kim JW, Baek SH, Kim TW, Chang YI. Comparison of stability between cylindrical and conical type mini-implants. Mechanical and histological properties. Angle Orthod 2008;78(4):692-698.

297. Romanos GE, Basha-Hijazi A, Gupta B, Ren YF, Malmstrom H. Role of clinician's experience and implant design on implant stability. An ex vivo study in artificial soft bones. Clin Implant Dent Relat Res 2014;16(2):166-171.

298. Romanos GE, Ciornei G, Jucan A, Malmstrom H, Gupta B. In vitro assessment of primary stability of Straumann $(\mathrm{R})$ implant designs. Clin Implant Dent Relat Res 2014;16(1):89-95.

299. Toyoshima T, Tanaka H, Ayukawa Y, Howashi M, Masuzaki T, Kiyosue T, Koyano K, Nakamura S. Primary Stability of a Hybrid Implant Compared with Tapered and Cylindrical Implants in an Ex Vivo Model. Clin Implant Dent Relat Res 2015;17(5):950-956.

300. Adell R, Lekholm U, Rockler B, Brånemark PI. A 15-year study of osseointegrated implants in the treatment of the edentulous jaw. Int $J$ Oral Surg 1981;10(6):387-416.

301. Bornstein MM, Valderrama P, Jones AA, Wilson TG, Seibl R, Cochran DL. Bone apposition around two different sandblasted and acidetched titanium implant surfaces: a histomorphometric study in canine mandibles. Clin Oral Implants Res 2008;19(3):233-241.

302. Bornstein MM, Wittneben JG, Bragger U, Buser D. Early loading at 21 days of non-submerged titanium implants with a chemically modified sandblasted and acid-etched surface: 3-year results of a prospective study in the posterior mandible. J Periodontol 2010;81(6):809-818. 
303. Sammons RL, Lumbikanonda N, Gross M, Cantzler P. Comparison of osteoblast spreading on microstructured dental implant surfaces and cell behaviour in an explant model of osseointegration. A scanning electron microscopic study. Clin Oral Implants Res 2005;16(6):657666.

304. Brash JL, Horbett TA. Proteins at interfaces: current issues and future prospects. In: Brash JL, Horbett TA, editors. Proteins at Interfaces: Physicochemical and Biochemical Studies. Washington D.C.: American Chemical Society; 1987. pp. 1-33.

305. Anselme K. Osteoblast adhesion on biomaterials. Biomaterials 2000;21(7):667-681.

306. Hing KA. Bone repair in the twenty-first century: biology, chemistry or engineering? Philos Trans A Math Phys Eng Sci 2004;362(1825):28212850.

307. Meirelles L, Albrektsson T, Kjellin P, Arvidsson A, Franke-Stenport $\mathrm{V}$, Andersson M, Currie F, Wennerberg A. Bone reaction to nano hydroxyapatite modified titanium implants placed in a gap-healing model. J Biomed Mater Res A 2008;87(3):624-631.

308. Meirelles L, Arvidsson A, Andersson M, Kjellin P, Albrektsson T, Wennerberg A. Nano hydroxyapatite structures influence early bone formation. J Biomed Mater Res A 2008;87(2):299-307.

309. Meirelles L, Currie F, Jacobsson M, Albrektsson T, Wennerberg A. The effect of chemical and nanotopographical modifications on the early stages of osseointegration. Int J Oral Maxillofac Implants 2008;23(4):641-647.

310. Meirelles L, Melin L, Peltola T, Kjellin P, Kangasniemi I, Currie F, Andersson M, Albrektsson T, Wennerberg A. Effect of hydroxyapatite and titania nanostructures on early in vivo bone response. Clin Implant Dent Relat Res 2008;10(4):245-254.

311. Wennerberg A, Albrektsson T. On implant surfaces: a review of current knowledge and opinions. Int J Oral Maxillofac Implants 2010;25(1):63-74.

312. Kilpadi DV, Lemons JE. Surface energy characterization of unalloyed titanium implants. J Biomed Mater Res 1994;28(12):1419-1425.

313. Textor M, Sittig C, Frauchiger V, Tosatti S, Brunette DM. Properties and biological significance of natural oxide films on titanium and its alloys. In: Brunette DM, Tengvall P, Textor M, Thomson P, editors. Titanium in Medicine. Berlin: Springer-Verlag; 2001. pp. 171-230.

314. Rupp F, Scheideler L, Olshanska N, de Wild M, Wieland M, GeisGerstorfer J. Enhancing surface free energy and hydrophilicity through chemical modification of microstructured titanium implant surfaces. $J$ Biomed Mater Res A 2006;76(2):323-334. 
315. Buser D, Broggini N, Wieland M, Schenk RK, Denzer AJ, Cochran DL, Hoffmann B, Lussi A, Steinemann SG. Enhanced bone apposition to a chemically modified SLA titanium surface. J Dent Res 2004;83(7):529533.

316. Ferguson SJ, Broggini N, Wieland M, de Wild M, Rupp F, GeisGerstorfer J, Cochran DL, Buser D. Biomechanical evaluation of the interfacial strength of a chemically modified sandblasted and acidetched titanium surface. J Biomed Mater Res A 2006;78(2):291-297.

317. Wilke HJ, Claes L, Steinemann S. The influence of various titanium surfaces on the interface shear strength between implants and bone. In: Heimke G, Soltesz U, Lee AJC, editors. Clinical Implant Materials (Advances in Biomaterials No. 9). Amsterdam: Elsevier Science Publishers BV; 1990. pp. 209-214.

318. Buser D, Schenk RK, Steinemann S, Fiorellini JP, Fox CH, Stich H. Influence of surface characteristics on bone integration of titanium implants. A histomorphometric study in miniature pigs. J Biomed Mater Res 1991;25(7):889-902.

319. Gotfredsen K, Wennerberg A, Johansson C, Skovgaard LT, HjortingHansen E. Anchorage of TiO2-blasted, HA-coated, and machined implants: an experimental study with rabbits. J Biomed Mater Res $1995 ; 29(10): 1223-1231$.

320. Wennerberg A, Albrektsson T, Andersson B, Krol JJ. A histomorphometric and removal torque study of screw-shaped titanium implants with three different surface topographies. Clin Oral Implants Res 1995;6(1):24-30.

321. Wennerberg A, Albrektsson T, Johansson C, Andersson B. Experimental study of turned and grit-blasted screw-shaped implants with special emphasis on effects of blasting material and surface topography. Biomaterials 1996;17(1):15-22.

322. Wennerberg A, Albrektsson T, Lausmaa J. Torque and histomorphometric evaluation of c.p. titanium screws blasted with 25- and 75-microns-sized particles of Al2O3. J Biomed Mater Res 1996;30(2):251-260.

323. Wennerberg A, Ektessabi A, Albrektsson T, Johansson C, Andersson B. A 1-year follow-up of implants of differing surface roughness placed in rabbit bone. Int J Oral Maxillofac Implants 1997;12(4):486-494.

324. Buser D, Nydegger T, Hirt HP, Cochran DL, Nolte LP. Removal torque values of titanium implants in the maxilla of miniature pigs. Int J Oral Maxillofac Implants 1998;13(5):611-619. 
325. Cochran DL, Schenk RK, Lussi A, Higginbottom FL, Buser D. Bone response to unloaded and loaded titanium implants with a sandblasted and acid-etched surface: a histometric study in the canine mandible. $J$ Biomed Mater Res 1998;40(1):1-11.

326. De Leonardis D, Garg AK, Pecora GE, Andreana S. Osseointegration of rough acid-etched implants: one-year follow-up of placement of 100 minimatic implants. Int J Oral Maxillofac Implants 1997;12(1):65-73.

327. Klokkevold PR, Nishimura RD, Adachi M, Caputo A.

Osseointegration enhanced by chemical etching of the titanium surface. A torque removal study in the rabbit. Clin Oral Implants Res 1997;8(6):442-447.

328. Chrcanovic BR, Martins MD. Study of the influence of acid etching treatments on the superficial characteristics of Ti. Materials Research 2014;17(2):373-380.

329. Chrcanovic BR, Wennerberg A, Martins MD. Influence of Temperature and Acid Etching Time on the Superficial Characteristics of Ti. Materials Research 2015;18(5):963-970.

330. Cochran DL, Buser D, ten Bruggenkate CM, Weingart D, Taylor TM, Bernard JP, Peters F, Simpson JP. The use of reduced healing times on ITI implants with a sandblasted and acid-etched (SLA) surface: early results from clinical trials on ITI SLA implants. Clin Oral Implants Res 2002;13(2):144-153.

331. Chrcanovic BR, Leão NLC, Martins MD. Influence of Different Acid Etchings on the Superficial Characteristics of Ti Sandblasted with A12O3. Materials Research 2013;16(5):1006-1014.

332. Sul YT, Johansson CB, Petronis S, Krozer A, Jeong Y, Wennerberg A, Albrektsson T. Characteristics of the surface oxides on turned and electrochemically oxidized pure titanium implants up to dielectric breakdown: the oxide thickness, micropore configurations, surface roughness, crystal structure and chemical composition. Biomaterials 2002;23(2):491-501.

333. Sul YT, Johansson C, Wennerberg A, Cho LR, Chang BS, Albrektsson T. Optimum surface properties of oxidized implants for reinforcement of osseointegration: surface chemistry, oxide thickness, porosity, roughness, and crystal structure. Int J Oral Maxillofac Implants 2005;20(3):349-359.

334. Frauchiger VM, Schlottig F, Gasser B, Textor M. Anodic plasmachemical treatment of CP titanium surfaces for biomedical applications. Biomaterials 2004;25(4):593-606.

335. Gottlander M, Albrektsson T. Histomorphometric studies of hydroxylapatite-coated and uncoated CP titanium threaded implants in bone. Int J Oral Maxillofac Implants 1991;6(4):399-404. 
336. Kim HS, Yang Y, Koh JT, Lee KK, Lee DJ, Lee KM, Park SW. Fabrication and characterization of functionally graded nano-micro porous titanium surface by anodizing. J Biomed Mater Res B Appl Biomater 2009;88(2):427-435.

337. Ellingsen JE, Johansson CB, Wennerberg A, Holmen A. Improved retention and bone-tolmplant contact with fluoride-modified titanium implants. Int J Oral Maxillofac Implants 2004;19(5):659-666.

338. Ramis JM, Taxt-Lamolle SF, Lyngstadaas SP, Reseland JE, Ellingsen JE, Monjo M. Identification of early response genes to roughness and fluoride modification of titanium implants in human osteoblasts. Implant Dent 2012;21(2):141-149.

339. Pham MH, Landin MA, Tiainen H, Reseland JE, Ellingsen JE, Haugen HJ. The effect of hydrofluoric acid treatment of titanium and titanium dioxide surface on primary human osteoblasts. Clin Oral Implants Res 2014;25(3):385-394.

340. Sul YT, Johansson CB, Albrektsson T. Oxidized titanium screws coated with calcium ions and their performance in rabbit bone. Int J Oral Maxillofac Implants 2002;17(5):625-634.

341. Kang BS, Sul YT, Johansson CB, Oh SJ, Lee HJ, Albrektsson T. The effect of calcium ion concentration on the bone response to oxidized titanium implants. Clin Oral Implants Res 2012;23(6):690-697.

342. Sul YT, Johansson C, Albrektsson T. Which surface properties enhance bone response to implants? Comparison of oxidized magnesium, TiUnite, and Osseotite implant surfaces. Int J Prosthodont 2006;19(4):319-328.

343. Sul YT, Jonsson J, Yoon GS, Johansson C. Resonance frequency measurements in vivo and related surface properties of magnesiumincorporated, micropatterned and magnesium-incorporated TiUnite, Osseotite, SLA and TiOblast implants. Clin Oral Implants Res 2009;20(10):1146-1155.

344. Galli S, Naito Y, Karlsson J, He W, Andersson M, Wennerberg A, Jimbo R. Osteoconductive Potential of Mesoporous Titania Implant Surfaces Loaded with Magnesium: An Experimental Study in the Rabbit. Clin Implant Dent Relat Res 2015;17(6):1048-1059.

345. Cecchinato F, Atefyekta S, Wennerberg A, Andersson M, Jimbo $\mathrm{R}$, Davies JR. Modulation of the nanometer pore size improves magnesium adsorption into mesoporous titania coatings and promotes bone morphogenic protein 4 expression in adhering osteoblasts. Dent Mater 2016;32(7):e148-158. 
346. Jemt T, Stenport V, Friberg B. Implant treatment with fixed prostheses in the edentulous maxilla. Part 1: implants and biologic response in two patient cohorts restored between 1986 and 1987 and 15 years later. Int J Prosthodont 2011;24(4):345-355.

347. Jemt T, Olsson M, Renouard F, Stenport V, Friberg B. Early Implant Failures Related to Individual Surgeons: An Analysis Covering 11,074 Operations Performed during 28 Years. Clin Implant Dent Relat Res 2016;18(5):861-872.

348. Gotfredsen K, Karlsson U. A prospective 5-year study of fixed partial prostheses supported by implants with machined and TiO2-blasted surface. J Prosthodont 2001;10(1):2-7.

349. Jungner M, Lundqvist P, Lundgren S. Oxidized titanium implants (Nobel Biocare TiUnite) compared with turned titanium implants (Nobel Biocare mark III) with respect to implant failure in a group of consecutive patients treated with early functional loading and twostage protocol. Clin Oral Implants Res 2005;16(3):308-312.

350. Degidi M, Iezzi G, Perrotti V, Piattelli A. Comparative analysis of immediate functional loading and immediate nonfunctional loading to traditional healing periods: a 5 -year follow-up of 550 dental implants. Clin Implant Dent Relat Res 2009;11(4):257-266.

351. Sivolella S, Stellini E, Testori T, Di Fiore A, Berengo M, Lops D. Splinted and unsplinted short implants in mandibles: a retrospective evaluation with 5 to 16 years of follow-up. J Periodontol 2013;84(4):502-512.

352. Dietrich UW, W. Zur Frage des Knochenabbaus bei IMZ-implantaten. Zeitschrift für Zahnärtzliche Implantologie 1992;8:240-245.

353. Quirynen M, Naert I, van Steenberghe D, Duchateau L, Darius P. Periodontal aspects of Bränemark and IMZ implants supporting overdentures: A comparative study. In: Laney W, Tolman D, editors. Tissue Integration in Oral, Orthopaedic and Maxillofacial Reconstruction. Chicago: Quintessence Co.; 1992. pp. 80-93.

354. Becker W, Becker BE, Ricci A, Bahat O, Rosenberg E, Rose LF, Handelsman M, Israelson $\mathrm{H}$. A prospective multicenter clinical trial comparing one- and two-stage titanium screw-shaped fixtures with one-stage plasma-sprayed solid-screw fixtures. Clin Implant Dent Relat Res 2000;2(3):159-165.

355. Teughels W, Van Assche N, Sliepen I, Quirynen M. Effect of material characteristics and/or surface topography on biofilm development. Clin Oral Implants Res 2006;17 Suppl 2:68-81.

356. Burgers R, Gerlach T, Hahnel S, Schwarz F, Handel G, Gosau M. In vivo and in vitro biofilm formation on two different titanium implant surfaces. Clin Oral Implants Res 2010;21(2):156-164. 
357. Lin HY, Liu Y, Wismeijer D, Crielaard W, Deng DM. Effects of oral implant surface roughness on bacterial biofilm formation and treatment efficacy. Int J Oral Maxillofac Implants 2013;28(5):12261231.

358. Berglundh T, Gotfredsen K, Zitzmann NU, Lang NP, Lindhe J. Spontaneous progression of ligature induced peri-implantitis at implants with different surface roughness: an experimental study in dogs. Clin Oral Implants Res 2007;18(5):655-661.

359. Dagorne C, Malet J, Bizouard G, Mora F, Range H, Bouchard P. Clinical evaluation of two dental implant macrostructures on periimplant bone loss: a comparative, retrospective study. Clin Oral Implants Res 2015;26(3):307-313.

360. Arnhart C, Dvorak G, Trefil C, Huber C, Watzek G, Zechner W. Impact of implant surface topography: a clinical study with a mean functional loading time of 85 months. Clin Oral Implants Res 2013;24(9):1049-1054.

361. De Bruyn H, Christiaens V, Doornewaard R, Jacobsson M, Cosyn J, Jacquet W, Vervaeke S. Implant surface roughness and patient factors on long-term peri-implant bone loss. Periodontol 2000 2017;73(1):218-227.

362. Foster J. Focus on quality. The Dentist; 2014.

363. Strub J, Rohner D, Schärer P. Die Versorgung des Lückengebisses mit implantatgetragenen Brücken. Eine longitudinalstudie über 7.5 Jahre. Z Zahnärztl Implantol 1987;3:242-254.

364. Schulte W. Das enossale Tübinger Implantat aus A12O3 (Frialit). Der Entwicklungsphase nach 6 Jahren. Zahnärztl Mitt 1981;71:1114-1122.

365. Schulte W. The intra-osseous Al2O3 (Frialit) Tuebingen implant. Developmental status after eight years (I-III). Quintessence Int 1984;15(1):1-39.

366. De Wijs FL, Van Dongen RC, De Lange GL, De Putter C. Front tooth replacement with Tubingen (Frialit) implants. J Oral Rehabil 1994;21(1):11-26.

367. Kohal RJ, Att W, Bachle M, Butz F. Ceramic abutments and ceramic oral implants. An update. Periodontol 2000 2008;47:224-243.

368. Lang NP, Pjetursson BE, Tan K, Bragger U, Egger M, Zwahlen M. A systematic review of the survival and complication rates of fixed partial dentures (FPDs) after an observation period of at least 5 years. II. Combined tooth--implant-supported FPDs. Clin Oral Implants Res 2004;15(6):643-653.

369. Malmqvist JP, Sennerby L. Clinical report on the success of 47 consecutively placed Core-Vent implants followed from 3 months to 4 years. Int J Oral Maxillofac Implants 1990;5(1):53-60. 
370. Fugazzotto PA, Wheeler SL, Lindsay JA. Success and failure rates of cylinder implants in type IV bone. J Periodontol 1993;64(11):10851087.

371. Albrektsson T. On long-term maintenance of the osseointegrated response. Aust Prosthodont J 1993;7 Suppl:15-24.

372. Wheeler SL. Eight-year clinical retrospective study of titanium plasmasprayed and hydroxyapatite-coated cylinder implants. Int J Oral Maxillofac Implants 1996;11(3):340-350.

373. Golec TS, Krauser JT. Long-term retrospective studies on hydroxyapatite coated endosteal and subperiosteal implants. Dent Clin North Am 1992;36(1):39-65.

374. Haugesund Byrett. Court Protocol of Norway. 2000.

375. Östman PO, Hellman M, Albrektsson T, Sennerby L. Direct loading of Nobel Direct and Nobel Perfect one-piece implants: a 1-year prospective clinical and radiographic study. Clin Oral Implants Res 2007;18(4):409-418.

376. Sennerby L, Rocci A, Becker W, Jonsson L, Johansson LA, Albrektsson T. Short-term clinical results of Nobel Direct implants: a retrospective multicentre analysis. Clin Oral Implants Res 2008;19(3):219-226.

377. Albrektsson T, Gottlow J, Meirelles L, Ostman PO, Rocci A, Sennerby L. Survival of NobelDirect implants: an analysis of 550 consecutively placed implants at 18 different clinical centers. Clin Implant Dent Relat Res 2007;9(2):65-70.

378. Glantz P, Klinge B, Åstrand P. Statements over comments from Nobel Biocare on the expert report concerning Nobel Direct 2006-08-31. In: Technology DoM, editor. Stockholm: Läkemedelsverket; 2006. p. 1-6.

379. Duddeck D, Maghaireh H, Faber FJ, Neugebauer J. SEM surface analyses of 120 sterile-packed implants. EDI Journal 2015;11(2):6475 .

380. Duddeck D, Neugebauer J. Surface analysis of sterile-package implants. EDI Journal 2015;11(2):54-63.

381. Hallab NJ, Jacobs JJ. Biologic effects of implant debris. Bull NYU Hosp Jt Dis 2009;67(2):182-188.

382. Granchi D, Ciapetti G, Amato I, Pagani S, Cenni E, Savarino L, Avnet S, Peris JL, Pellacani A, Baldini N, Giunti A. The influence of alumina and ultra-high molecular weight polyethylene particles on osteoblastosteoclast cooperation. Biomaterials 2004;25(18):4037-4045.

383. Vermes C, Chandrasekaran R, Jacobs JJ, Galante JO, Roebuck KA, Glant TT. The effects of particulate wear debris, cytokines, and growth factors on the functions of MG-63 osteoblasts. J Bone Joint Surg Am 2001;83-a(2):201-211. 
384. Pioletti DP, Leoni L, Genini D, Takei H, Du P, Corbeil J. Gene expression analysis of osteoblastic cells contacted by orthopedic implant particles. J Biomed Mater Res 2002;61(3):408-420.

385. Jacobs JJ, Urban RM, Gilbert JL, Skipor AK, Black J, Jasty M, Galante JO. Local and distant products from modularity. Clin Orthop Relat Res 1995;(319):94-105.

386. Peterson LJ. Antibiotic prophylaxis against wound infections in oral and maxillofacial surgery. J Oral Maxillofac Surg 1990;48(6):617-620.

387. Friberg B. Sterile operating conditions for the placement of intraoral implants. J Oral Maxillofac Surg 1996;54(11):1334-1336.

388. Morris HF, Ochi S, Plezia R, Gilbert H, Dent CD, Pikulski J, Lambert PM. AICRG, Part III: The influence of antibiotic use on the survival of a new implant design. J Oral Implantol 2004;30(3):144-151.

389. Laskin DM, Dent CD, Morris HF, Ochi S, Olson JW. The influence of preoperative antibiotics on success of endosseous implants at 36 months. Ann Periodontol 2000;5(1):166-174.

390. Isla A, Canut A, Gascon AR, Labora A, Ardanza-Trevijano B, Solinis MA, Pedraz JL. Pharmacokinetic/pharmacodynamic evaluation of antimicrobial treatments of orofacial odontogenic infections. Clin Pharmacokinet 2005;44(3):305-316.

391. Smith A, Bagg J. An update on antimicrobial chemotherapy: 3. Antimicrobial resistance and the oral cavity. Dent Update 1998;25(6):230-234.

392. Caiazzo A, Casavecchia P, Barone A, Brugnami F. A pilot study to determine the effectiveness of different amoxicillin regimens in implant surgery. J Oral Implantol 2011;37(6):691-696.

393. Brunski JB. Biomechanical factors affecting the bone-dental implant interface. Clin Mater 1992;10(3):153-201.

394. Szmukler-Moncler S, Piattelli A, Favero GA, Dubruille JH. Considerations preliminary to the application of early and immediate loading protocols in dental implantology. Clin Oral Implants Res 2000;11(1):12-25.

395. O'Sullivan D, Sennerby L, Meredith N. Measurements comparing the initial stability of five designs of dental implants: a human cadaver study. Clin Implant Dent Relat Res 2000;2(2):85-92.

396. Skalak R, Zhao Y. Interaction of force-fitting and surface roughness of implants. Clin Implant Dent Relat Res 2000;2(4):219-224.

397. Beer A, Gahleitner A, Holm A, Tschabitscher M, Homolka P. Correlation of insertion torques with bone mineral density from dental quantitative CT in the mandible. Clin Oral Implants Res 2003;14(5):616-620. 
398. Calandriello R, Tomatis M, Vallone R, Rangert B, Gottlow J. Immediate occlusal loading of single lower molars using Branemark System Wide-Platform TiUnite implants: an interim report of a prospective open-ended clinical multicenter study. Clin Implant Dent Relat Res 2003;5 Suppl 1:74-80.

399. Nikellis I, Levi A, Nicolopoulos C. Immediate loading of 190 endosseous dental implants: a prospective observational study of 40 patient treatments with up to 2-year data. Int J Oral Maxillofac Implants 2004;19(1):116-123.

400. Le Gall MG. Localized sinus elevation and osteocompression with single-stage tapered dental implants: technical note. Int J Oral Maxillofac Implants 2004;19(3):431-437.

401. Khayat PG, Arnal HM, Tourbah BI, Sennerby L. Clinical outcome of dental implants placed with high insertion torques (up to $176 \mathrm{Ncm}$ ). Clin Implant Dent Relat Res 2013;15(2):227-233.

402. Ottoni JM, Oliveira ZF, Mansini R, Cabral AM. Correlation between placement torque and survival of single-tooth implants. Int J Oral Maxillofac Implants 2005;20(5):769-776.

403. Trisi P, Perfetti G, Baldoni E, Berardi D, Colagiovanni M, Scogna G. Implant micromotion is related to peak insertion torque and bone density. Clin Oral Implants Res 2009;20(5):467-471.

404. Maló P, Rangert B, Dvarsater L. Immediate function of Branemark implants in the esthetic zone: a retrospective clinical study with 6 months to 4 years of follow-up. Clin Implant Dent Relat Res 2000;2(3):138-146.

405. Hui E, Chow J, Li D, Liu J, Wat P, Law H. Immediate provisional for single-tooth implant replacement with Branemark system: preliminary report. Clin Implant Dent Relat Res 2001;3(2):79-86.

406. Lorenzoni M, Pertl C, Zhang K, Wimmer G, Wegscheider WA. Immediate loading of single-tooth implants in the anterior maxilla. Preliminary results after one year. Clin Oral Implants Res 2003;14(2):180-187.

407. Neugebauer J, Traini T, Thams U, Piattelli A, Zoller JE. Peri-implant bone organization under immediate loading state. Circularly polarized light analyses: a minipig study. J Periodontol 2006;77(2):152-160.

408. Grandi T, Garuti G, Samarani R, Guazzi P, Forabosco A. Immediate loading of single post-extractive implants in the anterior maxilla: 12-month results from a multicenter clinical study. J Oral Implantol 2012;38 Spec No:477-484.

409. Halldin A. On a biomechanical approach to analysis of stability and load bearing capacity of oral implants [PhD thesis]. Malmö: Malmö University; 2015. 
410. Meredith N. A review of nondestructive test methods and their application to measure the stability and osseointegration of bone anchored endosseous implants. Crit Rev Biomed Eng 1998;26(4):275291.

411. Rasmusson L, Stegersjo G, Kahnberg KE, Sennerby L. Implant stability measurements using resonance frequency analysis in the grafted maxilla: a cross-sectional pilot study. Clin Implant Dent Relat Res 1999;1(2):70-74.

412. Rasmusson L, Kahnberg KE, Tan A. Effects of implant design and surface on bone regeneration and implant stability: an experimental study in the dog mandible. Clin Implant Dent Relat Res 2001;3(1):2-8.

413. Friberg B, Jisander S, Widmark G, Lundgren A, Ivanoff CJ, Sennerby $\mathrm{L}$, Thoren C. One-year prospective three-center study comparing the outcome of a "soft bone implant" (prototype Mk IV) and the standard Branemark implant. Clin Implant Dent Relat Res 2003;5(2):71-77.

414. Morris HF, Ochi S, Orenstein IH, Petrazzuolo V. AICRG, Part V: Factors influencing implant stability at placement and their influence on survival of Ankylos implants. J Oral Implantol 2004;30(3):162170.

415. Balshi SF, Allen FD, Wolfinger GJ, Balshi TJ. A resonance frequency analysis assessment of maxillary and mandibular immediately loaded implants. Int J Oral Maxillofac Implants 2005;20(4):584-594.

416. Sennerby L, Roos J. Surgical determinants of clinical success of osseointegrated oral implants: a review of the literature. Int J Prosthodont 1998;11(5):408-420.

417. Hallman M, Sennerby L, Lundgren S. A clinical and histologic evaluation of implant integration in the posterior maxilla after sinus floor augmentation with autogenous bone, bovine hydroxyapatite, or a 20:80 mixture. Int J Oral Maxillofac Implants 2002;17(5):635-643.

418. Divac M, Stawarczyk B, Sahrmann P, Attin T, Schmidlin PR. Influence of residual bone thickness on primary stability of hybrid self-tapping and cylindric non-self-tapping implants in vitro. Int J Oral Maxillofac Implants 2013;28(1):84-88.

419. Martinez H, Davarpanah M, Missika P, Celletti R, Lazzara R. Optimal implant stabilization in low density bone. Clin Oral Implants Res 2001;12(5):423-432.

420. Orsini E, Giavaresi G, Trire A, Ottani V, Salgarello S. Dental implant thread pitch and its influence on the osseointegration process: an in vivo comparison study. Int J Oral Maxillofac Implants 2012;27(2):383-392. 
421. Roberts WE, Smith RK, Zilberman Y, Mozsary PG, Smith RS. Osseous adaptation to continuous loading of rigid endosseous implants. Am J Orthod 1984;86(2):95-111.

422. Abuhussein H, Pagni G, Rebaudi A, Wang HL. The effect of thread pattern upon implant osseointegration. Clin Oral Implants Res 2010;21(2):129-136.

423. Sanz M, Alandez J, Lazaro P, Calvo JL, Quirynen M, van Steenberghe D. Histo-pathologic characteristics of peri-implant soft tissues in Branemark implants with 2 distinct clinical and radiological patterns. Clin Oral Implants Res 1991;2(3):128-134.

424. Quirynen M, Naert I, van Steenberghe D. Fixture design and overload influence marginal bone loss and fixture success in the Branemark system. Clin Oral Implants Res 1992;3(3):104-111.

425. Sagara M, Akagawa Y, Nikai H, Tsuru H. The effects of early occlusal loading on one-stage titanium alloy implants in beagle dogs: a pilot study. J Prosthet Dent 1993;69(3):281-288.

426. Becker W, Lekholm U, Dahlin C, Becker B, Donath K. The effect of clinical loading on bone regeneration by GTAM barriers: a study in dogs. Int J Oral Maxillofac Implants 1994;9(3):305-313.

427. Isidor F. Loss of osseointegration caused by occlusal load of oral implants. A clinical and radiographic study in monkeys. Clin Oral Implants Res 1996;7(2):143-152.

428. Isidor F. Histological evaluation of peri-implant bone at implants subjected to occlusal overload or plaque accumulation. Clin Oral Implants Res 1997;8(1):1-9.

429. Lioubavina-Hack N, Lang NP, Karring T. Significance of primary stability for osseointegration of dental implants. Clin Oral Implants Res 2006;17(3):244-250.

430. Orenstein IH, Tarnow DP, Morris HF, Ochi S. Factors affecting implant mobility at placement and integration of mobile implants at uncovering. J Periodontol 1998;69(12):1404-1412.

431. Glauser R, Rée A, Lundgren A, Gottlow J, Hämmerle CH, Schärer P. Immediate occlusal loading of Branemark implants applied in various jawbone regions: a prospective, 1-year clinical study. Clin Implant Dent Relat Res 2001;3(4):204-213.

432. Davies JE. Mechanisms of endosseous integration. Int J Prosthodont 1998;11(5):391-401.

433. Rotter BE, Blackwell R, Dalton G. Testing progressive loading of endosteal implants with the Periotest: a pilot study. Implant Dent 1996;5(1):28-32. 
434. Friberg B, Sennerby L, Meredith N, Lekholm U. A comparison between cutting torque and resonance frequency measurements of maxillary implants. A 20-month clinical study. Int J Oral Maxillofac Surg 1999;28(4):297-303.

435. Meredith N, Alleyne D, Cawley P. Quantitative determination of the stability of the implant-tissue interface using resonance frequency analysis. Clin Oral Implants Res 1996;7(3):261-267.

436. Meredith N. Assessment of implant stability as a prognostic determinant. Int J Prosthodont 1998;11(5):491-501.

437. Sennerby L, Meredith N. Resonance frequency analysis: measuring implant stability and osseointegration. Compend Contin Educ Dent 1998;19(5):493-498, 500, 502; quiz 504.

438. Friberg B, Sennerby L, Linden B, Grondahl K, Lekholm U. Stability measurements of one-stage Branemark implants during healing in mandibles. A clinical resonance frequency analysis study. Int J Oral Maxillofac Surg 1999;28(4):266-272.

439. Barewal RM, Oates TW, Meredith N, Cochran DL. Resonance frequency measurement of implant stability in vivo on implants with a sandblasted and acid-etched surface. Int J Oral Maxillofac Implants 2003;18(5):641-651.

440. Glauser R, Lundgren AK, Gottlow J, Sennerby L, Portmann M, Ruhstaller P, Hämmerle CH. Immediate occlusal loading of Branemark TiUnite implants placed predominantly in soft bone: 1 -year results of a prospective clinical study. Clin Implant Dent Relat Res 2003;5 Suppl 1:47-56.

441. Olsson M, Urde G, Andersen JB, Sennerby L. Early loading of maxillary fixed cross-arch dental prostheses supported by six or eight oxidized titanium implants: results after 1 year of loading, case series. Clin Implant Dent Relat Res 2003;5 Suppl 1:81-87.

442. Balshi TJ, Wolfinger GJ. Immediate placement and implant loading for expedited patient care: a patient report. Int J Oral Maxillofac Implants 2002;17(4):587-592.

443. Meredith N, Book K, Friberg B, Jemt T, Sennerby L. Resonance frequency measurements of implant stability in vivo. A cross-sectional and longitudinal study of resonance frequency measurements on implants in the edentulous and partially dentate maxilla. Clin Oral Implants Res 1997;8(3):226-233.

444. Meredith N, Friberg B, Sennerby L, Aparicio C. Relationship between contact time measurements and PTV values when using the Periotest to measure implant stability. Int J Prosthodont 1998;11(3):269-275. 
445. Werbitt MJ, Goldberg PV. The immediate implant: bone preservation and bone regeneration. Int J Periodontics Restorative Dent 1992;12(3):206-217.

446. Lindeboom JA, Tjiook Y, Kroon FH. Immediate placement of implants in periapical infected sites: a prospective randomized study in 50 patients. Oral Surg Oral Med Oral Pathol Oral Radiol Endod 2006;101(6):705-710.

447. De Bruyn H, Collaert B. Early loading of machined-surface Branemark implants in completely edentulous mandibles: healed bone versus fresh extraction sites. Clin Implant Dent Relat Res 2002;4(3):136-142.

448. Kourtis SG, Sotiriadou S, Voliotis S, Challas A. Private practice results of dental implants. Part I: survival and evaluation of risk factors--Part II: surgical and prosthetic complications. Implant Dent 2004;13(4):373-385.

449. Dhanrajani PJ, Al-Rafee MA. Single-tooth implant restorations: a retrospective study. Implant Dent 2005;14(2):125-130.

450. Horwitz J, Zuabi O, Peled M, Machtei EE. Immediate and delayed restoration of dental implants in periodontally susceptible patients: 1-year results. Int J Oral Maxillofac Implants 2007;22(3):423-429.

451. Deng F, Zhang H, Zhang H, Shao H, He Q, Zhang P. A comparison of clinical outcomes for implants placed in fresh extraction sockets versus healed sites in periodontally compromised patients: a 1-year follow-up report. Int J Oral Maxillofac Implants 2010;25(5):1036-1040.

452. Ji TJ, Kan JY, Rungcharassaeng K, Roe P, Lozada JL. Immediate loading of maxillary and mandibular implant-supported fixed complete dentures: a 1- to 10-year retrospective study. J Oral Implantol 2012;38 Spec No:469-476.

453. Polizzi G, Grunder U, Goene R, Hatano N, Henry P, Jackson WJ, Kawamura K, Renouard F, Rosenberg R, Triplett G, Werbitt M, Lithner B. Immediate and delayed implant placement into extraction sockets: a 5-year report. Clin Implant Dent Relat Res 2000;2(2):93-99.

454. De Rouck T, Collys K, Cosyn J. Single-tooth replacement in the anterior maxilla by means of immediate implantation and provisionalization: a review. Int J Oral Maxillofac Implants 2008;23(5):897-904.

455. Becker W, Goldstein M. Immediate implant placement: treatment planning and surgical steps for successful outcome. Periodontol 2000 2008;47:79-89.

456. Cosyn J, Vandenbulcke E, Browaeys H, Van Maele G, De Bruyn H. Factors associated with failure of surface-modified implants up to four years of function. Clin Implant Dent Relat Res 2012;14(3):347-358. 
457. Carlsson L, Rostlund T, Albrektsson B, Albrektsson T. Implant fixation improved by close fit. Cylindrical implant-bone interface studied in rabbits. Acta Orthop Scand 1988;59(3):272-275.

458. Ivanoff CJ, Sennerby L, Lekholm U. Influence of initial implant mobility on the integration of titanium implants. An experimental study in rabbits. Clin Oral Implants Res 1996;7(2):120-127.

459. Gillot L, Cannas B, Buti J, Noharet R. A retrospective cohort study of 113 patients rehabilitated with immediately loaded maxillary crossarch fixed dental prostheses in combination with immediate implant placement. Eur J Oral Implantol 2012;5(1):71-79.

460. Wang TM, Leu LJ, Wang J, Lin LD. Effects of prosthesis materials and prosthesis splinting on peri-implant bone stress around implants in poor-quality bone: a numeric analysis. Int J Oral Maxillofac Implants 2002;17(2):231-237.

461. Vogl S, Stopper M, Hof M, Wegscheider WA, Lorenzoni M. Immediate Occlusal versus Non-Occlusal Loading of Implants: A Randomized Clinical Pilot Study. Clin Implant Dent Relat Res 2015;17(3):589-597.

462. Albrektsson T, Jansson T, Lekholm U. Osseointegrated dental implants. Dent Clin North Am 1986;30(1):151-174.

463. Schropp L, Wenzel A, Kostopoulos L, Karring T. Bone healing and soft tissue contour changes following single-tooth extraction: a clinical and radiographic 12-month prospective study. Int J Periodontics Restorative Dent 2003;23(4):313-323.

464. Araújo MG, Lindhe J. Dimensional ridge alterations following tooth extraction. An experimental study in the dog. J Clin Periodontol 2005;32(2):212-218.

465. Raes F, Cosyn J, De Bruyn H. Clinical, aesthetic, and patient-related outcome of immediately loaded single implants in the anterior maxilla: a prospective study in extraction sockets, healed ridges, and grafted sites. Clin Implant Dent Relat Res 2013;15(6):819-835.

466. Covani U, Cornelini R, Barone A. Bucco-lingual bone remodeling around implants placed into immediate extraction sockets: a case series. J Periodontol 2003;74(2):268-273.

467. Calvo-Guirado JL, Boquete-Castro A, Negri B, Delgado Ruiz R, Gomez-Moreno G, Iezzi G. Crestal bone reactions to immediate implants placed at different levels in relation to crestal bone. A pilot study in Foxhound dogs. Clin Oral Implants Res 2014;25(3):344-351.

468. Caneva M, Salata LA, de Souza SS, Baffone G, Lang NP, Botticelli D. Influence of implant positioning in extraction sockets on osseointegration: histomorphometric analyses in dogs. Clin Oral Implants Res 2010;21(1):43-49. 
469. Tomasi C, Sanz M, Cecchinato D, Pjetursson B, Ferrus J, Lang NP, Lindhe J. Bone dimensional variations at implants placed in fresh extraction sockets: a multilevel multivariate analysis. Clin Oral Implants Res 2010;21(1):30-36.

470. Calvo-Guirado JL, Ortiz-Ruiz AJ, Negri B, Lopez-Mari L, RodriguezBarba C, Schlottig F. Histological and histomorphometric evaluation of immediate implant placement on a dog model with a new implant surface treatment. Clin Oral Implants Res 2010;21(3):308-315.

471. Weng D, Nagata MJ, Bell M, de Melo LG, Bosco AF. Influence of microgap location and configuration on peri-implant bone morphology in nonsubmerged implants: an experimental study in dogs. Int J Oral Maxillofac Implants 2010;25(3):540-547.

472. Weng D, Nagata MJ, Bosco AF, de Melo LG. Influence of microgap location and configuration on radiographic bone loss around submerged implants: an experimental study in dogs. Int J Oral Maxillofac Implants 2011;26(5):941-946.

473. Araújo MG, Linder E, Lindhe J. Bio-Oss collagen in the buccal gap at immediate implants: a 6-month study in the dog. Clin Oral Implants Res 2011;22(1):1-8.

474. Hsu KM, Choi BH, Ko CY, Kim HS, Xuan F, Jeong SM. Ridge alterations following immediate implant placement and the treatment of bone defects with Bio-Oss in an animal model. Clin Implant Dent Relat Res 2012;14(5):690-695.

475. Ozan O, Turkyilmaz I, Yilmaz B. A preliminary report of patients treated with early loaded implants using computerized tomographyguided surgical stents: flapless versus conventional flapped surgery. $J$ Oral Rehabil 2007;34(11):835-840.

476. Rocci A, Martignoni M, Gottlow J. Immediate loading in the maxilla using flapless surgery, implants placed in predetermined positions, and prefabricated provisional restorations: a retrospective 3-year clinical study. Clin Implant Dent Relat Res 2003;5 Suppl 1:29-36.

477. Arisan V, Karabuda CZ, Ozdemir T. Implant surgery using bone- and mucosa-supported stereolithographic guides in totally edentulous jaws: surgical and post-operative outcomes of computer-aided vs. standard techniques. Clin Oral Implants Res 2010;21(9):980-988.

478. Sunitha RV, Sapthagiri E. Flapless implant surgery: a 2-year follow-up study of 40 implants. Oral Surg Oral Med Oral Pathol Oral Radiol 2013;116(4):e237-243.

479. De Bruyn H, Atashkadeh M, Cosyn J, van de Velde T. Clinical outcome and bone preservation of single TiUnite implants installed with flapless or flap surgery. Clin Implant Dent Relat Res 2011;13(3):175-183. 
480. Chrcanovic BR, Oliveira DR, Custodio AL. Accuracy evaluation of computed tomography-derived stereolithographic surgical guides in zygomatic implant placement in human cadavers. J Oral Implantol 2010;36(5):345-355.

481. Gillot L, Cannas B, Friberg B, Vrielinck L, Rohner D, Pettersson A. Accuracy of virtually planned and conventionally placed implants in edentulous cadaver maxillae and mandibles: a preliminary report. $J$ Prosthet Dent 2014;112(4):798-804.

482. Lindeboom JA, van Wijk AJ. A comparison of two implant techniques on patient-based outcome measures: a report of flapless vs. conventional flapped implant placement. Clin Oral Implants Res 2010;21(4):366-370.

483. Van de Velde T, Glor F, De Bruyn H. A model study on flapless implant placement by clinicians with a different experience level in implant surgery. Clin Oral Implants Res 2008;19(1):66-72.

484. Campelo LD, Camara JR. Flapless implant surgery: a 10-year clinical retrospective analysis. Int J Oral Maxillofac Implants 2002;17(2):271276.

485. Chiapasco M, Zaniboni M. Clinical outcomes of GBR procedures to correct peri-implant dehiscences and fenestrations: a systematic review. Clin Oral Implants Res 2009;20 Suppl 4:113-123.

486. Annibali S, Ripari M, La Monaca G, Tonoli F, Cristalli MP. Local accidents in dental implant surgery: prevention and treatment. Int J Periodontics Restorative Dent 2009;29(3):325-331.

487. Berdougo M, Fortin T, Blanchet E, Isidori M, Bosson JL. Flapless implant surgery using an image-guided system. A 1- to 4-year retrospective multicenter comparative clinical study. Clin Implant Dent Relat Res 2010;12(2):142-152.

488. Katsoulis J, Avrampou M, Spycher C, Stipic M, Enkling N, MericskeStern R. Comparison of implant stability by means of resonance frequency analysis for flapless and conventionally inserted implants. Clin Implant Dent Relat Res 2012;14(6):915-923.

489. Wood DL, Hoag PM, Donnenfeld OW, Rosenfeld LD. Alveolar crest reduction following full and partial thickness flaps. J Periodontol 1972;43(3):141-144.

490. Covani U, Cornelini R, Barone A. Buccal bone augmentation around immediate implants with and without flap elevation: a modified approach. Int J Oral Maxillofac Implants 2008;23(5):841-846.

491. Maló P, Nobre M. Flap vs. flapless surgical techniques at immediate implant function in predominantly soft bone for rehabilitation of partial edentulism: a prospective cohort study with follow-up of 1 year. Eur J Oral Implantol 2008;1(4):293-304. 
492. Rousseau P. Flapless and traditional dental implant surgery: an open, retrospective comparative study. J Oral Maxillofac Surg 2010;68(9):2299-2306.

493. Van de Velde T, Sennerby L, De Bruyn H. The clinical and radiographic outcome of implants placed in the posterior maxilla with a guided flapless approach and immediately restored with a provisional rehabilitation: a randomized clinical trial. Clin Oral Implants Res 2010;21(11):1223-1233.

494. Heydenrijk K, Raghoebar GM, Meijer HJ, Van Der Reijden WA, Van Winkelhoff AJ, Stegenga B. Two-part implants inserted in a one-stage or a two-stage procedure. A prospective comparative study. J Clin Periodontol 2002;29(10):901-909.

495. Hall JA, Payne AG, Purton DG, Torr B. A randomized controlled clinical trial of conventional and immediately loaded tapered implants with screw-retained crowns. Int J Prosthodont 2006;19(1):17-19.

496. Schulte W, Heimke G. Das Tubinger Sofort-Implantat. Quintessenz 1976;27:17-23.

497. Östman PO, Hellman M, Sennerby L. Direct implant loading in the edentulous maxilla using a bone density-adapted surgical protocol and primary implant stability criteria for inclusion. Clin Implant Dent Relat Res 2005; 7 Suppl 1:S60-S69.

498. Buser D, Mericske-Stern R, Dula K, Lang NP. Clinical experience with one-stage, non-submerged dental implants. Adv Dent Res 1999;13:153-161.

499. Östman PO, Wennerberg A, Albrektsson T. Immediate occlusal loading of NanoTite PREVAIL implants: a prospective 1-year clinical and radiographic study. Clin Implant Dent Relat Res 2010;12(1):39-47.

500. Östman PO, Wennerberg A, Ekestubbe A, Albrektsson T. Immediate occlusal loading of NanoTite tapered implants: a prospective 1 -year clinical and radiographic study. Clin Implant Dent Relat Res 2013;15(6):809-818.

501. Engquist B, Astrand P, Anzén B, Dahlgren S, Engquist E, Feldmann H, Karlsson U, Nord PG, Sahlholm S, Svärdström P. Simplified methods of implant treatment in the edentulous lower jaw: a 3-year follow-up report of a controlled prospective study of one-stage versus two-stage surgery and early loading. Clin Implant Dent Relat Res 2005;7(2):95104.

502. Ericsson I, Persson LG, Berglundh T, Marinello CP, Lindhe J, Klinge B. Different types of inflammatory reactions in peri-implant soft tissues. $J$ Clin Periodontol 1995;22(3):255-261. 
503. Persson LG, Lekholm U, Leonhardt A, Dahlen G, Lindhe J. Bacterial colonization on internal surfaces of Branemark system implant components. Clin Oral Implants Res 1996;7(2):90-95.

504. Weber HP, Buser D, Donath K, Fiorellini JP, Doppalapudi V, Paquette DW, Williams RC. Comparison of healed tissues adjacent to submerged and non-submerged unloaded titanium dental implants. A histometric study in beagle dogs. Clin Oral Implants Res 1996;7(1):11-19.

505. Danser MM, van Winkelhoff AJ, van der Velden U. Periodontal bacteria colonizing oral mucous membranes in edentulous patients wearing dental implants. J Periodontol 1997;68(3):209-216.

506. Mombelli A, Marxer M, Gaberthuel T, Grunder U, Lang NP. The microbiota of osseointegrated implants in patients with a history of periodontal disease. J Clin Periodontol 1995;22(2):124-130.

507. Sbordone L, Barone A, Ciaglia RN, Ramaglia L, Iacono VJ. Longitudinal study of dental implants in a periodontally compromised population. J Periodontol 1999;70(11):1322-1329.

508. Maló P, Friberg B, Polizzi G, Gualini F, Vighagen T, Rangert B. Immediate and early function of Branemark System implants placed in the esthetic zone: a 1-year prospective clinical multicenter study. Clin Implant Dent Relat Res 2003;5 Suppl 1:37-46.

509. Schnitman PA, Wöhrle PS, Rubenstein JE. Immediate fixed interim prostheses supported by two-stage threaded implants: methodology and results. J Oral Implantol 1990;16(2):96-105.

510. Pilliar RM, Lee JM, Maniatopoulos C. Observations on the effect of movement on bone ingrowth into porous-surfaced implants. Clin Orthop Relat Res 1986;(208):108-113.

511. Horiuchi K, Uchida H, Yamamoto K, Sugimura M. Immediate loading of Branemark system implants following placement in edentulous patients: a clinical report. Int J Oral Maxillofac Implants 2000;15(6):824-830.

512. Chiapasco M, Abati S, Romeo E, Vogel G. Implant-retained mandibular overdentures with Branemark System MKII implants: a prospective comparative study between delayed and immediate loading. Int J Oral Maxillofac Implants 2001;16(4):537-546.

513. Szmukler-Moncler S, Salama H, Reingewirtz Y, Dubruille JH. Timing of loading and effect of micromotion on bone-dental implant interface: review of experimental literature. J Biomed Mater Res 1998;43(2):192203.

514. Siebers D, Gehrke P, Schliephake H. Immediate versus delayed function of dental implants: a 1- to 7-year follow-up study of 222 implants. Int J Oral Maxillofac Implants 2010;25(6):1195-1202. 
515. Romanos GE, Toh CG, Siar CH, Swaminathan D. Histologic and histomorphometric evaluation of peri-implant bone subjected to immediate loading: an experimental study with Macaca fascicularis. Int J Oral Maxillofac Implants 2002;17(1):44-51.

516. Romanos GE, Nentwig GH. Immediate versus delayed functional loading of implants in the posterior mandible: a 2-year prospective clinical study of 12 consecutive cases. Int J Periodontics Restorative Dent 2006;26(5):459-469.

517. Degidi M, Perrotti V, Piattelli A. Immediately loaded titanium implants with a porous anodized surface with at least 36 months of follow-up. Clin Implant Dent Relat Res 2006;8(4):169-177.

518. Meyer U, Joos U, Mythili J, Stamm T, Hohoff A, Fillies T, Stratmann U, Wiesmann HP. Ultrastructural characterization of the implant/ bone interface of immediately loaded dental implants. Biomaterials 2004;25(10):1959-1967.

519. Ghanavati F, Shayegh SS, Rahimi H, Sharifi D, Ghanavati F, Khalesseh $\mathrm{N}$, Eslami B. The effects of loading time on osseointegration and new bone formation around dental implants: a histologic and histomorphometric study in dogs. J Periodontol 2006;77(10):17011707.

520. Maló P, de Araújo Nobre M, Lopes A, Moss SM, Molina GJ. A longitudinal study of the survival of All-on-4 implants in the mandible with up to 10 years of follow-up. J Am Dent Assoc 2011;142(3):310320.

521. Friberg B, Ivanoff CJ, Lekholm U. Inferior alveolar nerve transposition in combination with Branemark implant treatment. Int J Periodontics Restorative Dent 1992;12(6):440-449.

522. Chrcanovic BR, Custodio AL. Inferior alveolar nerve lateral transposition. Oral Maxillofac Surg 2009;13(4):213-219.

523. Maló P, de Araújo Nobre M, Lopes A, Francischone C, Rigolizzo M. "All-on-4" immediate-function concept for completely edentulous maxillae: a clinical report on the medium (3 years) and long-term (5 years) outcomes. Clin Implant Dent Relat Res 2012;14 Suppl 1:e139150.

524. Chrcanovic BR, Abreu MH. Survival and complications of zygomatic implants: a systematic review. Oral Maxillofac Surg 2013;17(2):81-93.

525. Chrcanovic BR, Pedrosa AR, Neto Custodio AL. Zygomatic implants: a critical review of the surgical techniques. Oral Maxillofac Surg 2013;17(1):1-9.

526. Aparicio C, Perales P, Rangert B. Tilted implants as an alternative to maxillary sinus grafting: a clinical, radiologic, and periotest study. Clin Implant Dent Relat Res 2001;3(1):39-49. 
527. Crespi R, Vinci R, Cappare P, Romanos GE, Gherlone E. A clinical study of edentulous patients rehabilitated according to the "all on four" immediate function protocol. Int J Oral Maxillofac Implants 2012;27(2):428-434.

528. Zampelis A, Rangert B, Heijl L. Tilting of splinted implants for improved prosthodontic support: a two-dimensional finite element analysis. J Prosthet Dent 2007;97(6 Suppl):S35-43.

529. De Vico G, Bonino M, Spinelli D, Schiavetti R, Sannino G, Pozzi A, Ottria L. Rationale for tilted implants: FEA considerations and clinical reports. Oral Implantol (Rome) 2011;4(3-4):23-33.

530. Del Fabbro M, Ceresoli V. The fate of marginal bone around axial vs. tilted implants: A systematic review. Eur J Oral Implantol 2014;7(2):171-189.

531. Balshi TJ, Wolfinger GJ, Slauch RW, Balshi SF. A retrospective analysis of 800 Branemark System implants following the All-on-Four protocol. J Prosthodont 2014;23(2):83-88.

532. Canay S, Hersek N, Akpinar I, Asik Z. Comparison of stress distribution around vertical and angled implants with finite-element analysis. Quintessence Int 1996;27(9):591-598.

533. Watanabe F, Hata Y, Komatsu S, Ramos TC, Fukuda H. Finite element analysis of the influence of implant inclination, loading position, and load direction on stress distribution. Odontology 2003;91(1):31-36.

534. Lan TH, Pan CY, Lee HE, Huang HL, Wang CH. Bone stress analysis of various angulations of mesiodistal implants with splinted crowns in the posterior mandible: a three-dimensional finite element study. Int $J$ Oral Maxillofac Implants 2010;25(4):763-770.

535. Rangert BR, Sullivan RM, Jemt TM. Load factor control for implants in the posterior partially edentulous segment. Int J Oral Maxillofac Implants 1997;12(3):360-370.

536. Bellini CM, Romeo D, Galbusera F, Agliardi E, Pietrabissa R, Zampelis A, Francetti L. A finite element analysis of tilted versus nontilted implant configurations in the edentulous maxilla. Int J Prosthodont 2009;22(2):155-157.

537. Bellini CM, Romeo D, Galbusera F, Taschieri S, Raimondi MT, Zampelis A, Francetti L. Comparison of tilted versus nontilted implantsupported prosthetic designs for the restoration of the edentuous mandible: a biomechanical study. Int J Oral Maxillofac Implants 2009;24(3):511-517.

538. Lindquist LW, Rockler B, Carlsson GE. Bone resorption around fixtures in edentulous patients treated with mandibular fixed tissueintegrated prostheses. J Prosthet Dent 1988;59(1):59-63. 
539. Misch CM. Comparison of intraoral donor sites for onlay grafting prior to implant placement. Int J Oral Maxillofac Implants 1997;12(6):767-776.

540. Brånemark PI, Lindström J, Hallen $\mathrm{O}$, Breine U, Jeppson PH, Ohman A. Reconstruction of the defective mandible. Scand J Plast Reconstr Surg 1975;9(2):116-128.

541. Keller EE, Tolman D, Eckert S. Endosseous implant and autogenous bone graft reconstruction of mandibular discontinuity: a 12-year longitudinal study of 31 patients. Int J Oral Maxillofac Implants 1998;13(6):767-780.

542. Sethi A, Kaus T. Ridge augmentation using mandibular block bone grafts: preliminary results of an ongoing prospective study. Int J Oral Maxillofac Implants 2001;16(3):378-388.

543. Stellingsma K, Raghoebar GM, Meijer HJ, Stegenga B. The extremely resorbed mandible: a comparative prospective study of 2-year results with 3 treatment strategies. Int J Oral Maxillofac Implants 2004;19(4):563-577.

544. Pogrel MA, Podlesh S, Anthony JP, Alexander J. A comparison of vascularized and nonvascularized bone grafts for reconstruction of mandibular continuity defects. J Oral Maxillofac Surg 1997;55(11):1200-1206.

545. Foster RD, Anthony JP, Sharma A, Pogrel MA. Vascularized bone flaps versus nonvascularized bone grafts for mandibular reconstruction: an outcome analysis of primary bony union and endosseous implant success. Head Neck 1999;21(1):66-71.

546. Keller EE. Reconstruction of the severely atrophic edentulous mandible with endosseous implants: a 10-year longitudinal study. J Oral Maxillofac Surg 1995;53(3):305-320.

547. Verhoeven JW, Cune MS, Terlou M, Zoon MA, de Putter C. The combined use of endosteal implants and iliac crest onlay grafts in the severely atrophic mandible: a longitudinal study. Int J Oral Maxillofac Surg 1997;26(5):351-357.

548. Triplett RG, Schow SR. Autologous bone grafts and endosseous implants: complementary techniques. J Oral Maxillofac Surg 1996;54(4):486-494.

549. Lundgren S, Nystrom E, Nilson H, Gunne J, Lindhagen O. Bone grafting to the maxillary sinuses, nasal floor and anterior maxilla in the atrophic edentulous maxilla. A two-stage technique. Int J Oral Maxillofac Surg 1997;26(6):428-434. 
550. Neyt LF, De Clercq CA, Abeloos JV, Mommaerts MY. Reconstruction of the severely resorbed maxilla with a combination of sinus augmentation, onlay bone grafting, and implants. J Oral Maxillofac Surg 1997;55(12):1397-1401.

551. Chiapasco M, Abati S, Romeo E, Vogel G. Clinical outcome of autogenous bone blocks or guided bone regeneration with e-PTFE membranes for the reconstruction of narrow edentulous ridges. Clin Oral Implants Res 1999;10(4):278-288.

552. Raghoebar GM, Batenburg RH, Meijer HJ, Vissink A. Horizontal osteotomy for reconstruction of the narrow edentulous mandible. Clin Oral Implants Res 2000;11(1):76-82.

553. Bahat O, Fontanessi RV. Efficacy of implant placement after bone grafting for three-dimensional reconstruction of the posterior jaw. Int $J$ Periodontics Restorative Dent 2001;21(3):220-231.

554. Bell RB, Blakey GH, White RP, Hillebrand DG, Molina A. Staged reconstruction of the severely atrophic mandible with autogenous bone graft and endosteal implants. J Oral Maxillofac Surg 2002;60(10):1135-1141.

555. Jemt T, Lekholm U. Measurements of buccal tissue volumes at singleimplant restorations after local bone grafting in maxillas: a 3-year clinical prospective study case series. Clin Implant Dent Relat Res 2003;5(2):63-70.

556. Raghoebar GM, Schoen P, Meijer HJ, Stellingsma K, Vissink A. Early loading of endosseous implants in the augmented maxilla: a 1-year prospective study. Clin Oral Implants Res 2003;14(6):697-702.

557. Iizuka T, Smolka W, Hallermann W, Mericske-Stern R. Extensive augmentation of the alveolar ridge using autogenous calvarial split bone grafts for dental rehabilitation. Clin Oral Implants Res 2004;15(5):607-615.

558. Shirota T, Ohno K, Michi K, Tachikawa T. An experimental study of healing around hydroxylapatite implants installed with autogenous iliac bone grafts for jaw reconstruction. J Oral Maxillofac Surg 1991;49(12):1310-1315.

559. Rasmusson L, Meredith N, Kahnberg KE, Sennerby L. Effects of barrier membranes on bone resorption and implant stability in onlay bone grafts. An experimental study. Clin Oral Implants Res 1999;10(4):267-277.

560. Chiapasco M, Zaniboni M, Boisco M. Augmentation procedures for the rehabilitation of deficient edentulous ridges with oral implants. Clin Oral Implants Res 2006;17 Suppl 2:136-159. 
561. Clementini M, Morlupi A, Agrestini C, Barlattani A. Immediate versus delayed positioning of dental implants in guided bone regeneration or onlay graft regenerated areas: a systematic review. Int J Oral Maxillofac Surg 2013;42(5):643-650.

562. Friberg B. Bone augmentation for single tooth implants: A review of the literature. Eur J Oral Implantol 2016;9 Suppl 1:S123-134.

563. Froum SJ, Khouly I, Favero G, Cho SC. Effect of maxillary sinus membrane perforation on vital bone formation and implant survival: a retrospective study. J Periodontol 2013;84(8):1094-1099.

564. Silva LD, de Lima VN, Faverani LP, de Mendonca MR, Okamoto R, Pellizzer EP. Maxillary sinus lift surgery-with or without graft material? A systematic review. Int J Oral Maxillofac Surg 2016;45(12):15701576.

565. Zitzmann NU, Schärer P. Sinus elevation procedures in the resorbed posterior maxilla. Comparison of the crestal and lateral approaches. Oral Surg Oral Med Oral Pathol Oral Radiol Endod 1998;85(1):8-17.

566. Thor A, Sennerby L, Hirsch JM, Rasmusson L. Bone formation at the maxillary sinus floor following simultaneous elevation of the mucosal lining and implant installation without graft material: an evaluation of 20 patients treated with 44 Astra Tech implants. J Oral Maxillofac Surg 2007;65(7 Suppl 1):64-72.

567. Johansson LA, Isaksson S, Lindh C, Becktor JP, Sennerby L. Maxillary sinus floor augmentation and simultaneous implant placement using locally harvested autogenous bone chips and bone debris: a prospective clinical study. J Oral Maxillofac Surg 2010;68(4):837-844.

568. Sohn DS, Moon JW, Moon KN, Cho SC, Kang PS. New bone formation in the maxillary sinus using only absorbable gelatin sponge. J Oral Maxillofac Surg 2010;68(6):1327-1333.

569. Testori T, Weinstein RL, Taschieri S, Del Fabbro M. Risk factor analysis following maxillary sinus augmentation: a retrospective multicenter study. Int J Oral Maxillofac Implants 2012;27(5):11701176.

570. Moon JW, Sohn DS, Heo JU, Shin HI, Jung JK. New bone formation in the maxillary sinus using peripheral venous blood alone. J Oral Maxillofac Surg 2011;69(9):2357-2367.

571. Machtei EE, Horwitz J, Mahler D, Grossmann Y, Levin L. Third attempt to place implants in sites where previous surgeries have failed. J Clin Periodontol 2011;38(2):195-198.

572. Evian CI, Cutler SA. Direct replacement of failed CP titanium implants with larger-diameter, HA-coated Ti-6Al-4V implants: report of five cases. Int J Oral Maxillofac Implants 1995;10(6):736-743. 
573. Machtei EE, Mahler D, Oettinger-Barak O, Zuabi O, Horwitz J. Dental implants placed in previously failed sites: survival rate and factors affecting the outcome. Clin Oral Implants Res 2008;19(3):259264.

574. Grossmann Y, Levin L. Success and survival of single dental implants placed in sites of previously failed implants. J Periodontol 2007;78(9):1670-1674.

575. Kim YK, Park JY, Kim SG, Lee HJ. Prognosis of the implants replaced after removal of failed dental implants. Oral Surg Oral Med Oral Pathol Oral Radiol Endod 2010;110(3):281-286.

576. Alsaadi G, Quirynen M, van Steenberghe D. The importance of implant surface characteristics in the replacement of failed implants. Int J Oral Maxillofac Implants 2006;21(2):270-274.

577. Wagenberg B, Froum SJ. A retrospective study of 1925 consecutively placed immediate implants from 1988 to 2004. Int J Oral Maxillofac Implants 2006;21(1):71-80.

578. Antoun H, Karouni M, Abitbol J, Zouiten O, Jemt T. A retrospective study on 1592 consecutively performed operations in one private referral clinic. Part I: Early inflammation and early implant failures. Clin Implant Dent Relat Res 2017.

579. Quirynen M, Vogels R, Alsaadi G, Naert I, Jacobs R, van Steenberghe D. Predisposing conditions for retrograde peri-implantitis, and treatment suggestions. Clin Oral Implants Res 2005;16(5):599-608.

580. Chuang SK, Cai T, Douglass CW, Wei LJ, Dodson TB. Frailty approach for the analysis of clustered failure time observations in dental research. J Dent Res 2005;84(1):54-58.

581. He J, Zhao B, Deng C, Shang D, Zhang C. Assessment of implant cumulative survival rates in sites with different bone density and related prognostic factors: an 8-year retrospective study of 2,684 implants. Int J Oral Maxillofac Implants 2015;30(2):360-371.

582. Sakka S, Coulthard P. Bone quality: a reality for the process of osseointegration. Implant Dent 2009;18(6):480-485.

583. Friberg B, Sennerby L, Roos J, Lekholm U. Identification of bone quality in conjunction with insertion of titanium implants. A pilot study in jaw autopsy specimens. Clin Oral Implants Res 1995;6(4):213-219.

584. Jacobs R. Preoperative radiologic planning of implant surgery in compromised patients. Periodontol 2000 2003;33:12-25. 
585. Shibli JA, Mangano C, Mangano F, Rodrigues JA, Cassoni A, Bechara K, Ferreia JD, Dottore AM, Iezzi G, Piattelli A. Bone-to-implant contact around immediately loaded direct laser metal-forming transitional implants in human posterior maxilla. J Periodontol 2013;84(6):732-737.

586. Levin L, Sadet P, Grossmann Y. A retrospective evaluation of 1,387 single-tooth implants: a 6-year follow-up. J Periodontol 2006;77(12):2080-2083.

587. Machtei EE, Frankenthal S, Blumenfeld I, Gutmacher Z, Horwitz J. Dental implants for immediate fixed restoration of partially edentulous patients: a 1-year prospective pilot clinical trial in periodontally susceptible patients. J Periodontol 2007;78(7):1188-1194.

588. Todisco M, Trisi P. Bone mineral density and bone histomorphometry are statistically related. Int J Oral Maxillofac Implants 2005;20(6):898-904.

589. Jemt T, Stenport V. Implant treatment with fixed prostheses in the edentulous maxilla. Part 2: prosthetic technique and clinical maintenance in two patient cohorts restored between 1986 and 1987 and 15 years later. Int J Prosthodont 2011;24(4):356-362.

590. Jaffin RA, Berman CL. The excessive loss of Branemark fixtures in type IV bone: a 5-year analysis. J Periodontol 1991;62(1):2-4.

591. Ulm C, Kneissel M, Schedle A, Solar P, Matejka M, Schneider B, Donath K. Characteristic features of trabecular bone in edentulous maxillae. Clin Oral Implants Res 1999;10(6):459-467.

592. Ribeiro-Rotta RF, Lindh C, Rohlin M. Efficacy of clinical methods to assess jawbone tissue prior to and during endosseous dental implant placement: a systematic literature review. Int J Oral Maxillofac Implants 2007;22(2):289-300.

593. Johns RB, Jemt T, Heath MR, Hutton JE, McKenna S, McNamara DC, van Steenberghe D, Taylor R, Watson RM, Herrmann I. A multicenter study of overdentures supported by Branemark implants. Int J Oral Maxillofac Implants 1992;7(4):513-522.

594. Turkyilmaz I, McGlumphy EA. Influence of bone density on implant stability parameters and implant success: a retrospective clinical study. BMC Oral Health 2008;8:32.

595. Cordioli G, Majzoub Z. Heat generation during implant site preparation: an in vitro study. Int J Oral Maxillofac Implants 1997;12(2):186-193.

596. Lundskog J. Heat and bone tissue. An experimental investigation of the thermal properties of bone and threshold levels for thermal injury. Scand J Plast Reconstr Surg 1972;9:1-80. 
597. Eriksson RA, Albrektsson T, Magnusson B. Assessment of bone viability after heat trauma. A histological, histochemical and vital microscopic study in the rabbit. Scand J Plast Reconstr Surg 1984;18(3):261-268.

598. Bergkvist G, Koh KJ, Sahlholm S, Klintstrom E, Lindh C. Bone density at implant sites and its relationship to assessment of bone quality and treatment outcome. Int J Oral Maxillofac Implants 2010;25(2):321328.

599. Atsumi M, Park SH, Wang HL. Methods used to assess implant stability: current status. Int J Oral Maxillofac Implants 2007;22(5):743-754.

600. Lee S, Gantes B, Riggs M, Crigger M. Bone density assessments of dental implant sites: 3 . Bone quality evaluation during osteotomy and implant placement. Int J Oral Maxillofac Implants 2007;22(2):208212.

601. Ribeiro-Rotta RF, Lindh C, Pereira AC, Rohlin M. Ambiguity in bone tissue characteristics as presented in studies on dental implant planning and placement: a systematic review. Clin Oral Implants Res 2011;22(8):789-801.

602. Altman DG, Schulz KF, Moher D, Egger M, Davidoff F, Elbourne D, Gotzsche PC, Lang T. The revised CONSORT statement for reporting randomized trials: explanation and elaboration. Ann Intern Med 2001;134(8):663-694.

603. Moher D, Schulz KF, Altman DG. The CONSORT statement: revised recommendations for improving the quality of reports of parallel-group randomized trials. Ann Intern Med 2001;134(8):657-662.

604. Shapurian T, Damoulis PD, Reiser GM, Griffin TJ, Rand WM. Quantitative evaluation of bone density using the Hounsfield index. Int J Oral Maxillofac Implants 2006;21(2):290-297.

605. Holahan CM, Wiens JL, Weaver A, Assad D, Koka S. Relationship between systemic bone mineral density and local bone quality as effectors of dental implant survival. Clin Implant Dent Relat Res 2011;13(1):29-33.

606. Merheb J, Van Assche N, Coucke W, Jacobs R, Naert I, Quirynen M. Relationship between cortical bone thickness or computerized tomography-derived bone density values and implant stability. Clin Oral Implants Res 2010;21(6):612-617.

607. Rokn A, Rasouli Ghahroudi AA, Daneshmonfared M, Menasheof R, Shamshiri AR. Tactile sense of the surgeon in determining bone density when placing dental implant. Implant Dent 2014;23(6):697-703. 
608. Lindh C, Petersson A, Rohlin M. Assessment of the trabecular pattern before endosseous implant treatment: diagnostic outcome of periapical radiography in the mandible. Oral Surg Oral Med Oral Pathol Oral Radiol Endod 1996;82(3):335-343.

609. Trisi P, Rao W. Bone classification: clinical-histomorphometric comparison. Clin Oral Implants Res 1999;10(1):1-7.

610. Norton MR, Gamble C. Bone classification: an objective scale of bone density using the computerized tomography scan. Clin Oral Implants Res 2001;12(1):79-84.

611. Aranyarachkul P, Caruso J, Gantes B, Schulz E, Riggs M, Dus I, Yamada JM, Crigger M. Bone density assessments of dental implant sites: 2. Quantitative cone-beam computerized tomography. Int J Oral Maxillofac Implants 2005;20(3):416-424.

612. Horner K, Devlin H. The relationships between two indices of mandibular bone quality and bone mineral density measured by dual energy X-ray absorptiometry. Dentomaxillofac Radiol 1998;27(1):1721.

613. Shahlaie M, Gantes B, Schulz E, Riggs M, Crigger M. Bone density assessments of dental implant sites: 1. Quantitative computed tomography. Int J Oral Maxillofac Implants 2003;18(2):224-231.

614. Turkyilmaz I, Tozum TF, Tumer C, Ozbek EN. Assessment of correlation between computerized tomography values of the bone, and maximum torque and resonance frequency values at dental implant placement. J Oral Rehabil 2006;33(12):881-888.

615. Al-Askar M, O’Neill R, Stark PC, Griffin T, Javed F, Al-Hezaimi $\mathrm{K}$. Effect of single and contiguous teeth extractions on alveolar bone remodeling: a study in dogs. Clin Implant Dent Relat Res 2013;15(4):569-575.

616. Stedman RL. The chemical composition of tobacco and tobacco smoke. Chem Rev 1968;68(2):153-207.

617. Zevin S, Gourlay SG, Benowitz NL. Clinical pharmacology of nicotine. Clin Dermatol 1998;16(5):557-564.

618. Riebel GD, Boden SD, Whitesides TE, Hutton WC. The effect of nicotine on incorporation of cancellous bone graft in an animal model. Spine (Phila Pa 1976) 1995;20(20):2198-2202.

619. Chen F, Osterman AL, Mahony K. Smoking and bony union after ulnashortening osteotomy. Am J Orthop (Belle Mead NJ) 2001;30(6):486489.

620. Hagiwara S, Tsumura K. Smoking as a risk factor for bone mineral density in the heel of Japanese men. J Clin Densitom 1999;2(3):219222. 
621. Rapuri PB, Gallagher JC, Balhorn KE, Ryschon KL. Smoking and bone metabolism in elderly women. Bone 2000;27(3):429-436.

622. Forsmo S, Schei B, Langhammer A, Forsen L. How do reproductive and lifestyle factors influence bone density in distal and ultradistal radius of early postmenopausal women? The Nord-Trondelag Health Survey, Norway. Osteoporos Int 2001;12(3):222-229.

623. Medras M, Jankowska EA, Rogucka E. [The effect of smoking tobacco and drinking of alcohol and coffee on bone mineral density of healthy men 40 years of age]. Pol Arch Med Wewn 2000;103(3-4):187-193.

624. Hopper JL, Seeman E. The bone density of female twins discordant for tobacco use. N Engl J Med 1994;330(6):387-392.

625. Glassman SD, Anagnost SC, Parker A, Burke D, Johnson JR, Dimar JR. The effect of cigarette smoking and smoking cessation on spinal fusion. Spine (Phila Pa 1976) 2000;25(20):2608-2615.

626. Bain CA. Smoking and implant failure--benefits of a smoking cessation protocol. Int J Oral Maxillofac Implants 1996;11(6):756-759.

627. Bain CA. Implant installation in the smoking patient. Periodontol 2000 2003;33:185-193.

628. Meechan JG, Macgregor ID, Rogers SN, Hobson RS, Bate JP, Dennison M. The effect of smoking on immediate post-extraction socket filling with blood and on the incidence of painful socket. $\mathrm{Br} J$ Oral Maxillofac Surg 1988;26(5):402-409.

629. Holm G. Smoking as an additional risk for tooth loss. J Periodontol 1994;65(11):996-1001.

630. Bolin A, Eklund G, Frithiof L, Lavstedt S. The effect of changed smoking habits on marginal alveolar bone loss. A longitudinal study. Swed Dent J 1993;17(5):211-216.

631. Johnson GK, Guthmiller JM. The impact of cigarette smoking on periodontal disease and treatment. Periodontol 2000 2007;44:178-194.

632. Pereira ML, Carvalho JC, Peres F, Fernandes MH. Simultaneous effects of nicotine, acrolein, and acetaldehyde on osteogenic-induced bone marrow cells cultured on plasma-sprayed titanium implants. Int J Oral Maxillofac Implants 2010;25(1):112-122.

633. Lindquist LW, Carlsson GE, Jemt T. Association between marginal bone loss around osseointegrated mandibular implants and smoking habits: a 10-year follow-up study. J Dent Res 1997;76(10):1667-1674.

634. Engstrand P, Gröndahl K, Ohrnell LO, Nilsson P, Nannmark U, Brånemark PI. Prospective follow-up study of 95 patients with edentulous mandibles treated according to the Branemark Novum concept. Clin Implant Dent Relat Res 2003;5(1):3-10. 
635. van Steenberghe D, Molly L, Jacobs R, Vandekerckhove B, Quirynen $\mathrm{M}$, Naert I. The immediate rehabilitation by means of a readymade final fixed prosthesis in the edentulous mandible: a 1-year follow-up study on 50 consecutive patients. Clin Oral Implants Res 2004;15(3):360-365.

636. Agliardi E, Clericò M, Ciancio P, Massironi D. Immediate loading of full-arch fixed prostheses supported by axial and tilted implants for the treatment of edentulous atrophic mandibles. Quintessence Int 2010;41(4):285-293.

637. Grandi T, Guazzi P, Samarani R, Grandi G. Immediate loading of four (all-on-4) post-extractive implants supporting mandibular cross-arch fixed prostheses: 18 -month follow-up from a multicentre prospective cohort study. Eur J Oral Implantol 2012;5(3):277-285.

638. Klokkevold PR, Han TJ. How do smoking, diabetes, and periodontitis affect outcomes of implant treatment? Int J Oral Maxillofac Implants 2007;22 Suppl:173-202.

639. Ma L, Zwahlen RA, Zheng LW, Sham MH. Influence of nicotine on the biological activity of rabbit osteoblasts. Clin Oral Implants Res 2011;22(3):338-342.

640. Rawadi G, Vayssiere B, Dunn F, Baron R, Roman-Roman S. BMP-2 controls alkaline phosphatase expression and osteoblast mineralization by a Wnt autocrine loop. J Bone Miner Res 2003;18(10):1842-1853.

641. Deng ZL, Sharff KA, Tang N, Song WX, Luo J, Luo X, Chen J, Bennett E, Reid R, Manning D, Xue A, Montag AG, Luu HH, Haydon RC, He TC. Regulation of osteogenic differentiation during skeletal development. Front Biosci 2008;13:2001-2021.

642. De la Riva B, Sanchez E, Hernandez A, Reyes R, Tamimi F, LopezCabarcos E, Delgado A, Evora C. Local controlled release of VEGF and PDGF from a combined brushite-chitosan system enhances bone regeneration. J Control Release 2010;143(1):45-52.

643. Patel ZS, Young S, Tabata Y, Jansen JA, Wong ME, Mikos AG. Dual delivery of an angiogenic and an osteogenic growth factor for bone regeneration in a critical size defect model. Bone 2008;43(5):931-940.

644. Samee M, Kasugai S, Kondo H, Ohya K, Shimokawa H, Kuroda S. Bone morphogenetic protein-2 (BMP-2) and vascular endothelial growth factor (VEGF) transfection to human periosteal cells enhances osteoblast differentiation and bone formation. J Pharmacol Sci 2008;108(1):18-31.

645. Albrektsson T. Repair of bone grafts. A vital microscopic and histological investigation in the rabbit. Scand J Plast Reconstr Surg 1980;14(1):1-12. 
646. Albrektsson T. In vivo studies of bone grafts. The possibility of vascular anastomoses in healing bone. Acta Orthop Scand 1980;51(1):9-17.

647. Fang TD, Salim A, Xia W, Nacamuli RP, Guccione S, Song HM, Carano RA, Filvaroff EH, Bednarski MD, Giaccia AJ, Longaker MT. Angiogenesis is required for successful bone induction during distraction osteogenesis. J Bone Miner Res 2005;20(7):1114-1124.

648. Fleming JT, Barati MT, Beck DJ, Dodds JC, Malkani AL, Parameswaran D, Soukhova GK, Voor MJ, Feitelson JB. Bone blood flow and vascular reactivity. Cells Tissues Organs 2001;169(3):279284.

649. Lehr HA. Microcirculatory dysfunction induced by cigarette smoking. Microcirculation 2000;7(6 Pt 1):367-384.

650. Ma L, Zheng LW, Cheung LK. Inhibitory effect of nicotine on bone regeneration in mandibular distraction osteogenesis. Front Biosci 2007;12:3256-3262.

651. Ma L, Zheng LW, Sham MH, Cheung LK. Uncoupled angiogenesis and osteogenesis in nicotine-compromised bone healing. J Bone Miner Res 2010;25(6):1305-1313.

652. Wang Y, Wan C, Deng L, Liu X, Cao X, Gilbert SR, Bouxsein ML, Faugere MC, Guldberg RE, Gerstenfeld LC, Haase VH, Johnson RS, Schipani E, Clemens TL. The hypoxia-inducible factor alpha pathway couples angiogenesis to osteogenesis during skeletal development. $J$ Clin Invest 2007;117(6):1616-1626.

653. Kon T, Cho TJ, Aizawa T, Yamazaki M, Nooh N, Graves D, Gerstenfeld LC, Einhorn TA. Expression of osteoprotegerin, receptor activator of NF-kappaB ligand (osteoprotegerin ligand) and related proinflammatory cytokines during fracture healing. J Bone Miner Res 2001;16(6):1004-1014.

654. Keramaris NC, Calori GM, Nikolaou VS, Schemitsch EH, Giannoudis PV. Fracture vascularity and bone healing: a systematic review of the role of VEGF. Injury 2008;39 Suppl 2:S45-57.

655. Mountziaris PM, Mikos AG. Modulation of the inflammatory response for enhanced bone tissue regeneration. Tissue Eng Part B Rev 2008;14(2):179-186.

656. Geng Y, Savage SM, Razani-Boroujerdi S, Sopori ML. Effects of nicotine on the immune response. II. Chronic nicotine treatment induces T cell anergy. J Immunol 1996;156(7):2384-2390.

657. Balshe AA, Eckert SE, Koka S, Assad DA, Weaver AL. The effects of smoking on the survival of smooth- and rough-surface dental implants. Int J Oral Maxillofac Implants 2008;23(6):1117-1122. 
658. Sayardoust S, Gröndahl K, Johansson E, Thomsen P, Slotte C. Implant survival and marginal bone loss at turned and oxidized implants in periodontitis-susceptible smokers and never-smokers: a retrospective, clinical, radiographic case-control study. J Periodontol 2013;84(12):1775-1782.

659. Kan JY, Rungcharassaeng K, Lozada JL, Goodacre CJ. Effects of smoking on implant success in grafted maxillary sinuses. J Prosthet Dent 1999;82(3):307-311.

660. Kan JY, Rungcharassaeng K, Kim J, Lozada JL, Goodacre CJ. Factors affecting the survival of implants placed in grafted maxillary sinuses: a clinical report. J Prosthet Dent 2002;87(5):485-489.

661. Johnson GK, Slach NA. Impact of tobacco use on periodontal status. $J$ Dent Educ 2001;65(4):313-321.

662. Oates TW, Caraway D, Jones J. Relation between smoking and biomarkers of bone resorption associated with dental endosseous implants. Implant Dent 2004;13(4):352-357.

663. Talonpoika JT, Hamalainen MM. Type I collagen carboxyterminal telopeptide in human gingival crevicular fluid in different clinical conditions and after periodontal treatment. J Clin Periodontol 1994;21(5):320-326.

664. Bergström J, Keilani H, Lundholm C, Radestad U. Smokeless tobacco (snuff) use and periodontal bone loss. J Clin Periodontol 2006;33(8):549-554.

665. Holm H, Jarvis MJ, Russell MA, Feyerabend C. Nicotine intake and dependence in Swedish snuff takers. Psychopharmacology (Berl) 1992;108(4):507-511.

666. Fant RV, Henningfield JE, Nelson RA, Pickworth WB.

Pharmacokinetics and pharmacodynamics of moist snuff in humans. Tob Control 1999;8(4):387-392.

667. Beckett AH, Triggs EJ. Buccal absorption of basic drugs and its application as an in vivo model of passive drug transfer through lipid membranes. J Pharm Pharmacol 1967;19:Suppl:31S-41S.

668. Nair MK, Chetty DJ, Ho H, Chien YW. Biomembrane permeation of nicotine: mechanistic studies with porcine mucosae and skin. J Pharm Sci 1997;86(2):257-262.

669. Hirsch JM, Heyden G, Thilander H. A clinical, histomorphological and histochemical study on snuff-induced lesions of varying severity. J Oral Pathol 1982;11(5):387-398.

670. Grady D, Greene J, Daniels TE, Ernster VL, Robertson PB, Hauck W, Greenspan D, Greenspan J, Silverman S, Jr. Oral mucosal lesions found in smokeless tobacco users. J Am Dent Assoc 1990;121(1):117-123. 
671. Kaugars GE, Brandt RB, Chan W, Carcaise-Edinboro P. Evaluation of risk factors in smokeless tobacco-associated oral lesions. Oral Surg Oral Med Oral Pathol 1991;72(3):326-331.

672. Larsson A, Axell T, Andersson G. Reversibility of snuff dippers' lesion in Swedish moist snuff users: a clinical and histologic follow-up study. $J$ Oral Pathol Med 1991;20(6):258-264.

673. Little SJ, Stevens VJ, LaChance PA, Severson HH, Bartley MH, Lichtenstein E, Leben JR. Smokeless tobacco habits and oral mucosal lesions in dental patients. J Public Health Dent 1992;52(5):269-276.

674. Andersson G, Axell T. Clinical appearance of lesions associated with the use of loose and portion-bag packed Swedish moist snuff: a comparative study. J Oral Pathol Med 1989;18(1):2-7.

675. Andersson G, Axell T, Larsson A. Clinical classification of Swedish snuff dippers' lesions supported by histology. J Oral Pathol Med 1991;20(6):253-257.

676. Hugoson A, Rolandsson M. Periodontal disease in relation to smoking and the use of Swedish snus: epidemiological studies covering 20 years (1983-2003). J Clin Periodontol 2011;38(9):809-816.

677. Anand PS, Kamath KP, Bansal A, Dwivedi S, Anil S. Comparison of periodontal destruction patterns among patients with and without the habit of smokeless tobacco use--a retrospective study. J Periodontal Res 2013;48(5):623-631.

678. Rugh JD, Ohrbach R. Occlusal parafunction. In: Mohl N, Zarb GA, Carlsson GE, Rugh JD, editors. A Textbook of Occlusion. Chicago: Quintessence; 1988. pp. 249-261.

679. Tosun T, Karabuda C, Cuhadaroglu C. Evaluation of sleep bruxism by polysomnographic analysis in patients with dental implants. Int J Oral Maxillofac Implants 2003;18(2):286-292.

680. Kaptein ML, De Putter C, De Lange GL, Blijdorp PA. A clinical evaluation of 76 implant-supported superstructures in the composite grafted maxilla. J Oral Rehabil 1999;26(8):619-623.

681. Becker W, Becker BE, Newman MG, Nyman S. Clinical and microbiologic findings that may contribute to dental implant failure. Int J Oral Maxillofac Implants 1990;5(1):31-38.

682. Lobbezoo F, Brouwers JE, Cune MS, Naeije M. Dental implants in patients with bruxing habits. J Oral Rehabil 2006;33(2):152-159.

683. Ibañez JC, Tahhan MJ, Zamar JA, Menendez AB, Juaneda AM, Zamar NJ, Monqaut JL. Immediate occlusal loading of double acidetched surface titanium implants in 41 consecutive full-arch cases in the mandible and maxilla: 6- to 74-month results. J Periodontol 2005;76(11):1972-1981. 
684. Meyer G, Fanghanel J, Proff P. Morphofunctional aspects of dental implants. Ann Anat 2012;194(2):190-194.

685. Hämmerle CH, Wagner D, Brägger U, Lussi A, Karayiannis A, Joss A, Lang NP. Threshold of tactile sensitivity perceived with dental endosseous implants and natural teeth. Clin Oral Implants Res 1995;6(2):83-90.

686. Hsu YT, Fu JH, Al-Hezaimi K, Wang HL. Biomechanical implant treatment complications: a systematic review of clinical studies of implants with at least 1 year of functional loading. Int J Oral Maxillofac Implants 2012;27(4):894-904.

687. Kim Y, Oh TJ, Misch CE, Wang HL. Occlusal considerations in implant therapy: clinical guidelines with biomechanical rationale. Clin Oral Implants Res 2005;16(1):26-35.

688. Brunski JB. In vivo bone response to biomechanical loading at the bone/dental-implant interface. Adv Dent Res 1999;13:99-119.

689. Miyata T, Kobayashi Y, Araki H, Ohto T, Shin K. The influence of controlled occlusal overload on peri-implant tissue. Part 3: A histologic study in monkeys. Int J Oral Maxillofac Implants 2000;15(3):425-431.

690. Duyck J, Ronold HJ, Van Oosterwyck H, Naert I, Vander Sloten J, Ellingsen JE. The influence of static and dynamic loading on marginal bone reactions around osseointegrated implants: an animal experimental study. Clin Oral Implants Res 2001;12(3):207-218.

691. Duyck J, Vandamme K. The effect of loading on peri-implant bone: a critical review of the literature. J Oral Rehabil 2014;41(10):783-794.

692. Lavigne GJ, Manzini C. Bruxism. In: Kryger MH, Roth T, Dement WC, editors. Principles and Practice of Sleep Medicine. 3rd ed. Philadelphia: Saunders; 1999. pp. 773 -785.

693. Glaros AG. Incidence of diurnal and nocturnal bruxism. J Prosthet Dent 1981;45(5):545-549.

694. Johansson A, Omar R, Carlsson GE. Bruxism and prosthetic treatment: a critical review. J Prosthodont Res 2011;55(3):127-136.

695. Naert I, Quirynen M, van Steenberghe D, Darius P. A study of 589 consecutive implants supporting complete fixed prostheses. Part II: Prosthetic aspects. J Prosthet Dent 1992;68(6):949-956.

696. Bischof M, Nedir R, Abi Najm S, Szmukler-Moncler S, Samson J. A five-year life-table analysis on wide neck ITI implants with prosthetic evaluation and radiographic analysis: results from a private practice. Clin Oral Implants Res 2006;17(5):512-520.

697. Schneider D, Witt L, Hämmerle CH. Influence of the crown-to-implant length ratio on the clinical performance of implants supporting single crown restorations: a cross-sectional retrospective 5-year investigation. Clin Oral Implants Res 2012;23(2):169-174. 
698. Lobbezoo F, Ahlberg J, Glaros AG, Kato T, Koyano K, Lavigne GJ, de Leeuw R, Manfredini D, Svensson P, Winocur E. Bruxism defined and graded: an international consensus. J Oral Rehabil 2013;40(1):2-4.

699. Paesani DA, Lobbezoo F, Gelos C, Guarda-Nardini L, Ahlberg J, Manfredini D. Correlation between self-reported and clinically based diagnoses of bruxism in temporomandibular disorders patients. J Oral Rehabil 2013;40(11):803-809.

700. AASM. International Classification of Sleep Disorders, Revised: Diagnostic and Coding Manual. 3rd ed. Chicago: American Academy of Sleep Medicine; 2014.

701. Treede RD, Jensen TS, Campbell JN, Cruccu G, Dostrovsky JO, Griffin JW, Hansson P, Hughes R, Nurmikko T, Serra J. Neuropathic pain: redefinition and a grading system for clinical and research purposes. Neurology 2008;70(18):1630-1635.

702. Demenko V, Linetskiy I, Nesvit K, Shevchenko A. Ultimate masticatory force as a criterion in implant selection. J Dent Res 2011;90(10):12111215 .

703. McCoy G. Recognizing and managing parafunction in the reconstruction and maintenance of the oral implant patient. Implant Dent 2002;11(1):19-27.

704. Zitzmann NU, Berglundh T. Definition and prevalence of peri-implant diseases. J Clin Periodontol 2008;35(8 Suppl):286-291.

705. Pontoriero R, Tonelli MP, Carnevale G, Mombelli A, Nyman SR, Lang NP. Experimentally induced peri-implant mucositis. A clinical study in humans. Clin Oral Implants Res 1994;5(4):254-259.

706. Salvi GE, Aglietta M, Eick S, Sculean A, Lang NP, Ramseier CA. Reversibility of experimental peri-implant mucositis compared with experimental gingivitis in humans. Clin Oral Implants Res 2012;23(2):182-190.

707. Renvert S, Roos-Jansaker AM, Claffey N. Non-surgical treatment of peri-implant mucositis and peri-implantitis: a literature review. J Clin Periodontol 2008;35(8 Suppl):305-315.

708. Klinge B, Meyle J. Peri-implant tissue destruction. The Third EAO Consensus Conference 2012. Clin Oral Implants Res 2012;23 Suppl 6:108-110.

709. Lang NP, Berglundh T. Periimplant diseases: where are we now?-Consensus of the Seventh European Workshop on Periodontology. $J$ Clin Periodontol 2011;38 Suppl 11:178-181.

710. Ramanauskaite A, Tervonen T. The Efficacy of Supportive Peri-Implant Therapies in Preventing Peri-Implantitis and Implant Loss: a Systematic Review of the Literature. J Oral Maxillofac Res 2016;7(3):e12. 
711. Costa FO, Takenaka-Martinez S, Cota LO, Ferreira SD, Silva GL, Costa JE. Peri-implant disease in subjects with and without preventive maintenance: a 5-year follow-up. J Clin Periodontol 2012;39(2):173181.

712. Coli P, Christiaens V, Sennerby L, Bruyn H. Reliability of periodontal diagnostic tools for monitoring peri-implant health and disease. Periodontol 2000 2017;73(1):203-217.

713. Aguirre-Zorzano LA, Vallejo-Aisa FJ, Estefania-Fresco R. Supportive periodontal therapy and periodontal biotype as prognostic factors in implants placed in patients with a history of periodontitis. Med Oral Patol Oral Cir Bucal 2013;18(5):e786-792.

714. Rinke S, Ohl S, Ziebolz D, Lange K, Eickholz P. Prevalence of periimplant disease in partially edentulous patients: a practice-based cross-sectional study. Clin Oral Implants Res 2011;22(8):826-833.

715. Heitz-Mayfield LJ, Mombelli A. The therapy of peri-implantitis: a systematic review. Int J Oral Maxillofac Implants 2014;29 Suppl:325-345.

716. Hirschfeld L, Wasserman B. A long-term survey of tooth loss in 600 treated periodontal patients. J Periodontol 1978;49(5):225-237.

717. Becker W, Becker BE, Berg LE. Periodontal treatment without maintenance. A retrospective study in 44 patients. J Periodontol 1984;55(9):505-509.

718. Becker W, Berg L, Becker BE. The long term evaluation of periodontal treatment and maintenance in 95 patients. Int J Periodontics Restorative Dent 1984;4(2):54-71.

719. Lindhe J, Nyman S. Long-term maintenance of patients treated for advanced periodontal disease. J Clin Periodontol 1984;11(8):504-514.

720. Tønnesen H, Kehlet H. Preoperative alcoholism and postoperative morbidity. Br J Surg 1999;86(7):869-874.

721. Chen Y, Gao H, Yin Q, Chen L, Dong P, Zhang X, Kang J. ER stress activating ATF4/CHOP-TNF-alpha signaling pathway contributes to alcohol-induced disruption of osteogenic lineage of multipotential mesenchymal stem cell. Cell Physiol Biochem 2013;32(3):743-754.

722. Diamond T, Stiel D, Lunzer M, Wilkinson M, Posen S. Ethanol reduces bone formation and may cause osteoporosis. Am J Med 1989;86(3):282-288.

723. Wang Y, Li Y, Mao K, Li J, Cui Q, Wang GJ. Alcohol-induced adipogenesis in bone and marrow: a possible mechanism for osteonecrosis. Clin Orthop Relat Res 2003;(410):213-224. 
724. Wahl EC, Aronson J, Liu L, Liu Z, Perrien DS, Skinner RA, Badger TM, Ronis MJ, Lumpkin CK, Jr. Chronic ethanol exposure inhibits distraction osteogenesis in a mouse model: role of the TNF signaling axis. Toxicol Appl Pharmacol 2007;220(3):302-310.

725. Tønnesen H, Pedersen A, Jensen MR, Moller A, Madsen JC. Ankle fractures and alcoholism. The influence of alcoholism on morbidity after malleolar fractures. J Bone Joint Surg Br 1991;73(3):511-513.

726. Gonzalez-Calvín JL, Garcia-Sanchez A, Bellot V, Muñoz-Torres M, Raya-Alvarez E, Salvatierra-Rios D. Mineral metabolism, osteoblastic function and bone mass in chronic alcoholism. Alcohol Alcohol 1993;28(5):571-579.

727. Klein RF, Fausti KA, Carlos AS. Ethanol inhibits human osteoblastic cell proliferation. Alcohol Clin Exp Res 1996;20(3):572-578.

728. Turner RT, Wronski TJ, Zhang M, Kidder LS, Bloomfield SA, Sibonga JD. Effects of ethanol on gene expression in rat bone: transient dosedependent changes in mRNA levels for matrix proteins, skeletal growth factors, and cytokines are followed by reductions in bone formation. Alcohol Clin Exp Res 1998;22(7):1591-1599.

729. Farley JR, Fitzsimmons R, Taylor AK, Jorch UM, Lau KH. Direct effects of ethanol on bone resorption and formation in vitro. Arch Biochem Biophys 1985;238(1):305-314.

730. Friday KE, Howard GA. Ethanol inhibits human bone cell proliferation and function in vitro. Metabolism 1991;40(6):562-565.

731. Chavassieux P, Serre CM, Vergnaud P, Delmas PD, Meunier PJ. In vitro evaluation of dose-effects of ethanol on human osteoblastic cells. Bone Miner 1993;22(2):95-103.

732. Klein RF, Carlos AS. Inhibition of osteoblastic cell proliferation and ornithine decarboxylase activity by ethanol. Endocrinology 1995;136(8):3406-3411.

733. Dyer SA, Buckendahl P, Sampson HW. Alcohol consumption inhibits osteoblastic cell proliferation and activity in vivo. Alcohol 1998;16(4):337-341.

734. Torricelli P, Fini M, Giavaresi G, Borsari V, Rimondini L, Rimondini R, Carrassi A, Giardino R. Intermittent exposure to ethanol vapor affects osteoblast behaviour more severely than estrogen deficiency does in vitro study on rat osteoblasts. Toxicology 2007;237(1-3):168-176.

735. Rosa ML, Beloti MM, Prando N, Queiroz RH, de Oliveira PT, Rosa AL. Chronic ethanol intake inhibits in vitro osteogenesis induced by osteoblasts differentiated from stem cells. J Appl Toxicol 2008;28(2):205-211. 
736. Torricelli P, Fini M, Giavaresi G, Rimondini L, Tschon M, Rimondini R, Carrassi A, Giardino R. Chronic alcohol abuse and endosseous implants: linkage of in vitro osteoblast dysfunction to titanium osseointegration rate. Toxicology 2008;243(1-2):138-144.

737. Johnell O, Nilsson BE, Wiklund PE. Bone morphometry in alcoholics. Clin Orthop Relat Res 1982;(165):253-258.

738. Cheung RC, Gray C, Boyde A, Jones SJ. Effects of ethanol on bone cells in vitro resulting in increased resorption. Bone 1995;16(1):143147.

739. Trevisiol CH, Turner RT, Pfaff JE, Hunter JC, Menagh PJ, Hardin K, Ho E, Iwaniec UT. Impaired osteoinduction in a rat model for chronic alcohol abuse. Bone 2007;41(2):175-180.

740. Watson RR, Borgs P, Witte M, McCuskey RS, Lantz C, Johnson MI, Mufti SI, Earnest DL. Alcohol, immunomodulation, and disease. Alcohol Alcohol 1994;29(2):131-139.

741. Chiappelli F, Kung M, Lee P, Pham L, Manfrini E, Villanueva P. Alcohol modulation of human normal T-cell activation, maturation, and migration. Alcohol Clin Exp Res 1995;19(3):539-544.

742. Bombonato-Prado KF, Brentegani LG, Thomazini JA, Lachat JJ, Carvalho TL. Alcohol intake and osseointegration around implants: a histometric and scanning electron microscopy study. Implant Dent 2004;13(3):238-244.

743. Koo S, Konig B, Jr., Mizusaki CI, Allegrini S, Jr., Yoshimoto M, Carbonari MJ. Effects of alcohol consumption on osseointegration of titanium implants in rabbits. Implant Dent 2004;13(3):232-237.

744. Galindo-Moreno P, Fauri M, Avila-Ortiz G, Fernandez-Barbero JE, Cabrera-Leon A, Sanchez-Fernandez E. Influence of alcohol and tobacco habits on peri-implant marginal bone loss: a prospective study. Clin Oral Implants Res 2005;16(5):579-586.

745. Alissa R, Oliver RJ. Influence of prognostic risk indicators on osseointegrated dental implant failure: a matched case-control analysis. J Oral Implantol 2012;38(1):51-61.

746. de Deco CP, da Silva Marchini AM, Barbara MA, de Vasconcellos LM, da Rocha RF, Marchini L. Negative effects of alcohol intake and estrogen deficiency combination on osseointegration in a rat model. $J$ Oral Implantol 2011;37(6):633-639.

747. Mangione MP, Crowley-Matoka M. Improving pain management communication: how patients understand the terms "opioid" and “narcotic”. J Gen Intern Med 2008;23(9):1336-1338.

748. Daniell HW. Opioid osteoporosis. Arch Intern Med 2004;164(3):338; author reply 338 . 
749. Gordon CM, Nelson LM. Amenorrhea and bone health in adolescents and young women. Curr Opin Obstet Gynecol 2003;15(5):377-384.

750. Gordon CM. Clinical practice. Functional hypothalamic amenorrhea. N Engl J Med 2010;363(4):365-371.

751. Meczekalski B, Podfigurna-Stopa A, Genazzani AR. Hypoestrogenism in young women and its influence on bone mass density. Gynecol Endocrinol 2010;26(9):652-657.

752. Merza Z. Chronic use of opioids and the endocrine system. Horm Metab Res 2010;42(9):621-626.

753. Reddy RG, Aung T, Karavitaki N, Wass JA. Opioid induced hypogonadism. Bmi 2010;341:c4462.

754. Vuong C, Van Uum SH, O’Dell LE, Lutfy K, Friedman TC. The effects of opioids and opioid analogs on animal and human endocrine systems. Endocr Rev 2010;31(1):98-132.

755. Elhassan AM, Lindgren JU, Hultenby K, Bergstrom J, Adem A. Methionine-enkephalin in bone and joint tissues. J Bone Miner Res 1998;13(1):88-95.

756. Perez-Castrillon JL, Olmos JM, Gomez JJ, Barrallo A, Riancho JA, Perera L, Valero C, Amado JA, Gonzalez-Macias J. Expression of opioid receptors in osteoblast-like MG-63 cells, and effects of different opioid agonists on alkaline phosphatase and osteocalcin secretion by these cells. Neuroendocrinology 2000;72(3):187-194.

757. Daniell HW. Hypogonadism in men consuming sustained-action oral opioids. J Pain 2002;3(5):377-384.

758. Wilczek H, Stepan J. [Bone metabolism in individuals dependent on heroin and after methadone administration]. Cas Lek Cesk 2003;142(10):606-608.

759. Kinjo M, Setoguchi S, Schneeweiss S, Solomon DH. Bone mineral density in subjects using central nervous system-active medications. Am J Med 2005;118(12):1414.

760. Pedrazzoni M, Vescovi PP, Maninetti L, Michelini M, Zaniboni G, Pioli G, Costi D, Alfano FS, Passeri M. Effects of chronic heroin abuse on bone and mineral metabolism. Acta Endocrinol (Copenh) 1993;129(1):42-45.

761. Kim TW, Alford DP, Malabanan A, Holick MF, Samet JH. Low bone density in patients receiving methadone maintenance treatment. Drug Alcohol Depend 2006;85(3):258-262.

762. Di Marzo V, Bifulco M, De Petrocellis L. The endocannabinoid system and its therapeutic exploitation. Nat Rev Drug Discov 2004;3(9):771784 . 
763. Idris AI, van 't Hof RJ, Greig IR, Ridge SA, Baker D, Ross RA, Ralston SH. Regulation of bone mass, bone loss and osteoclast activity by cannabinoid receptors. Nat Med 2005;11(7):774-779.

764. Ofek O, Karsak M, Leclerc N, Fogel M, Frenkel B, Wright K, Tam J, Attar-Namdar M, Kram V, Shohami E, Mechoulam R, Zimmer A, Bab I. Peripheral cannabinoid receptor, CB2, regulates bone mass. Proc Natl Acad Sci U S A 2006;103(3):696-701.

765. Whyte LS, Ryberg E, Sims NA, Ridge SA, Mackie K, Greasley PJ, Ross RA, Rogers MJ. The putative cannabinoid receptor GPR55 affects osteoclast function in vitro and bone mass in vivo. Proc Natl Acad Sci U S A 2009;106(38):16511-16516.

766. Whyte LS, Ford L, Ridge SA, Cameron GA, Rogers MJ, Ross RA. Cannabinoids and bone: endocannabinoids modulate human osteoclast function in vitro. Br J Pharmacol 2012;165(8):2584-2597.

767. Bourne D, Plinke W, Hooker ER, Nielson CM. Cannabis use and bone mineral density: NHANES 2007-2010. Arch Osteoporos 2017;12(1):29.

768. Sophocleous A, Robertson R, Ferreira NB, McKenzie J, Fraser WD, Ralston SH. Heavy Cannabis Use Is Associated With Low Bone Mineral Density and an Increased Risk of Fractures. Am J Med 2017;130(2):214-221.

769. Balabanova S, Nowak R, Reinhardt G. [The role of calcium homeostasis hormones in the development of tolerance following drug and alcohol consumption]. Beitr Gerichtl Med 1989;47:379-383.

770. Rico H, Costales C, Cabranes JA, Escudero M. Lower serum osteocalcin levels in pregnant drug users and their newborns at the time of delivery. Obstet Gynecol 1990;75(6):998-1000.

771. Beresford JN, Gallagher JA, Poser JW, Russell RG. Production of osteocalcin by human bone cells in vitro. Effects of $1,25(\mathrm{OH}) 2 \mathrm{D} 3$, 24,25(OH)2D3, parathyroid hormone, and glucocorticoids. Metab Bone Dis Relat Res 1984;5(5):229-234.

772. Ohta T, Mori M, Ogawa K, Matsuyama T, Ishii S. Immunocytochemical localization of BGP in human bones in various developmental stages and pathological conditions. Virchows Arch A Pathol Anat Histopathol 1989;415(5):459-466.

773. Price PA, Otsuka AA, Poser JW, Kristaponis J, Raman N. Characterization of a gamma-carboxyglutamic acid-containing protein from bone. Proc Natl Acad Sci U S A 1976;73(5):1447-1451.

774. Price PA, Nishimoto SK. Radioimmunoassay for the vitamin K-dependent protein of bone and its discovery in plasma. Proc Natl Acad Sci U S A 1980;77(4):2234-2238. 
775. Seifert MF, Church MW. Long term effects of prenatal cocaine exposure on bone in rats. Life Sci 1991;49(8):569-574.

776. Ekfeldt A, Christiansson U, Eriksson T, Linden U, Lundqvist S, Rundcrantz T, Johansson LA, Nilner K, Billstrom C. A retrospective analysis of factors associated with multiple implant failures in maxillae. Clin Oral Implants Res 2001;12(5):462-467.

777. Nogueira-Filho Gda R, Cadide T, Rosa BT, Neiva TG, Tunes R, Peruzzo D, Nociti FH, Jr., Cesar-Neto JB. Cannabis sativa smoke inhalation decreases bone filling around titanium implants: a histomorphometric study in rats. Implant Dent 2008;17(4):461-470.

778. Nogueira-Filho GR, Todescan S, Shah A, Rosa BT, Tunes Uda R, Cesar Neto JB. Impact of cannabis sativa (marijuana) smoke on alveolar bone loss: a histometric study in rats. J Periodontol 2011;82(11):1602-1607.

779. Breivik T, Bogen IL, Haug KH, Fonnum F, Opstad PK, Eide DM, Myhre O. Effects of long-term exposure of 3,4-methylenedioxymethamphetamine (MDMA; “ecstasy") on neuronal transmitter transport, brain immuno-regulatory systems and progression of experimental periodontitis in rats. Neurochem Int 2014;72:30-36.

780. Montes CC, Alvim-Pereira F, de Castilhos BB, Sakurai ML, Olandoski M, Trevilatto PC. Analysis of the association of IL1B (C+3954T) and IL1RN (intron 2) polymorphisms with dental implant loss in a Brazilian population. Clin Oral Implants Res 2009;20(2):208-217.

781. Vaz P, Gallas MM, Braga AC, Sampaio-Fernandes JC, Felino A, Tavares P. IL1 gene polymorphisms and unsuccessful dental implants. Clin Oral Implants Res 2012;23(12):1404-1413.

782. Passarge E. Color Atlas of Genetics. 3rd ed. Stuttgart: Thieme; 2007.

783. Malo D, Skamene E. Genetic control of host resistance to infection. Trends Genet 1994;10(10):365-371.

784. Le Souef PN, Goldblatt J, Lynch NR. Evolutionary adaptation of inflammatory immune responses in human beings. Lancet 2000;356(9225):242-244.

785. Woo P. Cytokine polymorphisms and inflammation. Clin Exp Rheumatol 2000;18(6):767-771.

786. Taba M, Jr., Kinney J, Kim AS, Giannobile WV. Diagnostic biomarkers for oral and periodontal diseases. Dent Clin North Am 2005;49(3):551-571, vi.

787. Trindade R, Albrektsson T, Wennerberg A. Current concepts for the biological basis of dental implants: foreign body equilibrium and osseointegration dynamics. Oral Maxillofac Surg Clin North Am 2015;27(2):175-183. 
788. Trindade R, Albrektsson T, Tengvall P, Wennerberg A. Foreign Body Reaction to Biomaterials: On Mechanisms for Buildup and Breakdown of Osseointegration. Clin Implant Dent Relat Res 2016;18(1):192-203.

789. Page RC. The role of inflammatory mediators in the pathogenesis of periodontal disease. J Periodontal Res 1991;26(3 Pt 2):230-242.

790. Feloutzis A, Lang NP, Tonetti MS, Burgin W, Bragger U, Buser D, Duff GW, Kornman KS. IL-1 gene polymorphism and smoking as risk factors for peri-implant bone loss in a well-maintained population. Clin Oral Implants Res 2003;14(1):10-17.

791. Gruica B, Wang HY, Lang NP, Buser D. Impact of IL-1 genotype and smoking status on the prognosis of osseointegrated implants. Clin Oral Implants Res 2004;15(4):393-400.

792. Laine ML, Leonhardt A, Roos-Jansaker AM, Pena AS, van Winkelhoff AJ, Winkel EG, Renvert S. IL-1RN gene polymorphism is associated with peri-implantitis. Clin Oral Implants Res 2006;17(4):380-385.

793. Petkovic AB, Matic SM, Stamatovic NV, Vojvodic DV, Todorovic TM, Lazic ZR, Kozomara RJ. Proinflammatory cytokines (IL-1 beta and TNF-alpha) and chemokines (IL-8 and MIP-1alpha) as markers of periimplant tissue condition. Int J Oral Maxillofac Surg 2010;39(5):478485.

794. Shimpuku H, Nosaka Y, Kawamura T, Tachi Y, Shinohara M, Ohura K. Genetic polymorphisms of the interleukin-1 gene and early marginal bone loss around endosseous dental implants. Clin Oral Implants Res 2003;14(4):423-429.

795. Wilson TG, Jr., Nunn M. The relationship between the interleukin-1 periodontal genotype and implant loss. Initial data. J Periodontol 1999;70(7):724-729.

796. Rogers MA, Figliomeni L, Baluchova K, Tan AE, Davies G, Henry PJ, Price P. Do interleukin-1 polymorphisms predict the development of periodontitis or the success of dental implants? J Periodontal Res 2002;37(1):37-41.

797. Nosaka Y, Tachi Y, Shimpuku H, Kawamura T, Ohura K. Association of calcitonin receptor gene polymorphism with early marginal bone loss around endosseous implants. Int J Oral Maxillofac Implants 2002;17(1):38-43.

798. Shimpuku H, Nosaka Y, Kawamura T, Tachi Y, Shinohara M, Ohura $\mathrm{K}$. Bone morphogenetic protein-4 gene polymorphism and early marginal bone loss around endosseous implants. Int J Oral Maxillofac Implants 2003;18(4):500-504.

799. Santos MC, Campos MI, Souza AP, Trevilatto PC, Line SR. Analysis of MMP-1 and MMP-9 promoter polymorphisms in early osseointegrated implant failure. Int J Oral Maxillofac Implants 2004;19(1):38-43. 
800. Leite MF, Santos MC, de Souza AP, Line SR. Osseointegrated implant failure associated with MMP-1 promotor polymorphisms (-1607 and -519). Int J Oral Maxillofac Implants 2008;23(4):653-658.

801. Casado PL, Aguiar DP, Costa LC, Fonseca MA, Vieira TC, AlvimPereira CC, Alvim-Pereira F, Deeley K, Granjeiro JM, Trevilatto PC, Vieira AR. Different contribution of BRINP3 gene in chronic periodontitis and peri-implantitis: a cross-sectional study. BMC Oral Health 2015;15:33.

802. Dos Santos MC, Campos MI, Souza AP, Scarel-Caminaga RM, Mazzonetto R, Line SR. Analysis of the transforming growth factorbeta 1 gene promoter polymorphisms in early osseointegrated implant failure. Implant Dent 2004;13(3):262-269.

803. Cury PR, Joly JC, Freitas N, Sendyk WR, Nunes FD, de Araujo NS. Effect of tumor necrosis factor-alpha gene polymorphism on periimplant bone loss following prosthetic reconstruction. Implant Dent 2007;16(1):80-88.

804. Dereka X, Mardas N, Chin S, Petrie A, Donos N. A systematic review on the association between genetic predisposition and dental implant biological complications. Clin Oral Implants Res 2012;23(7):775-788.

805. Knox D, Schacht C. Choices in Relationships: An Introduction to Marriage and the Family. 11 ed. Boston: Cengage Learning; 2012.

806. “About Biology of Sex Differences: Aims \& Scope”, Biology of Sex Differences. 2015 [cited 7 January 2015]; Available from: http://www. bsd-journal.com/about\#aimsscope

807. Marx RE. A decade of bisphosphonate bone complications: what it has taught us about bone physiology. Int J Oral Maxillofac Implants 2014;29(2):e247-258.

808. Riggs BL. The mechanisms of estrogen regulation of bone resorption. $J$ Clin Invest 2000;106(10):1203-1204.

809. Bonnick SL. Monitoring osteoporosis therapy with bone densitometry: a vital tool or regression toward mediocrity? J Clin Endocrinol Metab 2000;85(10):3493-3495.

810. Friberg B, Ekestubbe A, Mellstrom D, Sennerby L. Branemark implants and osteoporosis: a clinical exploratory study. Clin Implant Dent Relat Res 2001;3(1):50-56.

811. McGrath C, Bedi R. Measuring the impact of oral health on quality of life in Britain using OHQoL-UK(W). J Public Health Dent 2003;63(2):73-77.

812. Shiau HJ, Reynolds MA. Sex differences in destructive periodontal disease: exploring the biologic basis. J Periodontol 2010;81(11):15051517. 
813. Eke PI, Dye BA, Wei L, Thornton-Evans GO, Genco RJ. Prevalence of periodontitis in adults in the United States: 2009 and 2010. J Dent Res 2012;91(10):914-920.

814. Freitag-Wolf S, Dommisch H, Graetz C, Jockel-Schneider Y, Harks I, Staufenbiel I, Meyle J, Eickholz P, Noack B, Bruckmann C, Gieger C, Jepsen S, Lieb W, Schreiber S, Konig IR, Schaefer AS. Genome-wide exploration identifies sex-specific genetic effects of alleles upstream NPY to increase the risk of severe periodontitis in men. J Clin Periodontol 2014;41(12):1115-1121.

815. Becker ST, Beck-Broichsitter BE, Graetz C, Dorfer CE, Wiltfang J, Hasler R. Peri-implantitis versus periodontitis: functional differences indicated by transcriptome profiling. Clin Implant Dent Relat Res 2014;16(3):401-411.

816. Lee DW. Validated surrogate endpoints needed for peri-implantitis. Evid Based Dent 2011;12(1):7.

817. Mendoza AR, Newcomb GM, Nixon KC. Compliance with supportive periodontal therapy. J Periodontol 1991;62(12):731-736.

818. Checchi L, Pelliccioni GA, Gatto MR, Kelescian L. Patient compliance with maintenance therapy in an Italian periodontal practice. J Clin Periodontol 1994;21(5):309-312.

819. Novaes AB, Jr., de Lima FR, Novaes AB. Compliance with supportive periodontal therapy and its relation to the bleeding index. $J$ Periodontol 1996;67(10):976-980.

820. Ojima M, Hanioka T, Shizukuishi S. Survival analysis for degree of compliance with supportive periodontal therapy. J Clin Periodontol 2001;28(12):1091-1095.

821. Frisch E, Ziebolz D, Vach K, Ratka-Kruger P. Supportive post-implant therapy: patient compliance rates and impacting factors: 3-year followup. J Clin Periodontol 2014;41(10):1007-1014.

822. Demetriou N, Tsami-Pandi A, Parashis A. Compliance with supportive periodontal treatment in private periodontal practice. A 14-year retrospective study. J Periodontol 1995;66(2):145-149.

823. Demirel K, Efeodlu A. Retrospective evaluation of patient compliance with supportive periodontal treatment. J Nihon Univ Sch Dent 1995;37(3):131-137.

824. Soolari A, Rokn AR. Adherence to periodontal maintenance in Tehran, Iran. A 7-year retrospective study. Quintessence Int 2003;34(3):215219.

825. Famili P, Short E. Compliance with periodontal maintenance at the University of Pittsburgh: Retrospective analysis of 315 cases. Gen Dent 2010;58(1):e42-47. 
826. Gokulanathan S, Balan N, Aravind RJ, Thangavelu K. Patient compliance and supportive periodontal therapy: Study among young adults of Namakkal district. J Pharm Bioallied Sci 2014;6(Suppl 1):S171-173.

827. Jamison CL, Bray KK, Rapley JW, Macneill SR, Williams KB. Analysis of patient factors impacting duration of periodontal maintenance appointments: an exploratory study. J Dent Hyg 2014;88(2):87-99.

828. Jamal A, Agaku IT, O’Connor E, King BA, Kenemer JB, Neff L. Current cigarette smoking among adults--United States, 2005-2013. MMWR Morb Mortal Wkly Rep 2014;63(47):1108-1112.

829. Vervaeke S, Collaert B, Cosyn J, Deschepper E, De Bruyn H. A multifactorial analysis to identify predictors of implant failure and peri-implant bone loss. Clin Implant Dent Relat Res 2015;17 Suppl 1:e298-307.

830. Statistiska centralbyrain. Alkohol-och tobaksbruk. Artikelnummer: LE114SA0701. Stockholm: Statistiska centralbyrån; 2007.

831. Chang M, Chronopoulos V, Mattheos N. Impact of excessive occlusal load on successfully-osseointegrated dental implants: a literature review. J Investig Clin Dent 2013;4(3):142-150.

832. Frost HM. Wolff's Law and bone's structural adaptations to mechanical usage: an overview for clinicians. Angle Orthod 1994;64(3):175-188.

833. Cosme DC, Baldisserotto SM, Canabarro Sde A, Shinkai RS. Bruxism and voluntary maximal bite force in young dentate adults. Int $\mathrm{J}$ Prosthodont 2005;18(4):328-332.

834. Tuxen A, Bakke M, Pinholt EM. Comparative data from young men and women on masseter muscle fibres, function and facial morphology. Arch Oral Biol 1999;44(6):509-518.

835. Manfredini D, Winocur E, Guarda-Nardini L, Paesani D, Lobbezoo F. Epidemiology of bruxism in adults: a systematic review of the literature. J Orofac Pain 2013;27(2):99-110.

836. Roccuzzo M, Bonino F, Aglietta M, Dalmasso P. Ten-year results of a three arms prospective cohort study on implants in periodontally compromised patients. Part 2: clinical results. Clin Oral Implants Res 2012;23(4):389-395.

837. Ong CT, Ivanovski S, Needleman IG, Retzepi M, Moles DR, Tonetti MS, Donos N. Systematic review of implant outcomes in treated periodontitis subjects. J Clin Periodontol 2008;35(5):438-462.

838. Apse P, Ellen RP, Overall CM, Zarb GA. Microbiota and crevicular fluid collagenase activity in the osseointegrated dental implant sulcus: a comparison of sites in edentulous and partially edentulous patients. $J$ Periodontal Res 1989;24(2):96-105. 
839. Karoussis IK, Muller S, Salvi GE, Heitz-Mayfield LJA, Bragger U, Lang NP. Association between periodontal and peri-implant conditions: a 10-year prospective study. Clin Oral Implants Res 2004;15(1):1-7.

840. Cecchinato D, Marino M, Lindhe J. Bone loss at implants and teeth in the same segment of the dentition in partially dentate subjects. Clin Oral Implants Res 2016.

841. Makris GP, Saffar JL. Disturbances in bone remodeling during the progress of hamster periodontitis. A morphological and quantitative study. J Periodontal Res 1985;20(4):411-420.

842. De Boever AL, Quirynen M, Coucke W, Theuniers G, De Boever JA. Clinical and radiographic study of implant treatment outcome in periodontally susceptible and non-susceptible patients: a prospective long-term study. Clin Oral Implants Res 2009;20(12):1341-1350.

843. Heitz-Mayfield LJ. Disease progression: identification of high-risk groups and individuals for periodontitis. J Clin Periodontol 2005;32 Suppl 6:196-209.

844. Ramseier CA. Potential impact of subject-based risk factor control on periodontitis. J Clin Periodontol 2005;32 Suppl 6:283-290.

845. Heasman L, Stacey F, Preshaw PM, McCracken GI, Hepburn S, Heasman PA. The effect of smoking on periodontal treatment response: a review of clinical evidence. J Clin Periodontol 2006;33(4):241-253.

846. Karoussis IK, Salvi GE, Heitz-Mayfield LJ, Bragger U, Hammerle $\mathrm{CH}$, Lang NP. Long-term implant prognosis in patients with and without a history of chronic periodontitis: a 10 -year prospective cohort study of the ITI Dental Implant System. Clin Oral Implants Res 2003;14(3):329-339.

847. Koldsland OC, Scheie AA, Aass AM. Prevalence of Implant Loss and the Influence of Associated Factors. J Periodontol 2009;80(7):10691075.

848. Aglietta M, Siciliano VI, Rasperini G, Cafiero C, Lang NP, Salvi GE. A 10-year retrospective analysis of marginal bone-level changes around implants in periodontally healthy and periodontally compromised tobacco smokers. Clin Oral Implants Res 2011;22(1):47-53.

849. Nitzan D, Mamlider A, Levin L, Schwartz-Arad D. Impact of smoking on marginal bone loss. Int J Oral Maxillofac Implants 2005;20(4):605609.

850. Hardt CRE, Grondahl K, Lekholm U, Wennstrom JL. Outcome of implant therapy in relation to experienced loss of periodontal bone support - A retrospective 5-year study. Clin Oral Implants Res 2002;13(5):488-494. 
851. Fardal O, Linden GJ. Tooth loss and implant outcomes in patients refractory to treatment in a periodontal practice. J Clin Periodontol 2008;35(8):733-738.

852. Matarasso S, Rasperini G, Siciliano VI, Salvi GE, Lang NP, Aglietta M. A 10-year retrospective analysis of radiographic bone-level changes of implants supporting single-unit crowns in periodontally compromised vs. periodontally healthy patients. Clin Oral Implants Res 2010;21(9):898-903.

853. Rosenberg ES, Cho SC, Elian N, Jalbout ZN, Froum S, Evian CI. A comparison of characteristics of implant failure and survival in periodontally compromised and periodontally healthy patients: a clinical report. Int J Oral Maxillofac Implants 2004;19(6):873-879.

854. Gianserra R, Cavalcanti R, Oreglia F, Manfredonia MF, Esposito M. Outcome of dental implants in patients with and without a history of periodontitis: a 5-year pragmatic multicentre retrospective cohort study of 1727 patients. Eur J Oral Implantol 2010;3(4):307-314.

855. Levin L, Ofec R, Grossmann Y, Anner R. Periodontal disease as a risk for dental implant failure over time: a long-term historical cohort study. J Clin Periodontol 2011;38(8):732-737.

856. Periodontology AAo. The pathogenesis of periodontal diseases. $J$ Periodontol 1999;70(4):457-470.

857. Evian CI, Emling R, Rosenberg ES, Waasdorp JA, Halpern W, Shah S, Garcia M. Retrospective analysis of implant survival and the influence of periodontal disease and immediate placement on long-term results. Int J Oral Maxillofac Implants 2004;19(3):393-398.

858. Rosenquist B. A comparison of various methods of soft tissue management following the immediate placement of implants into extraction sockets. Int J Oral Maxillofac Implants 1997;12(1):43-51.

859. Mombelli A, van Oosten MA, Schurch E, Jr., Land NP. The microbiota associated with successful or failing osseointegrated titanium implants. Oral Microbiol Immunol 1987;2(4):145-151.

860. George K, Zafiropoulos GG, Murat Y, Hubertus S, Nisengard RJ. Clinical and microbiological status of osseointegrated implants. $J$ Periodontol 1994;65(8):766-770.

861. Karoussis IK, Kotsovilis S, Fourmousis I. A comprehensive and critical review of dental implant prognosis in periodontally compromised partially edentulous patients. Clin Oral Implants Res 2007;18(6):669679.

862. Carcuac O, Berglundh T. Composition of human peri-implantitis and periodontitis lesions. J Dent Res 2014;93(11):1083-1088. 
863. Yerit KC, Posch M, Seemann M, Hainich S, Dortbudak O, Turhani D, Ozyuvaci H, Watzinger F, Ewers R. Implant survival in mandibles of irradiated oral cancer patients. Clin Oral Implants Res 2006;17(3):337-344.

864. Vissink A, Burlage FR, Spijkervet FK, Jansma J, Coppes RP. Prevention and treatment of the consequences of head and neck radiotherapy. Crit Rev Oral Biol Med 2003;14(3):213-225.

865. Chrcanovic BR, Reher P, Sousa AA, Harris M. Osteoradionecrosis of the jaws--a current overview--part 1: Physiopathology and risk and predisposing factors. Oral Maxillofac Surg 2010;14(1):3-16.

866. Chrcanovic BR, Reher P, Sousa AA, Harris M. Osteoradionecrosis of the jaws--a current overview--Part 2: dental management and therapeutic options for treatment. Oral Maxillofac Surg 2010;14(2):81-95.

867. Salinas TJ, Desa VP, Katsnelson A, Miloro M. Clinical evaluation of implants in radiated fibula flaps. J Oral Maxillofac Surg 2010;68(3):524-529.

868. Jacobsson M. On Bone Behaviour After Irradiation [PhD thesis]. Göteborg, Sweden: University of Göteborg; 1985.

869. Marx RE, Johnson RP. Studies in the radiobiology of osteoradionecrosis and their clinical significance. Oral Surg Oral Med Oral Pathol 1987;64(4):379-390.

870. Scully C, Epstein JB. Oral health care for the cancer patient. Eur J Cancer B Oral Oncol 1996;32b(5):281-292.

871. Granström G, Tjellström A, Albrektsson T. Postimplantation irradiation for head and neck cancer treatment. Int J Oral Maxillofac Implants 1993;8(5):495-501.

872. Larsen PE, Stronczek MJ, Beck FM, Rohrer M. Osteointegration of implants in radiated bone with and without adjunctive hyperbaric oxygen. J Oral Maxillofac Surg 1993;51(3):280-287.

873. Marx RE, Ames JR. The use of hyperbaric oxygen therapy in bony reconstruction of the irradiated and tissue-deficient patient. J Oral Maxillofac Surg 1982;40(7):412-420.

874. Johnsson K, Hansson A, Granström G, Jacobsson M, Turesson I. The effects of hyperbaric oxygenation on bone-titanium implant interface strength with and without preceding irradiation. Int J Oral Maxillofac Implants 1993;8(4):415-419.

875. Andersson G, Andreasson L, Bjelkengren G. Oral implant rehabilitation in irradiated patients without adjunctive hyperbaric oxygen. Int J Oral Maxillofac Implants 1998;13(5):647-654. 
876. Coulthard P, Esposito M, Worthington HV, Jokstad A. Interventions for replacing missing teeth: hyperbaric oxygen therapy for irradiated patients who require dental implants. Cochrane Database Syst Rev 2002;(3):Cd003603.

877. Granström G, Jacobsson M, Tjellström A. Titanium implants in irradiated tissue: benefits from hyperbaric oxygen. Int J Oral Maxillofac Implants 1992;7(1):15-25.

878. Albrektsson T, Dahl E, Enbom L, Engevall S, Engquist B, Eriksson AR, Feldmann G, Freiberg N, Glantz PO, Kjellman O, et al. Osseointegrated oral implants. A Swedish multicenter study of 8139 consecutively inserted Nobelpharma implants. J Periodontol 1988;59(5):287-296.

879. Franzén L, Rosenquist JB, Rosenquist KI, Gustafsson I. Oral implant rehabilitation of patients with oral malignancies treated with radiotherapy and surgery without adjunctive hyperbaric oxygen. Int J Oral Maxillofac Implants 1995;10(2):183-187.

880. Eckert SE, Desjardins RP, Keller EE, Tolman DE. Endosseous implants in an irradiated tissue bed. J Prosthet Dent 1996;76(1):45-49.

881. Weischer T, Schettler D, Mohr C. Concept of surgical and implantsupported prostheses in the rehabilitation of patients with oral cancer. Int J Oral Maxillofac Implants 1996;11(6):775-781.

882. Esser E, Wagner W. Dental implants following radical oral cancer surgery and adjuvant radiotherapy. Int J Oral Maxillofac Implants 1997;12(4):552-557.

883. Jisander S, Grenthe B, Alberius P. Dental implant survival in the irradiated jaw: a preliminary report. Int J Oral Maxillofac Implants 1997;12(5):643-648.

884. Keller EE, Tolman DE, Zuck SL, Eckert SE. Mandibular endosseous implants and autogenous bone grafting in irradiated tissue: a 10-year retrospective study. Int J Oral Maxillofac Implants 1997;12(6):800813.

885. Marker P, Siemssen SJ, Bastholt L. Osseointegrated implants for prosthetic rehabilitation after treatment of cancer of the oral cavity. Acta Oncol 1997;36(1):37-40.

886. Niimi A, Fujimoto T, Nosaka Y, Ueda M. A Japanese multicenter study of osseointegrated implants placed in irradiated tissues: a preliminary report. Int J Oral Maxillofac Implants 1997;12(2):259-264.

887. Roumanas ED, Markowitz BL, Lorant JA, Calcaterra TC, Jones NF, Beumer J, 3rd. Reconstructed mandibular defects: fibula free flaps and osseointegrated implants. Plast Reconstr Surg 1997;99(2):356-365. 
888. Brogniez V, Lejuste P, Pecheur A, Reychler H. Dental prosthetic reconstruction of osseointegrated implants placed in irradiated bone. Int J Oral Maxillofac Implants 1998;13(4):506-512.

889. Esser E, Neukirchen S, Wagner W. Vergleichende Untersuchungen von Brånemark-Implantaten im bestrahlten und nicht bestrahlten Unterkiefer. Mund Kiefer Gesichtschir 1999;3 Suppl 1:S125-129.

890. Bodard AG, Gourmet R, Lucas R, Bonnet E, Breton P. Implants dentaires en territoire irradié. Serié de 33 patients. Rev Stomatol Chir Maxillofac 2006;107(3):137-142; discussion 143-134.

891. Landes CA, Kovacs AF. Comparison of early telescope loading of nonsubmerged ITI implants in irradiated and non-irradiated oral cancer patients. Clin Oral Implants Res 2006;17(4):367-374.

892. Schepers RH, Slagter AP, Kaanders JH, van den Hoogen FJ, Merkx MA. Effect of postoperative radiotherapy on the functional result of implants placed during ablative surgery for oral cancer. Int J Oral Maxillofac Surg 2006;35(9):803-808.

893. Nelson K, Heberer S, Glatzer C. Survival analysis and clinical evaluation of implant-retained prostheses in oral cancer resection patients over a mean follow-up period of 10 years. J Prosthet Dent 2007;98(5):405-410.

894. Schoen PJ, Raghoebar GM, Bouma J, Reintsema H, Vissink A, Sterk W, Roodenburg JL. Rehabilitation of oral function in head and neck cancer patients after radiotherapy with implant-retained dentures: effects of hyperbaric oxygen therapy. Oral Oncol 2007;43(4):379-388.

895. Schoen PJ, Raghoebar GM, Bouma J, Reintsema H, Burlage FR, Roodenburg JL, Vissink A. Prosthodontic rehabilitation of oral function in head-neck cancer patients with dental implants placed simultaneously during ablative tumour surgery: an assessment of treatment outcomes and quality of life. Int J Oral Maxillofac Surg 2008;37(1):8-16.

896. Heberer S, Kilic S, Hossamo J, Raguse JD, Nelson K. Rehabilitation of irradiated patients with modified and conventional sandblasted acidetched implants: preliminary results of a split-mouth study. Clin Oral Implants Res 2011;22(5):546-551.

897. Linsen SS, Martini M, Stark H. Long-term results of endosteal implants following radical oral cancer surgery with and without adjuvant radiation therapy. Clin Implant Dent Relat Res 2012;14(2):250-258.

898. Mancha de la Plata M, Gias LN, Diez PM, Munoz-Guerra M, Gonzalez-Garcia R, Lee GY, Castrejon-Castrejon S, Rodriguez-Campo FJ. Osseointegrated implant rehabilitation of irradiated oral cancer patients. J Oral Maxillofac Surg 2012;70(5):1052-1063. 
899. Granström G, Tjellström A, Brånemark PI. Osseointegrated implants in irradiated bone: a case-controlled study using adjunctive hyperbaric oxygen therapy. J Oral Maxillofac Surg 1999;57(5):493-499.

900. Schoen PJ, Reintsema H, Raghoebar GM, Vissink A, Roodenburg JL. The use of implant retained mandibular prostheses in the oral rehabilitation of head and neck cancer patients. A review and rationale for treatment planning. Oral Oncol 2004;40(9):862-871.

901. Haas I, Hauser U, Ganzer U. The dilemma of follow-up in head and neck cancer patients. Eur Arch Otorhinolaryngol 2001;258(4):177183.

902. Kwakman JM, Freihofer HP, van Waas MA. Osseointegrated oral implants in head and neck cancer patients. Laryngoscope 1997;107(4):519-522.

903. Mericske-Stern R, Perren R, Raveh J. Life table analysis and clinical evaluation of oral implants supporting prostheses after resection of malignant tumors. Int J Oral Maxillofac Implants 1999;14(5):673-680.

904. King MA, Casarett GW, Weber DA. A study of irradiated bone: I. histopathologic and physiologic changes. J Nucl Med 1979;20(11):1142-1149.

905. Wächter RS, P. Möglichkeiten und Grenzen enossaler Implantate bei der oralen Rehabilitation von Tumorpatienten nach Bestrahlung. $Z$ Zahnärztl Implantol 1994;10:171-176.

906. Visch LL, van Waas MA, Schmitz PI, Levendag PC. A clinical evaluation of implants in irradiated oral cancer patients. J Dent Res 2002;81(12):856-859.

907. Sammartino G, Marenzi G, Cioffi I, Tete S, Mortellaro C. Implant therapy in irradiated patients. J Craniofac Surg 2011;22(2):443-445.

908. Klein MO, Grotz KA, Walter C, Wegener J, Wagner W, Al-Nawas B. Functional rehabilitation of mandibular continuity defects using autologous bone and dental implants - prognostic value of bone origin, radiation therapy and implant dimensions. Eur Surg Res 2009;43(3):269-275.

909. Werkmeister R, Szulczewski D, Walteros-Benz P, Joos U. Rehabilitation with dental implants of oral cancer patients. J Craniomaxillofac Surg 1999;27(1):38-41.

910. Cooper JS, Fu K, Marks J, Silverman S. Late effects of radiation therapy in the head and neck region. Int J Radiat Oncol Biol Phys 1995;31(5):1141-1164.

911. Nguyen TD, Panis X, Froissart D, Legros M, Coninx P, Loirette M. Analysis of late complications after rapid hyperfractionated radiotherapy in advanced head and neck cancers. Int J Radiat Oncol Biol Phys 1988;14(1):23-25. 
912. Jacobsson M, Kalebo P, Albrektsson T, Turesson I. Provoked repetitive healing of mature bone tissue following irradiation. A quantitative investigation. Acta Radiol Oncol 1986;25(1):57-62.

913. Jacobsson M, Tjellström A, Thomsen P, Albrektsson T, Turesson I. Integration of titanium implants in irradiated bone. Histologic and clinical study. Ann Otol Rhinol Laryngol 1988;97(4 Pt 1):337-340.

914. Nishimura RD, Roumanas E, Beumer J, 3rd, Moy PK, Shimizu KT. Restoration of irradiated patients using osseointegrated implants: current perspectives. J Prosthet Dent 1998;79(6):641-647.

915. Goto M, Jin-Nouchi S, Ihara K, Katsuki T. Longitudinal follow-up of osseointegrated implants in patients with resected jaws. Int J Oral Maxillofac Implants 2002;17(2):225-230.

916. Fenlon MR, Lyons A, Farrell S, Bavisha K, Banerjee A, Palmer RM. Factors affecting survival and usefulness of implants placed in vascularized free composite grafts used in post-head and neck cancer reconstruction. Clin Implant Dent Relat Res 2012;14(2):266-272.

917. Umstadt HE, Vollinger J, Muller HH, Austermann KH. Implantate in avaskulären Beckenknochentransplantaten - Prospektive Studie über 176 Implantate. Mund Kiefer Gesichtschir 1999;3 Suppl 1:S93-98.

918. Berggren A, Weiland AJ, Dorfman H. The effect of prolonged ischemia time on osteocyte and osteoblast survival in composite bone grafts revascularized by microvascular anastomoses. Plast Reconstr Surg 1982;69(2):290-298.

919. Albrektsson T, Zarb G, Worthington P, Eriksson AR. The long-term efficacy of currently used dental implants: a review and proposed criteria of success. Int J Oral Maxillofac Implants 1986;1(1):11-25.

920. McGhee MA, Stern SJ, Callan D, Shewmake K, Smith T. Osseointegrated implants in the head and neck cancer patient. Head Neck 1997;19(8):659-665.

921. Shah JP, Patel SG, Society AC. Cancer of the Head and Neck. Hamilton: BC Decker; 2001.

922. Chrcanovic BR, Pedrosa AR, Martins MD. Chemical and Topographic Analysis of Treated Surfaces of Five Different Commercial Dental Titanium Implants. Materials Research 2012;15(3):372-382.

923. Marx RE. Osteoradionecrosis: a new concept of its pathophysiology. $J$ Oral Maxillofac Surg 1983;41(5):283-288.

924. Friedlaender GE, Tross RB, Doganis AC, Kirkwood JM, Baron R. Effects of chemotherapeutic agents on bone. I. Short-term methotrexate and doxorubicin (adriamycin) treatment in a rat model. J Bone Joint Surg Am 1984;66(4):602-607. 
925. Young DR, Virolainen P, Inoue N, Frassica FJ, Chao EY. The shortterm effects of cisplatin chemotherapy on bone turnover. J Bone Miner Res 1997;12(11):1874-1882.

926. Teoh KH, Huryn JM, Patel S, Halpern J, Tunick S, Wong HB, Zlotolow IM. Implant prosthodontic rehabilitation of fibula freeflap reconstructed mandibles: a Memorial Sloan-Kettering Cancer Center review of prognostic factors and implant outcomes. Int J Oral Maxillofac Implants 2005;20(5):738-746.

927. Katsoulis J, Fierz J, Iizuka T, Mericske-Stern R. Prosthetic rehabilitation, implant survival and quality of life 2 to 5 years after resection of oral tumors. Clin Implant Dent Relat Res 2013;15(1):6472.

928. Accursi GE. Treatment outcomes with osseointegrated Branemark implants in diabetic patients: a retrospective study [PhD thesis]. Toronto: University of Toronto; 2000.

929. Oates TW, Huynh-Ba G, Vargas A, Alexander P, Feine J. A critical review of diabetes, glycemic control, and dental implant therapy. Clin Oral Implants Res 2013;24(2):117-127.

930. Danaei G, Finucane MM, Lu Y, Singh GM, Cowan MJ, Paciorek CJ, Lin JK, Farzadfar F, Khang YH, Stevens GA, Rao M, Ali MK, Riley LM, Robinson CA, Ezzati M. National, regional, and global trends in fasting plasma glucose and diabetes prevalence since 1980: systematic analysis of health examination surveys and epidemiological studies with 370 country-years and 2.7 million participants. Lancet 2011;378(9785):31-40.

931. Khader YS, Dauod AS, El-Qaderi SS, Alkafajei A, Batayha WQ. Periodontal status of diabetics compared with nondiabetics: a metaanalysis. J Diabetes Complications 2006;20(1):59-68.

932. Rothwell BR, Richard EL. Diabetes mellitus: medical and dental considerations. Spec Care Dentist 1984;4(2):58-65.

933. Frantzis TG, Reeve CM, Brown AL, Jr. The ultrastructure of capillary basement membranes in the attached gingiva of diabetic and nondiabetic patients with periodontal disease. J Periodontol 1971;42(7):406-411.

934. McMahon MM, Bistrian BR. Host defenses and susceptibility to infection in patients with diabetes mellitus. Infect Dis Clin North Am 1995;9(1):1-9.

935. Michaeli E, Weinberg I, Nahlieli O. Dental implants in the diabetic patient: systemic and rehabilitative considerations. Quintessence Int 2009;40(8):639-645. 
936. Siqueira JT, Cavalher-Machado SC, Arana-Chavez VE, Sannomiya P. Bone formation around titanium implants in the rat tibia: role of insulin. Implant Dent 2003;12(3):242-251.

937. de Morais JA, Trindade-Suedam IK, Pepato MT, Marcantonio E, Jr., Wenzel A, Scaf G. Effect of diabetes mellitus and insulin therapy on bone density around osseointegrated dental implants: a digital subtraction radiography study in rats. Clin Oral Implants Res 2009;20(8):796-801.

938. Dowell S, Oates TW, Robinson M. Implant success in people with type 2 diabetes mellitus with varying glycemic control: a pilot study. J Am Dent Assoc 2007;138(3):355-361; quiz 397-358.

939. Tawil G, Younan R, Azar P, Sleilati G. Conventional and advanced implant treatment in the type II diabetic patient: surgical protocol and long-term clinical results. Int J Oral Maxillofac Implants 2008;23(4):744-752.

940. Keller EE, Tolman DE, Eckert SE. Maxillary antral-nasal inlay autogenous bone graft reconstruction of compromised maxilla: a 12-year retrospective study. Int J Oral Maxillofac Implants 1999;14(5):707-721.

941. Morris HF, Ochi S, Winkler S. Implant survival in patients with type 2 diabetes: placement to 36 months. Ann Periodontol 2000;5(1):157165.

942. van Steenberghe D, Jacobs R, Desnyder M, Maffei G, Quirynen M. The relative impact of local and endogenous patient-related factors on implant failure up to the abutment stage. Clin Oral Implants Res 2002;13(6):617-622.

943. Doyle SL, Hodges JS, Pesun IJ, Baisden MK, Bowles WR. Factors affecting outcomes for single-tooth implants and endodontic restorations. J Endod 2007;33(4):399-402.

944. Alsaadi G, Quirynen M, Komarek A, van Steenberghe D. Impact of local and systemic factors on the incidence of late oral implant loss. Clin Oral Implants Res 2008;19(7):670-676.

945. Alsaadi G, Quirynen M, Michiles K, Teughels W, Komarek A, van Steenberghe D. Impact of local and systemic factors on the incidence of failures up to abutment connection with modified surface oral implants. J Clin Periodontol 2008;35(1):51-57.

946. Anner R, Grossmann Y, Anner Y, Levin L. Smoking, diabetes mellitus, periodontitis, and supportive periodontal treatment as factors associated with dental implant survival: a long-term retrospective evaluation of patients followed for up to 10 years. Implant Dent 2010;19(1):57-64. 
947. Bell CL, Diehl D, Bell BM, Bell RE. The immediate placement of dental implants into extraction sites with periapical lesions: a retrospective chart review. J Oral Maxillofac Surg 2011;69(6):1623-1627.

948. Grandi T, Guazzi P, Samarani R, Grandi G. A 3-year report from a multicentre randomised controlled trial: immediately versus early loaded implants in partially edentulous patients. Eur J Oral Implantol 2013;6(3):217-224.

949. Le BT, Follmar T, Borzabadi-Farahani A. Assessment of short dental implants restored with single-unit nonsplinted restorations. Implant Dent 2013;22(5):499-502.

950. Moy PK, Medina D, Shetty V, Aghaloo TL. Dental implant failure rates and associated risk factors. Int J Oral Maxillofac Implants 2005;20(4):569-577.

951. Cohen A, Horton ES. Progress in the treatment of type 2 diabetes: new pharmacologic approaches to improve glycemic control. Curr Med Res Opin 2007;23(4):905-917.

952. Goodson WH, Hunt TK. Wound healing in well-controlled diabetic men. Surg Forum 1984;35:614-616.

953. Lu H, Kraut D, Gerstenfeld LC, Graves DT. Diabetes interferes with the bone formation by affecting the expression of transcription factors that regulate osteoblast differentiation. Endocrinology 2003;144(1):346-352.

954. Kayal RA, Tsatsas D, Bauer MA, Allen B, Al-Sebaei MO, Kakar S, Leone CW, Morgan EF, Gerstenfeld LC, Einhorn TA, Graves DT. Diminished bone formation during diabetic fracture healing is related to the premature resorption of cartilage associated with increased osteoclast activity. J Bone Miner Res 2007;22(4):560-568.

955. Olson JW, Shernoff AF, Tarlow JL, Colwell JA, Scheetz JP, Bingham SF. Dental endosseous implant assessments in a type 2 diabetic population: a prospective study. Int J Oral Maxillofac Implants 2000;15(6):811818.

956. Krakauer JC, McKenna MJ, Buderer NF, Rao DS, Whitehouse FW, Parfitt AM. Bone loss and bone turnover in diabetes. Diabetes 1995;44(7):775-782.

957. Nevins ML, Karimbux NY, Weber HP, Giannobile WV, Fiorellini JP. Wound healing around endosseous implants in experimental diabetes. Int J Oral Maxillofac Implants 1998;13(5):620-629.

958. Takeshita F, Murai K, Iyama S, Ayukawa Y, Suetsugu T. Uncontrolled diabetes hinders bone formation around titanium implants in rat tibiae. A light and fluorescence microscopy, and image processing study. $J$ Periodontol 1998;69(3):314-320. 
959. Kwon PT, Rahman SS, Kim DM, Kopman JA, Karimbux NY, Fiorellini JP. Maintenance of osseointegration utilizing insulin therapy in a diabetic rat model. J Periodontol 2005;76(4):621-626.

960. Oates TW, Dowell S, Robinson M, McMahan CA. Glycemic control and implant stabilization in type 2 diabetes mellitus. J Dent Res 2009;88(4):367-371.

961. van Steenberghe D, Quirynen M, Molly L, Jacobs R. Impact of systemic diseases and medication on osseointegration. Periodontol 2000 2003;33:163-171.

962. Gruber HE. Bone and the immune system. Proc Soc Exp Biol Med 1991;197(3):219-225.

963. Bab IA, Einhorn TA. Regulatory role of osteogenic growth polypeptides in bone formation and hemopoiesis. Crit Rev Eukaryot Gene Expr 1993;3(1):31-46.

964. Ershler WB, Harman SM, Keller ET. Immunologic aspects of osteoporosis. Dev Comp Immunol 1997;21(6):487-499.

965. Alsaadi G, Quirynen M, Komarek A, van Steenberghe D. Impact of local and systemic factors on the incidence of oral implant failures, up to abutment connection. J Clin Periodontol 2007;34(7):610-617.

966. Bliziotes MM, Eshleman AJ, Zhang XW, Wiren KM. Neurotransmitter action in osteoblasts: expression of a functional system for serotonin receptor activation and reuptake. Bone 2001;29(5):477-486.

967. Westbroek I, van der Plas A, de Rooij KE, Klein-Nulend J, Nijweide PJ. Expression of serotonin receptors in bone. J Biol Chem 2001;276(31):28961-28968.

968. Battaglino R, Fu J, Spate U, Ersoy U, Joe M, Sedaghat L, Stashenko P. Serotonin regulates osteoclast differentiation through its transporter. $J$ Bone Miner Res 2004;19(9):1420-1431.

969. Gustafsson BI, Thommesen L, Stunes AK, Tommeras K, Westbroek I, Waldum HL, Slordahl K, Tamburstuen MV, Reseland JE, Syversen U. Serotonin and fluoxetine modulate bone cell function in vitro. J Cell Biochem 2006;98(1):139-151.

970. Warden SJ, Nelson IR, Fuchs RK, Bliziotes MM, Turner CH. Serotonin (5-hydroxytryptamine) transporter inhibition causes bone loss in adult mice independently of estrogen deficiency. Menopause 2008;15(6):1176-1183.

971. Warden SJ, Robling AG, Sanders MS, Bliziotes MM, Turner CH. Inhibition of the serotonin (5-hydroxytryptamine) transporter reduces bone accrual during growth. Endocrinology 2005;146(2):685-693. 
972. Yadav VK, Ryu JH, Suda N, Tanaka KF, Gingrich JA, Schutz G, Glorieux FH, Chiang CY, Zajac JD, Insogna KL, Mann JJ, Hen R, Ducy P, Karsenty G. Lrp5 controls bone formation by inhibiting serotonin synthesis in the duodenum. Cell 2008;135(5):825-837.

973. Cohen J, Cohen P, West SG, Aiken LS. Applied multiple regression/ correlation analysis for the behavioral sciences. 3rd edition ed. Mahwah, New Jersey: Lawrence Erlbaum; 2003.

974. Spangler JG, Konen JC. Oral health behaviors in medical patients with diabetes mellitus. J Dent Hyg 1994;68(6):287-293.

975. Deinzer R, Hilpert D, Bach K, Schawacht M, Herforth A. Effects of academic stress on oral hygiene--a potential link between stress and plaque-associated disease? J Clin Periodontol 2001;28(5):459-464.

976. Axtelius B, Soderfeldt B, Nilsson A, Edwardsson S, Attstrom R. Therapy-resistant periodontitis. Psychosocial characteristics. J Clin Periodontol 1998;25(6):482-491.

977. Persson GR, Persson RE, MacEntee CI, Wyatt CC, Hollender LG, Kiyak HA. Periodontitis and perceived risk for periodontitis in elders with evidence of depression. J Clin Periodontol 2003;30(8):691-696.

978. Moss ME, Beck JD, Kaplan BH, Offenbacher S, Weintraub JA, Koch GG, Genco RJ, Machtei EE, Tedesco LA. Exploratory case-control analysis of psychosocial factors and adult periodontitis. J Periodontol 1996;67(10 Suppl):1060-1069.

979. Kopic S, Geibel JP. Gastric acid, calcium absorption, and their impact on bone health. Physiol Rev 2013;93(1):189-268.

980. Graziani G, Como G, Badalamenti S, Finazzi S, Malesci A, Gallieni M, Brancaccio D, Ponticelli C. Effect of gastric acid secretion on intestinal phosphate and calcium absorption in normal subjects. Nephrol Dial Transplant 1995;10(8):1376-1380.

981. O’Connell MB, Madden DM, Murray AM, Heaney RP, Kerzner LJ. Effects of proton pump inhibitors on calcium carbonate absorption in women: a randomized crossover trial. Am J Med 2005;118(7):778-781.

982. Schinke T, Schilling AF, Baranowsky A, Seitz S, Marshall RP, Linn T, Blaeker M, Huebner AK, Schulz A, Simon R, Gebauer M, Priemel M, Kornak U, Perkovic S, Barvencik F, Beil FT, Del Fattore A, Frattini A, Streichert T, Pueschel K, Villa A, Debatin KM, Rueger JM, Teti A, Zustin J, Sauter G, Amling M. Impaired gastric acidification negatively affects calcium homeostasis and bone mass. Nat Med 2009;15(6):674681.

983. Alonso-Coello P, Garcia-Franco AL, Guyatt G, Moynihan R. Drugs for pre-osteoporosis: prevention or disease mongering? Bmj 2008;336(7636):126-129. 
984. Edwards MH, McCrae FC, Young-Min SA. Alendronate-related femoral diaphysis fracture--what should be done to predict and prevent subsequent fracture of the contralateral side? Osteoporos Int 2010;21(4):701-703.

985. Sobacchi C, Schulz A, Coxon FP, Villa A, Helfrich MH. Osteopetrosis: genetics, treatment and new insights into osteoclast function. Nat Rev Endocrinol 2013;9(9):522-536.

986. Benford HL, Frith JC, Auriola S, Monkkonen J, Rogers MJ. Farnesol and geranylgeraniol prevent activation of caspases by aminobisphosphonates: biochemical evidence for two distinct pharmacological classes of bisphosphonate drugs. Mol Pharmacol 1999;56(1):131-140.

987. Frith JC, Monkkonen J, Blackburn GM, Russell RG, Rogers MJ. Clodronate and liposome-encapsulated clodronate are metabolized to a toxic ATP analog, adenosine 5'-(beta, gamma-dichloromethylene) triphosphate, by mammalian cells in vitro. J Bone Miner Res 1997;12(9):1358-1367.

988. Sezer O, Heider U, Zavrski I, Kuhne CA, Hofbauer LC. RANK ligand and osteoprotegerin in myeloma bone disease. Blood 2003;101(6):2094-2098.

989. Jones DH, Nakashima T, Sanchez OH, Kozieradzki I, Komarova SV, Sarosi I, Morony S, Rubin E, Sarao R, Hojilla CV, Komnenovic V, Kong YY, Schreiber M, Dixon SJ, Sims SM, Khokha R, Wada T, Penninger JM. Regulation of cancer cell migration and bone metastasis by RANKL. Nature 2006;440(7084):692-696.

990. Hughes DE, MacDonald BR, Russell RG, Gowen M. Inhibition of osteoclast-like cell formation by bisphosphonates in long-term cultures of human bone marrow. J Clin Invest 1989;83(6):1930-1935.

991. Hughes DE, Wright KR, Uy HL, Sasaki A, Yoneda T, Roodman GD, Mundy GR, Boyce BF. Bisphosphonates promote apoptosis in murine osteoclasts in vitro and in vivo. J Bone Miner Res 1995;10(10):14781487.

992. Hofbauer LC, Kuhne CA, Viereck V. The OPG/RANKL/RANK system in metabolic bone diseases. J Musculoskelet Neuronal Interact 2004;4(3):268-275.

993. Mashiba T, Hirano T, Turner CH, Forwood MR, Johnston CC, Burr DB. Suppressed bone turnover by bisphosphonates increases microdamage accumulation and reduces some biomechanical properties in dog rib. J Bone Miner Res 2000;15(4):613-620.

994. Kwon TG, Lee CO, Park JW, Choi SY, Rijal G, Shin HI. Osteonecrosis associated with dental implants in patients undergoing bisphosphonate treatment. Clin Oral Implants Res 2014;25(5):632-640. 
995. Grant BT, Amenedo C, Freeman K, Kraut RA. Outcomes of placing dental implants in patients taking oral bisphosphonates: a review of 115 cases. J Oral Maxillofac Surg 2008;66(2):223-230.

996. Porras AG, Holland SD, Gertz BJ. Pharmacokinetics of alendronate. Clin Pharmacokinet 1999;36(5):315-328.

997. Marx RE, Sawatari Y, Fortin M, Broumand V. Bisphosphonateinduced exposed bone (osteonecrosis/osteopetrosis) of the jaws: risk factors, recognition, prevention, and treatment. J Oral Maxillofac Surg 2005;63(11):1567-1575.

998. Migliorati CA, Casiglia J, Epstein J, Jacobsen PL, Siegel MA, Woo SB. Managing the care of patients with bisphosphonate-associated osteonecrosis: an American Academy of Oral Medicine position paper. J Am Dent Assoc 2005;136(12):1658-1668.

999. Green JR. Bisphosphonates: preclinical review. Oncologist 2004;9 Suppl 4:3-13.

1000. Fleisch H, Russell RG, Straumann F. Effect of pyrophosphate on hydroxyapatite and its implications in calcium homeostasis. Nature 1966;212(5065):901-903.

1001. Weycker D, Macarios D, Edelsberg J, Oster G. Compliance with drug therapy for postmenopausal osteoporosis. Osteoporos Int 2006;17(11):1645-1652.

1002. Lin JH, Russell G, Gertz B. Pharmacokinetics of alendronate: an overview. Int J Clin Pract Suppl 1999;101:18-26.

1003. Ruggiero SL, Mehrotra B, Rosenberg TJ, Engroff SL. Osteonecrosis of the jaws associated with the use of bisphosphonates: a review of 63 cases. J Oral Maxillofac Surg 2004;62(5):527-534.

1004. Woo SB, Hande K, Richardson PG. Osteonecrosis of the jaw and bisphosphonates. N Engl J Med 2005;353(1):99-102; discussion 199102.

1005. Parfitt AM, Mundy GR, Roodman GD, Hughes DE, Boyce BF. A new model for the regulation of bone resorption, with particular reference to the effects of bisphosphonates. J Bone Miner Res 1996;11(2):150159.

1006. Fournier P, Boissier S, Filleur S, Guglielmi J, Cabon F, Colombel $\mathrm{M}$, Clezardin P. Bisphosphonates inhibit angiogenesis in vitro and testosterone-stimulated vascular regrowth in the ventral prostate in castrated rats. Cancer Res 2002;62(22):6538-6544.

1007. Zahid TM, Wang BY, Cohen RE. Influence of bisphosphonates on alveolar bone loss around osseointegrated implants. J Oral Implantol 2011;37(3):335-346. 
1008. Duarte PM, de Vasconcelos Gurgel BC, Sallum AW, Filho GR, Sallum EA, Nociti FH, Jr. Alendronate therapy may be effective in the prevention of bone loss around titanium implants inserted in estrogendeficient rats. J Periodontol 2005;76(1):107-114.

1009. Giro G, Goncalves D, Sakakura CE, Pereira RM, Marcantonio Junior $\mathrm{E}$, Orrico SR. Influence of estrogen deficiency and its treatment with alendronate and estrogen on bone density around osseointegrated implants: radiographic study in female rats. Oral Surg Oral Med Oral Pathol Oral Radiol Endod 2008;105(2):162-167.

1010. Jeffcoat MK. Safety of oral bisphosphonates: controlled studies on alveolar bone. Int J Oral Maxillofac Implants 2006;21(3):349-353.

1011. Fugazzotto PA, Lightfoot WS, Jaffin R, Kumar A. Implant placement with or without simultaneous tooth extraction in patients taking oral bisphosphonates: postoperative healing, early follow-up, and the incidence of complications in two private practices. J Periodontol 2007;78(9):1664-1669.

1012. Bell BM, Bell RE. Oral bisphosphonates and dental implants: a retrospective study. J Oral Maxillofac Surg 2008;66(5):1022-1024.

1013. Kasai T, Pogrel MA, Hossaini M. The prognosis for dental implants placed in patients taking oral bisphosphonates. J Calif Dent Assoc 2009;37(1):39-42.

1014. Koka S, Babu NM, Norell A. Survival of dental implants in postmenopausal bisphosphonate users. J Prosthodont Res 2010;54(3):108111.

1015. Shabestari GO, Shayesteh YS, Khojasteh A, Alikhasi M, Moslemi N, Aminian A, Masaeli R, Eslami B, Treister NS. Implant placement in patients with oral bisphosphonate therapy: a case series. Clin Implant Dent Relat Res 2010;12(3):175-180.

1016. Famili P, Quigley S, Mosher T. Survival of dental implants among postmenopausal female dental school patients taking oral bisphosphonates: a retrospective study. Compend Contin Educ Dent 2011;32(6):E106109.

1017. Leonida A, Vescovi P, Baldoni M, Rossi G, Lauritano D. Immediate loading in mandible full-arch: pilot study in patients with osteoporosis in bisphosphonate therapy. J Oral Implantol 2012;38(1):85-94.

1018. Memon S, Weltman RL, Katancik JA. Oral bisphosphonates: early endosseous dental implant success and crestal bone changes. A retrospective study. Int J Oral Maxillofac Implants 2012;27(5):12161222.

1019. Yip JK, Borrell LN, Cho SC, Francisco H, Tarnow DP. Association between oral bisphosphonate use and dental implant failure among middle-aged women. J Clin Periodontol 2012;39(4):408-414. 
1020. Wagenberg BD, Froum SJ, Eckert SE. Long-term bone stability assessment around 1,187 immediately placed implants with 1 - to 22year follow-up. Int J Oral Maxillofac Implants 2013;28(2):605-612.

1021. AAOMS. American Association of Oral and Maxillofacial Surgeons position paper on bisphosphonate-related osteonecrosis of the jaws. $J$ Oral Maxillofac Surg 2007;65(3):369-376.

1022. Abtahi J, Agholme F, Sandberg O, Aspenberg P. Effect of local vs. systemic bisphosphonate delivery on dental implant fixation in a model of osteonecrosis of the jaw. J Dent Res 2013;92(3):279-283.

1023. Weiss RB. Miscellaneous Toxicities. In: DeVita VTJH, S.; Rosenberg, S.A., editor. Cancer. Principles and Practice of Oncology. 5th ed. Philadelphia-New York: Lippincott-Raven; 1997. pp. 3217.

1024. Young DR, Shih LY, Rock MG, Frassica FJ, Virolainen P, Chao EY. Effect of cisplatin chemotherapy on extracortical tissue formation in canine diaphyseal segmental replacement. J Orthop Res 1997;15(5):773-780.

1025. Karr RA, Kramer DC, Toth BB. Dental implants and chemotherapy complications. J Prosthet Dent 1992;67(5):683-687.

1026. Sager RD, Theis RM. Dental implants placed in a patient with multiple myeloma: report of case. J Am Dent Assoc 1990;121(6):699-701.

1027. Steiner M, Windchy A, Gould AR, Kushner GM, Weber R. Effects of chemotherapy in patients with dental implants. J Oral Implantol 1995;21(2):142-147.

1028. McDonald AR, Pogrel MA, Sharma A. Effects of chemotherapy on osseointegration of implants: a case report. J Oral Implantol 1998;24(1):11-13.

1029. Aldegheri A, Beloni D, Blanc JL, Kaplanski P, Legre R, Zanaret M. La réhabilitation dentaire par fixtures ostéo-intégrables: traitement des cancers oro-maxillo-faciaux. Etude préliminaire de 7 cas. Rev Stomatol Chir Maxillofac 1996;97(2):108-116.

1030. Ihara K, Goto M, Miyahara A, Toyota J, Katsuki T. Multicenter experience with maxillary prostheses supported by Branemark implants: a clinical report. Int J Oral Maxillofac Implants 1998;13(4):531-538.

1031. Mincey BA. Osteoporosis in women with breast cancer. Curr Oncol Rep 2003;5(1):53-57.

1032. Pfeilschifter J, Diel IJ. Osteoporosis due to cancer treatment: pathogenesis and management. J Clin Oncol 2000;18(7):1570-1593. 
1033. Vehmanen L, Saarto T, Elomaa I, Makela P, Valimaki M, Blomqvist C. Long-term impact of chemotherapy-induced ovarian failure on bone mineral density (BMD) in premenopausal breast cancer patients. The effect of adjuvant clodronate treatment. Eur J Cancer 2001;37(18):2373-2378.

1034. Leder BZ, LeBlanc KM, Schoenfeld DA, Eastell R, Finkelstein JS. Differential effects of androgens and estrogens on bone turnover in normal men. J Clin Endocrinol Metab 2003;88(1):204-210.

1035. Lafferty KJ, Boel JF, Hodgkin P. Cyclosporin A (CsA): models for mechanism of action. Transplant Proc 1983;15:2230-2241.

1036. Jowell PS, Epstein S, Fallon MD, Reinhardt TA, Ismail F. 1,25-Dihydroxyvitamin D3 modulates glucocorticoid-induced alteration in serum bone Gla protein and bone histomorphometry. Endocrinology 1987;120(2):531-536.

1037. Keown PA, Stiller CR. Cyclosporine: a double-edged sword. Hosp Pract (Off Ed) 1987;22(5):207-215, 219-220.

1038. Buchinsky FJ, Ma Y, Mann GN, Rucinski B, Bryer HP, Romero DF, Jee WS, Epstein S. T lymphocytes play a critical role in the development of cyclosporin A-induced osteopenia. Endocrinology 1996;137(6):22782285.

1039. Movsowitz C, Epstein S, Fallon M, Ismail F, Thomas S. Cyclosporin-A in vivo produces severe osteopenia in the rat: effect of dose and duration of administration. Endocrinology 1988;123(5):2571-2577.

1040. Horowitz M, Vignery A, Gershon RK, Baron R. Thymus-derived lymphocytes and their interactions with macrophages are required for the production of osteoclast-activating factor in the mouse. Proc Natl Acad Sci U S A 1984;81(7):2181-2185.

1041. Aubia J, Masramón J, Serrano S, Lloveras J, Marinoso LL, Bourbigot B, Moal MC, Cledes J. Bone histology in renal transplant patients receiving cyclosporin. Lancet 1988;1(8593):1048-1049.

1042. Cueto-Manzano AM, Konel S, Hutchison AJ, Crowley V, France MW, Freemont AJ, Adams JE, Mawer B, Gokal R. Bone loss in long-term renal transplantation: histopathology and densitometry analysis. Kidney Int 1999;55(5):2021-2029.

1043. Orcel P, Denne MA, de Vernejoul MC. Cyclosporin-A in vitro decreases bone resorption, osteoclast formation, and the fusion of cells of the monocyte-macrophage lineage. Endocrinology 1991;128(3):1638-1646.

1044. Fu E, Hsieh YD, Nieh S, Wikesjo UM, Liu D. Effects of cyclosporin A on alveolar bone: an experimental study in the rat. J Periodontol 1999;70(2):189-194. 
1045. Duarte PM, Nogueira Filho GR, Sallum EA, de Toledo S, Sallum AW, Nociti Junior FH. The effect of an immunosuppressive therapy and its withdrawal on bone healing around titanium implants. A histometric study in rabbits. J Periodontol 2001;72(10):1391-1397.

1046. Sakakura CE, Margonar R, Holzhausen M, Nociti FH, Jr., Alba RC, Jr., Marcantonio E, Jr. Influence of cyclosporin A therapy on bone healing around titanium implants: a histometric and biomechanic study in rabbits. J Periodontol 2003;74(7):976-981.

1047. Sakakura CE, Margonar R, Sartori R, Morais JA, Marcantonio E, Jr. The influence of cyclosporin a on mechanical retention of dental implants previously integrated to the bone: a study in rabbits. $J$ Periodontol 2006;77(12):2059-2062.

1048. Feehally J, Walls J, Mistry N, Horsburgh T, Taylor J, Veitch PS, Bell PR. Does nifedipine ameliorate cyclosporin A nephrotoxicity? $\mathrm{Br}$ Med J (Clin Res Ed) 1987;295(6593):310.

1049. Duriez J, Flautre B, Blary MC, Hardouin P. Effects of the calcium channel blocker nifedipine on epiphyseal growth plate and bone turnover: a study in rabbit. Calcif Tissue Int 1993;52(2):120-124.

1050. Ritchie CK, Maercklein PB, Fitzpatrick LA. Direct effect of calcium channel antagonists on osteoclast function: alterations in bone resorption and intracellular calcium concentrations. Endocrinology 1994;135(3):996-1003.

1051. Redlich K, Pietschmann P, Stulc T, Peterlik M. Comparative study on the effect of calcium channel blockers on basal and parathyroid hormone-induced bone resorption in vitro. Pharmacol Toxicol 1997;80(6):262-265.

1052. Duarte PM, Nogueira Filho GR, Sallum EA, Sallum AW, Nociti Junior FH. Short-term immunosuppressive therapy does not affect the density of the pre-existing bone around titanium implants placed in rabbits. Pesqui Odontol Bras 2003;17(4):362-366.

1053. Gu L, Yu YC. Clinical outcome of dental implants placed in liver transplant recipients after 3 years: a case series. Transplant Proc 2011;43(7):2678-2682.

1054. Montebugnoli L, Venturi M, Cervellati F. Bone response to submerged implants in organ transplant patients: a prospective controlled study. Int J Oral Maxillofac Implants 2012;27(6):1494-1500.

1055. Montebugnoli L, Venturi M, Cervellati F, Servidio D, Vocale C, Pagan F, Landini MP, Magnani G, Sambri V. Peri-Implant Response and Microflora in Organ Transplant Patients 1 Year after Prosthetic Loading: A Prospective Controlled Study. Clin Implant Dent Relat Res 2015;17(5):972-982. 
1056. Hosoki M, Nishigawa K, Miyamoto Y, Ohe G, Matsuka Y. Allergic contact dermatitis caused by titanium screws and dental implants. $J$ Prosthodont Res 2016;60(3):213-219.

1057. Fleischmann P. Zur Frage der Gefährlichkeit kleinster Quecksilbermengen. Dtsch Med Wochenschr 1928;54:304-307.

1058. Blumenthal F, Jaffe K. Amalgamplomben als Ursache von Quecksilberdermatitis. Dtsch Med Wochenschr 1929;55:1720-1721.

1059. Lundström IM. Allergy and corrosion of dental materials in patients with oral lichen planus. Int J Oral Surg 1984;13(1):16-24.

1060. Magnusson B, Bergman M, Bergman B, Soremark R. Nickel allergy and nickel-containing dental alloys. Scand J Dent Res 1982;90(2):163167.

1061. Hubler WR, Jr., Hubler WR, Sr. Dermatitis from a chromium dental plate. Contact Dermatitis 1983;9(5):377-383.

1062. Feng B, Weng J, Yang BC, Chen JY, Zhao JZ, He L, Qi SK, Zhang XD. Surface characterization of titanium and adsorption of bovine serum albumin. Materials Characterization 2002;49(2):129-137.

1063. Wennerberg A, Ide-Ektessabi A, Hatkamata S, Sawase T, Johansson C, Albrektsson T, Martinelli A, Sodervall U, Odelius H. Titanium release from implants prepared with different surface roughness. Clin Oral Implants Res 2004;15(5):505-512.

1064. Fage SW, Muris J, Jakobsen SS, Thyssen JP. Titanium: a review on exposure, release, penetration, allergy, epidemiology, and clinical reactivity. Contact Dermatitis 2016;74(6):323-345.

1065. Cadosch D, Al-Mushaiqri MS, Gautschi OP, Meagher J, Simmen HP, Filgueira L. Biocorrosion and uptake of titanium by human osteoclasts. J Biomed Mater Res A 2010;95(4):1004-1010.

1066. Jacobs JJ, Skipor AK, Black J, Urban R, Galante JO. Release and excretion of metal in patients who have a total hip-replacement component made of titanium-base alloy. J Bone Joint Surg Am 1991;73(10):1475-1486.

1067. Jacobs JJ, Skipor AK, Patterson LM, Hallab NJ, Paprosky WG, Black J, Galante JO. Metal release in patients who have had a primary total hip arthroplasty. A prospective, controlled, longitudinal study. J Bone Joint Surg Am 1998;80(10):1447-1458.

1068. Jacobs JJ, Silverton C, Hallab NJ, Skipor AK, Patterson L, Black J, Galante JO. Metal release and excretion from cementless titanium alloy total knee replacements. Clin Orthop Relat Res 1999;(358):173-180.

1069. Savarino L, Tigani D, Greco M, Baldini N, Giunti A. The potential role of metal ion release as a marker of loosening in patients with total knee replacement: a cohort study. J Bone Joint Surg Br 2010;92(5):634-638. 
1070. Torgersen S, Gjerdet NR, Erichsen ES, Bang G. Metal particles and tissue changes adjacent to miniplates. A retrieval study. Acta Odontol Scand 1995;53(2):65-71.

1071. Woodman JL, Jacobs JJ, Galante JO, Urban RM. Metal ion release from titanium-based prosthetic segmental replacements of long bones in baboons: a long-term study. J Orthop Res 1984;1(4):421-430.

1072. Weingart D, Steinemann S, Schilli W, Strub JR, Hellerich U, Assenmacher J, Simpson J. Titanium deposition in regional lymph nodes after insertion of titanium screw implants in maxillofacial region. Int J Oral Maxillofac Surg 1994;23(6 Pt 2):450-452.

1073. Frisken KW, Dandie GW, Lugowski S, Jordan G. A study of titanium release into body organs following the insertion of single threaded screw implants into the mandibles of sheep. Aust Dent J 2002;47(3):214-217.

1074. Schliephake H, Reiss G, Urban R, Neukam FW, Guckel S. Metal release from titanium fixtures during placement in the mandible: an experimental study. Int J Oral Maxillofac Implants 1993;8(5):502-511.

1075. Meningaud JP, Poupon J, Bertrand JC, Chenevier C, Galliot-Guilley M, Guilbert F. Dynamic study about metal release from titanium miniplates in maxillofacial surgery. Int J Oral Maxillofac Surg 2001;30(3):185-188.

1076. Savarino L, Greco M, Cenni E, Cavasinni L, Rotini R, Baldini N, Giunti A. Differences in ion release after ceramic-on-ceramic and metal-on-metal total hip replacement. Medium-term follow-up. J Bone Joint Surg Br 2006;88(4):472-476.

1077. Lugowski SJ, Smith DC, McHugh AD, Van Loon JC. Release of metal ions from dental implant materials in vivo: determination of $\mathrm{Al}, \mathrm{Co}, \mathrm{Cr}, \mathrm{Mo}, \mathrm{Ni}, \mathrm{V}$, and Ti in organ tissue. J Biomed Mater Res 1991;25(12):1443-1458.

1078. Bianco PD, Ducheyne P, Cuckler JM. Local accumulation of titanium released from a titanium implant in the absence of wear. J Biomed Mater Res 1996;31(2):227-234.

1079. Lee S, Goh BT, Lai SH, Tideman H, Stoelinga PJ, Jansen JA. Periimplant and systemic release of metallic elements following insertion of a mandibular modular endoprosthesis in Macaca fascicularis. Acta Biomater 2009;5(9):3640-3646.

1080. Gil FJ, Sanchez LA, Espias A, Planell JA. In vitro corrosion behaviour and metallic ion release of different prosthodontic alloys. Int Dent J 1999;49(6):361-367.

1081. Huang HH, Chiu YH, Lee TH, Wu SC, Yang HW, Su KH, Hsu CC. Ion release from $\mathrm{NiTi}$ orthodontic wires in artificial saliva with various acidities. Biomaterials 2003;24(20):3585-3592. 
1082. Müller K, Valentine-Thon E. Hypersensitivity to titanium: clinical and laboratory evidence. Neuro Endocrinol Lett 2006;27 Suppl 1:31-35.

1083. Thomas P, Bandl WD, Maier S, Summer B, Przybilla B. Hypersensitivity to titanium osteosynthesis with impaired fracture healing, eczema, and T-cell hyperresponsiveness in vitro: case report and review of the literature. Contact Dermatitis 2006;55(4):199-202.

1084. Egusa H, Ko N, Shimazu T, Yatani H. Suspected association of an allergic reaction with titanium dental implants: a clinical report. $J$ Prosthet Dent 2008;100(5):344-347.

1085. Valentine-Thon E, Schiwara HW. Validity of MELISA for metal sensitivity testing. Neuro Endocrinol Lett 2003;24(1-2):57-64.

1086. Valentine-Thon E, Müller K, Guzzi G, Kreisel S, Ohnsorge P, Sandkamp M. LTT-MELISA is clinically relevant for detecting and monitoring metal sensitivity. Neuro Endocrinol Lett 2006;27 Suppl 1:17-24.

1087. Sicilia A, Cuesta S, Coma G, Arregui I, Guisasola C, Ruiz E, Maestro A. Titanium allergy in dental implant patients: a clinical study on 1500 consecutive patients. Clin Oral Implants Res 2008;19(8):823-835.

1088. Degidi M, Artese L, Scarano A, Perrotti V, Gehrke P, Piattelli A. Inflammatory infiltrate, microvessel density, nitric oxide synthase expression, vascular endothelial growth factor expression, and proliferative activity in peri-implant soft tissues around titanium and zirconium oxide healing caps. J Periodontol 2006;77(1):73-80.

1089. Barwacz CA, Brogden KA, Stanford CM, Dawson DV, Recker EN, Blanchette D. Comparison of pro-inflammatory cytokines and bone metabolism mediators around titanium and zirconia dental implant abutments following a minimum of 6 months of clinical function. Clin Oral Implants Res 2015;26(4):e35-41.

1090. Coen G. Leptin and bone metabolism. J Nephrol 2004;17(2):187-189.

1091. Zeadin MG, Butcher MK, Shaughnessy SG, Werstuck GH. Leptin promotes osteoblast differentiation and mineralization of primary cultures of vascular smooth muscle cells by inhibiting glycogen synthase kinase (GSK)-3beta. Biochem Biophys Res Commun 2012;425(4):924-930.

1092. Flatebø RS, Johannessen AC, Gronningsaeter AG, Boe OE, Gjerdet NR, Grung B, Leknes KN. Host response to titanium dental implant placement evaluated in a human oral model. J Periodontol 2006;77(7):1201-1210.

1093. Titanium Dioxide Manufacturers Association. About titanium dioxide. 2013 [cited 201723 March 2017]; Available from: http://www.tdma. info/images/Documents/About_TiO2_Brochure___July_2013.pdf 
1094. European Union. Commission Regulation (EU) No 1129/2011 of 11 November 2011 amending Annexe II to Regulation (EC) No 1333/2008 of the European parliament and of the council by establishing a union list of food additives. Off J Eur Union 2011;L295:1-177.

1095. Peters RJ, van Bemmel G, Herrera-Rivera Z, Helsper HP, Marvin HJ, Weigel S, Tromp PC, Oomen AG, Rietveld AG, Bouwmeester H. Characterization of titanium dioxide nanoparticles in food products: analytical methods to define nanoparticles. J Agric Food Chem 2014;62(27):6285-6293.

1096. Lomer MC, Thompson RP, Commisso J, Keen CL, Powell JJ. Determination of titanium dioxide in foods using inductively coupled plasma optical emission spectrometry. Analyst 2000;125(12):23392343.

1097. Weir A, Westerhoff P, Fabricius L, Hristovski K, von Goetz N. Titanium dioxide nanoparticles in food and personal care products. Environ Sci Technol 2012;46(4):2242-2250.

1098. Rompelberg C, Heringa MB, van Donkersgoed G, Drijvers J, Roos A, Westenbrink S, Peters R, van Bemmel G, Brand W, Oomen AG. Oral intake of added titanium dioxide and its nanofraction from food products, food supplements and toothpaste by the Dutch population. Nanotoxicology 2016;10(10):1404-1414.

1099. Oomen AG, Bennink M, van Engelen JGM, M. SAJA. Nanomaterial in consumer products. Detection, characterization and interpretation. RIVM report 320029001/2011. Bilthoven, the Netherlands: National Institute for Public Health and the Environment (RIVM); 2011.

1100. Shi H, Magaye R, Castranova V, Zhao J. Titanium dioxide nanoparticles: a review of current toxicological data. Part Fibre Toxicol 2013;10:15.

1101. Iavicoli I, Leso V, Fontana L, Bergamaschi A. Toxicological effects of titanium dioxide nanoparticles: a review of in vitro mammalian studies. Eur Rev Med Pharmacol Sci 2011;15(5):481-508.

1102. Iavicoli I, Leso V, Bergamaschi A. Toxicological Effects of Titanium Dioxide Nanoparticles: A Review of In Vivo Studies. Journal of Nanomaterials 2012.

1103. Geraets L, Oomen AG, Krystek P, Jacobsen NR, Wallin H, Laurentie M, Verharen HW, Brandon EF, de Jong WH. Tissue distribution and elimination after oral and intravenous administration of different titanium dioxide nanoparticles in rats. Part Fibre Toxicol 2014;11:30.

1104. Haug RH. Retention of asymptomatic bone plates used for orthognathic surgery and facial fractures. J Oral Maxillofac Surg 1996;54(5):611-617. 
1105. Hallab N, Merritt K, Jacobs JJ. Metal sensitivity in patients with orthopaedic implants. J Bone Joint Surg Am 2001;83-a(3):428-436.

1106. Matthew I, Frame JW. Allergic responses to titanium. J Oral Maxillofac Surg 1998;56(12):1466-1467.

1107. Bilhan H, Bural C, Geckili O. Titanium hypersensitivity. A hidden threat for dental implant patients? N Y State Dent J 2013;79(4):38-43.

1108. Chaturvedi T. Allergy related to dental implant and its clinical significance. Clin Cosmet Investig Dent 2013;5:57-61.

1109. Schramm M, Pitto RP. Clinical relevance of allergological tests in total hip joint replacement. In: Willmann G, Zweymüller K, editors. Bioceramics in Hip Joint Replacement. New York: Thieme; 2000. pp. 101-106.

1110. Thomas P. Allergological aspects of implant biocompatibility. In: Willmann KZG, Zweymüller K, editors. Bioceramics in Hip Joint Replacement. New York: Thieme; 2000. pp. 117-121.

1111. Brunski JB, Puleo DA, Nanci A. Biomaterials and biomechanics of oral and maxillofacial implants: current status and future developments. Int J Oral Maxillofac Implants 2000;15(1):15-46.

1112. Akagawa Y, Abe Y. Titanium: the ultimate solution or an evolutionary step? Int J Prosthodont 2003;16 Suppl:28-29; discussion 47-51.

1113. Bass JK, Fine H, Cisneros GJ. Nickel hypersensitivity in the orthodontic patient. Am J Orthod Dentofacial Orthop 1993;103(3):280-285.

1114. Johansson CB, Hansson HA, Albrektsson T. Qualitative interfacial study between bone and tantalum, niobium or commercially pure titanium. Biomaterials 1990;11(4):277-280.

1115. Matsuno H, Yokoyama A, Watari F, Uo M, Kawasaki T. Biocompatibility and osteogenesis of refractory metal implants, titanium, hafnium, niobium, tantalum and rhenium. Biomaterials 2001;22(11):1253-1262.

1116. Schwartz-Arad D, Mardinger O, Levin L, Kozlovsky A, Hirshberg A. Marginal bone loss pattern around hydroxyapatite-coated versus commercially pure titanium implants after up to 12 years of follow-up. Int J Oral Maxillofac Implants 2005;20(2):238-244.

1117. Simunek A, Kopecka D, Cierny M, Krulichova I. A six-year study of hydroxyapatite-coated root-form dental implants. West Indian Med J 2005;54(6):393-397.

1118. Artzi Z, Carmeli G, Kozlovsky A. A distinguishable observation between survival and success rate outcome of hydroxyapatitecoated implants in 5-10 years in function. Clin Oral Implants Res 2006;17(1):85-93. 
1119. Kohal RJ, Weng D, Bachle M, Strub JR. Loaded custom-made zirconia and titanium implants show similar osseointegration: an animal experiment. J Periodontol 2004;75(9):1262-1268.

1120. Sennerby L, Dasmah A, Larsson B, Iverhed M. Bone tissue responses to surface-modified zirconia implants: A histomorphometric and removal torque study in the rabbit. Clin Implant Dent Relat Res 2005;7 Suppl 1:S13-20.

1121. Johansson P, Jimbo R, Kjellin P, Currie F, Chrcanovic BR, Wennerberg A. Biomechanical evaluation and surface characterization of a nanomodified surface on PEEK implants: a study in the rabbit tibia. Int J Nanomedicine 2014;9:3903-3911.

1122. The glossary of prosthodontic terms. J Prosthet Dent 2005;94(1):1092.

1123. Birdi H, Schulte J, Kovacs A, Weed M, Chuang SK. Crown-to-implant ratios of short-length implants. J Oral Implantol 2010;36(6):425-433.

1124. Schillingburg HT, Hobo S, Whitsett LD, Jacobi R, Brackett SE. Treatment Planning for the Replacement of Missing Teeth. Fundamentals of Fixed Prosthodontics. 3rd ed. Chicago: Quintessence; 1997. pp. 85-103.

1125. Rosenstiel SF, Land MF, Fujimoto J. Diagnostic Casts and Related Procedures. Contemporary Fixed Prosthodontics. 4th ed. St. Louis: Elsevier; 2006. pp. 42-81.

1126. Grossmann Y, Sadan A. The prosthodontic concept of crown-to-root ratio: a review of the literature. J Prosthet Dent 2005;93(6):559-562.

1127. Newman NG, Takei HH, Carranza AC. Carranza's Clinical Periodontology. 9th ed. St. Louis: Elsevier; 2002.

1128. Misch CE, Steignga J, Barboza E, Misch-Dietsh F, Cianciola LJ, Kazor C. Short dental implants in posterior partial edentulism: a multicenter retrospective 6-year case series study. J Periodontol 2006;77(8):13401347.

1129. Rieger MR, Mayberry M, Brose MO. Finite element analysis of six endosseous implants. J Prosthet Dent 1990;63(6):671-676.

1130. Kitamura E, Stegaroiu R, Nomura S, Miyakawa O. Biomechanical aspects of marginal bone resorption around osseointegrated implants: considerations based on a three-dimensional finite element analysis. Clin Oral Implants Res 2004;15(4):401-412.

1131. Misch CE, Suzuki JB, Misch-Dietsh FM, Bidez MW. A positive correlation between occlusal trauma and peri-implant bone loss: literature support. Implant Dent 2005;14(2):108-116.

1132. Yang TC, Maeda Y, Gonda T. Biomechanical rationale for short implants in splinted restorations: an in vitro study. Int J Prosthodont 2011;24(2):130-132. 
1133. Ganeles J, Zollner A, Jackowski J, ten Bruggenkate C, Beagle J, Guerra F. Immediate and early loading of Straumann implants with a chemically modified surface (SLActive) in the posterior mandible and maxilla: 1-year results from a prospective multicenter study. Clin Oral Implants Res 2008;19(11):1119-1128.

1134. Rokni S, Todescan R, Watson P, Pharoah M, Adegbembo AO, Deporter D. An assessment of crown-to-root ratios with short sintered porous-surfaced implants supporting prostheses in partially edentulous patients. Int J Oral Maxillofac Implants 2005;20(1):69-76.

1135. Blanes RJ, Bernard JP, Blanes ZM, Belser UC. A 10-year prospective study of ITI dental implants placed in the posterior region. II: Influence of the crown-to-implant ratio and different prosthetic treatment modalities on crestal bone loss. Clin Oral Implants Res 2007;18(6):707-714.

1136. Schulte J, Flores AM, Weed M. Crown-to-implant ratios of single tooth implant-supported restorations. J Prosthet Dent 2007;98(1):1-5.

1137. Urdaneta RA, Rodriguez S, McNeil DC, Weed M, Chuang SK. The effect of increased crown-to-implant ratio on single-tooth locking-taper implants. Int J Oral Maxillofac Implants 2010;25(4):729-743.

1138. Quaranta A, Piemontese M, Rappelli G, Sammartino G, Procaccini M. Technical and biological complications related to crown to implant ratio: a systematic review. Implant Dent 2014;23(2):180-187.

1139. Sun SP, Moon IS, Park KH, Lee DW. Effect of Crown to Implant Ratio and Anatomical Crown Length on Clinical Conditions in a Single Implant: A Retrospective Cohort Study. Clin Implant Dent Relat Res 2015;17(4):724-731.

1140. Misch CE, Goodacre CJ, Finley JM, Misch CM, Marinbach M, Dabrowsky T, English CE, Kois JC, Cronin RJ, Jr. Consensus conference panel report: crown-height space guidelines for implant dentistry-part 2. Implant Dent 2006;15(2):113-121.

1141. Nissan J, Ghelfan O, Gross O, Priel I, Gross M, Chaushu G. The effect of crown/implant ratio and crown height space on stress distribution in unsplinted implant supporting restorations. J Oral Maxillofac Surg 2011;69(7):1934-1939.

1142. Anitua E, Alkhraist MH, Pinas L, Begona L, Orive G. Implant survival and crestal bone loss around extra-short implants supporting a fixed denture: the effect of crown height space, crown-to-implant ratio, and offset placement of the prosthesis. Int J Oral Maxillofac Implants 2014;29(3):682-689.

1143. Misch CE, Bidez MW. Implant-protected occlusion: a biomechanical rationale. Compendium 1994;15(11):1330, 1332, 1334 passim; quiz 1344. 
1144. Smith RA, Berger R, Dodson TB. Risk factors associated with dental implants in healthy and medically compromised patients. Int J Oral Maxillofac Implants 1992;7(3):367-372.

1145. Kinsel RP, Liss M. Retrospective analysis of 56 edentulous dental arches restored with 344 single-stage implants using an immediate loading fixed provisional protocol: statistical predictors of implant failure. Int J Oral Maxillofac Implants 2007;22(5):823-830.

1146. Bass SL, Triplett RG. The effects of preoperative resorption and jaw anatomy on implant success. A report of 303 cases. Clin Oral Implants Res 1991;2(4):193-198.

1147. Scurria MS, Morgan ZVt, Guckes AD, Li S, Koch G. Prognostic variables associated with implant failure: a retrospective effectiveness study. Int J Oral Maxillofac Implants 1998;13(3):400-406.

1148. Chuang SK, Wei LJ, Douglass CW, Dodson TB. Risk factors for dental implant failure: a strategy for the analysis of clustered failure-time observations. J Dent Res 2002;81(8):572-577.

1149. Ferrigno N, Laureti M, Fanali S, Grippaudo G. A long-term followup study of non-submerged ITI implants in the treatment of totally edentulous jaws. Part I: Ten-year life table analysis of a prospective multicenter study with 1286 implants. Clin Oral Implants Res 2002;13(3):260-273.

1150. Moheng P, Feryn JM. Clinical and biologic factors related to oral implant failure: a 2-year follow-up study. Implant Dent 2005;14(3):281-288.

1151. Palmqvist S, Owall B, Schou S. A prospective randomized clinical study comparing implant-supported fixed prostheses and overdentures in the edentulous mandible: prosthodontic production time and costs. Int J Prosthodont 2004;17(2):231-235.

1152. Hosseini M, Worsaae N, Schiodt M, Gotfredsen K. A 1-year randomised controlled trial comparing zirconia versus metal-ceramic implant supported single-tooth restorations. Eur J Oral Implantol 2011;4(4):347-361.

1153. Nissan J, Narobai D, Gross O, Ghelfan O, Chaushu G. Long-term outcome of cemented versus screw-retained implant-supported partial restorations. Int J Oral Maxillofac Implants 2011;26(5):1102-1107.

1154. Sherif S, Susarla SM, Hwang JW, Weber HP, Wright RF. Clinician- and patient-reported long-term evaluation of screw- and cement-retained implant restorations: a 5-year prospective study. Clin Oral Investig 2011;15(6):993-999.

1155. Vigolo P, Mutinelli S, Givani A, Stellini E. Cemented versus screwretained implant-supported single-tooth crowns: a 10-year randomised controlled trial. Eur J Oral Implantol 2012;5(4):355-364. 
1156. Crespi R, Cappare P, Gastaldi G, Gherlone EF. Immediate occlusal loading of full-arch rehabilitations: screw-retained versus cementretained prosthesis. An 8-year clinical evaluation. Int J Oral Maxillofac Implants 2014;29(6):1406-1411.

1157. Degidi M, Piattelli A. Immediate functional and non-functional loading of dental implants: a 2- to 60-month follow-up study of 646 titanium implants. J Periodontol 2003;74(2):225-241.

1158. Misch CE. Nonfunctional immediate teeth. Dent Today 1998;17(6):8891.

1159. Degidi M, Nardi D, Piattelli A. A comparison between immediate loading and immediate restoration in cases of partial posterior mandibular edentulism: a 3-year randomized clinical trial. Clin Oral Implants Res 2010;21(7):682-687.

1160. Lindeboom JA, Frenken JW, Dubois L, Frank M, Abbink I, Kroon FH. Immediate loading versus immediate provisionalization of maxillary single-tooth replacements: a prospective randomized study with BioComp implants. J Oral Maxillofac Surg 2006;64(6):936-942.

1161. Skalak R. Biomechanical considerations in osseointegrated prostheses. J Prosthet Dent 1983;49(6):843-848.

1162. Sones AD. Complications with osseointegrated implants. J Prosthet Dent 1989;62(5):581-585.

1163. Kallus T, Bessing C. Loose gold screws frequently occur in full-arch fixed prostheses supported by osseointegrated implants after 5 years. Int J Oral Maxillofac Implants 1994;9(2):169-178.

1164. Sahin S, Cehreli MC. The significance of passive framework fit in implant prosthodontics: current status. Implant Dent 2001;10(2):8592.

1165. Taylor TD, Agar JR, Vogiatzi T. Implant prosthodontics: current perspective and future directions. Int J Oral Maxillofac Implants 2000;15(1):66-75.

1166. Manzella C, Bignardi C, Burello V, Carossa S, Schierano G. Method to improve passive fit of frameworks on implant-supported prostheses: An in vitro study. J Prosthet Dent 2016;116(1):52-58.

1167. Spazzin AO, Bacchi A, Trevisani A, Farina AP, Dos Santos MB. Fit Analysis of Different Framework Fabrication Techniques for ImplantSupported Partial Prostheses. Int J Prosthodont 2016;29(4):351-353.

1168. Carlsson L. Built-in strain and untoward forces are the inevitable companions of prosthetic misfit. Nobelpharma News. 1994:5.

1169. Smedberg JI, Nilner K, Rangert B, Svensson SA, Glantz SA. On the influence of superstructure connection on implant preload: a methodological and clinical study. Clin Oral Implants Res 1996;7(1):55-63. 
1170. Richter EJ. In vivo horizontal bending moments on implants. Int J Oral Maxillofac Implants 1998;13(2):232-244.

1171. White SN, Caputo AA, Anderkvist T. Effect of cantilever length on stress transfer by implant-supported prostheses. J Prosthet Dent 1994;71(5):493-499.

1172. Brunski JB, Hipp JA. In vivo forces on endosteal implants: a measurement system and biomechanical considerations. J Prosthet Dent 1984;51(1):82-90.

1173. Assif D, Marshak B, Horowitz A. Analysis of load transfer and stress distribution by an implant-supported fixed partial denture. J Prosthet Dent 1996;75(3):285-291.

1174. Bryant SR. Oral Implant Outcomes Predicted by Age- and Site-specific Aspects of Bone Condition [PhD thesis]. Toronto: University of Toronto; 2001.

1175. Wang JH, Judge R, Bailey D. A 5-Year Retrospective Assay of Implant Treatments and Complications in Private Practice: The Restorative Complications of Single and Short-Span Implant-Supported Fixed Prostheses. Int J Prosthodont 2016;29(5):435-444.

1176. Preiskel HW, Tsolka P. Treatment outcomes in implant therapy: the influence of surgical and prosthodontic experience. Int J Prosthodont 1995;8(3):273-279.

1177. Lambert PM, Morris HF, Ochi S. Positive effect of surgical experience with implants on second-stage implant survival. J Oral Maxillofac Surg 1997;55(12 Suppl 5):12-18.

1178. Morris HF, Manz MC, Tarolli JH. Success of multiple endosseous dental implant designs to second-stage surgery across study sites. $J$ Oral Maxillofac Surg 1997;55(12 Suppl 5):76-82.

1179. Melo MD, Shafie H, Obeid G. Implant survival rates for oral and maxillofacial surgery residents: a retrospective clinical review with analysis of resident level of training on implant survival. J Oral Maxillofac Surg 2006;64(8):1185-1189.

1180. Weyant RJ. Characteristics associated with the loss and peri-implant tissue health of endosseous dental implants. Int J Oral Maxillofac Implants 1994;9(1):95-102.

1181. Birkmeyer JD, Finks JF, O’Reilly A, Oerline M, Carlin AM, Nunn AR, Dimick J, Banerjee M, Birkmeyer NJ. Surgical skill and complication rates after bariatric surgery. N Engl J Med 2013;369(15):1434-1442.

1182. Luft HS, Bunker JP, Enthoven AC. Should operations be regionalized? The empirical relation between surgical volume and mortality. $N$ Engl J Med 1979;301(25):1364-1369. 
1183. Birkmeyer JD, Stukel TA, Siewers AE, Goodney PP, Wennberg DE, Lucas FL. Surgeon volume and operative mortality in the United States. N Engl J Med 2003;349(22):2117-2127.

1184. Hannan EL, Kilburn H, Jr., O’Donnell JF, Lukacik G, Shields EP. Adult open heart surgery in New York State. An analysis of risk factors and hospital mortality rates. Jama 1990;264(21):2768-2774.

1185. Hall BL, Hamilton BH. New information technology systems and a Bayesian hierarchical bivariate probit model for profiling surgeon quality at a large hospital. The Quarterly Review of Economics and Finance 2004;44(3):410-429.

1186. Albrektsson T. Is surgical skill more important for clinical success than changes in implant hardware? Clin Implant Dent Relat Res 2001;3(4):174-175.

1187. Zarb GA, Schmitt A. The longitudinal clinical effectiveness of osseointegrated dental implants: the Toronto study. Part III: Problems and complications encountered. J Prosthet Dent 1990;64(2):185-194.

1188. Renouard F, Amalberti R, Renouard E. Are "Human Factors" the Primary Cause of Complications in the Field of Implant Dentistry? Int J Oral Maxillofac Implants 2017;32(2):e55-e61.

1189. Martin LN, Delgado MR. The influence of emotion regulation on decision-making under risk. J Cogn Neurosci 2011;23(9):2569-2581.

1190. Mather M, Lighthall NR. Both Risk and Reward are Processed Differently in Decisions Made Under Stress. Curr Dir Psychol Sci 2012;21(2):36-41.

1191. Starcke K, Brand M. Decision making under stress: a selective review. Neurosci Biobehav Rev 2012;36(4):1228-1248.

1192. Renouard F, Charrier JG. The Search for the Weakest Link: An Introduction to Human Factors: Ewenn éd.; 2011.

1193. Albrektsson T, Wennerberg A. Oral implant surfaces: Part 1--review focusing on topographic and chemical properties of different surfaces and in vivo responses to them. Int J Prosthodont 2004;17(5):536-543.

1194. Albrektsson T, Wennerberg A. Oral implant surfaces: Part 2--review focusing on clinical knowledge of different surfaces. Int J Prosthodont 2004;17(5):544-564.

1195. Shalabi MM, Gortemaker A, Van't Hof MA, Jansen JA, Creugers NH. Implant surface roughness and bone healing: a systematic review. $J$ Dent Res 2006;85(6):496-500.

1196. Adell R, Eriksson B, Lekholm U, Brånemark PI, Jemt T. Long-term follow-up study of osseointegrated implants in the treatment of totally edentulous jaws. Int J Oral Maxillofac Implants 1990;5(4):347-359. 
1197. Weyant RJ, Burt BA. An assessment of survival rates and withinpatient clustering of failures for endosseous oral implants. J Dent Res $1993 ; 72(1): 2-8$.

1198. Jemt T, Hager P. Early complete failures of fixed implant-supported prostheses in the edentulous maxilla: a 3-year analysis of 17 consecutive cluster failure patients. Clin Implant Dent Relat Res 2006;8(2):77-86.

1199. Schwartz-Arad D, Laviv A, Levin L. Failure causes, timing, and cluster behavior: an 8-year study of dental implants. Implant Dent 2008;17(2):200-207.

1200. Lin WS, Ercoli C, Lowenguth R, Yerke LM, Morton D. Oral rehabilitation of a patient with bruxism and cluster implant failures in the edentulous maxilla: a clinical report. J Prosthet Dent 2012;108(1):1-8.

1201. Tonetti MS. Determination of the success and failure of root-form osseointegrated dental implants. Adv Dent Res 1999;13:173-180.

1202. Roos-Jansåker AM, Lindahl C, Renvert H, Renvert S. Nine- to fourteen-year follow-up of implant treatment. Part II: presence of periimplant lesions. J Clin Periodontol 2006;33(4):290-295.

1203. Fransson C, Lekholm U, Jemt T, Berglundh T. Prevalence of subjects with progressive bone loss at implants. Clin Oral Implants Res 2005;16(4):440-446.

1204. Heitz-Mayfield LJ. Peri-implant diseases: diagnosis and risk indicators. J Clin Periodontol 2008;35(8 Suppl):292-304.

1205. Lindhe J, Berglundh T, Ericsson I, Liljenberg B, Marinello C. Experimental breakdown of peri-implant and periodontal tissues. A study in the beagle dog. Clin Oral Implants Res 1992;3(1):9-16.

1206. Albouy JP, Abrahamsson I, Persson LG, Berglundh T. Spontaneous progression of peri-implantitis at different types of implants. An experimental study in dogs. I: clinical and radiographic observations. Clin Oral Implants Res 2008;19(10):997-1002.

1207. Albouy JP, Abrahamsson I, Persson LG, Berglundh T. Spontaneous progression of ligatured induced peri-implantitis at implants with different surface characteristics. An experimental study in dogs II: histological observations. Clin Oral Implants Res 2009;20(4):366-371.

1208. Albouy JP, Abrahamsson I, Persson LG, Berglundh T. Implant surface characteristics influence the outcome of treatment of peri-implantitis: an experimental study in dogs. J Clin Periodontol 2011;38(1):58-64.

1209. Donath K. Pathogenesis of bony pocket formation around dental implants. J Dent Assoc S Afr 1992;47(5):204-208. 
1210. Thiele A, Birkenroth U, Bloching M, Knipping S.

Fremderkörperreaktion nach implantation eines biocompatiblen Osteosynthese systeme. HNO 2008;56:545-548.

1211. Christiansen RJ. Metal release from implants and its effects on the immune system [PhD thesis]. Lyngby: Technical University of Denmark; 2016.

1212. Landgraeber S, Jager M, Jacobs JJ, Hallab NJ. The pathology of orthopedic implant failure is mediated by innate immune system cytokines. Mediators Inflamm 2014;2014:185150.

1213. Chvartszaid D, Koka S, Zarb G. Osseointegration failure. In: Zarb G, Albrektsson T, Baker G, Eckert SE, Stanford C, editors. Osseointegration: On Continuing Synergies in Surgery, Prosthodontics, Biomaterials. 1st ed. Hanover Park: Quintessence Pub Co; 2008. p. 157-164.

1214. Velasquez-Plata D, Lutonsky J, Oshida Y, Jones R. A close-up look at an implant fracture: a case report. Int J Periodontics Restorative Dent 2002;22(5):483-491.

1215. Traini T, De Paoli S, Caputi S, Iezzi G, Piattelli A. Collagen fiber orientation near a fractured dental implant after a 5-year loading period: case report. Implant Dent 2006;15(1):70-76.

1216. Goodacre CJ, Kan JY, Rungcharassaeng K. Clinical complications of osseointegrated implants. J Prosthet Dent 1999;81(5):537-552.

1217. Levine RA, Clem DS, 3rd, Wilson TG, Jr., Higginbottom F, Solnit G. Multicenter retrospective analysis of the ITI implant system used for single-tooth replacements: results of loading for 2 or more years. Int J Oral Maxillofac Implants 1999;14(4):516-520.

1218. Rangert B, Jemt T, Jorneus L. Forces and moments on Branemark implants. Int J Oral Maxillofac Implants 1989;4(3):241-247.

1219. Capodiferro S, Favia G, Scivetti M, De Frenza G, Grassi R. Clinical management and microscopic characterisation of fatigue-induced failure of a dental implant. Case report. Head Face Med 2006;2:18.

1220. Balshi TJ. Preventing and resolving complications with osseointegrated implants. Dent Clin North Am 1989;33(4):821-868.

1221. Morgan MJ, James DF, Pilliar RM. Fractures of the fixture component of an osseointegrated implant. Int J Oral Maxillofac Implants 1993;8(4):409-414.

1222. Cho HW, Dong JK, Jin TH, Oh SC, Lee HH, Lee JW. A study on the fracture strength of implant-supported restorations using milled ceramic abutments and all-ceramic crowns. Int J Prosthodont 2002;15(1):9-13. 
1223. Yokoyama K, Ichikawa T, Murakami H, Miyamoto Y, Asaoka K. Fracture mechanisms of retrieved titanium screw thread in dental implant. Biomaterials 2002;23(12):2459-2465.

1224. Eckert SE, Meraw SJ, Cal E, Ow RK. Analysis of incidence and associated factors with fractured implants: a retrospective study. Int J Oral Maxillofac Implants 2000;15(5):662-667.

1225. Rangert B, Krogh PH, Langer B, Van Roekel N. Bending overload and implant fracture: a retrospective clinical analysis. Int J Oral Maxillofac Implants 1995;10(3):326-334.

1226. Ivanoff CJ, Grondahl K, Bergstrom C, Lekholm U, Branemark PI. Influence of bicortical or monocortical anchorage on maxillary implant stability: a 15-year retrospective study of Branemark System implants. Int J Oral Maxillofac Implants 2000;15(1):103-110.

1227. Virdee P, Bishop K. A review of the aetiology and management of fractured dental implants and a case report. $\mathrm{Br}$ Dent $\mathrm{J}$ 2007;203(8):461-466.

1228. Balshi TJ. An analysis and management of fractured implants: a clinical report. Int J Oral Maxillofac Implants 1996;11(5):660-666.

1229. Gibney K. Fracture of the body of an implant and its management--a case history. Br Dent J 2004;197(10):615-617.

1230. Ibbott CG. In vivo fracture of a basket-type osseointegrating dental implant: a case report. Int J Oral Maxillofac Implants 1989;4(3):255256.

1231. Piattelli A, Scarano A, Piattelli M, Vaia E, Matarasso S. Hollow implants retrieved for fracture: a light and scanning electron microscope analysis of 4 cases. J Periodontol 1998;69(2):185-189.

1232. Tagger Green N, Machtei EE, Horwitz J, Peled M. Fracture of dental implants: literature review and report of a case. Implant Dent 2002;11(2):137-143.

1233. Pylant T, Triplett RG, Key MC, Brunsvold MA. A retrospective evaluation of endosseous titanium implants in the partially edentulous patient. Int J Oral Maxillofac Implants 1992;7(2):195-202.

1234. Brunel G, Armand S, Miller N, Rue J. Histologic analysis of a fractured implant: a case report. Int J Periodontics Restorative Dent 2000;20(5):520-526.

1235. Meijer HJ, Kuiper JH, Starmans FJ, Bosman F. Stress distribution around dental implants: influence of superstructure, length of implants, and height of mandible. J Prosthet Dent 1992;68(1):96-102.

1236. Carr AB, Stewart RB. Full-arch implant framework casting accuracy: preliminary in vitro observation for in vivo testing. J Prosthodont 1993;2(1):2-8. 
1237. Watanabe F, Uno I, Hata Y, Neuendorff G, Kirsch A. Analysis of stress distribution in a screw-retained implant prosthesis. Int J Oral Maxillofac Implants 2000;15(2):209-218.

1238. Jemt T, Linden B, Lekholm U. Failures and complications in 127 consecutively placed fixed partial prostheses supported by Branemark implants: from prosthetic treatment to first annual checkup. Int J Oral Maxillofac Implants 1992;7(1):40-44.

1239. Jemt T, Lekholm U. Oral implant treatment in posterior partially edentulous jaws: a 5-year follow-up report. Int J Oral Maxillofac Implants 1993;8(6):635-640.

1240. Gunne J, Jemt T, Linden B. Implant treatment in partially edentulous patients: a report on prostheses after 3 years. Int J Prosthodont 1994;7(2):143-148.

1241. Lekholm UvS, D.; Herrmann, I.; Bolender, C.; Folmer, T.; Gunne, J.; Henry, P.; Higuchi, K.; Laney, W.R.; Lindén, U. Osseointegrated Implants in the Treatment of Partially Edentulous Jaws: A Prospective 5-Year Multicenter Study. Int J Oral Maxillofac Implants 1994;9(6):627-635.

1242. Rangert B, Sullivan R. Biomechanical principles preventing prosthetic overload induced by bending. Nobelpharma News 1993:4-5.

1243. Naert I, Quirynen M, van Steenberghe D, Darius P. A six-year prosthodontic study of 509 consecutively inserted implants for the treatment of partial edentulism. J Prosthet Dent 1992;67(2):236-245.

1244. Quirynen M, Naert I, van Steenberghe D, Dekeyser C, Callens A. Periodontal aspects of osseointegrated fixtures supporting a partial bridge. An up to 6-years retrospective study. J Clin Periodontol 1992;19(2):118-126.

1245. Allen PF, McMillan AS, Smith DG. Complications and maintenance requirements of implant-supported prostheses provided in a UK dental hospital. Br Dent J 1997;182(8):298-302.

1246. Williams DN. The hydrogen embrittlement of titanium alloys. J Inst Metals 1962;91:147-152.

1247. Yokoyama K, Hamada K, Moriyama K, Asaoka K. Degradation and fracture of $\mathrm{Ni}-\mathrm{Ti}$ superelastic wire in an oral cavity. Biomaterials 2001;22(16):2257-2262.

1248. Larson F, Bland B, Beaty K, Heylmun T. Relation between titanium alloy properties and dental implant fatigue strength. J Dent Res $1995 ; 74(($ special issue $)): 112$.

1249. Rangert B, Forsmalm G. Strength characteristics of CP titanium. Nobelpharma News. 1994:7.

1250. Wise MD. Failure in the Restored Dentition: Management and Treatment. London: Quintessence; 1995. 
1251. Sugerman PB, Barber MT. Patient selection for endosseous dental implants: oral and systemic considerations. Int J Oral Maxillofac Implants 2002;17(2):191-201.

1252. Balshi TJ, Wolfinger GJ. Management of the posterior maxilla in the compromised patient: historical, current, and future perspectives. Periodontol 2000 2003;33:67-81.

1253. Hwang D, Wang HL. Medical contraindications to implant therapy: part I: absolute contraindications. Implant Dent 2006;15(4):353-360.

1254. Hwang D, Wang HL. Medical contraindications to implant therapy: Part II: Relative contraindications. Implant Dent 2007;16(1):13-23.

1255. Diz P, Scully C, Sanz M. Dental implants in the medically compromised patient. J Dent 2013;41(3):195-206.

1256. Scully C. Medical problems in dentistry. 6th edition ed. London: Churchill Livingstone; 2010.

1257. Gornitsky M, Hammouda W, Rosen H. Rehabilitation of a hemophiliac with implants: a medical perspective and case report. $J$ Oral Maxillofac Surg 2005;63(5):592-597.

1258. Bacci C, Berengo M, Favero L, Zanon E. Safety of dental implant surgery in patients undergoing anticoagulation therapy: a prospective case-control study. Clin Oral Implants Res 2011;22(2):151-156.

1259. Zola MB. Staged sinus augmentation and implant placement in a patient with osteogenesis imperfecta. J Oral Maxillofac Surg 2000;58(4):443-447.

1260. Lee CY, Ertel SK. Bone graft augmentation and dental implant treatment in a patient with osteogenesis imperfecta: review of the literature with a case report. Implant Dent 2003;12(4):291-295.

1261. Binger T, Rucker M, Spitzer WJ. Dentofacial rehabilitation by osteodistraction, augmentation and implantation despite osteogenesis imperfecta. Int J Oral Maxillofac Surg 2006;35(6):559-562.

1262. Prabhu N, Duckmanton N, Stevenson AR, Cameron A. The placement of osseointegrated dental implants in a patient with type IV B osteogenesis imperfecta: a 9-year follow-up. Oral Surg Oral Med Oral Pathol Oral Radiol Endod 2007;103(3):349-354.

1263. Payne MA, Postlethwaite KR, Smith DG, Nohl FS. Implant-supported rehabilitation of an edentate patient with osteogenesis imperfecta: a case report. Int J Oral Maxillofac Implants 2008;23(5):947-952.

1264. Wannfors K, Johansson C, Donath K. Augmentation of the mandible via a "tent-pole" procedure and implant treatment in a patient with type III osteogenesis imperfecta: clinical and histologic considerations. Int J Oral Maxillofac Implants 2009;24(6):1144-1148. 
1265. Friberg B. Branemark system implants and rare disorders: a report of six cases. Int J Periodontics Restorative Dent 2013;33(2):139-148.

1266. Friberg B. Treatment with dental implants in patients with severe osteoporosis: a case report. Int J Periodontics Restorative Dent 1994;14(4):348-353.

1267. Eder A, Watzek G. Treatment of a patient with severe osteoporosis and chronic polyarthritis with fixed implant-supported prosthesis: a case report. Int J Oral Maxillofac Implants 1999;14(4):587-590.

1268. Ella B, Lasserre JF, Blanchard JP, Fricain JC. A 4-year follow-up of two complete mandibular implant-supported removable prostheses in a patient with severe rheumatoid polyarthritis: case report. Int J Oral Maxillofac Implants 2011;26(2):e19-22.

1269. Barker D, Nohl FS, Postlethwaite KR, Smith DG. Case report of multiple implant failure in a patient with ankylosing spondylitis. Eur J Prosthodont Restor Dent 2008;16(1):20-23.

1270. Krennmair G, Seemann R, Piehslinger E. Dental implants in patients with rheumatoid arthritis: clinical outcome and peri-implant findings. $J$ Clin Periodontol 2010;37(10):928-936.

1271. Weinlander M, Krennmair G, Piehslinger E. Implant prosthodontic rehabilitation of patients with rheumatic disorders: a case series report. Int J Prosthodont 2010;23(1):22-28.

1272. Bornstein MM, Cionca N, Mombelli A. Systemic conditions and treatments as risks for implant therapy. Int J Oral Maxillofac Implants 2009;24 Suppl:12-27.

1273. Dao TT, Anderson JD, Zarb GA. Is osteoporosis a risk factor for osseointegration of dental implants? Int J Oral Maxillofac Implants 1993;8(2):137-144.

1274. Dvorak G, Arnhart C, Heuberer S, Huber CD, Watzek G, Gruber R. Peri-implantitis and late implant failures in postmenopausal women: a cross-sectional study. J Clin Periodontol 2011;38(10):950-955.

1275. Al-Sabbagh M, Robinson FG, Romanos G, Thomas MV. Osteoporosis and bisphosphonate-related osteonecrosis in a dental school implant patient population. Implant Dent 2015;24(3):328-332.

1276. Siebert T, Jurkovic R, Statelova D, Strecha J. Immediate Implant Placement in a Patient With Osteoporosis Undergoing Bisphosphonate Therapy: 1-Year Preliminary Prospective Study. J Oral Implantol 2015;41 Spec No:360-365.

1277. Merheb J, Temmerman A, Rasmusson L, Kubler A, Thor A, Quirynen M. Influence of Skeletal and Local Bone Density on Dental Implant Stability in Patients with Osteoporosis. Clin Implant Dent Relat Res 2016;18(2):253-260. 
1278. Temmerman A, Rasmusson L, Kubler A, Thor A, Quirynen M. An open, prospective, non-randomized, controlled, multicentre study to evaluate the clinical outcome of implant treatment in women over 60 years of age with osteoporosis/osteopenia: 1-year results. Clin Oral Implants Res 2017;28(1):95-102.

1279. Goss A, Bartold M, Sambrook P, Hawker P. The nature and frequency of bisphosphonate-associated osteonecrosis of the jaws in dental implant patients: a South Australian case series. J Oral Maxillofac Surg 2010;68(2):337-343.

1280. Mattheos N, Caldwell P, Petcu EB, Ivanovski S, Reher P. Dental implant placement with bone augmentation in a patient who received intravenous bisphosphonate treatment for osteoporosis. J Can Dent Assoc 2013;79:d2.

1281. Coutinho AE, Chapman KE. The anti-inflammatory and immunosuppressive effects of glucocorticoids, recent developments and mechanistic insights. Mol Cell Endocrinol 2011;335(1):2-13.

1282. Buchman AL. Side effects of corticosteroid therapy. J Clin Gastroenterol 2001;33(4):289-294.

1283. Bencharit S, Reside GJ, Howard-Williams EL. Complex prosthodontic treatment with dental implants for a patient with polymyalgia rheumatica: a clinical report. Int J Oral Maxillofac Implants 2010;25(6):1241-1245.

1284. Fujimoto T, Niimi A, Sawai T, Ueda M. Effects of steroid-induced osteoporosis on osseointegration of titanium implants. Int J Oral Maxillofac Implants 1998;13(2):183-189.

1285. Werner SB, Tessler J, Guglielmotti MB, Cabrini RL. Effect of dexamethasone on osseointegration: a preliminary experimental study. J Oral Implantol 1996;22(3-4):216-219.

1286. Shibuya Y, Takata N, Takeuchi J, Tsuji K, Ishida S, Kobayashi M, Suzuki H, Hasegawa T, Kamae I, Komori T. Analysis of the 619 Branemark System TiUnite implants: a retrospective study. Kobe J Med Sci 2012;58(1):E19-28.

1287. Khadivi V, Anderson J, Zarb GA. Cardiovascular disease and treatment outcomes with osseointegration surgery. J Prosthet Dent 1999;81(5):533-536.

1288. Nobre Mde A, Malo P, Goncalves Y, Sabas A, Salvado F. Outcome of dental implants in diabetic patients with and without cardiovascular disease: A 5-year post-loading retrospective study. Eur J Oral Implantol 2016;9(1):87-95.

1289. Fox RI. Sjogren's syndrome. Lancet 2005;366(9482):321-331. 
1290. Fox PC, Bowman SJ, Segal B, Vivino FB, Murukutla N, Choueiri K, Ogale S, McLean L. Oral involvement in primary Sjogren syndrome. J Am Dent Assoc 2008;139(12):1592-1601.

1291. Binon PP, Fowler CN. Implant-supported fixed prosthesis treatment of a patient with Sjogren's syndrome: a clinical report. Int J Oral Maxillofac Implants 1993;8(1):54-58.

1292. Payne AG, Lownie JF, Van Der Linden WJ. Implant-supported prostheses in patients with Sjogren's syndrome: a clinical report on three patients. Int J Oral Maxillofac Implants 1997;12(5):679-685.

1293. Isidor F, Brondum K, Hansen HJ, Jensen J, Sindet-Pedersen S. Outcome of treatment with implant-retained dental prostheses in patients with Sjogren syndrome. Int J Oral Maxillofac Implants 1999;14(5):736743.

1294. Binon PP. Thirteen-year follow-up of a mandibular implant-supported fixed complete denture in a patient with Sjogren's syndrome: a clinical report. J Prosthet Dent 2005;94(5):409-413.

1295. Öczakir C, Balmer S, Mericske-Stern R. Implant-prosthodontic treatment for special care patients: a case series study. Int J Prosthodont 2005;18(5):383-389.

1296. Spinato S, Soardi CM, Zane AM. A mandibular implant-supported fixed complete dental prosthesis in a patient with Sjogren syndrome: case report. Implant Dent 2010;19(3):178-183.

1297. de Mendonca Invernici M, Finger Stadler A, Vale Nicolau G, Naval Machado MA, Soares de Lima AA, Compagnoni Martins M. Management of Sjogren's Syndrome Patient: A Case Report of Prosthetic Rehabilitation with 6-Year Follow-Up. Case Rep Dent 2014;2014:761251.

1298. Chatzistavrianou D, Shahdad S. Implant Treatment in Patients with Sjogren's Syndrome: A Review of the Literature and Two Clinical Case Reports. Eur J Prosthodont Restor Dent 2016;24(1):40-46.

1299. Chochlidakis K, Ercoli C, Elad S. Challenges in implant-supported dental treatment in patients with Sjogren's syndrome: A case report and literature review. Quintessence Int 2016;47(6):515-524.

1300. Cuifen L, Guoguang P, Yuanhua F, Xingxiang H. [Dental implantation in a patient with Sjogren's syndrome: a case report]. Hua Xi Kou Qiang Yi Xue Za Zhi 2017;35(1):108-111.

1301. Albrecht K, Callhoff J, Westhoff G, Dietrich T, Dorner T, Zink A. The Prevalence of Dental Implants and Related Factors in Patients with Sjogren Syndrome: Results from a Cohort Study. J Rheumatol 2016;43(7):1380-1385. 
1302. Korfage A, Raghoebar GM, Arends S, Meiners PM, Visser A, Kroese FG, Bootsma H, Vissink A. Dental Implants in Patients with Sjogren's Syndrome. Clin Implant Dent Relat Res 2016;18(5):937-945.

1303. Stevenson GC, Riano PC, Moretti AJ, Nichols CM, Engelmeier RL, Flaitz CM. Short-term success of osseointegrated dental implants in HIV-positive individuals: a prospective study. J Contemp Dent Pract 2007;8(1):1-10.

1304. Oliveira MA, Gallottini M, Pallos D, Maluf PS, Jablonka F, Ortega KL. The success of endosseous implants in human immunodeficiency viruspositive patients receiving antiretroviral therapy: a pilot study. $\mathrm{J} \mathrm{Am}$ Dent Assoc 2011;142(9):1010-1016.

1305. Gay-Escoda C, Perez-Alvarez D, Camps-Font O, Figueiredo R. Longterm outcomes of oral rehabilitation with dental implants in HIVpositive patients: A retrospective case series. Med Oral Patol Oral Cir Bucal 2016;21(3):e385-391.

1306. Gherlone EF, Cappare P, Tecco S, Polizzi E, Pantaleo G, Gastaldi G, Grusovin MG. A Prospective Longitudinal Study on Implant Prosthetic Rehabilitation in Controlled HIV-Positive Patients with 1-Year FollowUp: The Role of CD4+ Level, Smoking Habits, and Oral Hygiene. Clin Implant Dent Relat Res 2016;18(5):955-964.

1307. Gherlone EF, Cappare P, Tecco S, Polizzi E, Pantaleo G, Gastaldi G, Grusovin MG. Implant Prosthetic Rehabilitation in Controlled HIV-Positive Patients: A Prospective Longitudinal Study with 1-Year Follow-Up. Clin Implant Dent Relat Res 2016;18(4):725-734.

1308. May MC, Andrews PN, Daher S, Reebye UN. Prospective cohort study of dental implant success rate in patients with AIDS. Int J Implant Dent 2016;2(1):20.

1309. Rajnay ZW, Hochstetter RL. Immediate placement of an endosseous root-form implant in an HIV-positive patient: report of a case. $J$ Periodontol 1998;69(10):1167-1171.

1310. Baron M, Gritsch F, Hansy AM, Haas R. Implants in an HIV-positive patient: a case report. Int J Oral Maxillofac Implants 2004;19(3):425430 .

1311. Shetty K, Achong R. Dental implants in the HIV-positive patient--case report and review of the literature. Gen Dent 2005;53(6):434-437; quiz 438, 446.

1312. Achong RM, Shetty K, Arribas A, Block MS. Implants in HIV-positive patients: 3 case reports. J Oral Maxillofac Surg 2006;64(8):1199-1203.

1313. Strietzel FP, Rothe S, Reichart PA, Schmidt-Westhausen AM. Implantprosthetic treatment in HIV-infected patients receiving highly active antiretroviral therapy: report of cases. Int J Oral Maxillofac Implants 2006;21(6):951-956. 
1314. Kolhatkar S, Khalid S, Rolecki A, Bhola M, Winkler JR. Immediate dental implant placement in HIV-positive patients receiving highly active antiretroviral therapy: a report of two cases and a review of the literature of implants placed in HIV-positive individuals. J Periodontol 2011;82(3):505-511.

1315. Castellanos-Cosano L, Núñez-Vázquez RJ, Segura-Egea JJ, TorresLagares D, Corcuera-Flores JR, Machuca-Portillo G. Protocol for oral implant rehabilitation in a hemophilic HIV-positive patient with type C hepatitis. Implant Dent 2014;23(5):622-625.

1316. Romanos GE, Goldin E, Marotta L, Froum S, Tarnow DP. Immediate loading with fixed implant-supported restorations in an edentulous patient with an HIV infection: a case report. Implant Dent 2014;23(1):8-12.

1317. Vidal F, Vidal R, Bochnia J, de Souza RC, Goncalves LS. Dental implants and bone augmentation in HIV-infected patients under HAART: Case report and review of the literature. Spec Care Dentist 2017.

1318. Adbulwassie H, Dhanrajani PJ, Jiffry A. Papillon-Lefevre syndrome. Reappraisal of etiology, clinical features and treatment. II. Oral rehabilitation using osseointegrated implants. Indian J Dent Res 1996;7(2):63-70.

1319. Ullbro C, Crossner CG, Lundgren T, Stalblad PA, Renvert S. Osseointegrated implants in a patient with Papillon-Lefevre syndrome. A 4 1/2-year follow up. J Clin Periodontol 2000;27(12):951-954.

1320. Woo I, Brunner DP, Yamashita DD, Le BT. Dental implants in a young patient with Papillon-Lefevre syndrome: a case report. Implant Dent 2003;12(2):140-144.

1321. Etöz OA, Ulu M, Kesim B. Treatment of patient with Papillon-Lefevre syndrome with short dental implants: a case report. Implant Dent 2010;19(5):394-399.

1322. Ahmadian L, Monzavi A, Arbabi R, Hashemi HM. Full-mouth rehabilitation of an edentulous patient with Papillon-Lefevre syndrome using dental implants: a clinical report. J Prosthodont 2011;20(8):643648.

1323. Senel FC, Altintas NY, Bagis B, Cankaya M, Pampu AA, Satiroglu I, Senel AC. A 3-year follow-up of the rehabilitation of Papillon-Lefevre syndrome by dental implants. J Oral Maxillofac Surg 2012;70(1):163167.

1324. Al Farraj AlDosari A. Oral rehabilitation of a case of Papillon-Lefevre syndrome with dental implants. Saudi Med J 2013;34(4):424-427. 
1325. Alzoubi F, Bedrossian E, Wong A. Oral rehabilitation of patients with Chediak-Higashi syndrome using zygoma and root form implantsupported fixed prostheses: A report of two patients. J Prosthet Dent 2016;116(6):831-835.

1326. Rogers JO. Implant-stabilized complete mandibular denture for a patient with cerebral palsy. Dent Update 1995;22(1):23-26.

1327. López-Jiménez J, Romero-Dominguez A, Gimenez-Prats MJ. Implants in handicapped patients. Med Oral 2003;8(4):288-293.

1328. Ekfeldt A, Zellmer M, Carlsson GE. Treatment with implantsupported fixed dental prostheses in patients with congenital and acquired neurologic disabilities: a prospective study. Int J Prosthodont 2013;26(6):517-524.

1329. Ekfeldt A. Early experience of implant-supported prostheses in patients with neurologic disabilities. Int J Prosthodont 2005;18(2):132-138.

1330. Addy L, Korszun A, Jagger RG. Dental implant treatment for patients with psychiatric disorders. Eur J Prosthodont Restor Dent 2006;14(2):90-92.

1331. Feijoo JF, Limeres J, Diniz M, Del Llano A, Seoane J, Diz P. Osseointegrated dental implants in patients with intellectual disability: a pilot study. Disabil Rehabil 2012;34(23):2025-2030.

1332. Ambard A, Mueninghoff L. Rehabilitation of a bulimic patient using endosteal implants. J Prosthodont 2002;11(3):176-180.

1333. Heckmann SM, Heckmann JG, Weber HP. Clinical outcomes of three Parkinson's disease patients treated with mandibular implant overdentures. Clin Oral Implants Res 2000;11(6):566-571.

1334. Kubo K, Kimura K. Implant surgery for a patient with Parkinson's disease controlled by intravenous midazolam: a case report. Int J Oral Maxillofac Implants 2004;19(2):288-290.

1335. Cune MS, Strooker H, van der Reijden WA, de Putter C, Laine ML, Verhoeven JW. Dental implants in persons with severe epilepsy and multiple disabilities: a long-term retrospective study. Int J Oral Maxillofac Implants 2009;24(3):534-540.

1336. Del Rio Highsmith J. Manual de implantoprótesis para el desdentado total. Madrid: Avances Médico-Dentales; 1995.

1337. Guckes AD, Scurria MS, King TS, McCarthy GR, Brahim JS. Prospective clinical trial of dental implants in persons with ectodermal dysplasia. J Prosthet Dent 2002;88(1):21-25.

1338. Ekstrand K, Thomsson M. Ectodermal dysplasia with partial anodontia: prosthetic treatment with implant fixed prosthesis. ASDC J Dent Child 1988;55(4):282-284. 
1339. Bergendal T, Eckerdal O, Hallonsten AL, Koch G, Kurol J, Kvint S. Osseointegrated implants in the oral habilitation of a boy with ectodermal dysplasia: a case report. Int Dent J 1991;41(3):149-156.

1340. Davarpanah M, Moon JW, Yang LR, Celletti R, Martinez H. Dental implants in the oral rehabilitation of a teenager with hypohidrotic ectodermal dysplasia: report of a case. Int J Oral Maxillofac Implants 1997;12(2):252-258.

1341. Kearns G, Sharma A, Perrott D, Schmidt B, Kaban L, Vargervik K. Placement of endosseous implants in children and adolescents with hereditary ectodermal dysplasia. Oral Surg Oral Med Oral Pathol Oral Radiol Endod 1999;88(1):5-10.

1342. Sweeney IP, Ferguson JW, Heggie AA, Lucas JO. Treatment outcomes for adolescent ectodermal dysplasia patients treated with dental implants. Int J Paediatr Dent 2005;15(4):241-248.

1343. Garagiola U, Maiorana C, Ghiglione V, Marzo G, Santoro F, Szabo G. Osseointegration and guided bone regeneration in ectodermal dysplasia patients. J Craniofac Surg 2007;18(6):1296-1304.

1344. Rad AS, Siadat H, Monzavi A, Mangoli AA. Full mouth rehabilitation of a hypohidrotic ectodermal dysplasia patient with dental implants: a clinical report. J Prosthodont 2007;16(3):209-213.

1345. Bergendal B, Ekman A, Nilsson P. Implant failure in young children with ectodermal dysplasia: a retrospective evaluation of use and outcome of dental implant treatment in children in Sweden. Int J Oral Maxillofac Implants 2008;23(3):520-524.

1346. Kirmeier R, Gluhak C, Marada P, Wegscheider WA, Eskici A, Jakse $\mathrm{N}$. Oral rehabilitation of adult twins with severe lack of bone due to hypohidrotic ectodermal dysplasia--a 12-month follow-up. J Oral Maxillofac Surg 2009;67(1):189-194.

1347. Grecchi F, Zingari F, Bianco R, Zollino I, Casadio C, Carinci F. Implant rehabilitation in grafted and native bone in patients affected by ectodermal dysplasia: evaluation of 78 implants inserted in 8 patients. Implant Dent 2010;19(5):400-408.

1348. Koyuncuoglu CZ, Metin S, Saylan I, Calisir K, Tuncer O, Kantarci A. Full-mouth rehabilitation of a patient with ectodermal dysplasia with dental implants. J Oral Implantol 2014;40(6):714-721.

1349. Bergendal B, Bjerklin K, Bergendal T, Koch G. Dental Implant Therapy for a Child with X-linked Hypohidrotic Ectodermal Dysplasia--Three Decades of Managed Care. Int J Prosthodont 2015;28(4):348-356.

1350. Wu Y, Wang XD, Wang F, Huang W, Zhang Z, Zhang Z, Kaigler D, Zou D. Restoration of Oral Function for Adult Edentulous Patients with Ectodermal Dysplasia: A Prospective Preliminary Clinical Study. Clin Implant Dent Relat Res 2015;17 Suppl 2:e633-642. 
1351. Revanappa PD, Gaur S, Srivastava R, Patil A, Prithviraj SD. Ectodermal Dysplasia: Management of Knife-Edged Irregular Ridge and Its Rehabilitation with Hybrid Implant Prosthesis. J Clin Diagn Res 2016;10(5):Zd05-07.

1352. Maiorana C, Poli PP, Poggio C, Barbieri P, Beretta M. Oral Rehabilitation of a Patient With Ectodermal Dysplasia Treated With Fresh-Frozen Bone Allografts and Computer-Guided Implant Placement: A Clinical Case Report. J Oral Maxillofac Surg 2017.

1353. Larrazabal-Morón C, Boronat-Lopez A, Peñarrocha-Diago M, Peñarrocha-Diago M. Oral rehabilitation with bone graft and simultaneous dental implants in a patient with epidermolysis bullosa: a clinical case report. J Oral Maxillofac Surg 2009;67(7):1499-1502.

1354. Peñarrocha-Diago M, Serrano C, Sanchis JM, Silvestre FJ, Bagan JV. Placement of endosseous implants in patients with oral epidermolysis bullosa. Oral Surg Oral Med Oral Pathol Oral Radiol Endod 2000;90(5):587-590.

1355. Lee H, Al Mardini M, Ercoli C, Smith MN. Oral rehabilitation of a completely edentulous epidermolysis bullosa patient with an implantsupported prosthesis: a clinical report. J Prosthet Dent 2007;97(2):6569.

1356. Peñarrocha M, Larrazabal C, Balaguer J, Serrano C, Silvestre J, Bagan JV. Restoration with implants in patients with recessive dystrophic epidermolysis bullosa and patient satisfaction with the implant-supported superstructure. Int J Oral Maxillofac Implants 2007;22(4):651-655.

1357. Peñarrocha M, Rambla J, Balaguer J, Serrano C, Silvestre J, Bagan JV. Complete fixed prostheses over implants in patients with oral epidermolysis bullosa. J Oral Maxillofac Surg 2007;65(7 Suppl 1):103106.

1358. Muller F, Bergendal B, Wahlmann U, Wagner W. Implant-supported fixed dental prostheses in an edentulous patient with dystrophic epidermolysis bullosa. Int J Prosthodont 2010;23(1):42-48.

1359. Oliveira MA, Ortega KL, Martins FM, Maluf PS, Magalhaes MG. Recessive dystrophic epidermolysis bullosa--oral rehabilitation using stereolithography and immediate endosseous implants. Spec Care Dentist 2010;30(1):23-26.

1360. Peñarrocha-Oltra D, Peñarrocha-Diago M, Balaguer-Martinez J, Ata-Ali J, Peñarrocha-Diago M. Full-arch fixed prosthesis supported by four implants in patients with recessive dystrophic epidermolysis bullosa. Oral Surg Oral Med Oral Pathol Oral Radiol Endod 2011;112(2):e4-10. 
1361. Peñarrocha-Oltra D, Aloy-Prosper A, Ata-Ali J, Peñarrocha-Diago M, Peñarrocha-Diago M. Implants placed simultaneously with particulated bone graft in patients diagnosed with recessive dystrophic epidermolysis bullosa. J Oral Maxillofac Surg 2012;70(1):e51-57.

1362. Agustín-Panadero R, Gomar-Vercher S, Peñarrocha-Oltra D, GuzmánLetelier M, Peñarrocha-Diago M. Fixed full-arch implant-supported prostheses in a patient with epidermolysis bullosa: a clinical case history report. Int J Prosthodont 2015;28(1):33-36.

1363. Czerninski R, Eliezer M, Wilensky A, Soskolne A. Oral lichen planus and dental implants--a retrospective study. Clin Implant Dent Relat Res 2013;15(2):234-242.

1364. Simark-Mattsson C, Bergenholtz G, Jontell M, Eklund C, Seymour GJ, Sugerman PB, Savage NW, Dahlgren UI. Distribution of interleukin-2, $-4,-10$, tumour necrosis factor-alpha and transforming growth factorbeta mRNAs in oral lichen planus. Arch Oral Biol 1999;44(6):499507.

1365. Lodi G, Scully C, Carrozzo M, Griffiths M, Sugerman PB, Thongprasom K. Current controversies in oral lichen planus: report of an international consensus meeting. Part 1. Viral infections and etiopathogenesis. Oral Surg Oral Med Oral Pathol Oral Radiol Endod 2005;100(1):40-51.

1366. Taubman MA, Kawai T. Involvement of T-lymphocytes in periodontal disease and in direct and indirect induction of bone resorption. Crit Rev Oral Biol Med 2001;12(2):125-135.

1367. Esposito SJ, Camisa C, Morgan M. Implant retained overdentures for two patients with severe lichen planus: a clinical report. J Prosthet Dent 2003;89(1):6-10.

1368. Reichart PA. Oral lichen planus and dental implants. Report of 3 cases. Int J Oral Maxillofac Surg 2006;35(3):237-240.

1369. Hernández G, Lopez-Pintor RM, Arriba L, Torres J, de Vicente JC. Implant treatment in patients with oral lichen planus: a prospectivecontrolled study. Clin Oral Implants Res 2012;23(6):726-732.

1370. López-Jornet P, Camacho-Alonso F, Sánchez-Siles M. Dental implants in patients with oral lichen planus: a cross-sectional study. Clin Implant Dent Relat Res 2014;16(1):107-115.

1371. Little JA, Falace D, Miller C, Rhodus NL. Dental management of the medically compromised patient. St. Louis: Mosby; 2003.

1372. Meijer GJ, Cune MS. [Surgical dilemmas. Medical restrictions and risk factors]. Ned Tijdschr Tandheelkd 2008;115(12):643-651. 

PAPERS I-IX 

I 



\author{
Review \\ Reasons for failures of oral implants \\ B. R. CHRCANOVIC*, T. ALBREKTSSON*^ $\&$ A. WENNERBERG* *Department of \\ Prosthodontics, Faculty of Odontology, Malmö University, Malmö, and 'Department of Biomaterials, Göteborg University, Göteborg, \\ Sweden
}

SUMMARY This study reviews the literature regarding the factors contributing to failures of dental implants. An electronic search was undertaken including papers from 2004 onwards. The titles and abstracts from these results were read to identify studies within the selection criteria. All reference lists of the selected studies were then hand-searched, this time without time restrictions. A narrative review discussed some findings from the first two parts where separate data from non-comparative studies may have indicated conclusions different from those possible to draw in the systematic analysis. It may be suggested that the following situations are correlated to increase the implant failure rate: a low insertion torque of implants that are planned to be immediately or early loaded, inexperienced surgeons inserting the implants, implant insertion in the maxilla, implant insertion in the posterior region of the jaws, implants in heavy smokers, implant insertion in bone qualities type III and IV, implant insertion in places with small bone volumes, use of shorter length implants, greater number of implants placed per patient, lack of initial implant stability, use of cylindrical (nonthreaded) implants and prosthetic rehabilitation with implant-supported overdentures. Moreover, it may be suggested that the following situations may be correlated with an increase in the implant failure rate: use of the non-submerged technique, immediate loading, implant insertion in fresh extraction sockets, smaller diameter implants. Some recently published studies suggest that modern, moderately rough implants may present with similar results irrespective if placed in maxillas, in smoking patients or using only short implants.

KEYWORDS: dental implants, failure, associated conditions, systematic review

Accepted for publication 8 February 2014

\section{Introduction}

Implant failure may be of a primary or secondary nature. In addition, marginal bone loss around oral implants may continue and create a secondary failure (Fig. 1). It is commonly difficult to assess the proper reason for implant failure. For instance, in the case of primary failures, that is, those implants that never osseointegrate, an alleged reason for the observed failure is overheating/poor surgery. However, from a strict scientific point of view, there is little evidence incriminating poor surgery as the cause of primary failures. A minimal demand for believing in the the- ory of poor surgery would be that this is noted in the clinical records at the time of implant placement. This is seldom the case. Instead, the poor surgery explanation is made retrospectively, perhaps in the lack of other evidence. In reality, we do not know why some implants fail primarily but fortunately the frequency of such failures is small, in the range of $1-2 \%$ in most clinical reports. Another substantial controversy relates to the reason for marginal bone loss, which may end in clinical failure. In the recent literature, it has been assumed that an alleged primary disease entitled peri-implantitis is the original reason behind marginal bone loss around oral implants $(1,2)$. Some 


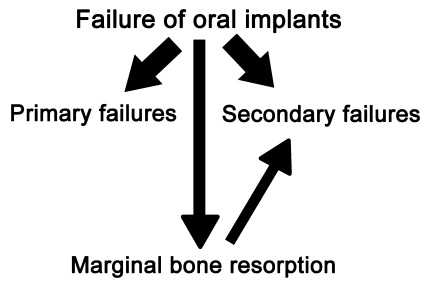

Fig. 1. Failure of oral implants.

authors have even suggested that any marginal bone loss after the first year of clinical function must depend on peri-implantitis (3). No $<8$ different definitions exist of what is to be regarded as peri-implantitis (4). In reality, the entire discussion about periimplantitis as a primary disease is probably misconceived (5-7). The reason for onset of marginal bone loss around implants is in all probability dependent on a complication to treatment, not on disease phenomenon. Infection and similar problems around oral implants is a secondary, not a primary phenomenon (7). Marginal bone loss has been demonstrated to be initiated by poor clinical handling, use of poor implant designs or by treating complicated patients. At a later stage patient disease, rapidly changing loading situations or reactions to cement particles accidentally embedded in the soft tissues represent other reasons for start of bone loss. Having said this, such conditions left untreated may secondarily result in aggravated bone resorption and peri-implantitis further complicating the clinical picture. Osseointegration is nothing but a foreign body response, and long-term clinical function is dependent on tissue equilibrium (7).

Secondary failure of an oral implant is in the great majority of cases preceded by marginal bone resorption, hence the importance of understanding the background to it. Chvartszaid et al. (8) assumed that if small summed trauma to the tissues than minor bone resorption occurred, but if a summed large trauma prevailed, then implant failure would follow. Trauma to the tissues that may disturb the maintained foreign body equilibrium that we call osseointegration is sometimes easy to understand such as inadequate surgery burning away the relevant cells needed for bone repair, but in other cases may be more difficult to identify. In this paper we have subdivided reasons for failure under subheadings such as surgical, location, patient, implant, prosthetic and other conditions. In the major, systematic part of our review, we have scrutinised comparative studies (with test and control situations) to present an attempt to a deeper understanding of failure mechanisms. However, as the great majority of clinical studies relate to turned, machined implants, this approach presented with some limitations with respect to understanding failure mechanisms of our present times when such implants have been largely replaced by modern, moderately rough devices. We have therefore added a narrative review of the outcome of such modern implants alone. This latter part of the paper is not based only on test and control situations as such an approach would have resulted in the inclusion of too few studies. Even if the quality of the evidence in the first, systematic part of our review is greater than found in the latter narrative review, we regarded the inclusion of some older papers of now withdrawn implant systems as well as more recent publications on modern implants to be important in our aim to present an as updated version of failure analyses as is possible.

\section{Materials and methods}

\section{Objectives}

The purpose of this study is to review the literature regarding the factors contributing to failures of osseointegrated endosseous dental implants. The aetiopathogenesis of failing/failed implants was evaluated in relation to clinical findings. The following questions were raised and will be evaluated: What are the main factors that may exert influence on the failure of endosseous dental implants? How these influences possibly affect the osseointegration?

\section{Data source and search strategies}

An electronic search was undertaken in August 2013 in the PubMed website (U.S. National Library of Medicine, National Institutes of Health; Bethesda, Maryland, USA). The last complementary check-up for newly published papers within the topic was performed in October 2013. The following filters from the referred website were selected: the item 'Clinical Trial' within 'Article types' and the item 'English' within 'Languages'. Only the papers from 2004 
onwards were included at the first part of the screening process. The following terms were used in the search strategy:

\{Subject AND Adjective\}

\{Subject: (dental implant [text words])

AND

Adjective: (failure [text words])

NOT

(grafting OR irradiated OR sinus lift [text words])\}

Some procedures were a reason for exclusion of studies because the influence of the irradiation of the bone-grafting materials and the added surgical procedure cannot be quantified or eliminated.

The publication had to be included in the electronic database to be considered in the review. All reference lists of the selected studies were then hand-searched for additional papers that might meet the eligibility criteria for inclusion in this study. The titles and abstracts (when available) from these results were read by the authors for identifying studies meeting the eligibility criteria. For studies appearing to meet the inclusion criteria, or for which there were insufficient data in the title and abstract to make a clear decision, the full report was obtained and assessed.

In the second part of the screening process, a handsearching process called 'snowballing' included papers that may have showed significant results implying that certain factors may lead to implant failure, with no restrictions concerning the year of publication.

In the third part of the screening process, literature review papers about factors associated with implant failures were used to identify other possible factors that were not enumerated in the two previous screening processes.

Contact with authors for possible missing data was not performed. Disagreement regarding the inclusion or exclusion of the retrieved articles was resolved by a discussion between the three reviewers.

\section{Inclusion criteria}

Eligibility criteria included controlled clinical human studies, either randomised or not. Regarding the type of intervention, endosseous root form titanium or titanium alloy implants to replace teeth were taken into consideration. All outcomes reporting on failure of implants, including clinical, radiographic and implant fracture, were considered. For the studies publishing more than one paper but with different follow-up periods, only the publication with the longest (the last) follow-up period was considered. For this review, implant failure represents the complete loss of the implant.

\section{Exclusion criteria}

Simple case reports, not controlled case series, and animal studies were not included. Comparisons of failure rates between teeth and implants in studies performing the same prosthetic treatment were not considered, nor studies evaluating prostheses supported by the combination of teeth and implants. Controlled studies not reporting implant failures rates were excluded. Studies reporting the use of implants for anchorage of maxillofacial prostheses, use of zygomatic implants, transitional/provisional implants, zirconia implants, and/or orthodontic implants or any other use were excluded. Studies performing grafting procedures concomitantly or previously to implant insertion surgery were excluded. Studies evaluating the survival/failure rates of implants inserted in patients previously submitted to radiotherapy in the head and neck region were excluded.

\section{Data collection and synthesis}

A sum-up of the clinical outcomes will be given in most cases, usually without a detailed analysis of the individual papers.

We did another type of screening in the third part of the present paper. The first two parts of this contribution deal with comparative studies that have been selected and summarised in a systematic manner. In contrast, the third part represents a narrative review where other papers than comparative ones are included too. This third part will discuss some findings from the first two parts where separate data from non-comparative studies may have indicated conclusions different from those possible to draw in the systematic analysis. In addition, the third part of our paper will discuss marginal bone loss which may lead to secondary failure of the implant. For example, data of a short-term nature ( 5 years follow-up or less) may have indicated quite positive a clinical outcome of some implant types such as IMZ or hydroxyapatitecoated implants to mention but a few. In the first two 
parts of this publication, known problems with such systems may have passed unnoticed unless comparative studies indicated some problems. However, from non-comparative studies of the same implant systems, we have learned about severe problems in the form of marginal bone loss with the same implants. This will be reported in part 3 of this contribution.

\section{Results}

The study selection process is summarised in Fig. 2. The first part of the search strategy resulted in 304 papers from the period 2004 to 2013. The three reviewers independently screened the abstracts for those articles related to the focus questions. Of the 304 studies found, 87 were excluded for not being controlled studies, 24 for performing graft procedures in the implant site, 15 for using other types of implants (zygomatic, transitional, zirconia or orthodontic), two for comparing failure rates between teeth and implants in with the same prosthetic treatment or prostheses supported by the combination of teeth and implants, 42 for not evaluating implant failure and 16 for being the same study but with earlier follow-up periods. Thus, the first part of the screening process resulted in 118 eligible publications. Additional handsearching of the reference lists of selected studies yielded 67 additional papers from 2004 onwards, and 126 papers published before 2004 (already excluding 12 papers for being the same study but with earlier follow-up periods). The second part of the screening

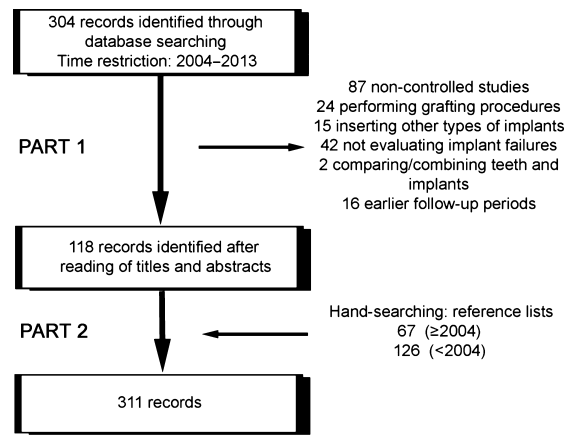

Fig. 2. Study screening process. process added more 193 publications, totalling 311 publications.

\section{Surgical conditions}

Prophylactic antibiotics versus placebo. Even though oral surgeons often prescribe antibiotics routinely following implant surgery, the usefulness of pre- and postoperative antibiotics in reducing the failure rates of endosseous implants remained unclear and unsubstantiated for several years. Some studies tried to evaluate whether the use of antibiotics may or may not influence the survival of dental implants.

Comparing the implant failure rates in patients receiving pre-operative antibiotics versus in patients who did not receive (or received a placebo), it was observed that antibiotics administered pre-operatively statistically improved implant survival in some studies $(9,10)$.

However, it was observed for most of the studies that there was no significant difference regarding the survival rate between the two groups (11-18), even though in one of these studies (15), four times more patients in the placebo group experienced implant failures than in the antibiotic group. Post-operative antibiotics did not influence the results of implant failure rates in one study (9). The drawback of these studies is the fact that the regimens used by each study for each patient varied somewhat by antibiotic type, dosage and time of administration.

From the results of these studies, it can be concluded that there is some evidence suggesting that pre-operative antibiotics significantly reduce failures of dental implants placed in ordinary conditions, but it is still unknown whether post-operative antibiotics are beneficial. Although the exact mechanism by which pre-operative antibiotics produce this effect is not known, it may be that a more aseptic local environment during the time of implant placement and in the immediate perioperative period results in improved healing and, ultimately, a better state of osseointegration (10).

Different types/regimen of antibiotic therapy. Instead of evaluating whether the use of antibiotics may or may not influence the survival of dental implants, some studies assessed whether the use of different regimen of antibiotic therapy could affect the implant survival. 
Lambert et al. (19) evaluated the influence of smoking on the survival of 2887 implants. At 36 months, implants in smokers failed $14.9 \%$ of the time when pre-operative antibiotics were not given or given at an ineffective dosage. Non-smokers or those who have quit had a $7.5 \%$ failure rate when not given pre-operative antibiotics. Smokers not given pre-operative antibiotics were almost three times more likely to have implant failures than those provided preoperative antibiotic coverage. When pre-operative antibiotics were used, the failure rate for both smokers and non-smoker/quit subjects was the same, decreasing to $4.7 \%$.

Wagenberg and Froum (20) retrospectively evaluated 1925 immediately placed implants. Patients unable to utilise post-surgical amoxicillin were 3.34 times as likely to experience implant failure as patients who received amoxicillin. Patients with an allergy to penicillin $(8.52 \%$ of failure) were 5.7 times more likely to experience implant failures due to infection than patients without allergy to penicillin (2.95\% of failure).

However, it can be observed from the results of six other studies that, in conjunction with routine oral implant surgery procedures, the adoption of different antibiotic prophylaxis regimens does not affect the implant failure rate. These different antibiotic prophylaxis regimens could be a single pre-operative dose versus $3(21), 7(22,23)$ or 10 days of administration (24), no antibiotic treatment versus a single pre-operative dose versus a preoperative dose plus post-operative antibiotic treatment for 7 days versus no pre-operative dose and post-operative antibiotic treatment for 7 days (25), a single pre-operative dose versus a pre-operative dose plus post-operative antibiotic treatment for 5 days versus no pre-operative dose and post-operative antibiotic treatment for 5 days (26).

Submerged versus non-submerged implants. Two different methods of implant insertion have been developed: the two-stage surgical technique (in which the implant is inserted in one session and the abutment is connected in another surgical procedure (submerged implant), and the one-stage surgery (non-submerged implant). The use of one-stage surgery has gained more and more interest as it reduces surgical intervention, thus reducing surgical time and patient discomfort, and produces healed and healthy peri-implant mucosa at the time of prosthetic rehabilitation (27). Several clinical studies were published comparing these two different surgical approaches.

Many studies observed that implant placement according to single-stage surgery (non-submerged implant) has the same predictability as two-stage surgery (submerged implant), with a high survival rate (27-42). However, many other studies showed that there is a greater risk of implant failures with a nonsubmerged technique compared with a submerged technique (43-50).

Flapless versus conventional flapped surgery. When placing dental implants, a flap is usually increased to better visualise the area. There is also the possibility to work blindly and insert implants without flap elevation (flapless surgery). Currently, some software systems using computed tomography scans have been proposed to aid in planning surgery and to produce surgical drilling guides to transfer the planned position to the surgical field. These guides are manufactured in such a way that they match the location, trajectory and depth of the planned implant with a high degree of precision. As the dental practitioner places the implants, the guides stabilise the drilling by restricting the degrees of freedom of the drill trajectory and depth (51). Some studies have compared the survival rate of implants using these two techniques.

By observing the results from the available controlled studies on the subject, it can be stated that most studies reported that implant placement according to flapless surgery has comparable results with the conventional flapped surgery, with a high survival rate (52-67). The one exception is the study of Sennerby et al. (68), which showed a higher failure probability for the implants inserted through the flapless technique.

Different insertion torques. Only two studies evaluating the survival rates of dental implants placed under different insertion torques were published, whereas one study (24) evaluated cutting torque measurements during implant placement.

Friberg et al. (24) evaluated cutting torque measurements during implant placement and correlated these observations with implant failure. According to the technique, the consumed electric current during lowspeed threading of implant sites is registered and the 
true cutting resistance of bone is calculated. The results of the study showed that the mean cutting torque values for the 14 implants that failed and the 398 successful implants were similar.

In the study of Ottoni et al. (69), implants were restored within a 24 -h period after placement with a provisional crown receiving occlusal load (test group), and this situation was compared with implants restored after a healing of 3-6 months (control group; 23 implants for each group). The primary stability was standardised with a minimum insertion torque of $20 \mathrm{Ncm}$. Relative risk of implant failure was associated with insertion torque in the test group but not so in the control group. To achieve osseointegration, it was found that an insertion torque above $32 \mathrm{Ncm}$ was necessary. It was observed that the insertion torque was associated with the risk potential, which can be decreased by $20 \%$ per $9.8 \mathrm{Ncm}$ added. The authors suggested that, in cases of early loading, an appropriate initial insertion torque must be applied to decrease the implant failure rates.

Cannizzaro et al. (70) compared the outcome of immediately loaded single implants inserted with medium (from 25 to $35 \mathrm{Ncm}$ ) or high insertion torques $(>80 \mathrm{Ncm})$, in a split-mouth design, 50 implants in each group. The patients were followed for 6 months after loading. Seven implants failed to osseointegrate when inserted with a torque between 25 and $35 \mathrm{Ncm}$ versus none of the implants inserted with a torque $>35 \mathrm{Ncm}$. The authors concluded that it is preferable to insert single implants with a high insertion torque in order to minimise early implant failures, when loading them immediately.

By observing the results of these two last studies, it can be suggested that a low insertion torque of implants that are planned to be early or immediately loaded increases the implant failure rate.

Bone condensing versus bone drilling. Marković et al. (71) compared the stability of implants placed by bone condensing (implant sites were prepared by pilot drill, followed by condensers of increasing diameter) versus the standard drilling technique in the posterior edentulous maxilla, in a split-mouth design, 24 implants for each group. Patients were followed until 6 weeks after surgery, when the final prosthetic rehabilitation was performed. Even though no implants were lost, the short period of follow-up is a considerable limitation of this study.
Standard drilling protocol versus adapted bone drilling method in low-density bone. In the study of Alghamdi et al. (72), 26 implants were surgically placed according to the standard drilling protocol (control group) and 26 were placed in low-density bone using an adapted bone drilling method (test group), which consisted of undersizing the bone drilling of the implant bed. Widening of the implant bed with osteotomes or tapping was not used in any of the cases. None of the implants failed at the end of 1 year. The results of this study suggest that placement of implants by an adapted drilling technique in sites with poor bone density does not increase/decrease the implant failure rate.

Implants inserted with versus without piezoelectric surgery split crest. Piezoelectric surgery (PES) uses a modulated ultrasonic frequency that permits a highly precise and safe hard tissue-cutting technique allegedly avoiding nerve, vessel and soft tissue injuries, which may result in a low bleeding tendency. Because of this potential advantage, it was hypothesised by one study that the use of PES in surgical insertion of implants would favour the survival outcome. Danza et al. (73) evaluated whether implants $(N=234,86$ patients) inserted into crests split using PES $(N=21)$ have a comparable outcome to those inserted into unsplit bone. The patients were followed for a mean of 13 months. Only nine of 234 implants were lost, all from the unsplit group, but no statistical difference was demonstrated. Even though it was showed that no implants inserted by PES were lost, the small sample size of this group may be considered a limitation of this study.

Intra- or post-operative complications. In the study of Strietzel et al. (74), with 1554 implants followed for a mean period of $6 \cdot 2$ years, a significant correlation between the occurrence of intra- or post-operative complications (perforation of nasal floor or maxillary sinus, suture spreading, oedema requiring therapy, sensation deficit and infection) and implant loss was established. The occurrence of complications increased the risk of implant loss 3.4 times $\left(P_{n}=1554=0.001\right)$ and 4.8 times $\left(P_{n}=504=0.011\right)$.

Two surgical guide systems. Abboud et al. (75) compared the survival rates of the immediately loaded dental implants inserted with the help of two different 
stereolithographic surgical guide systems, NobelGuide (41 implants) and SimPlant (34 implants). The patients received pre-fabricated provisional restorations. One of the NobelGuide-inserted implants failed, with a cumulative survival rate (CSR) of $97.6 \%$ for this group. No implant failures were observed in the other group.

Fresh extraction sockets versus healed sites. Alveolar ridge resorption after tooth extraction may considerably reduce the residual bone volume and affect the favourable positioning of implants, which is required to produce optimal restoration. Thus, some authors advocated immediate placement, which has some advantages (76). Several studies have compared the clinical outcomes of implants placed in fresh extraction sockets versus in sites left to heal from few weeks to several months.

Some studies showed that there is a greater risk of implant failures with insertion in fresh extraction sockets compared with placement in healed sites (77-82). On the other side, few studies showed that there is a greater risk of implant failures with an early insertion (within 6-8 weeks after tooth loss) (83) or late insertion (more than 9 months after tooth loss) (84) compared with an immediate insertion. However, most of the studies observed that the implants placed into fresh extraction sockets have similar failure rates as the implants placed in healed sites $(68,85-103)$.

Immediate placement in sockets with versus without periapical pathology. Truninger et al. (104) compared the clinical outcomes of implants placed in fresh extraction sockets with $(N=17)$ or without $(N=17)$ periapical pathology, patients in need of single-tooth replacement. Before the implant insertion, careful debridement of the extraction socket was performed. Of the 34 patients, four test and one control patient had to be withdrawn from the study due to the inability to obtain primary implant stability. The residual 29 implants revealed a survival rate of $100 \%$ 3 years after placement.

Socket depth. Grunder et al. (87) assessed the clinical outcomes of 264 implants placed in fresh extraction sites, in healed sites and with membranes placed over the extraction site. There was no clinical difference of the implant failure rate when compared to the socket depth in which the implants had been placed.
Ridge-expansion-with osteotome-only versus combined ridge split-and-osteotome procedure. In the study of Payne et al. (105), 40 edentulous participants with mandibular two-implant overdentures were allocated to two groups, either a ridge-expansion-with osteotome-only (OO; $N=69$ ) procedure or a combined ridge splitand-osteotome procedure (ORS; $N=48$ ), depending on ridge bucco-palatal width and the degree of ridge resorption detected radiographically. Of the 11 implant failures, $8(16.7 \%)$ happened in the ORS group (in type C/D bone quantity) and $3(4.3 \%)$ in OO group, the difference being statistically significant $(P=0.03)$.

Surgeon's surgical experience. In the study of Adell et al. (106), the material was divided into four groups (development group and routine groups I, II and III) designated by time periods of fixture placement. Routine group III represented those patients completed in the most recent group, when providers had achieved the most experience. They reported 5-year CSR for implants in the mandible for the developmental group to be $75 \%$. However, routine groups I-III were $91 \%$, $98 \%$ and $99 \%$, respectively.

Block and Kent (107) compared their CSR for hydroxyapatite (HA-coated) implants placed in two different periods. The first one, called 'developmental period' between 1985 and 1988 (4- to 8-year followup), was compared with the second one, called 'recent period' between 1989 and 1991 (1- to 4-year follow-up). The CSR for the developmental period was $86.5 \%$, improving to $97.6 \%$ for the recent period.

Minsk et al. (108), followed for 6 years 1263 implants inserted by 80 different operators with a wide range of clinical experience in 380 patients. The results indicated that the use of implants by operators with different levels of experience did not affect the implant survival rates.

Lambert et al. (109) evaluated the risk of an implant to fail according to the surgeons' experience. Implants placed by inexperienced surgeons $\quad(<50$ implants; $N=1260$; failure rate: $3.5 \%$ ) failed twice as often as those placed by experienced surgeons $(\geq 50$ implants; $N=1381$; failure rate: $1.8 \% ; P<0.05)$. Implants placed during the first $6,8,10,12$ and 16 cases were compared with all others. The greatest difference was seen between the first nine cases and all others $(P=0.001)$, with later cases failing significantly less often. Inexperienced surgeons had more failures 
in the first nine cases $(5.9 \%)$ than more experienced surgeons $(2 \cdot 4 \%)$.

Three of the four available studies evaluating the influence of the surgeon's surgical experience of the implant failure rates indicated that inexperienced surgeons tend to have more failures than more experienced surgeons.

\section{Location conditions}

Maxilla versus mandible. Since the first studies evaluating implant clinical outcomes have been published, most of the authors report the implant survival/failure rates relative to the jaw where the implants are inserted.

Most of the studies observed that implants fail more when inserted in the maxilla in comparison with implants inserted in the mandible, with statistically significant difference in the implant failures rates $(24$, 37, 74, 80, 81, 86, 90, 96, 110-141). Other studies presented similar findings, however, without demonstrating significant differences between the two jaws $(19,88,106,142-164)$. A smaller number of studies found the opposite that implants inserted in the mandible tend to fail more $(68,165-172)$. One study showed the same implant failure rates for both jaws (173).

The opinion of the majority of the studies is that the maxilla has a less favourable bone texture than mandibles, with low density of medullary bone and thin cortical plates (80). Jaws with poor bone quality may be at risk of establishing initial instability of the implants and a lack of resistance to mechanical stresses, thus also resulting in early implant failures (80, 113). Failure could also be related to the fact that short implants are more commonly placed in the posterior maxilla due to limited bone dimensions because of the pneumatisation of the maxillary sinus.

Region of the jaws. Several researchers tried to verify whether there is a difference in the implant failure rates depending on the region of the jaws where the implants are inserted.

Based on the reviewed studies, it can be clearly stated that the implantation site influences implant failure rates. Several studies reported that there was no significant difference when the implant failure rates in the maxilla and in the mandible were partitioned by position $(90,116,123,132,153,154,158,168$,
172-175). Three studies compared the failure rates within the posterior region, showing that the failure rate in the molar area was higher than that in the premolar area (176-178). One study (86) even showed that implants placed in the anterior region of the maxilla failed significantly more often than those placed in the posterior region; the same was not observed for the mandible, but the investigated implant was later withdrawn due to poor results. Another study (170) showed the same, but in the other jaw - implant failures in the mandible were more frequent in the anterior zone than in the posterior region; in the maxilla, all failures occurred in the posterior zone. Other few studies (136, 179, 180) observed that a significantly higher early implant failure rate was found in the anterior regions of maxilla and mandible compared with the posterior regions.

However, the majority of the studies observed that the greater failure rate is found in posterior regions $(46,74,87,88,91,113,114,117,156,157,169$, 181-187). This may be attributable to a combination of the demanding pre-conditions often present in the posterior maxilla, such as barely sufficient bone volume, poor bone quality and high functional forces (156). The cortical layer of both jaws tends to become thinner and more porous posteriorly. Moreover, posterior implants have to withstand the heaviest loading and are in general short due to insufficient quantity of available bone (the maxillary sinus and the inferior alveolar nerve are the main anatomical limitations). The high survival rates presented in the canine and/or premolar maxillary area in some studies $(20,113,179,186)$ could be attributed to favourable bone density and adequate bone quantity due to relatively rare anatomical limitations. One study (20) found high failure rates in the mandibular anterior, which was suggested to be caused by overheating of the bone when long implants (15-18 mm) were placed (20). SánchezGarcés et al. (188) followed for a mean of 81 months 273 implants $\leq 10 \mathrm{~mm}$ in length (from 5 to $10 \mathrm{~mm}$ ), placed in patients with severe alveolar bone resorption. The relative percentage of failure in the upper molar zone was slightly higher than in the mandibular molar region $(8.97 \%$ versus $7.98 \%)$. When the implants located in the position of the first upper and lower molars were considered separately, the failure rate increased to $9.5 \%$. The failure rate recorded in the region of the lower premolars was 
doubled compared with the region of the upper premolars.

\section{Patient conditions}

Male versus female. Some studies showed that gender was a risk factor for failure where females had higher failure rates than males $(74,116,189)$. In contrast, other studies reported higher failures rates for males compared with females $(20,129,136,151)$. However, most of the studies did not find a significant effect of patient's sex on the failure of implants $(19,37,46$, $47,86,115,119,120,122,138,153,156,158,170$, $173,179,190)$.

Age of the patient. The success of osseointegration depends in part on the state of the host bone and its healing capacity. Osteoporosis, which is a disorder characterised by a generalised diminution in bone mass and bone density, may therefore represent a risk factor for osseointegration (190). As the prevalence of osteoporosis rises with age, some researchers investigated whether the age of the patient may have some influence on the implant failure rates.

Few studies showed that there is an increase in implant failures with age $(168,191)$. On the other hand, most studies have shown that there is no correlation between implant failure and age of the patient $(20,46,47,86,112,115,119,120,122,129,145$, $158,170,190)$.

Smokers versus non-smokers. As cigarette smoking has been shown to adversely affect wound healing, it has emerged as a factor that may affect the implant survival outcomes (136). Several studies investigated whether there is a greater incidence of implant failure in patients who smoke compared with non-smokers.

The review of these studies shows that cigarette smoking appears to have a statistically significant detrimental effect on implant survival, even after accounting for some potentially confounding variables. Of the 27 studies reviewed, 22 showed that smoking influences negatively the survival rate of the implants $(19,46,47,49,90,117,119,128,129,133$, $136,164,173,192-200)$, and five studies showed that smoking does not significantly influence the survival rate of the implants $(20,36,108,132,170)$. It has been shown that a smoking cessation protocol described in one study (193) demonstrated consider- able promise in improving the success rates of implant integration in previous smokers.

Periodontally compromised versus non-compromised situations. Some evidence supports the concept that patients with a history of periodontal disease may experience increased implant failure rates when undergoing implant therapy. In many studies comparing the implant failure rates of implants inserted in periodontally health patients and in periodontally compromised patients, it was suggested that failed implants were concentrated in patients with a relatively high initial plaque index (151), in patients that had their tooth (or teeth) extracted due to periodontitis $(20,87,88)$, in periodontally 'maintained' (168), 'susceptible' (201), 'compromised' (187) patients, patients who had periodontal disease (89) and in patients with aggressive periodontitis instead of in patients with chronic periodontitis (131).

However, conclusions need to be interpreted with caution, because some studies showed that a previous history of periodontal disease $(198,202,203)$ may not have a significant impact on implant failures. Furthermore, a recent genetical study found clear differences between periodontitis and peri-implantitis (204).

It might be difficult to assume a causal association between implant failure and a previous history of periodontal disease (205). However, it can be speculated that periodontitis-affected tissues might have had a negative local influence, also for the presence of infrabony defects; this could increase the gap between bone and implant (206) or jeopardise the achievement of primary stability (207) both at immediate and early implant placement.

Bone quality. Bone quality is believed to be one of the most important aetiological factors for early implant failures. Bone quality is categorised into four groups, from type I to IV: (i) type I, homogeneous cortical bone; (ii) type II, thick cortical bone with marrow cavity; (iii) type III, thin cortical bone with dense trabecular bone of good strength; and (iv) Type IV, very thin cortical bone with low-density trabecular bone of poor strength. Several studies evaluated the influence of bone quality on the implant failure rates.

Receptor bone quality is a decisive factor for implant success. Analysis of the reviewed studies showed that the outcome of implant treatment could be related to bone quality. It was observed by many 
studies that there is an increased implant failure rate when implants are inserted in bone qualities type III and IV $(126,151,154,156,184,191,197,208)$ or in bone quality type IV $(90,113,114,120,135$, 160). Few studies reported a higher implant failure rate for implants inserted in bone quality type II (24) or type III $(87,167)$. Some observed that insertion in bone type I (or 'dense bone') may also result in increased implant failure rate $(113,160,172)$. The bone quality type I is more commonly found in the anterior mandible, where usually there is more bone available to insert long implants. Thus, this may be related to overheating of the bone when long implants are placed. Research has demonstrated that thermal damage at the drilling site inhibits the regenerative response in bone healing, slowing the process of osseointegration and potentially resulting in implant mobility (209). Some studies (36, 145, $173,176,178$ ) indicated that the bone quality did not significantly influence the failure rate of the implants.

The combination of poor bone quality and a shorter implant length leads to diminished mechanical stability at the time of fixation and during the subsequent osseointegration period.

Jaw bone volumeljaw bone resorption. Bone volume, together with bone quality, is generally believed to be one of the most important aetiological factors for early implant failures. Several studies evaluated the influence of bone volume - or bone quantity, based on the Lekholm and Zarb (210) system of bone classification - on the implant failure rates.

Many studies found that there was a significant higher implant failure rate when small bone volumes are present for implant insertion $(111,120,126,135$,
191). For many others $(145,167,178)$, there were no statistical significant differences.

Figure 3 shows implants inserted in a maxilla with small quantity of bone (courtesy of Dr. Anders Örtorp).

Bruxism. Glauser et al. (156) evaluated 41 patients who received 127 immediately loaded implants. Their results showed that implants in patients with a parafunctional habit (bruxers) were lost more frequently than those placed in patients with no parafunction ( $41 \%$ versus $12 \%$ ).

The higher failure rate among the bruxers is not so surprising because a high and unpredictable or uncontrolled loading of the implant could lead to micromotions above the critical limit, resulting in fibrous encapsulation of the implant instead of osseointegration (156). It was suggested that early or immediate loading per se is not detrimental for osseointegration, unless excessive micromotions occur at the boneimplant interface during the healing phase (211).

Number of present teeth/edentulism status. The implant failure rate in the partially edentulous jaw was not significantly different from that in the totally edentulous jaw in some studies $(24,116)$. It was also not significantly different when comparing the failure rates of implants placed to restore edentulous jaws versus posterior partial edentulism versus a single-tooth space versus an intermediate space (86). However, some observed that implants placed in completely edentulous jaws demonstrated higher failure rates than implants placed in partially edentulous jaws $(128,168)$. Kourtis et al. (90) observed in their study that that single-tooth implants showed statistically significant lower failure rate and better survival rate

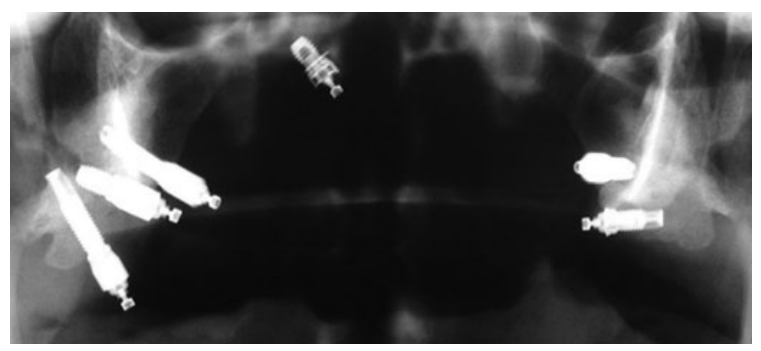

Fig. 3. Tilted implants inserted in a maxilla with small quantity of bone (courtesy of Dr. Anders Örtorp). 
probability compared with implants used in partially or completely edentulous patients. The Cox proportional hazard regression analyses performed in one study (49) showed that implants inserted in subjects with 20 or more teeth present were explanted at a rate that was 3.8 times higher than in subjects with fewer teeth present.

The observation that implants placed in completely edentulous jaws demonstrated higher failure rates than implants placed in partially edentulous jaws in some studies may be related to the fact that the dental status has a high influence on the jaw anatomy (212). When there is insufficient bone volume and grafting procedures are not performed, short implants are probably the only option, and shorter implants usually have higher failure rates than longer ones (consult subtopic 'Implant length').

Ethnicity. Lambert et al. (19) evaluated the influence of smoking on the survival of 2887 implants, followed for 3 years. When analysing ethnic groups, they observed that implants in white smokers were 1.3 times more likely to fail, in Hispanics four times, in blacks 2.4 times and in others 1.8 times more likely to fail than in non-smoker/quit subjects.

Oral hygiene. Kourtis et al. (90) evaluated the clinical outcomes of 1692 implants. The oral hygiene was evaluated subjectively as good, medium or insufficient in all recall appointments. The failed implants in patients with good oral hygiene were $13(17.6 \%$ of all failures), in patients with a medium level of oral hygiene $27(36.5 \%)$, and in patients with insufficient oral hygiene, 34 failures $(45.9 \%)$ were noticed. There was a statistically significant difference between failures in good-medium and insufficient oral hygiene groups compared with the chi-squared test $\left(P<0.001, \chi^{2}=61.55\right)$. The failure rate in good or medium oral hygiene groups was $2.5 \%$ and $2.9 \%$, respectively, and in patients with insufficient oral hygiene, almost four times higher $(13.8 \%)$. The survival rate probability was also influenced accordingly.

Figure 4 shows mandibular dental implants in a patient with poor oral hygiene habits (a). Despite poor oral hygiene, marginal bone levels were maintained around the implants (b).

Patients with versus without oral lichen planus. Hernández et al. (213) compared the implant survival rate in
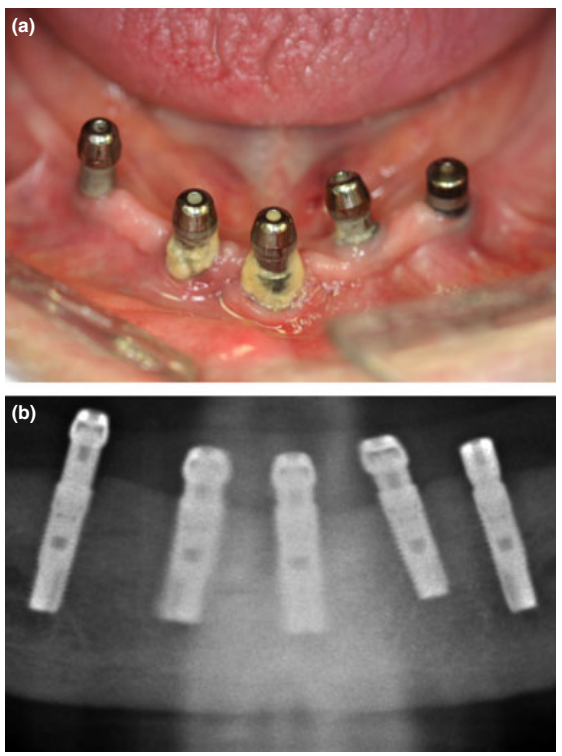

Fig. 4. Dental implants in a patient with poor oral hygiene habits (a). Despite poor oral hygiene, marginal bone levels were maintained around the implants (b).

18 patients diagnosed with oral lichen planus (OLP, 56 implants) and 18 control patients (62 implants). To obtain a uniform sample, only patients with originally erosive forms of OLP were included in the study and the type, length and diameter of the implants were similar in both groups. The implant survival rate was $100 \%$ for the OLP group and $96.8 \%$ in the control group.

The results of the only prospective controlled study on the subject seem to demonstrate that the presence of OLP is not associated with a higher prevalence of implant failure.

Compromised medical status/systemic conditions. The influence of general health problems on the implant failure rates is still poorly documented, with only few controlled studies available.

Smith et al. (115) observed that the number of medical problems (diabetes, oestrogen replacement and corticosteroid therapy) and the physical status of the 
patient according to the American Society of Anaesthesiologists classification system were not statistically associated with an increased risk of implant failure. Wagenberg and Froum (20) also did not find significant difference in implant failure rate associated with any medical condition (osteoporosis or taking bisphosphonates) of the patients included in the study.

Morris et al. (214) attempted to determine whether type 2 diabetes represents a significant risk factor to the long-term clinical performance of dental implants. A model assuming independence showed that implants in type 2 patients have significantly more failures $(P=0 \cdot 020)$.

Kourtis et al. (90) evaluated the clinical outcomes of 1692 implants. From this total, 56 (3.3\%) were placed in patients with diabetes mellitus, $11(0.7 \%)$ in patients who had previously undergone chemotherapy and $6(0.4 \%)$ in patients who had metabolic disturbances. The failure rate was increased with statistical significance among patients $(P<0.001)$ with metabolic diseases (e.g. thyroid gland dysfunction). No statistical significance was noted among failures in patients with diabetes.

Alsaadi et al. (215) assessed the influence of systemic and local bone and intra-oral factors on the occurrence of early 720 TiUnite implant failures. Between the systemic factors, the authors assessed hypertension, cardiac problems, gastric problems, osteoporosis, hypo- or hyperthyroid, hypercholesterolaemia, asthma, diabetes types I or II, Crohn's disease, rheumatoid arthritis, chemotherapy, hysterectomy and intake of medication (antidepressants, steroids, hormone replacement). Owing to the very few failures $(1.9 \%)$, no definitive conclusion concerning statistical significance can be achieved. However, a tendency for more failures was noticed for hormone replacement, gastric problems, Crohn's disease, diabetes I and radical hysterectomy.

Anner et al. (198) observed that diabetes was not associated with long-term implant survival in a sample size of 1626 implants followed for an average of 30 months.

\section{Implant conditions}

Implant length. It has long been an axiom of implant dentistry that as many implants as possible of maximum length should be used. Advantages of increased implant length include increased initial stability, long- term resistance to bending moment forces, expedited healing and a decreased risk of movement at the interface (216). A number of researchers evaluated whether the survival of the shorter implants was comparable with the longer implants.

From the review of these studies, it can be concluded that is highly suggestive that implant length is a significant factor in implant survival. In most studies, there are significant findings of decreased survival with shorter length $(<10 \mathrm{~mm})$ implants compared with their longer $(\geq 10 \mathrm{~mm})$ counterparts $(47,49,81$, $86,87,90,113,116,117,122,125,126,132,133$, $136,149,151,154,157,158,166-168,170,176,178$, 180, 182-185, 189, 191, 216-218). Some studies even showed that there is a progressive increase in the implant failure rates as the implant length decreases (117, 122, 125, 146, 149, 157, 178, 183, 185, 216). Two studies compared the implant failure rates between short implants $[8 \mathrm{~mm} \times 10 \mathrm{~mm}$ by Levine et al. (169); $7 \mathrm{~mm} \times 8.5 \mathrm{~mm}$ by Goené et al. (160)], and both studies showed increased implant failure rates for the shortest implants.

One interesting observation resulted from the study of Naert et al. (158), saying that by decreasing the implant length by $1 \mathrm{~mm}$ increases the hazard rate $0 \cdot 16$ times.

Few studies observed that the implant length did not appear to significantly affect the implant failure rate $(36,91,123,129,130,156,171,173,174,179$, 187, 188).

Figure 5 shows a bone resorption around a short turned surface implant (courtesy of Dr. Björn Gjelvold).

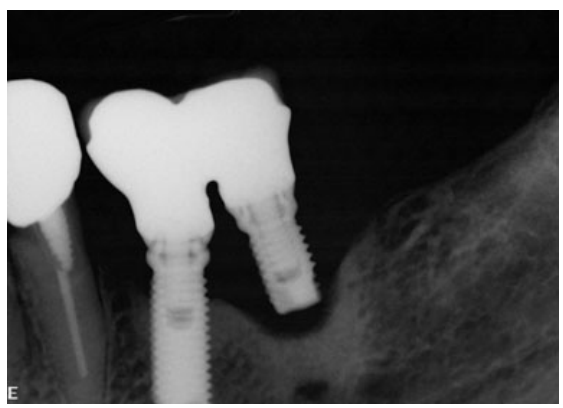

Fig. 5. Bone resorption around a short turned surface implant (courtesy of Dr. Björn Gjelvold). 
Implant width/diameter. The clinical use of several designs of oral implants has become highly predictable related to limitations of the geometry and volume of the alveolar bone. To reduce the risk of failure of endosseous implants used for posterior applications, wide-diameter implants have been suggested. The use of a $5 \cdot 0$-mm-diameter implant that is $6 \mathrm{~mm}$ long increases the surface area available to contact the bone similar to that of a 3.75-mm-diameter implant that is $10 \mathrm{~mm}$ in length (216). Several studies assessed whether the survival of the wider implants was comparable with the narrower implants.

It can be concluded that is suggestive that implant diameter is a significant factor in implant survival. Implants with wider diameter seemed to achieve better results than standard ones of corresponding lengths. In several studies, there are significant findings of increased failure with smaller diameter implants, either the comparison being between 3.3 versus $4.0 \mathrm{~mm}(86,116), 3.75$ versus $4.0 \mathrm{~mm}$ (151, 154), $\leq 4$ versus $>4 \mathrm{~mm}(185), 3.3$ versus 3.75 versus $4.0 \mathrm{~mm}$ (182), 3.75 versus 4.0 versus $5.0 \mathrm{~mm}$ (178), 3.3 versus 4.0 versus $5.0 \mathrm{~mm}(180), 3.0-3.9$ versus

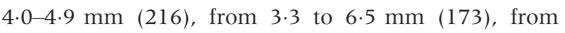
3.0 to $5.0 \mathrm{~mm}(219)$, or between implants from 3.25 to 6.0-mm-diameter implants (132).

On the other side, studies also showed a significant higher implant failure rate for wider diameter implants, either the comparison being between 3.75 versus 4.0 versus $5.0 \mathrm{~mm}(167,220,221), 4.0$ versus $5.0 \mathrm{~mm}$ (156), or between implants from 3.25- to 6.0mm-diameter implants (171). A reason for this may be, as observed by Shin et al. (221) in a multivariate analysis, a predictive relationship between overall CSR and the ratio of implant volume to remaining bone volume.

Many showed no statistically significant difference in implant failure rates between narrow- and widediameter implants, either the comparison being between 3.3 versus $4.0 \mathrm{~mm}$ (86), from 3.25 to $5 \mathrm{~mm}$ $(129,179), 3.75$ versus $4.0(81), 3.75$ versus 4.0 versus $5.0 \mathrm{~mm}$ (187), from 3.5 to $6 \mathrm{~mm}(91), 4.0$ versus 5.0 versus $6.0 \mathrm{~mm}(36)$, or between 3.3 - versus 4.1 versus $4 \cdot 8$-mm-diameter implants (170).

Initial stability. The presence of implant mobility at the initial stage of treatment is believed to represent a serious risk to its eventual integration and long-term survival.
Analysis on implant level did (222) or did not (156) show significantly higher failure rates for implants that had initial poor stability in comparison with implants that had good and very good initial stability.

Details from the other studies are important. In the study of Friberg et al. (113), 69 (of 4641) implants failed, and in 22 of the failures (32\%), the implants were placed in sites with extremely soft bone and/or where initial implant stability was not achieved.

Orenstein et al. (223) followed 2641 implants in order to examine the likelihood for an implant that was mobile at placement to osseointegrate, according to the surface modification of the implants. Seventysix $(93.8 \%)$ of the 81 mobile implants were integrated at uncovering compared with $97.5 \%$ for the 2560 immobile implants. Of the 54 HA-coated implants that were mobile at placement, all (100\%) integrated, while only $17(81.5 \%)$ of the 22 mobile nonHA-coated implants integrated $(P=0.003)$.

One study (224) used resonance frequency analysis to verify whether the implant stability has some influence on the possible differences between failing and successful implants. It was observed that after 1 month, the resonance frequency analysis of failing implants was significantly lower than found with the successful ones. Nedir et al. (225) showed that after 1 year of loading, all implants loaded after 3 months of healing with an initial of ISQ $\geq 49$, and all implants immediately loaded with an initial ISQi $\geq 54$ achieved and maintained osseointegration (ISQ - implant stability quotient).

Rodrigo et al. (226) followed 4114 consecutive SLA Straumann implants to evaluate primary stability, which was classified in four categories, depending on the degree of implant rotation when tightening the healing cap: A (no rotation at all), B (light rotation with a feeling of resistance), C (rotation without resistance) and D (rotation and lateral oscillation). A number of 3899 implants was classified as stable (A) and 213 as unstable (B-D), showing survival rates of $99.1 \%$ and $97.2 \%$, respectively. In the case of the unstable implants B $(N=158), \mathrm{C}(N=51)$ and D $(N=4)$, they had a survival rate of $98 \cdot 1 \%, 94.1 \%$ and $100 \%$, respectively. All these differences were statistically significant $(P<0.009)$. Using a resonance frequency analysis device, a threshold value of ISQ $=60$ was used to stratify implants by stability (stable/non-stable), in both group 1 (resonance frequency analysis method used at the day of the sur- 
gery) and group 2 (resonance frequency analysis method used at restoration placement) measurements. At the group 1 measurement, there was no significant association between primary stability and implant survival $(P<0.753)$. However, in the group 2 , the association was significant $(P<0 \cdot 001)$.

Primary implant stability is determined by the bone density, the implant design and the surgical technique. These studies show a correlation between implant stability and implant survival and probably reflect the importance of an undisturbed healing to achieve adequate osseointegration.

Threaded versus cylindrical implants. Four studies showed that there is a greater risk of implant failures with insertion of cylindrical implants when compared to threaded implants $(123,168,227,228)$. The statistical analysis of four other studies indicated that the type of implant (cylindrical versus threaded implant) did not influence the failure rate of the implants (33, $36,152,229$ ).

It is important to observe that we only report comparative studies of implant failure under this heading, that is, in this the systematic part of our review, we are not discussing potential problems with marginal bone loss that may occur to some implant systems. However, early survival rates may be unaffected in comparison with the ones where bone resorption has resulted in higher failure rates.

According to Karoussis et al. (228), the possible reasons for inferior survival rates of cylindrical implants in comparison with threaded implants are largely unknown and may only be speculated upon.

Implant thread type/design. When standard and selftapping implants were compared, studies observed that the implant failure rate of standard implants was higher than that of self-tapping implants $(217,230)$. Others found that the difference in the implant failure rate was not significant $(82,87,158)$.

When a comparison of variable-thread design versus standard tapered implants was made, no significant difference in implant failure rates was observed (231).

A comparison of different implants showed no statistical significant difference in implant failure rates between IMZ and Brånemark implants (232), Astra and ITI (124), IMZ, Brånemark and ITI (233), Brånemark and ITI $(30,32,34)$, titanium-blasted Astra Tech and turned Brånemark (234), Brånemark and South- ern (105), Frialit-2 and IMZ (173), cylindrical ITI and tapered ITI (235), and between ITI, SwissPlus and Astra (236).

A statistical significant difference was found in the comparison of implant failure rates between Steri-Oss versus Southern implants (favouring Southern implants) (237), Astra and ITI (favouring Astra implants) (49), and between straight-walled and tapered Osseotite (favouring straight-walled) (238).

It is important to stress that a comparison of results between these studies is difficult to make, because each study performed prosthetic rehabilitations on these implants under several configurations, the loading time after healing and the samples size varied, the bone quality in which these implants were inserted was different.

Number of implants placed per patient. Smith et al. (115) studied 313 implants. The univariate analysis of risk factors showed that the number of implants placed per patient did correlate with implant failure. The patients receiving from 4.1 implants had a greater probability of having failures than the patients receiving until 2.2 implants $(P=0.016)$.

Naert et al. (158) studied the outcome of 1956 implants, and according to their results, the higher the number of implants a patient has, the higher the hazard rate $(P=0.005)$. Increasing the number of implants by one increases the hazard rate 0.14 times.

One may hypothesise that placing multiple implants requires more mucoperiosteal stripping, compromising blood supply, more operating time and more contamination of the wound, all of which may contribute to the increased complication rate (115).

Implant surface. There have been constant efforts to improve osseointegration by modifying the surface properties of titanium, because this is where early interactions occur between the implant and the surrounding tissues following placement. Surface properties such as topography physics and chemistry may affect protein adsorption, cell-surface interaction and peri-implant tissue development, which are all relevant to the functionality of the implant or device and may have an influence on osseointegration, host response to the implant and subsequent treatment outcomes.

Titanium with different surface modifications shows a wide range of chemical, physical properties, and surface topographies or morphologies, depending on 
how they are prepared and handled $(239,240)$. It is not clear whether, in general, one surface modification is better than another.

No statistically significant difference was observed in comparisons of implant failure rates between turned and HA-coated implants (174, 187), titanium plasma-sprayed (TPS) and HA-coated implants (122), turned and grit-blasted $(241,242)$, turned and oxidised (243-245), turned and 'roughened' (36), turned and anodised (246-249), SLA and modSLA (250), grit-blasted and calcium-incorporated (251), and turned and acid-etched surfaces (252).

Statistically significant difference was observed in comparisons of implant failure rates between HA-coated and non-HA-coated implants (favouring HA-coated) (223), turned and TPS (favouring TPS) (253), turned and anodised (favouring anodised) $(37,188,254)$, turned and 'rough' (favouring rough) $(20,184)$.

One study did not inform whether there was statistical significant difference in the implant failure rates between turned and oxidised surfaces (162), but showed an implant failure rate of $9 \%$ for turned and $0 \%$ for oxidised implants. Another study showed implant failure rates of $37.5 \%$ and $0 \%$ for turned and oxidised-surface implants (255), but also did not inform whether there was statistical significant difference in the implant failure rates. However, the authors (255) decided to abandon the use of turned implants, due to an unacceptably high failure rate, when correlated with accepted criteria for assessment of implant performance.

Tilted versus axially placed implants. The technique of tilting of posterior implants was developed for improving bone anchorage and prosthesis support and avoiding bone graft procedures. There was no significant statistical difference in implant failure rates when comparing implants placed in tilted positions with axially placed implants in all available controlled studies $(140,228,256-260)$. A confounding factor can be the fact that most of the studies splinted the tilted implants with the axially placed implants to provide full-arch restorations in all $(140,258-261)$ or most patients of the study (256).

Figure 3 shows tilted implants inserted in a maxilla after partial maxillectomy (courtesy of Dr. Anders Örtorp).

One-piece versus two-piece implants. Östman et al. (262) compared the immediate loading of one- $(N=115)$ and two-piece $(N=380)$ implants, followed for at least 1 year. Six $(5 \cdot 2 \%)$ and five $(1.3 \%)$ implants failed in the one- and two-piece implants groups, respectively. The authors stated that factors such as implant design, insertion depth, rough surface towards the mucosa, in situ preparation and immediate loading may have an influence on the clinical outcome.

Wide versus double implants. Bahat and Handelsman (263) compared the clinical outcomes of the placement of different combinations of implants at one site in the posterior areas: (A) 59 single 5 -mm implants, (B) 20 pairs of 5 -mm implants, (C) $345-\mathrm{mm}$ implant paired with a 3.75- or 4-mm implant and (D) 162 double 3.75- or 4-mm implants. After a mean followup of 13-37 months (depending on the group), the number of failed implants was 2 (group A), 0 (group B), 2 (group C) and 5 (group D). The failure rate for all 5 -mm implants was $2 \cdot 3 \%$, and that for all double implants was $1 \cdot 6 \%$.

Time since implant placement. Lemmerman and Lemmerman (36) evaluated the clinical outcomes of 1003 implants. The statistical analysis indicated that the time since implant placement was the only variable that influenced the success rate of the implants, showing an increasing failure rate with time.

\section{Prosthetic conditions}

Occlusal versus non-occlusal loading. Most of the studies showed no statistically significant differences in implant failure rates between functional (with occlusal contacts) immediate loading and non-functional (without occlusal contacts) immediate loading (186, 187, 264-267). One study (268) observed implant failure rates of $0 \%$ and $6.7 \%$ for non-occlusal and occlusal immediate loading, respectively, but there was no information whether the difference was statistically significant or not.

The results of these studies tend to suggest that the differences in immediate loading between occlusal and non-occlusal loading may not have a detrimental effect on the survival of dental implants.

Cemented versus screw-retained implant. Three controlled studies (269-271) evaluated the differences in the clinical outcomes between cemented versus 
screw-retained implant restorations, and all three observed no statistically significant difference in implant failure rates between the two techniques.

Cement remnants may indeed cause marginal bone loss due to a foreign body on another foreign body reaction, but failure rates will increase first after many years in situ.

Type of prosthesis. Several studies observed statistically significant difference in the implant failure rates between implants used to support different prosthetic configurations, being overdentures versus fixed fullarch prostheses, favouring fixed prostheses (191), overdenture versus fixed partial prostheses, favouring fixed prostheses (122), overdentures versus all types of fixed prostheses (single-tooth, partial, full-arch), favouring fixed prostheses $(46,145,182)$, overdentures versus fixed full-arch bridges, favouring fixed prostheses (but only in the maxilla) (157), overdentures versus all types of fixed prostheses, favouring overdentures and full-arch fixed prostheses (173), and single- versus multiple-implant-supported fixed prostheses, favouring single-tooth prostheses (68).

Many studies did not observe statistically significant differences in the implant failure rates between implants used to support different prosthetic configurations, being fixed prosthesis versus single-tooth restorations (272), single crowns versus fixed partial prosthesis (158), overdenture versus fixed full-arch prosthesis (273), single- versus multiple-implant-supported fixed prosthesis (20) and all-ceramic versus metal-ceramic implant-supported single-tooth restorations (274).

In one study (275), no comparisons were possible between the groups (overdenture with a gold bar versus CAD/CAM-fabricated implant overdenture with a titanium bar versus CAD/CAM implant-supported fixed prosthesis) concerning implant failure rates, because the only implant failure occurred before the loading of the implants.

All-on-2 versus all-on-4 implants mandibular cross-arch fixed prostheses. Cannizzaro et al. (276) followed 60 patients who received mandibular cross-arch fixed prostheses immediately loaded supported by 2 $(N=30)$ or $4(N=30)$ implants. One year after loading, no implant failure had occurred.

Different crown-to-implant ratio. Because of vertical loss of the alveolar bone after tooth extraction, the supracrestal part of the implant bone reconstruction is often long in relation to the clinical crowns of the remaining dentition and to the supporting implant. Clinicians tend to insert the longest implants possible, presuming a higher success rate with increasing crown-to-implant length ratio (C/I ratio) (200). Three studies tested whether or not a higher $\mathrm{C} / \mathrm{I}$ ratio is associated with higher implant failure rates.

In the study of Blanes et al. (277), a total of 51 implants $(26.5 \%)$ showed a clinical $\mathrm{C} / \mathrm{I}$ ratio $\geq 2$, and three implants failed in this group, giving a CSR of $94 \cdot 1 \%$. Schulte et al. (278) retrospectively evaluated 889 single-tooth implants and observed that the mean $\mathrm{C} / \mathrm{I}$ ratio of implants in function was $1.3: 1$, and the mean C/I ratio of failed implants was $1 \cdot 4: 1$. Schneider et al. (200) observed no statistically significant influence of the technical (the top of the implant shoulder was used as a transition between the crown and the implant) and biological (the reference used for the calculation was the initial peri-implant marginal bone level) C/I ratio on the implant survival. Although Cox regression analysis revealed a higher biological $\mathrm{C} / \mathrm{I}$ ratio to negatively be associated with implant failure, this association was not statistically significant.

From the results of the three published controlled studies on the subject, it is suggested that implant restorations with high clinical or anatomical C/I ratios do not demonstrate lower survival or success rates as compared with implant restorations with low C/I ratios.

Splinted versus unsplinted implants. Splinted implants are usually indicated in clinical situations where there is a risk of mechanical overloading to reduce the forces on implants and surrounding tissues. It has been suggested that splinting implants helps to distribute functional loads and therefore decrease the implant failure rates. Some studies evaluated whether this is true or not.

Of the four studies comparing the splinting versus non-splinting of implants influence on implant failure rates, one got no conclusive results (279), because the implant failures happened at the stage-two surgery (thus, before the prosthetic rehabilitation), one found no difference between the approaches (280), one favoured unsplinted implants (158) and another favoured the splinted implants (197). Due to the small number of controlled studies on the subject and to the divergent results, it is impossible to suggest that 
one technique may cause higher implant failure rates when compared to the other one.

Number of implants for overdentures. It is generally agreed that removable mandibular dentures retained by some implants (overdentures) are more satisfactory and functional than conventional dentures. Some researchers have been questioning whether the variation in the number of implants used to support a mandibular overdenture may influence the implant failure rates.

There seems to be no statistically significant difference in implant failure rates between the use of one or two implants (281), or two or four implants (282284) to support mandibular overdentures. In one study, the implant failures occurred before the loading, thus no comparisons were possible between the groups concerning implant survival (285). It is important to note that the attachment system used varied among studies, generally being ball-attachment in studies comparing one and two implants, and bar-clip attachments in studies comparing two and four implants. A study comparing mandibular overdentures being supported by one or four implants has not been performed so far.

Type of overdenture attachment. Several studies observed no statistically significant difference in the implant failure rates between implants used to support overdentures using different attachment systems, being between gold-alloy bar without extension versus gold-alloy bar with extension versus non-connected abutments with different attachments (111), bar with clips versus ball attachments $(126,286)$, ball versus magnet attachments (287), magnets versus ball attachments versus straight bars (288), bar versus telescopic crowns (289) and ball versus resilient telescopic crown (290). Only one study (291) found significant difference in implant failure rates between standard ball attachments $(25 \%)$, large ball attachments $(0 \%)$ and Locator attachments $(0 \%)$. In one study (292), the failure of implants occurred during the healing period, and none after the prosthetic constructions and loading, which prevents any reliable comparison between the techniques concerning the implant survival.

Morse taper connection versus conventional abutments. Pieri et al. (293) compared the clinical outcomes of sin- gle implants immediately placed and restored 4 months later either with Morse taper connection or conventional abutments (20 implants each group). A provisional screw-retained crown was placed and adjusted for non-functional loading within $24 \mathrm{~h}$. After 1 year, no implants were lost in the conventional abutments group, whereas one implant failed in the Morse taper connection group.

Immediatelearly/delayed/late loading. In implantology, an undisturbed healing period was previously thought needed to ensure osseointegration. A modified protocol with immediate or early loading has been tested, in order to achieve a more rapid treatment and to reduce discomfort of wearing removable appliances during the healing period.

Some studies observed that failure rates for immediately loaded implants were significantly higher than implants loaded after a healing period of 6 weeks (294), 3 months (45), 4 months (295), 6 months (69, 296). The difference in implant failure rates was also significant in comparison of loading of implants within 6 weeks versus loaded after 6 weeks (219).

However, most of the studies showed no statistically significant difference in comparison of implant failure rates of implants loaded after several different healing periods, the comparisons being same day of surgery versus next day (297), immediately versus 7 days (298), immediately versus within 2 weeks (299), immediately versus 1 month $(300,301)$, immediately versus 6 weeks (302-305), immediately versus 2 months $(306,307)$, immediately versus $3-4$ months $(55,82,103,268,308-316)$, immediately versus 6 months $(187,317)$, immediately versus after 4-8 months (318), immediately versus 1 week versus 3-4 months (319), within 9-18 days versus 2.5-5 months (320), 10 days versus 3 months (321), within 2 weeks versus 6 weeks-6 months (68), within 20 days versus after 4 months (31), within 3 weeks versus 3 months $(48,322), 6$ versus 12 weeks $(237,323), 6$ weeks versus 6 months (324).

CNC (or laser-welded) titanium frameworks versus cast gold-alloy frameworks. Titanium frameworks have for more than 20 years been used as an option to goldalloy castings to restore edentulous patients with screw-retained implant-supported prostheses (164). Few controlled clinical studies comparing the two techniques were published. 
One study showed that there is a greater risk of implant failures with the implants supporting prostheses with laser-welded titanium frameworks compared with implants supporting conventional gold-alloy frameworks (325). Other studies did not show significant difference in implant failure rates between the uses of these two frameworks $(326,327)$, or between computer numeric control-milled titanium frameworks versus conventional gold-alloy frameworks (164).

\section{Other conditions}

Contamination with pre-fabricated stainless steel guide versus no guide. Jofré et al. (328) evaluated the 2-year survival rate of splinted mini-implants that came into contact with stainless steel prior to their insertion into the anterior mandible. A total of 46 implants were inserted using a pre-fabricated stainless steel guide (group 1), whereas 44 implants were placed without a guide (group 2). One implant failed $(97 \cdot 8 \%$ survival rate) in group 1, and four failed in group 2 (90.9\% survival rate). Energy dispersive X-ray spectroscopy analysis revealed carbon and oxygen on all implants. On the implants that were in contact with stainless steel, additional elements were identified, including silica, calcium, iron and chromium. The authors concluded that contact between mini-implants and stainless steel surgical guides does not seem to generate contamination that compromises the survival of mini-implants.

Control versus immediate orthodontic loading. Palagi et al. (329) evaluated 20 implants under immediate prosthetic and orthodontic forces after a follow-up period of at least 2 years. All implants received screwed provisional crowns immediately after surgery. The implants were randomly divided into two groups: the control group (nine implants), with a healing period of 4 months, and the immediate orthodontic loading group (11 implants), and the survival rates were $88.9 \%$ and $90.9 \%$, respectively.

Internal sinus lift without graft material versus implants in native posterior maxilla. In the study of Gabbert et al. (330), 36 patients received 92 screw-shaped implants in combination with an internal sinus lift without graft material, while 44 patients received 77 implants in the native posterior maxilla. After a mean followup was 1.2 years, four implants $(4.3 \%)$ failed in the internal sinus lift and $2(2 \cdot 6 \%)$ in the native maxilla group.

Reason for tooth extraction. Grunder et al. (87) assessed the clinical outcomes of 264 implants placed in fresh extraction sites, in healed sites, and with membranes placed over the extraction site. More implants were lost if the reason for tooth extraction was periodontitis $(10 \cdot 2 \%)$ when compared with trauma $(0 \%)$, root fracture $(0 \%)$, periapical inflammation $(0 \%)$ and caries $(5.0 \%)$. If teeth were extracted for a combination of reasons, in four of six failures, periodontitis was one of the reasons for tooth extraction. Only three of the 14 patients having an implant failure had no history of periodontitis before tooth extraction.

Narrow versus wide edentulous maxilla crest. Friberg and Jemt (218) compare the outcome of implants placed in edentulous maxillae with either wide (226 implants) or narrow (279 implants) jaw shapes and followed clinically up to 7 years. The implant CSR at 7 years was of $94.6 \%$ and $93.6 \%$ for wide and narrow crests, respectively.

\section{Narrative review with focus on oral implant failures and marginal bone resorption in relation to some findings in the systematic review}

The previous part of this paper represents a systematic screening process review on reasons for implant failure. We have added this narrative review on implant failures and bone resorption to ensure that our paper is to date and that we will not miss any important reasons for implant problems. This narrative part is of a lower level of evidence than found in the first part. We will start by commenting on some findings relative to the systematic review of the present paper where non-comparative data are available, at times pointing in a different direction than described in the systematic review. We will then comment on marginal bone resorption that, if unstoppable, certainly may result in secondary failures of implants.

The most commonly used material for oral implants is commercially pure (c.p.) titanium found in more than $95 \%$ of all annually placed implants. C.p. titanium is an excellent implant material where a dense oxide layer will make it biocompatible (331). However, recently there have been reports of a possible 
allergy to titanium (332). Titanium allergy cannot be excluded as a reason for implant loss (333), but a number of reports including the so-called Melisa test for evaluating allergies (334) have been criticised for lack of scientific correlates. In the past, aluminium oxides were used as implant materials too (335). However, ceramic materials share the shortcoming of ageing in the body with due possible subsequent implant fractures, a known fact for aluminium oxides and a clear long-term risk with zirconium materials too (6). In fact, implant fractures with titanium implants were not uncommon in the infancy of osseointegration (110), but are much more uncommon today when we use titanium grade 3 or 4 materials instead of c.p. titanium grade 1 originally preferred by Brånemark et al. (336).

In the systematic review, an obvious conclusion is that maxillary implants perform less well than mandibular ones. This statement is based on machined and other old implant surfaces and has been thoroughly documented. However, with modern moderately rough surfaces, the situation may have changed. In one study from the Brånemark clinic based on thousands of implants, it was clearly indicated that early maxillary failures dropped rapidly with the introduction of modern moderately rough surfaces (337). The drop in maxillary failures was so substantial that there seemed to be no differences in clinical outcome between mandibular and maxillary implants with the more modern implants. A recent overview displayed similar findings; different types of modern implant brands characterised by a moderately rough surface had significantly improved clinical outcome in the maxilla compared with the situation when older implants types were preferred, R. Jimbo, T. Albrektsson, unpublished data. A very similar observation was made with short implants where the systematic review of the present paper pointed to increased failure rates of short implants, a finding that may be disputed with the use of modern implants. It seems indeed to be the case that the modern surfaces, known to display a stronger bone to implant contact than found with the old standard of machined implants, compensate for the minimal osseointegrated area of the short implants. However, modern implants seem to have a very similar clinical outcome as older types of implants in uncompromised situations such as placement between the mental foraminae of the mandible. The advantage of the modern implants is limited to compromised situations such as patient smoking, implant placement in irradiated areas and applied direct loading in addition to benefits with maxillary and short implants.

Focusing on implant designs, we have solid information that cylindrical implants without threads do not work well in the long run. With the possible exception of one porous-coated oral implant system, such unthreaded designs have been more or less withdrawn from clinical usage due to marginal bone resorption $(338-340)$. The precise reason for clinical failure with unthreaded oral implant systems is difficult to assess. Is the reported high marginal bone loss related to the cylindrical shape of the implant per se or to the relatively rough plasma-sprayed surface typical for such implant designs or even a combination of these two characteristics? We have now come over to the second part of this narrative review; marginal bone resorption and have already learnt that the type of implant is indeed important for bone maintenance but only as one factor. In fact, there are other factors behind marginal bone loss, such as clinical handling and state of the patient, as described by Albrektsson et al. (341). Some clinicians have simply more marginal bone loss and higher failure rates than others. Albrektsson (342) investigated the clinical outcome of all implants placed at the Brånemark clinic in 1986 and found that one surgeon (who was not inexperienced) was alone responsible for the majority of failures with the implants and also responsible for the majority of those implants that demonstrated undue marginal bone resorption. The old machined Brånemark implant was the only one used in this study as well as in a study by Bryant (343) who reported of a correlation between implant outcome/marginal bone resorption and the individual surgeon who had placed the implants as well as the initial prosthodontist who had taken care of the patients. Everyone is not a good surgeon even if he or she has had proper training and experience. Similar viewpoints of the close correlation between implant problems and the involved clinician were discussed in a recent book pointing to the human factor being of main importance for failure (344). The combined findings in these reports point to the importance of proper clinical handling to avoid implant failure and that poor clinical techniques may be behind a large number of implant failures and cases that later develop peri-implantitis (342-344). Another example of the importance of clinical handling for avoidance of mar- 
ginal bone loss and failure emanate from experience with the Nobel Direct system (219). This implant system showed either failure or rapid marginal bone loss in about one-third of placed implants. However, the problem seemed not to have to do with the implant design per se but with the clinical procedures; clinicians were recommended to grind down the implant in situ (which of course caused severe bone vibrations and due destruction) and then load the implants immediately. This is a vicious combination of drastic clinical handling after placement with a direct loading procedure that in itself presents a threat to implant survival $(68,219)$. Another threat to implant survival related to clinical handling may come from inexperienced clinicians (6). It is also important that the whole team could plan and design a prosthetic suprastructure in a way that it will be possible for the patient to perform an adequate routine oral hygiene. For example, nonconnected implants are generally considered to facilitate optimal oral hygiene by the patients because of superior accessibility.

All these examples of increased failure rates and/or increased marginal bone resorption point to the need for a shift of focus away from assumed similarities between teeth and implants as the background for implant problems. There are, in fact, very little if any evidence of a true connection between periodontitis and the start of marginal bone loss around oral implants. Furthermore, such a connection is most unlikely in the light of the very different interfacial situations between the tooth and the implant. The former is anchored in a periodontal ligament that allows movement and is characterised by a rich vascularisation and innervation. This stands in stark contrast to the implant that is clinically stable and has an interfacial low blood circulation and an almost total lack of interfacial nerves. In recent studies, distinctly different genetical patterns were observed between periodontitis and peri-implantitis (204), and the implant was found representative of a foreign body reaction (7). The successful implant is one that maintains osseointegration, a typical foreign body response to the hardware that is shielded off from the tissues by embedment in bone. We see a similar foreign body reaction to Bioss particles, likewise separated from the tissues by bony encapsulation (345). What may disturb the foreign body equilibrium leading to marginal bone loss has to part already been mentioned (unsuitable implant designs, poor clinical handling and patients with poor bone quality/quantity) but do also include cement remnants in the soft tissues, suddenly changed loading patterns or certain types of patient disease $(6,7)$.

The end result of marginal bone loss may indeed be peri-implantitis that may threaten implant survival, but the start of the problem has nothing to do with periodontitis, at least we lack any evidence of this as of today. To maintain oral implants in the body, we have to have a foreign body equilibrium without or with only very little marginal bone resorption. More severe marginal bone resorption will lead to peri-implantitis that may start as an aggravation of the inflammatory response inevitable to any foreign body such as an oral implant. However, as demonstrated by pioneers behind reactions to foreign bodies (346-348), the presence of a foreign body may render the tissues to react with a much lowered resistance to infection. Foreign bodies in form of sutures were combined with bacterial exposition and resulted in severe infections, whereas the same amount of injected bacteria caused no visible harm if no foreign body was present (346-348). Future research will be guided by injecting a defined amount of bacteria in animals with or without an oral implant to find out whether Koch's postulates (349) apply to oral implants or not.

Understanding the mechanisms behind marginal bone resorption will help in minimising the problem in the future. The proper therapy to established peri-implantitis may be difficult to identify, but the clinician may act rapidly when pre-stages to periimplantitis is evidenced by an onset of bone resorption. Take, for example, the case of cement remnants that really represent a foreign body on another foreign body. In case such cement particles are carefully removed from the soft tissues, we may establish a secondary foreign body equilibrium if with some bone resorption. With other words, we act before a permanent state of peri-implantitis has been established. Another situation may be the patient who rapidly loses teeth around a single implant; if the body has no time to adapt to the new loading situation marginal bone resorption may follow, and in this situation, it may prove needed to temporarily unload the implant to give bone tissue time to gradually adapt to the new situation. These two examples point to the necessity to be clinically active in cases of early bone resorption instead of falsely believing that we have a situation beyond clinical control. 


\section{Concluding remarks}

Implant therapy is, today, a routinely used treatment option, frequently used even for young individuals. The implants must therefore ideally function for decades. Ten years or more clinical outcomes with modern, moderately rough implants point to a summed frequency of implant failure and peri-implantitis to be within $5 \%$ of all placed implants (5). Thus, one shortcoming of studies on implant failures is related to time - 5-year survival rates of a particular implant system may be quite acceptable, but ongoing bone resorption may nevertheless present with poor longterm results of the same design.

There are other limitations of clinical studies such as (i) study conducted in a single centre; (ii) controlled studies but not randomised; (iii) the lack of double blindness; (iv) limited number of patients/ sample size, which may be too low to detect a statistically significant difference in implant failures between the different procedures/conditions being compared and may not have been scientifically sound. The small sample size limited the power of the tests; therefore, the lack of statistical significance taken alone is not strong evidence of similarity between the groups; and (v) statistical method used for evaluation of implant failure/survival rates.

Concerning the statistical method, the typical method of calculating survival in most studies is a simple ratio of the number of implants removed to the total number of implants placed, which is misleading because it does not take into consideration the effects of time. The life-table analysis would be a more appropriate method (122). This method calculates the number of failures in a defined interval versus the number of implants at risk during that interval, and this ratio is then cumulated over the study period. At each succeeding interval, the number of failed implants and the number of implants lost to follow-up are subtracted from the number of implants at risk from the preceding interval. A characteristic feature of this progression is that the number of implants at risk decreases with time. Therefore, the confidence interval for the mean survival increases as a function of time. As a consequence, similar absolute differences between two populations may be statistically significant at an early interval but not significant at later intervals (122). However, one shortcoming of the CSR table is the lack of separation between implants yet not followed up for a given time limit and implants that are unaccounted for in dropout patients. There is a risk that the latter category of unaccounted for implants may present with higher failure rates than those implants that are yet not followed up for a specific time but still remain in the study.

Some of the factors compared in the present review were only covered in a couple of papers, and hence, findings from such comparative studies would present only weak evidence for the preference of one particular approach.

Considering these limitations and according to the findings of the present study, it may be suggested that the following situations are correlated to increase the implant failure rate: a low insertion torque of implants that are planned to be immediately or early loaded, inexperienced surgeons inserting the implants, implant insertion in the maxilla, implant insertion in the posterior region of the jaws, implants in heavy smokers, implant insertion in bone qualities type III and IV, implant insertion in places with small bone volumes, use of shorter length implants, greater number of implants placed per patient, lack of initial implant stability, use of cylindrical (non-threaded) implants and prosthetic rehabilitation with implant-supported overdentures. Moreover, it may be suggested that the following situations may be correlated with an increase in the implant failure rate: use of the non-submerged technique, immediate loading, implant insertion in fresh extraction sockets, smaller diameter implants.

The higher failure rate documented for shorter implants compared with longer ones may be related to compromised placement in restricted anatomical sites and to the reduced surface area available to contact the bone. The combination of poor bone quality and shorter implant length has been recognised as providing less mechanical stability at the time of implant placement and during the osseointegration period. This emphasises the rather deleterious combination of a small bone volume and a soft bone texture, when using short implants.

Some recently published studies of the narrative part of our review suggest that modern, moderately rough implants may present with similar results irrespective if placed in maxillas, in smoking patients or using only short implants.

\section{Ethical approval}

Not applicable. 


\section{Source of funding}

This work was supported by CNPq, Conselho Nacional de Desenvolvimento Científico e Tecnológico - Brazil.

\section{Conflict of interests}

There is no conflict of interest.

\section{References}

1. Roos-Jansåker AM, Renvert H, Lindahl C, Renvert S. Nine- to fourteen-year follow-up of implant treatment Part III: factors associated with peri-implant lesions. J Clin Periodontol. 2006;33:296-301.

2. Koldsland OC, Scheie AA, Aass AM. Prevalence of peri-implantitis related to severity of the disease with different degrees of bone loss. J Periodontol. 2010;81:231238.

3. Fransson C, Lekholm U, Jemt T, Berglundh T. Prevalence of subjects with progressive bone loss at implants. Clin Oral Implants Res. 2005;16:440-446.

4. Rosen P, Clem D, Cochran D, Froum S, McAllister B, Renvert $\mathrm{S}$ et al. Peri-implant mucositis and peri-implantitis: a current understanding of their diagnoses and clinical implications. J Periodontol. 2013;84:436-443.

5. Albrektsson T, Buser D, Sennerby L. Crestal bone loss and oral implants. Clin Implant Dent Relat Res. 2012;14:783791.

6. Qian J, Wennerberg A, Albrektsson T. Reasons for marginal bone loss around oral implants. Clin Implant Dent Relat Res. 2012;14:792-807.

7. Albrektsson T, Dahlin C, Jemt T, Sennerby L, Turri A, Wennerberg A. Is marginal bone loss around oral implants the result of a provoked foreign body reaction? Clin Implant Dent Relat Res. 2013; doi: 10.1111/cid.12142.

8. Chvartszaid D, Koka S, Zarb G. Osseointegration failures. In: Zarb GA, Albrektsson T, Baker G, Eckert SE, Stanford C, Tarnow DP, Wennerberg A, eds. Osseointegration - on continuing synergies in surgery, prosthodontics, and biomaterials. Chicago (IL): Quintessence; 2008:157-164.

9. Dent CD, Olson JW, Farish SE, Bellome J, Casino AJ, Morris HF et al. The influence of preoperative antibiotics on success of endosseous implants up to and including stage II surgery: a study of 2,641 implants. J Oral Maxillofac Surg. 1997; 55:19-24.

10. Laskin DM, Dent CD, Morris HF, Ochi S, Olson JW. The influence of preoperative antibiotics on success of endosseous implants at 36 months. Ann Periodontol. 2000;5:166-174.

11. Gynther GW, Köndell PÅ, Moberg LE. Dental implant installation without antibiotic prophylaxis. Oral Surg Oral Med Oral Pathol Oral Radiol Endod. 1998;85:509-511.

12. Morris HF, Ochi S, Plezia R, Gilbert H, Dent CD, Pikulski J et al. AICRG, Part III: the influence of antibiotic use on the survival of a new implant design. J Oral Implantol. 2004;30:144-151.

13. Abu-Ta'a M, Quirynen $M$, Teughels $W$, van Steenberghe D. Asepsis during periodontal surgery involving oral implants and the usefulness of peri-operative antibiotics: a prospective, randomized, controlled clinical trial. J Clin Periodontol. 2008;35:58-63.

14. Anitua E, Aguirre JJ, Gorosabel A, Barrio P, Errazquin $\mathrm{JM}$, Román $\mathrm{P}$ et al. A multicentre placebo-controlled randomised clinical trial of antibiotic prophylaxis for placement of single dental implants. Eur J Oral Implantol. 2009;2:283-292.

15. Esposito M, Cannizzaro G, Bozzoli P, Checchi L, Ferri V, Landriani $\mathrm{S}$ et al. Effectiveness of prophylactic antibiotics at placement of dental implants: a pragmatic multicentre placebo-controlled randomised clinical trial. Eur J Oral Implantol. 2010;3:135-143.

16. Nolan R, Kemmoona M, Polyzois I, Claffey N. The influence of prophylactic antibiotic administration on postoperative morbidity in dental implant surgery. A prospective double blind randomized controlled clinical trial. Clin Oral Implants Res. 2014;25:252-259.

17. Tan WC, Ong M, Han J, Mattheos N, Pjetursson BE, Tsai AY et al., on Behalf of the ITI Antibiotic Study Group. Effect of systemic antibiotics on clinical and patientreported outcomes of implant therapy - a multicenter randomized controlled clinical trial. Clin Oral Implants Res. 2014;2:185-193.

18. Zinser MJ, Randelzhofer P, Kuiper L, Zöller JE, De Lange GL. The predictors of implant failure after maxillary sinus floor augmentation and reconstruction: a retrospective study of 1045 consecutive implants. Oral Surg Oral Med Oral Pathol Oral Radiol. 2013;115:571582.

19. Lambert PM, Morris HF, Ochi S. The influence of smoking on 3-year clinical success of osseointegrated dental implants. Ann Periodontol. 2000; 5:79-89.

20. Wagenberg B, Froum SJ. A retrospective study of 1,925 consecutively placed immediate implants from 1988 to 2004. Int J Oral Maxillofac Implants. 2006;21:71-80.

21. El-Kholey KE. Efficacy of two antibiotic regimens in the reduction of early dental implant failure: a pilot study. Int J Oral Maxillofac Surg. 2014;43:487-490.

22. Binahmed A, Stoykewych A, Peterson L. Single preoperative dose versus long-term prophylactic antibiotic regimens in dental implant surgery. Int $\mathrm{J}$ Oral Maxillofac Implants. 2005;20:115-117.

23. Kashani H, Dahlin C, Bengt A. Influence of different prophylactic antibiotic regimens on implant survival rate: a retrospective clinical study. Clin Implant Dent Relat Res. 2005; 7:32-35.

24. Friberg B, Sennerby L, Gröndahl K, Bergström C, Bäck T, Lekholm U. On cutting torque measurements during implant placement: a 3-year clinical prospective study. Clin Implant Dent Relat Res. 1999;1:75-83.

25. Caiazzo A, Casavecchia P, Barone A, Brugnami F. A pilot study to determine the effectiveness of different amoxicil- 
lin regimens in implant surgery. $\mathrm{J}$ Oral Implantol. 2011;37:691-696.

26. Karaky AE, Sawair FA, Al-Karadsheh OA, Eimar HA, Algarugly SA, Baqain ZH. Antibiotic prophylaxis and early dental implant failure: a quasi-random controlled clinical trial. Eur J Oral Implantol. 2011;4:31-38.

27. Eliasson A, Narby B, Ekstrand K, Hirsch J, Johansson A, Wennerberg A. A 5-year prospective clinical study of submerged and nonsubmerged Paragon system implants in the edentulous mandible. Int J Prosthodont. 2010;23:231238.

28. Tarnow DP, Emtiaz S, Classi A. Immediate loading of threaded implants at stage 1 surgery in edentulous arches: ten consecutive case reports with 1- to 5-year data. Int J Oral Maxillofac Implants. 1997;12:319-324.

29. Collaert B, De Bruyn H. Comparison of Brånemark fixture integration and short-term survival using one-stage or two-stage surgery in completely and partially edentulous mandibles. Clin Oral Implants Res. 1998;9:131-135.

30. Becker W, Becker BE, Ricci A, Bahat O, Rosenberg E, Rose LF et al. A prospective multicenter clinical trial comparing one- and two-stage titanium screw-shaped fixtures with one-stage plasma-sprayed solid-screw fixtures. Clin Implant Dent Relat Res. 2000;2:159-165.

31. Ericsson I, Randow K, Nilner K, Petersson A. Early functional loading of Brånemark dental implants. A 5-year follow-up study. Clin Implant Dent Relat Res. 2000;2:70-77.

32. Moberg LE, Kondell PA, Sagulin GB, Bolin A, Heimdahl A, Gynther GW. Brånemark system and ITI dental implant system for treatment of mandibular edentulism. A comparative randomized study: 3-year follow-up. Clin Oral Implants Res. 2001;12:450-461.

33. Heydenrijk K, Raghoebar GM, Meijer HJ, van der Reijden WA, van Winkelhoff AJ, Stegenga B. Two-stage IMZ implants and ITI implants inserted in a single-stage procedure. A prospective comparative study. Clin Oral Implants Res. 2002;13:371-380.

34. Åstrand P, Engquist B, Anzén B, Bergendal T, Hallman M, Karlsson $\mathrm{U}$ et al. A three-year follow-up report of a comparative study of ITI Dental Implants and Brånemark System implants in the treatment of the partially edentulous maxilla. Clin Implant Dent Relat Res. 2004;6:130-141.

35. Cecchinato D, Olsson C, Lindhe J. Submerged or nonsubmerged healing of endosseous implants to be used in the rehabilitation of partially dentate patients. J Clin Periodontol. 2004;31:299-308.

36. Lemmerman KJ, Lemmerman NE. Osseointegrated dental implants in private practice: a long-term case series study. J Periodontol. 2005;76:310-319.

37. Balshi SF, Wolfinger GJ, Balshi TJ. A retrospective analysis of 44 implants with no rotational primary stability used for fixed prosthesis anchorage. Int $\mathrm{J}$ Oral Maxillofac Implants. 2007;22:467-471.

38. Cecchinato D, Bengazi F, Blasi G, Botticelli D, Cardarelli I, Gualini F. Bone level alterations at implants placed in the posterior segments of the dentition: outcome of submerged/non-submerged healing. A 5-year multicenter, randomized, controlled clinical trial. Clin Oral Implants Res. 2008;19:429-431.

39. Cordaro L, Torsello F, Roccuzzo M. Clinical outcome of submerged versus non-submerged implants placed in fresh extraction sockets. Clin Oral Implants Res. 2009;20:13071313.

40. Prosper L, Redaelli S, Pasi M, Zarone F, Radaelli G, Gherlone EF. A randomized prospective multicenter trial evaluating the platform-switching technique for the prevention of postrestorative crestal bone loss. Int J Oral Maxillofac Implants. 2009;24:299-308.

41. Tallarico M, Vaccarella A, Marzi GC, Alviani A, Campana V. A prospective case-control clinical trial comparing 1and 2-stage Nobel Biocare TiUnite implants: resonance frequency analysis assessed by Osstell Mentor during integration. Quintessence Int. 2011;42:635-644.

42. Tallarico M, Vaccarella A, Marzi GC. Clinical and radiological outcomes of 1 - versus 2-stage implant placement: 1year results of a randomised clinical trial. Eur J Oral Implantol. 2011;4:13-20.

43. Balshi TJ, Wolfinger GJ. Immediate loading of Brånemark implants in edentulous mandibles: a preliminary report. Implant Dent. 1997;6:83-88.

44. Ericsson I, Randow K, Nilner K, Petersson A. Some clinical and radiographical features of submerged and nonsubmerged titanium implants. A 5-year follow-up study. Clin Oral Implants Res. 1997;8:422-426.

45. Schnitman PA, Wöhrle PS, Rubenstein JE, Da Silva JD, Wang N-H. Ten year results for Brånemark implants immediately loaded with fixed prostheses at implant placement. Int J Oral Maxillofac Implants. 1997;12:495503.

46. Chuang SK, Wei LJ, Douglass CW, Dodson TB. Risk factors for dental implant failure: a strategy for the analysis of clustered failure time observations. J Dent Res. 2002;81:572-577.

47. Baelum V, Ellegaard B. Implant survival in periodontally compromised patients. J Periodontol. 2004;75:1404-1412.

48. Engquist B, Astrand P, Anzén B, Dahlgren S, Engquist E, Feldmann $\mathrm{H}$ et al. Simplified methods of implant treatment in the edentulous lower jaw: a 3-year follow-up report of a controlled prospective study of one-stage versus two-stage surgery and early loading. Clin Implant Dent Relat Res. 2005;7:95-104.

49. Ellegaard B, Baelum V, Kølsen-Petersen J. Non-grafted sinus implants in periodontally compromised patients: a time-to-event analysis. Clin Oral Implants Res. 2006; 17:156-164.

50. Becktor JP, Isaksson S, Billström C. A prospective multicenter study using two different surgical approaches in the mandible with turned Brånemark implants: conventional loading using fixed prostheses. Clin Implant Dent Relat Res. 2007;9:179-185.

51. Chrcanovic BR, Oliveira DR, Custódio AL. Accuracy evaluation of computed tomography-derived stereolithographic surgical guides in zygomatic implant placement in human cadavers. J Oral Implantol. 2010;36:345-355. 
52. Nkenke E, Eitner S, Radespiel-Tröger M, Vairaktaris E, Neukam FW, Fenner M. Patient-centred outcomes comparing transmucosal implant placement with an open approach in the maxilla: a prospective, nonrandomized pilot study. Clin Oral Implants Res. 2007;18:197-203.

53. Ozan O, Turkyilmaz I, Yilmaz B. A preliminary report of patients treated with early loaded implants using computerized tomography-guided surgical stents: flapless versus conventional flapped surgery. J Oral Rehabil. 2007:34:835-840.

54. Villa R, Rangert B. Immediate and early function of implants placed in extraction sockets of maxillary infected teeth: a pilot study. J Prosthet Dent. 2007;97:S96-S108.

55. Cannizzaro G, Leone M, Consolo U, Ferri V, Esposito M. Immediate functional loading of implants placed with flapless surgery versus conventional implants in partially edentulous patients: a 3-year randomized controlled clinical trial. Int J Oral Maxillofac Implants. 2008;23:867-875.

56. Covani U, Cornelini R, Barone A. Buccal bone augmentation around immediate implants with and without flap elevation: a modified approach. Int J Oral Maxillofac Implants. 2008;23:841-846.

57. Maló P, Nobre MD. Flap vs. flapless surgical techniques at immediate implant function in predominantly soft bone for rehabilitation of partial edentulism: a prospective cohort study with follow-up of 1 year. Eur J Oral Implantol. 2008;1:293-304.

58. Danza M, Zollin I, Carinci F. Comparison between implants inserted with and without computer planning and custom model coordination. J Craniofac Surg. 2009;20:1086-1092.

59. Arisan V, Karabuda CZ, Ozdemir T. Implant surgery using bone- and mucosasupported stereolithographic guides in totally edentulous jaws: surgical and post-operative outcomes of computer-aided vs. standard techniques. Clin Oral Implants Res. 2010;21:980-988.

60. Berdougo $M$, Fortin $T$, Blanchet $E$, Isidori M, Bosson JL. Flapless implant surgery using an image-guided system. A 1- to 4-year retrospective multicenter comparative clinical study. Clin Implant Dent Relat Res. 2010;12:142-152.

61. Rousseau P. Flapless and traditional dental implant surgery: an open, retrospective comparative study. J Oral Maxillofac Surg. 2010;68:2299-2306.

62. Van de Velde T, Sennerby L, De Bruyn H. The clinical and radiographic outcome of implants placed in the posterior maxilla with a guided flapless approach and immediately restored with a provisional rehabilitation: a randomized clinical trial. Clin Oral Implants Res. 2010;21:1223-1233.

63. Cannizzaro G, Felice P, Leone M, Checchi V, Esposito M. Flapless versus open flap implant surgery in partially edentulous patients subjected to immediate loading: 1year results from a split-mouth randomised controlled trial. Eur J Oral Implantol. 2011;4:177-188.

64. De Bruyn H, Atashkadeh M, Cosyn J, van de Velde T. Clinical outcome and bone preservation of single TiUnite ${ }^{\mathrm{TM}}$ implants installed with flapless or flap surgery. Clin Implant Dent Relat Res. 2011;13:175-183.
65. Al-Juboori MJ, Bin Abdulrahaman S, Jassan A. Comparison of flapless and conventional flap and the effect on crestal bone resorption during a 12-week healing period. Dent Implantol Update. 2012;23:9-16.

66. Katsoulis J, Avrampou M, Spycher C, Stipic M, Enkling N, Mericske-Stern R. Comparison of implant stability by means of resonance frequency analysis for flapless and conventionally inserted implants. Clin Implant Dent Relat Res. 2012; 14:915-923.

67. Sunitha RV, Sapthagiri E. Flapless implant surgery: a 2year follow-up study of 40 implants. Oral Surg Oral Med Oral Pathol Oral Radiol. 2013;116:e237-e243.

68. Sennerby L, Rocci A, Becker W, Jonsson L, Johansson LA, Albrektsson T. Short-term clinical results of Nobel Direct implants: a retrospective multicentre analysis. Clin Oral Implants Res. 2008;19:219-226.

69. Ottoni JM, Oliveira ZF, Mansini R, Cabral AM. Correlation between placement torque and survival of singletooth implants. Int $\mathrm{J}$ Oral Maxillofac Implants. 2005;20:769-776

70. Cannizzaro G, Leone M, Ferri V, Viola P, Federico G, Esposito $\mathrm{M}$. Immediate loading of single implants inserted flapless with medium or high insertion torque: a 6-month follow-up of a split-mouth randomised controlled trial. Eur J Oral Implantol. 2012;5:333-342.

71. Marković A, Calasan D, Colić S, Stojčev-Stajčić L, Janjić B, Mišić T. Implant stability in posterior maxilla: bonecondensing versus bone-drilling: a clinical study. Oral Surg Oral Med Oral Pathol Oral Radiol Endod. 2011;112:557-563.

72. Alghamdi $\mathrm{H}$, Anand PS, Anil S. Undersized implant site preparation to enhance primary implant stability in poor bone density: a prospective clinical study. J Oral Maxillofac Surg. 2011;69:e506-e512.

73. Danza M, Guidi R, Carinci F. Comparison between implants inserted into piezo split and unsplit alveolar crests. J Oral Maxillofac Surg. 2009;67:2460-2465.

74. Strietzel FP, Lange KP, Svegar M, Hartmann HJ, Küchler I. Retrospective evaluation of the success of oral rehabilitation using the Frialit-2 implant system. Part 1: influence of topographic and surgical parameters. Int J Prosthodont. 2004;17:187-194

75. Abboud M, Wahl G, Guirado JL, Orentlicher G. Application and success of two stereolithographic surgical guide systems forimplant placement with immediate loading. Int J Oral Maxillofac Implants. 2012;27:634643.

76. Chrcanovic BR, Martins MD, Wennerberg A. Immediate placement of implants into infected sites: a systematic review. Clin Implant Dent Relat Res. 2013; doi: 10.1111/ cid. 12098.

77. Maló P, Rangert B, Dvärsäter L. Immediate function of Brånemark implants in the esthetic zone: a retrospective clinical study with 6 months to 4 years of follow-up. Clin Implant Dent Relat Res. 2000;2:138-146.

78. Chaushu G, Chaushu S, Tzohar A, Dayan D. Immediate loading of single-tooth implants: immediate vs. non- 
immediate implantation. A clinical report. Int J Oral Maxillofac Implants. 2001;16:267-272.

79. De Bruyn H, Collaert B. Early loading of machined-surface Brånemark implants in completely edentulous mandibles: healed bone versus fresh extraction sites. Clin Implant Dent Relat Res. 2002;4:136-142.

80. Horwitz J, Zuabi O, Peled M, Machtei EE. Immediate and delayed restoration of dental implants in periodontally suscepticle patients: 1-year results. Int J Oral Maxillofac Implants. 2007:22:423-429.

81. Deng F, Zhang H, Zhang H, Shao H, He Q, Zhang P. A comparison of clinical outcomes for implants placed in fresh extraction sockets versus healed sites in periodontally compromised patients: a 1-year follow-up report. Int J Oral Maxillofac Implants. 2010;25:1036-1040.

82. Zafiropoulos GG, Deli G, Bartee BK, Hoffmann O. Singletooth implant placement and loading in fresh and regenerated extraction sockets. Five-year results: a case series using two different implant designs. J Periodontol. 2010;81:604-615.

83. Watzek G, Haider R, Mensdorff-Pouilly N, Haas R. Immediate and delayed implantation for complete restoration of the jaw following extraction of all residual teeth: a retrospective study comparing different types of serial immediate implantation. Int $\mathrm{J}$ Oral Maxillofac Implants. 1995; 10:561-567.

84. Gomez-Roman G, Schulte W, D'Hoedt B, Axman-Krcmar D. The Frialit-2 implant system: five-year clinical experience in single tooth and immediately postextraction applications. Int J Oral Maxillofac Implants. 1997;12:299-309.

85. Mensdorff-Pouilly N, Haas R, Mailath G, Watzek G. The immediate implant. A retrospective study comparing the different types of immediate implantation. Int J Oral Maxillofac Implants. 1994;9:571-578.

86. Haas R, Mensdorff-Pouilly N, Mailath G, Watzek G. Success of 1,920 IMZ implants followed for up to 100 months. Int J Oral Maxillofac Implants. 1996;1 1:581-588.

87. Grunder U, Polizzi G, Goené R, Hatano N, Henry P, Jackson WJ et al. A 3-year prospective multicenter follow-up report on the immediate and delayed-immediate placement of implants. Int $\mathrm{J}$ Oral Maxillofac Implants. 1999;14:210-216

88. Polizzi G, Grunder U, Goené R, Hatano N, Henry P, Jackson WJ et al. Immediate and delayed implant placement into extraction sockets: a 5-year report. Clin Implant Dent Relat Res. 2000;2:93-99.

89. Evian CI, Emling R, Rosenberg ES, Waasdorp JA, Halpern $\mathrm{W}$, Shah $\mathrm{S}$ et al. Retrospective analysis of implant survival and the influence of periodontal disease and immediate placement on long-term results. Int J Oral Maxillofac Implants. 2004; 19:393-398.

90. Kourtis SG, Sotiriadou S, Voliotis S, Challas A. Private practice results of dental implants. Part I: survival and evaluation of risk factors-Part II: surgical and prosthetic complications. Implant Dent. 2004;13:373-385.

91. Davarpanah M, Caraman M, Szmukler-Moncler S, Jakubowicz-Kohen B, Alcolforado G. Preliminary data of a prospective clinical study on the Osseotite NT implant: 18month follow-up. Int $\mathrm{J}$ Oral Maxillofac Implants. 2005;20:448-454.

92. Schropp L, Kostopoulos L, Wenzel A, Isidor F. Clinical and radiographic performance of delayed-immediate single-tooth implant placement associated with peri-implant bone defects. A 2-year prospective, controlled, randomized follow-up report. J Clin Periodontol. 2005;32:480487.

93. Lindeboom JA, Tjiook Y, Kroon FH. Immediate placement of implants in periapical infected sites: a prospective randomized study in 50 patients. Oral Surg Oral Med Oral Pathol Oral Radiol Endod. 2006;101:705-710.

94. Peñarrocha-Diego M, Carrillo-Garcîa C, Boronat-Lopez A, García-Mira B. Comparative study of wide-diameter implants placed after dental extraction and implants positioned in mature bone for molar replacement. Int $\mathrm{J}$ Oral Maxillofac Implants. 2008;23:497-501.

95. Ribeiro FS, Pontes AE, Marcantonio E, Piattelli A, Neto RJ, Marcantonio E Jr. Success rate of immediate nonfunctional loaded single-tooth implants: immediate versus delayed implantation. Implant Dent. 2008;17:109-117.

96. Schropp L, Isidor F. Clinical outcome and patient satisfaction following full-flap elevation for early and delayed placement of single-tooth implants: a 5-year randomized study. Int J Oral Maxillofac Implants. 2008;23:733-743.

97. Pieri F, Aldini NN, Fini M, Corinaldesi G. Immediate occlusal loading of immediately placed implants supporting fixed restorations in completely edentulous arches: a 1-year prospective pilot study. J Periodontol. 2009;80:411-421.

98. Cehreli MC, Kökat AM, Uysal S, Akca K. Spontaneous early exposure and marginal bone loss around conventionally and early-placed submerged implants: a doubleblind study. Clin Oral Implants Res. 2010;21:1327-1333.

99. Aguirre-Zorzano LA, Rodríguez-Andrés C, Estefanía-Fresco R, Fernández-Jiménez A. Immediate temporary restoration of single-tooth implants: prospective clinical study. Med Oral Patol Oral Cir Bucal. 2011;16:e794-e799.

100. Gillot L, Noharet R, Buti J, Cannas B. A retrospective cohort study of 105 patients rehabilitated with immediately loaded mandibular cross-arch bridges in combination with immediate implant placement. Eur J Oral Implantol. 2011;4:247-253.

101. Barbier L, Abeloos J, De Clercq C, Jacobs R. Peri-implant bone changes following tooth extraction, immediate placement and loading of implants in the edentulous maxilla. Clin Oral Investig. 2012;16:1061-1070.

102. Raes F, Cooper LF, Tarrida LG, Vandromme H, De Bruyn H. A case-control study assessing oral-health-related quality of life after immediately loaded single implants in healed alveolar ridges or extraction sockets. Clin Oral Implants Res. 2012;23:602-608.

103. Vandeweghe S, Ackermann A, Bronner J, Hattingh A, Tschakaloff A, De Bruyn H. A retrospective, multicenter study on a novo wide-body implant for posterior regions. Clin Implant Dent Relat Res. 2012;14:281-292. 
104. Truninger TC, Philipp AO, Siegenthaler DW, Roos $M$, Hämmerle $\mathrm{CH}$, Jung RE. A prospective, controlled clinical trial evaluating the clinical and radiological outcome after 3 years of immediately placed implants in sockets exhibiting periapical pathology. Clin Oral Implants Res. 2011;22:20-27

105. Payne AG, Tawse-Smith A, Thomson WM, Duncan WD, Kumara R. One-stage surgery and early loading of three implants for maxillary overdentures: a 1-year report. Clin Implant Dent Relat Res. 2004;6:61-74.

106. Adell R, Eriksson B, Lekholm U, Brånemark PI, Jemt T. A long-term follow-up study of osseointegrated implants in the treatment of totally edentulous jaws. Int J Oral Maxillofac Implants. 1990;5:347-359.

107. Block MS, Kent JN. Long-term follow-up on hydroxylapatite-coated cylindrical dental implants: a comparison between developmental and recent periods. J Oral Maxillofac Surg. 1994;52:937-943.

108. Minsk L, Polson AM, Weisgold A, Rose LF, Sanavi F, Baumgarten $\mathrm{H}$ et al. Outcome failures of endosseous implants from a clinical training center. Compend Contin Educ Dent. 1996; 17:848-850.

109. Lambert P, Morris H, Shigeru O. Positive effect of surgical experience with implants on second-stage implant survival. J Oral Maxillofac Surg. 1997;55:12-18.

110. Adell R, Lekholm U, Rockler B, Brånemark PI. A 15-year study of osseointegrated implants in the treatment of the edentulous jaw. Int J Oral Surg. 1981;10:387-416.

111. Engquist B, Bergendal T, Kallus T, Linden U. A retrospective multicenter evaluation of osseointegrated implants supporting overdentures. Int J Oral Maxillofac Implants. 1988;3:129-134

112. Köndell PA, Nordenram A, Landt H. Titanium implants in the treatment of edentulousness: influence of patient's age on prognosis. Gerodontics. 1988;4:280-284.

113. Friberg B, Jemt T, Lekholm U. Early failures in 4,641 consecutively placed Brånemark dental implants: a study from stage 1 surgery to the connection of completed prostheses. Int J Oral Maxillofac Implants. 1991;6:142-146.

114. Jaffin RA, Berman CL. The excessive loss of Branemark fixtures in type IV bone: a 5-year analysis. J Periodontol. $1991 ; 62: 2-4$.

115. Smith R, Berger R, Dodson T. Risk factors associated with dental implants in healthy and medically compromised patients. Int J Oral Maxillofac Implants. 1992;7:367-372.

116. Babbush CA, Shimura M. Five-year statistical and clinical observations with the IMZ two-stage osseointegrated implant system. Int $\mathrm{J}$ Oral Maxillofac Implants. $1993 ; 8: 245-253$.

117. Bain CA, Moy PK. The association between the failure of dental implans and cigarette smoking. Int J Oral Maxillofac Implants. 1993;8:609-615.

118. Zarb GA, Schmitt A. The longitudinal clinical effectiveness of osseointegrated dental implants in posterior partially edentulous patients. Int J Prosthodont. 1993;6:189-196.

119. De Bruyn H, Collaert B. The effect of smoking on early implant failure. Clin Oral Implants Res. 1994;5:260-264.
120. Hutton JE, Heath MR, Chai JY, Harnett J, Jemt T, Johns $\mathrm{RB}$ et al. Factors related to success and failure rates at 3 year follow-up in a multicenter study of overdentures supported by Brånemark implants. Int $\mathrm{J}$ Oral Maxillofac Implants. 1995; 10:33-42.

121. Jemt T, Chai J, Harnett J, Heath MR, Hutton JE, Johns RB et al. A 5-year prospective multicenter follow-up report on overdentures supported by osseointegrated implants. Int J Oral Maxillofac Implants. 1996;11:291298.

122. Wheeler SL. Eight-year clinical retrospective study of titanium plasma-sprayed and hydroxyapatite-coated cylinder implants. Int J Oral Maxillofac Implants. 1996;11:340350.

123. Buser D, Mericske-Stern R, Bernard JP, Behneke A, Behneke N, Hirt HP et al. Long-term evaluation of non-submerged ITI implants. Part 1: 8-year life table analysis of a prospective multi-center study with 2359 implants. Clin Oral Implants Res. 1997;8:161-172.

124. Ellegaard B, Baelum V, Karring T. Implant therapy in periodontally compromised patients. Clin Oral Implant Res. 1997;8:180-188.

125. Roos J, Sennerby L, Lekholm U, Jemt T, Gröndahl K, Albrektsson T. A qualitative and quantitative method for evaluating implant success- a 5-year retrospective analysis. Int J Oral Maxillofac Implants. 1997;12:504-514.

126. Bergendal T, Engquist B. Implant-supported overdentures: a longitudinal prospective study. Int J Oral Maxillofac Implants. 1998; 13:253-262.

127. Becker N, Becker BE, Alsuwyed A, Al-Mubarak S. Longterm evaluation of 282 implants in maxillary and mandibular molar positions: a prospective study. J Periodontol. 1999;70:896-901.

128. Hultin M, Fischer J, Gustafsson A, Kallus T, Klinge B. Factors affecting late fixture loss and marginal bone loss around teeth and dental implants. Clin Implant Dent Relat Res. 2000;2:203-208.

129. Schwartz-Arad D, Grossman Y, Chaushu G. The clinical effectiveness of implants placed immediately into fresh extraction sites of molar teeth. J Periodontol. 2000;71:839-844.

130. Eckert SE, Meraw SJ, Weaver AL, Lohse CM. Early experience with wide-platform Mk II implants. Part I: implant survival. Part II: evaluation of risk factors involving implant survival. Int $\mathrm{J}$ Oral Maxillofac Implants. $2001 ; 16: 208-216$.

131. Mengel R, Schröder T, Flores-de-Jacoby L. Osseointegrated implants in patients treated for generalized chronic periodontitis and generalized aggressive periodontitis: 3 and 5-year results of a prospective long-term study. J Periodontol. 2001;72:977-989.

132. Testori T, Wiseman L, Woolfe S, Porter SS. A prospective multicenter clinical study of the Osseotite implant: fouryear interim report. Int $\mathrm{J}$ Oral Maxillofac Implants. 2001;16:193-200.

133. Mordenfeld MH, Johansson A, Hedin M, Billstrom C, Fyrberg KA. A retrospective clinical study of wide-diameter 
implants used in posterior edentulous areas. Int J Oral Maxillofac Implants. 2004; 19:387-392.

134. Balshi SF, Allen FD, Wolfinger GJ, Balshi TJ. A resonance frequency analysis assessment of maxillary and mandibular immediately loaded implants. Int $\mathrm{J}$ Oral Maxillofac Implants. 2005;20:584-594.

135. Friberg B, Dahlin C, Widmark G, Ostman PO, Billström C. One-year results of a prospective multicenter study on Brånemark System implants with a TiUnite surface. Clin Implant Dent Relat Res. 2005;7:S70-S75.

136. DeLuca S, Habsha E, Zarb GA. The effect of smoking on osseointegrated dental implants. Part I: implant survival. Int J Prosthodont. 2006;19:491-498.

137. Binahmed A, Stoykewych A, Hussain A, Love B, Pruthi V. Long-term follow-up of hydroxyapatite-coated dental implants-a clinical trial. Int $\mathrm{J}$ Oral Maxillofac Implants. 2007;22:963-968.

138. Jebreen SE, Khraisat A. Multicenter retrospective study of ITI implant-supported posterior partial prosthesis in Jordan. Clin Implant Dent Relat Res. 2007;9:89-93.

139. Levine RA, Ganeles J, Jaffin RA, Clem DS III, Beagle JR, Keller GW. Multicenter retrospective analysis of wideneck dental implants for single molar replacement. Int $\mathrm{J}$ Oral Maxillofac Implants. 2007;22:736-742.

140. Testori T, Del Fabbro M, Capelli M, Zuffetti F, Francetti L, Weinstein RL. Immediate occlusal loading and tilted implants for the rehabilitation of the atrophic edentulous maxilla: 1-year interim results of a multicenter prospective study. Clin Oral Implants Res. 2008;19:227232.

141. Mangano C, Mangano F, Shibli JA, Tettamanti L, Figliuzzi M, d'Avila S et al. Prospective evaluation of 2,549 Morse taper connection implants: 1- to 6-year data. J Periodontol. 2011;82:52-61

142. Albrektsson T. A multicenter report on osseointegrated oral implants. J Prosthet Dent. 1988;60:75-84.

143. Jemt T, Lekholm U, Adell R. Osseointegrated implants in the treatment of partially edentulous patients: a preliminary study on 876 consecutively placed fixtures. Int J Oral Maxillofac Implants. 1989;4:211-217.

144. van Steenberghe D. A retrospective multicenter evaluation of the survival rate of osseointegrated fixtures supporting fixed partial prostheses in the treatment of partial edentulism. J Prosthet Dent. 1989;61:217-222.

145. Bass SL, Triplett RG. The effects of preoperative resorption and jaw anatomy on implant success. A report of 303 cases. Clin Oral Implants Res. 1991;2:193-198.

146. Naert I, Quirynen M, van Steenberghe D, Darius P. A sixyear prosthetic study of 509 consecutively inserted implants for the treatment of partial edentulism. J Prosthet Dent. 1992;67:236-245.

147. Fugazzotto PA, Gulbransen HJ, Wheeler SL, Lindsay JA. The use of IMZ osseointegrated implants in partially and completely edentulous patients: success and failure rates of 2,023 implant cylinders up to $60+$ months in function. Int J Oral Maxillofac Implants. 1993;8:617621.
148. Jemt T, Lekholm U. Oral implant treatment in posterior partially edentulous jaws: a 5-year follow-up report. Int $\mathrm{J}$ Oral Maxillofac Implants. 1993;8:635-640.

149. Nevins M, Langer B. The successful application of osseointegrated implants to the posterior jaw: a long-term retrospective study. Int $\mathrm{J}$ Oral Maxillofac Implants. 1993;8:428-432.

150. Faehn O, Siddiqui AA, Lazzara R, Feldman S. A four-year retrospective study of $3 \mathrm{i}$ threaded implants. Aust Prosthodont J. 1995;9:9-15.

151. Higuchi KW, Folmer T, Kultje C. Implant survival rates in partially edentulous patients: a 3-year prospective multicenter study. J Oral Maxillofac Surg. 1995;53:264-268.

152. Lazzara R, Seddiqui AA, Binon P, Feldman SA, Weiner R, Phillips $\mathrm{R}$ et al. Retrospective multicenter analysis of $3 \mathrm{i}$ endosseous dental implants placed over a five-year period. Clin Oral Implants Res. 1996;7:73-83.

153. Wyatt CCL, Zarb GA. Treatment outcomes of patients with implant - supported fixed partially prostheses. Int J Oral Maxillofac Implants. 1998;13:204-211.

154. Lekholm U, Gunne J, Henry P, Higuchi K, Lindén U, Bergström C et al. Survival of the Brånemark implant in partially edentulous jaws. A prospective 10-year prospective multicenter study. Int $\mathrm{J}$ Oral Maxillofac Implants. 1999; 14:639-645.

155. Davarpanah M, Martinez H, Celletti R, Alcoforado G, Tecucianu JF, Etienne D. Osseotite implant: 3-year prospective multicenter evaluation. Clin Implant Dent Relat Res. 2001;3:111-118.

156. Glauser R, Ree A, Lundgren A, Gottlow J, Hammerle CH, Scharer P. Immediate occlusal loading of Brånemark implants applied in various jawbone regions: a prospective, 1-year clinical study. Clin Implant Dent Relat Res. 2001;3:204-213.

157. Ferrigno N, Laureti M, Fanali S, Grippaudo G. A longterm follow-up study of non-submerged ITI implants in the treatment of totally edentulous jaws. Part 1: ten-year life table analysis of a prospective multicenter study with 1286 implants. Clin Oral Implants Res. 2002;13:260-273.

158. Naert I, Koutsikakis G, Duyck J, Quirynen M, Jacobs R, van Steenberghe D. Biologic outcome of implant-supported restorations in the treatment of partial edentulism. Part 1: a longitudinal clinical evaluation. Clin Oral Implants Res. 2002;13:381-389.

159. Fugazzotto PA, Vlassis J, Butler B. ITI implant use in private practice: clinical results with 5,526 implants followed up to $72+$ months in function. Int $\mathrm{J}$ Oral Maxillofac Implants. 2004; 19:408-412.

160. Goené R, Bianchesi C, Hüerzeler M, Del Lupo R, Testori $\mathrm{T}$, Davarpanah $\mathrm{M}$ et al. Performance of short implants in partial restorations: 3-year follow-up of Osseotite implants. Implant Dent. 2005;14:274-280.

161. Rasmusson L, Roos J, Bystedt H. A 10-year follow-up study of titanium dioxide-blasted implants. Clin Implant Dent Relat Res. 2005;7:36-42.

162. Malo P, de Araujo Nobre M, Rangert B. Implants placed in immediate function in periodontally compromised sites: 
a five-year retrospective and one-year prospective study. J Prosthet Dent. 2007;97:S86-S95.

163. Mangano C, Mangano F, Shibli JA, Luongo G, De Franco M, Briguglio $\mathrm{F}$ et al. Prospective clinical evaluation of 201 direct laser metal forming implants: results from a 1-year multicenter study. Lasers Med Sci. 2012;27:181-189.

164. Örtorp A, Jemt T. CNC-milled titanium frameworks supported by implants in the edentulous jaw: a 10-year comparative clinical study. Clin Implant Dent Relat Res. 2012;14:88-99.

165. Cox J, Zarb GA. The longitudinal clinical efficacy of osseointegrated dental implants: a 3-year report. Int J Oral Maxillofac Implants. 1987;2:91-100.

166. Aparicio C, Orozco P. Use of 5-mm-diameter implants: periotest values related to a clinical and radiographic evaluation. Clin Oral Implants Res. 1998;9:398-406.

167. Ivanoff CJ, Gröndahl K, Sennerby L, Bergström C, Lekholm U. Influence of variations in implant diameters: a 3to 5-year retrospective clinical report. Int J Oral Maxillofac Implants. 1999; 14:173-180.

168. Brocard D, Barthet P, Baysse E, Duffort JF, Eller P, Justumus $\mathrm{P}$ et al. A multicenter report on 1,022 consecutively placed ITI implants: a 7-year longitudinal study. Int J Oral Maxillofac Implants. 2000; 15:691-700.

169. Levine RA, Clem D, Beagle J, Ganeles J, Johnson P, Solnit $\mathrm{G}$ et al. Multicenter retrospective analysis of the solidscrew ITI implant for posterior single-tooth replacements. Int J Oral Maxillofac Implants. 2002;17:550-556.

170. Penarrocha M, Guarinos J, Sanchis JM, Balaguer J. A retrospective study (1994-1999) of 441 ITI implants in 114 patients followed-up during an average of 2.3 years. Med Oral. 2002;7:144-155.

171. Testori T, Del Fabbro M, Feldman S, Vincenzi G, Sullivan D, Rossi R Jr et al. A multicenter prospective evaluation of 2-months loaded OsseotiteA implants placed in the posterior jaws: 3-year follow-up results. Clin Oral Implants Res. 2002;13:154-161.

172. Sullivan D, Vincenzi G, Feldman S. Early loading of Osseotite implants 2 months after placement in the maxilla and mandible: a 5-year report. Int J Oral Maxillofac Implants. 2005;20:905-912

173. Moheng P, Feryn JM. Clinical and biologic factors related to oral implant failure: a 2-year follow-up study. Implant Dent. 2005;14:281-288.

174. Evian CI. A comparison of hydroxyapatite-coated MicroVvent and pure titanium Swede-Vent implants. Int J Oral Maxillofac Implants. 1996;11:639-644.

175. Grunder U, Gaberthuel T, Boitel N, Imoberdorf $M$, Meyenberg $\mathrm{K}$, Andreoni $\mathrm{C}$ et al. Evaluating the clinical performance of the Osseotite implant: defining prosthetic predictability. Compend Contin Educ Dent. 1999;20:628$633,636,638-40$.

176. Bahat $\mathrm{O}$. Treatment planning and placement of implants in the posterior maxillae: report of 732 consecutive Nobelpharma implants. Int $\mathrm{J}$ Oral Maxillofac Implants. $1993: 8: 151-161$.
177. Levine RA, Clem DS III, Wilson TG Jr, Higginbottom F, Solnit G. Multicenter retrospective analysis of the ITI implant system used for single-tooth replacements. Results of loading for 2 or more years. Int $\mathrm{J}$ Oral Maxillofac Implants. 1999; 14:516-520.

178. Bahat $\mathrm{O}$. Brånemark system implants in the posterior maxilla: clinical study of 660 implants followed for 5-12 years. Int J Oral Maxillofac Implants. 2000;15:646-653.

179. Levin L, Sadet P, Grossmann Y. A retrospective evaluation of 1387 single-tooth implants: a 6-year followup. J Periodontol. 2006;77:2080-2083.

180. Olate S, Lyrio MC, de Moraes M, Mazzonetto R, Moreira RW. Influence of diameter and length of implant on early dental implant failure. $\mathrm{J}$ Oral Maxillofac Surg. 2010:68:414-419.

181. Stultz ER, Lofland R, Sendax VI, Hornbuckle C. A multicenter 5-year retrospective success analysis of 6,200 Integral implants. Compend Contin Educ Dent. 1993;14:478486.

182. Scurria MS, Morgan V, Guckes AD, Li S, Koch G. Prognostic variables associated with implant failure: a retrospective effectiveness study. Int $\mathrm{J}$ Oral Maxillofac Implants. 1998; 13:400-406.

183. Weng D, Jacobson Z, Tarnow D, Hürzeler MB, Faehn O, Sanavi $\mathrm{F}$ et al. A prospective multicenter clinical trial of $3 \mathrm{i}$ machined-surface implants: results after 6 years of followup. Int J Oral Maxillofac Implants. 2003;18:417-423.

184. Feldman S, Boitel N, Weng D, Kohles SS, Stach RM. Fiveyear survival distributions of short-length (10 $\mathrm{mm}$ or less) machined-surfaced and Osseotite implants. Clin Implant Dent Relat Res. 2004;6:16-23.

185. Rosenberg ES, Cho SC, Elian N, Jalbout ZN, Froum S, Evian CI. A comparison of characteristics of implant failure and survival in periodontally compromised and periodontally health patients: a clinical report. Int $\mathrm{J}$ Oral Maxillofac Implants. 2004;19:873-879.

186. Machtei EE, Frankenthal S, Blumenfeld I, Gutmacher Z, Horwitz J. Dental implants for immediate fixed restoration of partially edentulous patients: a 1-year prospective pilot clinical trial in periodontally susceptible patients. J Periodontol. 2007;78:1188-1194.

187. Degidi M, Iezzi G, Perrotti V, Piattelli A. Comparative analysis of immediate functional loading and immediate nonfunctional loading to traditional healing periods: a 5year follow-up of 550 dental implants. Clin Implant Dent Relat Res. 2009;11:257-266.

188. Sánchez-Garcés MA, Costa-Berengue X, Gay-Escoda C. Short implants: a descriptive study of 273 implants. Clin Implant Dent Relat Res. 2012;14:508-516.

189. Jemt T, Book K, Lindén B, Urde G. Failures and complications in 92 consecutively inserted overdentures supported by Brånemark implants in severely resorbed edentulous maxillae: a study from prosthetic treatment to first annual check-up. Int J Oral Maxillofac Implants. 1992;7:162-167.

190. Dao TT, Anderson JD, Zarb GA. Is osteoporosis a risk factor for osseointegration of dental implants? Int J Oral Maxillofac Implants. 1993;8:137-144. 
191. Jemt T, Lekholm U. Implant treatment in edentulous maxillae. A 5-year follow-up report on patients with different degrees of jaw resorption. Int $\mathrm{J}$ Oral Maxillofac Implants. 1995;10:303-311.

192. Gorman LM, Lambert PM, Morris HF, Ochi S, Winkler S. The effect of smoking on implant survival at second stage surgery: DICRG Interim Report No. 5. Dental Implant Clinical Research Group. Implant Dent. 1994;3:165-168.

193. Bain CA. Smoking and implant failure - benefits of a smoking cessation protocol. Int $\mathrm{J}$ Oral Maxillofac Implants. 1996;11:756-759.

194. Lindquist LW, Carlsson GE, Jemt T. Association between marginal bone loss around osseointegrated mandibular implants and smoking habits: a 10-year follow-up study. J Dent Res. 1997;76:1667-1674.

195. Wallace RH. The relationship between cigarette smoking and dental implant failure. Eur J Prosthodont Restor Dent. 2000;8:103-106.

196. Schwartz-Arad D, Samet N, Samet N, Mamlider A. Smoking and complications of endosseous dental implants. J Periodontol. 2002;73:153-157.

197. Rocci A, Martignoni M, Gottlow J. Immediate loading in the maxilla using flapless surgery, implants placed in predetermined positions, and prefabricated provisional restorations: a retrospective 3-year clinical study. Clin Implant Dent Relat Res. 2003:5:29-36.

198. Anner R, Grossmann Y, Anner Y, Levin L. Smoking, diabetes mellitus, periodontitis, and supportive periodontal treatment as factors associated with dental implant survival: a long-term retrospective evaluation of patients followed for up to 10 years. Implant Dent. 2010;19:57-64.

199. Cavalcanti R, Oreglia F, Manfredonia MF, Gianserra R, Esposito $M$. The influence of smoking on the survival of dental implants: a 5-year pragmatic multicentre retrospective cohort study of 1727 patients. Eur J Oral Implantol. 2011;4:39-45.

200. Schneider D, Witt L, Hämmerle CH. Influence of the crown-to-implant length ratio on the clinical performance of implants supporting single crown restorations: a crosssectional retrospective 5-year investigation. Clin Oral Implants Res. 2012;23:169-174

201. Hardt CR, Grondahl K, Lekholm U, Wennstrom JL. Outcome of implant therapy in relation to experienced loss of periodontal bone support: a retrospective 5-year study. Clin Oral Implants Res. 2002;13:488-494.

202. Mengel R, Kreuzer G, Lehmann KM, Flores-de-Jacoby L. A telescopic crown concept for the restoration of partially edentulous patients with aggressive generalized periodontitis: a 3-year prospective longitudinal study. Int J Periodontics Restorative Dent. 2007;27:231-239.

203. Gianserra R, Cavalcanti R, Oreglia F, Manfredonia MF, Esposito $\mathrm{M}$. Outcome of dental implants in patients with and without a history of periodontitis: a 5-year pragmatic multicentre retrospective cohort study of 1727 patients. Eur J Oral Implantol. 2010;3:307-314.

204. Becker ST, Beck-Broichsitter BE, Graetz C, Dörfer CE, Wiltfang J, Häsler R. Peri-implantitis versus periodontitis: func- tional differences indicated by transcriptome profiling. Clin Implant Dent Relat Res. 2012, doi: 10.1111/cid.12001

205. Mengel R, Stelzel M, Hasse C, Flores-de-Jacoby L. Osseointegrated implants in patients treated for generalized severe adult periodontitis. An interim report. J Periodontol. 1996;67:782-787.

206. Carlsson L, Rostlund T, Albrektsson B, Albrektsson T. Implant furation improved by close fit. Cylindrical implant bone interface studied in rabbits. Acta Orthop Scand. 1988;59:272-275.

207. Ivanoff CJ, Sennerby L, Lekholm U. Influence of initial implant mobility on the integration of titanium implants. An experimental study in rabbits. Clin Oral Implants Res. 1996;7:120-127.

208. Holahan CM, Wiens JL, Weaver A, Assad D, Koka S. Relationship between systemic bone mineral density and local bone quality as effectors of dental implant survival. Clin Implant Dent Relat Res. 2011;13:29-33.

209. Eriksson RA, Albrektsson T. The effect of heat on bone regeneration: an experimental study in rabbits using the bone growth chamber. J Oral Maxillofac Surg. 1984:42:705-711.

210. Lekholm U, Zarb G. Patient selection and preparation. In: Brånemark P-I, Zarb G, Albrektsson T, eds. Tissue-integrated prostheses. Chicago (IL): Quintessence; 1985:199211.

211. Szmukler-Moncler S, Salama H, Reingewirtz Y, Dubruille $\mathrm{JH}$. Timing of loading and effect of micromotion on bonedental implant interface. Review of experimental literature. J Biomed Mater Res. 1998;43:192-203.

212. Chrcanovic BR, Abreu MH, Custódio AL. Morphological variation in dentate and edentulous human mandibles. Surg Radiol Anat. 2011;33:203-213.

213. Hernández G, Lopez-Pintor RM, Arriba L, Torres J, de Vicente JC. Implant treatment in patients with oral lichen planus: a prospective-controlled study. Clin Oral Implants Res. 2012;23:726-732.

214. Morris HF, Ochi S, Winkler S. Implant survival in patients with type 2 diabetes: placement to 36 months. Ann Periodontol. 2000; 5:157-165.

215. Alsaadi G, Quirynen M, Michiles K, Teughels W, Komarek A, van Steenberghe D. Impact of local and systemic factors on the incidence of failures up to abutment connection with modified surface oral implants. J Clin Periodont. 2008;35:51-57.

216. Winkler S, Morris HF, Ochi S. Implant survival to 36 months as related to length and diameter. Ann Periodontol. 2000;5:22-31.

217. Quirynen M, Naert I, van Steenberghe D. Fixture design and overload influence of marginal bone loss and fixture success in the Brånemark system. Clin Oral Implants Res. 1992;3:104-111.

218. Friberg B, Jemt T. Turned Brånemark System implants in wide and narrow edentulous maxillae: a retrospective clinical study. Clin Implant Dent Relat Res. 2008;10:78-85.

219. Albrektsson T, Gottlow J, Meirelles L, Ostman PO, Rocci A, Sennerby L. Survival of NobelDirect implants: an 
analysis of 550 consecutively placed implants at 18 different clinical centers. Clin Implant Dent Relat Res. 2007;9:65-70.

220. Wennerberg A, Jemt T. Complications in partially edentulous implant patients: a 5-year retrospective follow-up study of 133 patients supplied with unilateral maxillary prostheses. Clin Implant Dent Relat Res. 1999;1:49-56.

221. Shin SW, Bryant SR, Zarb GA. A retrospective study on the treatment outcome of wide-bodied implants. Int J Prosthodont. 2004;17:52-58.

222. Morris HF, Ochi S, Orenstein IH, Petrazzuolo V. AICRG, Part V: factors influencing implant stability at placement and their influence on survival of Ankylos implants. J Oral Implantol. 2004;30:162-170.

223. Orenstein IH, Tarnow DP, Morris HF, Ochi S. Factors affecting implant mobility at placement and integration of mobile implants at uncovering. J Periodontol. 1998;69:1404-1412.

224. Glauser R, Sennerby L, Meredith N, Ree A, Lundgren A, Gottlow $\mathrm{J}$ et al. Resonance frequency analysis of implants subjected to immediate or early functional occlusal loading. Successful vs. failing implants. Clin Oral Implants Res. 2004;15:428-434.

225. Nedir R, Bischof M, Szmukler-Moncler S, Bernard JP, Samson J. Predicting osseointegration by means of implant primary stability. Clin Oral Implants Res. 2004; 15:520-528.

226. Rodrigo D, Aracil L, Martin C, Sanz M. Diagnosis of implant stability and its impact on implant survival: a prospective case series study. Clin Oral Implants Res. 2010;21:255-261

227. Spiekermann H, Jansen VK, Richter EJ. A 10-year followup study of IMZ and TPS implants in the edentulous mandible using bar-retained overdentures. Int J Oral Maxillofac Implants. 1995; 10:231-243.

228. Karoussis IK, Brägger U, Salvi GE, Bürgin W, Lang NP. Effect of implant design on survival and success rates of titanium oral implants: a 10-year prospective cohort study of the ITI Dental Implant System. Clin Oral Implants Res. 2004;15:8-17.

229. Meijer HJ, Raghoebar GM, Van't Hof MA, Visser A. A controlled clinical trial of implant-retained mandibular overdentures: 10 years' results of clinical aspects and aftercare of IMZ implants and Brånemark implants. Clin Oral Implants Res. 2004;15:421-427.

230. Friberg B, Jisander S, Widmark G, Lundgren AK, Ivanoff CJ, Sennerby L et al. One-year prospective three-center study comparing the outcome of a 'soft bone implant' (Prototype Mk IV) and the standard Branemark Implant. Clin Implant Dent Relat Res. 2003;5:71-77.

231. Arnhart C, Kielbassa AM, Martinez-de Fuentes R, Goldstein $\mathrm{M}$, Jackowski J, Lorenzoni $\mathrm{M}$ et al. Comparison of variable-thread tapered implant designs to a standard taperedimplant design after immediate loading. A 3-year multicentre randomised controlled trial. Eur J Oral Implantol. 2012;5:123-136.
232. Geertman ME, Boerrigter EM, Van Waas MA, van Oort RP. Clinical aspects of a multicenter clinical trial of implant-retained mandibular overdentures in patients with severely resorbed mandibles. J Prosthet Dent. 1996;75:194-204.

233. Batenburg R, Meijer H, Raghoebar G, van Oort R, Boering G. Mandibular overdentures supported by two Brånemark, IMZ or IT1 implants. A prospective comparative preliminary study: one year results. Clin Oral Implants Res. 1998;9:374-383.

234. Åstrand P, Engquist B, Dahlgren S, Gröndahl K, Engquist E, Feldmann H. Astra Tech and Brånemark system implants: a 5-year prospective study of marginal bone reactions. Clin Oral Implants Res. 2004;15:413-420.

235. Lang NP, Tonetti MS, Suvan JE, Pierre Bernard J, Botticelli D, Fourmousis I et al., European Research Group on Periodontology. Immediate implant placement with transmucosal healing in areas of aesthetic priority. A multicentre randomized-controlled clinical trial I. Surgical outcomes. Clin Oral Implants Res. 2007; 18:188-196.

236. Akoglu B, Ucankale M, Ozkan Y, Kulak-Ozkan Y. Fiveyear treatment outcomes with three brands of implants supporting mandibular overdentures. Int J Oral Maxillofac Implants. 2011;26:188-194.

237. Tawse-Smith A, Payne AGT, Kumara R, Thomson WM. Early loading of unsplinted implants supporting mandibular overdentures using a one-stage operative procedure with two different implant systems: a 2-year report. Clin Implant Dent Relat Res. 2002;4:33-42.

238. Menicucci G, Pachie E, Lorenzetti M, Migliaretti G, Carossa S. Comparison of primary stability of straight-walled and tapered implants using an insertion torque device. Int J Prosthodont. 2012;25:465-471.

239. Chrcanovic BR, Pedrosa AR, Martins MD. Chemical and topographic analysis of treated surfaces of five different commercial dental titanium implants. Mater Res. 2012;15:372-382.

240. Chrcanovic BR, Leão NLC, Martins MD. Influence of different acid etchings on the superficial characteristics of Ti sandblasted with Al2O3. Mater Res. 2013;16:10061014

241. Gotfredsen K, Karlsson U. A prospective 5-year study of fixed partial prostheses supported by implants with machined and $\mathrm{TiO}_{2}$-blasted surface. J Prosthodont. 2001;10:2-7.

242. Vroom MG, Sipos P, de Lange GL, Gründemann LJ, Timmerman MF, Loos BG et al. Effect of surface topography of screw-shaped titanium implants in humans on clinical and radiographic parameters: a 12-year prospective study. Clin Oral Implants Res. 2009;20:1231-1239.

243. Jungner M, Lundqvist $P$, Lundgren S. Oxidized titanium implants (Nobel Biocare Ti- Unite) compared with turned titanium implants (Nobel Biocare mark III) with respect to implant failure in a group of consecutive patients treated with early functional loading and two-stage protocol. Clin Oral Implants Res. 2005;16:308-312. 
244. Renouard F, Nisand D. Short implants in the severely resorbed maxilla: a 2-year retrospective clinical study. Clin Implant Dent Relat Res. 2005; 7:S104-S110.

245. Östman PO, Hellman M, Sennerby L. Immediate occlusal loading of implants in the partially edentate mandible- a prospective 1-year radiographic. Int $\mathrm{J}$ Oral Maxillofac Implants. 2008;23:315-322.

246. Fröberg KK, Lindh C, Ericsson I. Immediate loading of Brånemark System Implants: a comparison between TiUnite and turned implants placed in the anterior mandible. Clin Implant Dent Relat Res. 2006;8:187-197.

247. Balshe AA, Assad DA, Eckert SE, Koka S, Weaver AL. A retrospective study of the survival of smooth- and roughsurface dental implants. Int J Oral Maxillofac Implants. 2009;24:1113-1118.

248. Fung K, Marzola R, Scotti R, Tadinada A, Schincaglia GP. A 36-month randomized controlled split-mouth trial comparing immediately loaded titanium oxide-anodized and machined implants supporting fixed partial dentures in the posterior mandible. Int $\mathrm{J}$ Oral Maxillofac Implants. 2011;26:631-638.

249. Van Assche N, Coucke W, Teughels W, Naert I, Cardoso MV, Quirynen M. RCT comparing minimally with moderately rough implants. Part 1: clinical observations. Clin Oral Implants Res. 2012;23:617-624.

250. Karabuda ZC, Abdel-Haq J, Arisan V. Stability, marginal bone loss and survival of standard and modified sandblasted, acid-etched implants in bilateral edentulous spaces: a prospective 15-month evaluation. Clin Oral Implants Res. 2011;22:840-849.

251. Esposito M, Grusovin MG, Pellegrino G, Soardi E, Felice P. Safety and effectiveness of maxillary early loaded titanium implants with a novel nanostructured calciumincorporated surface (Xpeed): 1-year results from a pilot multicenter randomised controlled trial. Eur J Oral Implantol. 2012;5:241-249.

252. Sivolella S, Stellini E, Testori T, Di Fiore A, Berengo M, Lops D. Splinted and unsplinted short implants in mandibles: a retrospective evaluation with 5 to 16 years of follow-up. J Periodontol. 2013;84:502-512.

253. Jaffin RA, Kumar A, Berman CL. Immediate loading of implants in partially and fully edentulous jaws: a series of 27 case reports. J Periodontol. 2000;71:833-838.

254. Rocci A, Martignoni M, Gottlow J. Immediate loading of Brånemark System TiUnite and machined-surface implants in the posterior mandible: a randomized openended clinical trial. Clin Implant Dent Relat Res. 2003:5:57-63.

255. Liddelow G, Henry P. The immediately loaded single implant-retained mandibular overdenture: a 36-month prospective study. Int J Prosthodont. 2010;23:13-21.

256. Krekmanov L, Kahn M, Rangert B, Lindström H. Tilting of posterior mandibular and maxillary implants for improved prosthesis support. Int J Oral Maxillofac Implants. 2000; 15:405-414

257. Aparicio C, Perales P, Rangert B. Tilted implants as an alternative to maxillary sinus grafting: a clinical, radio- logic, and periotest study. Clin Implant Dent Relat Res. 2001;3:39-49.

258. Francetti L, Agliardi E, Testori T, Romeo D, Taschieri S, Del Fabbro M. Immediate rehabilitation of the mandible with fixed full prosthesis supported by axial and tilted implants: interim results of single cohort prospective study. Clin Implant Dent Relat Res. 2008;10:255-263.

259. Agliardi E, Clericò M, Ciancio P, Massironi D. Immediate loading of full-arch fixed prostheses supported by axial and tilted implants for the treatment of edentulous atrophic mandibles. Quintessence Int. 2010;41:285-293.

260. Maló P, de Araújo Nobre M, Lopes A, Francischone C, Rigolizzo M. "All-on-4" immediate-function concept for completely edentulous maxillae: a clinical report on the medium ( 3 years) and long-term ( 5 years) outcomes. Clin Implant Dent Relat Res. 2012;14:e139-e150.

261. Crespi R, Vinci R, Capparé P, Romanos GE, Gherlone E. A clinical study of edentulous patients rehabilitated according to the "all on four" immediate function protocol. Int J Oral Maxillofac Implants. 2012;27:428-434.

262. Östman PO, Hellman M, Albrektsson T, Sennerby L. Direct loading of Nobel Direct and Nobel Perfect one-piece implants: a 1-year prospective clinical and radiographic study. Clin Oral Implants Res. 2007;18:409-418.

263. Bahat O, Handelsman M. Use of wide implants and double implants in the posterior jaw: a clinical report. Int $\mathrm{J}$ Oral Maxillofac Implants. 1996;11:379-386.

264. Degidi M, Piattelli A. Immediate functional and non-functional loading of dental implants: a 2- to 60-month followup study of 646 titanium implants. J Periodontol. 2003;74:225-241.

265. Degidi M, Piattelli A. Comparative analysis study of 702 dental implants subjected to immediate functional loading and immediate nonfunctional loading to traditional healing periods with a follow-up of up to 24 months. Int J Oral Maxillofac Implants. 2005;20:99-107.

266. Lindeboom JA, Frenken JW, Dubois L, Frank M, Abbink I, Kroon FH. Immediate loading versus immediate provisionalization of maxillary single-tooth replacement: a prospective randomized study with BioCom implants. J Oral Maxillofac Surg. 2006;64:936-942.

267. Degidi M, Nardi D, Piattelli A. A comparison between immediate loading and immediate restoration in cases of partial posterior mandibular edentulism: a 3-year randomized clinical trial. Clin Oral Implants Res. 2010;21: 682-687.

268. Margossian P, Mariani P, Stephan G, Margerit J, Jorgensen C. Immediate loading of mandibular dental implants in partially edentulous patients: a prospective randomized comparative study. Int J Periodontics Restorative Dent. 2012;32:e51-e58.

269. Nissan J, Narobai D, Gross O, Ghelfan O, Chaushu G. Long-term outcome of cemented versus screw-retained implant-supported partial restorations. Int J Oral Maxillofac Implants. 2011;26:1102-1107.

270. Sherif S, Susarla SM, Hwang JW, Weber HP, Wright RF. Clinician- and patient-reported long-term evaluation of 
screw- and cement-retained implant restorations: a 5-year prospective study. Clin Oral Investig. 2011;15:993-999.

271. Vigolo P, Mutinelli S, Givani A, Stellini E. Cemented versus screw-retained implant-supported single-tooth crowns: a 10-year randomised controlled trial. Eur J Oral Implantol. 2012;5:355-364.

272. Parein A, Ecket S, Wollan P, Keller E. Implant reconstruction in the posterior mandible: a long-term retrospective study. J Prosthet Dent. 1997;78:34-42.

273. Palmqvist S, Owall B, Schou S. A prospective randomized clinical study comparing implant-supported fixed prostheses and overdentures in the edentulous mandible: prosthodontic production time and costs. Int $\mathrm{J}$ Prosthodont. 2004; 17:231-235.

274. Hosseini M, Worsaae N, Schiodt M, Gotfredsen K. A 1year randomised controlled trial comparing zirconia versus metal-ceramicimplant supported single-tooth restorations. Eur J Oral Implantol. 2011;4:347-361.

275. Katsoulis J, Brunner A, Mericske-Stern R. Maintenance of implant-supported maxillary prostheses: a 2-year controlled clinical trial. Int $\mathrm{J}$ Oral Maxillofac Implants. 2011;26:648-656.

276. Cannizzaro G, Felice P, Soardi E, Ferri V, Leone M, Lazzarini $\mathrm{M}$ et al. Immediate loading of 2 (all-on-2) versus 4 (all-on-4) implants placed with a flapless technique supporting mandibular cross-arch fixed prostheses-1-year results from a pilot randomised controlled trial. Eur J Oral Implantol. 2013;6:121-131.

277. Blanes RJ, Bernard JP, Blanes ZM, Belser UC. A 10-year prospective study of ITI dental implants placed in the posterior region. II: Influence of the crown-to-implant ratio and different prosthetic treatment modalities on crestal bone loss. Clin Oral Implants Res. 2007; 18:707-714.

278. Schulte J, Flores AM, Weed M. Crown-to-implant ratios of single tooth implant-supported restorations. J Prosthet Dent. 2007;98:1-5.

279. Vigolo P, Zaccaria M. Clinical evaluation of marginal bone level change of multiple adjacent implants restored with splinted and nonsplinted restorations: a 5-year prospective study. Int J Oral Maxillofac Implants. 2010;25:1189-1194.

280. Naert I, Gizani S, Vuylsteke M, van Steenberge D. A 5 year randomized clinical trial on the influence of splinted and unsplinted oral implants in the mandibular overdenture therapy. Part I: peri-implant outcome. Clin Oral Implants Res. 1998;9:170-177.

281. Kronström M, Davis B, Loney R, Gerrow J, Hollender L. A prospective randomized study on the immediate loading of mandibular overdentures supported by one or two implants: a 12-month follow-up report. Int J Oral Maxillofac Implants. 2010;25:181-188.

282. Mau J, Behneke A, Behneke N, Fritzemeier CU, GomezRoman G, d'Hoedt B et al. Randomized multicenter comparison of 2 IMZ and 4 TPS screw implants supporting bar-retained overdentures in 425 edentulous mandibles. Int J Oral Maxillofac Implants. 2003;18:835-847.

283. Meijer HJ, Raghoebar GM, Batenburg RH, Visser A, Vissink A. Mandibular overdentures supported by two or four endosseous implants: a 10-year clinical trial. Clin Oral Implants Res. 2009;20:722-728.

284. de Jong MH, Wright PS, Meijer HJ, Tymstra N. Posterior mandibular residual ridge resorption in patients with overdentures supported by two or four endosseous implants in a 10-year prospective comparative study. Int J Oral Maxillofac Implants. 2010;25:1168-1174.

285. Walton JN, Glick N, Macentee MI. A randomized clinical trial comparing patient satisfaction and prosthetic outcomes with mandibular overdentures retained by one or two implants. Int J Prosthodont. 2009; 22:331-339.

286. Gotfredsen K, Holm B. Implant-supported mandibular overdentures retained with ball or bar attachments: a randomized prospective 5-year study. Int $\mathrm{J}$ Prosthodont. 2000; 13:125-130.

287. Davis DM, Packer ME. Mandibular overdentures stabilized by Astra Tech implants with either ball attachments or magnets: 5-year results. Int J Prosthodont. 1999;12:222229.

288. Naert I, Gizani S, Vuylsteke M, Van Steenberghe D. A 5 year prospective randomized clinical trial on the influence of splinted and unsplinted oral implants retaining a mandibular overdenture: prosthetic aspects and patient satisfaction. J Oral Rehabil. 1999;26:195-202.

289. Eitner S, Schlegel A, Emeka N, Holst S, Will J, Hamel J. Comparing bar and double-crown attachments in implant-retained prosthetic reconstruction: a follow-up investigation. Clin Oral Implants Res. 2008;19:530-537.

290. Krennmair G, Seemann R, Weinländer M, Piehslinger E. Comparison of ball and telescopic crown attachments in implant-retained mandibular overdentures: a 5-year prospective study. Int $\mathrm{J}$ Oral Maxillofac Implants. 2011;26:598-606.

291. Alsabeeha NH, Payne AG, De Silva RK, Thomson WM. Mandibular single-implant overdentures: preliminary results of a randomised-control trial on early loading with different implant diameters and attachment systems. Clin Oral Implants Res. 2011;22:330-337.

292. Wismeijer D, van Waas MAJ, Mulder J, Vermeeren JIJ, Kalk W. Clinical and radiological results of patients treated with three treatment modalities for overdentures on implants of the ITI Dental Implant System: a randomized controlled clinical trial. Clin Oral Implants Res. 1999;10:297-306.

293. Pieri F, Aldini NN, Marchetti C, Corinaldesi G. Influence of implant-abutment interface design on bone and soft tissue levels around immediately placed and restored singletooth implants: a randomized controlled clinical trial. Int J Oral Maxillofac Implants. 2011;26:169-178.

294. Zembić A, Glauser R, Khraisat A, Hämmerle CH. Immediate vs. early loading of dental implants: 3 -year results of a randomized controlled clinical trial. Clin Oral Implants Res. 2010;21:481-489.

295. Oh TJ, Shotwell JL, Billy EJ, Wang HL. Effect of flapless implant surgery on soft tissue profile: a randomized controlled clinical trial. J Periodontol. 2006;77:874-882. 
296. Ericsson I, Nilson H, Lindh T, Nilner K, Randow K. Immediate functional loading of Brånemark single tooth implants. An 18 months' clinical pilot follow-up study. Clin Oral Implants Res. 2000;11:26-33.

297. Testori T, Del Fabbro M, Galli F, Francetti L, Taschieri S, Weinstein R. Immediate occlusal loading the same day or the after implant placement: comparison of 2 different time frames in total edentulous lower jaws. J Oral Implantol. 2004;30:307-313.

298. Vanden Bogaerde L, Rangert B, Wendelhag I. Immediate/ early function of Brånemark System TiUnite implants in fresh extraction sockets in maxillae and posterior mandibles: an 18-month prospective clinical study. Clin Implant Dent Relat Res. 2005;7:S121-S130.

299. Misch CE, Degidi M. Five-year prospective study of immediate/early loading of fixed prostheses in completely edentulous jaws with a bone quality-based implant system. Clin Implant Dent Relat Res. 2003;5:17-28.

300. Ganeles J, Zöllner A, Jackowski J, ten Bruggenkate C, Beagle J, Guerra F. Immediate and early loading of Straumann implants with a chemically modified surface (SLActive) in the posterior mandible and maxilla: 1-year results from a prospective multicenter study. Clin Oral Implants Res. 2008;19:1119-1128.

301. Zöllner A, Ganeles J, Korostoff J, Guerra F, Krafft T, Brägger U. Immediate and early non-occlusal loading of Straumann implants with a chemically modified surface (SLActive) in the posterior mandible and maxilla: interim results from a prospective multicenter randomized-controlled study. Clin Oral Implants Res. 2008;19:442-450.

302. Achilli A, Tura F, Euwe E. Immediate/early function with tapered implants supporting maxillary and mandibular posterior fixed partial dentures: preliminary results of a prospective multicenter study. J Prosthet Dent. 2007;97: S52-S58.

303. Cannizzaro G, Leone $M$, Esposito $M$. Immediate versus early loading of two implants placed with a flapless technique supporting mandibular bar-retained overdentures: a single-blinded, randomised controlled clinical trial. Eur J Oral Implantol. 2008;1:33-43.

304. Cannizzaro G, Felice P, Leone M, Ferri V, Viola P, Esposito $\mathrm{M}$. Immediate versus early loading of $6.5 \mathrm{~mm}$-long flapless-placed single implants: a 4-year after loading report of a split-mouth randomised controlled trial. Eur J Oral Implantol. 2012;5:111-121

305. Merli M, Moscatelli M, Mariotti G, Piemontese M, Nieri M. Immediate versus early non-occlusal loading of dental implants placed flapless in partially edentulous patients: a 3-year randomized clinical trial. J Clin Periodontol. 2012:39:196-202.

306. Cannizzaro G, Torchio C, Leone M, Esposito M. Immediate versus early loading of flapless-placed implants supporting maxillary full-arch prostheses: a randomised controlled clinical trial. Eur J Oral Implantol. 2008;1:127-139.

307. Capelli M, Esposito M, Zuffetti F, Galli F, Del Fabbro M, Testori T. A 5-year report from a multicentre randomised clinical trial: immediate non-occlusal versus early loading of dental implants in partially edentulous patients. Eur J Oral Implantol. 2010;3:209-219.

308. Romeo E, Chiapasco M, Lazza A, Casentini P, Ghisolfi M, Iorio $\mathrm{M}$ et al. Implant-retained mandibular overdentures with ITI implants. Clin Oral Implants Res. 2002;13:495501.

309. Cannizzaro G, Leone M. Restoration of partially edentulous patients using dental implants with a microtextured surface: a prospective comparison of delayed and immediate full occlusal loading. Int $\mathrm{J}$ Oral Maxillofac Implants. 2003; 18:512-522.

310. Attard NJ, David LA, Zarb GA. Immediate loading of implants with mandibular overdentures: one-year clinical results of a prospective study. Int $\mathrm{J}$ Prosthodont. 2005; 18:463-470.

311. Romanos GE, Nentwig GH. Immediate versus delayed functional loading of implants in the posterior mandible: a 2-year prospective clinical study of 12 consecutive cases. Int J Periodontics Restorative Dent. 2006;26:459-469.

312. Güncü MB, Aslan Y, Tümer C, Güncü GN, Uysal S. Inpatient comparison of immediate and conventional loaded implants in mandibular molar sites within 12 months. Clin Oral Implants Res. 2008;19:335-341.

313. Schincaglia GP, Marzola R, Giovanni GF, Chiara CS, Scotti R. Replacement of mandibular molars with single-unit restorations supported by wide-body implants: immediate versus delayed loading. A randomized controlled study. Int J Oral Maxillofac Implants. 2008;23:474-480.

314. Prosper L, Crespi R, Valenti E, Capparé P, Gherlone E. Five-year follow-up of wide-diameter implants placed in fresh molar extraction sockets in the mandible: immediate versus delayed loading. Int $\mathrm{J}$ Oral Maxillofac Implants. 2010;25:607-612.

315. den Hartog L, Raghoebar GM, Stellingsma K, Vissink A, Meijer HJ. Immediate non-occlusal loading of single implants in the aesthetic zone: a randomized clinical trial. J Clin Periodontol. 2011;38:186-194.

316. Meloni SM, De Riu G, Pisano M, De Riu N, Tullio A. Immediate versus delayed loading of single mandibular molars. One-year results from a randomised controlled trial. Eur J Oral Implantol. 2012;5:345-353.

317. Hall JA, Payne AG, Purton DG, Torr B, Duncan WJ, De Silva RK. Immediately restored, single-tapered implants in the anterior maxilla: prosthodontic and aesthetic outcomes after 1 year. Clin Implant Dent Relat Res. 2007;9:34-45.

318. Chiapasco M, Abati S, Romeo E, Vogel G. Implantretained mandibular overdentures with Brånemark system MKII implants- a prospective comparative study between delayed and immediate loading. Int $\mathrm{J}$ Oral Maxillofac Implants. 2001;16:537-546.

319. De Smet E, Duyck J, Vander Sloten J, Jacobs R, Naert I. Timing of loading-immediate, early, or delayed-in the outcome of implants in the edentulous mandible: a prospective clinical trial. Int $\mathrm{J}$ Oral Maxillofac Implants. 2007;22:580-594

320. Fischer K, Stenberg T, Hedin M, Sennerby L. Five-year results from a randomized, controlled trial on early and 
delayed loading ofimplants supporting full-arch prosthesis in the edentulous maxilla. Clin Oral Implants Res. 2008; 19:433-441.

321. Turkyilmaz I, Tumer C. Early versus late loading of unsplinted TiUnite surface implants supporting mandibular overdentures: a 2-year report from a prospective study. J Oral Rehabil. 2007;34:773-780.

322. Røynesdal A-K, Amundrud B, Hannaes HR. A comparative clinical investigation of 2 early loaded IT1 dental implants supporting an overdenture in the mandible. Int $\mathrm{J}$ Oral Maxillofac Implants. 2001;16:246-251.

323. Payne AG, Tawse-Smith A, Duncan WD, Kumara R. Conventional and early loading of unsplinted iti implants supporting mandibular overdentures: two-year results of a prospective randomized clinical trial. Clin Oral Implants Res. 2002;13:603-609.

324. Turkyilmaz I, Avci M, Kuran S, Ozbek EN. A 4-year prospective clinical and radiological study of maxillary dental implantssupporting single-tooth crowns using early and delayed loading protocols. Clin Implant Dent Relat Res. 2007;9:222-227.

325. Örtorp A, Jemt T. Laser-welded titanium frameworks supported by implants in the partially edentulous mandible: a 10-year comparative follow-up study. Clin Implant Dent Relat Res. 2008;10:128-139.

326. Bergendal B, Palmqvist S. Laser-welded titanium frameworks for implant-supported fixed prostheses: a 5-year report. Int J Oral Maxillofac Implants. 1999;14:69-71

327. Örtorp A, Jemt T. Early laser-welded titanium frameworks supported by implants in the edentulous mandible: a $15-$ year comparative follow-up study. Clin Implant Dent Relat Res. 2009;11:311-322.

328. Jofré J, Conrady Y, Carrasco C. Survival of splinted miniimplants after contamination with stainless steel. Int $\mathrm{J}$ Oral Maxillofac Implants. 2010;25:351-356.

329. Palagi LM, Sabrosa CE, Gava EC, Baccetti T, Miguel JA. Long-term follow-up of dental single implants under immediate orthodontic load. Angle Orthod. 2010;80:807-811.

330. Gabbert O, Koob A, Schmitter M, Rammelsberg P. Implants placed in combination with an internal sinus lift without graft material: an analysis of short-term failure. $\mathrm{J}$ Clin Periodontol. 2009;36:177-183.

331. Albrektsson T, Brånemark PI, Hansson HA, Kasemo B, Larsson K, Lundström I et al. The interface zone of inorganic implants in vivo: titanium implants in bone. Ann Biomed Eng. 1983;11:1-27.

332. Sicilia A, Cuesta S, Coma G, Arregui I, Guisasola C, Ruiz $\mathrm{E}$ et al. Titanium allergy in dental implant patients: a clinical study on 1500 consecutive patients. Clin Oral Implants Res. 2008; 19:823-835.

333. Francisco H, van Assche N, Kapos T, Navarro J, Palarie V, Thoma D et al. EAOs second summer camp. Clin Oral Implants Res. 2013;24:S1-S5.

334. Valentine-Thon E, Schiwara HW. Validity of MELISA for metal sensitivity testing. Neuro Endocrinol Lett. 2003; $24: 57-64$
335. Schulte W, Heimke G. Das Tübingen Sofort-implantat. Quintessenz. 1976;27:17-23.

336. Brånemark PI, Hansson $\mathrm{BO}$, Adell R, Breine $\mathrm{U}$, Lindström $\mathrm{J}$, Hallén $\mathrm{O}$ et al. Osseointegrated implants in the treatment of the edentulous jaw. Experience from a 10-year period. Scand J Plast Reconstr Surg Suppl. 1977;16:1-132.

337. Olsson M, Stenport V, Jemt T. Incidence of first implant failure in relation to implant surface - a preliminary report on early failure. Abstract, Swed Dent J. 2012;36:221.

338. Malmquist J, Sennerby L. Clinical report on the success of 47 consecutively placed Core-Vent implants followed from 3 months to 4 years. Int $\mathrm{J}$ Oral Maxillofac Implants. 1990;5:53-60.

339. Albrektsson T. On long-term maintenance of the osseointegrated response. Aust Prosthodont J. 1993;7:15-24.

340. Albrektsson T. Hydroxypatite-coated implants: a case against their use. J Oral Maxillofac Surg. 1998;56:13121326.

341. Albrektsson T, Brånemark PI, Hansson HA, Lindström J. Osseointegrated titanium implants. Requirements for ensuring a long-lasting, direct bone-to-implant anchorage in man. Acta Orthop Scand. 1981;52:155-170.

342. Albrektsson T. Is surgical skill more important for clinical success than changes in implant hardware? Clin Implant Dent Relat Res. 2001;3:174-175.

343. Bryant SR. Oral implant outcomes predicted by age- and site specific aspects of bone condition. PhD Thesis, Department of Prosthocontics, University of Toronto, Toronto, Canada; 2001.

344. Renouard F, Charrier JG. The search for the weakest link: an introduction to human factors. Paris: Ewenn éd.; 2011:221.

345. Mordenfeld A, Hallman M, Johansson C, Albrektsson T Histological and histomorphometrical analyses of biopsies harvested 11 years after maxillary sinus floor augmentation with deproteinized and autogenous bone. Clin Oral Implant Res. 2010;21:961-970.

346. Elek SD, Conen PE. The virulence of Staphylococcus pyogenes for man; a study of the problems of wound infection. Br J Exp Pathol. 1957;38:573-586.

347. James RC, MacLeod CJ. Induction of staphylococcal infections in mice with small inocula introduced on sutures. Br J Exp Pathol. 1961;42:266-277.

348. Taubler JH, Kapral FA. Staphylococcal population changes in experimentally infected mice: infection with sutureadsorbed and unadsorbed organisms grown in vitro and in vivo. J Infect Dis. 1966;116:257-262.

349. Koch R. Über bakteriologische Forschung. Verhandlungen des X. Internationalen Medicinischen Congresses. Berlin: A. Hirschwald; 1890. Bd. I:35-47.

Correspondence: Bruno R. Chrcanovic, Department of Prosthodontics, Faculty of Odontology, Malmö University, Carl Gustafs väg 34, SE-20506 Malmö, Sweden. E-mails: bruno.chrcanovic@mah.se; brunochrcanovic@hotmail.com 
II 



\title{
Review
}

\section{Smoking and dental implants: A systematic review and meta-analysis}

\author{
Bruno Ramos Chrcanovic ${ }^{a,},{ }^{*}$ Tomas Albrektsson ${ }^{a, b}$, Ann Wennerberg ${ }^{a}$

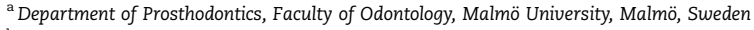 \\ ${ }^{\mathrm{b}}$ Department of Biomaterials, Göteborg University, Göteborg, Sweden
}

\section{A R T I C L E I N F O}

\section{Article history:}

Received 31 October 2014

Received in revised form

3 March 2015

Accepted 5 March 2015

\section{Keywords:}

Dental implants

Smoking

Implant failure rate

Postoperative infection

Marginal bone loss

Meta-analysis

\begin{abstract}
A B S T R A C T
Objective: Recent studies implicate smoking as a significant factor in the failure of dental implants. This review aims to test the null hypothesis of no difference in the implant failure rates, risk of postoperative infection, and marginal bone loss for smokers versus nonsmokers, against the alternative hypothesis of a difference.

Data: Main search terms used in combination: dental implant, oral implant, smoking, tobacco, nicotine, smoker, and non-smoker.

Sources: An electronic search was undertaken in September/2014 in PubMed/Medline, Web of Science, Cochrane Oral Health Group Trials Register plus hand-searching.

Study selection: Eligibility criteria included clinical human studies, either randomized or not. The search strategy resulted in 1432 publications, of which 107 were eligible, with 19,836 implants placed in smokers, with 1259 failures (6.35\%), and 60,464 implants placed in nonsmokers, with 1923 failures (3.18\%).

Conclusions: The insertion of implants in smokers significantly affected the failure rates, the risk of postoperative infections as well as the marginal bone loss. The results should be interpreted with caution due to the presence of uncontrolled confounding factors in the included studies.

Clinical significance: Smoking is a factor that has the potential to negatively affect healing and the outcome of implant treatment. It is important to perform an updated periodic review to synthesize the clinical research evidence relevant to the matter.
\end{abstract}

C 2015 Elsevier Ltd. All rights reserved.

\section{Introduction}

Nicotine is the most important constituent among more than 4000 potentially toxic substances in tobacco products. It is the main chemical component responsible for tobacco addiction, appears to mediate the haemodynamic effects of smoking, and has been implicated in the pathogenesis of numerous diseases. ${ }^{1}$ Studies have also demonstrated the detrimental effects of smoking on oral health. A clinical study ${ }^{2}$ observed that smokers had a higher prevalence of moderate and severe periodontitis and higher prevalence and extent of attachment loss and gingival recession than non-smokers, suggesting poorer periodontal health in smokers. In addition, smokers had a higher number of missing teeth than non-smokers. Concerning the bone-implant interface, the deleterious effects

\footnotetext{
* Corresponding author. Tel.: +46 725541 545; fax: +46 406658503

E-mail addresses: bruno.chrcanovic@mah.se, brunochrcanovic@hotmail.com (B.R. Chrcanovic) http://dx.doi.org/10.1016/j.jdent.2015.03.003 0300-5712/C 2015 Elsevier Ltd. All rights reserved.
} 
of tobacco smoke reflects a series of direct and indirect systemic and local effects on bone metabolism. ${ }^{3}$ It has been strongly suggested that local exposure of the peri-implant tissues to tobacco products is the main factor leading to an overall increase in implant failure rate in smokers. ${ }^{4} \mathrm{~A}$ recent meta-analysis on the subject ${ }^{5}$ observed that smoking was associated with a higher risk of dental implant failure. However, the review was only able to include 33 studies, even though observational retrospective studies were eligible, according to the inclusion criteria. Moreover, the study did not evaluate the effects of smoking on marginal bone loss (MBL) around implants.

The ability to anticipate outcomes is an essential part of risk management in an implant practice. Recognizing conditions that place the patient at a higher risk of failure will allow the surgeon to make informed decisions and refine the treatment plan to optimize the outcome. ${ }^{6}$ The use of implant therapy in special populations requires consideration of potential benefits to be gained from the therapy. To better appreciate this potential, we conducted a systematic review and meta-analysis of both prospective and retrospective studies to compare the survival rate of dental implants, postoperative infection, and MBL between smokers and nonsmokers. The present meta-analysis included non-randomized studies and performed several sensitivity analyses, in order to verify whether the results were sensitive to restrictions on the data included.

\section{Materials and Methods}

This study followed the PRISMA statement guidelines. ${ }^{7}$ A review protocol does not exist.

\subsection{Objective}

The purpose of the present review was to test the nul hypothesis of no difference in the implant failure rates, postoperative infection, and MBL for smokers or non-smokers, against the alternative hypothesis of a difference. The focused question was elaborated by using the PICO format (participants, interventions, comparisons, and outcomes): in patients undergoing implant placement, are patients who smoke versus those who do not at higher risk for implant failure, postoperative infection, and greater MBL?

\subsection{Search Strategies}

See appendix-supplementary data

\subsection{Inclusion and Exclusion Criteria}

Eligibility criteria included clinical human studies, either randomized or not, providing outcome data for dental implant failure in smokers and non-smokers, in any group of patients (of any age, race, or sex), with no follow-up restrictions There were no time or language restrictions for the publications. For this review, patients smoking a minimum of one cigarette per day were classified as smokers, and implant failure represents the complete loss of the implant. Exclusion criteria were case reports, technical reports, biomechanical studies, finite element analysis (FEA) studies, animal studies, in vitro studies, and review papers.

\subsection{Study Selection}

The titles and abstracts of all reports identified through the electronic searches were read independently by three authors. For studies appearing to meet the inclusion criteria, or for which there were insufficient data in the title and abstract to make a clear decision, the full report was obtained. Disagreements were resolved by discussion between the authors.

\subsection{Quality Assessment}

Quality assessment of the studies was executed according to the Newcastle-Ottawa scale (NOS), which is a quality assessment tool to use when observational studies are also included in systematic reviews. ${ }^{8}$ The NOS calculates the study quality on the basis of three major components: selection, comparability, and outcome for cohort studies. It assigns a maximum of four stars for selection, a maximum of two stars for comparability, and a maximum of three stars for outcome. According to that quality scale, a maximum of nine stars/ points can be given to a study, and this score represents the highest quality, where six or more points were considered of high quality.

\subsection{Data Extraction and Meta-analysis}

From the studies included in the final analysis, the following data was extracted (when available): year of publication, study design, unicenter or multicenter study, country, setting (academic, institutional, industry, etc.), number of patients, type of smokers included in the study, patients' age, follow-up, days of antibiotic prophylaxis, mouth rinse, implant healing period, failed and placed implants, postoperative infection, marginal bone loss, implant surface modification, jaws receiving implants (maxilla and/or mandible), type of prosthetic rehabilitation, and opposing dentition. Only one reviewer performed the data extraction. Authors were contacted for possible missing data.

Implant failure and postoperative infection were the dichotomous outcomes measures evaluated. Weighted mean differences were used to construct forest plots of marginal bone loss, a continuous outcome. The statistical unit for all outcomes ('implant failure', 'marginal bone loss', and 'postoperative infection') was the implant. Whenever outcomes of interest were not clearly stated, the data were not used for analysis. The $I^{2}$ statistic was used to express the percentage of the total variation across studies due to heterogeneity, with $25 \%$ corresponding to low heterogeneity, $50 \%$ to moderate, and $75 \%$ to high. The inverse variance method was used for random-effects or fixed-effects model. Where statistically significant $(P<0.10)$ heterogeneity is detected, a randomeffects model was used to assess the significance of treatment effects. Where no statistically significant heterogeneity was found, analysis was performed using a fixed-effects model. ${ }^{9}$ The estimates of relative effect for dichotomous outcomes were expressed in risk ratio (RR) and in mean difference (MD) 
in millimetres for continuous outcomes, both with a $95 \%$ confidence interval (CI). Only if there were studies with similar comparisons reporting the same outcome measures was meta-analysis to be attempted. In the case where no events (or all events) are observed in both groups, the study provides no information about relative probability of the event and is automatically omitted from the meta-analysis. In this (these) case(s), the term 'not estimable' is shown under the column of RR of the forest plot table.

Sensitivity analysis tests were performed when possible, in order to verify whether the results were sensitive to restrictions on the data included. A funnel plot (plot of effect size versus standard error) was drawn. Asymmetry of the funnel plot may indicate publication bias and other biases related to sample size, although the asymmetry may also represent a true relationship between trial size and effect size. ${ }^{10}$

The data were analyzed using the statistical software Review Manager (version 5.3.3, The Nordic Cochrane Centre, The Cochrane Collaboration, Copenhagen, Denmark, 2014).

\section{Results}

\subsection{Literature Search}

The study selection process is summarized in Fig. 1. The search strategy resulted in 1432 papers. A number of 464 articles were cited in more than one research of terms (duplicates). The three reviewers independently screened the abstracts for those articles related to the focus question. Of the resulted 968 studies, 754 were excluded for not being related to the topic. Additional hand-searching of the reference lists of selected studies yielded 32 additional papers. The full-text reports of the remaining 246 articles led to the exclusion of 139 because they did not meet the inclusion criteria ( 80 papers did not inform the number of implants and/or failures in each group, 32 review papers, 20 papers not evaluating failures, two same studies published in a different journal, two histologic studies, one earlier follow-up, one gene expression profile study, and one case report paper). Thus, a total of 107 publications were included in the review.

\subsection{Description of the Studies}

Detailed data of the 107 included studies are listed in Table 1 and 2 (appendix-supplementary data). Four randomized clinical trials (RCT), ${ }^{11-14} 16$ controlled clinical trials (CCT), ${ }^{15-30} 16$ prospective studies, ${ }^{31-46}$ and 71 retrospective analyses ${ }^{47-117}$ were included in the meta-analysis. Seven CCTs ${ }^{15-17,22,27,29,30}$ were controlled for the patients' smoking habit. Four RCTs and nine of the CCTs included here were not controlled for the smoking habit

In total, 39 publications ${ }^{16,19,24,25,27-30,32,33,35-37,41,44,45,51,54}$ $59,61,62,64,65,68,70,73-77,82,85,94,99,103,104,111,112,117$ clearly defined what kind of smoking patients were included in their studies based on how many cigarettes the patients used to smoke per day.

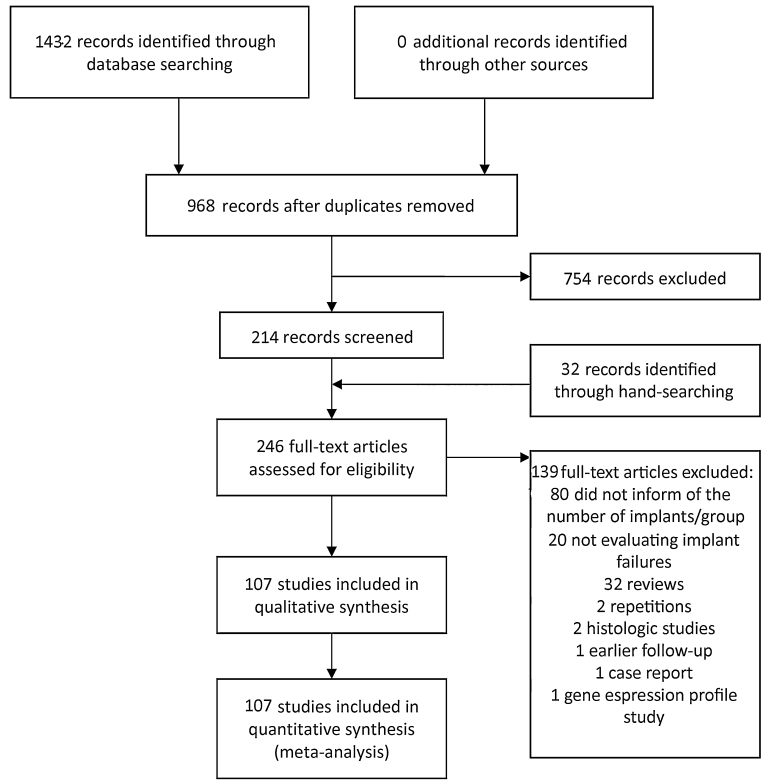

Fig. 1 - Study screening process. 
Three studies ${ }^{13,21,66}$ included light or heavy smokers 'without distinction', or the patients were classified as non-smokers, former smokers, and current smokers in two studies. ${ }^{55,67}$ Only 15 studies ${ }^{11,13,18,22,29,45,46,55,63,71,81,83,89,107,111}$ provided information about postoperative infection, with 65 occurrences in a total of 2580 patients receiving 7745 implants. In total, 18 studies $^{12,14,29,30,39,44,65,74,75,78,91,99,103-106,113,115}$ provided information about the marginal bone loss separated by groups and with mean values and standard deviation.

From the 107 included studies, three studies ${ }^{12,14,104}$ did not provide information about the implant failure rates separately between smokers and non-smokers, reporting information only about the marginal bone loss. From the 104 studies comparing the implant failure rates, a total of 19,836 dental implants were placed in smokers, with 1259 failures (6.35\%), and 60,464 implants were placed in non-smokers, with 1923 failures $(3.18 \%)$. There were no implant failures in five studies. $^{24,28,94,95,105}$ In total, 44 studies ${ }^{11,15,17,20,22,26,30,34,37-39}$, $43,45,47,48,51,57,61,65,67,69,73,74,77,80,81,83,84,87,88,90,93,96,99-103,106,107,110$, 112,116,117 informed whether there was a statistically significant difference or not between the implant failure rates between smokers and non-smokers, and $17^{20,22,26,30,34,39,61,69,73,81,83,88,90,99-101,110}$ of these studies did not find a statistically significant difference favouring smokers or non-smokers, on $\mathrm{e}^{65}$ found a statistically higher implants failure rate in non-smokers, while the other 26 studies found a statistically higher implants failure rate in smokers.

\subsection{Quality Assessment}

In total, 85 studies were of high quality and 22 were of moderate quality. The scores are summarized in Table 3 (appendix-supplementary data). The moderate quality of some studies is due to four main reasons: (a) the fact that the individuals were not representative from the general population seeking dental implant treatment, (b) the ascertainment of exposure is an issue in retrospective analyses given that this data is collected using questionnaires, (c) short follow-ups, and (d) a considerable number subjects lost to follow-up.

\subsection{Meta-analysis}

In this study, a random-effects model was used to evaluate the implant failure in the comparison between the procedures, since statistically heterogeneity was found $(P<0.00001$; $\left.I^{2}=51 \%\right)$. The insertion of dental implants in smokers statistically affected the implant failure rates $(P<0.00001$ Fig. 2). A RR of 2.23 ( $95 \%$ CI 1.96-2.53) implies that failures of implants inserted in smokers are 2.23 times likely to happen than failures of implants inserted in non-smokers; i.e. the insertion of implants in smokers increases the risk of implant failure by $123 \%$. The insertion of dental implants in smokers statistically affected the incidence of postoperative infections (RR 2.01, 95\% CI 1.09-3.72; $P=0.03$; heterogeneity: $P=0.63$; $I^{2}=0 \%$, fixed-effects model; and Fig. 3 ), as well as the marginal bone loss (MD 0.32, 95\% CI 0.21-0.43; $\mathrm{P}<0.00001$; heterogeneity: $P<0.00001 ; I^{2}=95 \%$, random-effects model; and Fig. 4).

Since the effect size could differ depending on the insertion of implants in bone areas of different quality, a sensitivity

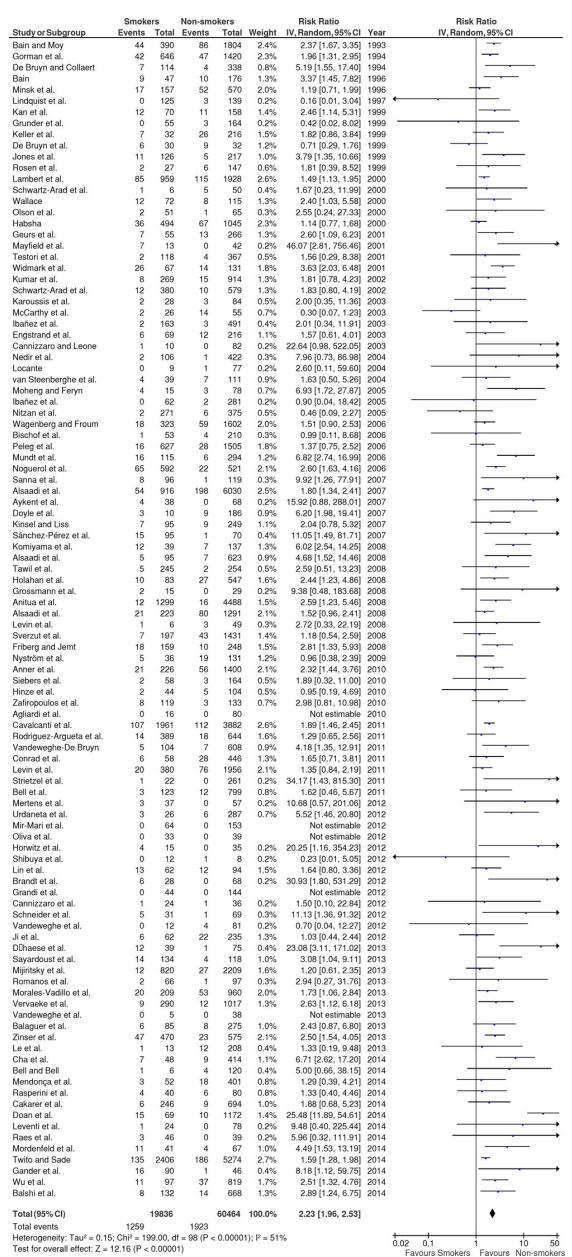

Fig. 2 - Forest plot for the event 'implant failure'.

analysis was performed. When only the studies inserting implants in maxillae were pooled, a RR of 2.22 resulted $(95 \% \mathrm{CI}$ 1.63-3.01; heterogeneity: $P=0.005 ; I^{2}=49 \%$, random-effects model; and Fig. 5-appendix-supplementary data), also statistically affecting the implant failure rates $(P<0.00001)$. When only the studies inserting implants in mandibles were pooled, a RR of 2.61 resulted ( $95 \%$ CI 0.92-7.39; heterogeneity: $P=0.09 ; I^{2}=48 \%$, random-effects model; and Fig. 6-appendixsupplementary data), not statistically affecting the implant failure rates $(P=0.07)$. 


\begin{tabular}{|c|c|c|c|c|c|c|c|c|c|c|}
\hline \multirow{3}{*}{ Study or Subgroup } & \multicolumn{2}{|c|}{ Smokers } & \multicolumn{2}{|c|}{ Non-smokers } & \multicolumn{3}{|c|}{ Risk Ratio } & \multirow{2}{*}{\multicolumn{2}{|c|}{$\begin{array}{c}\text { Risk Ratio } \\
\text { IV, Fixed, } 95 \% \mathrm{Cl}\end{array}$}} & \\
\hline & Events & Total & Events & Total & Weight & IV, Fixed, $95 \% \mathrm{Cl}$ & Year & & & \\
\hline & 0 & 10 & 0 & 82 & & Not estimable & 2003 & & & \\
\hline Peleg et al. & 13 & 627 & 14 & 1505 & $67.2 \%$ & $2.23[1.05,4.71]$ & 2006 & & & \\
\hline Aykent et al. & 0 & 38 & 1 & 68 & $3.7 \%$ & $0.59[0.02,14.13]$ & 2007 & & & \\
\hline Sverzut et al. & 3 & 197 & 15 & 1431 & $24.9 \%$ & $1.45[0.42,4.97]$ & 2008 & & & \\
\hline Rodriguez-Argueta et al. & 2 & 389 & 0 & 644 & $4.1 \%$ & $8.27[0.40,171.79]$ & 2011 & & & \\
\hline Cannizzaro et al. & 0 & 24 & 0 & 36 & & Not estimable & 2012 & & & \\
\hline Romanos et al. & 0 & 66 & 0 & 97 & & Not estimable & 2013 & & & \\
\hline Cha et al. & 0 & 48 & 0 & 414 & & Not estimable & 2014 & & & \\
\hline Total $(95 \% \mathrm{Cl})$ & & 1399 & & 4277 & $100.0 \%$ & $2.01[1.09,3.72]$ & & & & \\
\hline Total events & 18 & & 30 & & & & & & & \\
\hline $\begin{array}{l}\text { Heterogeneity: } \mathrm{Chi}^{2}=1.7 \\
\text { Test for overall effect: } \mathrm{Z}\end{array}$ & $\begin{array}{l}d f=3(P= \\
23(P=0\end{array}$ & $\begin{array}{l}=0.63) \\
0.03)\end{array}$ & $; 1^{2}=0 \%$ & & & & & $\begin{array}{ll}\qquad & 1 \\
0.01 & 0.1\end{array}$ & 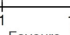 & $\frac{1}{10}$ \\
\hline
\end{tabular}

Fig. 3 - Forest plot for the event 'postoperative infection'.

\begin{tabular}{|c|c|c|c|c|c|c|c|c|c|c|c|c|}
\hline \multirow[b]{2}{*}{ Study or Subgroup } & \multicolumn{3}{|c|}{ Smokers } & \multicolumn{3}{|c|}{ Non-smokers } & \multicolumn{3}{|c|}{ Mean Difference } & \multirow{2}{*}{\multicolumn{3}{|c|}{$\begin{array}{l}\text { Mean Difference } \\
\text { IV, Random, } 95 \% \mathrm{Cl}\end{array}$}} \\
\hline & Mean & SD & Total & Mean & SD & Total & Weight & IV, Random, $95 \% \mathrm{Cl}$ & Year & & & \\
\hline Wennström et al. (5y) & 0.76 & 0.84 & 16 & 0.22 & 0.69 & 31 & $2.5 \%$ & $0.54[0.06,1.02]$ & 2004 & & & \\
\hline Wennström et al. (1y) & 0.41 & 0.78 & 17 & 0.3 & 0.51 & 34 & $2.9 \%$ & $0.11[-0.30,0.52]$ & 2004 & & & \\
\hline Nitzan et al. & 0.153 & 0.092 & 271 & 0.047 & 0.048 & 375 & $4.9 \%$ & $0.11[0.09,0.12]$ & 2005 & & - & \\
\hline Sánchez-Pérez et al. & 2.71 & 1.55 & 95 & 2.78 & 1.5 & 70 & $2.6 \%$ & $-0.07[-0.54,0.40]$ & 2007 & & & \\
\hline Sanna et al. & 2.6 & 1.6 & 25 & 1.3 & 1 & 14 & $1.3 \%$ & $1.30[0.48,2.12]$ & 2007 & & & \\
\hline Friberg and Jemt (1y,WCM) & 0.66 & 0.33 & 79 & 0.31 & 0.5 & 82 & $4.6 \%$ & $0.35[0.22,0.48]$ & 2008 & & & \\
\hline Friberg and Jemt (5y,NCM) & 0.74 & 0.48 & 56 & 0.58 & 0.6 & 103 & $4.4 \%$ & $0.16[-0.01,0.33]$ & 2008 & & & \\
\hline Friberg and Jemt (1y,NCM) & 0.38 & 0.37 & 80 & 0.25 & 0.45 & 166 & $4.7 \%$ & $0.13[0.02,0.24]$ & 2008 & & - & \\
\hline Friberg and Jemt (5y,WCM) & 0.78 & 0.49 & 52 & 0.68 & 0.78 & 90 & $4.1 \%$ & $0.10[-0.11,0.31]$ & 2008 & & & \\
\hline Nyström et al. (1y) & 2.53 & 0.16 & 36 & 2.55 & 0.11 & 131 & $4.8 \%$ & $-0.02[-0.08,0.04]$ & 2009 & & & \\
\hline Nyström et al. (10y) & 3.56 & 0.3 & 31 & 3.07 & 0.1 & 112 & $4.7 \%$ & $0.49[0.38,0.60]$ & 2009 & & & \\
\hline Vandeweghe-De Bruyn & 1.56 & 0.53 & 60 & 1.32 & 0.38 & 303 & $4.5 \%$ & $0.24[0.10,0.38]$ & 2011 & & & \\
\hline Stoker et al. (2ISB) & 1.17 & 1.12 & 22 & 0.83 & 0.9 & 44 & $2.2 \%$ & $0.34[-0.20,0.88]$ & 2012 & & & \\
\hline Stoker et al. (4ITB) & 2.46 & 2.75 & 52 & 1.24 & 0.75 & 84 & $1.4 \%$ & $1.22[0.46,1.98]$ & 2012 & & & \\
\hline Vandeweghe et al. & 0.38 & 1.11 & 12 & 0.48 & 0.82 & 81 & $1.8 \%$ & $-0.10[-0.75,0.55]$ & 2012 & & & \\
\hline Stoker et al. (2|BA) & 1.53 & 1.08 & 22 & 0.7 & 0.8 & 32 & $2.3 \%$ & $0.83[0.30,1.36]$ & 2012 & & & \\
\hline Sayardoust et al.(turned) & 1.54 & 0.21 & 78 & 0.84 & 0.14 & 66 & $4.8 \%$ & $0.70[0.64,0.76]$ & 2013 & & & - \\
\hline D' haese et al. & 0.62 & 1.05 & 39 & 0.36 & 0.89 & 75 & $3.0 \%$ & $0.26[-0.13,0.65]$ & 2013 & & & \\
\hline Wagenberg et al. & 0.65 & 1.11 & 184 & 0.51 & 0.76 & 1003 & $4.4 \%$ & $0.14[-0.03,0.31]$ & 2013 & & & \\
\hline Romanos et al.(mesial) & 0.45 & 1.4 & 66 & 0.565 & 0.996 & 97 & $3.0 \%$ & $-0.11[-0.51,0.28]$ & 2013 & & & \\
\hline Romanos et al.(distal) & 0.41 & 1.31 & 66 & 0.359 & 0.965 & 97 & $3.1 \%$ & $0.05[-0.32,0.42]$ & 2013 & & & \\
\hline Vervaeke et al. & 0.57 & 0.93 & 279 & 0.3 & 0.58 & 996 & $4.6 \%$ & $0.27[0.16,0.38]$ & 2013 & & & \\
\hline Vandeweghe et al. & 1.02 & 0.39 & 5 & 0.99 & 0.29 & 38 & $3.2 \%$ & $0.03[-0.32,0.38]$ & 2013 & & & \\
\hline Sayardoust et al.(oxid.) & 1.16 & 0.24 & 56 & 1.26 & 0.15 & 52 & $4.8 \%$ & $-0.10[-0.17,-0.03]$ & 2013 & & & \\
\hline Rasperini et al.(S,PHP) & 2.51 & 0.31 & 10 & 1.95 & 0.42 & 20 & $3.8 \%$ & $0.56[0.29,0.83]$ & 2014 & & & \\
\hline Leventi et al. & 1.35 & 1.06 & 24 & 1.25 & 1.06 & 78 & $2.5 \%$ & $0.10[-0.38,0.58]$ & 2014 & & & \\
\hline Raes et al. & 0.22 & 0.42 & 43 & -0.33 & 1.19 & 39 & $3.0 \%$ & $0.55[0.16,0.94]$ & 2014 & & & \\
\hline Rasperini et al. (N,PCP) & 3.47 & 1.09 & 10 & 2.32 & 0.41 & 20 & $1.6 \%$ & $1.15[0.45,1.85]$ & 2014 & & & \\
\hline Rasperini et al.(S,PCP) & 3.77 & 1.43 & 10 & 2.32 & 0.41 & 20 & $1.1 \%$ & $1.45[0.55,2.35]$ & 2014 & & & \\
\hline Rasperini et al. (N,PHP) & 2.65 & 0.41 & 10 & 1.43 & 0.38 & 20 & $3.5 \%$ & $1.22[0.92,1.52]$ & 2014 & & & \\
\hline Total $(95 \% \mathrm{Cl})$ & & & 1806 & & & 4373 & $100.0 \%$ & $0.32[0.21,0.43]$ & & & & \\
\hline $\begin{array}{l}\text { Heterogeneity: } \mathrm{Tau}^{2}=0.0 \\
\text { Test for overall effect: } Z=\end{array}$ & k & 001) & & & & & & & & Smok & irs $\mathrm{N}$ & $\mathrm{N}$ \\
\hline
\end{tabular}

Fig. 4 - Forest plot for the event 'marginal bone loss'.

Other sensitivity analyses were also performed, pooling studies evaluating different implant surface modification processes, there was a statistically significant difference between smokers and non-smokers when the only studies making use of turned implants were pooled (RR 2.17, 95\% CI 1.53-3.06, $P<0.0001$; heterogeneity: $P=0.001 ; I^{2}=64 \%$, random-effects model; and Fig. 7-appendix-supplementary data), acid-etched surface implants (RR 2.07, 95\% CI 1.20 3.58, and $P=0.009$; heterogeneity: $P=0.50 ; I^{2}=0 \%$, fixedeffects model; and Fig. 8-appendix-supplementary data), the same happening to sandblasted and acid-etched surface implants (RR 2.92, 95\% CI 1.60-5.34, and $P=0.0005$; heterogeneity: $P=0.02 ; I^{2}=50 \%$, random-effects model; and Fig. 9appendix-supplementary data), sandblasted and fluoridemodified surface implants (RR 4.18, 95\% CI 2.06-8.50, and $P<0.0001$; heterogeneity: $P=0.22 ; I^{2}=32 \%$, fixed-effects model; and Fig. 10-appendix-supplementary data), and oxidized surface implants (RR 5.07, 95\% CI 2.76-9.30, $P<0.00001$; heterogeneity: $P=0.35 ; I^{2}=10 \%$, fixed-effects model; and Fig. 11-appendix-supplementary data).

\subsection{Publication Bias}

The funnel plot for the studies reporting the outcome 'implant failure' did not show a clear asymmetry (Fig. 12), indicating possible absence of publication bias.

\section{Discussion}

In a meta-analysis, homogeneity implies a mathematical compatibility between the results of each individual trial. 


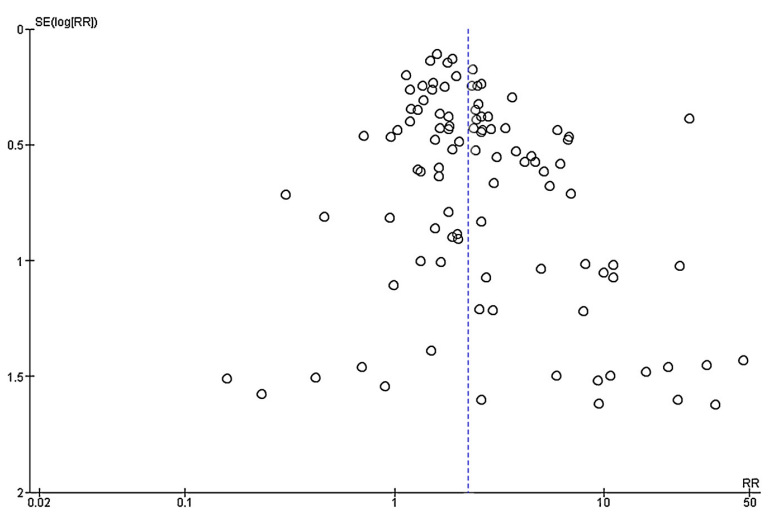

Fig. 12 - Funnel plot for the studies reporting the outcome event 'implant failure'.

Potential biases are likely to be greater for non-randomized studies compared with RCTs, so results should always be interpreted with caution when they are included in reviews and meta-analyses. ${ }^{10}$ However, narrowing the inclusion criteria increases homogeneity but also excludes the results of more trials, and thus risks the exclusion of significant data. ${ }^{118}$ This was the reason to include non-randomized studies in the present meta-analysis. The issue is important because meta-analyses are frequently conducted on a limited number of RCTs. In meta-analyses, such as these, adding more information from observational studies may aid in clinical reasoning and establish a more solid foundation for causal inferences. ${ }^{118}$

In the present meta-analysis, the statistical unit of analysis for 'implant failure' was the implant. It would be technically more correct to adjust for the effect of clustered, correlated observations; however, it is a challenging analytic method and the implant survival is so high that failing to adjust for clustered, correlated observations would have little effect on the estimate and deviation of survival. ${ }^{119}$

The results of the present study suggest that the insertion of dental implants in smokers affects implant failure rates, the risk of postoperative infection, and the MBL. The increase of implant failure rates due to smoking is hypothesized to be related mainly to the effect of smoking in osteogenesis and angiogenesis. It was shown ${ }^{120}$ that nicotine inhibited the gene expression of several enzymes that play an important role in the regulation of osteoblast proliferation, differentiation, and apoptosis, with subsequent important effects on bone formation and remodelling. ${ }^{121}$ Moreover, it was demonstrated ${ }^{122}$ that nicotine exposure has direct effects on blood vessels, producing vasoconstriction and systemic venoconstriction, which decreases blood perfusion and causes low oxygen and ischaemia. ${ }^{123}$ Besides carrying oxygen and nutrients to bone tissue, blood flow plays an active role in bone formation and remodelling by mediating the interactions among osteoblasts, osteocytes, osteoclasts, and vascular cells at a variety of levels. ${ }^{124}$
Furthermore, sensitivity analysis suggests that smoking significantly affects the survival of implants inserted only in the maxilla. The lack of statistical significance for the mandible is surprising but is most likely explained by the limited number of studies ${ }^{16,24,28,34,35}$ reporting implant survival for smokers and non-smokers exclusively in the inferior jaw. A previous review ${ }^{125}$ on the subject suggested that smoking may be a significant risk factor with an adverse effect on implant survival and success in areas of loose trabecular bone, but may not be as significant for good bone sites. It is important to stress that caution is required when sensitivity analyses are performed, because both type I and type II errors are likely given the multiple testing and the subgrouping. Moreover, these studies were never designed for showing these effects, and thus all the findings are presumably heavily biased.

Concerning the subgroup analyses for the different surfaces, sensitivity analyses suggest that smoking significantly affects the survival of implants submitted to any surface modification here reviewed (turned, acid-etched, sandblasted and acid-etched, sandblasted and fluoride-modified, and oxidized). The fact is that titanium with different surface modifications shows a wide range of chemical and physical properties, and surface topographies or morphologies, depending on how they are prepared and handled. ${ }^{126-128}$ It is known that the surface properties of dental implants, such as topography and chemistry are relevant for the osseointegration process and may influence the results. ${ }^{129}$ It seems evident from our results (Figs. 7-11) that smoking is associated with increased number of failures irrespective of the type of implant surface being investigated. Moreover, a higher risk ratio was observed for implants with roughened surfaces in comparison with turned implants in smokers. Having said this, there is some contradictory evidence published that smoking mainly is associated with older turned implant surfaces but not with more modern ones. Balshe et al. ${ }^{130}$ observed that smoking was not identified as significantly 
associated with implant failure among the moderately rough surface (anodized) implants, while it was associated with implant failure among the group with minimally rough surface implants. Even though Balshe et al.'s paper ${ }^{130}$ presented a great number of implants in their study $(n=4607)$, the results were not included in the present meta-analysis because the number of implants placed and the number of failures were not reported separately between smokers and non-smokers. The evidence presented by Balshe et al. ${ }^{130}$ did not fulfil all requirements to be included in the meta-analysis, but is nevertheless an important contribution since a great number of implants are being investigated. More recently, Sayardoust et al. ${ }^{103}$ showed that turned implants failed more frequently and lost more marginal bone in smokers, and that oxidized implants showed similar failure rates and bone loss in smokers and never-smokers. These contrasting results between the present meta-analysis and previous studies indicate that controversy still exists and that there is a need for more studies to evaluate the long-term outcome of implants with altered surface characteristics in smokers. ${ }^{125}$ The studies included here made use of implants with several different brands and surface treatments.

The results of the present study have to be interpreted with caution because of its limitations. First of all, all confounding factors may have affected the long-term outcomes and not just the fact that implants were placed in smokers or nonsmokers, and the impact of these variables on the implant survival rate, postoperative infection, and marginal bone loss ${ }^{131-138}$ is difficult to estimate if these confounding factors are not identified separately between the two different procedures in order to perform a meta-regression analysis. The studies included here have a considerable number of confounding factors, and most of the studies, if not all, did not inform how many implant were inserted and survived/lost in several different conditions. The use of grafting in some studies is a confounding risk factor, as well as the insertion of some or all implants in fresh extraction sockets, the insertion of implants in different locations, different healing periods, different prosthetic configurations, type of opposing dentition, different implant angulation ranges, splinting of the implants, and the presence of bruxers, or diabetics patients. The dose effect of smoking is another important consideration. There is evidence to suggest that smoking may have a dose-related effect on osseointegration. ${ }^{70}$ Unfortunately, not all studies included here reported the quantity of cigarettes smoked per day, and almost none reported the number of years those patients have smoked. The real fact is that individual patients sometimes present with more than one risk factor, and groups of patients are typically heterogeneous with respect to risk factors and susceptibilities so the specific effect of an individual risk factor could be isolated neither for individual studies nor for the present review. This is understandable and expected because study populations are typically representative of normal populations with various risk factors. ${ }^{125}$ To precisely assess the effect of a risk factor on implant outcomes, it would be ideal to eliminate all other risk factors from the study population. Not only does the coexistence of multiple risk factors within a study population create an inability to assess the specific effect of one individual risk factor, but there is a possibility that certain risk factors together may be more detrimental than the individual risk factors alone. ${ }^{125}$ The lack of control of the confounding factors limited the potential to draw robust conclusions. Second, most of the included studies had a retrospective design, and the nature of a retrospective study inherently results in flaws. These problems were manifested by the gaps in information and incomplete records. Furthermore, all data rely on the accuracy of the original examination and documentation. Items may have been excluded in the initial examination or not recorded in the medical chart. ${ }^{139-141}$ In a retrospective study, it is difficult to assess the adverse effects of smoking on the prognosis of implants purely on the basis of implant failure because of the multifactorial genesis of implant failure. ${ }^{6}$ Third, much of the research in the field is limited by small cohort size and short follow-up periods. It is important to stress that some publications included in this review have a short-term followup period, of up to 3 years. In a 12-month follow-up study, Kan et al. $^{51}$ reported a $93.04 \%$ success for non-smokers and an $82.82 \%$ success for smokers. In a second study by the authors, ${ }^{142}$ but now with a 60 -month follow-up, the success rate for the non-smokers was $82.7 \%$ and for smokers was $65.3 \%$. Thus, if one considers the difference in success rates for smokers and non-smokers with implants placed in loose trabecular bone sites that are followed over a longer period of time, the adverse effect of smoking may be more evident. A longer follow-up period can lead to an increase in the failure rate, especially if it extended beyond functional loading, because other prosthetic factors can influence implant failure from that point onward. This might have led to an underestimation of actual failures in some studies. However, it is hard to define what it would be considered as a short follow-up period to evaluate implant failures in smokers. Fourth, the criteria for the classification of patients as 'smokers' and 'non-smokers' were not always reported by the included studies, which probably resulted in a poor homogeneity of the study group. Fifth, most included studies are characterized by a low level of specificity, where the assessment of smoking as a complicating factor for dental implants was seldom the main focus of the investigation.

\section{Conclusion}

The results of the present review should be interpreted with caution due to the presence of uncontrolled confounding factors in the included studies. Within the limitations of the existing investigations, the results of the present study suggest that the insertion of dental implants in smokers affects the implant failure rates, the incidence of postoperative infections, as well as the marginal bone loss.

\section{Acknowledgements}

This work was supported by CNPq, Conselho Nacional de Desenvolvimento Científico e Tecnológico-Brazil. The authors would like to thank Dr. Rodolfo Gianserra, for having sent us his article, Mrs. Angela Ruban, who provided us some missing information about Dr. Devorah Schwartz-Arad's article, Dr. Derk Siebers, Dr. James S. Hodges, Dr. Ronen Ofec, Dr. David 
Schneider, and Dr. Swati Ahuja, who provided us some missing information about their studies, and Dr. Torsten Jemt, Dr. Miguel de Araújo Nobre, and Dr. Francesco Guido Mangano, who replied our e-mail, even though it was not possible for them to provide us the missing information.

\section{Appendix A. Supplementary data}

Supplementary material related to this article can be found, in the online version, at http://dx.doi.org/10.1016/j. jdent.2015.03.003.

1. Ciftci O, Gunday M, Caliskan M, Gullu H, Guven A Muderrisoglu H. Light cigarette smoking and vascular function. Acta Cardiologica 2013:68:255-61.

2. Albandar JM, Streckfus CF, Adesanya MR, Winn DM. Cigar, pipe, and cigarette smoking as risk factors for periodontal disease and tooth loss. Journal of Periodontology 2000;71:1874-81.

3. Pereira ML, Carvalho JC, Peres F, Fernandes MH. Simultaneous effects of nicotine, acrolein, and acetaldehyde on osteogenic-induced bone marrow cells cultured on plasma-sprayed titanium implants. International Journal of Oral and Maxillofacial Implants 2010;25:112-22.

4. Johnson GK, Guthmiller JM. The impact of cigarette smoking on periodontal disease and treatment. Periodontology 2000 2007;44:178-94

5. Chen H, Liu N, Xu X, Qu X, Lu E. Smoking, radiotherapy, diabetes and osteoporosis as risk factors for dental implant failure: a meta-analysis. PLOS ONE 2013;8:e71955.

6. Chrcanovic BR, Albrektsson T, Wennerberg A. Reasons for failures of oral implants. Journal of Oral Rehabilitation 2014;41:443-76.

7. Moher D, Liberati A, Tetzlaff J, Altman DG, Grp P. Preferred reporting items for systematic reviews and meta-analyses: the PRISMA statement. Annals of Internal Medicine 2009;151:264-9. W64.

8. Wells GA, Shea B, O'Connell D, Peterson J, Welch V, Losos $\mathrm{M}$, et al. The Newcastle-Ottawa Scale (NOS) for assessing the quality of nonrandomised studies in meta-analyses. 2000. http://www.ohri.ca/programs/clinical_epidemiology/ oxford.asp [cited 2014 August 27, 2014].

9. Egger M, Smith GD. Principles of and procedures for systematic reviews. In: Egger M, Smith GD, Altman DG, editors. Systematic reviews in health care: meta-analysis in context. London: BMJ books; 2003. p. 23-42.

10. Higgins JPT, Green S. Cochrane Collaboration Cochrane handbook for systematic reviews of interventions. Chichester, England; Hoboken, NJ: Wiley-Blackwell; 2008.

11. Jones JD, Lupori J, Van Sickels JE, Gardner W. A 5-year comparison of hydroxyapatite-coated titanium plasma sprayed and titanium plasma-sprayed cylinder dental implants. Oral Surgery Oral Medicine Oral Pathology Oral Radiology and Endodontics 1999;87:649-52.

12. Wennström JL, Ekestubbe A, Gröndahl K, Karlsson S, Lindhe J. Oral rehabilitation with implant-supported fixed partial dentures in periodontitis-susceptible subjects. A 5 -year prospective study. Journal of Clinical Periodontology 2004;31:713-24.
13. Cannizzaro G, Felice P, Leone M, Ferri V, Viola P, Esposito $\mathrm{M}$. Immediate versus early loading of $6.5 \mathrm{~mm}$-long flaplessplaced single implants: a 4-year after loading report of a split-mouth randomised controlled trial. European Journal of Oral Implantology 2012;5:111-21.

14. Stoker G, van Waas R, Wismeijer D. Long-term outcomes of three types of implant-supported mandibular overdentures in smokers. Clinical Oral Implants Research 2012;23:925-9.

15. Gorman LM, Lambert PM, Morris HF, Ochi S, Winkler S. The effect of smoking on implant survival at second-stage surgery: DICRG Interim Report No. 5. Dental Implant Clinical Research Group. Implant Dentistry 1994;3:165-8.

16. Lindquist LW, Carlsson GE, Jemt T. Association between marginal bone loss around osseointegrated mandibular implants and smoking habits: a 10-year follow-up study. Journal of Dental Research 1997:76:1667-74.

17. Lambert PM, Morris HF, Ochi S. The influence of smoking on 3-year clinical success of osseointegrated dental implants. Annals of Periodontology 2000;5:79-89.

18. Cannizzaro G, Leone M. Restoration of partially edentulous patients using dental implants with a microtextured surface: a prospective comparison of delayed and immediate full occlusal loading. International Journal of Oral and Maxillofacial Implants 2003;18:512-22.

19. Ibañez JC, Tahhan MJ, Zamar JA. Performance of double acid-etched surface external hex titanium implants in relation to one- and two-stage surgical procedures. Journal of Periodontology 2003;74:1575-81.

20. Karoussis IK, Salvi GE, Heitz-Mayfield LJ, Bragger U, Hammerle CH, Lang NP. Long-term implant prognosis in patients with and without a history of chronic periodontitis: a 10-year prospective cohort study of the ITI Dental Implant System. Clinical Oral Implants Research 2003;14:329-39.

21. Nedir R, Bischof M, Briaux JM, Beyer S, Szmukler-Moncler S, Bernard JP. A 7-year life table analysis from a prospective study on ITI implants with special emphasis on the use of short implants. Results from a private practice. Clinical Oral Implants Research 2004:15:150-7.

22. Peleg M, Garg AK, Mazor Z. Healing in smokers versus nonsmokers: survival rates for sinus floor augmentation with simultaneous implant placement. International Journal of Oral and Maxillofacial Implants 2006;21:551-9.

23. Tawil G, Younan R, Azar P, Sleilati G. Conventional and advanced implant treatment in the type II diabetic patient: surgical protocol and long-term clinical results. International Journal of Oral and Maxillofacial Implants 2008;23:744-52.

24. Agliardi E, Clerico M, Ciancio P, Massironi D. Immediate loading of full-arch fixed prostheses supported by axial and tilted implants for the treatment of edentulous atrophic mandibles. Quintessence International 2010;41:285-93.

25. Hinze M, Thalmair T, Bolz W, Wachtel H. Immediate loading of fixed provisional prostheses using four implants for the rehabilitation of the edentulous arch: a prospective clinical study. International Journal of Oral and Maxillofacial Implants 2010;25:1011-8.

26. Siebers D, Gehrke P, Schliephake H. Immediate versus delayed function of dental implants: a 1- to 7-year followup study of 222 implants. International Journal of Oral and Maxillofacial Implants 2010;25:1195-202.

27. Levin L, Ofec R, Grossmann Y, Anner R. Periodontal disease as a risk for dental implant failure over time: a long-term historical cohort study. Journal of Clinical Periodontology 2011;38:732-7.

28. Grandi T, Guazzi P, Samarani R, Grandi G. Immediate loading of four (all-on-4) post-extractive implants supporting mandibular cross-arch fixed prostheses: 
18-month follow-up from a multicentre prospective cohort study. European Journal of Oral Implantology 2012;5:277-85.

29. Romanos GE, Gaertner K, Aydin E, Nentwig GH. Long-term results after immediate loading of platform-switched implants in smokers versus nonsmokers with full-arch restorations. International Journal of Oral and Maxillofacial Implants 2013;28:841-5.

30. Raes S, Rocci A, Raes F, Cooper L, De Bruyn H, Cosyn J. A prospective cohort study on the impact of smoking on soft tissue alterations around single implants. Clinical Oral Implants Research 2014

31. De Bruyn H, Collaert B, Linden U, Johansson C, Albrektsson T. Clinical outcome of Screw Vent implants. A 7-year prospective follow-up study. Clinical Oral Implants Research 1999;10:139-48.

32. Grunder U, Gaberthuel T, Boitel N, Imoberdorf $M$ Meyenberg K, Andreoni C, et al. Evaluating the clinical performance of the Osseotite implant: defining prosthetic predictability. Compendium of Continuing Education in Dentistry 1999:20:628-33. 36, 38-40.

33. Testori T, Wiseman L, Woolfe S, Porter SS. A prospective multicenter clinical study of the Osseotite implant: fouryear interim report. International Journal of Oral and Maxillofacial Implants 2001;16:193-200.

34. Engstrand P, Gröndahl K, Ohrnell LO, Nilsson P, Nannmark U, Brånemark PI. Prospective follow-up study of 95 patients with edentulous mandibles treated according to the Branemark Novum concept. Clinical Implant Dentistry and Related Research 2003:5:3-10.

35. van Steenberghe D, Molly L, Jacobs R, Vandekerckhove B, Quirynen M, Naert I. The immediate rehabilitation by means of a ready-made final fixed prosthesis in the edentulous mandible: a 1-year follow-up study on 50 consecutive patients. Clinical Oral Implants Research 2004;15:360-5.

36. Ibañez JC, Tahhan MJ, Zamar JA, Menendez AB, Juaneda AM, Zamar NJ, et al. Immediate occlusal loading of double acid-etched surface titanium implants in 41 consecutive full-arch cases in the mandible and maxilla: 6- to 74-month results. Journal of Periodontology 2005;76:1972-81.

37. Moheng P, Feryn JM. Clinical and biologic factors related to oral implant failure: a 2-year follow-up study. Implant Dentistry 2005;14:281-8.

38. Alsaadi G, Quirynen M, Michiles K, Teughels W, Komarek A, van Steenberghe D. Impact of local and systemic factors on the incidence of failures up to abutment connection with modified surface oral implants. Journal of Clinical Periodontology 2008;35:51-7.

39. Nyström E, Nilson H, Gunne J, Lundgren S. Reconstruction of the atrophic maxilla with interpositional bone grafting/ Le Fort I osteotomy and endosteal implants: a 11-16 year follow-up. International Journal of Oral and Maxillofacial Surgery 2009;38:1-6.

40. Brandt R, Hollis S, Ahuja S, Adatrow P, Balanoff W. Shortterm objective and subjective evaluation of small-diameter implants used to support and retain mandibular prosthesis. Journal of the Tennessee Dental Association 2012;92:34-8. quiz 38-9.

41. Horwitz J, Levin L, Gabay E, Zuabi O, Machtei EE. Immediate restoration of delayed placement of dental implants in patients with treated periodontal disease: 1-year results. International Journal of Oral and Maxillofacial Implants 2012;27:1569-75.

42. Mertens C, Steveling HG, Stucke K, Pretzl B, Meyer-Baumer A. Fixed implant-retained rehabilitation of the edentulous maxilla: 11-year results of a prospective study. Clinical Implant Dentistry and Related Research 2012;14:816-27.

43. Balaguer J, Ata-Ali J, Penarrocha-Oltra D, Garcia B, Penarrocha M. Long-term survival rates of implants supporting overdentures. Journal of Oral Implantology 2013.
44. D'Haese J, Vervaeke S, Verbanck N, De Bruyn H. Clinical and radiographic outcome of implants placed using stereolithographic guided surgery: a prospective monocenter study. International Journal of Oral and Maxillofacial Implants 2013;28:205-15.

45. Cha HS, Kim A, Nowzari H, Chang HS, Ahn KM. Simultaneous sinus lift and implant installation: prospective study of consecutive two hundred seventeen sinus lift and four hundred sixty-two implants. Clinical Implant Dentistry and Related Research 2014;16:337-47.

46. Mordenfeld A, Albrektsson T, Hallman M. A 10-year clinical and radiographic study of implants placed after maxillary sinus floor augmentation with an 80:20 mixture of deproteinized bovine bone and autogenous bone. Clinical Implant Dentistry and Related Research 2014;16:435-46.

47. Bain CA, Moy PK. The association between the failure of dental implants and cigarette smoking. International Journal of Oral and Maxillofacial Implants 1993;8:609-15.

48. De Bruyn H, Collaert B. The effect of smoking on early implant failure. Clinical Oral Implants Research 1994:5:260-4.

49. Bain CA. Smoking and implant failure-benefits of a smoking cessation protocol. International Journal of Oral and Maxillofacial Implants 1996;11:756-9.

50. Minsk L, Polson AM, Weisgold A, Rose LF, Sanavi F, Baumgarten $\mathrm{H}$, et al. Outcome failures of endosseous implants from a clinical training center. Compendium of Continuing Education in Dentistry 1996;17:848-50. 52-4, 56 passim.

51. Kan JY, Rungcharassaeng K, Lozada JL, Goodacre CJ. Effects of smoking on implant success in grafted maxillary sinuses. Journal of Prosthetic Dentistry 1999;82:307-11.

52. Keller EE, Tolman DE, Eckert SE. Maxillary antral-nasal inlay autogenous bone graft reconstruction of compromised maxilla: a 12-year retrospective study. International Journal of Oral and Maxillofacial Implants 1999:14:707-21.

53. Rosen PS, Summers R, Mellado JR, Salkin LM, Shanaman $\mathrm{RH}$, Marks MH, et al. The bone-added osteotome sinus floor elevation technique: multicenter retrospective report of consecutively treated patients. International Journal of Oral and Maxillofacial Implants 1999;14:853-8.

54. Habsha E. Survival of osseointegrated dental implants in smokers and non-smokers. Toronto: University of Toronto; 2000.

55. Olson JW, Dent CD, Morris HF, Ochi S. Long-term assessment (5-71 months) of endosseous dental implants placed in the augmented maxillary sinus. Annals of Periodontology 2000;5:152-6.

56. Schwartz-Arad D, Grossman Y, Chaushu G. The clinical effectiveness of implants placed immediately into fresh extraction sites of molar teeth. Journal of Periodontology 2000;71:839-44.

57. Wallace RH. The relationship between cigarette smoking and dental implant failure. European Journal of Prosthodontics and Restorative Dentistry 2000;8:103-6.

58. Geurs NC, Wang IC, Shulman LB, Jeffcoat MK. Retrospective radiographic analysis of sinus graft and implant placement procedures from the Academy of Osseointegration Consensus Conference on Sinus Grafts. International Journal of Periodontics and Restorative Dentistry 2001;21:517-23.

59. Mayfield LJ, Skoglund A, Hising P, Lang NP, Attstrom R. Evaluation following functional loading of titanium fixtures placed in ridges augmented by deproteinized bone mineral. A human case study. Clinical Oral Implants Research 2001;12:508-14

60. Widmark G, Andersson B, Carlsson GE, Lindvall AM, Ivanoff CJ. Rehabilitation of patients with severely resorbed maxillae by means of implants with or without bone grafts: a 3- to 5-year follow-up clinical report. 
International Journal of Oral and Maxillofacial Implants 2001;16:73-9.

61. Kumar A, Jaffin RA, Berman C. The effect of smoking on achieving osseointegration of surface-modified implants: a clinical report. International Journal of Oral and Maxillofacial Implants 2002;17:816-9.

62. Schwartz-Arad D, Samet N, Samet N, Mamlider A. Smoking and complications of endosseous dental implants. Journal of Periodontology 2002;73:153-7.

63. McCarthy C, Patel RR, Wragg PF, Brook IM. Sinus augmentation bone grafts for the provision of dental implants: report of clinical outcome. International Journal of Oral and Maxillofacial Implants 2003;18:377-82.

64. Locante WM. Single-tooth replacements in the esthetic zone with an immediate function implant: a preliminary report. Journal of Oral Implantology 2004;30:369-75.

65. Nitzan D, Mamlider A, Levin L, Schwartz-Arad D. Impact of smoking on marginal bone loss. International Journal of Oral and Maxillofacial Implants 2005;20:605-9.

66. Bischof M, Nedir R, Abi Najm S, Szmukler-Moncler S, Samson J. A 5-year life-table analysis on wide neck ITI implants with prosthetic evaluation and radiographic analysis: results from a private practice. Clinical Oral Implants Research 2006;17:512-20.

67. Mundt T, Mack F, Schwahn C, Biffar R. Private practice results of screw-type tapered implants: survival and evaluation of risk factors. International Journal of Oral and Maxillofacial Implants 2006;21:607-14.

68. Noguerol B, Munoz R, Mesa F, de Dios Luna J, O'Valle F. Early implant failure. Prognostic capacity of Periotest: retrospective study of a large sample. Clinical Oral Implants Research 2006;17:459-64.

69. Wagenberg B, Froum SJ. A retrospective study of 1925 consecutively placed immediate implants from 1988 to 2004. International Journal of Oral and Maxillofacial Implants 2006;21:71-80.

70. Alsaadi G, Quirynen M, Komarek A, van Steenberghe D. Impact of local and systemic factors on the incidence of oral implant failures, up to abutment connection. Journal of Clinical Periodontology 2007;34:610-7.

71. Aykent F, Inan O, Ozyesil AG, Alptekin NO. A 1- to 12-year clinical evaluation of 106 endosseous implants supporting fixed and removable prostheses. International Journal of Periodontics and Restorative Dent 2007;27:358-67.

72. Doyle SL, Hodges JS, Pesun IJ, Baisden MK, Bowles WR. Factors affecting outcomes for single-tooth implants and endodontic restorations. Journal of Endodontics 2007;33:399-402

73. Kinsel RP, Liss M. Retrospective analysis of 56 edentulous dental arches restored with 344 single-stage implants using an immediate loading fixed provisional protocol: statistical predictors of implant failure. International Journal of Oral and Maxillofacial Implants 2007;22:823-30.

74. Sánchez-Pérez A, Moya-Villaescusa MJ, Caffesse RG. Tobacco as a risk factor for survival of dental implants. Journal of Periodontology 2007;78:351-9.

75. Sanna AM, Molly L, van Steenberghe D. Immediately loaded CAD-CAM manufactured fixed complete dentures using flapless implant placement procedures: a cohort study of consecutive patients. Journal of Prosthetic Dentistry 2007;97:331-9

76. Alsaadi G, Quirynen M, Komarek A, van Steenberghe D. Impact of local and systemic factors on the incidence of late oral implant loss. Clinical Oral Implants Research 2008;19:670-6.

77. Anitua E, Orive G, Aguirre JJ, Ardanza B, Andia I. 5-year clinical experience with BTI dental implants: risk factors for implant failure. Journal of Clinical Periodontology 2008;35:724-32.
78. Friberg B, Jemt T. Turned Branemark System implants in wide and narrow edentulous maxillae: a retrospective clinical study. Clinical Implant Dentistry and Related Research 2008;10:78-85.

79. Grossmann Y, Levin L, Sadan A. A retrospective case series of implants used to restore partially edentulous patients with implant-supported removable partial dentures: 31month mean follow-up results. Quintessence International 2008;39:665-71.

80. Holahan CM, Koka S, Kennel KA, Weaver AL, Assad DA, Regennitter FJ, et al. Effect of osteoporotic status on the survival of titanium dental implants. International Journal of Oral and Maxillofacial Implants 2008;23:905-10.

81. Komiyama A, Klinge B, Hultin M. Treatment outcome of immediately loaded implants installed in edentulous jaws following computer-assisted virtual treatment planning and flapless surgery. Clinical Oral Implants Research 2008;19:677-85.

82. Levin L, Hertzberg R, Har-Nes S, Schwartz-Arad D. Longterm marginal bone loss around single dental implants affected by current and past smoking habits. Implant Dentistry 2008;17:422-9.

83. Sverzut AT, Stabile GA, de Moraes M, Mazzonetto R, Moreira RW. The influence of tobacco on early dental implant failure. Journal of Oral and Maxillofacial Surgery 2008;66:1004-9.

84. Anner R, Grossmann Y, Anner Y, Levin L. Smoking, diabetes mellitus, periodontitis, and supportive periodontal treatment as factors associated with dental implant survival: a long-term retrospective evaluation of patients followed for up to 10 years. Implant Dentistry 2010;19:57-64.

85. Zafiropoulos GG, Deli G, Bartee BK, Hoffmann O. Singletooth implant placement and loading in fresh and regenerated extraction sockets. A 5-year results: a case series using two different implant designs. Journal of Periodontology 2010;81:604-15.

86. Bell CL, Diehl D, Bell BM, Bell RE. The immediate placement of dental implants into extraction sites with periapical lesions: a retrospective chart review. Journal of Oral and Maxillofacial Surgery 2011;69:1623-7.

87. Cavalcanti R, Oreglia F, Manfredonia MF, Gianserra R, Esposito M. The influence of smoking on the survival of dental implants: a 5-year pragmatic multicentre retrospective cohort study of 1727 patients. European Journal of Oral Implantology 2011;4:39-45.

88. Conrad HJ, Jung J, Barczak M, Basu S, Seong WJ. Retrospective cohort study of the predictors of implant failure in the posterior maxilla. International Journal of Oral and Maxillofacial Implants 2011;26:154-62.

89. Rodriguez-Argueta OF, Figueiredo R, Valmaseda-Castellon E, Gay-Escoda C. Postoperative complications in smoking patients treated with implants: a retrospective study. Journal of Oral and Maxillofacial Surgery 2011;69:2152-7.

90. Strietzel FP, Karmon B, Lorean A, Fischer PP. Implantprosthetic rehabilitation of the edentulous maxilla and mandible with immediately loaded implants: preliminary data from a retrospective study, considering time of implantation. International Journal of Oral and Maxillofacial Implants 2011;26:139-47.

91. Vandeweghe S, De Bruyn $\mathrm{H}$. The effect of smoking on early bone remodeling on surface modified Southern Implants(R). Clinical Implant Dentistry and Related Research 2011;13:206-14

92. Ji TJ, Kan JY, Rungcharassaeng K, Roe P, Lozada JL. Immediate loading of maxillary and mandibular implantsupported fixed complete dentures: a 1- to 10-year retrospective study. Journal of Oral Implantology 2012:469-76. 38 Spec No: 
93. Lin TH, Chen L, Cha J, Jeffcoat M, Kao DW, Nevins M, et al. The effect of cigarette smoking and native bone height on dental implants placed immediately in sinuses grafted by hydraulic condensation. International Journal of Periodontics and Restorative Dentistry 2012;32:255-61.

94. Mir-Mari J, Mir-Orfila P, Valmaseda-Castellon E, GayEscoda C. Long-term marginal bone loss in 217 machinedsurface implants placed in 68 patients with 5-9 years of follow-up: a retrospective study. International Journal of Oral and Maxillofacial Implants 2012;27:1163-9.

95. Oliva J, Oliva X, Oliva JD. All-on-three delayed implant loading concept for the completely edentulous maxilla and mandible: a retrospective 5-year follow-up study. International Journal of Oral and Maxillofacial Implants 2012;27:1584-92.

96. Schneider D, Witt L, Hämmerle CH. Influence of the crownto-implant length ratio on the clinical performance of implants supporting single crown restorations: a crosssectional retrospective 5-year investigation. Clinical Oral Implants Research 2012;23:169-74.

97. Shibuya Y, Takeuchi Y, Asai T, Takeuchi J, Suzuki H, Komori T. Maxillary sinus floor elevation combined with a vertical onlay graft. Implant Dentistry 2012;21:91-6.

98. Urdaneta RA, Daher S, Leary J, Emanuel KM, Chuang SK. The survival of ultrashort locking-taper implants. International Journal of Oral and Maxillofacial Implants 2012;27:644-54.

99. Vandeweghe S, Ackermann A, Bronner J, Hattingh A, Tschakaloff A, De Bruyn H. A retrospective, multicenter study on a novo wide-body implant for posterior regions. Clinical Implant Dentistry and Related Research 2012;14:281-92.

100. Le BT, Follmar T, Borzabadi-Farahani A. Assessment of short dental implants restored with single-unit nonsplinted restorations. Implant Dentistry 2013;22:499-502.

101. Mijiritsky E, Mazor Z, Lorean A, Levin L. Implant diameter and length influence on survival: interim results during the first 2 years of function of implants by a single manufacturer. Implant Dentistry 2013;22:394-8.

102. Morales-Vadillo R, Leite FP, Guevara-Canales J, Netto HD, Miranda Chaves M, Cruz F, et al. Retrospective study of the survival and associated risk factors of wedge-shaped implants. International Journal of Oral and Maxillofacial Implants 2013;28:875-82.

103. Sayardoust S, Gröndahl K, Johansson E, Thomsen P, Slotte C. Implant survival and marginal bone loss at turned and oxidized implants in periodontitis-susceptible smokers and never-smokers: a retrospective, clinical, radiographic case-control study. Journal of Periodontology 2013;84:1775-82.

104. Wagenberg BD, Froum SJ, Eckert SE. Long-term bone stability assessment around 1,187 immediately placed implants with 1- to 22-year follow-up. International Journal of Oral and Maxillofacial Implants 2013;28:605-12.

105. Vandeweghe S, Nicolopoulos C, Thevissen E, Jimbo R, Wennerberg A, De Bruyn H. Immediate loading of screwretained all-ceramic crowns in immediate versus delayed single implant placement. International Journal of Prosthodontics 2013;26:458-64.

106. Vervaeke S, Collaert B, Cosyn J, Deschepper E, De Bruyn H. A multifactorial analysis to identify predictors of implant failure and peri-implant bone loss. Clinical Implant Dentistry and Related Research 2013.

107. Zinser MJ, Randelzhofer P, Kuiper L, Zoller JE, De Lange GL. The predictors of implant failure after maxillary sinus floor augmentation and reconstruction: a retrospective study of 1045 consecutive implants. Oral Surgery Oral Medicine Oral Pathology Oral Radiology 2013:115:571-82.

108. Balshi TJ, Wolfinger GJ, Slauch RW, Balshi SF. A retrospective analysis of 800 Branemark System implants following the all-on-four protocol. Journal of Prosthodontics 2014;23:83-8.

109. Bell C, Bell RE. Immediate restoration of NobelActive implants placed into fresh extraction sites in the anterior maxilla. Journal of Oral Implantology 2014:40:455-8.

110. Cakarer S, Selvi F, Can T, Kirli I, Palancioglu A, Keskin B, et al. Investigation of the risk factors associated with the survival rate of dental implants. Implant Dentistry 2014:23:328-33.

111. Doan NV, Du Z, Reher P, Xiao Y. Flapless dental implant surgery: a retrospective study of 1,241 consecutive implants. International Journal of Oral and Maxillofacial Implants 2014:29:650-8.

112. Gander T, Studer S, Studer G, Gratz KW, Bredell M. Medium-term outcome of Astra Tech implants in head and neck oncology patients. International Journal of Oral and Maxillofacial Surgery 2014;43:1381-5.

113. Leventi E, Malden NJ, Lopes VR. Peri-implant bone-level reduction in relation to hydroxyapatite-coated dental implants that act as mandibular overdenture retainers: results at 6-10 years. Journal of Prosthetic Dentistry 2014;112:792-7.

114. Mendonc JA, Francischone CE, Senna PM, Matos de Oliveira AE, Sotto-Maior BS. A retrospective evaluation of the survival rates of splinted and non-splinted short dental implants in posterior partially edentulous jaws. Journal of Periodontology 2014;85:787-94.

115. Rasperini G, Siciliano VI, Cafiero C, Salvi GE, Blasi A, Aglietta M. Crestal bone changes at teeth and implants in periodontally healthy and periodontally compromised patients. A 10-year comparative case-series study. Journal of Periodontology 2014;85:e152-9.

116. Twito D, Sade P. The effect of cigarette smoking habits on the outcome of dental implant treatment. PeerJ 2014;2:e546.

117. Wu X, Al-Abedalla K, Rastikerdar E, Abi Nader S, Daniel NG, Nicolau B, et al. Selective serotonin reuptake inhibitors and the risk of osseointegrated implant failure: a cohort study. Journal of Dental Research 2014;93:1054-61.

118. Shrier I, Boivin JF, Steele RJ, Platt RW, Furlan A, Kakuma R, et al. Should meta-analyses of interventions include observational studies in addition to randomized controlled trials? A critical examination of underlying principles. American Journal of Epidemiology 2007;166:1203-9.

119. Chuang SK, Tian L, Wei LJ, Dodson TB. Kaplan-Meier analysis of dental implant survival: a strategy for estimating survival with clustered observations. Journal of Dental Research 2001;80:2016-20.

120. Ma L, Zwahlen RA, Zheng LW, Sham MH. Influence of nicotine on the biological activity of rabbit osteoblasts. Clinical Oral Implants Research 2011;22:338-42.

121. Deng ZL, Sharff KA, Tang N, Song WX, Luo J, Luo X, et al. Regulation of osteogenic differentiation during skeletal development. Frontiers in Bioscience 2008;13:2001-21.

122. Ma L, Zheng LW, Sham MH, Cheung LK. Uncoupled angiogenesis and osteogenesis in nicotine-compromised bone healing. Journal of Bone and Mineral Research 2010;25:1305-13.

123. Wang Y, Wan C, Deng L, Liu X, Cao X, Gilbert SR, et al. The hypoxia-inducible factor alpha pathway couples angiogenesis to osteogenesis during skeletal development. Journal of Clinical Investigation 2007;117:1616-26.

124. Fleming JT, Barati MT, Beck DJ, Dodds JC, Malkani AL, Parameswaran D, et al. Bone blood flow and vascular reactivity. Cells Tissues Organs 2001;169:279-84.

125. Klokkevold PR, Han TJ. How do smoking, diabetes, and periodontitis affect outcomes of implant treatment. International Journal of Oral and Maxillofacial Implants 2007;22(Suppl.):173-202. 
126. Chrcanovic BR, Pedrosa AR, Martins MD. Chemical topographic analysis of treated surfaces of five different commercial dental titanium implants. Materials Research 2012;15:372-82

127. Chrcanovic BR, Leão NLC, Martins MD. Influence of different acid etchings on the superficial characteristics of Ti sandblasted with $\mathrm{Al}_{2} \mathrm{O}_{3}$. Materials Research 2013;16:1006-14

128. Chrcanovic BR, Martins MD. Study of the influence of acid etching treatments on the superficial characteristics of Ti. Materials Research 2014;17:373-80.

129. Wennerberg A, Albrektsson T. On implant surfaces: review of current knowledge and opinions. International Journal of Oral and Maxillofacial Implants 2010;25:63-74.

130. Balshe AA, Eckert SE, Koka S, Assad DA, Weaver AL. The effects of smoking on the survival of smooth- and roughsurface dental implants. International Journal of Oral and Maxillofacial Implants 2008;23:1117-22.

131. Chrcanovic BR, Albrektsson T, Wennerberg A. Flapless versus conventional flapped dental implant surgery: a meta-analysis. PLoS ONE 2014;9:e100624.

132. Chrcanovic BR, Albrektsson T, Wennerberg A. Immediate nonfunctional versus immediate functional loading and dental implant failure rates: a systematic review and metaanalysis. Journal of Dentistry 2014;42:1052-9.

133. Chrcanovic BR, Albrektsson T, Wennerberg A. Prophylactic antibiotic regimen and dental implant failure: a metaanalysis. Journal of Oral Rehabilitation 2014;41:941-56.

134. Chrcanovic BR, Albrektsson T, Wennerberg A. Periodontally compromised vs. periodontally healthy patients and dental implants: a systematic review and meta-analysis. Journal of Dentistry 2014;42:1509-27.

135. Chrcanovic BR, Albrektsson T, Wennerberg A. Diabetes and oral implant failure: a systematic review. Journal of Dental Research 2014;93:859-67.

136. Chrcanovic BR, Albrektsson T, Wennerberg A. Dental implants inserted in fresh extraction sockets versus healed sites: a systematic review and meta-analysis. Journal of Dentistry 2015;43:16-41.

137. Chrcanovic BR, Albrektsson T, Wennerberg A. Tilted versus axially placed dental implants: a meta-analysis. Journal of Dentistry 2015;43:149-70.

138. Chrcanovic BR, Albrektsson T, Wennerberg A. Platform switch and dental implants: a meta-analysis. Journal of Dentistry 2015. http://dx.doi.org/10.1016/j.jdent.2014.12.013.

139. Chrcanovic BR, Abreu MH, Freire-Maia B, Souza LN. Facial fractures in children and adolescents: a retrospective study of 3 years in a hospital in Belo Horizonte, Brazil. Dental Traumatology 2010;26:262-70.

140. Chrcanovic BR, Souza LN, Freire-Maia B, Abreu MH. Facial fractures in the elderly: a retrospective study in a hospital in Belo Horizonte, Brazil. Journal of Trauma 2010;69:E73-8.

141. Chrcanovic BR, Abreu MH, Freire-Maia B, Souza LN. 1,454 mandibular fractures: a 3-year study in a hospital in Belo Horizonte, Brazil. Journal of Cranio-maxillofacial Surgery 2012;40:116-23.

142. Kan JY, Rungcharassaeng K, Kim J, Lozada JL, Goodacre CJ. Factors affecting the survival of implants placed in grafted maxillary sinuses: a clinical report. Journal of Prosthetic Dentistry 2002;87:485-9. 


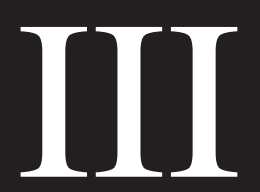





\title{
Factors Influencing Early Dental Implant Failures
}

\author{
B.R. Chrcanovic', J. Kisch ${ }^{2}$, T. Albrektsson ${ }^{1,3}$, \\ and A. Wennerberg'
}

\begin{abstract}
The purpose of the present study was to assess the influence of local and systemic factors on the occurrence of dental implant failures up to the second-stage surgery (abutment connection). This retrospective study is based on 2,670 patients who received 10,096 implants and were consecutively treated with implant-supported prostheses between 1980 and 2014 at I specialist clinic. Several anatomic-, patient-, health-, and implant-related factors were collected. Descriptive statistics were used to describe the patients and implants. Univariate and multivariate logistic regression models were used at the patient level as well as the implant level to evaluate the effect of explanatory variables on the failure of implants up to abutment connection. A generalized estimating equation method was used for the implant-level analysis to account for the fact that repeated observations (several implants) were available for a single patient. Overall, 642 implants (6.36\%) failed, of which $176(1.74 \%)$ in 139 patients were lost up to second-stage surgery. The distribution of implants in sites of different bone quantities and qualities was quite similar between implants lost up to and after abutment connection. Smoking and the intake of antidepressants were the statistically significant predictors in the multivariate model (ClinicalTrials.gov NCT02369562).
\end{abstract}

Keywords: endosseous dental implantation, osseointegration, treatment outcome, adverse effects, survival rate, multivariate analysis

\section{Introduction}

Nowadays dental implant placement is an effective and predictable treatment modality for replacing missing teeth in fully and partially edentulous patients. Nevertheless, failures still happen despite high implant survival and success rates (Chrcanovic et al. 2014). Failures of dental implants can be subdivided into early and late failures, depending on whether they occur before or at abutment connection surgery (early) or after occlusal loading by a prosthetic restoration (late). This subdivision is relevant because it suggests that failures in these 2 distinct periods may be associated with different factors. An early failure of an implant results from an inability to establish an intimate bone-to-implant contact. In this case, bone healing after implant insertion is impaired and may be influenced by local and systemic factors (Alsaadi et al. 2007). Systemic diseases and compromising risky habits may affect oral tissues by increasing their susceptibility to other diseases or by interfering with wound healing. Surgical conditions, submission to radiotherapy, and the intake of medications by the patient may play a role on the outcome of implants. When it comes to late implant failures, oral microbial environment, parafunctional habits, and prosthetic rehabilitation variables are also taken into account. Although many studies have shown the influence of local and systemic factors in the long-term outcome of dental implants (Chrcanovic et al. 2014), less is known concerning the factors affecting the initial phases of osseointegration. The aim of the present study was to assess the influence of local and systemic factors on the occurrence of implant failures up to second-stage surgery (abutment connection).

\section{Materials and Methods}

\section{Materials}

This retrospective study is based on all 2,670 patients who received 10,096 implants and were consecutively treated with implant-supported prostheses between 1980 and 2014 at 1 specialist clinic (Clinic for Prosthodontics, Centre of Dental Specialist Care, Malmö, Sweden). The study was approved by the Regional Ethical Review Board in Lund (Dnr 2014/598, Dnr 2015/72). This study is in concordance with the STROBE (Strengthening the Reporting of Observational Studies in Epidemiology) guidelines for observational studies.

\section{Inclusion and Exclusion Criteria}

All implants that had failed up to second-stage surgery (abutment connection) were included. Therefore, there is a minimum risk of selection bias, since all implants with early failure were selected from all implants ever inserted in this clinic.

'Department of Prosthodontics, Faculty of Odontology, Malmö University, Malmö, Sweden

${ }^{2}$ Clinic for Prosthodontics, Centre of Dental Specialist Care, Malmö, Sweden

${ }^{3}$ Department of Biomaterials, Göteborg University, Göteborg, Sweden

Corresponding Author:

B.R. Chrcanovic, Department of Prosthodontics, Faculty of Odontology, Malmö University, Carl Gustafs väg 34, SE-214 21, Malmö, Sweden. Emails: bruno.chrcanovic@mah.se; brunochrcanovic@hotmail.com 
Only modern endosseous dental implants with cylindrical or conical design were considered. Zygomatic implants were not included in the study, as well as implants that were detected in radiographies, but that did not have basic information about them in the patients' files.

\section{Definitions}

An implant was considered a failure if presenting signs and symptoms led to implant removal. Thus, a failed implant in our study is equal to a lost implant.

\section{Data Collection}

The following data were collected: implant surface (turned/ machined or enlarged surfaces, the latter including sandblasted, acid-etched, sandblasted + acid-etched, anodized, hydroxyapatitecoated surfaces), implant system (Nobel turned, Nobel TiUnite, Astra TiOblast, Astra Osseospeed, Straumann SLA/SLActive/ Roxlid, XIVE/Frialit-2, other), implant length and diameter, implant design (cylindrical or conical), prescription of antibiotics (the prophylactic antibiotic regimen usually started 1 to $2 \mathrm{~h}$ before surgery and went from 5 to $7 \mathrm{~d}$ postoperatively), bone graft procedures, reason for tooth extraction (periodontal disease, fracture/trauma, advanced caries, agenesia, other), implant jaw location (maxilla or mandible), number of implants in maxilla or mandible, anterior or posterior location of the implant (locations between 13 and 23 and between 33 and 43 were considered anterior location), patient's sex, age of the patient at the implant insertion surgery, number of days until failure, time between loss of an implant and replacement by another one, and follow-up time. Bone quantity and quality of the treated jaws were classified at the time of surgery according to the Lekholm and Zarb (1985) classification.

General health and behavioral history were collected from the patients' files. The presence of a medicament list in the patients' records was used to correlate the use of certain drugs to specific health conditions. The following health factors were assessed: diabetes type I or II, hypertension, hypercholesterolemia, hypothyroidism, asthma, psoriasis, chemotherapy, and irradiation of the head-neck region. The patients were also classified according to the intake of the following medication types: antidepressants, immunosuppressive drugs, bisphosphonates, antithrombotic agents (antiplatelet, anticoagulant, thrombolytic drugs), hormone replacement therapy in women, and medicaments to reduce gastric acid production. The following behavioral factors were assessed: smoking habits, use of snuff, bruxism.

\section{Statistical Analyses}

The mean, standard deviation, and percentages were presented as descriptive statistics. Logistic regression models were used at the patient level as well as the implant level. The patientlevel analysis considered the patient as the statistical unit, with patients presenting or not presenting implant failures. Regression at the patient level was used to evaluate the effect of explanatory health variables on the failure of implants up to abutment connection - that is, health factors that are inherently associated to the patient, not to the implant. First, a univariate effect of each health factor on the implant failure was evaluated. Odds ratios and their $95 \%$ confidence intervals were computed. The Wald test based on robust standard errors was used to assess the significance of each factor. A factor was excluded from further multivariate analysis if the univariate logistic regression resulted in a clearly nonsignificant odds ratio $(P>0.1)$. In the second step, a multivariate logistic regression gave the effects on different risk factors when controlling for other factors. The results of the final model were presented as an estimated odds ratio of each significant prognostic variable $(P<0.05)$.

An implant-level model having the implant as the statistical unit was performed to assess the effects of the implant-related and local bone factors on the failure of implants up to abutment connection, also including the health variables. A generalized estimating equation (GEE) method was used to account for the fact that repeated observations (several implants) were available for a single patient. Because the outcomes relating to implants within a single patient must be more closely correlated to each other than the outcomes of implants in separate patients, ignoring these correlations could result in a bias in $P$ value computations (Shintani 2014). All models were adjusted for clustering of subject and implants in a binary logistic regression model through GEE with a binomial distribution and a logit link function, while assuming an exchangeable working correlation structure to assess the relationship between implant failure up to abutment connection (dependent variable) and the risk factors (independent variables). Initially, a univariate GEE on each variable was performed. To verify multicollinearity, a correlation matrix of all predictor variables with a significant odds ratio ( $P$ value cutoff point of 0.1$)$ identified in the univariate GEE was scanned to see whether there were some high correlations among the predictors. Collinearity statistics obtaining variance inflation factor and tolerance statistic were also performed to detect more subtle forms of multicollinearity. Then, a multivariable model with a forced entry method was used to evaluate the effect of the factors that were univariately significant $(P<0.1)$ and did not present multicollinearity. A Wald chi-square test was used to analyze the statistical significance of each parameter within the model. The results of the final model were presented as an estimated odds ratio of each significant prognostic variable $(P<0.05)$. Inc.).

All data were statistically analyzed with SPSS 22 (SPSS

\section{Results}

Overall, 642 of 10,096 implants $(6.36 \%)$ failed. From this total of 642 lost implants, 176 implants in 139 patients were lost up to the second-stage surgery ( 65 men and 74 women; mean age at the time of implant surgery, $54.1 \pm 16.3 \mathrm{y}$; minimum, $17.7 \mathrm{y}$; maximum, $90.1 \mathrm{y}$ ). This corresponds to a failure rate up to abutment connection of $1.74 \%$ at the implant level and $5.21 \%$ 
Table I. Comparison of Implants That Failed up to and after Abutment Connection.

\begin{tabular}{|c|c|c|c|c|c|c|c|}
\hline & A & B & C & D & $\mathrm{E}$ & Total & $\%$ \\
\hline & \multicolumn{7}{|c|}{ Up to Abutment Connection ${ }^{a}$} \\
\hline 1 & 0 & 0 & 1 & 6 & 1 & 8 & 4.8 \\
\hline 2 & 4 & 30 & 10 & 3 & 2 & 49 & 29.7 \\
\hline 3 & 6 & 29 & 26 & 12 & 1 & 74 & 44.9 \\
\hline 4 & 0 & 4 & 12 & 10 & 8 & 34 & 20.6 \\
\hline Total & 10 & 63 & 49 & 31 & 12 & 165 & 100 \\
\hline \multirow[t]{2}{*}{$\%$} & 6.0 & 38.2 & 29.7 & 18.8 & 7.3 & 100 & \\
\hline & \multicolumn{7}{|c|}{ After Abutment Connection ${ }^{b}$} \\
\hline I & 0 & I & 5 & 0 & I & 7 & 1.5 \\
\hline 2 & 7 & 67 & 37 & 12 & 3 & 126 & 27.8 \\
\hline 3 & 9 & 72 & 85 & 51 & 7 & 224 & 49.3 \\
\hline 4 & 0 & 16 & 24 & 32 & 25 & 97 & 21.4 \\
\hline Total & 16 & 156 & $|5|$ & 95 & 36 & 454 & 100 \\
\hline$\%$ & 3.5 & 34.4 & 33.3 & 20.9 & 7.9 & 100 & \\
\hline
\end{tabular}

According to the Lekholm and Zarb (1985) classification, bone quality is broken into 4 groups per the proportion and structure of compact and trabecular bone tissue: type I, large homogeneous cortical/compact bone; type 2, thick layer of compact bone surrounding a dense trabecular bone; type 3 , thin cortical layer surrounding a dense trabecular bone; type 4 , thin cortical layer surrounding a core of low-density trabecular bone. The quantity of jawbone is broken into 5 groups (A-E) based on the residual jaw shape following tooth extraction: bone classified as A presents the largest amount of bone among all classes, whereas bone classified as $E$ presents the lowest volume of bone.

${ }^{2}$ Missing information of bone quantity and quality: II implants.

bMissing information of bone quantity and quality: 12 implants.

at the patient level. The location of the implants was as follows: 73 in the anterior maxilla, 36 in the anterior mandible, 44 in the posterior maxilla, and 23 in the posterior mandible. Only 4 of the 176 failures up to the abutment connection occurred in nonsubmerged implants with a delayed loading protocol.

Table 1 shows a comparison of groups of implants failed up to and after abutment connection according to the distribution of implants with regard to the Lekholm and Zarb (1985) classification of bone quantity and quality. Note that the distribution of implants in sites of different bone quantities and qualities was quite similar between the groups.

The univariate binary logistic regression showed that the following predictors had a statistically significant odds ratio at the patient level (Table 2): smoking $(P<0.001)$, number of cigarettes per day $(P=0.002)$, the intake of antidepressants $(P=$ $0.002)$, and age of the patient at the time of the surgery $(P=$ 0.001 ). After only the variables with a statistically significant odds ratio were included in the multivariate binary logistic regression model (Table 3$)$, smoking $(P=0.003)$ and the intake of antidepressants $(P=0.009)$ continued to present a statistically significant odds ratio.

The univariate GEE model showed that the following predictors had a statistically significant odds ratio at the implant level (Table 4): smoking $(P<0.001)$, number of cigarettes per day $(P=0.040)$, hypothyroidism $(P=0.065)$, the intake of antidepressants $(P=0.046)$, implant jaw location $(P=0.064)$, bone quantity (quantity $\mathrm{A}$ as the reference category; quantity $\mathrm{C}$, $P=0.039$; quantity $\mathrm{D}, P<0.001$; quantity E, $P<0.001$ ), bone quality (quality 1 as the reference category; quality $4, P=$ 0.034), implant surface $(P<0.001)$, implant system (Nobel turned as the reference category; Nobel TiUnite, $P=0.002$ ), and bone graft procedures $(P=0.009)$. After only the variables with a statistically significant odds ratio were included in the multivariate GEE model (Table 5), only smoking $(P=0.022)$ continued to present a statistically significant odds ratio.

As none of the patients who presented a failure before abutment connection were taking bisphosphonates, this variable was not included in any statistical model.

\section{Discussion}

The aim of the present study was to assess the influence of local and systemic factors on the occurrence of implant failures up to second-stage surgery. As the study of failures only before the abutment connection limits the observation to the stage before the prosthetic treatment, confounding factors are eliminated. The regression analyses performed in this study tried to identify the factors that were possibly related to implant failure. The univariate regression assessed the relationship between each independent variable and implant failure separately, and the multivariate regression assessed the relationship of the variables that were univariately significant to implant failure, controlling for one another. In the multivariate model, only 2 variables were shown to exert some significant effect on the failures up to abutment connection: the intake of antidepressant drugs at the patient-level analysis and smoking at both the implant- and patient-level analyses.

Regarding the intake of antidepressants, there is biochemical and clinical evidence suggesting a relationship between the intake of such medicaments and the impairment of bone metabolism, which in theory could interfere with the osseointegration process. From the biochemical point of view, it is possible that neuroendocrine mechanisms related to the serotonin system could regulate osteoclast differentiation/activation 
Table 2. Univariate Binary Logistic Regression for Implant Failure up to Abutment Connection: Sex and Health Factors at the Patient Level.

\begin{tabular}{|c|c|c|}
\hline Factor & OR $(95 \% \mathrm{Cl})$ & $P$ Value \\
\hline \multicolumn{3}{|l|}{ Smoking } \\
\hline No & 1 & \\
\hline Yes & 3.065 (1.965 to 4.779$)$ & $<0.001$ \\
\hline Former smoker & $1.359(0.318$ to 5.799$)$ & 0.679 \\
\hline Cigarettes/day: increase by I & $1.043(1.015$ to 1.071$)$ & 0.002 \\
\hline \multicolumn{3}{|l|}{ Snuff } \\
\hline No & I & \\
\hline Yes & $1.363(0.578$ to 3.212$)$ & 0.479 \\
\hline \multicolumn{3}{|l|}{ Bruxism } \\
\hline No & 1 & \\
\hline Yes & $1.567(0.705$ to 3.485$)$ & 0.270 \\
\hline \multicolumn{3}{|l|}{ Diabetes } \\
\hline No & 1 & \\
\hline Type I & $0.775(0.104$ to 5.775$)$ & 0.804 \\
\hline Type II & $1.576(0.708$ to 3.507$)$ & 0.265 \\
\hline \multicolumn{3}{|l|}{ High blood pressure } \\
\hline No & I & \\
\hline Yes & $0.948(0.580$ to $\mathrm{I} .55 \mathrm{I})$ & 0.832 \\
\hline \multicolumn{3}{|l|}{ High cholesterol } \\
\hline No & I & \\
\hline Yes & 0.893 ( 0.442 to 1.807$)$ & 0.754 \\
\hline \multicolumn{3}{|l|}{ Hypothyroidism } \\
\hline No & 1 & \\
\hline Yes & $0.429(0.104$ to 1.768$)$ & $0.24 I$ \\
\hline \multicolumn{3}{|l|}{ Asthma } \\
\hline No & I & \\
\hline Yes & 1.471 (0.722 to 2.997$)$ & 0.288 \\
\hline \multicolumn{3}{|l|}{ Intake of antidepressants } \\
\hline No & I & \\
\hline Yes & 2.477 ( 1.385 to 4.429$)$ & 0.002 \\
\hline \multicolumn{3}{|l|}{ Irradiation } \\
\hline No & I & \\
\hline Yes & $1.076(0.255$ to 4.534$)$ & 0.920 \\
\hline \multicolumn{3}{|l|}{ Hormone replacement therapy } \\
\hline No & I & \\
\hline Yes & $1.452(0.516$ to 4.086$)$ & 0.480 \\
\hline \multicolumn{3}{|l|}{ Gastric } \\
\hline No & I & \\
\hline Yes & $1.569(0.739$ to 3.330$)$ & 0.241 \\
\hline \multicolumn{3}{|l|}{ Antithrombotics } \\
\hline No & I & \\
\hline Yes & 1.476 (0.875 to 2.489$)$ & 0.145 \\
\hline \multicolumn{3}{|l|}{ Immunosuppressive } \\
\hline No & 1 & \\
\hline Yes & $0.845(0.113$ to $6.32 \mathrm{I})$ & 0.870 \\
\hline \multicolumn{3}{|l|}{ Psoriasis } \\
\hline No & 1 & \\
\hline Yes & $2.719(0.612$ to 12.076$)$ & 0.189 \\
\hline \multicolumn{3}{|c|}{ 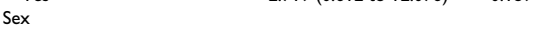 } \\
\hline Male & I & \\
\hline Female & $0.980(0.696$ to 1.380$)$ & 0.909 \\
\hline Age: increase by I & $1.014(1.006$ to 1.023$)$ & 0.001 \\
\hline
\end{tabular}

$95 \% \mathrm{Cl}, 95 \%$ confidence interval; OR, odds ratio.

because osteoclasts derive from hematopoietic cell precursors and a relationship between bone and the immune system has been established (Gruber 1991; Bab and Einhorn 1993; Ershler et al. 1997). Studies have identified a functional serotonin
Table 3. Multivariate Logistic Regression Model for Implant Failure up to Abutment Connection at the Patient Level.

\begin{tabular}{lcc}
\hline Factor & OR $(95 \% \mathrm{Cl})$ & $P$ Value \\
\hline Smoking & I & \\
$\quad$ No & $3.265(1.486$ to 7.173$)$ & 0.003 \\
$\quad$ Yes & $1.430(0.332$ to 6.156$)$ & 0.631 \\
$\quad$ Former smoker & $\quad$ I & \\
Cigarettes/day: increase by I & $0.990(0.943$ to 1.039$)$ & 0.676 \\
Intake of antidepressants & $2.438(1.251$ to 4.751$)$ & 0.009 \\
$\quad$ No & $1.005(0.992$ to 1.018$)$ & 0.451 \\
$\quad$ Yes &
\end{tabular}

Only the patient and health factors that were considered statistically significant $(P<0.1)$ in the univariate model and did not present multicollinearity were included in the multivariate model. $95 \% \mathrm{Cl}, 95 \%$ confidence interval; OR, odds ratio.

system in osteoblasts and osteoclasts (Bliziotes et al. 2001; Westbroek et al. 2001; Battaglino et al. 2004) in which the serotonin transporter and several receptors are expressed in osteoblasts as well as osteoclasts (Bliziotes et al. 2001; Westbroek et al. 2001). The presence of serotonin receptors and the serotonin transporter in bone raises the question whether medications that antagonize serotonin reuptake could influence bone metabolism. Peripheral serotonin signaling directly activates osteoblastic serotonin receptors to inhibit bone formation. Central serotonin signaling inhibits the sympathetic nervous system, thus alleviating the negative adrenergic tone on osteoblasts. In the situation of elevated serotonin levels that result from treatment with SSRIs (i.e., selective serotonin reuptake inhibitors-a class of antidepressant drugs), the negative skeletal effects of peripheral serotonin may outweigh the positive skeletal benefits resulting from the enhanced central serotonin antidepressant and antisympathetic activity (Ducy and Karsenty 2010).

In vitro studies have shown that activity of the serotonin transporter is required for osteoclast differentiation. While blockage of the serotonin transporter reduced osteoclast differentiation when fluoxetine, an antidepressant, was administered to produce micromolar $(\mu \mathrm{M})$ concentrations (Battaglino et al. 2004; Gustafsson et al. 2006), there was an increase in osteoclast differentiation for the same medicament in the nanomolar (nM) concentrations (Gustafsson et al. 2006). In vivo studies demonstrated detrimental effects of fluoxetine on trabecular architecture (Warden et al. 2008) and bone mineral density (Warden et al. 2005; Warden et al. 2008) in mice. Another in vivo study showed that serotonin acts on osteoblasts, inhibiting their proliferation (Yadav et al. 2008). These studies in animal models indicate a negative effect of SSRIs on bone mass and suggest that these antidepressants may possess direct antianabolic skeletal effects through the pharmacologic inhibition of the serotonin transporter.

From the clinical point of view, an association between antidepressants and decreased bone mineral density in humans has been suggested (Cauley et al. 2005; Richards et al. 2007; Williams et al. 2008). Moreover, bone mass has been shown to 
Table 4. Risk Factor Analysis for Implant Failure up to Abutment Connection with a Univariate Generalized Estimating Equations Logistic Regression Model at the Implant Level.

\begin{tabular}{|c|c|c|c|}
\hline Factor & Failure: Survival $^{\mathrm{a}}$ & OR $(95 \% \mathrm{Cl})$ & $P$ Value \\
\hline \multicolumn{4}{|l|}{ Smoking } \\
\hline No & $232: 4,125$ & 1 & \\
\hline Yes & 180: 1,838 & 2.709 ( 1.737 to 4.226$)$ & $<0.001$ \\
\hline Former smoker & 14: 186 & $2.159(0.463$ to 10.070$)$ & 0.327 \\
\hline Cigarettes/day: increase by I & & $1.024(1.001$ to 1.048$)$ & 0.040 \\
\hline \multicolumn{4}{|l|}{ Snuff } \\
\hline No & $380: 5,824$ & 1 & \\
\hline Yes & 27: 247 & $2.063(0.829$ to 5.139$)$ & 0.120 \\
\hline \multicolumn{4}{|l|}{ Bruxism } \\
\hline No & $361: 6,320$ & 1 & \\
\hline Yes & 85: 342 & $1.386(0.611$ to 3.142$)$ & 0.434 \\
\hline \multicolumn{4}{|l|}{ Sex } \\
\hline Male & 289: 4,47I & 1 & \\
\hline Female & $353: 4,983$ & $1.034(0.716$ to 1.494$)$ & 0.857 \\
\hline \multicolumn{4}{|l|}{ Diabetes } \\
\hline No & $404: 6,037$ & 1 & \\
\hline Type I & 7: 110 & $0.585(0.085$ to $4.05 \mathrm{I})$ & 0.588 \\
\hline Type II & 33: 477 & $1.574(0.669$ to 3.703$)$ & 0.298 \\
\hline \multicolumn{4}{|l|}{ High blood pressure } \\
\hline No & 295: 4,498 & I & \\
\hline Yes & $147: 2,10 \mid$ & 0.857 (0.508 to I.445) & 0.563 \\
\hline \multicolumn{4}{|l|}{ High cholesterol } \\
\hline No & $365: 5,652$ & 1 & \\
\hline Yes & 76: 910 & $0.806(0.362$ to 1.793$)$ & 0.596 \\
\hline \multicolumn{4}{|l|}{ Hypothyroidism } \\
\hline No & $419: 6,210$ & 1 & \\
\hline Yes & 24: 358 & 0.274 (0.069 to 1.086$)$ & 0.065 \\
\hline \multicolumn{4}{|l|}{ Asthma } \\
\hline No & $412: 6,056$ & 1 & \\
\hline Yes & 35: 527 & $1.559(0.75 \mathrm{I}$ to 3.237$)$ & 0.233 \\
\hline \multicolumn{4}{|l|}{ Intake of antidepressants } \\
\hline No & 358: 5,991 & 1 & \\
\hline Yes & 94: 584 & 1.735 (1.010 to 2.980$)$ & 0.046 \\
\hline \multicolumn{4}{|l|}{ Irradiation } \\
\hline No & $434: 6,443$ & 1 & \\
\hline Yes & 12: 172 & $0.760(0.188$ to 3.080$)$ & 0.701 \\
\hline \multicolumn{4}{|l|}{ Hormone replacement therapy } \\
\hline No & 508: 7,750 & 1 & \\
\hline Yes & 20: 227 & $1.472(0.478$ to 4.540$)$ & 0.501 \\
\hline \multicolumn{4}{|l|}{ Gastric } \\
\hline No & $368: 5,990$ & 1 & \\
\hline Yes & 58: 475 & 1.059 (0.510 to 2.199$)$ & 0.877 \\
\hline \multicolumn{4}{|l|}{ Antithrombotics } \\
\hline No & $304: 5,224$ & 1 & \\
\hline Yes & 128: 1,322 & $1.276(0.729$ to 2.233$)$ & 0.394 \\
\hline \multicolumn{4}{|l|}{ Immunosuppressive } \\
\hline No & $422: 6,428$ & 1 & \\
\hline Yes & 8: 87 & $0.614(0.092$ to 4.097$)$ & 0.615 \\
\hline \multicolumn{4}{|l|}{ Psoriasis } \\
\hline No & 428: 6,448 & 1 & \\
\hline Yes & 3: 72 & 1.974 (0.500 to 7.796$)$ & 0.332 \\
\hline Age: increase by I & & 0.997 (0.989 to 1.005$)$ & 0.463 \\
\hline Implant diameter: increase by I & & $0.982(0.455$ to 2.118$)$ & 0.963 \\
\hline Implant length: increase by I & & $0.945(0.862$ to 1.036$)$ & 0.230 \\
\hline Implant design & & & \\
\hline Cylindrical & $631: 9,018$ & 1 & \\
\hline Conical & II: 436 & $0.798(0.357$ to 1.787$)$ & 0.584 \\
\hline Location & & & \\
\hline Maxilla & 473: 5,303 & 1 & \\
\hline Mandible & $169: 4,151$ & 0.701 (0.48I to 1.021$)$ & 0.064 \\
\hline Anterior & 390: 5,702 & 1 & \\
\hline Posterior & $252: 3,752$ & 1.001 (0.708 to I.4I4) & 0.997 \\
\hline
\end{tabular}


Table 4. (continued)

\begin{tabular}{|c|c|c|c|}
\hline Factor & Failure: Survival $^{a}$ & OR $(95 \% \mathrm{Cl})$ & $P$ Value \\
\hline \multicolumn{4}{|l|}{ Bone quantity } \\
\hline$A$ & 26: 1,247 & 1 & \\
\hline B & $219: 4,482$ & $1.730(0.803$ to 3.730$)$ & 0.162 \\
\hline $\mathrm{C}$ & $200: 2,619$ & 2.255 (1.042 to 4.879$)$ & 0.039 \\
\hline $\mathrm{D}$ & $126: 735$ & $4.714(2.050$ to 10.840$)$ & $<0.001$ \\
\hline $\mathrm{E}$ & 48: 122 & $10.777(4.221$ to $27.5 \mid 8)$ & $<0.001$ \\
\hline \multicolumn{4}{|l|}{ Bone quality } \\
\hline I & 15: 37| & I & \\
\hline 2 & $175: 4,089$ & $0.674(0.213$ to 2.139$)$ & 0.504 \\
\hline 3 & 298: 4,234 & $0.952(0.317$ to 2.861$)$ & 0.930 \\
\hline 4 & $131: 511$ & $3.399(1.097$ to 10.537$)$ & 0.034 \\
\hline \multicolumn{4}{|l|}{ Reoperation } \\
\hline No & 596: 9,326 & 1 & \\
\hline Yes & 46: 128 & $0.824(0.084$ to $8.07 \mathrm{I})$ & 0.868 \\
\hline \multicolumn{4}{|l|}{ Implant surface } \\
\hline Turned & 493: 5,255 & 1 & \\
\hline Enlarged & 149: 4,199 & $0.494(0.34 \mid$ to 0.716$)$ & $<0.001$ \\
\hline \multicolumn{4}{|l|}{ Implant system } \\
\hline Nobel turned & 493: 5,255 & 1 & \\
\hline Nobel TiUnite & $102: 2,818$ & 0.501 ( 0.326 to 0.768$)$ & 0.002 \\
\hline Astra TiOblast & 24: 367 & $0.752(0.314$ to 1.798$)$ & 0.522 \\
\hline Astra Osseospeed & 4: 204 & $0.266(0.041$ to 1.749$)$ & 0.168 \\
\hline Straumann & 9: 325 & 0.324 (0.084 to I.248) & 0.101 \\
\hline XIVE/Frialit-2 & 6: 260 & $0.579(0.185$ to 1.813$)$ & 0.348 \\
\hline Other & 4: 226 & 0.373 (0.094 to 1.417$)$ & 0.159 \\
\hline \multicolumn{4}{|l|}{ Antibiotics } \\
\hline No & 47: 574 & I & \\
\hline Yes & 242: 4,309 & $0.773(0.410$ to 1.458$)$ & 0.427 \\
\hline \multicolumn{4}{|l|}{ Bone grafting } \\
\hline No & 550: 8,980 & I & \\
\hline Yes & $91: 473$ & $2.274(1.226$ to 4.216$)$ & 0.009 \\
\hline \multicolumn{4}{|c|}{ Reason for tooth extraction } \\
\hline Periodontal disease & 52: 892 & I & \\
\hline Fracture/trauma & 27: 558 & $1.006(0.331$ to 3.060$)$ & 0.991 \\
\hline Advanced caries & 9: 236 & $1.453(0.389$ to 5.424$)$ & 0.579 \\
\hline Agenesia & 25: 791 & $2.152(0.888$ to 5.216$)$ & 0.102 \\
\hline Other & 6: 199 & $1.129(0.245$ to 5.206$)$ & 0.876 \\
\hline
\end{tabular}

$95 \% \mathrm{Cl}, 95 \%$ confidence interval; OR, odds ratio.

${ }^{\mathrm{a}}$ The information for some conditions is unknown for a variable number of implants.

remain negatively associated with not only clinical depression but also depressive symptoms (Robbins et al. 2001; Williams et al. 2011). Bone quality has also been shown to be reduced among men and younger women with a history of mood disorders (Williams et al. 2013).

In some studies, smoking has been associated with depression (Escobedo et al. 1998; Hall et al. 1993). In the present study, smoking was identified as the other predictor to exert some statistically significant effect on the failures up to abutment connection. A recent meta-analysis analyzing $>100$ studies has shown that failures of implants inserted into smokers are 2.23 times more likely to happen than failures of implants inserted into nonsmokers (Chrcanovic et al. 2015). The increase of implant failure rates due to smoking is hypothesized to be related mainly to the effect of smoking in osteogenesis and angiogenesis (Ma et al. 2010). Concerning the effects on osteogenesis, Ma et al. (2011) showed that nicotine inhibited the gene expression of BMP-2, TGF-b1, PDGF-AA, and
VEGF in osteoblasts. BMP-2 is the most potent osteogenic induction factor regulating osteoblast differentiation, ALP expression, and subsequent mineralization (Rawadi et al. 2003). TGF-b1 is produced by osteoblasts and incorporated into the bone matrix. During bone remodeling, TGF-b1 plays an important role in the regulation of osteoblast proliferation, differentiation, and apoptosis, with subsequent important effects on bone formation and remodeling (Deng et al. 2008). PDGF and VEGF have angiogenic effects during bone healing (De la Riva et al. 2010). Whereas their expression can be detected in osteoblasts, they are considered to be able to regulate osteoblast activity as well. VEGF can interact synergistically with bone morphogenetic protein to promote skeletal development and bone healing by enhancing cell recruitment, prolonging cell survival, and increasing angiogenesis (Patel et al. 2008). Bone morphogenetic protein acts as an important regulator that stimulates production of VEGF in osteoblasts (Samee et al. 2008). Therefore, the inhibitive effect of nicotine 
on osteoblastic activity may contribute to the failure of dental implant osseointegration (Ma et al. 2011).

In addition, some studies showed that osteogenesis and angiogenesis are tightly coupled during bone formation and that angiogenesis plays a pivotal role in skeletal development and bone repair (Fang et al. 2005). Besides carrying oxygen and nutrients to bone tissue, blood flow plays an active role in bone formation and remodeling by mediating the interactions among osteoblasts, osteocytes, osteoclasts, and vascular cells at a variety of levels (Fleming et al. 2001). The deleterious effects of smoking have been shown on not only osteoblasts but also microcirculation-including morphologic aspects, particularly vessel wall injury and capillary loss, as well as functional aspects, predominantly changes in tissue perfusion and its regulatory mechanisms, notable reactive hyperemia, and sequestration of blood cells in the microcirculation (Lehr 2000). Studies (Ma et al. 2007; Ma et al. 2010) demonstrated that nicotine exposure has direct effects on blood vessels, producing vasoconstriction and systemic venoconstriction, which decrease blood perfusion and cause low oxygen and ischemia - the major stimulus for initiating the angiogenic cascade (Wang et al. 2007). Hypoxia and ischemia owing to nicotine exposure could stimulate HIF- $1 \alpha$ expression, leading to an increased expression of VEGF, which in turn stimulates angiogenesis. However, the enhanced vessel formation is incapable of compensating for the adverse effect of the reduced blood flow possibly caused by nicotine-induced vasoconstriction (Ma et al. 2010). Even though the increased expression of VEGF caused by hypoxia and ischemia may stimulate angiogenesis, it may also contribute to compromised bone healing because excessive VEGF may lead to impairment in bone formation - possibly by promoting mesenchymal stem cell differentiation toward an endothelial lineage (Kon et al. 2001), consequently reducing the availability of mesenchymal stem cells for osteogenic differentiation (Keramaris et al. 2008). Alternatively, excessive VEGF may increase recruitment of osteoclasts into the bone regeneration sites and lead to an excessive bone resorption (Keramaris et al. 2008). Another fact that should be taken into consideration is that the inflammatory response to bone trauma plays an important role in initiating the repair cascade. The trauma activates downstream factors (e.g., cytokines and growth factors) that recruit osteoprogenitor and mesenchymal cells to the injury site (Kon et al. 2001; Mountziaris and Mikos 2008). The problem is that nicotine is an anti-inflammatory agent (Geng et al. 1996).

Concerning the bone quantity and quality of the implant site, as the distribution of implants that failed up to abutment connection was similar to the implants failing after this procedure, poor bone was not likely a determinant factor to influence an early failure for the patients of the present study.

The limitations of the present study include that it is a retrospective study, which inherently results in flaws. These problems were manifested by the gaps in information and incomplete records. As all data rely on the accuracy of the original examination and documentation, items may have been excluded in the initial examination or not recorded in the dental/ medical chart. The lack of specific information characterizing
Table 5. Multivariate Generalized Estimating Equations Logistic Regression Model at the Implant Level.

\begin{tabular}{|c|c|c|}
\hline Factor & OR $(95 \% \mathrm{Cl})$ & $P$ Value \\
\hline \multicolumn{3}{|l|}{ Smoking } \\
\hline No & 1 & \\
\hline Yes & $2.120(1.113$ to 4.037$)$ & 0.022 \\
\hline Former smoker & 2.129 (0.338 to $13.41 \mathrm{I})$ & 0.421 \\
\hline Cigarettes/day: increase by I & 0.987 (0.952 to I.023) & 0.463 \\
\hline \multicolumn{3}{|l|}{ Hypothyroidism } \\
\hline No & 1 & \\
\hline Yes & $0.508(0.13 \mid$ to 1.966$)$ & 0.327 \\
\hline \multicolumn{3}{|l|}{ Intake of antidepressants } \\
\hline No & 1 & \\
\hline Yes & 1.742 (0.856 to 3.542$)$ & 0.126 \\
\hline \multicolumn{3}{|l|}{ Location } \\
\hline Maxilla & 1 & \\
\hline Mandible & $0.805(0.421$ to 1.537$)$ & 0.510 \\
\hline \multicolumn{3}{|l|}{ Bone quantity } \\
\hline A & 1 & \\
\hline B & $2.838(0.779$ to 10.343$)$ & 0.114 \\
\hline C & $2.538(0.670$ to 9.621$)$ & 0.171 \\
\hline$D$ & 3.621 (0.804 to I6.308) & 0.094 \\
\hline $\mathrm{E}$ & 2.197 (0.267 to 18.052) & 0.464 \\
\hline \multicolumn{3}{|l|}{ Bone quality } \\
\hline 1 & 1 & \\
\hline 2 & $1.176(0.168$ to 8.233$)$ & 0.870 \\
\hline 3 & $1.471(0.202$ to 10.696$)$ & 0.703 \\
\hline 4 & $4.679(0.591$ to 37.050$)$ & 0.144 \\
\hline \multicolumn{3}{|l|}{ Implant surface } \\
\hline Turned & 1 & \\
\hline Enlarged & $0.642(0.339$ to 1.213$)$ & 0.172 \\
\hline \multicolumn{3}{|l|}{ Implant system } \\
\hline Nobel turned & 1 & \\
\hline Nobel TiUnite & $0.642(0.339$ to 1.213$)$ & 0.172 \\
\hline Astra TiOblast & $0.602(0.152$ to 2.380$)$ & 0.469 \\
\hline Astra Osseospeed & $0.515(0.065$ to 4.098$)$ & 0.530 \\
\hline Straumann & 0.311 ( 0.040 to 2.397$)$ & 0.262 \\
\hline XIVE/Frialit-2 & $1.339(0.264$ to 6.800$)$ & 0.725 \\
\hline Others & $0.316(0.048$ to 2.094$)$ & 0.232 \\
\hline \multicolumn{3}{|l|}{ Bone grafting } \\
\hline No & 1 & \\
\hline Yes & $1.680(0.805$ to 3.507$)$ & 0.167 \\
\hline
\end{tabular}

Only the factors that were considered statistically significant $(P<0 . I)$ in the univariate model and did not present multicollinearity were included in the multivariate model.

$95 \% \mathrm{Cl}, 95 \%$ confidence interval; OR, odds ratio.

the statuses of the patients' systemic conditions and the dosages of their medications is a limitation also connected to the retrospective nature of this study. It is also important to stress that the different classes of antidepressants were prescribed to patients of the present study. Antidepressants are a large family of drugs, which have different biological properties and mechanisms of action that might have differently affected the bone metabolism and, consequently, the failures of dental implants. The present results might be applicable to the general population, since all patients ever treated with dental implants in the clinic were included in the database from which the implants with early failures were analyzed. No patient was excluded, regardless of any health or other condition. For that reason, selection bias was also minimized. 


\section{Conclusion}

Smoking and the intake of antidepressants are suggested to be potential influencing factors to the occurrence of implant failures up to abutment connection.

\section{Author Contributions}

B.R. Chrcanovic, contributed to conception and design, data acquisition, analysis, and interpretation, drafted and critically revised the manuscript; J. Kisch, T. Albrektsson, and A. Wennerberg, contributed to conception and design, data interpretation, critically revised the manuscript. All authors gave final approval and agree to be accountable for all aspects of the work.

\section{Acknowledgments}

This work was supported by research funds from the Oral Health Related Research by Region Skåne (Odontologisk Forskning i Region Skåne; OFRS 414321), Sweden, and from the Scientific Research Council of Sweden (Vetenskapsrådet; Dnr 2015-02971). This work was supported by Folktandvården $\mathrm{AB}$, Region Skåne, Sweden, and by $\mathrm{CNPq}$, Conselho Nacional de Desenvolvimento Científico e Tecnológico, Brazil. The authors declare no potential conflicts of interest with respect to the authorship and/or publication of this article.

\section{References}

Alsaadi G, Quirynen M, Komárek A, van Steenberghe D. 2007. Impact of local and systemic factors on the incidence of oral implant failures, up to abutment connection. J Clin Periodontol. 34(7):610-617.

Bab IA, Einhorn TA. 1993. Regulatory role of osteogenic growth polypeptides in bone formation and hemopoiesis. Crit Rev Eukaryot Gene Expr. 3(1):31-46.

Battaglino R, Fu J, Späte U, Ersoy U, Joe M, Sedaghat L, Stashenko P. 2004. Serotonin regulates osteoclast differentiation through its transporter. J Bone Miner Res. 19(9):1420-1431.

Bliziotes MM, Eshleman AJ, Zhang XW, Wiren KM. 2001. Neurotransmitter action in osteoblasts: expression of a functional system for serotonin receptor activation and reuptake. Bone. 29(5):477-486.

Cauley JA, Fullman RL, Stone KL, Zmuda JM, Bauer DC, Barrett-Connor E, Ensrud K, Lau EM, Orwoll ES; Mr. OS Research Group. 2005. Factors associated with the lumbar spine and proximal femur bone mineral density in older men. Osteoporos Int. 16(12):1525-1537.

Chrcanovic BR, Albrektsson T, Wennerberg A. 2014. Reasons for failures of oral implants. J Oral Rehabil. 41(6):443-476.

Chrcanovic BR, Albrektsson T, Wennerberg A. 2015. Smoking and dental implants: a systematic review and meta-analysis. J Dent. 43(5):487-498.

De la Riva B, Sánchez E, Hernández A, Reyes R, Tamimi F, López-Cabarcos E, Delgado A, Evora C. 2010. Local controlled release of VEGF and PDGF from a combined brushite-chitosan system enhances bone regeneration. J Control Release. 143(1):45-52.

Deng ZL, Sharff KA, Tang N, Song WX, Luo J, Luo X, Chen J, Bennett E, Reid $\mathrm{R}$, Manning D, et al. 2008. Regulation of osteogenic differentiation during skeletal development. Front Biosci. 13:2001-2021.

Ducy P, Karsenty G. 2010. The two faces of serotonin in bone biology. J Cell Biol. 191(1):7-13.

Ershler WB, Harman SM, Keller ET. 1997. Immunologic aspects of osteoporosis. Dev Comp Immunol. 21(6):487-499.

Escobedo LG, Reddy M, Giovino GA. 1998. The relationship between depressive symptoms and cigarette smoking in US adolescents. Addiction. 93(3):433-440.

Fang TD, Salim A, Xia W, Nacamuli RP, Guccione S, Song HM, Carano RA, Filvaroff EH, Bednarski MD, Giaccia AJ, et al. 2005. Angiogenesis is required for successful bone induction during distraction osteogenesis. J Bone Miner Res. 20(7):1114-1124.

Fleming JT, Barati MT, Beck DJ, Dodds JC, Malkani AL, Parameswaran D, Soukhova GK, Voor MJ, Feitelson JB. 2001. Bone blood flow and vascular reactivity. Cells Tissues Organs. 169(3):279-284

Geng Y, Savage SM, Razani-Boroujerdi S, Sopori ML. 1996. Effects of nicotine on the immune response: II. Chronic nicotine treatment induces T cell anergy. J Immunol. 156(7):2384-2390.
Gruber HE. 1991. Bone and the immune system. Proc Soc Exp Biol Med. 197(3):219-225

Gustafsson BI, Thommesen L, Stunes AK, Tommeras K, Westbroek I, Waldum HL, Slordahl K, Tamburstuen MV, Reseland JE, Syversen U. 2006. Serotonin and fluoxetine modulate bone cell function in vitro. $\mathrm{J} \mathrm{Cell}$ Biochem. 98(1):139-151.

Hall SM, Muñoz RF, Reus VI, Sees KL. 1993. Nicotine, negative affect, and depression. J Consult Clin Psychol. 61(5):761-767.

Keramaris NC, Calori GM, Nikolaou VS, Schemitsch EH, Giannoudis PY. 2008. Fracture vascularity and bone healing: a systematic review of the role of VEGF. Injury. 39 Suppl 2:S45-S57.

Kon T, Cho TJ, Aizawa T, Yamazaki M, Nooh N, Graves D, Gerstenfeld LC, Einhorn TA. 2001. Expression of osteoprotegerin, receptor activator of NF-kappaB ligand (osteoprotegerin ligand) and related proinflammatory cytokines during fracture healing. J Bone Miner Res. 16(6):1004-1014.

Lehr HA. 2000. Microcirculatory dysfunction induced by cigarette smoking. Microcirculation. 7(6 Pt 1):367-384.

Lekholm U, Zarb GA. 1985. Patient selection and preparation.In:Brånemark PI, Zarb GA, Albrektsson T, editors. Tissue integrated prostheses: osseointegration in clinical dentistry. Chicago (IL): Quintessence Publishing integration in clinical

Ma L, Zheng LW, Cheung LK. 2007. Inhibitory effect of nicotine on bone regeneration in mandibular distraction osteogenesis. Front Biosci. 12:3256-3262.

Ma L, Zheng LW, Sham MH, Cheung LK. 2010. Uncoupled angiogenesis and osteogenesis in nicotine-compromised bone healing. J Bone Miner Res. 25(6): 1305-1313.

Ma L, Zwahlen RA, Zheng LW, Sham MH. 2011. Influence of nicotine on the biological activity of rabbit osteoblasts. Clin Oral Implants Res. 22(3):338-342.

Mountziaris PM, Mikos AG. 2008. Modlati ountziaris PM, Mikos AG. 2008. Modulation of the inflammatory response fo enhanced bone tissue regeneration. Tissue Eng Part B Rev. 14(2):179-186. Patel ZS, Young S, Tabata Y, Jansen JA, Wong ME, Mikos AG. 2008. Dual
delivery of an angiogenic and an osteogenic growth factor for bone regendelivery of an angiogenic and an osteogenic growth factor fo
eration in a critical size defect model. Bone. 43(5):931-940.

eration in a critical size defect model. Bone. 43(5):931-940.
Rawadi G, Vayssière B, Dunn F, Baron R, Roman-Roman S. 2003. BMP-2 Rawadi G, Vayssière B, Dunn F, Baron R, Roman-Roman S. 2003. BMP-2 controls alkaline phosphatase expression and osteoblast min
Wnt autocrine loop. J Bone Miner Res. 18(10):1842-1853.

Richards JB, Papaioannou A, Adachi JD, Joseph L, Whitson HE, Prior JC Goltzman D; Canadian Multicentre Osteoporosis Study Research Group. 2007. Effect of selective serotonin reuptake inhibitors on the risk of fracture. Arch Intern Med. 167(2):188-194.

Robbins J, Hirsch C, Whitmer R, Cauley J, Harris T. 2001. The association of bone mineral density and depression in an older population. J Am Geriat Soc. 49(6): 732-736

Samee M, Kasugai S, Kondo H, Ohya K, Shimokawa H, Kuroda S. 2008. Bone morphogenetic protein-2 (BMP-2) and vascular endothelial growth factor (VEGF) transfection to human periosteal cells enhances osteoblast differentiation and bone formation. J Pharmacol Sci. 108(1): 18-31.

Shintani A. 2014. Primer of statistics in dental research: part II. J Prosthodont Res. 58(2):85-91.

Wang Y, Wan C, Deng L, Liu X, Cao X, Gilbert SR, Bouxsein ML, Faugere MC, Guldberg RE, Gerstenfeld LC, et al. 2007. The hypoxia-inducible factor alpha pathway couples angiogenesis to osteogenesis during skeletal development. J Clin Invest. 117(6):1616-1626.

Warden SJ, Nelson IR, Fuchs RK, Bliziotes MM, Turner CH. 2008. Serotonin (5-hydroxytryptamine) transporter inhibition causes bone loss in adult mice independently of estrogen deficiency. Menopause. 15(6):1176-1183.

Warden SJ, Robling AG, Sanders MS, Bliziotes MM, Turner CH. 2005. Inhibition of the serotonin (5-hydroxytryptamine) transporter reduces bone accrual during growth. Endocrinology. 146(2):685-693.

Westbroek I, van der Plas A, de Rooij KE, Klein-Nulend J, Nijweide PJ. 2001 Expression of serotonin receptors in bone. J Biol Chem. 276(31):2896128968.

Williams LJ, Bjerkeset O, Langhammer A, Berk M, Pasco JA, Henry MJ, Schei B, Forsmo S. 2011. The association between depressive and anxiety symptoms and bone mineral density in the general population: the HUNT Study. toms and bone mineral density in the

Williams LJ, Henry MJ, Berk M, Dodd S, Jacka FN, Kotowicz MA, Nicholson GC, Pasco JA. 2008. Selective serotonin reuptake inhibitor use and bone mineral density in women with a history of depression. Int Clin Psychopharmacol. 23(2):84-87.

Williams LJ, Pasco JA, Jacka FN, Hodge JM, Kotowicz MA, Berk M. 2013. Quantitative heel ultrasound (QUS) measures of bone quality in association with mood and anxiety disorders. J Affect Disord. 146(3):395-400.

Yadav VK, Ryu JH, Suda N, Tanaka KF, Gingrich JA, Schutz G, Glorieux FH, Chiang CY, Zajac JD, Insogna KL, et al. 2008. Lrp5 controls bone formation by inhibiting serotonin synthesis in the duodenum. Cell. 135(5):825 837. 


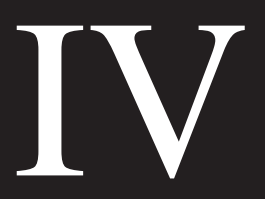





\section{CLINICAL ORAL IMPLANTS RESEARCH}

\author{
Bruno Ramos Chrcanovic \\ Jenö Kisch \\ Tomas Albrektsson \\ Ann Wennerberg
}

\section{Bruxism and dental implant treatment complications: a retrospective comparative study of 98 bruxer patients and a matched group}

Authors' affiliations:

Bruno Ramos Chrcanovic, Tomas Albrektsson, Ann Wennerberg, Department of Prosthodontics, Faculty of Odontology, Malmö University, Malmö, Swede

Ienö Kisch, Clinic for Prosthodontics, Centre of Dental Specialist Care, Malmö, Sweden Tomas Albrektsson, Department of Biomaterials, Göteborg University, Göteborg, Sweden

\section{Corresponding author:}

Bruno Ramos Chrcanovic, DDS, MSc

Department of Prosthodontics, Faculty of

Odontology

Malmö University

Carl Gustafs väg 34, SE-214 21, Malmö, Sweden

Tel.: +46725 541545

Fax: +46406658503

e-mails: bruno.chrcanovic@mah.se,

brunochrcanovic@hotmail.com
Date:

Accepted 6 March 2016

To cite this article:

Chrcanovic BR, Kisch J, Albrektsson T, Wennerberg A. retrospective comptimplant treatment complications: a matched group.

Clin. Oral Impl. Res. 00, 2016, 1

doi: $10.1111 / \mathrm{cl}$. 12844

Key words: bruxism, dental implant, implant failure, mechanical complications, risk factor

Abstract

Objectives: To analyze the complications of dental implant treatment in a group of patients with bruxism in comparison with a matched group of non-bruxers.

Material and methods: Patients being diagnosed as bruxers were identified within a group of patients consecutively treated with implant-supported prostheses at one specialist clinic, based on the most recent listed sign and symptoms of bruxism according to the International Classification of Sleep Disorders. A diagnostic grading system of "possible," "probable," and "definite" sleep or awake bruxism was used, according to a recent published international consensus. A case-control matching model was used to match the bruxers with a group of non-bruxers, based on five variables. Implant-, prosthetic-, and patient-related data were collected, as well as 14 mechanical complications, and compared between groups.

Results: Ninety-eight of 2670 patients were identified as bruxers. The odds ratio of implant failure in bruxers in relation to non-bruxers was 2.71 ( $95 \% \mathrm{Cl} 1.25,5.88)$. Considering the same number of patients with the same total number of implants equally distributed between groups, the bruxers group had a higher prevalence of mechanical complications in comparison with the non-bruxers group.

Conclusions: This study suggests that bruxism may significantly increase both the implant failure rate and the rate of mechanical and technical complications of implant-supported restorations. Other risk factors may also have influenced the results.

Bruxism is a repetitive jaw-muscle activity that is mainly characterized by teeth grinding and clenching, during sleep as well as during wakefulness (Lobbezoo et al. 2013, AASM 2014). It is reported that its prevalence could reach $20 \%$ among the adult population (Glaros 1981). Bruxism has been generally considered a clinical problem with possible detrimental consequences for dental, periodontal, and musculoskeletal tissues (Lobbezoo et al. 2006b|. These possible consequences made bruxism to be considered a contraindication for dental implant treatment, even though the evidence for this is usually based on clinical experience only (Lobbezoo et al. 2006a, b) Bruxism is suggested to cause excessive load of implant-supported rehabilitations, which may cause an implant fracture or may result in bone loss around the implants and subsequent implant failure.

The results of a recent meta-analysis on the subject (Chrcanovic et al. 2015a) found that the insertion of dental implants in patients being diagnosed with bruxism negatively affected the implant failure rates. However, the authors stressed that the effect size observed was not reliable due to a limited number of published studies, all characterized by a low level of specificity, and most of them dealing with a limited number of cases. Therefore, the real effect of bruxism on the survival of dental implants is still not well established.

The ability to anticipate outcomes is an essential part of risk management in an implant practice. Recognizing conditions that place the patient at a higher risk of failure will allow the surgeon to make informed decisions and refine the treatment plan to optimize the outcomes (Chrcanovic et al. 2014). Having said this and considering that several publications refer to bruxism as a risk factor capable of exerting a negative effect on the successful outcome of the long-term 
osseointegration of implants and on the outcome of implant-supported rehabilitations, the aim of this retrospective clinical study was to analyze the complications of dental implant treatment in a group of patients with bruxism in comparison with a matched group of non-bruxers.

\section{Material and methods}

\section{Aim of the study}

The aim of this retrospective study was to analyze the complications of dental implant treatment in a group of patients presenting bruxism in comparison with a matched group of non-bruxers. The focused question was elaborated using the PICO format (Participants, Interventions, Comparisons, Outcomes): "Do bruxers undergoing implant-prosthetic rehabilitation present a higher risk for implant failure and mechanical complications in comparison with patients not presenting bruxism?"

\section{Definitions}

Late biological complications were defined as pathological bone loss after osseointegration was obtained at an earlier stage, with possible subsequent implant failure. Implant failure represents the complete loss of the implant. Mechanical complications were defined as failure of one or more components of an implant system: fracture of an implant itself, loosening, loss or fracture of connecting screws or abutment screws, loosening, excessive wear or fracture of structural components in overdentures, excessive wear or fracture of suprastructural porcelain or acrylic teeth (Gothberg et al. 2003), hard splint fracture, and loss of implant's hole composite sealing.

For this study, the authors followed the definition of bruxism proposed by Lobbezoo et al. (2013): "Bruxism is a repetitive jawmuscle activity characterized by clenching or grinding of the teeth and/or by bracing or thrusting of the mandible. Bruxism has two distinct circadian manifestations: it can occur during sleep (indicated as sleep bruxism) or during wakefulness (indicated as awake bruxism)." As reliable and valid diagnostic tools for bruxism are scarce (Lobbezoo et al. 2013), a diagnostic grading system of "possible," "probable," and "definite" sleep or awake bruxism was used, as suggested for clinical and research purposes (Treede et al. 2008). According to an international consensus (Lobbezoo et al. 2013), "possible" sleep or awake bruxism should be based on self- report, by means of questionnaires and/or the anamnestic part of a clinical examination. "Probable" sleep or awake bruxism should be based on self-report plus the inspection part of a clinical examination. "Definite" sleep bruxism should be based on self-report, a clinical examination, and a polysomnographic recording, preferably along with audio/video recordings. As electromyography and/or polysomnography were not used due to high cost and limited availability, the patients of this study would only fall into the categories "possible" or "probable". Thus, a patient was considered as presenting bruxism based on self-report of clenching/grinding during sleep or during wakefulness, plus the inspection part of a clinical examination.

\section{Patients}

This retrospective study is based on all 2670 patients (1434 women, $1236 \mathrm{men}$ ) consecutively treated with implant-supported prostheses between 1980 and 2014 at one specialist clinic /Clinic for Prosthodontics, Centre of Dental Specialist Care, Malmö, Sweden). The study was approved by the Regional Ethical Review Board in Lund (Dnr 2014/598; Dnr 2015/72).

First, the patients' records were scrutinized looking for clinical notes and photographs that would suggest the possible/probable diagnosis of bruxism. All patients who were still alive and were diagnosed in the records as bruxers were considered for a clinical re-evaluation. The patients were contacted through a telephone call and were asked to attend a follow-up appointment.

\section{Patients willing to attend a follow-up visit}

The patients willing to come back signed an informed and written consent form approving the participation in the study and were clinically re-assessed. The self-conscience of the condition was evaluated with some questions, according to suggestions of a previous study (Paesani et al. 2013), questions like the following were asked: (i) Are you aware of the fact that you grind your teeth during sleep? (ii) Did anyone tell you that you grind your teeth during sleep? (iii) On morning awakening or on awakenings during the night, do you have your jaws thrust or braced? (iv) Do you clench your teeth while awake? (v) Do you grind your teeth while awake? All questions could be answered with either "yes" or "no". The patients were instructed to answer "yes" if considered their habit to be frequent enough to be clinically relevant (e.g. frequency of more than thrice a week and/or several hours per day) (Paesani et al. 2013). The clinical condition was re-evaluated to assess possible bruxismrelated signs and symptoms. The sign and symptoms of bruxism were listed according to the International Classification of Sleep Disorders (AASM 2014): (i) presence of regular or frequent tooth grinding sounds occurring during sleep, (ii) abnormal tooth wear consistent with above reports of tooth grinding during sleep, (iii) transient morning jaw muscle pain or fatigue; and/or temporal headache; and/or jaw locking on awakening consistent with above reports of tooth grinding during sleep. Moreover, clenching or grinding of the teeth and/or by bracing or thrusting of the mandible during wakefulness was also considered, according to a recent international consensus (Lobbezoo et al. 2013). The clinical examination was carried out by the same trained operator.

Patients not willing to attend a follow-up visit

The patients who were not willing to attend an additional visit were asked permission for the use of the information present in their records and were asked the same questions listed above. The answers were correlated with the clinical notes/findings registered in the patients' records.

\section{Deceased patients}

The diagnosis of bruxism in patients who were already deceased was based on notes of clinical assessment and photographs, present in the patients' records. Only patients with records showing a strong correlation between these two items were considered as possibly/ probably having the diagnosis of bruxism. Access to the patients' information was granted and approved by the Ethical Committee, provided that would not represent a threat to the patients' confidentiality.

The patients were not identifiable in any way, and a decoding list linking patient names and numbers was used and stored by the principal investigator, which was destroyed after completion of the study.

\section{Data collection}

Information on mechanical complications was collected for the implant restorations only, even though the patients may have had mechanical complications on prostheses made on natural teeth.

The following data were collected: implant surface /turned/machined or roughened surfaces, the latter including sandblasted, acidetched, sandblasted + acid-etched, anodized, hydroxyapatite-coated surfaces), implant length and diameter, implant jaw location 
(maxilla/mandible), anterior or posterior location of the implant (locations between 13-23 and 33-43 were considered anterior location), patient's sex, age of the patient at the implant insertion surgery, number of days until failure, follow-up time, prosthetic complications of mechanical nature, type of implant-supported prosthetic restoration (single crown, partial bridge with 2-6 prosthetic units, partial bridge with $7-10$ prosthetic units, full-arch, overdenture), and the number of bruxers with a hard stabilization splint for nightly use. Bone quantity and quality of the treated jaws were classified at the time of surgery according to Lekholm \& Zarb (1985) classification.

The general health and the behavioral history of the patients were collected from the patients' files. The presence of a medicament list in the patients' records was also use to correlate the use of certain drugs to specific health conditions. The following health fac tors were assessed: diabetes types I or II, hypertension, hypercholesterolemia, hypothy roidism, asthma, psoriasis, chemotherapy, and irradiation of the head-neck region. The patients were also classified according to the intake of the following medication types: antidepressants, immunosuppressive drugs, bisphosphonates, antithrombotic agents (antiplatelet, anticoagulant, thrombolytic drugs hormone replacement therapy in women, and medicaments to reduce the acid gastric production. The following behavioral factors were assessed: smoking habits and the use of snuff.

\section{Formation of a matched group}

As the division of all initial patients into groups would generate extremely unbalanced groups and the variance was not homogenous between them, the two groups were therefore not expected to be comparable with respect to important covariates (D'Agostino 1998), and then methods were used to match patients and implants between bruxers to non-bruxers patients. Matching ensures that any differences between the study and the control groups are not a result of differences on the matching variables, thus reducing selection bias.

From the group of patients not presenting bruxism, a control group was included with the same number of patients as in the study group. The matching was performed using the "case control matching" function in SPSS, and the matches were selected on the basis of similarities in (i) patients' gender, (ii) patients' age at the time of the surgery, (iii) total follow-up time, (iv) number of implants in the maxilla, and $(\mathrm{v})$ number of implants in the mandible. As there were no perfect matches in a first matching attempt considering all five variables, some tolerance was set for four predictors: \pm 7 years for the patients' age, \pm 500 days for the total followup time, and \pm 2 implants in maxilla and mandible. Thus, a little variance of these predictors between the groups was expected. In the case of the patients' gender, the number of males and females in each group was set to be equal.

\section{Statistical analyses}

The mean, standard deviation, and percentages were presented as descriptive statistics. Kolmogorov-Smirnov test was performed to evaluate the normal distribution of the variables, and Levene's test evaluated homoscedasticity. The performed tests for two independent groups were Student's $t$-tes or Mann-Whitney test, and paired-samples $t$-test or Wilcoxon signed-rank test for two dependent groups, depending on the normality. Pearson's chi-square test or Fisher's exact test was used in the analysis of contingency tables of categorical data of independen groups, and McNemar's test for dependent groups. The degree of statistical significance was considered $P<0.05$. All data were statistically analyzed using the Statistical Package for the Social Sciences (SPSS) version 22 software (SPSS Inc., Chicago, IL, USA).

\section{Results}

Ninety-nine patients were initially identified with the condition. As one patient diagnosed once as bruxer did not fulfill what we established as a person with the habit, 98 of the 2670 patients were identified as bruxers. The information of being a bruxers or non-bruxer was ultimately available for 1915 patients, which represents $5.12 \%$ of this population. All patients diagnosed with bruxism reported that they already had the habit prior to implant treatment. Eleven (75 implants) of the 98 patients were no longer alive at the time of the study data collection, and three patients had moved out of the country. The remaining 84 patients agreed to participate in

\section{Table 1. Number of implants placed in each region, in bruxers and non-bruxers}

\begin{tabular}{lccl}
\hline Region & Bruxers $n(\%)$ & Non-bruxers $n(\%)$ & Total $n(\%)$ \\
\hline Anterior maxilla & $168(39.4)$ & $148(34.7)$ & $316(37.0)$ \\
Anterior mandible & $41(9.6)$ & $102(23.9)$ & $143(16.7)$ \\
Posterior maxilla & $118(27.6)$ & $96(22.5)$ & $214(25.1)$ \\
Posterior mandible & $100(23.4)$ & $81(18.9)$ & $181(21.2)$ \\
Total & $427(100)$ & $427(100)$ & $854(100)$ \\
\hline
\end{tabular}

3 | Clin. Oral Impl. Res. 0, 2016/1-9 
Table 2. Implant diameter and length in bruxers and non-bruxers

\begin{tabular}{|c|c|c|c|}
\hline Implant & $\begin{array}{l}\text { Bruxers } \\
\text { mean } \pm \text { SD (median, min, max) } \\
(\mathrm{mm})\end{array}$ & $\begin{array}{l}\text { Non-bruxers } \\
\text { mean } \pm \text { SD (median, min, max) } \\
(\mathrm{mm})\end{array}$ & $P$-value* \\
\hline Diameter & $3.79 \pm 0.23(3.75,3.0,5.0)$ & $3.75 \pm 0.15(3.75,3.3,5.0)$ & 0.001 \\
\hline Length & $12.53 \pm 2.14(13.0,7.0,18.0)$ & $13.20 \pm 2.14(13.0,7.0,20.0)$ & $<0.001$ \\
\hline
\end{tabular}

Table 3. Comparison of the number of implant-supported prosthetic restorations performed between bruxers and non-bruxers

\begin{tabular}{|c|c|c|c|c|}
\hline \multirow[b]{2}{*}{$\begin{array}{l}\text { Prosthetic } \\
\text { restoration }\end{array}$} & \multicolumn{2}{|l|}{ Bruxers } & \multicolumn{2}{|l|}{ Non-bruxers } \\
\hline & $\begin{array}{l}\text { Number of } \\
\text { restorations } \\
\text { (n) }\end{array}$ & $\begin{array}{l}\text { Prosthetic elements } \\
\text { (teeth) Acrylicl } \\
\text { porcelain* }(n)\end{array}$ & $\begin{array}{l}\text { Number of } \\
\text { restorations } \\
\text { (n) }\end{array}$ & $\begin{array}{l}\text { Prosthetic } \\
\text { elements } \\
\text { (teeth) } \\
\text { Acrylic/ } \\
\text { porcelain* }(n)\end{array}$ \\
\hline Single crown & 69 & $0 / 69$ & 55 & $0 / 55$ \\
\hline $\begin{array}{l}\text { Partial fixed, } 2-6 \\
\text { prosthetic units }\end{array}$ & 53 & $11 / 173$ & 38 & $11 / 104$ \\
\hline $\begin{array}{l}\text { Partial fixed, } 7-10 \\
\text { prosthetic units }\end{array}$ & 3 & $9 / 16$ & 3 & $17 / 7$ \\
\hline Full-arch fixed & 26 & $254 / 30$ & 45 & $436 / 73$ \\
\hline Overdenture & 1 & $12 / 0$ & 3 & $36 / 0$ \\
\hline Total & 152 & $286 / 288$ & 144 & $500 / 239$ \\
\hline
\end{tabular}

Table 4. Comparison of survival and failure of implants between bruxers and non-bruxers at the patient level, based on all patients of the database

\begin{tabular}{|c|c|c|c|c|c|}
\hline Implants & Bruxers $n(\%)$ & Non-bruxers $n(\%)$ & $P$-value & Unknown* & Total \\
\hline No implant failures & $73(74.5)$ & $1618(89.0)$ & & $653(86.5)$ & $2344(87.8)$ \\
\hline At least one implant failure & $25(25.5)$ & $199(11.0)$ & $<0.001^{* *}$ & $102(13.5)$ & $326(12.2)$ \\
\hline Odds ratio $(95 \% \mathrm{Cl})^{* * * *}$ & \multicolumn{2}{|c|}{$2.78(1.73,4.49)$} & & & \\
\hline Total & $98(100)$ & $1817(100)$ & & $755(100)$ & $2670(100)$ \\
\hline \multicolumn{6}{|c|}{$\begin{array}{l}\text { Bruxers, } 427 \text { implants in total ( } 342 \text { survived, } 69 \text { failed due to lose/lack of osseointegration, } 16 \\
\text { implants fractured). } \\
\text { Non-bruxers, } 6681 \text { implants in total ( } 6320 \text { survived, } 347 \text { failed due to lose/lack of osseointegration, } \\
14 \text { implants fractured). } \\
\text { Unknown for the condition, } 2988 \text { implants in total ( } 2792 \text { survived, } 186 \text { failed due to lose/lack of } \\
\text { osseointegration, } 10 \text { implants fractured). } \\
\text { * Unknown for the condition of bruxism. } \\
\text { **Pearson's chi-square test. } \\
\text { ***The odds ratio considered all failures (lost/lack of osseointegration + fractures) into account. }\end{array}$} \\
\hline
\end{tabular}

same number of implants as in some fullarch fixed rehabilitations.

Table 4 shows a comparison of the survival/failure rates in the group of bruxers and non-bruxers before matching the groups, and Table 5 shows the same comparison after matching. The odds ratios of implant failure in bruxers in relation to non-bruxers before and after the group matching were 2.78 and 2.71 , respectively. Implants in bruxers presented a statistically significantly $(P=0.001$, Wilcoxon signed-rank test) longer mean time from insertion to failure $(1840 \pm 2009$ days, median 1123, minimum 84, maximum 8840 ) in comparison with non-bruxers $634=$ 757 days, median 186 , minimum 24 , maxi mum 2295). Table 6 shows a comparison of

implant failures between bruxers and nonbruxers, according to different implant lengths, diameters, and surfaces. A higher failure rate of short implants in bruxers in comparison with longer implants was observed. The difference in implant failure rates between the groups tended to decrease as the implant length increased, that is, $25.7 \%$ vs. $2.8 \%(7-10 \mathrm{~mm}$ long implants $18.4 \%$ vs. $3.3 \%$ (11-13 mm long implants), and $17.3 \% \quad$ vs. $4.2 \% \quad(15-20 \mathrm{~mm}$ long implants), in bruxers vs. non-bruxers, respectively. Moreover, a lower failure rate of rough-surface implants in comparison with turned-surface implants was found. However, the group of turned-surface implants had a mean follow-up of $6134 \pm 2224$ days (median
6048) against $2197+1491$ days (median 1792) for the group of implants with a roughened surface $(P<0.001$; Mann-Whitney test $)$. Table 7 shows a comparison of groups according to the distribution of implants with regard to the classification of bone quantity and quality (Lekholm \& Zarb 1985). It can be observed that a greater percentage of implants were placed in bone sites with quantities $\mathrm{D}$ and $\mathrm{E}(P<0.001 ;$ McNemar's test) and qualities 3 and $4(P=0.052$; McNemar's test) in the bruxers patients group, in comparison with the non-bruxers group. However, the difference was shown to not be significant for bone quality.

There was a statistically significantly unbalanced distribution of the systemic conditions and habits for the patients between the two groups (bruxers vs. non-bruxers) (Table 8). Other conditions did not include enough cases to perform a direct comparison, such as irradiation of the head-neck region (two patients with four implants in non-bruxers group), chemotherapy (one patient with four implants in bruxers group), intake of bisphosphonates (one patient with six implants in bruxers groupl, intake of hormone replacement therapy in women (four patients with 20 implants in bruxers groupl, intake of immunosuppressive medication (one patient with two implants in bruxers and one patient with five implants in non-bruxers group), and psoriasis (one patient with two implants in bruxers and one patient with four implant in nonbruxers group).

Considering the same number of patients with the same total number of implants equally distributed between groups, the bruxers group had a higher prevalence of mechanical complications in comparison with the non-bruxers group (Table 9). The odds ratio was calculated for the complications with known total number of units in each group, that is, number of acrylic/porcelain teeth, number of fixed prostheses, and the number of screws. The calculated odds ratios showed a higher statistically significant probability $(P<0.001)$ of these five complications to happen in bruxers.

\section{Discussion}

The aim of this retrospective clinical study was to analyze the complications of dental implant treatment in a group of patients presenting bruxism in comparison with a matched group of non-bruxers, and the results showed a statistically higher implant 
Table 5. Comparison of survival and failure rates of implants inserted in bruxers and a matched group of non-bruxers

\begin{tabular}{|c|c|c|c|c|}
\hline Implants & Bruxers $n(\%)$ & Non-bruxers $n(\%)$ & $P$-value & Total \\
\hline No implant failures & $73(74.5)$ & $87(88.8)$ & & $160(81.6)$ \\
\hline At least one implant failure & $25(25.5)$ & $11(11.2)$ & $<0.001^{*}$ & $36(18.4)$ \\
\hline Odds ratio $(95 \% \mathrm{Cl})^{* *}$ & $2.71(1.25,5.88)$ & & 0.01 & \\
\hline Total & $98(100)$ & $98(100)$ & & $196(100)$ \\
\hline \multicolumn{5}{|c|}{$\begin{array}{l}\text { Bruxers - } 427 \text { implants in total ( } 342 \text { survived, } 69 \text { failed due to lose/lack of osseointegration, } 16 \\
\text { implants fractured). } \\
\text { Non-bruxers }-427 \text { implants in total ( } 412 \text { survived, } 15 \text { failed due to lose/lack of osseointegration, no } \\
\text { implants fractured). } \\
\text { *McNemar's test. }\end{array}$} \\
\hline
\end{tabular}

Table 6. Comparison of implant failures between bruxers and non-bruxers, according to different implant lengths, diameters, and surfaces

\begin{tabular}{|c|c|c|c|c|c|}
\hline Implants & $\begin{array}{l}\text { Bruxers failure/ } \\
\text { total }(\%)\end{array}$ & & $\begin{array}{l}\text { Non-bruxers } \\
\text { failure/total (\%) }\end{array}$ & & $P$-value ${ }^{*}$ \\
\hline 7-10 mm long & $27 / 105(25.7)$ & & $2 / 71(2.8)$ & & $<0.001$ \\
\hline $11-13 \mathrm{~mm}$ long & 38/207 (18.4) & & $7 / 212(3.3)$ & & $<0.001$ \\
\hline $15-20 \mathrm{~mm}$ long & 19/110 (17.3) & & $6 / 142(4.2)$ & & $<0.001$ \\
\hline $3.00-3.50 \mathrm{~mm}$ wide & 9/37 (24.3) & & $0 / 29(0)$ & & 0.002 \\
\hline $3.75-4.30 \mathrm{~mm}$ wide & 74/381 (19.4) & & 14/394 (3.6) & & $<0.001$ \\
\hline $4.50-5.00 \mathrm{~mm}$ wide & $2 / 9(22.2)$ & & $1 / 4(25)$ & & 1.000 \\
\hline Turned-surface & 57/173 (32.9) & & $12 / 227(5.3)$ & & $<0.001$ \\
\hline Roughened-surface & $28 / 254(11)$ & $<0.001^{* * *}$ & $3 / 200(1.5)$ & $0.037^{* * *}$ & $<0.001$ \\
\hline \multicolumn{6}{|c|}{$\begin{array}{l}\text { *McNemar's test. } \\
\text { **Pearson's chi-square test. Comparison of failures between turned- and roughened-surface implants } \\
\text { in bruxers. } \\
\text { ***Fischer's exact test. Comparison of failures between turned- and roughened-surface implants in } \\
\text { non-bruxers. }\end{array}$} \\
\hline
\end{tabular}

Table 7. Comparison of groups according to the distribution of implants with regard to Lekholm \& Zarb (1985) classification of bone quantity and quality.

\begin{tabular}{|c|c|c|c|c|c|c|}
\hline & A & B & C & D & $\mathrm{E}$ & Total \\
\hline \multicolumn{7}{|c|}{ Implants in bruxers patients } \\
\hline 1 & 0 & 5 & 7 & 0 & 0 & 12 \\
\hline 2 & $35(3)$ & $103(8)$ & $30(11)$ & 1 & 0 & $169(22)$ \\
\hline 3 & 10 & $78(11)$ & $76(11)$ & $28(15)$ & $4(1)$ & $196(38)$ \\
\hline 4 & 0 & 3 & $22(12)$ & $16(8)$ & $8(5)$ & $49(25)$ \\
\hline Total & $45(3)$ & $189(19)$ & $135(34)$ & $45(23)$ & $12(6)$ & $426(85)$ \\
\hline \multicolumn{7}{|c|}{ Missing information: one implant } \\
\hline \multicolumn{7}{|c|}{ Implants in non-bruxers patients } \\
\hline 1 & 3 & 6 & 5 & 0 & 0 & 14 \\
\hline 2 & 21 & $106(4)$ & 57 & 8 & 0 & $192(4)$ \\
\hline 3 & $25(3)$ & $131(3)$ & $43(3)$ & $11(1)$ & 0 & $210(10)$ \\
\hline 4 & 0 & 2 & $7(1)$ & 2 & 0 & $11(1)$ \\
\hline Total & $49(3)$ & $245(7)$ & $112(4)$ & $21(1)$ & 0 & 427 \\
\hline No $\mathrm{mi}$ & nformat & & & & & \\
\hline
\end{tabular}

failure rate in the group of bruxers. Although a recent review (Manfredini et al. 2014) has concluded that is unlikely that bruxism would be considered a risk factor for a higher implant failure, based on the identified studies that considered bruxism as presenting no or uncertain risk factor for biological complications in implant-supported restorations, there are some studies that corroborate our results. Wannfors et al. (2000) and Glauser et al. (2001) reported a significant relationship between bruxism and implant failure after the implants have been functional for
1 year, as well as Ji et al. (Ji et al. 2012), with a mean follow-up of 42.1 months.

These results are believed to be partly related to the decreased proprioception of implants in comparison with teeth. The periodontal ligament of natural teeth provides the central nerve system with feedback for sensory perception and motor control (Meyer et al. 2012), whereas the proprioception around dental implants is limited because of the absence of a periodontal ligament, causing lower tactile sensitivity (Hämmerle et al. 1995). Consequently, the proprioceptive feed- back mechanisms to the jaw-closing muscles are limited as well. It is therefore not unlikely that forces that are applied to implants during bruxism are even larger than those exerted during mastication (Lobbezoo et al. 2006a), making them more prone to occlusal overload and possible subsequent failure (Meyer et al. 2012), as well as more subject to mechanical complications.

When it comes to fracture of implants and their components, it has been suggested that these are associated with overloads created by a combination of parafunctional stresses, cantilevers, posterior implant location and diameter, bone resorption, and possible misfit in superstructures (Balshi 1996). A superstructure with non-passive entry creates undesirable stress, which has often been linked to implant fractures (Adell et al. 1981). Thus, bruxism may have not been the only factor involved in the fractures. Two studies reported the absence of relationship between bruxism and mechanical complications (Tawil et al. 2006; Schneider et al. 2012), and one investigation reported uncertain findings (Wahlström et al. 2010). However, some studies suggested that bruxism may be a risk factor for fractures of ceramics (Kinsel \& Lin 2009) and, in general, for the need for technical interventions on implant-supported restorations (Brägger et al. 2001; De Boever et al. 2006; Maló et al. 2011). The results of the present study add evidence to suggest that bruxism may be an important contributor to the rate of mechanical complications in implant-supported restorations, as well as for a higher prevalence of implant fracture.

Considering the whole population treated in the present study's clinic, there was a lower percentage of women presenting with bruxism ( 49 of $1433,3.42 \%$ ) than men (49 of $1236,3.96 \%$ ). This is in contrast to Manfredini et al. (2004), who observed a strong difference in the prevalence of bruxism between female $(57.8 \%)$ and male $(25.5 \%)$ patients, who attended a neuroscience clinic. This female predilection was also observed in a study adopting clinical and/or interviewbased diagnosis of bruxism (Jensen et al. 1993). On the other hand, there was no gender predilection found in a study of polisomnographically diagnosed sleep bruxism (Lavigne et al. 1996). Thus, it can be seen that the difference in prevalence in the present study is opposite to what has been usually observed in the literature. This may be explained by the fact that the present study population may not be representative of the general population, comprising only patients being treated with dental implants. 
Table 8. Comparison of survival and failure of dental implants between bruxers and non-bruxers, according to health condition and patients' habits " $n$ " represents the number of patients, and the number between parentheses represents the number of ing to

\begin{tabular}{|c|c|c|c|c|c|c|c|}
\hline & \multicolumn{3}{|l|}{ Bruxer patients } & \multicolumn{3}{|l|}{ Non-bruxer patients } & \multirow[b]{2}{*}{$P$-value ${ }^{*}$} \\
\hline & $\begin{array}{l}\text { No implant failures } \\
n \text { (implants) }\end{array}$ & $\begin{array}{l}\text { At least one implant } \\
\text { failure } n \text { (implants) }\end{array}$ & $\begin{array}{l}\text { Total } n \\
\text { (implants) }\end{array}$ & $\begin{array}{l}\text { No implant } \\
\text { failures } n \text { (implants) }\end{array}$ & $\begin{array}{l}\text { At least one } \\
\text { implant failure } \\
n \text { (implants) }\end{array}$ & $\begin{array}{l}\text { Total } n \\
\text { (implants) }\end{array}$ & \\
\hline \multicolumn{8}{|c|}{ High blood pressure } \\
\hline No & $48(209)$ & $15(58)$ & $63(267)$ & $63(291)$ & $6(7)$ & $69(298)$ & \\
\hline Yes & $21(97)$ & $5(14)$ & $26(111)$ & $20(98)$ & $5(8)$ & $25(106)$ & \\
\hline Total** & $69(306)$ & $20(72)$ & 89 (378) & $83(389)$ & $11(15)$ & $94(404)$ & \\
\hline Unknown & $4(36)$ & $5(13)$ & $9(49)$ & $4(23)$ & $0(0)$ & $4(23)$ & \\
\hline $\begin{array}{l}\text { Patients/Group } \\
\text { Diabetes }\end{array}$ & \multicolumn{3}{|c|}{ High blood pressure -26. Normal -63} & \multicolumn{3}{|c|}{ High blood pressure -25. Normal - 69} & $<0.001$ \\
\hline & $60(260)$ & $15(58)$ & $75(318)$ & $81(385)$ & $11(15)$ & $92(400)$ & \\
\hline Type 1 & $1(4)$ & $2(5)$ & $3(9)$ & $1(2)$ & $0(0)$ & $1(2)$ & \\
\hline Type 2 & $8(42)$ & $3(9)$ & $11(51)$ & $2(13)$ & $0(0)$ & $2(13)$ & \\
\hline Total ${ }^{* *}$ & $69(310)$ & $20(72)$ & $89(382)$ & $84(400)$ & $11(15)$ & 95 (415) & \\
\hline Unknown & $4(36)$ & $5(13)$ & $9(49)$ & $3(12)$ & $0(0)$ & $3(12)$ & \\
\hline Patients/Group & \multicolumn{3}{|c|}{ Diabetes - 14. Normal - 75} & \multicolumn{3}{|c|}{ Diabetes - 3. Normal - 92} & $<0.001$ \\
\hline \multicolumn{8}{|l|}{ High cholesterol } \\
\hline No & $54(235)$ & $16(54)$ & 70 (289) & $78(360)$ & $9(13)$ & $87(373)$ & \\
\hline Yes & $15(71)$ & $4(18)$ & $19(89)$ & $5(29)$ & $2(2)$ & $7(31)$ & \\
\hline Total** & $69(306)$ & $20(72)$ & $89(378)$ & $83(389)$ & $11(15)$ & 94 (404) & \\
\hline Unknown & $4(36)$ & $5(13)$ & $9(49)$ & $4(23)$ & $0(0)$ & $4(23)$ & \\
\hline Patients/Group & \multirow{2}{*}{\multicolumn{3}{|c|}{ High cholesterol - 19. Normal - 70}} & \multicolumn{3}{|c|}{ High cholesterol - 7. Normal - 87} & $<0.001$ \\
\hline \multicolumn{5}{|c|}{ 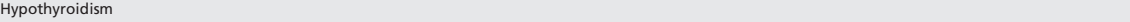 } & & & \\
\hline No & $63(281)$ & $18(66)$ & $81(347)$ & 79 (369) & $11(15)$ & $90(384)$ & \\
\hline Yes & $6(27)$ & $3(8)$ & $9(35)$ & $4(20)$ & $0(0)$ & $4(20)$ & \\
\hline Total*** & $69(308)$ & $21(74)$ & $8(382)$ & 83 (389) & $11(15)$ & 94 (404) & \\
\hline Unknown & $4(34)$ & $4(11)$ & $8(45)$ & $4(23)$ & $0(0)$ & $4(23)$ & \\
\hline Patients/Group & \multicolumn{3}{|c|}{ Hypothyroidism -9. No -81} & \multirow{2}{*}{\multicolumn{3}{|c|}{ Hypothyroidism - 4. No -90}} & $<0.001$ \\
\hline Asthma & & & & & & & \\
\hline No & $60(274)$ & $19(66)$ & $79(340)$ & 79 (356) & $9(10)$ & $88(366)$ & \\
\hline Yes & $9(41)$ & $2(12)$ & $11(53)$ & 4 (33) & $2(5)$ & $6(38)$ & \\
\hline Total** & $69(315)$ & $21(78)$ & 90 (393) & $83(389)$ & $11(15)$ & $94(404)$ & \\
\hline Unknown & $4(27)$ & $4(7)$ & $8(34)$ & $4(23)$ & $0(0)$ & $4(23)$ & \\
\hline Patients/Group & Asthma -11 . No -79 & & & Asthma - 6. No -88 & & & $<0.001$ \\
\hline Intake of medicam & its for depression & & & & & & \\
\hline No & $57(238)$ & $16(43)$ & $73(281)$ & $80(375)$ & $10(11)$ & 90 (386) & \\
\hline Yes & $12(77)$ & $5(35)$ & $17(112)$ & $3(14)$ & $1(4)$ & $4(18)$ & \\
\hline Total** & $69(315)$ & $21(78)$ & $90(393)$ & $83(389)$ & $11(15)$ & $94(404)$ & \\
\hline Unknown & $4(27)$ & $4(7)$ & $8(34)$ & $4(23)$ & $0(0)$ & $4(23)$ & \\
\hline Patients/Group & Depression - 17. No - & & & Depression - 4. No- & & & $<0.001$ \\
\hline Intake of medicam & its to reduce the acid & astric production & & & & & \\
\hline No & $63(269)$ & $16(57)$ & 79 (326) & $80(376)$ & $9(13)$ & 89 (389) & \\
\hline Yes & $6(37)$ & $4(15)$ & $10(52)$ & $3(13)$ & $2(2)$ & $5(15)$ & \\
\hline Total** & $69(306)$ & $20(72)$ & 89 (378) & $83(389)$ & $11(15)$ & $94(404)$ & \\
\hline Unknown & $4(36)$ & $5(13)$ & $9(49)$ & $4(23)$ & $0(0)$ & $4(23)$ & \\
\hline Patients/Group & Intake -10. No -79 & & & Intake -5. No -89 & & & $<0.001$ \\
\hline Intake of antithror & otic agents & & & & & & \\
\hline No & $59(237)$ & $15(54)$ & 74 (291) & $78(340)$ & $7(8)$ & $85(348)$ & \\
\hline Yes & $10(69)$ & $5(18)$ & $15(87)$ & $5(49)$ & $4(7)$ & $9(56)$ & \\
\hline Total** & 69 (306) & $20(72)$ & 89 (378) & $83(389)$ & $11(15)$ & 94 (404) & \\
\hline Unknown & $4(36)$ & $5(13)$ & $9(49)$ & $4(23)$ & $0(0)$ & $4(23)$ & \\
\hline Patients/Group & Intake -15. No -74 & & & Intake -9. No -85 & & & $<0.001$ \\
\hline Smoking & & & & & & & \\
\hline No & $42(189)$ & $12(43)$ & $54(232)$ & $63(273)$ & $7(8)$ & $70(281)$ & \\
\hline Yes & $19(76)$ & $7(32)$ & $26(108)$ & $19(117)$ & $4(7)$ & $23(124)$ & \\
\hline Former smoker & $4(37)$ & $1(2)$ & $5(39)$ & $0(0)$ & $0(0)$ & $0(0)$ & \\
\hline Total** & 65 (302) & $20(77)$ & 85 (379) & $82(390)$ & $11(15)$ & $93(405)$ & \\
\hline Unknown & $8(40)$ & $5(8)$ & $13(48)$ & $5(22)$ & $0(0)$ & $5(22)$ & \\
\hline Patients/Group & Smoker - 26. No smo & er -54 & & Smoker - 23. No smok & $r-70$ & & 0.001 \\
\hline Snuff & & & & & & & \\
\hline No & $62(274)$ & $16(59)$ & 78 (333) & $75(367)$ & $10(14)$ & $85(381)$ & \\
\hline Yes & $3(19)$ & $3(12)$ & $6(31)$ & $7(23)$ & $1(1)$ & $8(24)$ & \\
\hline Total** & 65 (293) & $19(71)$ & $84(364)$ & $82(390)$ & $11(15)$ & $93(405)$ & \\
\hline Unknown & $8(49)$ & $6(14)$ & $14(63)$ & $5(22)$ & $0(0)$ & $5(22)$ & \\
\hline Patients/Group & Snuff -6 . No -78 & & & Snuff -8 . No -85 & & & $<0.001$ \\
\hline
\end{tabular}


Table 9. Comparison of the number of mechanical complications between bruxers and non-bruxers patients groups in the implant-supported prosthetic
supported prosthetic restorations are not included)

\begin{tabular}{lllll}
\hline Complication & Bruxers & Non-bruxers & Odds ratio $(95 \% \mathrm{Cl})$ & $P$-value \\
\hline Acrylic teeth fractured/lost $(n)$ & 154 & 44 & $12.091(8.211,17.805) \dagger$ & $<0.001$ \\
Segment of prosthesis' acrylic & 15 & 5 & & \\
$\quad$ fractured $(n)$ & & & & \\
Implant's hole sealing lost $(n)$ & 43 & 21 & & \\
Porcelain fracture $(n)$ & 50 & 4 & $12.342(4.388,34.719) \dagger$ & $<0.001$ \\
Prosthesis fractured* $(n)$ & 17 & 1 & & \\
Prosthesis loose $(n)$ & 73 & 15 & $7.862(4.215,14.662) \ddagger$ & $<0.001$ \\
Fixed prosthesis fell out/came off $(n)$ & 2 & 0 & & \\
Screw fractured $(n)$ & $96(61 / 35) \uparrow$ & $3(2 / 1) \uparrow$ & $40.991(12.875,130.509) \S$ & $<0.001$ \\
Screw loose $(n)$ & 54 & 11 & $5.475(2.821,10.627) \S$ & $<0.001$ \\
Screw lost $(n)$ & 8 & 0 & & \\
Fractured implant & 16 & 0 & & \\
Abutment deformed $(n)$ & 16 & 0 & & \\
Overdenture component fractured $(n)$ & 7 & 6 & & \\
Hard splint fractured $(n)$ & 2 & - & & \\
\hline
\end{tabular}

*Prosthesis with complete transversal (buccal-lingual) fracture.

*The total number of acrylic/porcelain teeth was considered for the calculation of the odds ratio. the total number of fixed prostheses (excluding overdentures) was considered for the calculation of the odds ratio.

$\S$ The total number of screws was considered for the calculation of the odds ratio.

$\sigma(n 1 / n 2)$ - the first number between parenthesis $(n 1)$ represents the number of fractures of prosthetic screws and the second number (n2) represents the number of fractures of abutment screws.

It was suggested that one of the most important factors responsible for implant failures is probably the local anatomic structure regarding bone quality and quantity, or rather the lack thereof (Ekfeldt et al. 2001). The present study observed that a greater percentage of implants were placed in bone sites having been classified as quantities D and E in the bruxer patients group, in comparison with the non-bruxer group, with the difference showing to be statistically significant. This fact may also have exerted some influence on the difference in implant failure rates.

It was also observed that the patients' medically related conditions and habits had statistically significantly different numbers of patients included in the two groups (bruxers vs. non-bruxers), which may also have influenced the implant failure rates. Depression is a condition of great interest, due to its suggested strong association with bruxism. A study by Manfredini et al. (2004) found significant differences between bruxers and controls regarding the presence of depressive symptoms, such as manic symptoms, stress sensitivity, and anxious expectation. This may be related to the fact that disturbances in the central neurotransmitter system may be involved in the etiology of bruxism (Lobbezoo et al. 1997; Lavigne et al. 2003). In addition, results from pharmacological studies suggest a possible role for selective serotonin reuptake inhibitors, a class of antidepressants, which can exacerbate manic symptoms in bipolar individuals (Craddock et al. 2001) and can also cause bruxism after long-term usage (Lobbezoo et al. 2001). Dopa- mine agonists, such as amphetamine, which can induce manic symptoms (Sokoloff et al. 1990) and tooth grinding may also play a possible role (Milosevic et al. 1999). AlonsoNavarro et al. (2009) reported a case of a 62-year-old man who developed severe bruxism that began 2 weeks after starting a therapy with venlafaxine because of depression and anxiety. After venlafaxine withdrawal, bruxism improved gradually and disappeared 2 months later.

The present results documented a higher failure rate of short implants in bruxers in comparison with longer implants, which tended to decrease as the implant length increased. Implants with regular diameter $(3.75-4.30 \mathrm{~mm})$ had a lower failure rate than bruxers. The total number of implants of wide diameter $(4.50-5.00 \mathrm{~mm})$ was considered too low to possibly draw any conclusion concerning this group. The diameter and length of an implant (Himmlová et al. 2004), as well as thread pitch, shape, and surface treatment (Abuhussein et al. 2010), are modifiers that affect load transfer characteristics between implant and bone. Longer implants with a larger diameter help to keep the stresses in the bone as low as possible (el Askary et al. 1999). Moreover, implants classified as having roughened surfaces had a statistically significantly lower probability to fail in com parison with turned implants, in both bruxers and non-bruxers. It has been shown that the osseointegration process is influenced by sevthem the surface texture (Albrektsson \& narrow diameter implants $(3.00-3.50 \mathrm{~mm})$ in eral factors (Chrcanovic et al. 2014), among
Wennerberg 2004; Chrcanovic et al. 2015b). There is supportive evidence for a positive relationship between an improved bone healing around implants and its surface roughness (Shalabi et al. 2006), which enhances the process of osseointegration. However, it is also important to note that the group of turned-surface implants had statistically significantly longer mean follow-up time than the group of enlarged surface implants, which can lead to an increase in the failure rate.

The nightly use of a hard acrylic occlusal guard by the present sample of bruxers was found to be erratic in this study. Thus, a proper evaluation of the effect of nightguard use on implant failure and/or mechanical complication rates was not possible. It is important to note that there are conflicting results regarding the clinical efficacy of occlusal splints (Dao \& Lavigne 1998; List \& Axelsson 2010) and a lack of knowledge about the potential mechanisms that explain their success in the treatment of bruxism. This has led to the understanding that these devices represent a nonspecific therapy (Dao \& Lavigne 1998), being restricted to the prevention and/or limitation of dental wear potentially induced by bruxism (Dao \& Lavigne 1998; Ommerborn et al. 2011).

Although associated with a number of clinical problems, including orofacial pain, tooth wear, and failing dental restorative treatments, bruxism remains difficult to manage in effective and safe ways (Lobbezoo et al. 2006b). Unfortunately, there is a scarcity of reliable and valid diagnostic tools for the condition (Lobbezoo et al. 2013). Without a "definite" diagnosis of bruxism been established in the present study, it is acknowledged that some of the outcomes illustrated in some of the clinical cases may be due to such loadincreasing or material-related factors, rather than to bruxism per se (Johansson et al. 2011). Some of the assessment procedures here performed may also have some limitations, as for example, difficulties in distinguishing between functional and nonfunctional tooth wear during a clinical evaluation (Manfredini et al. 2004). Another limitation of the present study is its retrospective nature, which inherently results in flaws. These problems were manifested by the gaps in information and incomplete records.

\section{Conclusions}

The present study suggests that bruxism may significantly increase both the implant 
failure rate and the rate of mechanical and technical complications of implant-supported restorations. Other risk factors may also have influenced the results.

Acknowledgements: Funding and support: This work was supported by research funds from the Oral Health

\section{References}

AASM (2014) International Classification of Slee Disorders, Revised: Diagnostic and Coding Manual, 3rd edition. Chicago: American Academy of Sleep Medicine.

Abuhussein, H., Pagni, G., Rebaudi, A. \& Wang H.L. (2010) The effect of thread pattern upon implant osseointegration. Clinical Oral Implant Research 21: 129-136.

Adell, R., Lekholm, U., Rockler, B. \& Brånemark, P.I. (1981) A 15-year study of osseointegrate implants in the treatment of the edentulous jaw. International Journal of Oral Surgery 10: 387-416. Albrektsson, T. \& Wennerberg, A. (2004) Ora implant surfaces: part 2-review focusing on clincal knowledge of different surfaces. Internation Journal of Prosthodontics 17: 544-564.

Alonso-Navarro, H., Martin-Prieto, M., RuizEzquerro, J.J. \& Jimenez-Jimenez, F.J. (2009) Bruxism possibly induced by venlafaxine. Clinical Neuropharmacology 32: 111-112.

el Askary, A.S., Meffert, R.M. \& Griffin, T. (1999) Why do dental implants fail? Part i. Implant Dentistry 8: 173-185.

Balshi, T.J. (1996) An analysis and management of fractured implants: a clinical report. International Journal of Oral and Maxillofacial Implants 11: 660-666.

Brägger, U., Aeschlimann, S., Burgin, W Hämmerle, C.H. \& Lang, N.P. (2001) Biologica and technical complications and failures wit fixed partial dentures (fpd) on implants and teeth after four to five years of function. Clinical Oral Implants Research 12: 26-34

Chrcanovic, B.R., Albrektsson, T. \& Wennerberg A. (2014) Reasons for failures of oral implants. Journal of Oral Rehabilitation 41: 443-476.

Chrcanovic, B.R., Albrektsson, T. \& Wennerberg A. (2015a) Bruxism and dental implants: a metaanalysis. Implant Dentistry 24: 505-516.

Chrcanovic, B.R., Wennerberg, A. \& Martins, M.D. (2015b) Influence of temperature and acid etchin time on the superficial characteristics of ti. Mate rials Research 18: 963-970.

Craddock, N., Dave, S. \& Greening, J. (2001) Association studies of bipolar disorder. Bipolar Disor ders 3: 284-298.

D'Agostino, R.B., Jr. (1998) Propensity score methods for bias reduction in the comparison of treatment to a non-randomized control group. Statistics in Medicine 17: 2265-2281.

Dao, T.T. \& Lavigne, G.J. (1998) Oral splints: the crutches for temporomandibular disorders an bruxism? Critical Reviews in Oral Biology and Medicine 9: 345-361.
Related Research by Region Skåne (Odontologisk Forskning Skåne), Sweden, and from the Scientific Research Council of Sweden (Vetenskapsrådet, Dnr 2015-02971). This work was supported by Folktandvården $\mathrm{AB}$, Region Skåne, Sweden and by CNPq, Conselho Nacional de Desenvolvimento Científico e Tecnológico, Brazil. Trial registration at the

De Boever, A.L., Keersmaekers, K., Vanmaele, G. Kerschbaum, T., Theuniers, G. \& De Boever, J.A (2006) Prosthetic complications in fixed endosseous implant-borne reconstructions after an observations period of at least 40 months. Journal of Oral Rehabilitation 33: 833-839.

Ekfeldt, A., Christiansson, U., Eriksson, T., Linden, U., Lundqvist, S., Rundcrantz, T., Johansson, L.A., Nilner, K. \& Billstrom, C. (2001) A retrospective analysis of factors associated with multiple implant failures in maxillae. Clinical Oral Implants Research 12: 462-467.

Glaros, A.G. (1981) Incidence of diurnal and nocturnal bruxism. Tournal of Prosthetic Dentistry 45 545-549.

Glauser, R., Rée, A., Lundgren, A., Gottlow, I. Hämmerle, C.H. \& Schärer, P. (2001) Immediate occlusal loading of branemark implants applied in various jawbone regions: a prospective, 1-year clinical study. Clinical Implant Dentistry and Related Research 3: 204-213.

Gothberg, C., Bergendal, T. \& Magnusson, T. (2003) Complications after treatment with implant-supported fixed prostheses: a retrospective study. International Journal of Prosthodontics 16: 201 207.

Hämmerle, C.H., Wagner, D., Brägger, U., Lussi, A. Karayiannis, A., Joss, A. \& Lang, N.P. (1995) Threshold of tactile sensitivity perceived with dental endosseous implants and natural teeth. Clinical Oral Implants Research 6: 83-90.

Himmlová, L., Dostalova, T., Kacovsky, A. \& Konvickova, S. (2004) Influence of implant length and diameter on stress distribution: a finite element analysis. Journal of Prosthetic Dentistry 91: 2025.

Jensen, R., Rasmussen, B.K., Pedersen, B., Lous, I. \& Olesen, J. (1993) Prevalence of oromandibular dysfunction in a general population. Journal of Orofacial Pain 7: 175-182

Ji, T.J., Kan, J.Y., Rungcharassaeng, K., Roe, P. \& Lozada, J.L. (2012) Immediate loading of maxillary and mandibular implant-supported fixed complete dentures: a 1- to 10-year retrospective study. Journal of Oral Implantology 38(Spec No): 469-476.

Johansson, A., Omar, R. \& Carlsson, G.E. (2011) Bruxism and prosthetic treatment: a critical review. Journal of Prosthodontic Research 55: 127-136.

Kinsel, R.P. \& Lin, D. (2009) Retrospective analysis of porcelain failures of metal ceramic crowns and fixed partial dentures supported by 729 implants in 152 patients: patient-specific and implant-spe-
US National Institutes of Health (clinicaltrials.gov): NCT02369562.

\section{Declaration of conflicting interests}

There are no conflict of interests to declare.

cific predictors of ceramic failure. Journal of Prosthetic Dentistry 101: 388-394.

Lavigne, G.J., Kato, T., Kolta, A. \& Sessle, B.J. (2003) Neurobiological mechanisms involved in sleep bruxism. Critical Reviews in Oral Biology and Medicine 14: 30-46.

Lavigne, G.J., Rompre, P.H. \& Montplaisir, J.Y. (1996) Sleep bruxism: validity of clinical research diagnostic criteria in a controlled polysomnographic study. Journal of Dental Research 75: 546-552.

Lekholm, U. \& Zarb, G.A. (1985) Patient selection and preparation. In: Brånemark, P.I., Zarb, G.A. \&

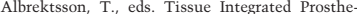
ses: Osseointegration in Clinical Dentistry, 199 209. Chicago: Quintessence Publishing Company. List, T. \& Axelsson, S. (2010) Management of tmd: evidence from systematic reviews and meta-analyses. Journal of Oral Rehabilitation 37: 430-451. Lobbezoo, F., Ahlberg, J., Glaros, A.G., Kato, T., Koyano, K., Lavigne, G.J., de Leeuw, R., Manfredini, D., Svensson, P. \& Winocur, E. (2013) Bruxism defined and graded: an international consensus. Journal of Oral Rehabilitation 40: 2-4. obbezoo, F., Brouwers, J.E., Cune, M.S. \& Naeije, M. (2006) Bental implants in patients with bruxing habits. Journal of Oral Rehabilitation 33: $152-159$

Lobbezoo, F., Soucy, J.P., Hartman, N.G., Montplaisir, J.Y. \& Lavigne, G.J. (1997) Effects of the d2 receptor agonist bromocriptine on sleep bruxism: report of two single-patient clinical trials. Journal of Dental Research 76: 1610-1614.

Lobbezoo, F., van Denderen, R.J., Verheij, J.G. \& Naeije, M. (2001) Reports of ssri-associated bruxism in the family physician's office. Journal of Orofacial Pain 15: 340-346.

Lobbezoo, F., Van Der Zaag, J. \& Naeije, M. (2006b) Bruxism: its multiple causes and its effects on dental implants - an updated review. Journal of Oral Rehabilitation 33: 293-300.

Maló, P., Nobre, M. \& Lopes, A. (2011) The rehabilitation of completely edentulous maxillae with different degrees of resorption with four or more immediately loaded implants: a 5-year retrospective study and a new classification. European Journal of Oral Implantology 4: 227-243.

Manfredini, D., Landi, N., Romagnoli, M. \& Bosco, M. (2004) Psychic and occlusal factors in bruxers. Australian Dental Journal 49: 84-89.

Manfredini, D., Poggio, C.E. \& Lobbezoo, F. (2014) Is bruxism a risk factor for dental implants? A systematic review of the literature. Clinical Implant Dentistry and Related Research 16: 460-469. 
Meyer, G., Fanghanel, I. \& Proff, P. (2012) Morphofunctional aspects of dental implants. Annals of Anatomy - Anatomischer Anzeiger 194: 190-194. Milosevic, A., Agrawal, N., Redfearn, P. \& Mair, L. (1999) The occurrence of toothwear in users of ecstasy (3,4-methylenedioxymethamphetamine). Community Dentistry and Oral Epidemiology 27: 283-287.

Ommerborn, M.A., Taghavi, J., Singh, P., Handschel, J., Depprich, R.A. \& Raab, W.H. (2011) Therapies most frequently used for the management of bruxism by a sample of german dentists. Journal of Prosthetic Dentistry 105: 194-202.

Paesani, D.A., Lobbezoo, F., Gelos, C., Guarda-Natdini, L., Ahlberg, J. \& Manfredini, D. (2013) Correlation between self-reported and clinically based diagnoses of bruxism in temporomandibular disorders patients. Journal of Oral Rehabilita tion 40: 803-809.
Schneider, D., Witt, L. \& Hämmerle, C.H. (2012) Influence of the crown-to-implant length ratio on the clinical performance of implants supporting single crown restorations: a cross-sectional retrospective 5-year investigation. Clinical Oral Implants Research 23: 169-174.

Shalabi, M.M., Gortemaker, A, Van't Hof, M.A. Jansen, J.A. \& Creugers, N.H. (2006) Implant surface roughness and bone healing: a systematic review. Journal of Dental Research 85 496-500.

Sokoloff, P., Giros, B., Martres, M.P., Bouthenet, M.L. \& Schwartz, J.C. (1990) Molecular cloning and characterization of a novel dopamine recepto (d3) as a target for neuroleptics. Nature 347: 146 151

Tawil, G., Aboujaoude, N. \& Younan, R. (2006) Influence of prosthetic parameters on the survival and complication rates of short implants. Interna- tional Journal of Oral and Maxillofacial Implants 21: 275-282.

Treede, R.D., Jensen, T.S., Campbell, J.N., Cruccu, G., Dostrovsky, J.O., Griffin, J.W., Hansson, P., Hughes, R., Nurmikko, T. \& Serra, J. (2008) Neuropathic pain: redefinition and a grading system for clinical and research purposes. Neurology 70 : 1630-1635.

Wahlström, M., Sagulin, G.B. \& Jansson, L.E. (2010) Clinical follow-up of unilateral, fixed dental prosthesis on maxillary implants. Clinical Oral Implants Research 21: 1294-1300.

Wannfors, K., Johansson, B., Hallman, M. \& Strandkvist, T. (2000) A prospective randomized study of 1- and 2-stage sinus inlay bone grafts: 1-year follow-up International Journal of Oral and Maxillofacial Implants 15: 625-632. 



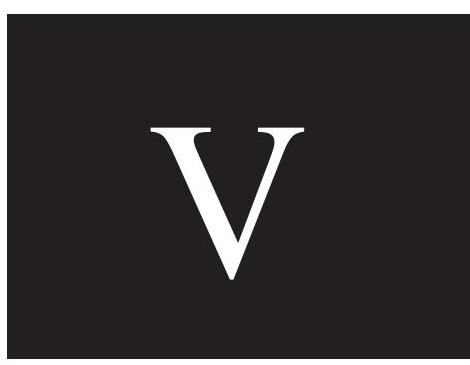



The intake of proton pump inhibitors is associated with an increased risk of dental implant failure

\author{
Bruno Ramos Chrcanovic ${ }^{1 *}$ \\ Jenö Kisch ${ }^{2}$ \\ Tomas Albrektsson ${ }^{3}$ \\ Ann Wennerberg ${ }^{4}$
}

${ }^{1}$ DDS, MSc, PhD student; Department of Prosthodontics, Faculty of Odontology, Malmö University, Malmö, Sweden

${ }^{2}$ DDS; Clinic for Prosthodontics, Centre of Dental Specialist Care, Malmö, Sweden

${ }^{3} \mathrm{MD}, \mathrm{PhD}$; Retired Professor and Former Head, Department of Biomaterials, Göteborg University, Göteborg, Sweden; Guest Professor, Department of Prosthodontics, Faculty of Odontology, Malmö University, Malmö, Sweden

${ }^{4}$ DDS, PhD; Professor and Head, Department of Prosthodontics, Faculty of Odontology, Malmö University, Malmö, Sweden

* Corresponding author:

Bruno Ramos Chrcanovic, Department of Prosthodontics, Faculty of Odontology, Malmö University, Carl Gustafs väg 34, SE-214 21, Malmö, Sweden. bruno.chrcanovic@mah.se; brunochrcanovic@hotmail.com Mobile: +46 725541545 Fax: +46 406658503 


\section{Abstract}

Purpose. To investigate the association between the intake of pump proton inhibitors (PPIs) and the risk of dental implant failure.

Materials and Methods. The present retrospective cohort study is based on the patients consecutively treated with implant-supported/retained prostheses at one specialist clinic. Modern endosseous dental implants with cylindrical or conical design were included. Only complete cases were considered, i.e. only those implants with information available for all variables were included in the regression model analysis. Zygomatic implants and Implants detected in radiographies, but without basic information about them in the patients' files were excluded from the study. Implantand patient-related data were collected. Multilevel mixed effects parametric survival analysis was used to test the association between PPIs exposure (predictor variable) and risk of implant failure (outcome variable) adjusting for several potential confounders (other variables).

Results. A total of 3,559 implants were placed in 999 patients, with 178 implants reported as failures. The implant failure rates were $12.0 \%$ (30/250) for PPIs users and $4.5 \%(148 / 3309)$ for nonusers. Forty-five (25.3\%) out of 178 failed implants were lost up to the abutment connection (6 in PPIs users, 39 in nonusers), with an early:late failure ratio of $0.34: 1$. The intake of PPIs has shown to be statistically significantly for implant survival rate (HR 2.811;95\%Cl 1.139, 6.937; $P=0.025$ ). Bruxism, smoking, implant length, prophylactic antibiotic regimen, and implant location were also identified as factors that statistically significantly affected the implant survival rate.

Conclusion. This study suggests that the intake of PPIs may be associated with an increased risk of dental implant failure.

\section{Keywords}

Dental implant; failure; risk factors; proton pump inhibitors; multivariate analysis; multilevel mixed effects parametric survival analysis 


\section{Introduction}

Dental implants are an effective and predictable treatment modality for replacing missing teeth in both fully and partially edentulous patients. However, failures can occur. ${ }^{1}$ Several risk factors have been suggested to influence the failure of implants. Surgical conditions, submission to radiotherapy, the oral microbial environment, parafunctional habits, and prosthetic variables are some of these factors. Systemic diseases and habits such as smoking may affect oral tissues by increasing their susceptibility to other diseases or by interfering with wound healing. The intake by the patient of medications that directly or indirectly affect the bone metabolism may also play a role on the outcome of implants. ${ }^{2}$ Among the drugs commonly prescribed nowadays, we find the proton pump inhibitors (PPIs).

Proton pump inhibitors (PPIs) are commonly used for the treatment of acid-related disorders, such as gastroesophageal reflux disease or gastric ulcer disease, by inhibiting the acid output of the stomach. The higher probability of losing an implant in patients taking medicaments to reduce the acid gastric production has been suggested to be related to observations indicating that a reduction of gastric acidity may impair effective calcium uptake through the intestines. ${ }^{3}$ As the calcium balance is essential for the maintenance of bone health, it seems reasonable to believe that the unbalance of calcium may to some degree affect osseointegration.

There is still no consensus on the influence of PPIs on the risk of dental implant failure. The aim of this retrospective study was to investigate the association between the intake of PPIs and the risk of dental implant failure, and to describe and compare the group of patients taking PPIs with the one not taking this class of drugs. In this study, it was hypothesized that the intake of PPIs might be associated with an increased risk of dental implant failure. 


\section{Materials and Methods}

\section{Study design/sample}

This study was designed as a retrospective cohort study, and is based on 10,096 implants placed in all 2,670 patients consecutively treated with implant-supported prostheses between 1980 and 2014 at one specialist clinic (Clinic for Prosthodontics, Centre of Dental Specialist Care, Malmö, Sweden).

Inclusion criteria:

- All modern endosseous dental implants with cylindrical or conical design;

- Complete cases only, i.e. only those implants with information available for all variables here included (see Variables below) were included in the regression model analysis.

Exclusion criteria:

- Zygomatic implants;

- Implants detected in radiographies, but without basic information about them in the patients' files.

Each patient received oral prophylaxis by a dental hygienist within 6 months after the final implant-supported/retained restoration and enrolled in a prophylaxis schedule based on their individual needs.

\section{Variables}

For this study, the PPIs status was the predictor variable. Patients who reported to be taking this type of medication during the presurgery appointment ( 1 to 2 weeks prior to implant placement) were defined as PPIs users. The PPIs verified included omeprazole, lansoprazole, dexlansoprazole, esomeprazole, pantoprazole, rabeprazole, and ilaprazole.

The outcome variable was implant failure. An implant was considered a failure if presenting signs and symptoms that led to implant removal. Thus, a failed implant in our study is equal to a lost implant. 
Other variables included: implant surface (machined or rough surfaces, specifically sandblasted, acid-etched, sandblasted + acid-etched, anodized, hydroxyapatite-coated surfaces), implant system (Nobel machined, Nobel TiUnite [Nobel Biocare, Göteborg, Sweden], Astra TiOblast, Astra Osseospeed, XIVE/Frialit-2 [Dentsply, Mölndal, Sweden], Straumann SLA/SLActive/Roxlid [Straumann, Basel, Switzerland], other), implant length (three categories: 6.0-10.0, 10.5-14.0, 15.0$20.0 \mathrm{~mm}$ ), implant diameter (three categories: 3.00-3.50, 3.70-4.10, 4.20-5.00 mm), prescription of prophylactic antibiotics (starting 1-2 hours before surgery and continuing for 5-7 days), bone graft procedures, implant jaw location (maxilla or mandible, anterior or posterior; region from a canine tooth to the contralateral canine tooth was considered anterior location), patient's gender, age of the patient at the implant insertion surgery (three categories: $\leq 30,30<x \leq 60,>60$ years), time period until failure, and follow-up time (baseline = day of implant installation). Health factors, medical disorders, and patients' habits known or suspected to substantially affect bone metabolism were evaluated: smoking, bruxism, and the intake of PPIs, antidepressants, immunosuppressives, bisphosphonates, antihypertensive drugs, and antithrombotic agents (antiplatelet, anticoagulant, thrombolytic drugs).

\section{Data collection methods}

The dental records of all patients ever treated with implants in the aforementioned clinic were read in order to collect the data. The data were directly entered into a SPSS file (SPSS software, version 23, SPSS Inc., Chicago, IL, USA) as the files were being read. The general health and the behavioral history of the patients and correlation of drugs to specific health conditions were collected from the patients' files.

\section{Data analyses}

Descriptive statistics were used to describe the basic features of the data. Differences between implants of PPIs users and nonusers were compared with the student's $t$-test or Mann- 
Whitney test for continuous variables, depending on the normality, and the Pearson's chi-squared or Fisher's exact tests for categorical variables, depending on the expected count of events in a $2 \times 2$ contingency table. Comparison between PPI drugs users and nonusers in terms of demographic systemic conditions and other factors were done; odds ratios (OR) and their $95 \%$ confidence intervals $(\mathrm{Cl})$ were computed.

Multilevel mixed effects parametric survival analysis ${ }^{4,5}$ was performed to assess the association between PPIs intake (predictor variable) and dental implant failure (outcome variable), accounting for the fact that repeated observations (several implants) were available for a single patient (cluster effect). Because there was little prior knowledge about the appropriate shape of survival probability, parametric frailty models were extended including five different parametric models (Weibull, Exponential, Log logistic, Log Normal, and Gamma) to allow any number of normally distributed random effects. Akaike information criteria (AIC) were used to choose the best fit survival model. ${ }^{6}$ The intake of PPIs was the exposure variable, and all analyses were adjusted for the following potential confounders (other variables): patient's gender and age, implant surface, implant type, implant length, implant diameter, implant location, bone augmentation, smoking habits, bruxism, and the intake of prophylactic antibiotics, antidepressants, immunosuppressives, bisphosphonates, antihypertensive drugs, and antithrombotic agents. In order to verify multicollinearity, a correlation matrix of all of the predictor variables with a significant odds ratio $(P$ value cut-off point of 0.1 ) was scanned, to see whether there were some high correlations among the predictors. Collinearity statistics obtaining variance inflation factor (VIF) and tolerance statistic were also performed to detect more subtle forms of multicollinearity. The results of the final model were presented as an estimated hazard ratio (HR) of each significant prognostic variable.

A $P$-value of $P<0.05$ was considered statistically significant. All data were statistically analyzed using the software Stata version 14 (StataCorp LP, College Station, TX, USA) and SPSS version 23 (SPSS Inc., Chicago, IL, USA). The study was approved by the Regional Ethical Review 
Board in Lund (Dnr 2014/598; Dnr 2015/72), and is in concordance with the STROBE guidelines for observational studies.

\section{Results}

The number of implants with information available for all variables totaled 3,559, placed in 999 patients, with 178 implants reported as failures and removed. There were 1,773 implants in 479 men (mean age $\pm S D$ 60.4 \pm 15.9 , min-max 15.9-90.2) and 1786 implants in 520 women (mean age $\pm S D$ 59.6 \pm 15.0 , min-max 14.9-90.8). Sixty-seven PPIs users received a total of 250 implants whereas 932 nonusers received 3,309 implants. The number of implants per patient ranged from 1 to 20 (mean \pm SD $3.46 \pm 2.64$ ). The mean \pm SD follow-up time was $94.8 \pm 78.7$ months [median $71.4,25^{\text {th }}$ percentile $30.4,75^{\text {th }}$ percentile 138.9 ] (PPIs users: $88.8 \pm 80.5$ months, median $52.3,25^{\text {th }}$ percentile $26.9,75^{\text {th }}$ percentile 115.3 , nonusers: $95.2 \pm 78.6$ months, median $72.2,25^{\text {th }}$ percentile $31.2,75^{\text {th }}$ percentile 139.2; $P=0.072$; Mann-Whitney test). The implant failure rates were $12.0 \%(30 / 250)$ for people taking PPIs and 4.5\% (148/3309) for people not taking these drugs $(P<0.001$; Pearson's chisquared test). The 178 failed implants were lost at a mean \pm SD of $37.6 \pm 53.8$ months (min-max, 0.8 316.4). Forty-five (25.3\%) out of 178 failed implants were lost up to the abutment connection procedure or $2^{\text {nd }}$ stage surgery (6 in PPIs users, 39 in nonusers). Eighty-five out of $178(47.8 \%)$ failed implants were lost until 1 year after surgery. All implants were inserted with open flapped surgery. Twenty implants were immediately loaded (1 in PPIs users, 19 in nonusers). Eleven implants were placed in fresh extraction sockets, all in nonusers of PPIs. A number of 113 implants were nonsubmerged (13 in PPIs users, 100 in nonusers). The abutment connection surgery was performed after a mean \pm SD healing time of $5.2 \pm 1.8$ and $5.2 \pm 2.1$ months for the PPIs users and nonusers, respectively $(P=0.588$; Mann-Whitney test $)$.

The comparison between patients taking and not taking PPIs (Table 1) showed that people from the age range $30<x \leq 60$ and older than 60 years were more likely to take PPIs than younger patients ( $\leq 30)$. Moreover, shorter implants were more common in PPIs users than in nonusers. There 
were more bruxers, and people taking antihypertensive, antidepressants, and antithrombotic drugs among the PPIs users. The two groups of patients were comparable in terms of the factors gender, implant diameter, implant surface, implant location, smoking habits, bone augmentation procedures, prophylactic antibiotic regimen, and intake of bisphosphonates and immunosuppressives, and for most of the different implant types (Table 1).

The multilevel mixed effects parametric survival analysis adjusted for the aforementioned confounders using Weibull model (Table 2) based on AIC selection showed that the intake of PPIs statistically significantly affected the implant survival rate. Bruxism, smoking, implant length, prophylactic antibiotic regimen, and implant location were also identified as factors that statistically significantly affected the implant survival rate. Age, gender, implant diameter, implant surface, implant type, bone augmentation, former smokers, and the intake of antihypertensive, antidepressants, antithrombotic, and immunosuppressive drugs did not statistically significantly affect the implant survival rate (Table 2). Multicollinearity was not detected.

\section{Discussion}

The results of the present study suggested that the intake of PPIs might be associated with an increased risk of dental implant failure. Moreover, the statistical model also suggested bruxism, smoking, implant length, prophylactic antibiotic regimen, and implant location as potential factors exerting a statistically significant influence on dental implant failures.

Some reasons could theoretically account for the suggested association between PPIs intake and the increased likelihood of dental implant failures. The most prominent hypothesis assumes that the reduced acidity in the stomach impairs the intestinal absorption of dietary calcium. Thus, there can be a decreased calcium absorption under PPI therapy ${ }^{7-9}$. Graziani et al. ${ }^{7}$ observed that postprandial calcium concentrations did not increase in subjects on a PPI regime (omeprazole $20 \mathrm{mg}$ $3 x$ daily), whereas control subjects demonstrated a clear spike in serum calcium levels. They as well observed that the urine calcium excretion in the PPI group was also reduced in comparison to the 
control group. $\mathrm{O}^{\prime}$ Connell et al. $^{8}$ measured the intestinal calcium absorption and observed that 7 days of PPI (omeprazole $20 \mathrm{mg}$ once a day) intake significantly reduced calcium absorption in elderly women under fasting conditions compared with the placebo group. Schinke et al. ${ }^{9}$ showed that genetically manipulated mice to be achlorhydric (with absence of hydrochloric acid from gastric juice) presented decreased serum calcium levels and developed osteoporosis and secondary hyperparathyroidism in an effort to maintain calcium balance. As the calcium balance is essential for the maintenance of bone health, it seems reasonable to believe that the unbalance of calcium may to some degree affect osseointegration.

Bruxism was shown to significantly affect the implant failure rates negatively, agreeing with the results of a recent meta-analysis ${ }^{10}$ and two clinical trials ${ }^{11,12}$ on the subject. This is due to the fact that bruxism is suggested to cause excessive load of implant-supported rehabilitations, which may cause an implant fracture or may result in bone loss around the implants and subsequent implant failure. ${ }^{13}$ Smoking was also shown as a factor affecting negatively the failure rates. A metaanalysis analyzing more than 100 studies has shown that failures of implants inserted in smokers are 2.23 times likely to happen than failures of implants inserted in non-smokers. ${ }^{14}$ The increase of implant failure rates due to smoking is hypothesized to be related mainly to the effect of smoking in osteogenesis and angiogenesis. ${ }^{15}$ Moreover, smokers' health behavior and attitudes appear to be less favorable to oral health than those of non-smokers. ${ }^{16}$ Studies have shown that smokers brush and floss their teeth less frequently than non-smokers ${ }^{17}$ and have dental visits less frequently than do non-smokers. ${ }^{18}$ With regard to lower hazard ratios for longer implants, although some good results can be obtained with the use of shorter implants, they seem to fail more often than longer ones. ${ }^{19}$ Increased initial stability, long-term resistance to bending moment forces, expedited healing, and a decreased risk of movement at the interface are listed as advantages of increased implant length. ${ }^{20}$ When it comes to the statistically reduced failures rates when a prophylactic antibiotic regimen was prescribed for the surgery, it has been suggested that the use of antibiotics in healthy patients significantly decreases implant failures. ${ }^{21}$ Implants inserted in the anterior mandible 
presented a statistically lower risk of failure when compared to those inserted in the anterior maxilla. The maxilla has a less favorable bone texture than mandibles, with low density of medullary bone and thin cortical plates. ${ }^{22}$ The region of the anterior mandible is usually considered as having an osseous structure more propitious to achieve primary instability of implants. Jaws with poor bone quality may be at risk of establishing initial instability of the implants and a lack of resistance to mechanical stresses, thus also resulting in early implant failures. ${ }^{22,23}$

The limitations of the present study include the fact that this is a retrospective study, which inherently results in flaws, manifested by the gaps in information and incomplete records. The lack of specific information characterizing the some patients' systemic conditions status and the medications dosage are limitations also connected to the retrospective nature of this study.

\section{Conclusions}

It is suggested that the intake of PPIs may be associated with an increased risk of dental implant failure. 


\section{Acknowledgements}

Funding and support: This work was supported by research funds from the Oral Health Related Research by Region Skåne (Odontologisk Forskning i Region Skåne, OFRS 414321), Sweden, and from the Scientific Research Council of Sweden (Vetenskapsrådet, Dnr 2015-02971). This work was supported by Folktandvården $A B$, Region Skåne, Sweden and by CNPq, Conselho Nacional de Desenvolvimento Científico e Tecnológico, Brazil.

Trial registration at the U.S. National Institutes of Health (clinicaltrials.gov): NCT02369562

Declaration of conflicting interests: There are no conflicts of interest to declare.

\section{References}

1. Chrcanovic BR, Albrektsson T, Wennerberg A. Reasons for failures of oral implants. J Oral Rehabil 2014;41:443-476.

2. Chrcanovic BR, Kisch J, Albrektsson T, Wennerberg A. Factors Influencing Early Dental Implant Failures. Journal of dental research 2016;95:995-1002.

3. Kopic S, Geibel JP. Gastric acid, calcium absorption, and their impact on bone health. Physiol Rev 2013;93:189-268.

4. Crowther MJ. Multilevel mixed-effects parametric survival analysis. United Kingdom Stata Users' Group Meeting. London, UK, 2013.

5. Crowther MJ, Look MP, Riley RD. Multilevel mixed effects parametric survival models using adaptive Gauss-Hermite quadrature with application to recurrent events and individual participant data meta-analysis. Stat Med 2014;33:3844-3858. 
6. Fang Y. Asymptotic equivalence between cross-validations and akaike information criteria in mixed-effects models. J Data Sci 2011;9:15-21.

7. Graziani G, Como G, Badalamenti S, Finazzi S, Malesci A, Gallieni M, et al. Effect of gastric acid secretion on intestinal phosphate and calcium absorption in normal subjects. Nephrol Dial Transplant 1995;10:1376-1380.

8. O'Connell MB, Madden DM, Murray AM, Heaney RP, Kerzner LJ. Effects of proton pump inhibitors on calcium carbonate absorption in women: a randomized crossover trial. Am J Med $2005 ; 118: 778-781$.

9. Schinke T, Schilling AF, Baranowsky A, Seitz S, Marshall RP, Linn T, et al. Impaired gastric acidification negatively affects calcium homeostasis and bone mass. Nat Med 2009;15:674-681.

10. Chrcanovic BR, Albrektsson T, Wennerberg A. Bruxism and Dental Implants: A Meta-Analysis. Implant Dent 2015;24:505-516.

11. Chrcanovic BR, Kisch J, Albrektsson T, Wennerberg A. Bruxism and dental implant treatment complications: a retrospective comparative study of 98 bruxer patients and a matched group. Clin Oral Implants Res 2016. doi: 10.1111/clr.12844

12. Chrcanovic BR, Kisch J, Albrektsson T, Wennerberg A. Bruxism and dental implant failures: a multilevel mixed effects parametric survival analysis approach. Journal of Oral Rehabilitation 2016. doi: $10.1111 /$ joor.12431

13. Lobbezoo F, Brouwers JE, Cune MS, Naeije M. Dental implants in patients with bruxing habits. J Oral Rehabil 2006;33:152-159.

14. Chrcanovic BR, Albrektsson T, Wennerberg A. Smoking and dental implants: A systematic review and meta-analysis. J Dent 2015;43:487-498.

15. Ma L, Zheng LW, Sham MH, Cheung LK. Uncoupled angiogenesis and osteogenesis in nicotinecompromised bone healing. J Bone Miner Res 2010;25:1305-1313.

16. Telivuo M, Kallio P, Berg MA, Korhonen HJ, Murtomaa H. Smoking and oral health: a population survey in Finland. J Public Health Dent 1995;55:133-138. 
17. Andrews JA, Severson HH, Lichtenstein E, Gordon JS. Relationship between tobacco use and self-reported oral hygiene habits. J Am Dent Assoc 1998;129:313-320.

18. Attwood D, West P, Blinkhorn AS. Factors associated with the dental visiting habits of adolescents in the west of Scotland. Community Dent Health 1993;10:365-373.

19. Pommer B, Frantal S, Willer J, Posch M, Watzek G, Tepper G. Impact of dental implant length on early failure rates: a meta-analysis of observational studies. J Clin Periodontol 2011;38:856-863.

20. Winkler S, Morris HF, Ochi S. Implant survival to 36 months as related to length and diameter. Ann Periodontol 2000;5:22-31.

21. Chrcanovic BR, Albrektsson T, Wennerberg A. Prophylactic antibiotic regimen and dental implant failure: a meta-analysis. J Oral Rehabil 2014;41:941-956.

22. Horwitz J, Zuabi O, Peled M, Machtei EE. Immediate and delayed restoration of dental implants in periodontally susceptible patients: 1-year results. Int J Oral Maxillofac Implants 2007;22:423429.

23. Friberg B, Jemt T, Lekholm U. Early failures in 4,641 consecutively placed Branemark dental implants: a study from stage 1 surgery to the connection of completed prostheses. Int J Oral Maxillofac Implants 1991;6:142-146. 


\section{Tables}

Table 1. Description of the cohort by patients $(n=999)$ between proton pump inhibitors (PPIs) users and nonusers. The number between parentheses represents the number of implants.

\begin{tabular}{|c|c|c|c|c|}
\hline \multirow[t]{2}{*}{ Variables } & \multicolumn{2}{|c|}{$\begin{array}{c}\text { Proton pump inhibitors - patients } \\
\text { (implants) }\end{array}$} & \multirow[t]{2}{*}{ Odds Ratio $(95 \% \mathrm{Cl})$} & \multirow[t]{2}{*}{$p$ value } \\
\hline & Users & Nonusers & & \\
\hline \multicolumn{5}{|l|}{ Age (years) } \\
\hline$\leq 30$ & $1(6)$ & $159(248)$ & 1 & \\
\hline $30<x \leq 60$ & $24(95)$ & $361(1163)$ & $10.571(1.418,78.818)$ & 0.021 \\
\hline$>60$ & 42 (149) & 412 (1898) & $16.209(2.212,118.764)$ & 0.006 \\
\hline \multicolumn{5}{|l|}{ Gender } \\
\hline Male & $28(113)$ & $451(1660)$ & 1 & \\
\hline Female & $39(137)$ & 481 (1649) & $1.306(0.790,2.158)$ & 0.297 \\
\hline \multicolumn{5}{|l|}{ Implant length $(\mathrm{mm})^{*}$} \\
\hline $6.0-10.0$ & $29(73)$ & $306(644)$ & 1 & \\
\hline $10.5-14.0$ & $52(128)$ & $677(1621)$ & $0.810(0.505,1.302)$ & 0.385 \\
\hline $15.0-20.0$ & $19(49)$ & $430(1044)$ & $0.466(0.257,0.847)$ & 0.012 \\
\hline \multicolumn{5}{|l|}{$\begin{array}{l}\text { Implant diameter } \\
(\mathrm{mm})^{*}\end{array}$} \\
\hline $3.00-3.50$ & $6(16)$ & $129(290)$ & 1 & \\
\hline $3.70-4.10$ & $61(228)$ & 806 (2951) & $1.627(0.689,3.841)$ & 0.267 \\
\hline $4.20-5.00$ & $5(6)$ & $54(68)$ & $1.991(0.583,6.801)$ & 0.272 \\
\hline \multicolumn{5}{|l|}{ Implant surface* } \\
\hline Machined & $16(90)$ & 333 (1523) & 1 & \\
\hline Rough & $51(160)$ & $622(1786)$ & $1.706(0.958,3.039)$ & 0.070 \\
\hline \multicolumn{5}{|l|}{ Implant type* } \\
\hline Nobel Machined & $16(90)$ & $333(1523)$ & 1 & \\
\hline Nobel TiUnite & 34 (114) & 466 (1300) & $1.519(0.825,2.796)$ & 0.180 \\
\hline Astra TiOblast & $2(3)$ & $38(158)$ & $1.095(0.243,4.948)$ & 0.906 \\
\hline Astra Osseospeed & $3(6)$ & $19(56)$ & $3.286(0.881,12.264)$ & 0.077 \\
\hline Straumann & $5(13)$ & $27(73)$ & $3.854(1.311,11.327)$ & 0.014 \\
\hline XiVE/Frialit-2 & $0(0)$ & $9(33)$ & - & - \\
\hline Other & $9(24)$ & 77 (166) & $2.433(1.036,5.711)$ & 0.041 \\
\hline \multicolumn{5}{|l|}{ Implant location* } \\
\hline Anterior maxilla & $31(85)$ & 458 (1129) & 1 & \\
\hline Posterior maxilla & $32(51)$ & $360(653)$ & $1.313(0.786,2.193)$ & 0.298 \\
\hline Anterior mandible & $20(62)$ & $235(888)$ & $1.257(0.701,2.254)$ & 0.442 \\
\hline Posterior mandible & $24(52)$ & 302 (639) & $1.174(0.676,2.040)$ & 0.569 \\
\hline \multicolumn{5}{|l|}{ Bone augmentation* } \\
\hline No & $64(221)$ & 900 (3201) & 1 & \\
\hline Yes & $7(29)$ & $62(108)$ & $1.588(0.698,3.611)$ & 0.270 \\
\hline \multicolumn{5}{|l|}{$\begin{array}{l}\text { Prophylactic } \\
\text { antibiotics* }\end{array}$} \\
\hline No & $7(20)$ & $112(323)$ & 1 & \\
\hline Yes & $60(230)$ & $838(2986)$ & $1.146(0.511,2.568)$ & 0.741 \\
\hline \multicolumn{5}{|l|}{ Smoking } \\
\hline No & 47 (147) & $666(2236)$ & 1 & \\
\hline Yes & $16(90)$ & $247(991)$ & $0.918(0.511,1.649)$ & 0.774 \\
\hline
\end{tabular}




\begin{tabular}{|c|c|c|c|c|}
\hline Former smoker & $4(13)$ & $19(82)$ & $2.983(0.975,9.125)$ & 0.055 \\
\hline \multicolumn{5}{|l|}{ Bruxism } \\
\hline No & 59 (219) & $884(3156)$ & 1 & \\
\hline Yes & $8(31)$ & $48(153)$ & $2.497(1.129,5.522)$ & 0.024 \\
\hline \multicolumn{5}{|c|}{ Antihypertensive drugs } \\
\hline No & $33(116)$ & $683(2291)$ & 1 & \\
\hline Yes & $34(134)$ & 249 (1018) & $2.826(1.713,4.662)$ & $<0.001$ \\
\hline \multicolumn{5}{|l|}{ Antidepressants } \\
\hline No & $48(165)$ & $858(3050)$ & 1 & \\
\hline Yes & $19(85)$ & $74(259)$ & $4.590(2.565,8.213)$ & $<0.001$ \\
\hline \multicolumn{5}{|l|}{ Bisphosphonates } \\
\hline No & $66(246)$ & 914 (3257) & 1 & \\
\hline Yes & $1(4)$ & $18(52)$ & $0.769(0.101,5.853)$ & 0.800 \\
\hline \multicolumn{5}{|l|}{ Antithrombotic drugs } \\
\hline No & $43(166)$ & 793 (2736) & 1 & \\
\hline Yes & $24(84)$ & $139(573)$ & $3.184(1.873,5.415)$ & $<0.001$ \\
\hline \multicolumn{5}{|l|}{ Immunosuppressives } \\
\hline No & 65 (238) & 923 (3269) & 1 & \\
\hline Yes & $2(12)$ & $9(40)$ & $3.156(0.668,14.907)$ & 0.147 \\
\hline
\end{tabular}

Table 2. Multilevel survival analysis for dental implant failure in terms of different factors (total number of implants $=3,559)$. Hazard ratios $(H R)$ were performed using multilevel mixed effects parametric survival analysis.

\begin{tabular}{|c|c|c|c|c|c|}
\hline Factor & $\begin{array}{l}\text { Number of } \\
\text { failed } \\
\text { implants (\%) }\end{array}$ & $\begin{array}{l}\text { Number of } \\
\text { survived } \\
\text { implants (\%) }\end{array}$ & Failure (\%) & $\begin{array}{c}p \\
\text { value }\end{array}$ & $\mathrm{HR}(95 \% \mathrm{Cl})$ \\
\hline \multicolumn{6}{|l|}{ Proton pump } \\
\hline \multicolumn{6}{|l|}{ inhibitors } \\
\hline No & $148(83.1)$ & 3161 (93.5) & 4.5 & & 1 \\
\hline Yes & 30 (16.9) & $220(6.5)$ & 12.0 & 0.025 & $2.811(1.139,6.937)$ \\
\hline \multicolumn{6}{|l|}{ Age (years) } \\
\hline$\leq 30$ & $10(5.6)$ & $244(7.2)$ & 3.9 & & 1 \\
\hline $30<x \leq 60$ & $101(56.7)$ & $1157(34.2)$ & 8.0 & 0.876 & $0.925(0.346,2.474)$ \\
\hline$>60$ & $67(37.6)$ & $1980(58.6)$ & 3.3 & 0.099 & $0.418(0.148,1.179)$ \\
\hline \multicolumn{6}{|l|}{ Gender } \\
\hline Male & $78(43.8)$ & $1695(50.1)$ & 4.4 & & 1 \\
\hline Female & $100(56.2)$ & $1686(49.9)$ & 5.6 & 0.940 & $1.022(0.582,1.793)$ \\
\hline \multicolumn{6}{|c|}{ Implant length (mm) } \\
\hline $6.0-10.0$ & $75(42.1)$ & $642(19.0)$ & 10.5 & & 1 \\
\hline $10.5-14.0$ & $67(37.7)$ & $1682(49.7)$ & 3.8 & $<0.001$ & $0.394(0.254,0.611)$ \\
\hline $15.0-20.0$ & $36(20.2)$ & $1057(31.3)$ & 3.3 & $<0.001$ & $0.298(0.172,0.515)$ \\
\hline Implant diamet & & & & & \\
\hline
\end{tabular}




\begin{tabular}{|c|c|c|c|c|c|}
\hline \multicolumn{6}{|l|}{$(\mathrm{mm})$} \\
\hline $3.00-3.50$ & $19(10.7)$ & $287(8.5)$ & 6.2 & & 1 \\
\hline $3.70-4.10$ & $157(88.2)$ & $3022(89.4)$ & 4.9 & 0.171 & $0.546(0.229,1.298)$ \\
\hline $4.20-5.00$ & $2(1.1)$ & $72(2.1)$ & 2.7 & 0.520 & $0.546(0.087,3.448)$ \\
\hline \multicolumn{6}{|l|}{ Implant surface } \\
\hline Machined & $122(68.5)$ & $1491(44.1)$ & 7.6 & & 1 \\
\hline Rough & $56(31.5)$ & 1890 (55.9) & 2.9 & 0.114 & $0.300(0.068,1.333)$ \\
\hline \multicolumn{6}{|l|}{ Implant type } \\
\hline Nobel Machined & $122(68.5)$ & 1491 (44.1) & 7.6 & & 1 \\
\hline Nobel TiUnite & 35 (19.7) & $1379(40.8)$ & 2.5 & 0.871 & $1.132(0.254,5.037)$ \\
\hline Astra TiOblast & $12(6.8)$ & $149(4.4)$ & 7.5 & 0.579 & $1.733(0.249,12.070)$ \\
\hline Astra Osseospeed & $1(0.6)$ & $61(1.8)$ & 1.6 & 0.584 & $0.447(0.025,7.991)$ \\
\hline Straumann & $4(2.2)$ & $82(2.4)$ & 4.7 & 0.792 & $0.746(0.085,6.549)$ \\
\hline XiVE/Frialit-2 & $0(0)$ & $33(1.0)$ & 0 & - & - \\
\hline Other & $4(2.2)$ & $186(5.5)$ & 2.1 & - & - \\
\hline \multicolumn{6}{|l|}{ Implant location } \\
\hline Anterior maxilla & $73(41.0)$ & 1141 (33.7) & 6.0 & & 1 \\
\hline Posterior maxilla & $41(23.0)$ & $663(19.6)$ & 5.8 & 0.530 & $0.864(0.550,1.361)$ \\
\hline Anterior mandible & $25(14.1)$ & $925(27.4)$ & 2.6 & 0.049 & $0.537(0.288,0.999)$ \\
\hline Posterior mandible & 39 (21.9) & $652(19.3)$ & 5.6 & 0.418 & $0.788(0.443,1.402)$ \\
\hline \multicolumn{6}{|l|}{ Bone augmentation } \\
\hline No & $163(91.6)$ & $3259(96.4)$ & 4.8 & & 1 \\
\hline Yes & $15(8.4)$ & $122(3.6)$ & 10.9 & 0.398 & $1.478(0.597,3.655)$ \\
\hline \multicolumn{6}{|l|}{$\begin{array}{l}\text { Prophylactic } \\
\text { antibiotics }\end{array}$} \\
\hline No & $24(13.5)$ & $319(9.4)$ & 7.0 & & 1 \\
\hline Yes & $154(86.5)$ & $3062(90.6)$ & 4.8 & 0.046 & $0.496(0.249,0.987)$ \\
\hline \multicolumn{6}{|l|}{ Smoking } \\
\hline No & $85(47.7)$ & $2298(68.0)$ & & & 1 \\
\hline Yes & $82(46.1)$ & 999 (29.5) & 7.6 & 0.003 & $2.363(1.336,4.180)$ \\
\hline Former smoker & $11(6.2)$ & $84(2.5)$ & 11.6 & 0.276 & $2.302(0.514,10.306)$ \\
\hline \multicolumn{6}{|l|}{ Bruxism } \\
\hline No & $155(87.1)$ & $3220(95.2)$ & 4.6 & & 1 \\
\hline Yes & 23 (12.9) & $161(4.8)$ & 12.5 & 0.032 & $2.892(1.098,7.618)$ \\
\hline \multicolumn{6}{|l|}{$\begin{array}{l}\text { Antihypertensive } \\
\text { drugs }\end{array}$} \\
\hline No & $127(71.3)$ & $2280(67.4)$ & 5.3 & & 1 \\
\hline Yes & $51(28.7)$ & 1101 (32.6) & 4.4 & 0.662 & $0.855(0.423,1.727)$ \\
\hline \multicolumn{6}{|l|}{ Antidepressants } \\
\hline No & $146(82.0)$ & 3069 (90.8) & 4.5 & & 1 \\
\hline Yes & $32(18.0)$ & $312(9.2)$ & 9.3 & 0.711 & $1.178(0.495,2.804)$ \\
\hline \multicolumn{6}{|l|}{ Bisphosphonates } \\
\hline No & $178(100)$ & 3325 (98.3) & 5.1 & & 1 \\
\hline Yes & $0(0)$ & $56(1.7)$ & 0 & - & - \\
\hline \multicolumn{6}{|l|}{ Antithrombotic drugs } \\
\hline No & $140(78.7)$ & 2762 (81.7) & 4.8 & & 1 \\
\hline Yes & $38(21.3)$ & $619(18.3)$ & 5.8 & 0.625 & $1.223(0.546,2.740)$ \\
\hline \multicolumn{6}{|l|}{ Immunosuppressives } \\
\hline No & $171(96.1)$ & 3336 (98.7) & 4.9 & & 1 \\
\hline Yes & $7(3.9)$ & $45(1.3)$ & 13.5 & 0.706 & $1.491(0.187,11.875)$ \\
\hline
\end{tabular}




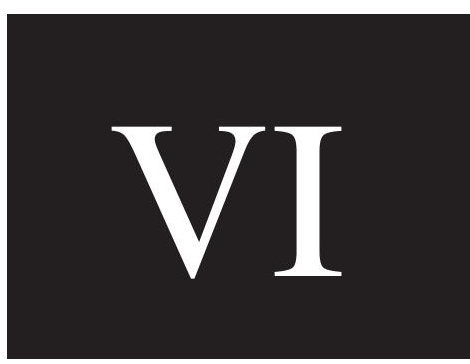





\section{Is the intake of selective serotonin reuptake inhibitors associated with an increased risk of dental implant failure?}

B.R. Chrcanovic, J. Kisch, T. Albrektsson, A. Wennerberg: Is the intake of selective serotonin reuptake inhibitors associated with an increased risk of dental implant failure?. Int. J. Oral Maxillofac. Surg. 2017; 46: 782-788. (C) 2017 International Association of Oral and Maxillofacial Surgeons. Published by Elsevier Ltd. All rights reserved.

Abstract. The aim of this retrospective study was to investigate the association between the intake of selective serotonin reuptake inhibitors (SSRIs) and the risk of dental implant failure. Patients were included if they were taking SSRIs only and no other medication, did not present any other systemic condition or compromising habits (bruxism, smoking, snuff), and complied with the use of prophylactic antibiotics for implant surgery. The multivariate generalized estimating equation (GEE) method and multilevel mixed-effects parametric survival analysis were used to test the association between SSRI exposure (predictor variable) and the risk of implant failure (outcome variable), adjusting for several potential confounders (other variables). The total number of implants with information available and meeting the necessary eligibility criteria was 931 ( 35 failures). These were placed in 300 patients. The implant failure rate was $12.5 \%$ for SSRI users and $3.3 \%$ for nonusers $(P=0.007)$. Kaplan-Meier analysis showed a statistically significant difference in the cumulative survival rate $(P<0.001)$. The multivariate GEE model did not show a statistically significant association between SSRI intake and implant failure $(P=0.530)$, nor did the multilevel model $(P=0.125)$. It is suggested that the intake of SSRIs may not be associated with an increased risk of dental implant failure.
B. R. Chrcanovic ${ }^{1}$, J. Kisch ${ }^{2}$, T. Albrektsson ${ }^{1,3}$, A. Wennerberg ${ }^{1}$ ${ }^{1}$ Department of Prosthodontics, Faculty of Odontology, Malmö University, Malmö,

Sweden; ${ }^{2}$ Clinic for Prosthodontics, Centre of Dental Specialist Care, Malmö, Sweden; ${ }^{3}$ Department of Biomaterials, Gothenburg University, Göteborg, Sweden
Key words: dental implant; implant failure; selective serotonin reuptake inhibitors; multiselective serotonin reuptake inhibitors; multivariate generalized estimating equation analysis; multilevel mixed-effects parametric survival analysis.

Accepted for publication 26 January 2017 Available online 20 February 2017
Nowadays dental implant placement is an effective and predictable treatment modality for replacing missing teeth in both fully and partially edentulous patients. Nevertheless, failures still happen despite high implant survival and success rates. ${ }^{1}$ Several risk factors have been suggested to influence the failure of implants. Surgical conditions, radiotherapy, the oral microbial environment, parafunctional habits, and prosthetic variables are some of these factors. Systemic diseases and compromising risky habits may affect the oral tissues by increasing their susceptibility to other diseases or by interfering 
with wound healing. The patient's intake of medications that directly or indirectly affect bone metabolism may also play a role in the outcome of implants. ${ }^{2}$

Among the drugs commonly prescribed today are the selective serotonin reuptake inhibitors (SSRIs). SSRIs are a class of drugs typically used as antidepressants in the treatment of major depressive and anxiety disorders. Studies have shown that the use of antidepressants predicts decreased bone mineral density in women, ${ }^{3}$ and both depression and the use of antidepressants are suggested to be possible risk factors for osteoporosis in men. ${ }^{4}$ It is possible that neuroendocrine mechanisms related to the serotonin system could regulate osteoclast differentiation/activation, because osteoclasts derive from haematopoietic cell precursors and a relationship between bone and the immune system has been established. $^{5-7}$ Studies have identified a functional serotonin system in osteoblasts and osteoclasts, ${ }^{8-10}$ in which the serotonin transporter and several receptors are expressed in osteoblasts as well as in osteoclasts. ${ }^{9,10}$ The presence of serotonin receptors and the serotonin transporter in bone raises the question whether medications that antagonize serotonin reuptake could influence bone metabolism.

It has been shown in in vitro studies that activity of the serotonin transporter is required for osteoclast differentiation. While blockage of the serotonin transporter was found to reduce osteoclast differentiation when fluoxetine, an antidepressant, was administered to produce micromolar concentrations, ${ }^{8,11}$ there was an increase in osteoclast differentiation for the same medication in the nanomolar concentrations. ${ }^{11}$ In vivo studies have demonstrated detrimental effects of fluoxetine on the trabecular architecture ${ }^{12}$ and on bone mineral density ${ }^{12,13}$ in mice. Another in vivo study showed that serotonin acts on osteoblasts, inhibiting their proliferation. ${ }^{14}$ These animal studies indicate a negative effect of SSRIs on bone mass and suggest that these antidepressants may possess direct anti-anabolic skeletal effects through the pharmacological inhibition of the serotonin transporter.

Therefore, the intake of SSRIs could in theory interfere with the osseointegration process. In the case of dental implants in particular, the findings of recent studies suggest that treatment with antidepressants is associated with an increased risk of failure of osseointegrated implants, ${ }^{2,15}$ while others have not found a relationship between these two factors. ${ }^{16-18}$ Thus, there is still no clear consensus on the influence of antidepressants on the risk of dental implant failure.

As the recognition of conditions that place the patient at a higher risk of failure will allow the surgeon to make informed decisions and refine the treatment plan to optimize the clinical outcome, the purpose of this study was to investigate the association between the intake of SSRIs and the risk of dental implant failure. It was hypothesized that patients taking SSRIs would have a higher implant failure rate than patients not taking this class of drugs. The specific aims of the study were to compare the implant failure rates between users and non-users of SSRIs, and to estimate the influence of several variables on the prevalence of implant failure in regression models, with the intake of these medications as the predictor variable.

\section{Materials and methods}

\section{Study design/sample}

A retrospective cohort study was designed and implemented to address the research purposes. The study population comprised all patients treated consecutively with implant-supported prostheses between 1980 and 2014 at one specialist clinic (Clinic for Prosthodontics, Centre of Dental Specialist Care, Malmö, Sweden).

To be included in the study sample, the patient had to be taking only SSRIs and no other medication and not present any other systemic condition. The analysis was based on complete cases only; i.e. only those implants with information available for all variables investigated here (see section on Data collection below) were included in the analysis. As it has been suggested that the use of antibiotics in healthy patients significantly decreases early implant failure, ${ }^{19}$ all patients had to have taken prophylactic antibiotics for implant surgery in order to be included. All modern endosseous dental implants with a cylindrical or conical design were included.

Patients were excluded as study subjects if they presented a severe systemic disease (American Society of Anesthesiologists physical status III or IV) or had been subjected to irradiation of the head and neck region, were pregnant, alcoholic, bruxers, or smokers, presented a medical disorder known to substantially affect bone metabolism (such as hyperthyroidism, hypothyroidism, vitamin D deficiency, osteomalacia, osteoporosis, Paget's disease, cancer (excluding non-melanoma skin cancer), diabetes), or were taking corticosteroids, antihypertensive drugs, immunosuppressive drugs, antithrombotic agents (antiplatelet, anticoagulant, thrombolytic drugs), antiepileptic drugs, proton pump inhibitors, bisphosphonates, medications for asthma, or medications to decrease high levels of cholesterol. Thus, the status 'taking SSRIs' was isolated as much as possible from the influence of other systemic conditions or medications. Zygomatic implants were not included in the study, nor were implants detected in radiographs but without basic information about them in the patient's files.

In accordance with the standard protocol at the study clinic, the patients' dental hygiene was followed up by a dental hygienist within 6 months after the final implant-supported/retained restoration. Each patient then attended a dental hygiene recall programme based on individual needs.

The trial from which data in this study were derived is registered with the US National Institutes of Health (clinicaltrials.gov, NCT02369562).

\section{Variables}

In this study, the patient's SSRI status was the predictor variable. SSRI users were defined as patients who reported taking this type of medication during the presurgery appointment that was scheduled 1 to 2 weeks prior to implant placement. The SSRIs verified included citalopram, dapoxetine, escitalopram, fluoxetine, fluvoxamine, indalpine, paroxetine, sertraline, venlafaxine, and zimelidine.

The outcome variable was implant failure. An implant was considered a failure in the presence of signs and symptoms that led to implant removal, including lack or loss of osseointegration, implant mobility, continuous pain, advanced marginal bone loss, and refractory infection.

The following factors were the other variables investigated: implant surface (turned/machined or enlarged surfaces, the latter including sandblasted, acidetched, sandblasted + acid-etched, anodized), implant length (three categories: $6.0-10.0,10.5-14.0,15.0-20.0 \mathrm{~mm})$, implant diameter (three categories: 3.00 $3.50,3.70-4.10,4.20-5.00 \mathrm{~mm}$ ), prescription of antibiotics (the prophylactic antibiotic regimen was usually started $1-2 \mathrm{~h}$ before surgery and continued for 5-7 days postoperatively), bone graft procedures, implant jaw location (maxilla/mandible), anterior or posterior location of the implant (locations 13-23 and 33-43 were considered anterior), patient sex, patient age at implant insertion surgery (three categories: $\leq 30,>30$ to $\leq 60,>60$ years), 
number of days until failure, and the duration of follow-up.

\section{Data collection methods}

The dental records of all patients ever treated with implants at the study clinic were read in order to collect the data. Th data were entered directly into an SPSS file as the files were being read (IBM SPSS Statistics version 23; IBM Corp., Armonk NY, USA). In order to identify patient with the systemic conditions that would exclude them from the analysis, the general health and behavioural history of each patient was collected from the patient files. The presence of a medication list in the patient records was also used to correlate the use of certain drugs to specific health conditions.

\section{Data analyses}

With regard to descriptive statistics, the mean \pm standard deviation or number and percentage were recorded. Differences between implants placed in SSRI users and SSRI non-users were compared with the Student $t$-test or Mann-Whitney test for continuous variables, depending on the normality of the data, and with the Pearson $\chi^{2}$ test or Fisher's exact test for categorical variables, depending on the expected count of events in a $2 \times 2$ contingency table. Comparisons were made between SSRI users and SSRI non-users in terms of demographic systemic conditions and other factors; odds ratios (OR) and their 95\% confidence intervals (CI) were computed.

An implant-level model with the implant as the statistical unit was performed in order to assess the effects of age, sex, implant length, implant diameter, implant surface, implant location, and bone aug mentation on the failure of implants in patients taking SSRIs. A multivariate generalized estimating equation (GEE) method was used to account for the fact that repeated observations (several implants) were available for a single patient. All models were adjusted for clustering of subject and implants in a binary logistic regression model using GEE with a binomial distribution and a logit link function, while assuming an exchangeable working correlation structure, to assess the relationship between implant failure (dependent variable) and the risk factors (independent variables). A Wald $\chi^{2}$ test was used to analyze the statistical significance of each parameter within the model.

Furthermore, a multilevel mixed-effects parametric survival analysis was used ${ }^{20,21}$ - a patient-based multilevel analysis - to assess the association between SSRI intake and dental implant failure, accounting for the fact that repeated observations (several implants) were available for a single patient (cluster effect). Because there was little prior knowledge about the appropriate shape of survival probability, parametric frailty models including five different parametric models (Weibull, exponential, log-logistic, log-normal, and gamma) were extended to allow any number of normally distributed random effects. The Akaike information criterion (AIC) was used to choose the best fit survival model. ${ }^{22}$ The intake of SSRIs was the exposure variable, and all analyses were adjusted for the following potential confounders: age, sex, implant length, implant diameter, implant surface, implant location, and bone augmentation.

In order to verify multicollinearity, a correlation matrix of all of the predictor variables with a significant OR $(P$-value cut-off point of 0.1 ) was scanned to determine whether there were some high correlations among the predictors. Collinearity statistics obtaining the variance inflation factor (VIF) and tolerance statistics were also performed to detect more subtle forms of multicollinearity. The results of the final multivariate GEE model were presented as the estimated $\mathrm{OR}$, and the results of the multilevel mixed-effects parametric survival analysis were presented as the estimated hazard ratio (HR) of each significant prognostic variable $(P<0.05)$

Kaplan-Meier survival curves were plotted to describe the cumulative proportion of dental implant failure stratified by use of SSRIs (yes/no), and a comparison among groups was analyzed by log-rank test.

The level of statistical significance was set at $P<0.05$. All data were analyzed statistically using the software Stata version 14 (StataCorp LP, College Station, TX, USA) and IBM SPSS Statistics version 23 (IBM Corp., Armonk, NY, USA). The study was approved by the Regional Ethics Review Board in Lund and was performed in accordance with the STROBE guidelines for observational studies.

\section{Results}

Following the application of the eligibility criteria, a total of 931 implants placed in 300 patients were included in the study; 35 implant failures were reported. Of these 931 implants, 460 were placed in 145 men (mean age $55.9 \pm 18.5$, range 15.9-82.6 years) and 471 were placed in 155 women (mean age 56.0 \pm 17.8 , range 14.9-90.8 years; $P=0.665$, Mann-Whitney test). A total of 48 implants were placed in 18 SSRI users, whereas 883 implants were placed in 282 non-users. The number of implants per patient ranged from 1 to 15 (mean $3.10 \pm 2.49$ ). The mean follow-up time was $2741 \pm 2258$ days $\quad(1578 \pm 1717$ days for SSRI users, $2804 \pm 2267$ days for non-users; $P<0.000$, Mann-Whitney test).

The implant failure rate was $12.5 \%(6 /$ 48 ) for SSRI users and 3.3\% (29/883) for non-users $(P=0.007$, Fisher's exact test). The 35 failed implants were lost at a mean of $756 \pm 958$ days (range 24 3980 days). Eleven of the 35 failed implants $(31.4 \%)$ were lost prior to the abutment connection procedure or second stage surgery (one in SSRI users, 10 in non-users). Eighteen of the 35 failed implants $(51.4 \%)$ were lost up to 1 year after surgery.

All implants were inserted with open flap surgery. Nine implants were immediately loaded and three implants were placed in fresh extraction sockets, all in SSRI non-users. Thirty-five implants were non-submerged (five in SSRI users, 30 in non-users). The abutment connection surgery was performed after a mean healing time of $159 \pm 63$ days for the SSRI users and $150 \pm 59$ days for the non-users ( $P=0.012$, Mann-Whitney test). All 469 'turned/machined' implants were Brånemark implants (Nobel Biocare AB, Göteborg, Sweden). The 'enlargedsurface' implants were mostly Brånemark MKIII implants with a TiUnite surface $(n=386)$; the rest $(n=76)$ were Astra TiOblast, OsseoSpeed, XiVE, Frialit-2 (Dentsply Implants, Mölndal, Sweden) and Straumann SLA (Straumann, Basel, Switzerland) implants. There were no hydroxyapatite-coated surface implants in this group.

Comparisons between users and nonusers of SSRIs in terms of demographic systemic conditions and other factors ( $\mathrm{Ta}$ ble 1) showed that people from the age range $>30$ to $\leq 60$ years and those $>60$ years were more likely to be taking SSRIs than younger patients ( $\leq 30$ years), although this did not reach statistical significance. More women were taking SSRIs than men. Furthermore, fewer implants were placed in the mandible in relation to the anterior maxilla, and fewer patients were followed up for longer periods in the SSRI user group than in the non-user group, and these differences were statistically significant. The groups of SSRI users and non-users were comparable in terms of the other factors. 
Table 1. Description of the cohort by implants $(n=931)$ between users and non-users of SSRIs.

\begin{tabular}{|c|c|c|c|c|}
\hline \multirow{2}{*}{ Variables } & \multicolumn{2}{|l|}{ SSRIs } & \multirow{2}{*}{ OR $(95 \% \mathrm{CI})$} & \multirow{2}{*}{$P$-value } \\
\hline & Users, $n(\%)$ & Non-users, $n(\%)$ & & \\
\hline \multicolumn{5}{|l|}{ Age (years) } \\
\hline$\leq 30$ & $3(6.3)$ & $127(14.4)$ & 1 & \\
\hline$>30$ to $\leq 60$ & $21(43.8)$ & $311(35.2)$ & $2.859(0.838-9.752)$ & 0.093 \\
\hline$>60$ & $24(50.0)$ & $445(50.4)$ & $2.283(0.677-7.705)$ & 0.183 \\
\hline \multicolumn{5}{|l|}{ Sex } \\
\hline Male & $3(6.3)$ & $457(51.8)$ & 1 & \\
\hline Female & $45(93.8)$ & $426(48.2)$ & $16.092(4.964-52.166)$ & $<0.001$ \\
\hline \multicolumn{5}{|l|}{ Implant length (mm) } \\
\hline $6.0-10.0$ & $11(22.9)$ & $179(20.3)$ & 1 & \\
\hline $10.5-14.0$ & $27(56.3)$ & $425(48.1)$ & $1.034(0.502-2.129)$ & 0.928 \\
\hline $15.0-20.0$ & $10(20.8)$ & $279(31.6)$ & $0.583(0.243-1.402)$ & 0.228 \\
\hline \multicolumn{5}{|l|}{ Implant diameter $(\mathrm{mm})$} \\
\hline $3.00-3.50$ & $6(12.5)$ & $85(9.6)$ & 1 & \\
\hline $3.70-4.10$ & $42(87.5)$ & $774(87.7)$ & $0.769(0.318-1.861)$ & 0.560 \\
\hline $4.20-5.00$ & $0(0)$ & $24(2.7)$ & - & - \\
\hline \multicolumn{5}{|l|}{ Implant surface } \\
\hline Turned & $22(45.8)$ & $447(50.6)$ & 1 & \\
\hline Enlarged & $26(54.2)$ & $436(49.4)$ & $1.212(0.676-2.170)$ & 0.519 \\
\hline \multicolumn{5}{|l|}{ Implant location } \\
\hline Anterior maxilla & $27(56.3)$ & $308(34.9)$ & 1 & \\
\hline Posterior maxilla & $9(18.8)$ & $158(17.9)$ & $0.650(0.298-1.415)$ & 0.278 \\
\hline Anterior mandible & $6(12.5)$ & $235(26.6)$ & $0.291(0.118-0.717)$ & 0.007 \\
\hline Posterior mandible & $6(12.5)$ & $182(20.6)$ & $0.376(0.152-0.928)$ & 0.034 \\
\hline \multicolumn{5}{|l|}{ Bone augmentation } \\
\hline No & $48(100)$ & $852(96.5)$ & 1 & \\
\hline Yes & $0(0)$ & $31(3.5)$ & - & - \\
\hline \multicolumn{5}{|c|}{ Follow-up time (years) } \\
\hline$\leq 1$ & $7(14.6)$ & $91(10.3)$ & 1 & \\
\hline$>1$ to $\leq 5$ & $32(66.7)$ & $307(34.8)$ & $1.355(0.579-3.172)$ & 0.484 \\
\hline$>5$ to $\leq 10$ & $4(8.3)$ & $207(23.4)$ & $0.251(0.072-0.879)$ & 0.031 \\
\hline$>10$ & $5(10.4)$ & $278(31.5)$ & $0.234(0.072-0.755)$ & 0.015 \\
\hline
\end{tabular}

SSRIs, selective serotonin reuptake inhibitors; OR, odds ratio; CI, confidence interval.

The multivariate GEE model (Table 2) showed that the intake of SSRIs did not exert a statistically significant influence on implant failure, nor did age, sex, implant diameter, or bone augmentation. Howev- er, longer implants failed less in comparison to shorter ones, as did enlargedsurface implants in relation to turned implants and implants placed in the anterior mandible in relation to the anteri-

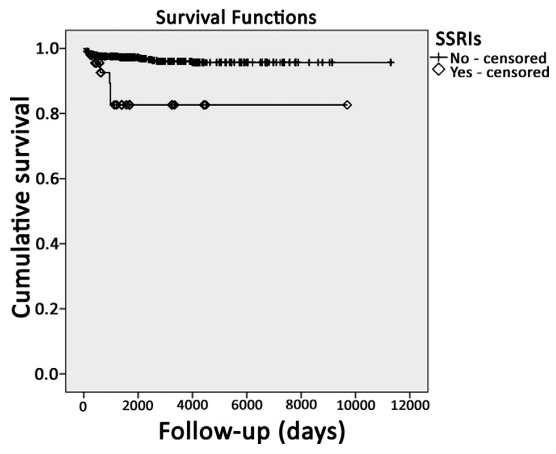

Fig. 1. Kaplan-Meier curves comparing the cumulative survival between implants placed in users and non-users of selective serotonin reuptake inhibitors (SSRIs). or maxilla. Furthermore, implants failed less in those with longer follow-ups in comparison to shorter periods of observation.

The multilevel mixed-effects parametric survival analysis (Table 3 ) adjusted for the aforementioned confounders using a Weibull model based on the AIC selection, showed that the intake of SSRIs did not significantly affect the implant survival rate, and nor did age, sex, implant diameter, implant surface, and implant location. Longer implants had a statistically significant HR, as did bone augmentation procedures.

Figure 1 shows a comparison of the Kaplan-Meier curves between the users and non-users of SSRIs (log-rank test); a statistically significant difference in the cumulative implant survival was found between the groups $(P<0.001)$.

\section{Discussion}

The purpose of this study was to investigate the association between the intake of SSRIs and the risk of dental implant failure. It was hypothesized that patients taking SSRIs would have a higher implant failure rate than patients not taking this class of drugs. A comparison of the implant failure rates between SSRI users and non-users was performed, as well as an estimation of the influence of several variables on the prevalence of implant failure in regression models, with the intake of these medications as the predictor variable.

Four different statistical methods were used to assess this relationship, with two analyses identifying an association and the other two not finding such an association. A statistically significant association between the intake of SSRIs and an increased risk of implant failure was found using Fisher's exact test. However, this test does not take into consideration the other confounders, the factor time, and the cluster effect, i.e. the fact that several implants were available for a single patient. The Kaplan-Meier analysis showed a statistically significant difference in the cumulative survival rate between SSRI users and non-users. Although this test considers the factor time, it does not consider the influence of the other variables and the cluster effect. The multivariate GEE method did not find a statistically significant association, but although analyzing the influence of the other confounders on implant failure, the model takes the factor time as a simple confounder. The multilevel mixed-effects parametric survival analysis could represent the best 
Table 2. Multivariate generalized estimating equation (GEE) logistic regression model (total implants, $n=931$ ).

\begin{tabular}{|c|c|c|c|}
\hline Variables & Failed/survived, $n / n$ & OR $(95 \% \mathrm{CI})$ & $P$-value \\
\hline \multicolumn{4}{|l|}{ SSRIs } \\
\hline No & $29 / 854$ & 1 & \\
\hline Yes & $6 / 42$ & $2.316(0.168-31.932)$ & 0.530 \\
\hline \multicolumn{4}{|l|}{ Age (years) } \\
\hline$\leq 30$ & $4 / 126$ & 1 & \\
\hline$>30$ to $\leq 60$ & $23 / 309$ & $1.936(0.318-11.781)$ & 0.474 \\
\hline$>60$ & $8 / 461$ & $0.224(0.026-1.916)$ & 0.172 \\
\hline \multicolumn{4}{|l|}{ Sex } \\
\hline Male & $10 / 450$ & 1 & \\
\hline Female & $25 / 446$ & $1.846(0.655-5.203)$ & 0.246 \\
\hline \multicolumn{4}{|l|}{ Implant length (mm) } \\
\hline $6.0-10.0$ & $18 / 172$ & 1 & \\
\hline $10.5-14.0$ & $12 / 440$ & $0.203(0.077-0.536)$ & 0.001 \\
\hline $15.0-20.0$ & $5 / 284$ & $0.172(0.036-0.827)$ & 0.028 \\
\hline \multicolumn{4}{|l|}{ Implant diameter (mm) } \\
\hline $3.00-3.50$ & $3 / 88$ & 1 & \\
\hline $3.70-4.10$ & $31 / 785$ & $0.880(0.124-6.222)$ & 0.898 \\
\hline $4.20-5.00$ & $1 / 23$ & $1.665(0.113-24.545)$ & 0.710 \\
\hline \multicolumn{4}{|l|}{ Implant surface } \\
\hline Turned & $21 / 448$ & 1 & \\
\hline Enlarged & $14 / 448$ & $0.085(0.015-0.495)$ & 0.006 \\
\hline \multicolumn{4}{|l|}{ Implant location } \\
\hline Anterior maxilla & $15 / 320$ & 1 & \\
\hline Posterior maxilla & $6 / 161$ & $0.341(0.097-1.197)$ & 0.093 \\
\hline Anterior mandible & $3 / 238$ & $0.124(0.019-0.805)$ & 0.029 \\
\hline Posterior mandible & $11 / 177$ & $0.490(0.101-2.386)$ & 0.377 \\
\hline \multicolumn{4}{|l|}{ Bone augmentation } \\
\hline No & $32 / 868$ & 1 & \\
\hline Yes & $3 / 28$ & $3.220(0.698-14.847)$ & 0.134 \\
\hline \multicolumn{4}{|l|}{ Follow-up time (years) } \\
\hline$\leq 1$ & $18 / 80$ & 1 & \\
\hline$>1$ to $\leq 5$ & $11 / 328$ & $0.088(0.026-0.295)$ & $<0.001$ \\
\hline$>5$ to $\leq 10$ & $5 / 206$ & $0.069(0.012-0.415)$ & 0.004 \\
\hline$>10$ & $1 / 282$ & $0.002(0.000-0.047)$ & $<0.001$ \\
\hline
\end{tabular}

SSRIs, selective serotonin reuptake inhibitors; OR, odds ratio; CI, confidence interval.

analysis; it showed no statistically significant association between the intake of SSRIs and a higher risk of dental implant failure.

The statistical models identified implant length, implant surface, implant location, bone augmentation, and the duration of follow-up as potential factors exerting a statistically significant influence on denta implant failure. Increased initial stability, long-term resistance to bending moment forces, expedited healing, and a decreased risk of movement at the interface are listed as advantages of increased implant length, which could increase the chance of a bette implant clinical outcome. ${ }^{23}$ Implants classified as having an enlarged surface showed a significantly lower probability of failing in comparison to turned implants. There is supportive evidence for a positive relationship between improved bone healing around implants and the roughness of the implant surface, ${ }^{24,25}$ which enhances the process of osseointegration. It is known that the surface properties of dental implants, such as their topography and chemistry, are relevant for the osseointegration process, influencing ionic interactions, protein adsorption, and cellular activity at the surface. $^{26-28}$

Regarding the implant location, the maxilla has a less favourable bone texture ullary bone and thin cortical Plates. ${ }^{29}$ The anterior mandible region is usually considered to have an osseous structure more favourable to achieving primary stability of implants. Jaws with poor bone quality may be at risk of establishing initial instability of the implants and a lack of resistance to mechanical stresses, thus resulting in early implant failure. ${ }^{29,30}$

The greater risk of implant failure at sites submitted to bone augmentation procedures may be related to the fact that these implants were placed in areas of insufficient bone volume and/or remaining defects, which could indicate a more challenging healing situation in comparison to implants placed in healed sites. ${ }^{31,32}$ If the than the mandible, with low density med- need for augmentation arises as a result of bone loss due to periodontal disease, infection, or osteoporosis, it is possible that these conditions will affect the successful integration of the graft. ${ }^{33}$

The influence of the follow-up time on the failure rate, with a lower risk of failure with a longer follow-up, may be connected to the fact that $31.4 \%$ of the implants were lost prior to the abutment connection procedure and $51.4 \%$ up to 1 year after surgery.

Studies in the field of dentistry have already assessed whether the intake of antidepressants might be associated with an increased risk of implant failure. Alsaadi et al. evaluated the impact of local and systemic factors on the incidence of oral implant failure up to abutment connection, finding no relationship between these two factors. ${ }^{16,18}$ The same group of researchers also evaluated the impact of the same factors on the incidence of late oral implant failure, again finding no relationship. ${ }^{17}$ However, these three studies did not specify whether the medications used were SSRIs only or included other classes of antidepressant. A very recent cohort study evaluated the same conditions as these two previous studies, i.e. failure up to abutment connection, with several implant- and health-related factors as confounders and not limiting antidepressants to the SSRI class. ${ }^{2}$ In contrast to Alsaadi et al., ${ }^{16-18}$ an increased risk of implant failure was observed with the use of these medications. ${ }^{2}$

A study by $\mathrm{Wu}$ et al. is the only one among those previously published that has evaluated SSRIs only, excluding the other classes of antidepressant, and a statistically significant association was found. ${ }^{15}$ However, it is important to take into account certain aspects of the study by Wu et al. ${ }^{15} \mathrm{~A}$ table in that publication included nine factors other than the intake of SSRIs, which was presented alone in a different table. It is not entirely clear from the publication whether they included the intake of SSRIs in this final model or whether they analyzed the factor SSRIs alone in a crude model. If they did not analyze the factor SSRIs in the model with the other nine factors, this could have generated a misspecified model, i.e. one that is not complete and is missing key/important explanatory variables and so does not adequately represent what one is trying to model or trying to predict. Supposing that the variable SSRIs was not included in the model together with the other confounders, and if it were, the interaction of other confounders of strong effect on the outcome (implant diameter and smok- 
Table 3. Multilevel survival analysis for dental implant failure in terms of different factors (total implants, $n=931)$. Hazard ratios (HR) were determined using multilevel mixed-effects parametric survival analysis.

\begin{tabular}{|c|c|c|c|c|c|}
\hline \multirow{2}{*}{ Factor } & \multicolumn{2}{|c|}{ Implants, $n(\%)$} & \multirow{2}{*}{ Failure (\%) } & \multirow{2}{*}{$P$-value } & \multirow{2}{*}{ HR (95\% CI) } \\
\hline & Failed & Survived & & & \\
\hline \multicolumn{6}{|l|}{ SSRIs } \\
\hline No & $29(82.9)$ & $854(95.3)$ & 3.4 & & 1 \\
\hline Yes & $6(17.1)$ & $42(4.7)$ & 14.3 & 0.125 & $4.108(0.676-24.963)$ \\
\hline \multicolumn{6}{|l|}{ Age (years) } \\
\hline$\leq 30$ & $4(11.4)$ & $126(14.1)$ & 3.2 & & 1 \\
\hline$>30$ to $\leq 60$ & $23(65.7)$ & $309(34.5)$ & 7.4 & 0.662 & $1.426(0.290-7.008)$ \\
\hline$>60$ & $8(22.9)$ & $461(51.5)$ & 1.7 & 0.160 & $0.258(0.039-1.710)$ \\
\hline \multicolumn{6}{|r|}{ 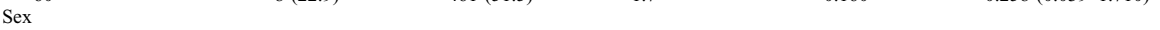 } \\
\hline Male & $10(28.6)$ & $450(50.2)$ & 2.2 & & 1 \\
\hline Female & $25(71.4)$ & $446(49.8)$ & 5.6 & 0.404 & $1.641(0.513-5.250)$ \\
\hline \multicolumn{6}{|l|}{ Implant length (mm) } \\
\hline $6.0-10.0$ & $18(51.4)$ & $172(19.2)$ & 10.5 & & 1 \\
\hline $10.5-14.0$ & $12(34.3)$ & $440(49.1)$ & 2.7 & 0.074 & $0.426(0.167-1.088)$ \\
\hline $15.0-20.0$ & $5(14.3)$ & $284(31.7)$ & 1.8 & 0.040 & $0.249(0.066-0.940)$ \\
\hline \multicolumn{6}{|l|}{ Implant diameter (mm) } \\
\hline $3.00-3.50$ & $3(8.6)$ & $88(9.8)$ & 3.4 & & 1 \\
\hline $3.70-4.10$ & $31(88.5)$ & 785 (87.6) & 3.9 & 0.816 & $0.812(0.141-4.697)$ \\
\hline $4.20-5.00$ & $1(2.9)$ & $23(2.6)$ & 4.3 & 0.945 & $0.900(0.045-18.139)$ \\
\hline \multicolumn{6}{|l|}{ Implant surface } \\
\hline Turned & $21(60.0)$ & $448(50.0)$ & 4.7 & & 1 \\
\hline Enlarged & $14(40.0)$ & $448(50.0)$ & 3.1 & 0.132 & $0.383(0.110-1.335)$ \\
\hline \multicolumn{6}{|l|}{ Implant location } \\
\hline Anterior maxilla & $15(42.9)$ & $320(35.7)$ & 4.7 & & 1 \\
\hline Posterior maxilla & $6(17.1)$ & $161(18.0)$ & 3.7 & 0.831 & $0.890(0.306-2.587)$ \\
\hline Anterior mandible & $3(8.6)$ & $238(26.6)$ & 1.3 & 0.167 & $0.307(0.058-1.637)$ \\
\hline Posterior mandible & $11(31.4)$ & $177(19.8)$ & 6.2 & 0.329 & $1.885(0.528-6.729)$ \\
\hline \multicolumn{6}{|l|}{ Bone augmentation } \\
\hline No & $32(91.4)$ & $868(96.9)$ & 3.7 & & 1 \\
\hline Yes & $3(8.6)$ & $28(3.1)$ & 10.7 & 0.036 & $8.538(1.154-63.170)$ \\
\hline
\end{tabular}

HR, hazard ratio; CI, confidence interval; SSRIs, selective serotonin reuptake inhibitors.

ing, in this case in particular) could have influenced the significance of the factor SSRIs. It might happen that when there is the inclusion of a new variable in a model, something that was previously statistically significant becomes non-significant. ${ }^{34}$ Thus, the results of the study by $\mathrm{Wu}$ et al. must be analyzed carefully. ${ }^{1}$

It is important to take into consideration that the alleged higher risk of dental implant failure could be associated with the patient's mental condition rather than by the intake of antidepressants per se. Some studies have demonstrated that psychosocial stress may induce neglect of oral hygiene, ${ }^{35,36}$ and that people suffering from psychosocial stress may show resistance to periodontal therapies. ${ }^{37}$ Thus, it is suggested that psychosocial stress may be an important risk factor in defining the progression of periodontitis, even if the patient is undergoing therapy. ${ }^{38}$ Moreover, another study showed depression to be associated with more extensive periodontitis, with continuous periodontal breakdown, and with differences in serum antibody titres to pathogens associated with periodontitis. ${ }^{39}$ It is a matter of debate whether the results relating depression to periodontitis (around teeth) could be similar around implants (peri-implantitis).

The limitations of the present study include the retrospective design, with gaps in information and incomplete records. As all data rely on the accuracy of the original examination and documentation, items may have been excluded in the initial examination or not recorded in the den$\mathrm{tal} /$ medical charts. The lack of specific information characterizing the systemic conditions status and the medication dosages of some patients represents a limitation also connected to the retrospective nature of this study. Moreover, as the status 'taking SSRIs' was completely isolated from the influence of other systemic conditions, medications, or compromising habits (bruxism, smoking, snuff) that could somehow have affected the bone metabolism and/or osseointegration, the group investigated in this study is, itself, not representative of the general population.

In conclusion, it is suggested that the intake of SSRIs may not be associated with an increased risk of dental implant failure. The statistical models also identified implant length, implant surface, implant location, bone augmentation, and the duration of follow-up as potential factors exerting a statistically significant influence on dental implant failure. New research efforts on the subject should be concentrated in prospective studies with a larger cohort size presenting more balanced study groups.

\section{Funding}

This work was supported by research funds from the Oral Health Related Research by Region Skåne, Sweden (Odontologisk Forskning i Region Skåne, OFRS 414321), and from the Scientific Research Council of Sweden (Vetenskapsrådet, Dnr 2015-02971). This work was supported by Folktandvården $\mathrm{AB}$, Region Skåne, Sweden, and by CNPq, Conselho Nacional de Desenvolvimento Científico e Tecnológico, Brazil.

\section{Competing interests}

None declared.

\section{Ethical approval}

Regional Ethics Review Board, Lund, Sweden (Dnr 2014/598; Dnr 2015/72). 


\section{Patient consent}

Not required.

\section{References}

1. Chrcanovic BR, Albrektsson T, Wennerberg A. Reasons for failures of oral implants. Oral Rehabil 2014:41:443-76.

2. Chrcanovic BR, Kisch J, Albrektsson T, Wennerberg A. Factors influencing early dental implant failures. I Dent Res 2016;95:995-1002.

3. Mezuk B, Eaton WW, Golden SH, Wand G Lee HB. Depression, antidepressants, and bone mineral density in a population-based cohort. J Gerontol A Biol Sci Med Sci 2008; $63: 1410-5$

4. Rauma PH, Pasco JA, Berk M, Stuart AL Koivumaa-Honkanen H, Honkanen RJ, Hodge JM, Williams LJ. The association between major depressive disorder, use of antidepressants and bone mineral density (BMD) in men. $J$ Musculoskelet Neuronal Interact 2015;15:177-85.

5. Bab IA, Einhorn TA. Regulatory role of osteogenic growth polypeptides in bone formation and hemopoiesis. Crit Rev Eukaryo Gene Expr 1993;3:31-46.

6. Ershler WB, Harman SM, Keller ET. Immunologic aspects of osteoporosis. Dev Comp Immunol 1997;21:487-99.

7. Gruber HE. Bone and the immune system. Proc Soc Exp Biol Med 1991:197:219-25.

8. Battaglino R, Fu J, Spate U, Ersoy U, Joe M, Sedaghat L, Stashenko P. Serotonin regulate osteoclast differentiation through its transporter. J Bone Miner Res 2004:19:1420-31.

9. Bliziotes MM, Eshleman AJ, Zhang XW Wiren KM. Neurotransmitter action in osteoblasts: expression of a functional system fo serotonin receptor activation and reuptake. Bone 2001;29:477-86.

10. Westbroek I, van der Plas A, de Rooij KE, Klein-Nulend J, Nijweide PJ. Expression of serotonin receptors in bone. $J$ Biol Chem 2001;276:28961-8

11. Gustafsson BI, Thommesen L, Stunes AK, Tommeras K, Westbroek I, Waldum HL, Slørdahl K, Tamburstuen MV, Reselan JE, Syversen U. Serotonin and fluoxetine modulate bone cell function in vitro. $J \mathrm{Cel}$ Biochem 2006:98:139-51.

12. Warden SJ, Nelson IR, Fuchs RK, Bliziote MM, Turner CH. Serotonin (5-hydroxytryptamine) transporter inhibition causes bone loss in adult mice independently of estroge deficiency. Menopause 2008;15:1176-83.

13. Warden SJ, Robling AG, Sanders MS, Bliziotes MM, Turner $\mathrm{CH}$. Inhibition of the serotonin (5-hydroxytryptamine) transporte reduces bone accrual during growth. Endo crinology 2005:146:685-93.

14. Yadav VK, Ryu JH, Suda N, Tanaka KF, Gingrich JA, Schütz G, Glorieux FH, Chian CY, Zajac JD, Insogna KL, Mann JJ, Hen R,
Ducy P, Karsenty G. Lrp5 controls bone formation by inhibiting serotonin synthesis in the duodenum. Cell 2008;135:825-37.

15. Wu X, Al-Abedalla K, Rastikerdar E, Abi Nader S, Daniel NG, Nicolau B, Tamimi F. Selective serotonin reuptake inhibitors and the risk of osseointegrated implant failure: a cohort study, J Dent Res 2014;93:1054-61.

16. Alsaadi G, Quirynen M, Komarek A, van Steenberghe D. Impact of local and systemic factors on the incidence of oral implant failures, up to abutment connection. $J$ Clin Periodontol 2007;34:610-7.

17. Alsaadi G, Quirynen M, Komarek A, van Steenberghe D. Impact of local and systemic factors on the incidence of late oral implant loss. Clin Oral Implants Res 2008;19:670-6.

18. Alsaadi G, Quirynen M, Michiles K, Teughels W, Komarek A, van Steenberghe D. Impact of local and systemic factors on the incidence of failures up to abutment connection with modified surface oral implants. J Clin Periodontol 2008;35:51-7.

19. Chrcanovic BR, Albrektsson T, Wennerberg A. Prophylactic antibiotic regimen and dental implant failure: a meta-analysis. J Oral Rehabil 2014;41:941-56.

20. Crowther MJ. Multilevel mixed-effects parametric survival analysis. United Kingdom Stata Users' Group Meeting, London, UK, 2013.

21. Crowther MJ, Look MP, Riley RD. Multilevel mixed effects parametric survival models using adaptive Gauss-Hermite quadrature with application to recurrent events and individual participant data meta-analysis. Stat Med 2014:33:3844-58.

22. Fang Y. Asymptotic equivalence between cross-validations and Akaike information criteria in mixed-effects models. $J$ Data Sci 2011;9:15-21.

23. Winkler S, Morris HF, Ochi S. Implant survival to 36 months as related to length and diameter. Ann Periodontol 2000;5:22-31.

24. Albrektsson T, Wennerberg A. Oral implant surfaces: part 2-review focusing on clinical knowledge of different surfaces. Int $J$ Prosthodont 2004:17:544-64

25. Shalabi MM, Gortemaker A, Van't Hof MA, Jansen JA, Creugers NH. Implant surface roughness and bone healing: a systematic review, J Dent Res 2006;85:496-500.

26. Chrcanovic BR, Leão NL, Martins MD. Influence of different acid etchings on the superficial characteristics of $\mathrm{Ti}$ sandblasted with $\mathrm{Al}_{2} \mathrm{O}_{3}$. Materials Research 2013;16:1006-14

27. Chrcanovic BR, Martins MD. Study of the influence of acid etching treatments on the superficial characteristics of Ti. Materials Research 2014;17:373-80.

28. Chrcanovic BR, Wennerberg A, Martin MD. Influence of temperature and acid etching time on the superficial characteristics of Ti. Materials Research 2015;18:963-70.
29. Horwitz J, Zuabi O, Peled M, Machtei EE. Immediate and delayed restoration of denta implants in periodontally susceptible patients: 1-year results. Int J Oral Maxillofac Implants 2007;22:423-9.

30. Friberg B, Jemt T, Lekholm U. Early failure in 4,641 consecutively placed Brånemark dental implants: a study from stage 1 surgery to the connection of completed prostheses. Int J Oral Maxillofac Implants 1991;6:142-

31. Zumstein T, Billstrom C, Sennerby L. A 4- to 5-year retrospective clinical and radiographic study of Neoss implants placed with or without GBR procedures. Clin Implant Dent Relat Res 2012;14:480-90.

32. Chrcanovic BR, Albrektsson T, Wennerberg A. Dental implants inserted in fresh extraction sockets versus healed sites: a systematic review and meta-analysis. $J$ Dent 2015; 43:16-41

33. Porter JA, von Fraunhofer JA. Success or failure of dental implants? A literature review with treatment considerations. Gen Dent 2005;53:423-32. quiz 433, 446.

34. Cohen J, Cohen P, West SG, Aiken LS. Applied multiple regression/correlation analysis for the behavioral sciences. 3rd edn. Mahwah. NJ: Lawrence Erlbaum; 2003

35. Deinzer R, Hilpert D, Bach K, Schawacht M, Herforth A. Effects of academic stress on oral hygiene- a potential link between stress and plaque-associated disease? J Clin Periodontol 2001:28:459-64.

36. Spangler JG, Konen JC. Oral health behaviors in medical patients with diabetes mellitus. J Dent Hyg 1994;68:287-93.

37. Axtelius B, Soderfeldt B, Nilsson A, Edwardsson S, Attstrom R. Therapy-resistant periodontitis. Psychosocial characteristics. J Clin Periodontol 1998;25:482-91.

38. Persson GR, Persson RE, MacEntee CI, Wyatt CC, Hollender LG, Kiyak HA. Periodontitis and perceived risk for periodontitis in elders with evidence of depression. J Clin Periodontol 2003:30:691-6.

39. Moss ME, Beck JD, Kaplan BH, Offenbacher S, Weintraub JA, Koch GG, Genco RJ, Machtei EE, Tedesco LA. Exploratory casecontrol analysis of psychosocial factors and adult periodontitis. J Periodontol 1996;67:1060-9.

Address:

Bruno Ramos Chrcanovic

Department of Prosthodontics

Faculty of Odontology

Malmö University

Carl Gustafs väg 34

Malmö

SE-214 21

Sweden

Tel: +46725 $541545 \cdot$ Fax: +46406658503

E-mails: bruno.chrcanovic@mah.se,

brunochrcanovic@hotmail.com 

VII 



\section{Impact of different surgeons on dental implant failure}

Bruno Ramos Chrcanovic ${ }^{1 *}$

Jenö Kisch ${ }^{2}$

Tomas Albrektsson ${ }^{1,3}$

Ann Wennerberg ${ }^{1}$

${ }^{1}$ Department of Prosthodontics, Faculty of Odontology, Malmö University, Malmö, Sweden

${ }^{2}$ Clinic for Prosthodontics, Centre of Dental Specialist Care, Malmö, Sweden

${ }^{3}$ Department of Biomaterials, Gothenburg University, Göteborg, Sweden

* Corresponding author:

Bruno Ramos Chrcanovic, Department of Prosthodontics, Faculty of Odontology, Malmö University,

Carl Gustafs väg 34, SE-214 21, Malmö, Sweden. bruno.chrcanovic@mah.se; brunochrcanovic@hotmail.com Mobile: +46 725541545 Fax: +46 406658503 


\section{Abstract}

Aims. To assess the influence of several factors on dental implant failure prevalence, with special consideration on the placement of implants by different dental surgeons.

Methods. This retrospective study is based on 2,670 patients who received 10,096 implants at one specialist clinic. Only the data of patients and implants treated by surgeons who had inserted a minimum of 200 Implants at the clinic were included. Kaplan-Meier curves were stratified with respect to the individual surgeon. A generalized estimating equation (GEE) method was used, to account for the fact that repeated observations (several implants) were placed in a single patient. The factors bone quantity/quality, implant location, implant surface, and implant system were analyzed with descriptive statistics separately for each individual surgeon.

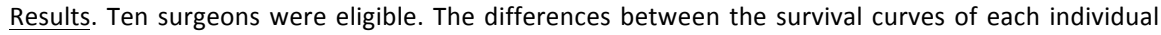
were statistically significant. The multivariate GEE model showed the following variables to be statistically significant: surgeon, bruxism, intake of antidepressants, location, implant length, and implant system. The surgeon with the highest absolute number of failures was also the one who inserted most implants in sites of poor bone and used turned implants in most cases, whereas the surgeon with the lowest absolute number of failures used mainly modern implants. Separate survival analyses of turned and modern implants stratified for the individual surgeon showed statistically significant differences in cumulative survival.

Conclusion. Different levels of failure incidence could be observed between the surgeons, occasionally reaching significant levels. Although a direct causal relationship could not be ascertained, the results of the present study suggest that the surgeons' technique, skills, and/or judgment may negatively influence the implant survival rates.

\section{Keywords}

Dental implants; osseointegration; surgeons; predictors; survival rate 


\section{Introduction}

Some studies have assumed that the operator skill and/or experience is of importance for achieving high implant success rates and that a learning curve does exist for dental implant therapy. ${ }^{1-}$

${ }^{4}$ However, this subject is not without controversy, and a significant association of 'experienced surgeon-less implant failures' has not been verified by all studies. Melo et al. ${ }^{5}$ found no statistically significant difference in implant survival rates among oral and maxillofacial residents of different levels of training. However, the authors stressed that this observation may be due to the fact that more complicated cases were assigned to surgeons with more experience in implant surgery. Another study ${ }^{6}$ observed that clinical experience had no significant impact on implant survival, at least not on the basis of multivariate analyses. The authors suggested that a significant difference in favor of inexperienced surgeons based on univariate methods could be explained by the fact that the more challenging cases with poor bone quality and/or quantity had been treated by the academic staff; whereas, standard cases had been usually treated by clinicians-in-training.

Since studies of clinical experience may be skewed by the fact that difficult patients are selected for the experienced clinician, another potential clinician-related factor would be the skill of the surgeon, that may depend on other reasons than mere experience. Having this in mind, the aim of the present retrospective study was to assess, among other factors, the influence of the placement of dental implants by different dental surgeons on the implant failure prevalence.

\section{Materials and Methods}

Objective. The purpose of the present study was to assess the influence of several factors on dental implant failure prevalence, with special consideration on the placement of implants by different dental surgeons. The main aim was to test the null hypothesis of a difference in the implant failure rates for different surgeons, against the alternative hypothesis of no difference.

Materials. This retrospective study is based on all 2,670 patients who received 10,096 implants, and were consecutively treated with implant-supported prostheses between 1980 and 
2014 at one specialist clinic (Clinic for Prosthodontics, Centre of Dental Specialist Care, Malmö, Sweden). The study was approved by the Regional Ethical Review Board in Lund (Dnr 2014/598; Dnr 2015/72).

Inclusion and exclusion criteria. To be included in the study, a surgeon needed to have inserted a minimum of 200 Implants in the clinic. Only modern endosseous dental implants with cylindrical or conical design were considered. Zygomatic implants were not included in the study, as well as implants detected in radiographies, but without basic information about them in the patients' files.

Definitions. An implant was considered a failure if presenting signs and symptoms that led to implant removal, including lack or loss of osseointegration, implant mobility, continuous pain, advanced marginal bone loss, refractory infection, and implant fracture.

Data collection. The dental records of all patients ever treated with implants in the aforementioned clinic were read in order to collect the data. The data were directly entered into a SPSS file (SPSS software, version 23, SPSS Inc., Chicago, IL, USA) as the files were being read.

The following data were collected: implant surface (turned/machined or enlarged surfaces, the latter including sandblasted, acid-etched, sandblasted + acid-etched, anodized or hydroxyapatite-coated surfaces), implant system (Nobel turned, Nobel TiUnite, Astra TiOblast/Osseospeed, Straumann SLA/SLActive/Roxlid, XIVE/Frialit-2, others), implant length and diameter, implant design (cylindrical or conical), prescription of antibiotics (the prophylactic antibiotic regimen was usually starting 1-2 hours before surgery and lasting for 5-7 days postoperatively), bone graft procedures, reason for tooth extraction (periodontal disease, fracture/trauma, advanced caries, tooth agenesis, others), implant jaw location (maxilla or mandible), anterior or posterior location of the implant (locations between 13-23 and between 33-43 were considered anterior location), patient's sex, age of the patient at the time for implant surgery, number of days until failure, and follow-up time. Periapical radiographic images were used to classify the bone sites at the time of surgery according to the Lekholm and Zarb ${ }^{7}$ classification. According to 
this classification, bone quality is broken down into four groups according to the proportion and structure of compact and trabecular bone tissue: type 1 = large homogenous cortical/compact bone; type 2 = thick layer of compact bone surrounding a dense trabecular bone; type $3=$ thin cortical layer surrounding a dense trabecular bone; type 4 = thin cortical layer surrounding a core of low-density trabecular bone. The quantity of jawbone is broken down into five groups $(A, B, C, D$, and E), based on the residual jaw shape following tooth extraction. Bone classified as 'A' presents the largest amount of bone among all classes, whereas bone classified as ' $E$ ' presents the lowest volume of bone. The classification was performed based on the radiograms available for each and every implant included in the study, and was performed case by case at the same time by two researchers who were calibrated previously. Disagreements were resolved by discussion between them, until there was an agreement about the classification of each case.

The general health and the behavioral history of the patients were collected from the patients' files. The presence of a medicament list in the patients' records was used to correlate the use of certain drugs to specific health conditions. The following health factors were assessed: diabetes types I or II, hypertension, hypercholesterolemia, hypothyroidism, asthma, psoriasis, and irradiation of the head-neck region. The patients were also classified according to the intake of the following medication types: antidepressants, immunosuppressive drugs, antithrombotic agents (antiplatelet, anticoagulant, thrombolytic drugs), hormone replacement therapy (HRT) in women, and medicaments to reduce the acid gastric production (proton pump inhibitors - PPI). The following behavioral factors were assessed: smoking habits, use of snuff, bruxism.

Statistical analyses. The mean, standard deviation, and percentages were presented as descriptive statistics. Survival analysis stratified in relation to the surgeon was performed using the Kaplan-Meier analysis, and a comparison among groups was analyzed using the log-rank test. Survival analyses stratified in relation to each surgeon were also performed for turned and enlarged-surface implant groups. Logistic regression models were used and a generalized estimating equation (GEE) method was performed to account for the fact that repeated observations (several implants) were 
available for a single patient. All models were adjusted for clustering of subject and implants in a binary logistic regression model using GEE with a binomial distribution and a logit link function, while assuming an exchangeable working correlation structure to assess the relationship between implant failure (dependent variable) and the risk factors (independent variables). Initially a univariate GEE on each of the variables was performed. In order to verify multicollinearity, a correlation matrix of all of the predictor variables with a significant odds ratio (OR; $P$-value cut-off point of 0.1$)$ identified in the univariate GEE was scanned, to see whether there were some high correlations among the predictors. Collinearity statistics obtaining variance inflation factor (VIF) and tolerance statistic were also performed to detect more subtle forms of multicollinearity. Then a multivariable model with a forced entry method was used to evaluate the effect of the factors that were univariately significant $(P<0.1)$ and did not present multicollinearity. A Wald chi-square test was used to analyze the statistical significance of each parameter within the model. The results of the final model were presented as an estimated OR of each significant prognostic variable $(P<0.05)$. The factors bone quantity/quality, implant location, and implant surface, and implant system were analyzed separately for each individual surgeon, with descriptive statistics. All data were statistically analyzed using the SPSS version 23 software (SPSS Inc., Chicago, IL, USA).

\section{Results}

Ten surgeons were identified as having inserted a minimum of 200 Implants in the clinic, totaling 8930 implants in 2340 patients. Figure 1 shows a comparison of the Kaplan-Meier curves between the surgeons $(P<0.001$, log-rank test). Table 1 shows detailed implant information for the surgeons, including the crude GEE analysis with a statistically significant difference for some surgeons in comparison to surgeon 1 , which was the category reference. The univariate GEE model showed that the other variables (Table 2) also had a statistically significant OR: smoking, bruxism, intake of antidepressants, Intake of PPIs, implant diameter, implant length, location (anterior maxilla as the reference category), bone quantity (quantity $A$ as the reference category), bone quality (quality 1 as 
the reference category), implant system (Nobel turned as the reference category), antibiotics, and bone grafting. Only the variables with a statistically significant odds ratio were included in the multivariate GEE model (Table 3). The following variables continued to be statistically significant: surgeon 3 in relation to surgeon 1, smoking, bruxism, intake of antidepressants, anterior mandible in relation to anterior maxilla, implants placed in bone quantity $D$ in relation to bone quantity $A$, implant length, and implant system (Nobel turned as the reference category; Nobel TiUnite, $P<0.001$; Astra TiOblast/Osseospeed, $P=0.035$; Straumann, $P=0.030$; other, $P=0.001)$. Only 129 implants ( $1.44 \%$ of 8,930) were installed before 1985 (before the Lekholm and Zarb classification), of which only 6 (4.65\% of 129$)$ were considered as failures.

The separate descriptive analysis of the variables for each surgeon (Table 4) showed an unequal distribution of implants placed in sites of poor bone (bone quantity $D / E$, bone quality 4) among the surgeons, as well as for implant location and use of turned and enlarged-surface implants. From this point of view it can be seen, for example, that the surgeon with the highest absolute number of failures (surgeon $4-12.20 \%$ ) was also the one who mostly inserted implants in sites of poor bone and virtually used only turned implants (99.9\%). On the other hand, the surgeon with the lowest absolute number of failures (surgeon $10-1.79 \%$ ) mainly used modern implants (99.1\%). Moreover, surgeon 4 had the highest percentage of implants placed in poor bone in comparison to any other surgeon. Table 5 shows the failure and survival distribution of implants according to region and bone quality. Implants placed in poor bone quality in the maxilla had the highest failure rates.

In order to verify the survival of different implant types separately for each surgeon, survival analysis for turned (Figure 2) and enlarged-surface implants (Figure 3) were also performed $(P<0.001$ for both analyses, log-rank test). Due to an extremely reduced number of turned implants placed by surgeon ' 10 ', he was not included in the survival analysis of turned implants (Figure 2), the same happening to surgeons ' 4 ', ' 6 ', ' 7 ', and ' 9 ' when it comes to the enlarged-surface implants survival analysis (Figure 3). 


\section{Discussion}

The aim of the present study was to assess the influence of the placement of dental implants by different dental surgeons on the implants failure prevalence. We found clear differences in clinical outcome that were coupled to the responsible surgeon. Different levels of failure prevalence could be observed between the surgeons, occasionally reaching significant levels.

Besides the surgeon, the multivariate GEE model showed a statistically significant influence of bruxism, intake of antidepressants, implant system, implant length, and implant location on the implant failure prevalence. Bruxism was shown to significantly affect the implant failure prevalence negatively, agreeing with the results of the only meta-analysis on the subject ${ }^{8}$ and recent clinical trials. ${ }^{9}, 10$ This is due to the fact that bruxism is suggested to cause excessive load of implantsupported rehabilitations, which may cause an implant fracture or may result in bone loss around the implants and subsequent implant failure. ${ }^{11} \mathrm{~A}$ meta-analysis including more than 100 studies has shown that failures of implants inserted in smokers are 2.23 times likely to happen than failures of implants inserted in non-smokers. ${ }^{12}$ The increase of implant failure rates due to smoking is hypothesized to be related mainly to the effect of smoking in osteogenesis and angiogenesis. ${ }^{13}$ There are biochemical ${ }^{14,15}$ and clinical ${ }^{16,17}$ evidence suggesting a relationship between the intake of antidepressants and the impairment of bone metabolism, which in theory could interfere with the osseointegration process.

Concerning implant length, there was a decrease in the failure probability with the increase of the implant length. Although some good results can be obtained with the use of shorter implants, they seem to fail more often than longer ones, ${ }^{18}$ at least for the turned implants, which presented the majority of implants in the present study. Increased initial stability, long-term resistance to bending moment forces, expedited healing and a decreased risk of movement at the interface are listed as advantages of increased implant length. ${ }^{19}$ The fact that implants in the anterior mandible presented a lower risk of failure than those inserted in the anterior maxilla may also be associated 
with the implant length. Longer implants are placed in regions that usually provide more bone height, i.e. the anterior mandible. ${ }^{20}$

Although a direct causal relationship could not be ascertained, the results of the present study put some light into the matter of to what point the surgeons' technique, skills, and/or judgment may negatively influence the implant survival rates and prevalence.

A study ${ }^{1}$ comparing treatment outcomes in implant therapy between surgeons with a minimum of 2 years implant experience and surgeons just beginning involvement in implant techniques observed that the surgeon's experience had a major impact on the failure probability of unloaded implants. Lambert et al. ${ }^{2}$ observed that implants placed by inexperienced surgeons (who had placed $<50$ implants) failed twice as often as those placed by experienced surgeons (who had placed 50 or more implants). The results of Morris et al. ${ }^{3}$ showed that the percentage of failure for the less experienced surgeons was nearly twice that seen with the more experienced surgeons. Ji et al. ${ }^{4}$ reported in their study that the implant failure rate for 2 surgeons with $>5$ years of surgical experience was $2.4 \%$ ( 2 of 85 implants), whereas the remaining 18 surgeons, i.e. those with $\leq 5$ years of surgical experience, incurred an implant failure rate of $12.2 \%$ ( 26 of 212 implants). On the other hand, Morris et al. ${ }^{21}$ noticed an only slightly greater survival for implants placed by the more experienced dentists in their study, evaluating more than 2600 implants followed up for 3 years. Two other studies ${ }^{5,6}$ also observed that clinical experience had no significant impact on implant survival, but in these studies only experienced surgeons treated the complicates patients.

There is also a relationship between the quantity of surgical procedures performed and clinical outcomes. Although surgical volume is less important for simple procedures, it might be so for complex procedures. Procedure volume may be linked to other delivery factors associated with surgical outcomes, but it has been assumed that volume is important in large part because it serves as a proxy for operative proficiency. ${ }^{22}$

Besides the procedure volume and experience of the surgeon, the skill is another important factor to take into consideration. A considerable body of research suggests that some surgeons have 
better results than others, with some studies showing wide variation in risk-adjusted patient mortality across surgeons, as observed in studies on bariatric and open heart surgeries. ${ }^{22-24}$ Another example comes from the Swedish Hip Arthroplasty Register where about 13,000 hips placed in the country annually are followed up. Year after year some surgeons show less good clinical results than their peers, despite them using the same hip design. ${ }^{25}$

In many procedures, the technical skill of the operating surgeon may be an important determinant of the outcomes. Analyzing the outcome of about 1000 implants placed at the University Clinic of Göteborg in 1986, a particular surgeon with more than 3 years of clinical experience was found responsible for $40 \%$ of all failures as well as for a majority of the implants that showed marginal bone resorption. ${ }^{26}$ Bryant $^{27}$ likewise found a correlation between some surgeons and implant outcome and marginal bone loss. These data support the theory that implant bone loss may depend on minor trauma whereas implant failure may depend on a greater level of trauma. ${ }^{28}$ However, in the present study we did not investigate marginal bone resorption; survival of the implants was our key parameter. A high level of surgical skill may be essential in preventing intraoperative problems, such as failure to properly maintain or use equipment and instruments that may compromise drilling precision. If an imprecise implant site is prepared or if tapping procedures are excessive, initial implant stability and subsequent osseointegration may be compromised. A bony dehiscence or defect may occur as a result of incorrect implant placement. Moreover, an excessive countersinking may result in the loss of a superior cortical bone plate to stabilize the implant. The failure to preserve keratinized gingiva was even mentioned as a problem, as it may compromise maintenance of peri-implant tissue health in the long-term. ${ }^{29}$ Because failure to osseointegrate can, in part, be related to imprecise surgical technique, it follows that these complications are more likely to occur after operations performed by less skilled surgeons.

It is important to note some facts concerning the observed statistically significant difference in implant failure rates between some surgeons in the present study. The success of a technique depends on careful and scrupulous adherence to the prescribed clinical protocol by the combined 
surgical-prosthodontic team. Theoretically, oral implants may display marginal bone loss or fail due to different clinical skills or clinical judgement. ${ }^{30}$ The present authors observed that clinical judgment may be an important reason behind oral implant problems. Generally, clinical judgment may be defined as the application of information based on actual observation of a patient combined with subjective and objective data that lead to a conclusion. ${ }^{31}$ Everyone working in the health disciplines must realize the importance of clinical judgment, since not doing so may result in unnecessary implant failures. However, there is an obvious problem in measuring the level of clinical judgment. Judgment with due problems afterwards is certainly not limited to the clinical situation. What we call human error, for instance in a flight catastrophy ${ }^{32}$ is in many cases dependent more on judgment than on lack of particular skills. Skills can always be trained to an increased level of skill whereas our level of judgment is dependent on the individual human being and may be very hard to improve. In clinical dentistry, the success of a technique depends on careful and scrupulous adherence to the prescribed clinical protocol by the combined surgical/prosthodontic team. Failure in so doing may result in clinical problems due to either lacking skills about the clinical protocol or violations of it due to clinical judgment. The surgeon might display habits and choices that would, as for example, risk too much in inserting implants in sites with poor bone quantity/quality. The level of complexity of cases treated by different dentists may vary, and some professionals may be more willing to take more risky cases. The surgeons may overestimate their ability to execute a complex or high-risk procedure. $^{27,33}$ There are some categories of behavior that are frequently cited to increase the likelihood of one or more complications occurring during the implementation of a procedure, and such behaviors are also relevant in the dental/medical field and may make the surgeon less careful. ${ }^{32}$ The present study observed that the surgeon with the highest absolute number of failures was also the one who inserted most implants in sites of poor bone. Some surgeons might have had considerable experience with some implant systems but not with other systems. Moreover, some surgeons used old implants more often than other surgeons, but this is also related to the fact that some surgeons inserted more implants in the 1980's and 1990's. Modern implants having an 
enlarged surface had a statistically significant lower probability to fail in comparison to turned implants. It has been shown that the osseointegration process is influenced by several factors, among them the surface texture. ${ }^{34-36}$ There is supportive evidence for a positive relationship between an improved bone healing around implants and its surface roughness, ${ }^{37}$ which enhances the process of osseointegration. However, it is also important to note that the group of turned implants had statistically significant longer mean follow-up time than the group of enlarged surface implants, which can lead to an increase in the failure rate due to longer time at risk.

A separate analysis showed a far unequal distribution of implants placed in sites of poor bone (bone quantity $D / E$, bone quality 4) among the surgeons. The surgeon with the highest absolute number of failures was also the one who inserted most implants in sites of poor bone. It was suggested that one of the most important factors responsible for implant failures is probably the local anatomic structure regarding bone quality and quantity, or rather the lack thereof. ${ }^{38}$ Even if the Lekholm and Zarb ${ }^{7}$ paper was published first in 1985, the general knowledge that implants fared less well in poor bone was discussed among the few implant users of those days prior to 1985. Furthermore, in the material of the present paper only $1.44 \%$ (129 of 8,930 implants) were actually placed before 1985 and only 6 of those implants failed.

Unfortunately, it was not always possible in the present study to assess implant survival based on dentist experience in implant surgery, as this information was not found in the records. Dentists at the specialist clinic of the present study had a diverse background; some entered the specialist training program after only 2 years of working in general practice after leaving the dental school (minimum requirement to enter a specialist program in the country), whereas others had substantial experience from a private dental practice. It is important to remember that the present study includes implants placed as long ago as 1980, and some of the surgeons are already retired. We have lacked information of the particular previous experience of some surgeons. Other important issues include the fact that the experience of the surgeon may be masked by that of the restorative dentist, which was found to influence marginal bone loss and implant outcome in one study. ${ }^{27}$ With 
the existing data forms, the particular experience of each surgeon was difficult to ascertain, and any conclusions regarding experience drawn from these data must be considered with caution in mind. However, if one hypothetically assumes that the surgeons analyzed in the present study would have the same level of experience, and assuming all other factors equal, the variations in the failure rates could be, in part, explained by the surgeons' skill and/or judgment.

Another limitation of the present study is its retrospective nature, which inherently results in flaws. These problems were manifested by the gaps in information and incomplete records. As all data rely on the accuracy of the original examination and documentation, items may have been excluded in the initial examination or nor recorded in the dental/medical chart.

\section{Conclusions}

Different levels of dental implants failure prevalence could be observed between the surgeons, occasionally reaching significant levels. Although a direct causal relationship could not be ascertained, the results of the present study put some light into the matter of to what point the surgeons' poor technique, poor skills, and/or poor judgment may negatively influence the implant survival rates. 


\section{Acknowledgements}

Funding and support: This work was supported by research funds from the Oral Health Related Research by Region Skåne (Odontologisk Forskning i Region Skåne, OFRS 414321), Sweden, and from the Scientific Research Council of Sweden (Vetenskapsrådet, Dnr 2015-02971). This work was supported by Folktandvården AB, Region Skåne, Sweden and by CNPq, Conselho Nacional de Desenvolvimento Científico e Tecnológico, Brazil.

Trial registration at the U.S. National Institutes of Health (clinicaltrials.gov): NCT02369562

Declaration of conflicting interests: There are no conflicts of interest to declare.

\section{References}

1. Preiskel HW, Tsolka P. Treatment outcomes in implant therapy: the influence of surgical and prosthodontic experience. Int J Prosthodont 1995;8:273-279.

2. Lambert PM, Morris HF, Ochi S. Positive effect of surgical experience with implants on secondstage implant survival. J Oral Maxillofac Surg 1997;55:12-18.

3. Morris HF, Manz MC, Tarolli JH. Success of multiple endosseous dental implant designs to second-stage surgery across study sites. J Oral Maxillofac Surg 1997;55:76-82.

4. Ji TJ, Kan JY, Rungcharassaeng K, Roe P, Lozada JL. Immediate loading of maxillary and mandibular implant-supported fixed complete dentures: a 1- to 10-year retrospective study. J Oral Implantol 2012;38 Spec No:469-476.

5. Melo MD, Shafie H, Obeid G. Implant survival rates for oral and maxillofacial surgery residents: a retrospective clinical review with analysis of resident level of training on implant survival. J Oral Maxillofac Surg 2006;64:1185-1189.

6. Cosyn J, Vandenbulcke E, Browaeys H, Van Maele G, De Bruyn H. Factors associated with failure of surface-modified implants up to four years of function. Clin Implant Dent Relat Res 2012;14:347-358. 
7. Lekholm U, Zarb GA. Patient selection and preparation. In: Brånemark PI, Zarb GA, Albrektsson T (eds). Tissue integrated prostheses: osseointegration in clinical dentistry. Chicago: Quintessence Publishing Company, 1985:199-209.

8. Chrcanovic BR, Albrektsson T, Wennerberg A. Bruxism and Dental Implants: A Meta-Analysis. Implant Dent 2015;24:505-516.

9. Chrcanovic BR, Kisch J, Albrektsson T, Wennerberg A. Bruxism and dental implant treatment complications: a retrospective comparative study of 98 bruxer patients and a matched group. Clin Oral Implants Res 2016. doi: 10.1111/clr.12844

10. Chrcanovic BR, Kisch J, Albrektsson T, Wennerberg A. Bruxism and dental implant failures: a multilevel mixed effects parametric survival analysis approach. Journal of Oral Rehabilitation 2016. doi: $10.1111 /$ joor.12431

11. Lobbezoo F, Van Der Zaag J, Naeije M. Bruxism: its multiple causes and its effects on dental implants - an updated review. J Oral Rehabil 2006;33:293-300.

12. Chrcanovic BR, Albrektsson T, Wennerberg A. Smoking and dental implants: A systematic review and meta-analysis. J Dent 2015;43:487-498.

13. Ma L, Zheng LW, Sham MH, Cheung LK. Uncoupled angiogenesis and osteogenesis in nicotinecompromised bone healing. J Bone Miner Res 2010;25:1305-1313.

14. Ducy P, Karsenty G. The two faces of serotonin in bone biology. J Cell Biol 2010;191:7-13.

15. Gustafsson BI, Thommesen L, Stunes AK, Tommeras K, Westbroek I, Waldum HL, et al. Serotonin and fluoxetine modulate bone cell function in vitro. J Cell Biochem 2006;98:139-151.

16. Robbins J, Hirsch C, Whitmer R, Cauley J, Harris T. The association of bone mineral density and depression in an older population. J Am Geriatr Soc 2001;49:732-736.

17. Williams LJ, Bjerkeset O, Langhammer A, Berk M, Pasco JA, Henry MJ, et al. The association between depressive and anxiety symptoms and bone mineral density in the general population: the HUNT Study. J Affect Disord 2011;131:164-171. 
18. Pommer B, Frantal S, Willer J, Posch M, Watzek G, Tepper G. Impact of dental implant length on early failure rates: a meta-analysis of observational studies. J Clin Periodontol 2011;38:856-863.

19. Winkler S, Morris HF, Ochi S. Implant survival to 36 months as related to length and diameter. Ann Periodontol 2000;5:22-31.

20. Chrcanovic BR, Albrektsson T, Wennerberg A. Reasons for failures of oral implants. J Oral Rehabil 2014;41:443-476.

21. Morris HF, Ochi S, Winkler S. Implant survival in patients with type 2 diabetes: placement to 36 months. Ann Periodontol 2000;5:157-165.

22. Birkmeyer JD, Finks JF, O'Reilly A, Oerline M, Carlin AM, Nunn AR, et al. Surgical skill and complication rates after bariatric surgery. N Engl J Med 2013;369:1434-1442.

23. Hannan EL, Kilburn H, Jr., O'Donnell JF, Lukacik G, Shields EP. Adult open heart surgery in New York State. An analysis of risk factors and hospital mortality rates. Jama 1990;264:2768-2774.

24. Hall BL, Hamilton BH. New information technology systems and a Bayesian hierarchical bivariate probit model for profiling surgeon quality at a large hospital. The Quarterly Review of Economics and Finance 2004;44:410-429.

25. Malchau H, Herberts $P$, Ahnfelt L. Prognosis of total hip replacement in Sweden. Follow-up of 92,675 operations performed 1978-1990. Acta Orthop Scand 1993;64:497-506.

26. Albrektsson T. Is surgical skill more important for clinical success than changes in implant hardware? Clin Implant Dent Relat Res 2001;3:174-175.

27. Bryant SR. Oral Implant Outcomes Predicted by Age- and Site-specific Aspects of Bone Condition. Toronto: University of Toronto, 2001:462.

28. Chvartszaid D, Koka S, Zarb G. Osseointegration failure. In: Zarb G, Albrektsson T, Baker G, Eckert SE, Stanford C (eds). Osseointegration: On Continuing Synergies in Surgery, Prosthodontics, Biomaterials. Hanover Park: Quintessence Pub Co, 2008:157-164. 
29. Zarb GA, Schmitt A. The longitudinal clinical effectiveness of osseointegrated dental implants: the Toronto study. Part III: Problems and complications encountered. J Prosthet Dent 1990;64:185-194.

30. Qian J, Wennerberg A, Albrektsson T. Reasons for marginal bone loss around oral implants. Clin Implant Dent Relat Res 2012;14:792-807.

31. Mosby's Medical Dictionary. St. Louis: Mosby Elsevier, 2013.

32. Renouard F, Charrier JG. The Search for the Weakest Link: An Introduction to Human Factors: Ewenn éd., 2011.

33. Jemt T, Olsson M, Renouard F, Stenport V, Friberg B. Early Implant Failures Related to Individual Surgeons: An Analysis Covering 11,074 Operations Performed during 28 Years. Clin Implant Dent Relat Res 2016;18:861-872.

34. Albrektsson T, Wennerberg A. Oral implant surfaces: Part 2--review focusing on clinical knowledge of different surfaces. Int J Prosthodont 2004;17:544-564.

35. Chrcanovic BR, Martins MD. Study of the influence of acid etching treatments on the superficial characteristics of Ti. Materials Research 2014;17:373-380.

36. Chrcanovic BR, Wennerberg A, Martins MD. Influence of Temperature and Acid Etching Time on the Superficial Characteristics of Ti. Materials Research 2015;18:963-970.

37. Shalabi MM, Gortemaker A, Van't Hof MA, Jansen JA, Creugers NH. Implant surface roughness and bone healing: a systematic review. J Dent Res 2006;85:496-500.

38. Ekfeldt A, Christiansson U, Eriksson T, Linden U, Lundqvist S, Rundcrantz T, et al. A retrospective analysis of factors associated with multiple implant failures in maxillae. Clin Oral Implants Res 2001;12:462-467. 


\section{Tables}

Table 1. Univariate generalized estimating equations (GEE) logistic regression model for implant failure: different surgeons (OR - odds ratio).

\begin{tabular}{lccc}
\hline Surgeon & Failure/survival (\% failure) & OR $(95 \% \mathrm{Cl})$ & $P$-value \\
\hline 1 & $134 / 3418(3.77)$ & & \\
2 & $140 / 1413(9.01)$ & $2.477(1.655,3.707)$ & $<0.001$ \\
3 & $40 / 824(4.63)$ & $1.030(0.582,1.825)$ & 0.918 \\
4 & $85 / 612(12.20)$ & $3.301(1.923,5.665)$ & $<0.001$ \\
5 & $28 / 477(5.54)$ & $0.939(0.278,3.174)$ & 0.919 \\
6 & $35 / 412(7.83)$ & $2.112(1.010,4.414)$ & 0.047 \\
7 & $33 / 387(7.86)$ & $2.122(0.926,4.865)$ & 0.075 \\
8 & $25 / 368(6.36)$ & $1.617(0.718,3.638)$ & 0.246 \\
9 & $29 / 247(10.51)$ & $5.150(2.637,10.057)$ & $<0.001$ \\
10 & $4 / 219(1.79)$ & $0.248(0.009,6.654)$ & 0.406 \\
TOTAL & $553 / 8377(6.19)$ & & \\
\hline
\end{tabular}

Table 2. Risk factor analysis for implant failure including implants of the 10 surgeons, using a univariate generalized estimating equations (GEE) logistic regression model, at the implant-level (OR - odds ratio).

\begin{tabular}{|c|c|c|c|}
\hline Factor & Failure/survival* & OR $(95 \% \mathrm{Cl})$ & $P$-value \\
\hline \multicolumn{4}{|l|}{ Smoking } \\
\hline No & $204 / 3695$ & 1 & \\
\hline Yes & $155 / 1663$ & $1.903(1.322,2.738)$ & 0.001 \\
\hline Former smoker & $7 / 152$ & $1.661(0.632,4.366)$ & 0.304 \\
\hline \multicolumn{4}{|l|}{ Snuff } \\
\hline No & $334 / 5207$ & 1 & \\
\hline Yes & $19 / 235$ & $0.884(0.386,2.024)$ & 0.770 \\
\hline \multicolumn{4}{|l|}{ Bruxism } \\
\hline No & $310 / 5624$ & 1 & \\
\hline Yes & $72 / 318$ & $3.594(2.152,6.003)$ & $<0.001$ \\
\hline \multicolumn{4}{|l|}{ Gender } \\
\hline Male & $246 / 3975$ & 1 & \\
\hline Female & $307 / 4402$ & $1.190(0.894,1.584)$ & 0.233 \\
\hline \multicolumn{4}{|l|}{ Diabetes } \\
\hline No & $352 / 5387$ & 1 & \\
\hline Type I & $7 / 105$ & $1.473(0.426,5.092)$ & 0.541 \\
\hline Type II & $25 / 443$ & $0.857(0.477,1.541)$ & 0.607 \\
\hline \multicolumn{4}{|l|}{ High blood pressure } \\
\hline No & $262 / 3975$ & 1 & \\
\hline Yes & $121 / 1944$ & $0.916(0.636,1.319)$ & 0.636 \\
\hline \multicolumn{4}{|l|}{ High cholesterol } \\
\hline No & $325 / 5046$ & 1 & \\
\hline Yes & $57 / 848$ & $0.819(0.487,1.377)$ & 0.451 \\
\hline
\end{tabular}




\begin{tabular}{|c|c|c|c|}
\hline \multicolumn{4}{|l|}{ Hypothyroidism } \\
\hline No & $360 / 5582$ & 1 & \\
\hline Yes & $24 / 318$ & $1.344(0.669,2.700)$ & 0.406 \\
\hline \multicolumn{4}{|l|}{ Asthma } \\
\hline No & $360 / 5431$ & 1 & \\
\hline Yes & $24 / 469$ & $1.008(0.574,1.771)$ & 0.977 \\
\hline \multicolumn{4}{|l|}{ Intake of antidepressants } \\
\hline No & $307 / 5361$ & 1 & \\
\hline Yes & $79 / 529$ & $2.366(1.433,3.908)$ & 0.001 \\
\hline \multicolumn{4}{|l|}{ Irradiation } \\
\hline No & $375 / 5754$ & 1 & \\
\hline Yes & $11 / 160$ & $1.196(0.431,3.322)$ & 0.731 \\
\hline \multicolumn{4}{|c|}{ Hormone replacement therapy } \\
\hline No & $445 / 6886$ & 1 & \\
\hline Yes & $10 / 207$ & $0.690(0.339,1.403)$ & 0.306 \\
\hline \multicolumn{4}{|l|}{ Proton pump inhibitors } \\
\hline No & $321 / 5375$ & 1 & \\
\hline Yes & $46 / 428$ & $2.156(1.256,3.703)$ & 0.005 \\
\hline \multicolumn{4}{|l|}{ Antithrombotics } \\
\hline No & $262 / 4655$ & 1 & \\
\hline Yes & $107 / 1195$ & $1.273(0.836,1.939)$ & 0.261 \\
\hline \multicolumn{4}{|l|}{ Immunosuppressive } \\
\hline No & $359 / 5744$ & 1 & \\
\hline Yes & $8 / 79$ & $1.110(0.326,3.781)$ & 0.868 \\
\hline \multicolumn{4}{|l|}{ Psoriasis } \\
\hline No & $365 / 5760$ & 1 & \\
\hline Yes & $3 / 68$ & $1.217(0.375,3.944)$ & 0.744 \\
\hline \multicolumn{4}{|l|}{ Age } \\
\hline Increase by 1 & & $0.997(0.989,1.004)$ & 0.360 \\
\hline \multicolumn{4}{|l|}{ Implant diameter } \\
\hline Increase by 1 & & $2.284(1.142,4.567)$ & 0.019 \\
\hline \multicolumn{4}{|l|}{ Implant length } \\
\hline Increase by 1 & & $0.885(0.824,0.951)$ & 0.001 \\
\hline \multicolumn{4}{|l|}{ Implant design } \\
\hline Cylindrical & $544 / 7970$ & 1 & \\
\hline Conical & $9 / 407$ & $0.238(0.057,0.997)$ & 0.050 \\
\hline \multicolumn{4}{|l|}{ Location } \\
\hline Anterior maxilla & $263 / 2989$ & 1 & \\
\hline Anterior mandible & $65 / 2097$ & $0.468(0.283,0.774)$ & 0.003 \\
\hline Posterior maxilla & $137 / 1611$ & $1.331(0.998,1.776)$ & 0.052 \\
\hline Posterior mandible & $88 / 1680$ & $0.697(0.457,1.062)$ & 0.093 \\
\hline \multicolumn{4}{|l|}{ Bone quantity } \\
\hline A & $23 / 1032$ & 1 & \\
\hline B & $182 / 4112$ & $1.646(0.892,3.039)$ & 0.111 \\
\hline C & $172 / 2252$ & $2.949(1.586,5.482)$ & 0.001 \\
\hline D & $113 / 652$ & $7.386(3.876,14.075)$ & $<0.001$ \\
\hline $\mathrm{E}$ & $42 / 115$ & $11.735(4.911,28.040)$ & $<0.001$ \\
\hline \multicolumn{4}{|l|}{ Bone quality } \\
\hline 1 & $14 / 343$ & 1 & \\
\hline 2 & $145 / 3719$ & $1.167(0.390,3.493)$ & 0.782 \\
\hline 3 & $251 / 3615$ & $1.539(0.507,4.669)$ & 0.446 \\
\hline
\end{tabular}




\begin{tabular}{|c|c|c|c|}
\hline 4 & $122 / 486$ & $6.464(2.015,20.735)$ & 0.002 \\
\hline \multicolumn{4}{|l|}{ Implant system } \\
\hline Nobel turned & $440 / 4871$ & 1 & \\
\hline Nobel TiUnite & $84 / 2492$ & $0.301(0.183,0.497)$ & $<0.001$ \\
\hline Astra & $14 / 379$ & $0.324(0.088,1.190)$ & 0.090 \\
\hline \multicolumn{4}{|c|}{ TiOblast/Osseospeed** } \\
\hline Straumann & $6 / 179$ & $0.141(0.011,1.809)$ & 0.132 \\
\hline XIVE/Frialit-2 & $5 / 232$ & $0.092(0.002,4.755)$ & 0.236 \\
\hline Other & $4 / 224$ & $0.145(0.042,0.495)$ & 0.002 \\
\hline \multicolumn{4}{|l|}{ Antibiotics } \\
\hline No & $46 / 548$ & 1 & \\
\hline Yes & $218 / 4081$ & $0.590(0.386,0.903)$ & 0.015 \\
\hline \multicolumn{4}{|l|}{ Bone grafting } \\
\hline No & $474 / 7978$ & 1 & \\
\hline Yes & $79 / 388$ & $2.184(1.235,3.862)$ & 0.007 \\
\hline \multicolumn{4}{|c|}{ Reason for tooth extraction } \\
\hline Periodontal disease & $44 / 766$ & 1 & \\
\hline Fracture/trauma & $24 / 489$ & $1.072(0.583,1.969)$ & 0.823 \\
\hline Advanced caries & $7 / 231$ & $0.827(0.366,1.867)$ & 0.647 \\
\hline Tooth agenesis & $19 / 595$ & $0.508(0.234,1.106)$ & 0.088 \\
\hline Other & $6 / 180$ & $0.680(0.273,1.695)$ & 0.408 \\
\hline
\end{tabular}

* The information for some conditions is unknown for a variable number of implants

** Unfortunately, it was not possible to run the regression having Astra TiOblast and Astra Osseospeed as different categories within the parameter 'Implant system', even knowing that they are implants of different designs and surface treatments, although of the same manufacturer. The reason was because of the problem of quasi-complete separation, since there were no events of failure for Astra Osseospeed. The numbers were (failure/survival): Astra TiOblast (14/288) and Astra Osseospeed (0/91)

Table 3. Multivariate generalized estimating equations (GEE) logistic regression model at the implantlevel. Only the factors that were considered statistically significant $(P<0.1)$ in the univariate model and didn't present multicollinearity were included in the multivariate model (OR - odds ratio).

\begin{tabular}{lll}
\hline Factor & OR $(95 \% \mathrm{Cl})$ & $P$-value \\
\hline Surgeon & 1 & \\
1 & $1.148(0.693,1.902)$ & 0.593 \\
2 & $2.834(1.394,5.764)$ & 0.004 \\
3 & $1.180(0.584,2.385)$ & 0.645 \\
4 & $0.795(0.233,2.712)$ & 0.713 \\
5 & $1.243(0.518,2.982)$ & 0.627 \\
6 & $1.566(0.769,3.189)$ & 0.216 \\
7 & $1.153(0.277,4.801)$ & 0.845 \\
8 & $1.377(0.549,3.452)$ & 0.495 \\
9 & $0.583(0.076,4.471)$ & 0.604 \\
10 & & \\
Smoking & & \\
No & 1 & 0.001 \\
Yes & $1.875(1.273,2.763)$ & 0.712 \\
Former smoker & $1.239(0.397,3.869)$ & \\
\hline
\end{tabular}




\begin{tabular}{|c|c|c|}
\hline \multicolumn{3}{|l|}{ Bruxism } \\
\hline No & 1 & \\
\hline Yes & $3.165(1.740,5.758)$ & $<0.001$ \\
\hline \multicolumn{3}{|l|}{ Intake of antidepressants } \\
\hline No & 1 & \\
\hline Yes & $2.174(1.150,4.106)$ & 0.017 \\
\hline \multicolumn{3}{|l|}{ Proton pump inhibitors } \\
\hline No & 1 & \\
\hline Yes & $1.831(0.911,3.680)$ & 0.089 \\
\hline \multicolumn{3}{|l|}{ Location } \\
\hline Anterior maxilla & 1 & \\
\hline Anterior mandible & $0.588(0.362,0.956)$ & 0.032 \\
\hline Posterior maxilla & $0.910(0.666,1.243)$ & 0.554 \\
\hline Posterior mandible & $0.844(0.545,1.307)$ & 0.448 \\
\hline \multicolumn{3}{|l|}{ Bone quantity } \\
\hline A & 1 & \\
\hline B & $1.398(0.703,2.780)$ & 0.339 \\
\hline c & $1.544(0.773,3.083)$ & 0.218 \\
\hline D & $2.278(1.036,5.007)$ & 0.041 \\
\hline $\mathrm{E}$ & $1.845(0.431,7.900)$ & 0.409 \\
\hline \multicolumn{3}{|l|}{ Bone quality } \\
\hline 1 & 1 & \\
\hline 2 & $1.012(0.349,2.934)$ & 0.983 \\
\hline 3 & $0.958(0.325,2.823)$ & 0.938 \\
\hline 4 & $2.419(0.756,7.734)$ & 0.136 \\
\hline \multicolumn{3}{|l|}{ Implant diameter } \\
\hline Increase by 1 & $1.816(0.466,7.077)$ & 0.390 \\
\hline \multicolumn{3}{|l|}{ Implant length } \\
\hline Increase by 1 & $0.903(0.839,0.971)$ & 0.006 \\
\hline \multicolumn{3}{|l|}{ Implant design } \\
\hline Cylindrical & 1 & \\
\hline Conical & $1.036(0.365,2.943)$ & 0.947 \\
\hline \multicolumn{3}{|l|}{ Implant system } \\
\hline Nobel turned & 1 & \\
\hline Nobel TiUnite & $0.307(0.176,0.535)$ & $<0.001$ \\
\hline Astra TiOblast/Osseospeed** & $0.226(0.057,0.898)$ & 0.035 \\
\hline Straumann & $0.087(0.009,0.789)$ & 0.030 \\
\hline XIVE/Frialit-2 & $0.111(0.005,2.736)$ & 0.179 \\
\hline Other & $0.087(0.020,0.369)$ & 0.001 \\
\hline \multicolumn{3}{|l|}{ Bone grafting } \\
\hline No & 1 & \\
\hline Yes & $1.651(0.904,3.015)$ & 0.103 \\
\hline
\end{tabular}

The predictor 'Antibiotics' was shown to present multicollinearity and was excluded from this multivariate model

** Unfortunately, it was not possible to run the regression having Astra TiOblast and Astra Osseospeed as different categories within the parameter 'Implant system', even knowing that they are implants of different designs and surface treatments, although of the same manufacturer. The reason was because of the problem of quasi-complete separation, since there were no events of failure for Astra Osseospeed. The numbers were (failure/survival): Astra TiOblast (14/288) and Astra Osseospeed (0/91) 
Table 4. Distribution of the factors for each surgeon. The total number of implants might not add to 8,930 due to missing information. $n(\%) ; n$-implants.

\begin{tabular}{|c|c|c|c|c|c|}
\hline Surgeon & 1 & 2 & 3 & 4 & 5 \\
\hline & & \multicolumn{4}{|c|}{ Bone quantity } \\
\hline $\mathrm{A} / \mathrm{B} / \mathrm{C}$ & 3473 (97.9) & $1144(77.2)$ & $765(95.5)$ & $470(70.5)$ & $441(89.8)$ \\
\hline$D / E$ & $74(2.1)$ & $337(22.8)$ & $36(4.5)$ & $197(29.5)$ & $50(10.2)$ \\
\hline \multirow[t]{2}{*}{ Total } & $3547(100)$ & $1481(100)$ & $801(100)$ & $667(100)$ & $491(100)$ \\
\hline & & \multicolumn{4}{|c|}{ Bone quality } \\
\hline $1 / 2 / 3$ & 3441 (97.0) & $1283(86.6)$ & $780(97.4)$ & $498(74.7)$ & $481(98.0)$ \\
\hline 4 & $106(3.0)$ & $198(13.4)$ & $21(2.6)$ & $169(25.3)$ & $10(2.0)$ \\
\hline \multirow[t]{2}{*}{ Total } & $3547(100)$ & $1481(100)$ & $801(100)$ & $667(100)$ & $491(100)$ \\
\hline & & \multicolumn{4}{|c|}{ Implant location } \\
\hline Anterior maxilla & $1181(33.2)$ & $572(36.8)$ & $429(49.6)$ & $303(43.5)$ & $240(47.5)$ \\
\hline Anterior mandible & $1005(28.3)$ & $377(24.3)$ & $101(11.7)$ & $151(21.7)$ & $44(8.7)$ \\
\hline Posterior maxilla & $681(19.2)$ & 300 (19.3) & $195(22.6)$ & $144(20.6)$ & $139(27.5)$ \\
\hline Posterior mandible & 685 (19.3) & 304 (19.6) & $139(16.1)$ & $99(14.2)$ & $82(16.3)$ \\
\hline \multirow[t]{2}{*}{ Total } & $3552(100)$ & $1553(100)$ & $864(100)$ & $697(100)$ & $505(100)$ \\
\hline & & \multicolumn{4}{|c|}{ Implant surface } \\
\hline Turned & $1441(40.6)$ & 1497 (96.4) & $60(6.9)$ & $696(99.9)$ & $151(29.9)$ \\
\hline Enlarged & $2111(59.4)$ & $56(3.6)$ & $804(93.1)$ & $1(0.1)$ & $354(70.1)$ \\
\hline \multirow[t]{2}{*}{ Total } & $3552(100)$ & $1553(100)$ & $864(100)$ & $697(100)$ & $505(100)$ \\
\hline & & \multicolumn{4}{|c|}{ Implant system } \\
\hline Nobel turned & $1441(40.6)$ & $1497(96.4)$ & $60(6.9)$ & $696(99.9)$ & $151(29.9)$ \\
\hline Nobel TiUnite & $1644(46.3)$ & 45 (2.9) & $504(58.4)$ & $1(0.1)$ & $170(33.7)$ \\
\hline Astra & $195(5.5)$ & $5(0.3)$ & $37(4.3)$ & $0(0)$ & $117(23.2)$ \\
\hline Straumann & $41(1.1)$ & $6(0.4)$ & $84(9.7)$ & $0(0)$ & $36(7.1)$ \\
\hline XiVE/Frialit-2 & $21(0.6)$ & $0(0)$ & $177(20.5)$ & $0(0)$ & $31(6.1)$ \\
\hline Others & $210(5.9)$ & $0(0)$ & $2(0.2)$ & $0(0)$ & $0(0)$ \\
\hline Total & $3552(100)$ & $1553(100)$ & $864(100)$ & $697(100)$ & $505(100)$ \\
\hline \multicolumn{6}{|l|}{ Table 4. (continued) } \\
\hline \multirow[t]{2}{*}{ Surgeon } & 6 & 7 & 8 & 9 & 10 \\
\hline & \multicolumn{4}{|c|}{ Bone quantity } & \\
\hline $\mathrm{A} / \mathrm{B} / \mathrm{C}$ & $368(85.0)$ & 393 (93.6) & $329(86.8)$ & $214(80.8)$ & $176(83.4)$ \\
\hline$D / E$ & $65(15.0)$ & $27(6.4)$ & $50(13.2)$ & $51(19.2)$ & $35(16.6)$ \\
\hline \multirow[t]{2}{*}{ Total } & $433(100)$ & $420(100)$ & $379(100)$ & $265(100)$ & $211(100)$ \\
\hline & \multicolumn{4}{|c|}{ Bone quality } & \\
\hline $1 / 2 / 3$ & $422(97.5)$ & $379(90.2)$ & $350(92.3)$ & $243(91.7)$ & 210 (99.5) \\
\hline 4 & $11(2.5)$ & $41(9.8)$ & $29(7.7)$ & $22(8.3)$ & $1(0.5)$ \\
\hline \multirow[t]{2}{*}{ Total } & $433(100)$ & $420(100)$ & $379(100)$ & $265(100)$ & $211(100)$ \\
\hline & \multicolumn{4}{|c|}{ Implant location } & \\
\hline Anterior maxilla & $179(40.0)$ & $99(23.6)$ & $106(27.0)$ & $73(26.5)$ & $70(31.4)$ \\
\hline Anterior mandible & $78(17.5)$ & $143(34.0)$ & $127(32.3)$ & $88(31.9)$ & $48(21.5)$ \\
\hline Posterior maxilla & $110(24.6)$ & $39(9.3)$ & $58(14.8)$ & $36(13.0)$ & $46(20.6)$ \\
\hline Posterior mandible & $80(17.9)$ & $139(33.1)$ & $102(25.9)$ & $79(28.6)$ & $59(26.5)$ \\
\hline \multirow[t]{2}{*}{ Total } & $447(100)$ & $420(100)$ & $393(100)$ & $276(100)$ & $223(100)$ \\
\hline & \multicolumn{4}{|c|}{ Implant surface } & \\
\hline Turned & 442 (98.9) & $415(98.8)$ & $334(85.0)$ & $273(98.9)$ & $2(0.9)$ \\
\hline Enlarged & $5(1.1)$ & $5(1.2)$ & $59(15.0)$ & $3(1.1)$ & 221 (99.1) \\
\hline Total & $447(100)$ & $420(100)$ & $393(100)$ & $276(100)$ & $223(100)$ \\
\hline
\end{tabular}




\begin{tabular}{lccccc}
\hline & \multicolumn{5}{c}{ Implant system } \\
Nobel turned & $442(98.9)$ & $415(98.8)$ & $334(85.0)$ & $273(98.9)$ & $2(0.9)$ \\
Nobel TiUnite & $0(0)$ & $0(0)$ & $50(12.7)$ & $2(0.7)$ & $160(71.7)$ \\
Astra & $5(1.1)$ & $5(1.2)$ & $7(1.8)$ & $1(0.4)$ & $21(9.4)$ \\
Straumann & $0(0)$ & $0(0)$ & $2(0.5)$ & $0(0)$ & $16(7.2)$ \\
XiVE/Frialit-2 & $0(0)$ & $0(0)$ & $0(0)$ & $0(0)$ & $8(3.6)$ \\
Others & $0(0)$ & $0(0)$ & $0(0)$ & $0(0)$ & $16(7.2)$ \\
Total & $447(100)$ & $420(100)$ & $393(100)$ & $276(100)$ & $223(100)$ \\
\hline
\end{tabular}

Table 5. Failure/survival distribution of implants according to region and bone quality*.

\begin{tabular}{|c|c|c|c|c|c|}
\hline & \multicolumn{4}{|c|}{$\begin{array}{l}\text { Implant failure/survival - n (\% failure) } \\
\text { Bone quality }\end{array}$} & \multirow[b]{2}{*}{ Total } \\
\hline & 1 & 2 & 3 & 4 & \\
\hline Anterior maxilla & $0 / 14(0)$ & $53 / 1184$ (4.3) & $\begin{array}{c}118 / 1497 \\
(7.3)\end{array}$ & 74/204 (26.6) & $\begin{array}{c}245 / 2899 \\
(7.8)\end{array}$ \\
\hline $\begin{array}{l}\text { Anterior } \\
\text { mandible }\end{array}$ & $9 / 234(3.7)$ & $33 / 1248(2.6)$ & 18/507 (3.4) & $5 / 58$ (7.9) & $65 / 2047(3.1)$ \\
\hline Posterior maxilla & $0 / 6(0)$ & $11 / 291(3.6)$ & $81 / 1096$ (6.9) & $42 / 164(20.4)$ & $\begin{array}{l}134 / 1557 \\
(7.9)\end{array}$ \\
\hline $\begin{array}{l}\text { Posterior } \\
\text { mandible }\end{array}$ & $5 / 85(5.6)$ & 48/988 (4.6) & $34 / 510(6.2)$ & $1 / 60(1.6)$ & $88 / 1643(5.1)$ \\
\hline Total & $14 / 339(4.0)$ & $\begin{array}{c}145 / 3711 \\
(3.8)\end{array}$ & $\begin{array}{l}251 / 3610 \\
(6.5)\end{array}$ & $122 / 486(20.1)$ & $\begin{array}{l}532 / 8146 \\
(6.1)\end{array}$ \\
\hline
\end{tabular}

* The information of bone quality is unknown for a variable number of implants 


\section{Figures}

Figure 1. Kaplan-Meier curves comparing the survival of implants placed by different surgeons.

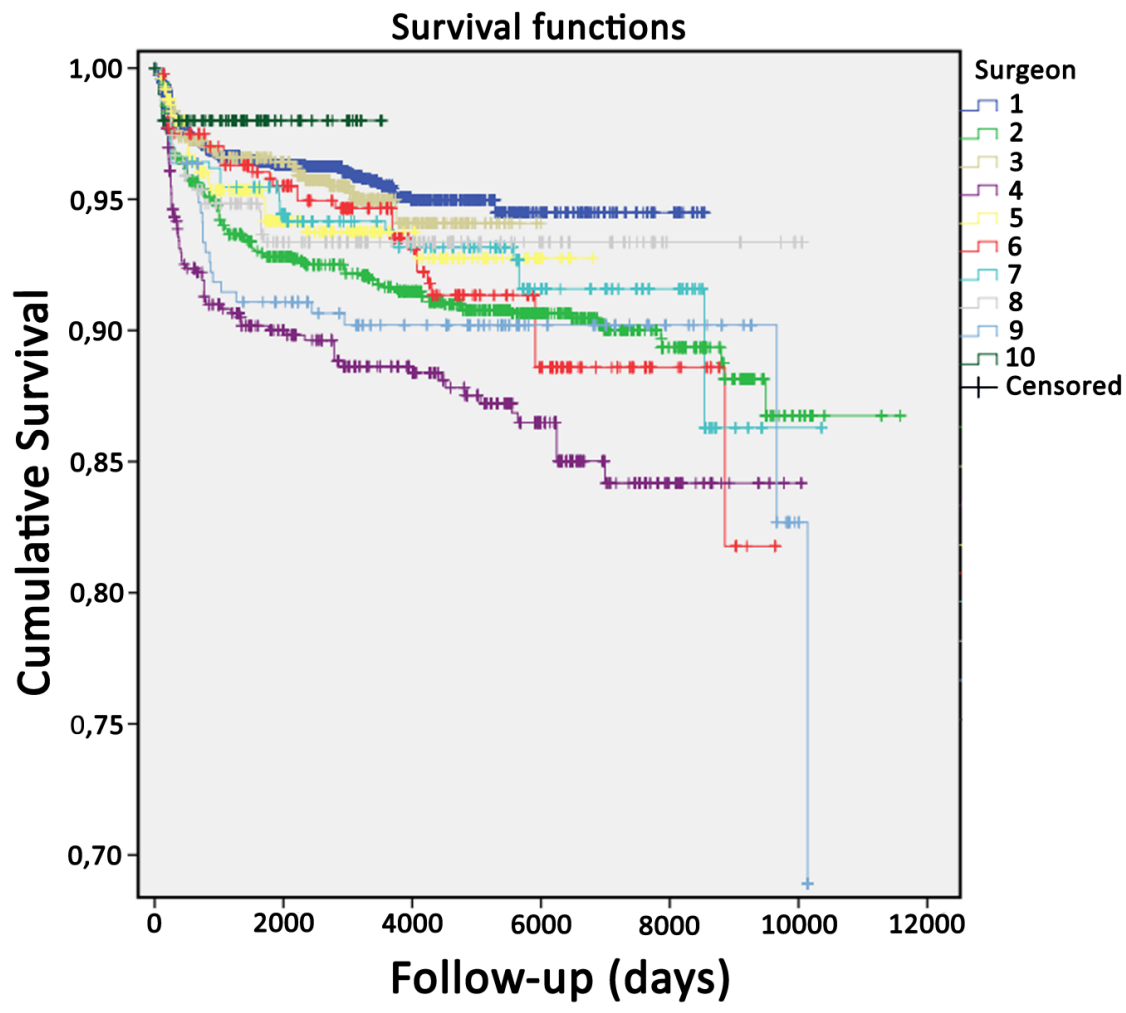


Figure 2. Kaplan-Meier curves comparing the survival of turned implants placed by different surgeons.

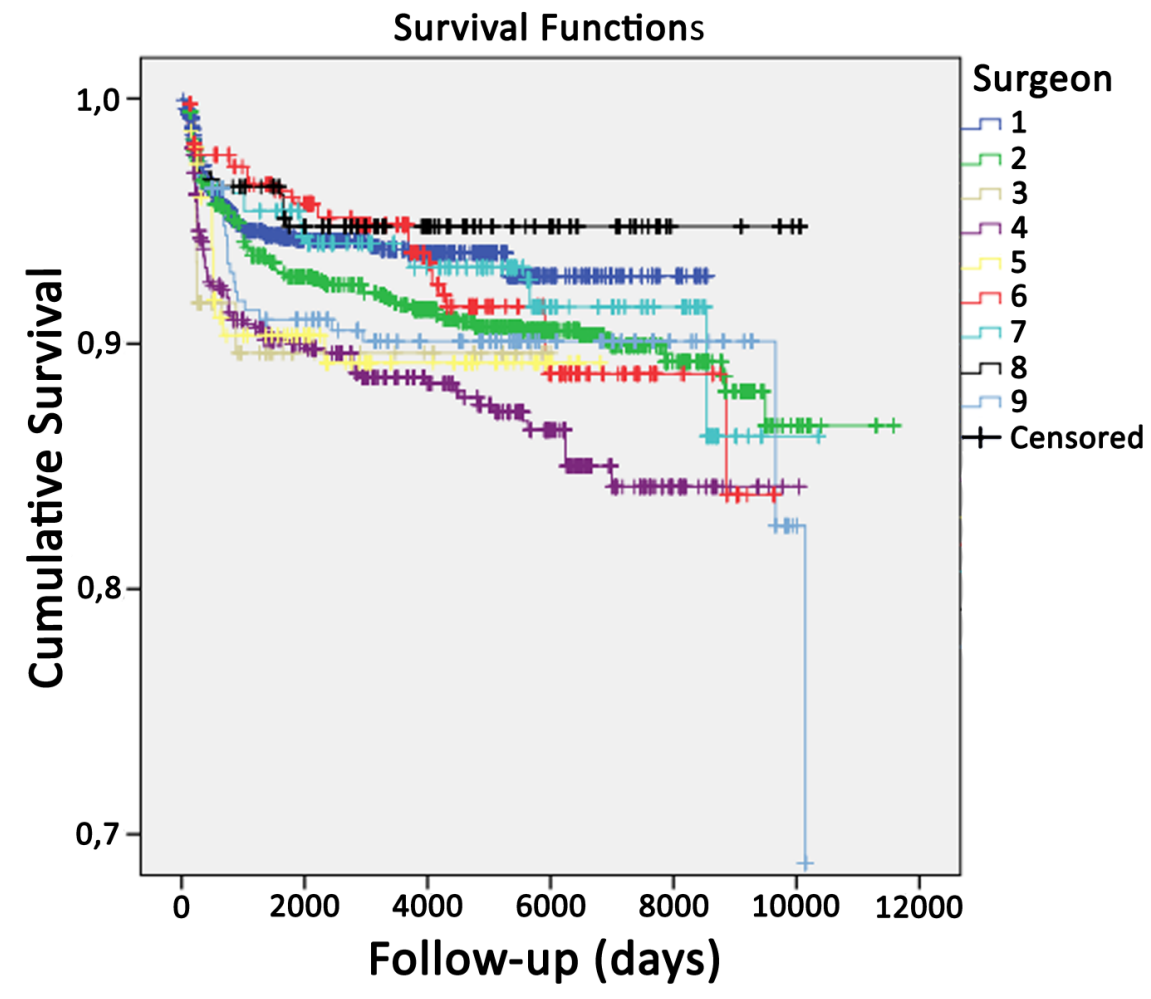


Figure 3. Kaplan-Meier curves comparing the survival of enlarged-surface implants placed by different surgeons.

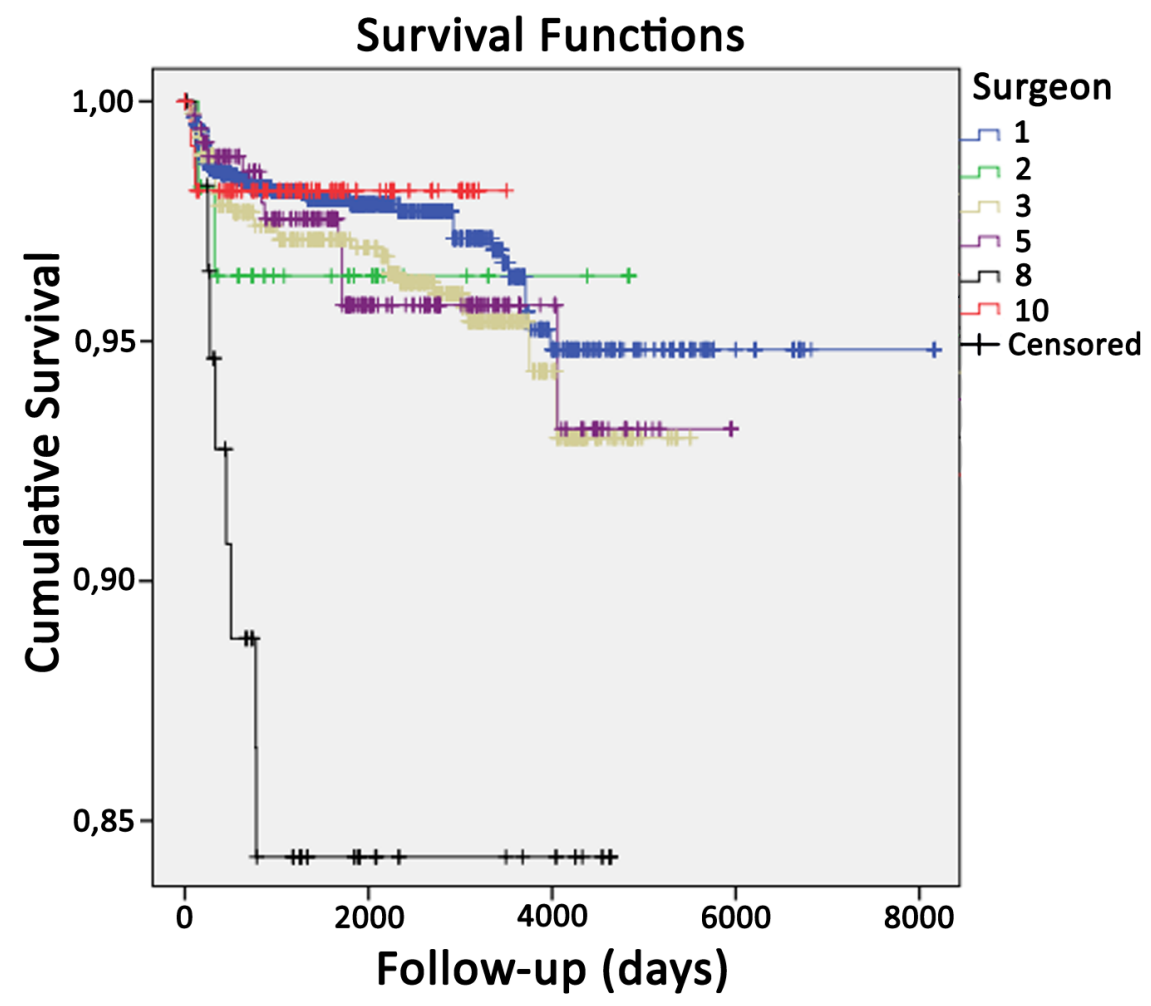




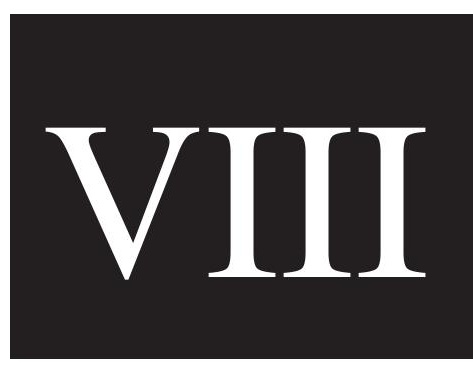





\title{
Analysis of risk factors for cluster behavior of dental implant failures
}

\author{
Bruno Ramos Chrcanovic, DDS, MSc, PhD student ${ }^{1}$ | Jenö Kisch, DDS ${ }^{2}$ | \\ Tomas Albrektsson, MD, PhD ${ }^{3,4}$ | Ann Wennerberg, DDS, PhD4
}

${ }^{1}$ Department of Prosthodontics, Faculty of Odontology, Malmö University, Malmö, Sweden

${ }^{2}$ Clinic for Prosthodontics, Centre of Dental Specialist Care, Malmö, Sweden

${ }^{3}$ Department of Biomaterials, Göteborg University, Göteborg, Sweden

${ }^{4}$ Department of Prosthodontics, Faculty of Odontology, Malmö University, Malmö, Sweden

Correspondence

Bruno Ramos Chrcanovic, Department of Prosthodontics, Faculty of Odontology, Malmö University, Carl Gustafs väg 34 , SE-214 21, Malmö, Sweden. Email: bruno.chrcanovic@mah.se; brunochrcanovic@hotmail.com

\section{Funding information}

Oral Health Related Research by Region Skåne (Odontologisk Forskning i Region Skåne, Grant/Award Number: OFRS 414321; Scientific Research Council of Sweden, Vetenskapsrådet, Grant/Award Number: Dnr 2015-02971; Folktandvården AB, Region Skåne, Sweden; CNPq, Conselho Nacional de Desenvolvimento Científico e Tecnológico, Grant/Award Number: 201318/2012-1

\begin{abstract}
Background: Some studies indicated that implant failures are commonly concentrated in few patients.

Purpose: To identify and analyze cluster behavior of dental implant failures among subjects of a retrospective study.

Materials and Methods: This retrospective study included patients receiving at least three implants only. Patients presenting at least three implant failures were classified as presenting a cluster behavior. Univariate and multivariate logistic regression models and generalized estimating equations analysis evaluated the effect of explanatory variables on the cluster behavior.

Results: There were 1406 patients with three or more implants (8337 implants, 592 failures) Sixty-seven $(4.77 \%)$ patients presented cluster behavior, with $56.8 \%$ of all implant failures. The intake of antidepressants and bruxism were identified as potential negative factors exerting a statistically significant influence on a cluster behavior at the patient-level. The negative factors at the implant-level were turned implants, short implants, poor bone quality, age of the patient, the intake of medicaments to reduce the acid gastric production, smoking, and bruxism.

Conclusions: A cluster pattern among patients with implant failure is highly probable. Factors of interest as predictors for implant failures could be a number of systemic and local factors, although a direct causal relationship cannot be ascertained.

KEYWORDS

cluster phenomenon, dental implant, implant failure, logistic models, multivariate analysis, risk factors
\end{abstract}

\section{1 | INTRODUCTION}

Results of some studies indicated that implant failures are commonly concentrated in few patients, rather than to be evenly distributed among all treated patients. ${ }^{1-6}$ Implant failures are not randomly distributed in all patients and a cluster behavior can occur. ${ }^{4}$ Cluster was once defined as more than one implant failure per patient, not necessarily in the same area or quadrant. ${ }^{5}$ As such, these failing patients have been described as "cluster patients," and even though they have seemingly been observed in a randomized pattern, it is reasonable to assume that the patient with failing implant has certain individual characteristics that separate them from the more successful implant patients, ${ }^{4}$ that is, these implant loss clusters happen in specific high-risk groups and individuals. $^{7}$

Common risk factors for implant failures are poor bone quantity and quality, implant insertion in the maxilla and in the posterior region of the jaws, heavy smoking, use of shorter length implants, untreated chronic periodontitis, irradiation of the head and neck region, lack of initial implant stability, a low insertion torque of implants that are planned to be immediately or early loaded, use of cylindrical (nonthreaded) implants, inexperienced surgeons conducting the surgery, greater number of implants placed per patient, implant insertion in fresh extraction sockets, and prosthetic rehabilitation with implant-supported overdentures. ${ }^{8,9}$ However, there is 
still no consensus on or scientific evidence for the etiology of clustering failure phenomena. ${ }^{6}$

The aim of this study was to retrospectively analyze cluster behavior of dental implant failures among patients, to assess the possible risk factors influencing this phenomenon, and to describe and compare this group of patients with one not presenting this behavior.

\section{MATERIALS AND METHODS}

\section{1 | Materials}

This retrospective study was based on all 2670 patients provided with implants, consecutively treated on a routine basis at one specialist clinic (Clinic for Prosthodontics, Centre of Dental Specialist Care, Malmö, Sweden) during the period from 1980 to 2014 . The study protocol was approved by the regional Ethical Committee, Lund, Sweden (Dnr 2014/ 598; Dnr 2015/72).

\section{2 | Definitions}

An implant was considered a failure if presenting signs and symptoms that led to implant removal. Thus, a failed implant in our study is equal to a lost implant. The failures were classified into two types: (1) implants lost due to lack/loss of osseointegration and (2) fractured implants. Primary failures were the failures occurring until/at the day of the $2^{\circ}$ stage surgery (abutment connection). A patient was considered as presenting a cluster behavior of failures when having at least three dental implant failures. In order to diagnose patients as bruxers, the authors followed the definition of bruxism proposed by Lobbezoo et al., ${ }^{10}$ and the sign and symptoms of bruxism were listed according to the International Classification of Sleep Disorders, ${ }^{11}$ following the same guidelines used in a recent study. ${ }^{12}$

\subsection{Inclusion and exclusion criteria}

Only patients receiving at least three implants were included. Patients with all modern types of implants with cylindrical or conical design were included. Zygomatic implants were not included in the study, as well as implants detected in radiographies, but without basic information about them in the patients' files.

\section{4 | Data collection}

The dental records of all patients ever treated with implants in the aforementioned clinic were read in order to collect the data. The data were directly entered into a SPSS file (SPSS software, version 23, SPSS Inc., Chicago, IL) as the files were being read. The following data were collected:

1. Implant-related factors: implant surface (turned/machined or enhanced surfaces, the latter including sandblasted, acid-etched, sandblasted + acid-etched, anodized, hydroxyapatite-coated surfaces), implant diameter and length, and implant design (cylindrical or conical);
2. Site-related factors: implant jaw location (maxilla/mandible), anterior or posterior location of the implant (sites from right canine to left canine teeth were considered anterior location), bone quantity and quality of the implant sites, which were classified at the time of surgery according to the Lekholm and Zarb ${ }^{13}$ classification, and implant sites with previous implant failures (reoperation).

3. Patient-related factors: patient's sex, age of the patient at the implant insertion surgery, general health, and behavioral history. The presence of a medicament list in the patients' records was also use to correlate the use of certain drugs to specific health conditions. Health factors assessed: diabetes types I or II, hypertension, hypercholesterolemia, hypothyroidism, asthma, psoriasis, chemotherapy, and irradiation of the head-neck region. The patients were also classified according to the intake of the following medication types: antidepressants, immunosuppressives, bisphosphonates, antithrombotic agents (antiplatelet, anticoagulant, thrombolytic drugs), hormone replacement therapy in women, and medicaments to reduce the acid gastric production. Behavioral factors assessed: smoking habits, use of snuff, bruxism.

4. Other factors: prescription of antibiotics (the prophylactic antibiotic regimen was usually starting 1-2 hours before surgery and going from 5 to 7 days postoperatively), bone graft procedures, reason for tooth extraction (periodontal disease, fracture/trauma, advanced caries, agenesia, other), type of implant-supported prosthetic restoration (single crown, partial bridge with 2-6 prosthetic elements, partial bridge with 7-10 prosthetic elements, full-arch, overdenture), number of days until failure, and follow-up time $(\leq 1$ year, 1 year $<x \leq 5$ years, 5 years $<x \leq 10$ years, 10 years $<x \leq 20$ years, $>20$ years).

As the standard protocol in the clinic, the patients' dental hygiene was followed up by a dental hygienist within 6 months after the final implant-supported/retained restoration. Each patient then attended a dental hygiene recall program based on individual needs.

\subsection{Statistical analyses}

The mean, standard deviation (SD), and percentage were calculated for several variables. Differences between implants of cluster and noncluster patients were compared with the student's t-test or Mann-Whitney test for continuous variables, depending on the normality and, the Pearson's chi-squared or Fisher's exact tests for categorical variables, depending on the number of samples in a $2 \times 2$ contingency table. Statistical significance was set at $P<.05$. Logistic regression models were used at the patient-level as well as at the implant-level. The patient-level analysis considered the patient as the statistical unit, with patients presenting or not presenting implant failures. Regression at the patient-level was used to evaluate the effect of explanatory health variables on the cluster behavior of implant failures, that is, health factors there are inherently associated to the patient, not to the implant. First, a univariate effect of each health factor on the implant failure was evaluated. Odds ratios (OR) and their $95 \%$ confidence intervals $(\mathrm{Cl})$ were computed. The Wald test based on robust standard errors was used to assess the significance of each factor. A factor was excluded from further multivariate 
analysis if the univariate logistic regression resulted in a clearly nonsignificant odds ratio $(P>.1)$. In the second step, a multivariate logistic regression gave the effects on different risk factors when controlling for other factors. The results of the final model were presented as an estimated OR of each significant prognostic variable $(P<.05)$.

An implant-level model was performed in order to assess the effects of the implant-related and local bone factor on the implant failures, also including the health variables. A generalized estimating equations (GEE) method was used to account for the fact that repeated observations (several implants) were available for a single patient. All models were adjusted for clustering of subject and implants in a binary logistic regression model using GEE with a binomial distribution and a logit link function, while assuming an exchangeable working correlation structure to assess the relationship between patients with cluster behavior and the risk factors. Initially, a univariate GEE on each of the variables was performed. In order to verify multicollinearity, a correlation matrix of all of the predictor variables with a significant odds ratio $(P$-value cut-off point of .1) identified in the univariate GEE was scanned, to see whether there were some high correlations among the predictors. Collinearity statistics obtaining variance inflation factor and tolerance statistic were also performed to detect more subtle forms of multicollinearity. Then, a multivariable model with a forced entry method was used to evaluate the effect of the factors that were univariately significant $(P<.1)$ and did not present multicollinearity. $A$ Wald chi-square test was used to analyze the statistical significance of each parameter within the model. The results of the final model were presented as an estimated $\mathrm{OR}$ of each significant prognostic variable $(P<.05)$.

SPSS software version 23 (SPSS Inc., Chicago, IL) was used for the statistical analyses.

\section{3 | RESULTS}

A number of 766 patients (390 men, 376 women) received only one implant, and presented 17 implant failures $(2.22 \%$; 15 lack/loss of osseointegration, 2 fractured implants), and 498 patients (221 men, 277 women) received two implants, and presented 37 implant failures (out of 996, 3.71\%; 35 lack/loss of osseointegration, 2 fractured implants). These patients with only one or two implants were not eligible for the analysis.

There were 1406 patients with three or more implants, totaling 8337 implants, with 592 implant failures (7.10\%; 556 lack/loss of osseointegration, 36 fractured implants). Sixty-seven (4.77\%) patients were identified as presenting cluster behavior, who received 620 implants, of which 331 failed (3.37\%; 315 lack/loss of osseointegration, 16 fractured implants). Thirty-two of these 67 patients were already deceased when the data collection was performed. There were 328 implants in 33 men (mean age \pm SD $62.1 \pm 10.3$, min-max 36.9-83.3) and 292 implants in 34 women (mean age \pm SD $59.5 \pm 9.2$, min-max $39.7-79.6)$. These $4.75 \%$ of the patients had $56.8 \%$ of all implant failures, when only the failures due to lack/loss of osseointegration were considered. The percentage is $56.0 \%$ when all failures are considered together (lack/loss of osseointegration + fracture of implants). Sixtyone (19.4\%) of the 315 implants with osseointegration failure in cluster patients were lost until or at the day of the second-stage surgery (primary failures), and $80.6 \%$ after loading. The 67 cluster patients had a mean implant failure rate of $53.4 \%$ (range $20-100 \%$ ). Twelve patients lost all their implants. One-hundred-and-thirty-three implants (40.2\%) were lost (lack/loss of osseointegration + fracture) until 1 year after surgery, $192(58.0 \%)$ later than 1 year of surgery, and there was no information for six implants (1.8\%).

A total of 1339 patients did not present a cluster behavior, and they received 7717 implants, of which 261 failed (3.38\%; 241 lack/loss of osseointegration, 20 fractured implants). In this group, $41.5 \%$ of the failures (100 out of 241 implant failures) occurred until/at the abutment connection (primary failures).

All implants were inserted with open flapped surgery. Only three implants were immediately loaded, all in noncluster patients, with no failures. The abutment connection surgery was performed after a mean \pm SD healing time of $184 \pm 66$ and $162 \pm 128$ days for the cluster and noncluster groups, respectively $(P<.001$; Mann-Whitney test). The number of nonsubmerged implants for the respective groups was 7 (out of $620 ; 1.13 \%$ ) and 289 (out of $7717 ; 3.74 \%$ ), and the number of implants placed in fresh extraction sockets was 6 (out of 620; $0.97 \%$ ) and 15 (out of $7717 ; 0.19 \%$ ). The cluster group presented 479 implants $(77.3 \%)$ with turned surfaces, whereas there were 4785 turned-surface implants $(62.0 \%)$ in the noncluster group $(P<.001$; Pearson's chi-squared test). The rest of the implants had some kind of enhanced surface. The group of turned-surface implants had a mean follow-up of $4678 \pm 2612$ days, against $2215 \pm 1474$ days for the group of enhanced-surface implants $(P<.001$; Mann-Whitney test). The mean length of the inserted implants was $12.5 \pm 2.7 \mathrm{~mm}$ for the cluster group and $12.9 \pm 2.4 \mathrm{~mm}$ for the noncluster group $(P=.008$; Mann-Whitney test). The mean diameter for cluster and noncluster groups were $3.76 \pm 0.16$ and $3.75 \pm 0.15$, respectively $(P=.042$; Mann-Whitney test).

Table 1 shows a comparison of groups according to the distribution of implants with regard to Lekholm and Zarb (1985) classification of bone quantity and quality. It can be observed that a greater percentage of implants were placed in bone sites having been classified as quantities $D$ and $E(P<.001$; Pearson's chi-squared test) and qualities 3 and $4(P<.001$; Pearson's chi-squared test $)$ in the cluster patients group, in comparison to the noncluster group. Table 2 shows a comparison of the difference in failure rates between implants groups of different surfaces, for cluster and noncluster patients, in relation to bone sites of different quantities/qualities, according to the Lekholm and Zarb (1985) classification.

The univariate binary logistic regression at the patient-level showed that the following predictors had a statistically significant odds ratio at the patient-level (Table 3): the intake of antidepressants, the intake of medicaments to reduce the acid gastric production, the intake of antithrombotic agents, smoking, number of cigarettes per day, and bruxism. Only the intake of antidepressants and bruxism continued to present a statistically significant odds ratio in the multivariate binary logistic regression model (Table 4). 
TABLE 1 Comparison of cluster and noncluster groups according to the distribution of implants with regard to Lekholm and Zarb classification of bone quantity and quality ${ }^{a}$

\begin{tabular}{|c|c|c|c|c|c|c|c|c|}
\hline & \multicolumn{6}{|c|}{ Cluster group } & \multirow[b]{2}{*}{$\%$ Group $^{b}$} & \multirow[b]{2}{*}{$\%$ Failed $^{c}$} \\
\hline & $A$ & B & C & D & $E$ & Total & & \\
\hline 1 & 0 & $1(1)$ & 4 & $6(3)$ & 0 & $11(4)$ & 1.8 & 36.4 \\
\hline 2 & 7 & $80(33)$ & $47(19)$ & $13(7)$ & $7(4)$ & $154(63)$ & 25.6 & 40.9 \\
\hline 3 & $7(3)$ & $102(48)$ & $114(59)$ & $64(40)$ & $6(6)$ & 293(156) & 48.7 & 53.2 \\
\hline 4 & 0 & $21(16)$ & $24(15)$ & $47(32)$ & $52(30)$ & $144(93)$ & 23.9 & 64.6 \\
\hline Total & $14(3)$ & 204(98) & 189(93) & $130(82)$ & $65(40)$ & $602(316)$ & 100 & 52.5 \\
\hline$\%$ Group $^{b}$ & 2.3 & 33.9 & 31.4 & 21.6 & 10.8 & 100 & & \\
\hline$\%$ Failed $^{c}$ & 21.4 & 48.0 & 49.2 & 63.1 & 61.5 & 52.5 & & \\
\hline
\end{tabular}

Missing information of bone quantity/quality: 18 implants, 15 failures

\begin{tabular}{|c|c|c|c|c|c|c|c|c|}
\hline & \multicolumn{6}{|c|}{ Noncluster group } & \multirow[b]{2}{*}{$\%$ Group $^{b}$} & \multirow[b]{2}{*}{$\%$ Failed $^{c}$} \\
\hline & $A$ & B & C & D & $\mathrm{E}$ & Total & & \\
\hline 1 & 19 & 56 & $90(6)$ & $141(3)$ & $41(2)$ & $347(11)$ & 4.6 & 3.2 \\
\hline 2 & $317(6)$ & $1858(50)$ & $814(27)$ & $147(8)$ & $26(1)$ & $3162(92)$ & 42.1 & 2.9 \\
\hline 3 & $344(9)$ & $1621(44)$ & $1266(45)$ & $308(20)$ & $10(2)$ & $3549(120)$ & 47.3 & 3.4 \\
\hline 4 & 19 & $106(3)$ & 193(18) & $104(9)$ & $28(3)$ & $450(33)$ & 6.0 & 7.3 \\
\hline Total & 699(15) & $3641(97)$ & $2363(96)$ & $700(40)$ & 105(8) & $7508(256)$ & 100 & 3.4 \\
\hline$\%$ Group $^{\mathrm{b}}$ & 9.3 & 48.5 & 31.5 & 9.3 & 1.4 & 100 & & \\
\hline$\%$ Failed $^{c}$ & 2.1 & 2.7 & 4.1 & 5.7 & 7.6 & 3.4 & & \\
\hline
\end{tabular}

Missing information of bone quantity/quality: 209 implants, 5 failures

The number between parentheses represents failures.

${ }^{a}$ According to the Lekholm and Zarb classification, bone quality is broken down into four groups according to the proportion and structure of compact and trabecular bone tissue: type 1 = large homogenous cortical/compact bone; type 2 = thick layer of compact bone surrounding a dense trabecular bone; type 3 = thin cortical layer surrounding a dense trabecular bone; type 4 = thin cortical layer surrounding a core of low-density trabecular bone. The quantity of jawbone is broken down into five groups (A, B, C, D, and E), based on the residual jaw shape following tooth extraction. Bone classified as "A" presents the largest amount of bone among all classes, whereas bone classified as "E" presents the lowest volume of bone.

bercentage of implants in each bone quantity/quality class, considering all implants in cluster or noncluster group as $100 \%$.

'Percentage of failed implants for each bone quantity/quality class.

TABLE 2 Comparison of the difference in failure rates (Pearson's chi-squared test) between implants groups of different surfaces, for cluster and noncluster patients, in relation to bone sites of different quantities/qualities, according to the Lekholm and Zarb classification

\begin{tabular}{|c|c|c|c|c|}
\hline \multirow[b]{3}{*}{ Bone } & \multirow[b]{3}{*}{ Group } & \multicolumn{2}{|l|}{ Implant } & \multirow[b]{3}{*}{$P$ value* } \\
\hline & & \multicolumn{2}{|c|}{ failure/total (\% failed) } & \\
\hline & & Turned & Enhanced & \\
\hline \multirow[t]{2}{*}{ Quantity $A / B / C$} & Cluster & $145 / 285$ (50.9\%) & $49 / 122(40.2 \%)$ & .047 \\
\hline & Noncluster & $154 / 4010(3.8 \%)$ & $54 / 2693(2.0 \%)$ & $<.001$ \\
\hline \multirow[t]{2}{*}{ Quantity D/E } & Cluster & $114 / 176(64.8 \%)$ & $8 / 19(42.1 \%)$ & .052 \\
\hline & Noncluster & $36 / 655$ (5.5\%) & $12 / 150(8.0 \%)$ & .243 \\
\hline \multirow[t]{2}{*}{ Quality $1 / 2$} & Cluster & $52 / 115(45.3 \%)$ & $15 / 50(30 \%)$ & .067 \\
\hline & Noncluster & $75 / 2129(3.5 \%)$ & $28 / 1380(2.0 \%)$ & .010 \\
\hline \multirow[t]{2}{*}{ Quality 3/4 } & Cluster & $207 / 346(59.8 \%)$ & $42 / 91(46.2 \%)$ & .019 \\
\hline & Noncluster & $115 / 2536(4.5 \%)$ & $38 / 1463(2.6 \%)$ & .002 \\
\hline
\end{tabular}

*Pearson's chi-squared test. 
TABLE 3 Univariate binary logistic regression for cluster behavior: gender and health factors at the patient-level (OR-odds ratio)

\begin{tabular}{|c|c|c|c|}
\hline Factor & $\begin{array}{l}\text { Patients } \\
\text { with } \geq 3 \\
\text { failures/0-2 } \\
\text { failures }^{\mathrm{a}}\end{array}$ & OR $(95 \% \mathrm{Cl})$ & $P$-value \\
\hline \multicolumn{4}{|l|}{ Gender } \\
\hline Male & $33 / 592$ & 1 & \\
\hline Female & $34 / 747$ & $0.817(0.500,1.334)$ & .418 \\
\hline \multicolumn{4}{|l|}{ Age } \\
\hline Increase by 1 & - & $1.011(0.994,1.028)$ & .196 \\
\hline \multicolumn{4}{|l|}{ Diabetes } \\
\hline No & $44 / 850$ & 1 & \\
\hline Type I & $1 / 18$ & $1.073(0.140,8.224)$ & .946 \\
\hline Type II & $4 / 72$ & $1.073(0.375,3.071)$ & .895 \\
\hline \multicolumn{4}{|c|}{ High blood pressure } \\
\hline No & $29 / 608$ & 1 & \\
\hline Yes & $20 / 331$ & $1.267(0.706,2.274)$ & .428 \\
\hline \multicolumn{4}{|l|}{ High cholesterol } \\
\hline No & $39 / 795$ & 1 & \\
\hline Yes & $10 / 138$ & $1.477(0.721,3.028)$ & .287 \\
\hline \multicolumn{4}{|l|}{ Hypothyroidism } \\
\hline No & $45 / 881$ & 1 & \\
\hline Yes & $4 / 54$ & $1.450(0.503,4.181)$ & .491 \\
\hline \multicolumn{4}{|l|}{ Asthma } \\
\hline No & $46 / 854$ & 1 & \\
\hline Yes & $4 / 81$ & $0.917(0.322,2.612)$ & .871 \\
\hline \multicolumn{4}{|c|}{ Intake of antidepressants } \\
\hline No & $38 / 853$ & 1 & \\
\hline Yes & $13 / 79$ & $3.694(1.889,7.223)$ & $<.001$ \\
\hline
\end{tabular}

\begin{tabular}{llll}
$\begin{array}{l}\text { Irradiation } \\
\text { No }\end{array}$ & $48 / 911$ & 1 & \\
Yes & $2 / 30$ & $1.265(0.294,5.451)$ & .752 \\
\hline $\begin{array}{lll}\text { Hormone replacement therapy } \\
\text { No }\end{array}$ & & \\
Yes & $56 / 1080$ & 1 & \\
Yes & $2 / 37$ & $1.042(0.245,4.435)$ & .955 \\
\hline
\end{tabular}

\begin{tabular}{llll} 
Gastric & & & \\
$\quad$ No & $39 / 850$ & 1 & \\
Yes & $8 / 71$ & $2.456(1.105,5.456)$ & .027 \\
Antithrombotics & & & \\
$\quad$ No & $32 / 723$ & 1 & \\
Yes & $16 / 209$ & $1.730(0.931,3.214)$ & .083 \\
\hline
\end{tabular}

\begin{tabular}{|llll|}
\hline $\begin{array}{l}\text { Immunosuppressive } \\
\text { No }\end{array}$ & $46 / 915$ & 1 & \\
\hline Yes & $2 / 11$ & $3.617(0.779,16.794)$ & .101 \\
\hline $\begin{array}{l}\text { Psoriasis } \\
\text { No } \\
\text { Yes }\end{array}$ & $48 / 915$ & 1 & \\
\hline $\begin{array}{l}\text { Bisphosphonates } \\
\text { No }\end{array}$ & $0 / 12$ & $0.000(0.000)$ & 1.000 \\
\hline Yes & $47 / 907$ & 1 & \\
\hline & $0 / 18$ & $0.000(0.000)$ & 1.000 \\
\hline
\end{tabular}

TABLE 3 (continued)

\begin{tabular}{|c|c|c|c|}
\hline Factor & $\begin{array}{l}\text { Patients } \\
\text { with } \geq 3 \\
\text { failures/0-2 } \\
\text { failures }^{\mathrm{a}}\end{array}$ & OR $(95 \% \mathrm{Cl})$ & $P$-value \\
\hline \multicolumn{4}{|l|}{ Smoking } \\
\hline No & $22 / 588$ & 1 & \\
\hline Yes & $26 / 259$ & $2.683(1.493,4.822)$ & .001 \\
\hline Former smoker & $1 / 32$ & $0.835(0.109,6.394)$ & .862 \\
\hline \multicolumn{4}{|l|}{ Cigarettes/day } \\
\hline Increase by 1 & - & $1.034(0.998,1.072)$ & .068 \\
\hline \multicolumn{4}{|l|}{ Snuff } \\
\hline No & $43 / 839$ & 1 & \\
\hline Yes & $3 / 28$ & $2.091(0.611,7.148)$ & .240 \\
\hline \multicolumn{4}{|l|}{ Bruxism } \\
\hline No & $38 / 901$ & 1 & \\
\hline Yes & $11 / 43$ & $6.065(2.901,12.681)$ & $<.001$ \\
\hline
\end{tabular}

aThe information for some conditions is unknown for a variable number of patients.

The univariate GEE model at the implant-level showed that the following predictors had a statistically significant odds ratio at the implant-level (Table 5): implant surface, implant length, implant location, bone quantity and quality, reoperation, age of the patient at the time of the surgery, the intake of antidepressants, the intake of medicaments to reduce the acid gastric production, smoking, bruxism, the use of prophylactic antibiotics, bone grafting, and follow-up time. The following factors remained statistically significant in the multivariate

TABLE 4 Multivariate logistic regression model for cluster behavior at the patient-level

\begin{tabular}{lll|}
\hline Factor & OR $(95 \% \mathrm{Cl})$ & P-value \\
\hline $\begin{array}{l}\text { Intake of antidepressants } \\
\text { No } \\
\text { Yes }\end{array}$ & 1 & \\
\hline $\begin{array}{l}\text { Gastric } \\
\text { No } \\
\text { Yes }\end{array}$ & $2.473(1.026,5.958)$ & .044 \\
\hline $\begin{array}{l}\text { Antithrombotics } \\
\text { No } \\
\text { Yes }\end{array}$ & 1 & \\
\hline $\begin{array}{l}\text { Smoking } \\
\text { No }\end{array}$ & $2.182(0.829,5.744)$ & .114 \\
\hline $\begin{array}{l}\text { Yes } \\
\text { Former smoker }\end{array}$ & 1 & \\
\hline $\begin{array}{l}\text { Cigarettes/day } \\
\text { Increase by 1 }\end{array}$ & $1.247(0.552,2.813)$ & .596 \\
\hline $\begin{array}{l}\text { Bruxism } \\
\text { No } \\
\text { Yes }\end{array}$ & 1 & $.2 .170(0.629,7.483)$ \\
\hline
\end{tabular}

Only the patient and health factors that were considered statistically significant $(P<.1)$ in the univariate model and did not present multicollinearity were included in the multivariate model (OR-odds ratio). 
TABLE 5 Risk factor analysis using a univariate generalized estimating equations logistic regression model, at the implant-level (OR-odds ratio)

\begin{tabular}{|c|c|c|c|c|}
\hline \multirow[t]{2}{*}{ Factor } & $\begin{array}{l}\text { Failure/survival in } \\
\text { cluster patients }^{\mathrm{a}}\end{array}$ & $\begin{array}{l}\text { Failure/survival in } \\
\text { noncluster patients }\end{array}$ & OR $(95 \% \mathrm{Cl})$ & $P$-value \\
\hline & \multicolumn{3}{|c|}{ Implant-related factors } & \\
\hline \multicolumn{5}{|l|}{ Implant surface ${ }^{\mathrm{b}}$} \\
\hline Turned & $274 / 205$ & $194 / 4591$ & 1 & \\
\hline Enhanced & $57 / 84$ & $67 / 2865$ & $0.286(0.190,0.430)$ & $<.001$ \\
\hline \multicolumn{5}{|l|}{ Implant diameter } \\
\hline Increase by 1 & - & - & $1.656(0.783,3.502)$ & .187 \\
\hline \multicolumn{5}{|l|}{ Implant length } \\
\hline Increase by 1 & - & - & $0.904(0.849,0.962)$ & .002 \\
\hline \multicolumn{5}{|l|}{ Implant design } \\
\hline Cylindrical & $330 / 281$ & $257 / 7297$ & 1 & \\
\hline \multirow[t]{2}{*}{ Conical } & $1 / 8$ & $4 / 159$ & $0.174(0.008,3.767)$ & .265 \\
\hline & \multicolumn{4}{|l|}{ Site-related factors } \\
\hline \multicolumn{5}{|l|}{ Location } \\
\hline Maxilla & $277 / 175$ & $160 / 3795$ & 1 & \\
\hline Mandible & $54 / 114$ & $101 / 3661$ & $0.454(0.334,0.617)$ & $<.001$ \\
\hline Anterior & $231 / 183$ & $121 / 4469$ & 1 & \\
\hline Posterior & $100 / 106$ & $140 / 2987$ & $1.233(1.007,1.510)$ & .043 \\
\hline \multicolumn{5}{|l|}{ Bone quantity } \\
\hline A & $3 / 11$ & $15 / 684$ & 1 & \\
\hline B & $98 / 106$ & $97 / 3544$ & $1.529(0.777,3.011)$ & .219 \\
\hline C & $93 / 96$ & $96 / 2267$ & $2.398(1.204,4.778)$ & .013 \\
\hline D & $82 / 48$ & $40 / 660$ & $4.913(2.346,10.289)$ & $<.001$ \\
\hline$E$ & $40 / 25$ & $8 / 97$ & $9.251(3.892,21.988)$ & $<.001$ \\
\hline \multicolumn{5}{|l|}{ Bone quality } \\
\hline 1 & $4 / 7$ & $11 / 336$ & 1 & \\
\hline 2 & $63 / 91$ & $92 / 3070$ & $1.193(0.439,3.243)$ & .730 \\
\hline 3 & $156 / 137$ & $120 / 3429$ & $1.640(0.610,4.413)$ & .327 \\
\hline 4 & $93 / 51$ & $33 / 417$ & $5.282(1.793,15.565)$ & .003 \\
\hline \multicolumn{5}{|l|}{ Reoperation } \\
\hline No & $228 / 240$ & $260 / 7392$ & 1 & \\
\hline \multirow[t]{2}{*}{ Yes } & $43 / 49$ & $1 / 64$ & $5.423(3.358,8.758)$ & $<.001$ \\
\hline & \multicolumn{4}{|c|}{ Patient-related factors } \\
\hline \multicolumn{5}{|l|}{ Gender } \\
\hline Male & $160 / 168$ & $108 / 3494$ & 1 & \\
\hline Female & $171 / 121$ & $153 / 3962$ & $1.052(0.784,1.411)$ & .735 \\
\hline \multicolumn{5}{|l|}{ Age } \\
\hline Increase by 1 & - & - & $0.981(0.972,0.990)$ & $<.001$ \\
\hline \multicolumn{5}{|l|}{ Diabetes } \\
\hline No & $226 / 174$ & $143 / 4701$ & 1 & \\
\hline Type I & $3 / 0$ & $4 / 97$ & $1.461(0.420,5.086)$ & .551 \\
\hline Type II & $15 / 21$ & $17 / 419$ & $0.968(0.558,1.679)$ & .908 \\
\hline \multicolumn{5}{|c|}{ High blood pressure } \\
\hline No & $162 / 96$ & $104 / 3394$ & 1 & \\
\hline Yes & $82 / 99$ & $59 / 1813$ & $0.899(0.625,1.293)$ & .567 \\
\hline \multicolumn{5}{|l|}{ High cholesterol } \\
\hline No & $198 / 152$ & $132 / 4382$ & 1 & \\
\hline Yes & $46 / 43$ & $30 / 789$ & $1.124(0.714,1.771)$ & .614 \\
\hline
\end{tabular}


CHRCANOVIC ET AL.

WILEY $\stackrel{7}{7}$

TABLE 5 (continued)

\begin{tabular}{|c|c|c|c|c|}
\hline Factor & $\begin{array}{l}\text { Failure/survival in } \\
\text { cluster patients }^{\mathrm{a}}\end{array}$ & $\begin{array}{l}\text { Failure/survival in } \\
\text { noncluster patients }\end{array}$ & OR $(95 \% \mathrm{Cl})$ & $P$-value \\
\hline \multicolumn{5}{|l|}{ Hypothyroidism } \\
\hline No & $231 / 179$ & $156 / 4893$ & 1 & \\
\hline Yes & $13 / 16$ & $8 / 286$ & $1.034(0.478,2.235)$ & .932 \\
\hline \multicolumn{5}{|l|}{ Asthma } \\
\hline No & $231 / 179$ & $151 / 4746$ & 1 & \\
\hline Yes & $19 / 25$ & $11 / 438$ & $0.799(0.427,1.493)$ & .481 \\
\hline \multicolumn{5}{|c|}{ Intake of antidepressants } \\
\hline No & $180 / 150$ & $145 / 4737$ & 1 & \\
\hline Yes & $77 / 65$ & $15 / 432$ & $2.523(1.527,4.167)$ & $<.001$ \\
\hline \multicolumn{5}{|l|}{ Irradiation } \\
\hline No & $241 / 190$ & $157 / 5062$ & 1 & \\
\hline Yes & $7 / 6$ & $5 / 151$ & $1.105(0.430,2.828)$ & .836 \\
\hline \multicolumn{5}{|c|}{ Hormone replacement therapy } \\
\hline No & $13 / 9$ & $8 / 190$ & 1 & \\
\hline Yes & $273 / 250$ & $192 / 6076$ & $1.130(0.505,2.531)$ & .766 \\
\hline \multicolumn{5}{|l|}{ Gastric } \\
\hline No & $195 / 157$ & $143 / 4705$ & 1 & \\
\hline Yes & $36 / 33$ & $17 / 395$ & $1.890(1.105,3.233)$ & .020 \\
\hline \multicolumn{5}{|l|}{ Antithrombotics } \\
\hline No & $151 / 127$ & $119 / 4007$ & 1 & \\
\hline Yes & $83 / 71$ & $43 / 1151$ & $1.289(0.862,1.928)$ & .217 \\
\hline \multicolumn{5}{|l|}{ Immunosuppressive } \\
\hline No & $227 / 188$ & $159 / 5071$ & 1 & \\
\hline Yes & $7 / 10$ & $1 / 59$ & $1.310(0.388,4.422)$ & .663 \\
\hline \multicolumn{5}{|l|}{ Psoriasis } \\
\hline No & $234 / 234$ & $159 / 5072$ & 1 & \\
\hline Yes & $0 / 0$ & $2 / 63$ & $0.000(0.000)$ & 1.000 \\
\hline \multicolumn{5}{|l|}{ Bisphosphonates } \\
\hline No & $232 / 190$ & $159 / 5044$ & 1 & \\
\hline Yes & $0 / 0$ & $1 / 80$ & $0.000(0.000)$ & 1.000 \\
\hline \multicolumn{5}{|l|}{ Smoking } \\
\hline No & $119 / 95$ & $96 / 3179$ & 1 & \\
\hline Yes & $115 / 102$ & $51 / 1495$ & $1.747(1.202,2.541)$ & .003 \\
\hline Former smoker & $7 / 5$ & $7 / 159$ & $1.312(0.555,3.106)$ & .536 \\
\hline \multicolumn{5}{|l|}{ Cigarettes/day } \\
\hline Increase by 1 & - & - & $1.014(0.990,1.038)$ & .262 \\
\hline \multicolumn{5}{|l|}{ Snuff } \\
\hline No & $206 / 173$ & $145 / 4620$ & 1 & \\
\hline Yes & $20 / 13$ & $5 / 152$ & $1.713(0.745,3.937)$ & .205 \\
\hline \multicolumn{5}{|l|}{ Bruxism } \\
\hline No & $177 / 155$ & $150 / 5001$ & 1 & \\
\hline \multirow[t]{2}{*}{ Yes } & $63 / 49$ & $20 / 242$ & $4.449(2.691,7.357)$ & $<.001$ \\
\hline & Other factors & & & \\
\hline \multicolumn{5}{|l|}{ Antibiotics } \\
\hline No & $20 / 20$ & $25 / 457$ & 1 & \\
\hline Yes & $130 / 117$ & $92 / 3505$ & $0.641(0.401,1.025)$ & .063 \\
\hline \multicolumn{5}{|l|}{ Bone grafting } \\
\hline No & $267 / 253$ & $239 / 7155$ & 1 & \\
\hline Yes & $64 / 36$ & $22 / 301$ & $2.139(1.083,4.226)$ & .029 \\
\hline
\end{tabular}


TABLE 5 (continued)

\begin{tabular}{|c|c|c|c|c|}
\hline Factor & $\begin{array}{l}\text { Failure/survival in } \\
\text { cluster patients }^{\mathrm{a}}\end{array}$ & $\begin{array}{l}\text { Failure/survival in } \\
\text { noncluster patients }^{\mathrm{a}}\end{array}$ & OR $(95 \% \mathrm{Cl})$ & $P$-value \\
\hline \multicolumn{5}{|l|}{ Reason for tooth extraction } \\
\hline Periodontal disease & $24 / 34$ & $28 / 791$ & 1 & \\
\hline Fracture/trauma & $1 / 0$ & $9 / 140$ & $1.633(0.809,3.297)$ & .171 \\
\hline Advanced caries & $6 / 4$ & $2 / 188$ & $0.878(0.381,2.020)$ & .759 \\
\hline Agenesia & $0 / 0$ & $17 / 361$ & $0.821(0.366,1.843)$ & .633 \\
\hline Other & $1 / 2$ & $3 / 52$ & $1.443(0.490,4.250)$ & .506 \\
\hline \multicolumn{5}{|l|}{ Prosthetic restoration } \\
\hline Single crown & $2 / 0$ & $22 / 414$ & 1 & \\
\hline Partial fixed, 2-6 units & $50 / 46$ & 68/1986 & $0.872(0.539,1.410)$ & .575 \\
\hline Partial fixed, $7-10$ units & $17 / 16$ & $9 / 328$ & $1.115(0.457,2.722)$ & .810 \\
\hline Full-arch fixed & $220 / 198$ & $134 / 4502$ & $1.031(0.626,1.696)$ & .905 \\
\hline Overdenture & $33 / 25$ & $15 / 169$ & $1.807(0.500,6.533)$ & .367 \\
\hline \multicolumn{5}{|l|}{ Time of follow-up } \\
\hline$\leq 1$ year & $6 / 4$ & $5 / 327$ & 1 & \\
\hline 1 year $<x \leq 5$ years & $49 / 55$ & $50 / 1742$ & $1.709(0.328,8.875)$ & .524 \\
\hline 5 years $<x \leq 10$ years & $69 / 69$ & $59 / 1916$ & $2.301(0.469,11.298)$ & .305 \\
\hline 10 years $<x \leq 20$ years & $123 / 106$ & $106 / 2686$ & $3.777(0.791,18.041)$ & .096 \\
\hline$>20$ years & $84 / 55$ & $42 / 784$ & $8.658(1.801,41.613)$ & .007 \\
\hline
\end{tabular}

${ }^{a}$ The information for some conditions is unknown for a variable number of implants.

'The turned implants group was comprised by Nobel turned implants only. The numbers (failure/survival) for the enhanced surface implants in cluster and noncluster patients were, respectively: Nobel TiUnite, 42/56 and 41/1989; Astra TiOblast, 12/12 and 11/285; Astra Osseospeed, 0/0 and 4/139; Straumann, 0/2 and 8/232; XIVE/Frialit-2, 0/6 and 2/89; other, 3/8 and 1/131.

GEE model (Table 6): turned implants, short implants, poor bone quality, age of the patient, the intake of medicaments to reduce the acid gastric production, smoking, and bruxism.

\section{I DISCUSSION}

The results of the present study showed profound differences of implant survival rate in different individuals, that is, less than $5 \%$ of patients showed about $56 \%$ of all implant failures, and this suggests a cluster behavior with regard to implant failures, most likely with multifactorial causes. ${ }^{8}$ The regression analyses performed in this study tried to identify the factors that could possibly be related to implant failure. The univariate regression assessed the relationship between each independent variable and implant failure separately, and the multivariate regression assessed the relationship of the variables that were univariately significant to implant failure, controlling for each other. At the patient-level, the multivariate regression model identified the intake of antidepressants and bruxism as potential negative factors exerting a statistically significant influence on the high failure rates in cluster patients. At the implant-level, the negative factors identified by the multivariate GEE model were turned implants, shorter implants, poor bone quality, younger patients, the intake of medicaments to reduce the acid gastric production, smoking, and bruxism.

Two health-related variables were shown to exert some significant effect on the cluster behavior. The first one was depression. Knowing that a loss of motivation is one of the volitional symptoms of depression, ${ }^{14}$ one may presume that depression could have a negative impact on oral hygiene. ${ }^{15}$ Kurer et al. ${ }^{16}$ found an association between mean depression scores and oral hygiene in a group of 51 regular dental attenders. Monteiro da Silva et al. ${ }^{17}$ found significantly increased depression and loneliness in patients with rapidly progressive adultonset periodontitis compared with a group with regular chronic adult periodontitis and a control group. Among the risk factors associated with periodontal disease and ultimate tooth loss, smoking is the best documented one. ${ }^{18}$ In some studies, smoking has also been associated with depression. ${ }^{19,20}$

Some reasons could theoretically account for the suggested association between PPIs intake and the increased likelihood of dental implant failures. The most prominent hypothesis assumes that the reduced acidity in the stomach impairs the intestinal absorption of dietary calcium. Thus, there can be a decreased calcium absorption under PPI therapy. ${ }^{21-23}$ As the calcium balance is essential for the maintenance of bone health, it seems reasonable to believe that the unbalance of calcium may to some degree affect osseointegration.

Concerning the patients' habits, bruxism was shown to significantly affect the implant failure rates negatively, agreeing with the results of the two very recent clinical trials assessing the effect of bruxism on dental implants. ${ }^{12,24}$ This gives a new perspective on the condition, which was, until very recently, considered to be not related with implant failures. ${ }^{25,26}$ Bruxism is suggested to generate overload of prosthetic rehabilitations on implants, which could possibly cause implant fracture or peri-implant marginal bone loss, ultimately resulting in implant failure. ${ }^{27}$ Implant failure may result in a "domino" effect that could lead to further implant failures in the same individual. ${ }^{5}$ This kind of effect was noticed in one study, ${ }^{4}$ where an observed pattern in the study group was that implant failures started in some patients predominantly in one quadrant, causing an unfavorable distribution of the remaining implants. Even if adjustments with shortening of the fixed 
TABLE 6 Multivariate generalized estimating equations logistic regression model at the implant-level

\begin{tabular}{|c|c|c|}
\hline Factor & OR $(95 \% \mathrm{Cl})$ & $P$-value \\
\hline \multicolumn{3}{|l|}{ Implant surface } \\
\hline Turned & 1 & \\
\hline Enhanced & $0.311(0.127,0.762)$ & .011 \\
\hline \multicolumn{3}{|l|}{ Implant length } \\
\hline Increase by 1 & $0.863(0.773,0.964)$ & .009 \\
\hline \multicolumn{3}{|l|}{ Location } \\
\hline Maxilla & 1 & \\
\hline Mandible & $0.789(0.452,1.375)$ & .403 \\
\hline Anterior & 1 & \\
\hline Posterior & $1.122(0.770,1.636)$ & .548 \\
\hline \multicolumn{3}{|l|}{ Bone quantity } \\
\hline A & 1 & \\
\hline B & $1.658(0.476,5.779)$ & .427 \\
\hline C & $1.136(0.297,4.341)$ & .852 \\
\hline D & $2.790(0.616,12.636)$ & .183 \\
\hline$E$ & $4.330(0.547,34.291)$ & .165 \\
\hline \multicolumn{3}{|l|}{ Bone quality } \\
\hline 1 & 1 & \\
\hline 2 & $1.094(0.387,3.088)$ & .866 \\
\hline 3 & $1.066(0.374,3.041)$ & .905 \\
\hline 4 & $4.023(1.133,14.286)$ & .031 \\
\hline \multicolumn{3}{|l|}{ Reoperation } \\
\hline No & 1 & \\
\hline Yes & $0.470(0.037,6.021)$ & .562 \\
\hline \multicolumn{3}{|l|}{ Age } \\
\hline Increase by 1 & $0.973(0.954,0.992)$ & .005 \\
\hline \multicolumn{3}{|l|}{ Intake of antidepressants } \\
\hline No & 1 & \\
\hline Yes & $1.077(0.423,2.747)$ & .876 \\
\hline \multicolumn{3}{|l|}{ Gastric } \\
\hline No & 1 & \\
\hline Yes & $2.135(1.001,4.064)$ & .049 \\
\hline \multicolumn{3}{|l|}{ Smoking } \\
\hline No & 1 & \\
\hline Yes & $1.975(1.137,3.429)$ & .016 \\
\hline Former smoker & $1.908(0.693,5.254)$ & .211 \\
\hline \multicolumn{3}{|l|}{ Bruxism } \\
\hline No & 1 & \\
\hline Yes & $5.200(2.235,12.097)$ & $<.001$ \\
\hline \multicolumn{3}{|l|}{ Antibiotics } \\
\hline No & 1 & \\
\hline Yes & $0.658(0.354,1.222)$ & .185 \\
\hline \multicolumn{3}{|l|}{ Bone grafting } \\
\hline No & 1 & \\
\hline Yes & $2.082(0.425,10.207)$ & .366 \\
\hline \multicolumn{3}{|l|}{ Time of follow-up } \\
\hline$\leq 1$ year & 1 & \\
\hline 1 year $<x \leq 5$ years & $4.356(0.290,65.416)$ & .287 \\
\hline 5 years $<x \leq 10$ years & $3.814(0.275,52.982)$ & .319 \\
\hline 10 years $<x \leq 20$ years & $3.075(0.230,41.066)$ & .396 \\
\hline$>20$ years & $7.289(0.541,98.232)$ & .134 \\
\hline
\end{tabular}

Only the factors that were considered statistically significant $(P<.1)$ in the univariate model and did not present multicollinearity were included in the multivariate model (OR-odds ratio). implant-supported prostheses were made, these situations indicate that an overload could be a contributing factor of importance for some of the cluster failures.

Other patients' habits such as smoking and the use of smokeless tobacco (snuff) were also analyzed. The results of the multivariate GEE logistic regression model suggest a statistically significant influence of smoking on the cluster behavior. A recent meta-analysis analyzing more than 100 studies has shown that failures of implants inserted in smokers are 2.23 times likely to happen than failures of implants inserted in nonsmokers. ${ }^{28}$ The increase of implant failure rates due to smoking is hypothesized to be related mainly to the effect of smoking in osteogenesis and angiogenesis. ${ }^{29}$ Moreover, smokers' health behavior and attitudes appear to be less favorable to oral health than those of nonsmokers. ${ }^{30}$ Studies have shown that smokers brush and floss their teeth less frequently than nonsmokers ${ }^{31}$ and have dental visits less frequently than do nonsmokers. ${ }^{32}$

With regard to the age of the patient, the results of the multivariate GEE model suggest that the odds of implant failure decrease by $3.3 \%$ for every 1 year increase in the patient's age. This could be related to the lower prevalence of bruxism among the elderly in relation to younger adult, ${ }^{33}$ to the ageing effect in oral physiology, resulting in less muscular strength and weaker mastication forces, ${ }^{34}$ and to a high prevalence of removable prosthesis opposing implant-supported restorations. $^{35}$

The patients of the cluster group presented, in comparison to the patients of the noncluster group, a statistically significant greater percentage of implants placed in sites of poor bone, that is, bone quantities $\mathrm{D}$ and $\mathrm{E}$, and bone qualities 3 and 4 . The multivariate GEE model showed a statistically significant negative influence of poor bone on the cluster behavior of implant failures. Poor bone site has been considered an important factor to influence implant survival negatively. ${ }^{3}$

Concerning the different implant surfaces, the higher failure rate of turned implants is hypothesized to be related to the small differences in the osseointegration process in relation to the enhanced-surface implants. The enhanced surfaces are designed to allow greater boneto-implant contact, and provide better possibilities for microbiomechanical retention due to larger surface and thus more retention for proteins to attach and new bone formation. ${ }^{36}$ The longer mean followup of the turned implants in comparison to the enhanced-surface implants group may also have some influence on these results, as a longer period of follow-up can result in an increased failure rate, especially if it extended beyond functional loading, because other prosthetic factors can influence implant failure from that point onward.

With regard to lower hazard ratios for longer implants, although some good results can be obtained with the use of shorter implants, they seem to fail more often than longer ones. ${ }^{37}$ Increased initial stability, long-term resistance to bending moment forces, expedited healing, and a decreased risk of movement at the interface are listed as advantages of increased implant length. ${ }^{38}$

The lack of specific information characterizing the patients' systemic conditions status and the medications dosage, as well as gaps in information in the dental records are limitations also connected to the 
retrospective nature of this study. Moreover, the implant primary stability was not analyzed, as well as the possible influence of the placement of implants by different surgeons/clinicians.

In the clear advantage of hindsight, it may be said that some patients should have been treated differently than what was done in the first place. Grafting procedures might have been advantageous in the cases with poor bone quantity and quality that displayed large number of failures. Parameters that can be available before the surgical and prosthodontics treatment would certainly be of importance to identify information to allow a careful discussion regarding risks and problems prior to surgery, and also to allow for measures to be taken to reduce the risk by, for example, choosing other surgical or prosthodontic techniques. ${ }^{4}$ The indication for the use of oral implants should sometimes be reconsidered when alternative prosthetic treatments are available and when possibly multiple interfering systemic or local factors are identified.

\section{5 | CONCLUSIONS}

A cluster pattern among patients with implant failure is highly probable. Factors of interest as predictors for implant failures could be a number of systemic and local factors, although a direct causal relationship cannot be ascertained. The present study identified shorter implants, turned implants, poor bone quality, age of the patient, the intake of antidepressants and of medicaments to reduce the acid gastric production, smoking, and bruxism as suggested potential factors exerting a statistically significant influence on the cluster behavior of dental implant failures.

\section{ACKNOWLEDGMENTS}

This work was supported by research funds from the Oral Health Related Research by Region Skåne (Odontologisk Forskning i Region Skåne, OFRS 414321), Sweden, and from the Scientific Research Council of Sweden (Vetenskapsrådet, Dnr 2015-02971). This work was supported by Folktandvården $\mathrm{AB}$, Region Skåne, Sweden and by CNPq, Conselho Nacional de Desenvolvimento Científico e Tecnológico, Brazil (201318/2012-1).

Trial registration at the U.S. National Institutes of Health (clinicaltrials.gov): NCT02369562.

\section{CONFLICT OF INTEREST}

There are no conflicts of interest to declare.

\section{REFERENCES}

[1] Adell R, Eriksson B, Lekholm U, Brånemark PI, Jemt $\mathrm{T}$. Long-term follow-up study of osseointegrated implants in the treatment of totally edentulous jaws. Int J Oral Maxillofac Implants. 1990;5: 347-359.

[2] Weyant RJ, Burt BA. An assessment of survival rates and withinpatient clustering of failures for endosseous oral implants. J Dent Res. 1993;72:2-8.
[3] Ekfeldt A, Christiansson U, Eriksson T, et al. A retrospective analysis of factors associated with multiple implant failures in maxillae. Clin Oral Implants Res. 2001;12:462-467.

[4] Jemt T, Hager P. Early complete failures of fixed implant-supported prostheses in the edentulous maxilla: a 3-year analysis of 17 consecutive cluster failure patients. Clin Implant Dent Relat Res. 2006;8:77-86.

[5] Schwartz-Arad D, Laviv A, Levin L. Failure causes, timing, and cluster behavior: an 8-year study of dental implants. Implant Dent. 2008;17:200-207.

[6] Lin WS, Ercoli C, Lowenguth R, Yerke LM, Morton D. Oral rehabilitation of a patient with bruxism and cluster implant failures in the edentulous maxilla: a clinical report. J Prosthet Dent. 2012;108:1-8.

[7] Tonetti MS. Determination of the success and failure of root-form osseointegrated dental implants. Adv Dent Res. 1999;13:173-180.

[8] Chrcanovic BR, Albrektsson T, Wennerberg A. Reasons for failures of oral implants. J Oral Rehabil. 2014;41:443-476.

[9] Chrcanovic BR, Kisch J, Albrektsson T, Wennerberg A. Factors influencing early dental implant failures. J Dent Res. 2016;95:995-1002.

[10] Lobbezoo F, Ahlberg J, Glaros AG, et al. Bruxism defined and graded: an international consensus. J Oral Rehabil. 2013;40:2-4.

[11] AASM. International Classification of Sleep Disorders, Revised: Diagnostic and Coding Manual. Chicago: American Academy of Sleep Medicine; 2014.

[12] Chrcanovic BR, Kisch J, Albrektsson T, Wennerberg A. Bruxism and dental implant treatment complications: a retrospective comparative study of 98 bruxer patients and a matched group. Clin Oral Implants Res. 2016 Mar 23. doi: 10.1111/clr.12844. [Epub ahead of print]

[13] Lekholm U, Zarb GA. Patient selection and preparation. In: Brånemark PI, Zarb GA, Albrektsson TS, eds. Proceedings of the Tissue integrated prostheses: osseointegration in clinical dentistry. Quintessence Publishing Company; 1985 Chicago, IL:199-209.

[14] Beck AT. Depression: Causes and Treatment. Philadelphia: University of Pennsylvania Press.

[15] Anttila SS, Knuuttila ML, Sakki TK. Relationship of depressive symptoms to edentulousness, dental health, and dental health behavior. Acta Odontol Scand. 2001;59:406-412.

[16] Kurer JR, Watts TL, Weinman J, Gower DB. Psychological mood of regular dental attenders in relation to oral hygiene behaviour and gingival health. J Clin Periodontol. 1995;22:52-55.

[17] Monteiro da Silva AM, Oakley DA, Newman HN, Nohl FS, Lloyd HM. Psychosocial factors and adult onset rapidly progressive periodontitis. J Clin Periodontol. 1996;23:789-794.

[18] Norderyd O, Hugoson A, Grusovin G. Risk of severe periodontal disease in a Swedish adult population. A longitudinal study. J Clin Periodontol. 1999;26:608-615.

[19] Hall SM, Munoz RF, Reus VI, Sees KL. Nicotine, negative affect, and depression. J Consult Clin Psychol. 1993;61:761-767.

[20] Escobedo LG, Reddy M, Giovino GA. The relationship between depressive symptoms and cigarette smoking in US adolescents. Addiction. 1998;93:433-440.

[21] Graziani G, Como G, Badalamenti S, et al. Effect of gastric acid secretion on intestinal phosphate and calcium absorption in normal subjects. Nephrol Dial Transplant. 1995;10:1376-1380

[22] O'Connell MB, Madden DM, Murray AM, Heaney RP, Kerzner LJ. Effects of proton pump inhibitors on calcium carbonate absorption in women: a randomized crossover trial. Am J Med. 2005;118:778-781.

[23] Schinke T, Schilling AF, Baranowsky A, et al. Impaired gastric acidification negatively affects calcium homeostasis and bone mass. Nat Med. 2009;15:674-681. 
[24] Chrcanovic BR, Kisch J, Albrektsson T, Wennerberg A. Bruxism and dental implant failures: a multilevel mixed effects parametric survival analysis approach. J Oral Rehabil. 2016;43:813-823.

[25] Manfredini D, Poggio CE, Lobbezoo F. Is bruxism a risk factor for dental implants? A systematic review of the literature. Clin Implant Dent Relat Res. 2014;16:460-469.

[26] Chrcanovic BR, Albrektsson T, Wennerberg A. Bruxism and dental implants: a meta-analysis. Implant Dent. 2015;24:505-516.

[27] Lobbezoo F, Brouwers JE, Cune MS, Naeije M. Dental implants in patients with bruxing habits. J Oral Rehabil. 2006;33:152-159.

[28] Chrcanovic BR, Albrektsson T, Wennerberg A. Smoking and dental implants: a systematic review and meta-analysis. J Dent. 2015;43: 487-498.

[29] Ma L, Zheng LW, Sham MH, Cheung LK. Uncoupled angiogenesis and osteogenesis in nicotine-compromised bone healing. J Bone Miner Res. 2010;25:1305-1313.

[30] Telivuo M, Kallio P, Berg MA, Korhonen HJ, Murtomaa H. Smoking and oral health: a population survey in Finland. J Public Health Dent. 1995;55:133-138.

[31] Andrews JA, Severson HH, Lichtenstein E, Gordon JS. Relationship between tobacco use and self-reported oral hygiene habits. J Am Dent Assoc. 1998;129:313-320.

[32] Attwood D, West P, Blinkhorn AS. Factors associated with the dental visiting habits of adolescents in the west of Scotland. Community Dent Health. 1993;10:365-373.
[33] Lavigne GJ, Montplaisir JY. Restless legs syndrome and sleep bruxism: prevalence and association among Canadians. Sleep. 1994;17: 739-743.

[34] Mioche L, Bourdiol P, Peyron MA. Influence of age on mastication: effects on eating behaviour. Nutr Res Rev. 2004;17:43-54.

[35] Osterberg T, Carlsson GE, Sundh V. Trends and prognoses of dental status in the Swedish population: analysis based on interviews in 1975 to 1997 by Statistics Sweden. Acta Odontol Scand. 2000;58: 177-182.

[36] Balshe AA, Assad DA, Eckert SE, Koka S, Weaver AL. A retrospective study of the survival of smooth- and rough-surface dental implants. Int J Oral Maxillofac Implants. 2009;24:1113-1118.

[37] Pommer B, Frantal S, Willer J, Posch M, Watzek G, Tepper G. Impact of dental implant length on early failure rates: a metaanalysis of observational studies. J Clin Periodontol. 2011;38:856863.

[38] Winkler S, Morris HF, Ochi S. Implant survival to 36 months as related to length and diameter. Ann Periodontol. 2000;5:22-31.

How to cite this article: Chrcanovic BR, Kisch J, Albrektsson T, Wennerberg A. Analysis of risk factors for cluster behavior of dental implant failures. Clin Implant Dent Relat Res. 2017;00:1-11. https://doi.org/10.1111/cid.12485 



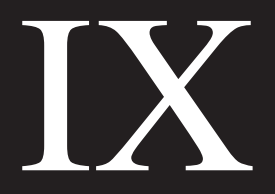



A retrospective study on clinical and radiological outcomes of oral implants in patients followed up for a minimum of 20 years

Bruno Ramos Chrcanovic ${ }^{1 *}$,

Jenö Kisch ${ }^{2}$

Tomas Albrektsson ${ }^{3}$

Ann Wennerberg ${ }^{4}$

${ }^{1}$ DDS, MSc, PhD student; Department of Prosthodontics, Faculty of Odontology, Malmö University, Malmö, Sweden

${ }^{2}$ DDS, OD. h.c.; Clinic for Prosthodontics, Centre of Dental Specialist Care, Malmö, Sweden

${ }^{3}$ MD, PhD, RCPSG; Department of Biomaterials, Göteborg University, Göteborg, Sweden; Department of Prosthodontics, Faculty of Odontology, Malmö University, Malmö, Sweden 4 DDS, PhD; Department of Prosthodontics, Faculty of Odontology, Malmö University, Malmö, Sweden

* Corresponding author:

Bruno Ramos Chrcanovic, Department of Prosthodontics, Faculty of Odontology, Malmö University, Carl Gustafs väg 34, SE-214 21, Malmö, Sweden. bruno.chrcanovic@mah.se; brunochrcanovic@hotmail.com Mobile: +46725 541545 Fax: +46 406658503 


\section{Abstract}

Purpose. To assess dental implant failure rates and marginal bone loss (MBL) of patients followed up for a minimum of 20 years.

Materials and Methods. This retrospective study is based on 2,670 patients who received 10,096 implants and were consecutively treated with implant-supported prostheses at one specialist clinic. Only implants placed in patients followed up for at least 20 years were included. Several anatomical-, patient-, health-, and implant-related factors were collected. Descriptive statistics and survival analyses were performed. Generalized estimating equations (GEE) analysis evaluated the effect of explanatory variables on implant failure. A number of 300 implants were randomly selected for MBL.

Results. A total of 1,045 implants placed in 227 patients were included. Implant location, irradiation, and bruxism were identified as the factors that statistically significantly affected the implant survival rate. Thirty-five percent of the implant failures occurred within the first year after implantation, and another $26.8 \%$ of the failures occurred in the second and third year. There was a CSR of $87.8 \%$ after 36 years of follow-up. In the last radiological follow-up, 35 implants (11.7\%) had bone gain, and 35 implants (11.7\%) presented at least $3 \mathrm{~mm}$ of MBL. Twenty-six out of 86 failed implants with available radiograms presented severe MBL in the last radiological register before implant failure.

Conclusions. Most of the implant failures occurred at the first few years after implantation, regardless of a very long follow-up. MBL can be insignificant in long term observations, but it may, nevertheless, be the cause of secondary failure of oral implants in some cases.

\section{Keywords}

Dental implants; implant failure; risk factors; marginal bone loss, long-term follow-up, multivariate analysis 


\section{Introduction}

Brånemark discovered the process of osseointegration in the 1960s and placed the first dental implant about 50 years ago. ${ }^{1}$ Since then dental implants supported by evidencebased data became an increasingly viable alternative to conventional prosthodontic treatment options. Thousands of clinical studies on dental implants have been carried out and published. Most studies present data on implant and prosthesis survival, as well as on bone response adjacent to the implants. However, many of the studies are on a relatively short- or medium-term basis. ${ }^{2}$ There is an increase in the volume of dental implants being placed and follow-up is essential to determine and predict a future clinical course. The patients receiving implants expect to keep them for years ever, and therefore it is more reliable to have a basis for dental implants prognosis with long follow-up studies.

During the first decades of osseointegration, the most commonly used implant in the world was the turned ("machined") Brånemark implant. This implant design showed quite good long term data with survival rates in the mandible above $90 \%$ and in the maxilla above $80 \%$ for a follow up time of 5 years or more. ${ }^{3-5}$ Having said this, there were some problems reported in soft maxillary bone ${ }^{6,7}$ and the old Brånemark implants showed less good clinical results if loaded prematurely or used in form of short implants. ${ }^{8}$ Another problem was reported with Brånemark implants; that of substantial marginal bone loss allegedly leading to disease entitled "Peri-implantitis". Roos-Jansåker et al. ${ }^{9}$ initially defined disease around oral implants based on bleeding on probing and $>1.8 \mathrm{~mm}$ of marginal bone loss at 9-14 years of follow up; they reported $6.6 \%$ of their Brånemark implants with disease. Fransson et al. ${ }^{10}$ saw any bone loss after the first year if combined with bleeding on probing and pus to be synonymous to implant disease and reported $12.4 \%$ of their 5-20 years followed up Brånemark implants with such problems.

These early reports of disease may not have survived the scrutiny of time, ${ }^{11}$ but previous reports have focused on the importance of careful monitoring of implant marginal bone loss as one way of documenting their long term performance. ${ }^{3,12}$ The latter authors reported that a successful implant would maximally lose $1 \mathrm{~mm}$ of bone during the implant's first year in function and less than $0.2 \mathrm{~mm}$ annually thereafter. Implants losing more bone would not be successful, but may certainly survive and function in the bone of the patient.

The purpose of the present study was to assess the dental implant failure rates and marginal bone loss (MBL) of patients followed up for a minimum of 20 years. By selecting 
such a long follow up time of implants placed at one specialist clinic in Malmö, the included implants are indeed old turned Brånemark ones $(n=1025)$ and a very small number of turned Astra implants $(n=20)$.

\section{Materials and Methods}

Materials. This retrospective study was based on all 2,670 patients provided with implants, consecutively treated on a routine basis at one specialist clinic (Clinic for Prosthodontics, Centre of Dental Specialist Care, Malmö, Sweden) during the period from 1980 to 2014. The study protocol was approved by the regional Ethical Committee, Lund, Sweden (Dnr 2014/598; Dnr 2015/72).

Definitions. An implant was considered a failure if presenting signs and symptoms that led to implant removal. Thus, a failed implant in our study is equal to a lost implant. The failures were classified into two types: (1) implants lost due to lack/loss of osseointegration and (2) fractured implants. Primary failures were those occurring until/at the day of the $2^{\circ}$ stage surgery (abutment connection).

We considered as severe MBL at least $1.0 \mathrm{~mm}$ of bone loss per year or a minimum bone loss of $1 / 3$ of the implant's full length in the last radiological register before implant failure.

Inclusion and exclusion criteria. Only implants placed in patients followed up for at least 20 years were included. Patients with all modern types of threaded implants with cylindrical or conical design were included. Zygomatic implants were not included in the study, as well as implants detected in radiographies, but without basic information about them in the patients' files.

A number of 300 implants were randomly selected for MBL. Only implants not lost and with baseline radiographs taken within 12 months after implant placement and with a minimum of 10 years of radiological follow-up were considered for MBL. Negative values correspond to bone loss. The marginal bone around the failed implants was also assessed.

Data collection. The dental records of all patients ever treated with implants in the aforementioned clinic were read in order to collect the data. The data were directly entered into a SPSS file (SPSS software, version 23, SPSS Inc., Chicago, IL, USA) as the files were being read. The following data were collected:

(a) Implant-related factors: implant diameter and length; 
(b) Site-related factors: implant jaw location (maxilla/mandible), anterior or posterior location of the implant (sites from right canine to left canine teeth were considered anterior location), and bone quantity and quality of the implant sites, which were classified at the time of surgery according to the Lekholm and Zarb ${ }^{13}$ classification;

(c) Patient-related factors: patient's sex, age of the patient at the implant insertion surgery, general health, and behavioral history. The presence of a medicament list in the patients' records was also use to correlate the use of certain drugs to specific health conditions. Health factors assessed: diabetes types I or II, hypertension, hypercholesterolemia, hypothyroidism, asthma, chemotherapy, and irradiation of the headneck region. The patients were also classified according to the intake of the following medication types: antidepressants, immunosuppressives, bisphosphonates, antithrombotic agents (antiplatelet, anticoagulant, thrombolytic drugs), hormone replacement therapy in women, and medicaments to reduce the acid gastric production. Behavioral factors assessed: smoking habits, use of snuff, bruxism;

(d) Other factors: prescription of antibiotics (the prophylactic antibiotic regimen was usually starting 1-2 hours before surgery and going from 5-7 days postoperatively), bone graft procedures, type of implant-supported prosthetic restoration (single crown, partial bridge with 2-6 prosthetic elements, partial bridge with 7-10 prosthetic elements, full-arch, overdenture), number of days until failure, and follow-up time.

As the standard protocol in the clinic, the patients' dental hygiene was followed up by a dental hygienist within 6 months after the final implant-supported/retained restoration. Each patient then attended a dental hygiene recall program based on individual needs.

Marginal bone level evaluation. Reproducible intra-oral radiographs were used. When there were no available digital radiographies from the baseline appointment, the analogue periapical radiographies were scanned at 1200 dpi (Epson Perfection V800 Photo Color Scanner; Nagano, Japan). Marginal bone level (MBL) was measured after calibration based on the inter-thread distance of the Nobel implants $(0.60 \mathrm{~mm})$. Measurements were taken from the implant-abutment junction to the marginal bone level, at both mesial and distal sides of each implant, and then the mean value of these two measurements was considered. MBL was calculated by comparing bone-to-implant contact levels to the radiographic baseline examination. The Image J software (National Institute of Health, Bethesda, USA) was used for all measurements. 
Statistical analyses. The mean, standard deviation (SD), and percentage were calculated for several variables. The Kolmogorov-Smirnov test was performed to evaluate the normal distribution of the variables, and Levene's test evaluated homoscedasticity. Differences between groups were compared with the student's t-test or Mann-Whitney test for continuous variables, depending on the normality. Survival analyses were performed. A life table was presented with cumulative survival rate (CSR), besides Kaplan-Meier analysis. Correlation and linear regression were performed to check the relationship between MBL and time of follow-up. An implant-level model was performed in order to assess the effects of the implant-related and local bone factors on the implant failures, also including health variables. A generalized estimating equations (GEE) method was used to account for the fact that repeated observations (several implants) were available for a single patient. All models were adjusted for clustering of subject and implants in a binary logistic regression model using GEE with a binomial distribution and a logit link function, while assuming an exchangeable working correlation structure. Initially a univariate GEE on each of the variables was performed. In order to verify multicollinearity, a correlation matrix of all of the predictor variables with a significant odds ratio ( $P$-value cut-off point of 0.1$)$ identified in the univariate GEE was scanned, to see whether there were some high correlations among the predictors. Collinearity statistics obtaining variance inflation factor (VIF) and tolerance statistic were also performed to detect more subtle forms of multicollinearity. Then a multivariable model with a forced entry method was used to evaluate the effect of the factors that were univariately significant $(P<0.1)$ and didn't present multicollinearity. A Wald chi-square test was used to analyze the statistical significance of each parameter within the model. The results of the final model were presented as an estimated OR of each significant prognostic variable $(P<0.05)$. SPSS software version 23 (SPSS Inc., Chicago, IL, USA) was used for the statistical analyses.

\section{Results}

Overall, 642 of 10,096 implants (6.36\%) failed. A number of 95 men (405 implants) and 132 women (640 implants) were followed up for at least 20 years and were included for analysis in the present study. The patients were followed-up for a mean $\pm S D$ of $291.0 \pm 33.7$ months (min-max, 244.4-437.5). These patients received 1,045 threaded implants with a cylindrical design - 1025 Nobel turned and 20 Astra turned implants. Fifty-eight patients 
presented 131 implants failures, 116 due to loss/lack of osseointegration and 15 failed fixtures due to implant fracture. There was a mean of 2.26 failures per patient (min-max, 19). Failures were recorded for 130 Nobel implants and 1 Astra implant. The failures happened at a mean $\pm S D$ of $54.3 \pm 67.0$ months ( $\min -\max , 1.2-294.7$ ) after implant insertion. The exact date of failure of 8 implants was not known; these failures were confirmed when these implants were no longer in place in subsequent radiograms. Nineteen failures happened before the abutment connection. 499 implants were installed in maxillae, of which 92 (18.4\%) failed (82 due to loss/lack of osseointegration, 10 fractured implants), and 546 implants were placed in mandibles, of which 39 (7.1\%) failed (34 due to loss/lack of osseointegration, 5 fractured implants).

Table 1 shows a comparison of groups according to the distribution of implants with regard to Lekholm and Zarb ${ }^{13}$ classification of bone quantity and quality. A greater percentage of failed implants were placed in bone sites having been classified as quantities $D$ and $E$ and bone quality 4 . Table 2 shows the life table for implant fracture. Thirty-five percent of the occurrences of failed implants occurred within the first year after implantation. Another $26.8 \%$ of the failures occurred in the second and third year after implant insertion. Thus, $59.3 \%$ of all failures occurred at the first three years after implantation. There was a CSR of $87.8 \%$ after 36 years of follow-up. Figure 1 shows the Kaplan-Meier analysis. No censored cases were observed before 20 years of follow-up, as only these cases were included in the present study.

All implants were inserted with open flapped surgery and had a delayed loading. Only 5 implants were inserted in fresh extraction sockets. The abutment connection surgery was performed after a mean \pm SD healing time of $167 \pm 56$ days. The mean length of the inserted implants was $12.4 \pm 2.7 \mathrm{~mm}$ and the mean diameter for was $3.74 \pm 0.09 \mathrm{~mm}$.

Seventy-four implants were used for single-crown restorations ( 3 failed due to loss/lack of osseointegration, 5 fractured implants), 242 implants for fixed partial prostheses of 2-6 prosthetic elements (23 failed due to loss/lack of osseointegration, 4 fractured implants), 25 implants fixed partial prostheses of 7-10 prosthetic elements ( 7 failed due to loss/lack of osseointegration, no fractured implants), 666 implants for full-arch fixed prostheses (69 failed due to loss/lack of osseointegration, 6 fractured implants), and 33 implants to support overdentures ( 9 failed due to loss/lack of osseointegration, no fractured 
implants). There was no information for 5 implants concerning the type of prosthodontic restoration performed.

The univariate GEE model at the implant-level showed that the following predictors had a statistically significant odds ratio at the implant-level (Table 3): location, bone quantity and quality, the intake of antidepressants, irradiation, the intake of medicaments to reduce the acid gastric production, bruxism, and the type of implant-supported prosthetic restoration. The following factors remained statistically significant in the multivariate GEE model (Table 4): location, irradiation, and bruxism.

Of the 4,691 implants that were placed until December 31st 1996, and then could theoretically reach the minimum of 20 years in the year of 2017, 2,762 implants (58.88\%) were placed in patients that were deceased by the first day of the year of 2017. Of these 2,762 implants, 2,402 (86.97\%) were placed in patients who died before a hypothetical 20 year follow-up. Patient referral to other clinics and other reasons accounted for 884 of the unaccounted for implants.

With regard to the MBL around the 300 randomly selected implants, all Nobel turned implants, 1948 measurements were performed, considering the several radiological followups for each implant. This resulted in a mean of 6.5 MBL measurements per implant. Each $M B L$ measurement consisted of a mean value of mesial + distal measurements. A number of $1032 \mathrm{MBL}$ measurements were performed for the 152 implants in the maxilla and $916 \mathrm{MBL}$ measurements for the 148 implants in the mandible. The baseline radiograms were taken at a mean $\pm S D$ of $7.0 \pm 2.4$ months (min-max, 0-12) after implant placement. The implants were radiologically followed up for mean \pm SD of $244.8 \pm 54.8$ months (min-max, 127.2-350.2). At the last follow-up, there was a mean $\pm S D M B L$ of $-1.405 \pm 1.504 \mathrm{~mm}$ ( $\min , \max ;-7.955,1.442$ ) for all implants $(n=300)$. These values at the last follow-up were $-1.391 \pm 1.691 \mathrm{~mm}(\mathrm{~min}$, max; $-7.955,1.442)$ for implants placed in maxillae $(n=152)$, and $-1.419 \pm 1.289 \mathrm{~mm}(\mathrm{~min}$, max; $7.128,0.858)$ for implants placed in mandibles $(n=148)$. The difference in MBL between implants placed in different jaws was not statistically significant at the last radiological follow-up ( $p=0.176$, Mann-Whitney test). The last radiological follow-up was performed at a mean $\pm S D$ of $258.9 \pm 54.0$ months (min-max, 123.1-350.2) for implants placed in maxillae and 229.4 \pm 52.7 months (min-max. 127.2-336.8) for implants in mandibles. This difference in the mean last follow-up between different jaws was statistically significant $(p<0.001$, MannWhitney test). 
In the last radiological follow-up (Table 5), it was observed that 35 implants (11.7\%) had bone gain (min-max 0.010-1.442 mm), 109 implants (36.3\%) displayed up to $1 \mathrm{~mm}$ of MBL, 84 implants (28\%) showed a MBL between 1 and $2 \mathrm{~mm}, 37$ implants (12.3\%) a MBL between 2 and $3 \mathrm{~mm}$, and 35 implants (11.7\%) with at least $3 \mathrm{~mm}$ of MBL, of which 7 implants (2.3\%) presented MBL of more than $6 \mathrm{~mm}$. It is important to stress that these 300 implants randomly selected for $\mathrm{MBL}$ measurements were among the implants that were not lost.

Figure 2 shows a scatter plot of all $1948 \mathrm{MBL}$ measurements, showing that there is an estimated trend to loss bone with time, reaching $1.98 \mathrm{~mm}$ of bone loss 30 years after implant installation. The linear regression equation ( $y=-0.35-0.00453 x)$ estimated a loss of $0.00453 \mathrm{~mm}$ of bone for every additional month of follow-up. MBL was moderately associated to the follow-up time $\left(R=-0.358, R^{2}=0.128, p<0.001\right.$, Pearson correlation).

The marginal bone around the 131 failed implants was also assessed. The radiological follow-up protocol at the clinic did not include the register of a radiogram at the implant placement day, beginning at the abutment connection day only. Thus, it was not able to evaluate the marginal bone condition around some implants, due to lack of radiograms, because they failed before the abutment connection (primary failures). In other cases, some patients received several implants, but the first radiological register of the implants was carried out only after abutment connection. These cases that presented no radiological register of the failed fixtures accounted for 37 implants. Then, of the 131 lost implants, 86 implants had available radiograms: 131 total implants -8 implants with unknown date of failure -37 implants without radiograms $=86$ implants with radiograms. Twenty-six out of these 86 implants with radiograms presented severe MBL. Figure 3 shows radiograms of some examples of implants with advanced MBL found in the present study.

\section{Discussion}

The results of the present study showed that most of the implant failures occurred at the first few years after implantation, regardless of a very long follow-up. The regression analysis performed in this study tried to identify the factors that could possibly be related to implant failure. The univariate regression assessed the relationship between each independent variable and implant failure separately, and the multivariate regression assessed the relationship of the variables that were univariately significant to implant 
failure, controlling for each other. The negative factors identified by the multivariate GEE model were implant location, irradiation, and bruxism. The improved survival rate of implants placed in the anterior mandible in relation to the anterior maxilla may be related to the usually improved bone quality and greater bone volume found in the anterior mandible, even years after teeth extraction in this region. ${ }^{14}$ When it comes to irradiation, it has been shown that it negatively affects the survival of implants, ${ }^{15}$ which is mostly related to the deleterious long-term effects of irradiation on the bone vascularization ${ }^{16}$ and bone-healing capacity, ${ }^{17}$ compromising the implantation site. Concerning the patients' habits, bruxism was shown to significantly affect the implant failure rates negatively, agreeing with the results of the two very recent clinical trials assessing the effect of bruxism on dental implants. ${ }^{18,}{ }^{19}$ Bruxism is suggested to generate overload of prosthetic rehabilitations on implants, which could possibly cause implant fracture or peri-implant marginal bone loss, ultimately resulting in implant failure..$^{20}$ Implant fractures noticed in the present study may further be related to the use of grade 1 titanium which is a less strong material than presently used grade 3-4 titanium.

About $12 \%$ of the implants in this study displayed more than $3 \mathrm{~mm}$ of marginal bone loss. However, this observation must be balanced against the observation that hexed Brånemark implants display an average of $1 \mathrm{~mm}$ of marginal bone loss already at 1 year of follow up, although the five year bone loss figures of the same implants indicated a relative steady state with respect to further bone loss. ${ }^{4}$ In contrast, implants with internal couplings instead of hexes only demonstrated an average 5-year bone loss of about $0.2 \mathrm{~mm}$. Furthermore, an average marginal bone loss of more than $3 \mathrm{~mm}$ need not represent disease or other clinical problems as indicated by Jemt et al. ${ }^{21}$ These authors followed up what happened to the allegedly sick implants of the study by Fransson et al. ${ }^{22}$ who saw $12.4 \%$ of their implants with $>2.4 \mathrm{~mm}$ of marginal bone loss coupled with bleeding on probing and increased pocket depths. Fransson's et al. ${ }^{22}$ implants were followed up for a median of 12.5 years. Jemt et al. ${ }^{21}$ reported that $91.3 \%$ of the allegedly sick implants of Fransson's et al. study $^{22}$ saw no significant further bone loss another 9.1 years (average) of follow-up, i.e. a total follow-up in the 20 -year range. A total of $95.4 \%$ of the implants with progressive bone loss were still functioning as part of support for dental bridges at 20+ years of follow-up. Based on these figures, it is obvious that one cannot judge disease on a millimeter report of 
bone loss. In addition, Coli et al. ${ }^{23}$ have recently questioned the relevance of using probes as criteria for disease around oral implants.

Therefore, we have to analyze other reasons than only disease for marginal bone loss. In a recent paper ${ }^{11}$ some cases with bone loss were reported to most likely be dependent on an age-related bone loss that may happen around implants as well as teeth.

The number of unaccounted for implants was quite high in the present report. However, when patients who died or were just referred to other clinics are concerned, these reasons for drop out are unlikely to skew the present analysis. It must be remembered that the Malmö clinic originally was the only implant active unit in the region, therefore when new clinics started with implants at a later time, it was quite natural that those clinics took over the controls of some patients.

The total failure rate of the Malmö material has been reported to $6.36 \%$. However, this failure rate is presumably greater in reality since some implants controlled at other clinics may have failed too. However, overall oral implants fare quite well, despite some of them seeing marginal bone loss of $3 \mathrm{~mm}$ of more. The term peri-implantitis indicative of disease of all implants with marginal bone loss has, to the knowledge of the present authors not been verified in clinical studies and it seems likely that the future will see improved ways of looking at the implants with bone loss. Physiological causes have been overlooked as one reason for bone loss and, furthermore, as described by orthopedic surgeons, some implants may display stress shielding; i.e. only the amount of bone needed for implant anchorage remains after long time.

\section{Conclusions}

Most of the implant failures occurred at the first few years after implantation, regardless of a very long follow-up. Implants in different jaw locations, irradiation, and bruxism were the factors suggested to affect the long-term survival of implants. Marginal bone loss can be insignificant in long term observations, but it may, nevertheless, be the cause of secondary failure of oral implants in some cases. 


\section{Acknowledgements}

Funding and support: This work was supported by research funds from the Oral Health Related Research by Region Skåne (Odontologisk Forskning i Region Skåne, OFRS 414321), Sweden, and from the Scientific Research Council of Sweden (Vetenskapsrådet, Dnr 201502971). This work was supported by Folktandvården AB, Region Skåne, Sweden and by CNPq, Conselho Nacional de Desenvolvimento Científico e Tecnológico, Brazil.

Trial registration at the U.S. National Institutes of Health (clinicaltrials.gov): NCT02369562 Declaration of conflicting interests: There are no conflicts of interest to declare.

\section{References}

1. Brånemark $\mathrm{Pl}$, Adell $\mathrm{R}$, Breine U, Hansson BO, Lindström J, Ohlsson A. Intra-osseous anchorage of dental prostheses. I. Experimental studies. Scand J Plast Reconstr Surg 1969; 3: 81-100.

2. Ekelund JA, Lindquist LW, Carlsson GE, Jemt T. Implant treatment in the edentulous mandible: a prospective study on Branemark system implants over more than 20 years. Int J Prosthodont 2003; 16: 602-608.

3. Albrektsson T, Zarb G, Worthington P, Eriksson AR. The long-term efficacy of currently used dental implants: a review and proposed criteria of success. Int J Oral Maxillofac Implants 1986; 1: 11-25.

4. Jimbo R, Albrektsson T. Long-term clinical success of minimally and moderately rough oral implants: a review of 71 studies with 5 years or more of follow-up. Implant Dent 2015; 24: 62-69.

5. Lindquist LW, Carlsson GE, Jemt T. Association between marginal bone loss around osseointegrated mandibular implants and smoking habits: a 10-year follow-up study. J Dent Res 1997; 76: 1667-1674.

6. Adell R, Lekholm U, Rockler B, Brånemark PI. A 15-year study of osseointegrated implants in the treatment of the edentulous jaw. Int J Oral Surg 1981; 10: 387-416.

7. Jaffin RA, Berman CL. The excessive loss of Branemark fixtures in type IV bone: a 5-year analysis. J Periodontol 1991; 62: 2-4.

8. Brånemark $\mathrm{PI}$, Hansson $\mathrm{BO}$, Adell R, Breine $\mathrm{U}$, Lindström J, Hallen $\mathrm{O}$, Ohman A. Osseointegrated implants in the treatment of the edentulous jaw. Experience from a 10-year period. Scand J Plast Reconstr Surg Suppl 1977; 16: 1-132. 
9. Roos-Jansåker AM, Lindahl C, Renvert $\mathrm{H}$, Renvert $\mathrm{S}$. Nine- to fourteen-year follow-up of implant treatment. Part II: presence of peri-implant lesions. J Clin Periodontol 2006; 33: 290-295.

10. Fransson C, Wennström J, Berglundh T. Clinical characteristics at implants with a history of progressive bone loss. Clin Oral Implants Res 2008; 19: 142-147.

11. Albrektsson T, Chrcanovic B, Östman PO, Sennerby L. Initial and long-term crestal bone responses to modern dental implants. Periodontol 2000 2017; 73: 41-50.

12. Albrektsson T, Zarb GA. Current interpretations of the osseointegrated response: clinical significance. Int J Prosthodont 1993; 6: 95-105.

13. Lekholm U, Zarb GA. Patient selection and preparation In: Brånemark PI, Zarb GA, Albrektsson Ts, eds. Proceedings of the Tissue integrated prostheses: osseointegration in clinical dentistry. Quintessence Publishing Company, 1985: 199-209.

14. Glauser R, Rée A, Lundgren A, Gottlow J, Hämmerle CH, Schärer P. Immediate occlusal loading of Branemark implants applied in various jawbone regions: a prospective, 1year clinical study. Clin Implant Dent Relat Res 2001; 3: 204-213.

15. Chrcanovic BR, Albrektsson T, Wennerberg A. Dental implants in irradiated versus nonirradiated patients: A meta-analysis. Head Neck 2016; 38: 448-481.

16. Yerit KC, Posch M, Seemann M, Hainich S, Dortbudak O, Turhani D, Ozyuvaci H, Watzinger F, Ewers R. Implant survival in mandibles of irradiated oral cancer patients. Clin Oral Implants Res 2006; 17: 337-344.

17. Marx RE, Johnson RP. Studies in the radiobiology of osteoradionecrosis and their clinical significance. Oral Surg Oral Med Oral Pathol 1987; 64: 379-390.

18. Chrcanovic BR, Kisch J, Albrektsson T, Wennerberg A. Bruxism and dental implant treatment complications: a retrospective comparative study of 98 bruxer patients and a matched group. Clin Oral Implants Res 2016.

19. Chrcanovic BR, Kisch J, Albrektsson T, Wennerberg A. Bruxism and dental implant failures: a multilevel mixed effects parametric survival analysis approach. J Oral Rehabil 2016; 43: 813-823.

20. Lobbezoo F, Brouwers JE, Cune MS, Naeije M. Dental implants in patients with bruxing habits. J Oral Rehabil 2006; 33: 152-159. 
21. Jemt T, Sundén Pikner S, Gröndahl K. Changes of Marginal Bone Level in Patients with "Progressive Bone Loss" at Branemark System(R) Implants: A Radiographic Follow-Up Study over an Average of 9 Years. Clin Implant Dent Relat Res 2015; 17: 619-628.

22. Fransson C, Lekholm U, Jemt T, Berglundh T. Prevalence of subjects with progressive bone loss at implants. Clin Oral Implants Res 2005; 16: 440-446.

23. Coli P, Christiaens V, Sennerby L, Bruyn H. Reliability of periodontal diagnostic tools for monitoring peri-implant health and disease. Periodontol 2000 2017; 73: 203-217. 


\section{Tables}

Table 1. Distribution of implants with regard to Lekholm and Zarb (1985) classification of bone quantity and quality. The number between parentheses represents failures.

\begin{tabular}{|c|c|c|c|c|c|c|c|c|}
\hline & A & B & C & D & $E$ & Total & $\begin{array}{c}\% \\
\text { Group** } \\
\end{array}$ & $\begin{array}{c}\% \\
\text { Failed } t \\
\end{array}$ \\
\hline 1 & 8 & 10 & $15(2)$ & $60(1)$ & $16(2)$ & 109(5) & 10.5 & 4.6 \\
\hline 2 & $51(5)$ & $162(21)$ & $147(13)$ & 19 & $19(4)$ & $398(43)$ & 38.1 & 10.8 \\
\hline 3 & 21 & 158(18) & $185(11)$ & $46(15)$ & $6(2)$ & $416(46)$ & 39.9 & 11.1 \\
\hline 4 & 2 & $19(6)$ & $37(7)$ & $35(10)$ & $27(14)$ & $120(37)$ & 11.5 & 30.8 \\
\hline Total & $82(5)$ & $349(45)$ & $384(33)$ & $160(26)$ & $68(22)$ & $1043(131)$ & 100 & 12.6 \\
\hline$\%$ & 7.9 & 33.5 & 36.8 & 15.3 & 6.5 & 100 & & \\
\hline \multicolumn{9}{|l|}{ Group** } \\
\hline$\%$ Failed $†$ & 6.1 & 12.9 & 8.6 & 16.3 & 32.4 & 12.6 & & \\
\hline \multicolumn{9}{|c|}{ Missing information of bone quantity/quality: $\mathbf{2}$ implants, $\mathbf{0}$ failures } \\
\hline
\end{tabular}

* According to the Lekholm and Zarb (1985) classification, bone quality is broken down into four groups according to the proportion and structure of compact and trabecular bone tissue: type $1=$ large homogenous cortical/compact bone; type 2 = thick layer of compact bone surrounding a dense trabecular bone; type 3 = thin cortical layer surrounding a dense trabecular bone; type $4=$ thin cortical layer surrounding a core of low-density trabecular bone. The quantity of jawbone is broken down into five groups $(A, B, C, D$, and $E)$, based on the residual jaw shape following tooth extraction. Bone classified as ' $A$ ' presents the largest amount of bone among all classes, whereas bone classified as ' $E$ ' presents the lowest volume of bone.

** Percentage of implants in each bone quantity/quality class, considering all implants as $100 \%$

+ Percentage of failed implants for each bone quantity/quality class 
Table 2. Life table for implant failure.

\begin{tabular}{|c|c|c|c|c|c|c|c|}
\hline $\begin{array}{l}\text { Interval } \\
\text { start } \\
\text { time } \\
\text { (years) }\end{array}$ & $\begin{array}{l}\text { Number } \\
\text { entering } \\
\text { interval }\end{array}$ & $\begin{array}{c}\text { Number } \\
\text { withdrawing } \\
\text { during } \\
\text { interval }\end{array}$ & $\begin{array}{l}\text { Number } \\
\text { exposed } \\
\text { to risk* }\end{array}$ & $\begin{array}{l}\text { Number } \\
\text { of } \\
\text { terminal } \\
\text { events } \\
\text { (failures) }\end{array}$ & $\begin{array}{l}\text { Proportion } \\
\text { terminating }\end{array}$ & $\begin{array}{l}\text { Proportion } \\
\text { surviving }\end{array}$ & $\begin{array}{c}\text { Cumulative } \\
\text { proportion } \\
\text { surviving } \\
\text { at end of } \\
\text { interval } \\
\end{array}$ \\
\hline 0 & 1037 & 0 & 1037.0 & 43 & .041 & .959 & .959 \\
\hline 1 & 994 & 0 & 994.0 & 17 & .017 & .983 & .942 \\
\hline 2 & 977 & 0 & 977.0 & 16 & .016 & .984 & .927 \\
\hline 3 & 961 & 0 & 961.0 & 8 & .008 & .992 & .919 \\
\hline 4 & 953 & 0 & 953.0 & 3 & .003 & .997 & .916 \\
\hline 5 & 950 & 0 & 950.0 & 5 & .005 & .995 & .911 \\
\hline 6 & 945 & 0 & 945.0 & 5 & .005 & .995 & .906 \\
\hline 7 & 940 & 0 & 940.0 & 2 & .002 & .998 & .905 \\
\hline 8 & 938 & 0 & 938.0 & 1 & .001 & 999 & .904 \\
\hline 9 & 937 & 0 & 937.0 & 2 & .002 & .998 & .902 \\
\hline 10 & 935 & 0 & 935.0 & 4 & .004 & .996 & .898 \\
\hline 11 & 931 & 0 & 931.0 & 2 & .002 & .998 & .896 \\
\hline 12 & 929 & 0 & 929.0 & 2 & .002 & .998 & .894 \\
\hline 13 & 927 & 0 & 927.0 & 2 & .002 & .998 & .892 \\
\hline 14 & 925 & 0 & 925.0 & 0 & 0.000 & 1.000 & .892 \\
\hline 15 & 925 & 0 & 925.0 & 3 & .003 & .997 & .889 \\
\hline 16 & 922 & 0 & 922.0 & 4 & .004 & .996 & .885 \\
\hline 17 & 918 & 0 & 918.0 & 0 & 0.000 & 1.000 & .885 \\
\hline 18 & 918 & 0 & 918.0 & 0 & 0.000 & 1.000 & .885 \\
\hline 19 & 918 & 5 & 915.5 & 1 & .001 & .999 & .884 \\
\hline 20 & 912 & 140 & 842.0 & 0 & 0.000 & 1.000 & .884 \\
\hline 21 & 772 & 112 & 716.0 & 1 & .001 & 999 & .883 \\
\hline 22 & 659 & 137 & 590.5 & 0 & 0.000 & 1.000 & .883 \\
\hline 23 & 522 & 129 & 457.5 & 0 & 0.000 & 1.000 & .883 \\
\hline 24 & 393 & 113 & 336.5 & 2 & .006 & .994 & .878 \\
\hline 25 & 278 & 84 & 236.0 & 0 & 0.000 & 1.000 & .878 \\
\hline 26 & 194 & 48 & 170.0 & 0 & 0.000 & 1.000 & .878 \\
\hline 27 & 146 & 46 & 123.0 & 0 & 0.000 & 1.000 & .878 \\
\hline 28 & 100 & 59 & 70.5 & 0 & 0.000 & 1.000 & .878 \\
\hline 29 & 41 & 23 & 29.5 & 0 & 0.000 & 1.000 & .878 \\
\hline 30 & 18 & 10 & 13.0 & 0 & 0.000 & 1.000 & .878 \\
\hline 31 & 8 & 0 & 8.0 & 0 & 0.000 & 1.000 & .878 \\
\hline 32 & 8 & 3 & 6.5 & 0 & 0.000 & 1.000 & .878 \\
\hline 33 & 5 & 0 & 5.0 & 0 & 0.000 & 1.000 & .878 \\
\hline 34 & 5 & 0 & 5.0 & 0 & 0.000 & 1.000 & .878 \\
\hline 35 & 5 & 5 & 2.5 & 0 & 0.000 & 1.000 & .878 \\
\hline
\end{tabular}

* The exact date of failure was unknown for 8 implants. Therefore, they were not considered here. If these 8 lost implants were included in the present life table, the CSR would be lower 
Table 3. Risk factor analysis using a univariate generalized estimating equations (GEE) logistic regression model, at the implant-level (OR - odds ratio).

\begin{tabular}{|c|c|c|c|}
\hline Factor & Failure/survival* & OR $(95 \% \mathrm{Cl})$ & $P$-value \\
\hline \multicolumn{4}{|c|}{ IMPLANT-RELATED FACTORS } \\
\hline \multicolumn{4}{|l|}{ Implant diameter } \\
\hline Increase by 1 & - & $1.134(0.045,28.288)$ & 0.939 \\
\hline \multicolumn{4}{|l|}{ Implant length } \\
\hline \multirow[t]{2}{*}{ Increase by 1} & - & $0.930(0.835,1.035)$ & 0.183 \\
\hline & \multicolumn{2}{|c|}{ SITE-RELATED FACTORS } & \\
\hline \multicolumn{4}{|l|}{ Location } \\
\hline Anterior Maxilla & $58 / 280$ & 1 & \\
\hline Posterior Maxilla & $34 / 127$ & $1.546(0.945,2.530)$ & 0.083 \\
\hline Anterior Mandible & $13 / 283$ & $0.302(0.141,0.647)$ & 0.002 \\
\hline Posterior Mandible & $26 / 224$ & $0.579(0.306,1.096)$ & 0.093 \\
\hline \multicolumn{4}{|l|}{ Bone quantity } \\
\hline$A$ & $5 / 77$ & 1 & \\
\hline B & $45 / 304$ & $1.564(0.315,7.758)$ & 0.584 \\
\hline C & $33 / 351$ & $1.743(0.367,8.269)$ & 0.484 \\
\hline $\mathrm{D}$ & $26 / 134$ & $2.788(0.567,13.714)$ & 0.207 \\
\hline$E$ & $22 / 46$ & $8.079(1.624,40.194)$ & 0.011 \\
\hline \multicolumn{4}{|l|}{ Bone quality } \\
\hline 1 & $5 / 104$ & 1 & \\
\hline 2 & $43 / 355$ & $2.369(0.556,10.097)$ & 0.244 \\
\hline 3 & $46 / 370$ & $1.805(0.445,7.319)$ & 0.409 \\
\hline \multirow[t]{2}{*}{4} & $37 / 83$ & $7.048(1.690,29.387)$ & 0.007 \\
\hline & \multicolumn{2}{|c|}{ PATIENT-RELATED FACTORS } & \\
\hline \multicolumn{4}{|l|}{ Gender } \\
\hline Male & $62 / 343$ & 1 & \\
\hline Female & $69 / 571$ & $0.672(0.359,1.258)$ & 0.214 \\
\hline \multicolumn{4}{|l|}{ Age } \\
\hline Increase by 1 & - & $0.998(0.980,1.015)$ & 0.781 \\
\hline \multicolumn{4}{|l|}{ Diabetes } \\
\hline No & $102 / 568$ & 1 & \\
\hline Type I & $0 / 0$ & - & \\
\hline Type II & $12 / 64$ & $1.080(0.393,2.970)$ & 0.882 \\
\hline \multicolumn{4}{|l|}{ High blood pressure } \\
\hline No & $83 / 418$ & 1 & \\
\hline Yes & $31 / 228$ & $0.734(0.363,1.484)$ & 0.390 \\
\hline \multicolumn{4}{|l|}{ High cholesterol } \\
\hline No & $93 / 512$ & 1 & \\
\hline Yes & $21 / 114$ & $1.196(0.514,2.780)$ & 0.678 \\
\hline \multicolumn{4}{|l|}{ Hypothyroidism } \\
\hline No & $109 / 580$ & 1 & \\
\hline Yes & $5 / 46$ & $0.605(0.153,2.391)$ & 0.474 \\
\hline \multicolumn{4}{|l|}{ Asthma } \\
\hline No & $101 / 577$ & 1 & \\
\hline Yes & $13 / 61$ & $1.339(0.452,3.965)$ & 0.598 \\
\hline \multicolumn{4}{|l|}{ Intake of antidepressants } \\
\hline No & $79 / 570$ & 1 & \\
\hline Yes & $35 / 58$ & $3.874(1.381,10.866)$ & 0.010 \\
\hline
\end{tabular}




\begin{tabular}{|c|c|c|c|}
\hline \multicolumn{4}{|l|}{ Irradiation } \\
\hline No & $112 / 625$ & 1 & \\
\hline Yes & $2 / 3$ & $4.255(2.996,6.043)$ & $<0.001$ \\
\hline \multicolumn{4}{|c|}{ Hormone replacement therapy } \\
\hline No & $117 / 744$ & 1 & \\
\hline Yes & $9 / 22$ & $2.111(0.641,6.956)$ & 0.219 \\
\hline \multicolumn{4}{|l|}{ Gastric } \\
\hline No & $90 / 556$ & 1 & \\
\hline Yes & $24 / 62$ & $2.917(1.161,7.324)$ & 0.023 \\
\hline \multicolumn{4}{|l|}{ Antithrombotics } \\
\hline No & $77 / 461$ & 1 & \\
\hline Yes & $38 / 169$ & $1.499(0.743,3.025)$ & 0.258 \\
\hline \multicolumn{4}{|l|}{ Immunosuppressive } \\
\hline No & $111 / 612$ & 1 & \\
\hline Yes & $3 / 10$ & $1.513(0.277,8.246)$ & 0.632 \\
\hline \multicolumn{4}{|l|}{ Bisphosphonates } \\
\hline No & $113 / 610$ & 1 & \\
\hline Yes & $1 / 18$ & $0.283(0.048,1.676)$ & 0.164 \\
\hline \multicolumn{4}{|l|}{ Smoking } \\
\hline No & $65 / 405$ & 1 & \\
\hline Yes & $42 / 164$ & $1.397(0.630,3.096)$ & 0.411 \\
\hline Former smoker & $3 / 11$ & $3.616(0.618,21.152)$ & 0.154 \\
\hline \multicolumn{4}{|l|}{ Cigarettes/day } \\
\hline Increase by 1 & - & $1.028(0.983,1.074)$ & 0.229 \\
\hline \multicolumn{4}{|l|}{ Snuff } \\
\hline No & $106 / 567$ & 1 & \\
\hline Yes & $4 / 13$ & $1.356(0.309,5.953)$ & 0.687 \\
\hline \multicolumn{4}{|l|}{ Bruxism } \\
\hline No & $79 / 583$ & 1 & \\
\hline \multirow[t]{2}{*}{ Yes } & $37 / 50$ & $4.414(1.840,10.590)$ & 0.001 \\
\hline & \multicolumn{2}{|c|}{ OTHER FACTORS } & \\
\hline \multicolumn{4}{|l|}{ Antibiotics } \\
\hline No & $12 / 87$ & 1 & \\
\hline Yes & $51 / 358$ & $0.754(0.298,1.908)$ & 0.551 \\
\hline \multicolumn{4}{|l|}{ Bone grafting } \\
\hline No & $100 / 864$ & 1 & \\
\hline Yes & $31 / 50$ & $2.753(0.753,10.062)$ & 0.126 \\
\hline \multicolumn{4}{|l|}{ Prosthetic restoration } \\
\hline Single crown & $8 / 66$ & 1 & \\
\hline $\begin{array}{l}\text { Partial fixed, 2-6 } \\
\text { units }\end{array}$ & $27 / 215$ & $1.007(0.347,2.921)$ & 0.990 \\
\hline $\begin{array}{l}\text { Partial fixed, 7-10 } \\
\text { units }\end{array}$ & $7 / 18$ & $2.192(0.371,12.966)$ & 0.387 \\
\hline Full-arch fixed & 75/591 & $1.033(0.387,2.755)$ & 0.948 \\
\hline Overdenture & $9 / 24$ & $4.686(1.185,18.528)$ & 0.028 \\
\hline
\end{tabular}

* The information for some conditions is unknown for a variable number of implants 
Table 4. Multivariate generalized estimating equations (GEE) logistic regression model at the implantlevel. Only the factors that were considered statistically significant $(P<0.1)$ in the univariate model and didn't present multicollinearity were included in the multivariate model (OR - odds ratio).

\begin{tabular}{|c|c|c|}
\hline Factor & OR $(95 \% \mathrm{Cl})$ & $P$-value \\
\hline \multicolumn{3}{|l|}{ Location } \\
\hline Anterior Maxilla & 1 & \\
\hline Posterior Maxilla & $1.388(0.784,2.458)$ & 0.260 \\
\hline Anterior Mandible & $0.264(0.109,0.643)$ & 0.003 \\
\hline Posterior Mandible & $0.488(0.232,1.030)$ & 0.060 \\
\hline \multicolumn{3}{|l|}{ Bone quantity } \\
\hline$A$ & 1 & \\
\hline B & $1.455(0.392,5.407)$ & 0.575 \\
\hline C & $1.078(0.281,4.142)$ & 0.913 \\
\hline D & $1.828(0.435,7.677)$ & 0.410 \\
\hline$E$ & $3.412(0.605,19.241)$ & 0.164 \\
\hline \multicolumn{3}{|l|}{ Bone quality } \\
\hline 1 & 1 & \\
\hline 2 & $1.228(0.318,4.744)$ & 0.766 \\
\hline 3 & $0.713(0.177,2.878)$ & 0.635 \\
\hline 4 & $1.335(0.285,6.267)$ & 0.714 \\
\hline \multicolumn{3}{|c|}{ Intake of antidepressants } \\
\hline No & 1 & \\
\hline Yes & $2.578(0.813,8.175)$ & 0.108 \\
\hline \multicolumn{3}{|l|}{ Irradiation } \\
\hline No & 1 & \\
\hline Yes & $13.560(6.058,30.354)$ & $<0.001$ \\
\hline \multicolumn{3}{|l|}{ Gastric } \\
\hline No & 1 & \\
\hline Yes & $2.097(0.685,6.416)$ & 0.194 \\
\hline \multicolumn{3}{|l|}{ Bruxism } \\
\hline No & 1 & \\
\hline Yes & $2.800(1.012,7.748)$ & 0.047 \\
\hline \multicolumn{3}{|l|}{ Prosthetic restoration } \\
\hline Single crown & 1 & \\
\hline $\begin{array}{l}\text { Partial fixed, 2-6 } \\
\text { units }\end{array}$ & $1.592(0.448,5.660)$ & 0.473 \\
\hline $\begin{array}{l}\text { Partial fixed, 7-10 } \\
\text { units }\end{array}$ & $0.520(0.051,5.315)$ & 0.582 \\
\hline Full-arch fixed & $1.788(0.456,7.016)$ & 0.405 \\
\hline Overdenture & $1.631(0.314,8.467)$ & 0.561 \\
\hline
\end{tabular}


Table 5. Marginal bone condition around 300 non-failed implants at the last radiological follow-up (mean of 244.8 months).

\begin{tabular}{lc}
\hline \multicolumn{1}{c}{ Marginal bone condition } & Number of implants (\%) \\
\hline Bone gain $(0.010-1.442 \mathrm{~mm})$ & $35(11.7 \%)$ \\
Bone loss up to $1 \mathrm{~mm}$ & $109(36.3 \%)$ \\
Bone loss between 1 and $2 \mathrm{~mm}$ & $84(28.0 \%)$ \\
Bone loss between 2 and $3 \mathrm{~mm}$ & $37(12.3 \%)$ \\
More than $3 \mathrm{~mm}$ of bone loss & $35^{*}(11.7 \%)$ \\
\hline
\end{tabular}

* Seven (2.3\% of the total number of implants) out of these 35 implants presented MBL of more than $6 \mathrm{~mm}$

\section{Figures}

Figure 1. Kaplan-Meier curve.

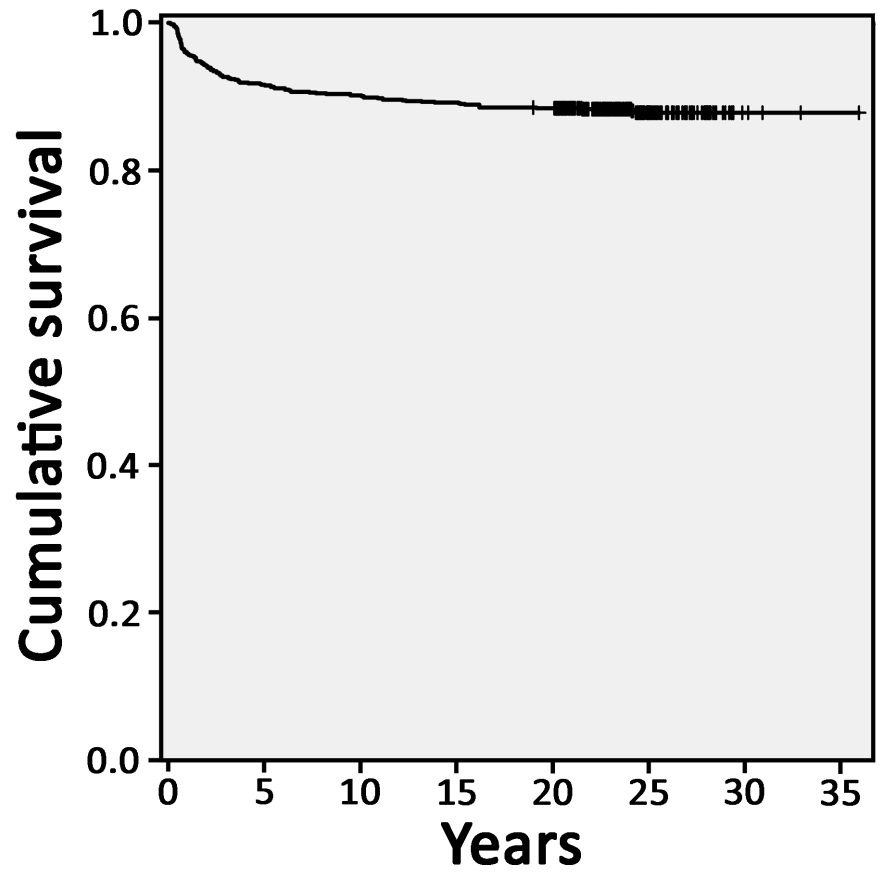


Figure 2. Scatter plot of 1948 marginal bone loss (MBL) measurements of 300 implants. The line represents the estimated $\mathrm{MBL}$ along the years of observation, according to the linear regression.

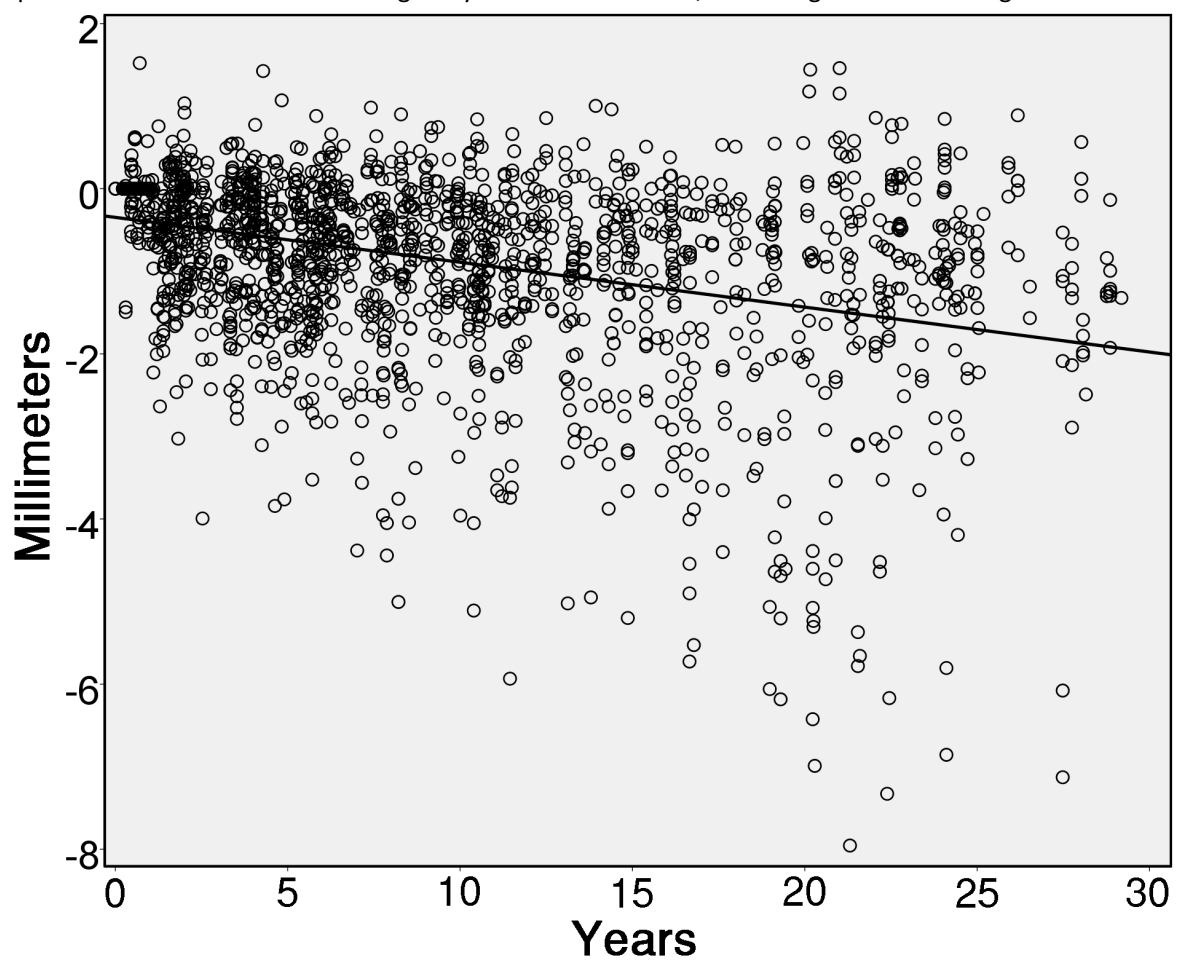

Figure 3. Examples of cases of severe MBL around implants that were eventually lost or fractured.
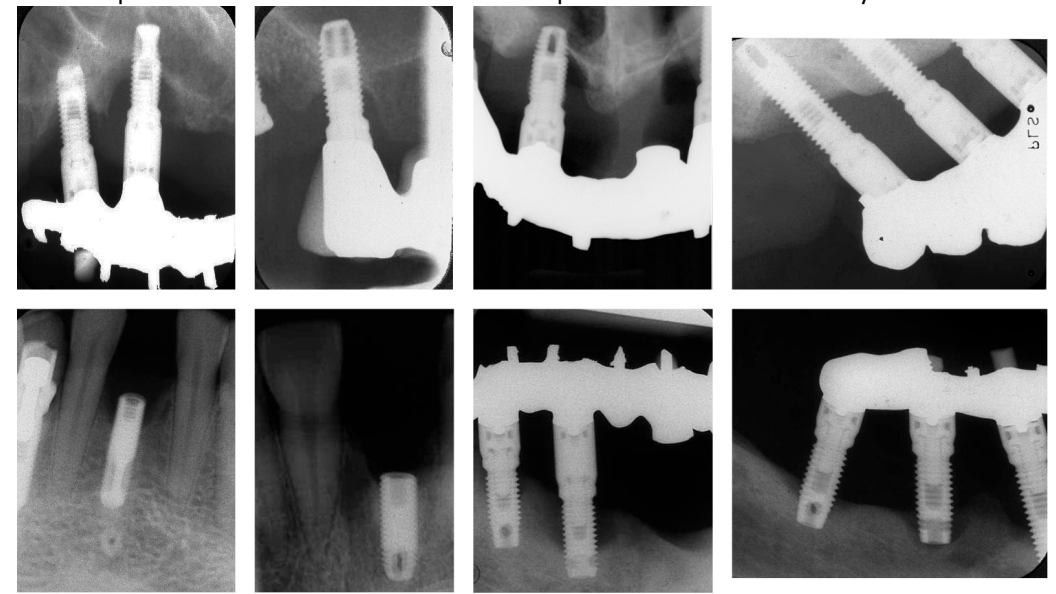


ISBN 978-9I-7IO4-766-3 (print)

ISBN 978-9I-7 IO4-767-O (pdf)

MALMÖ UNIVERSITY 20506 MALMÖ, SWEDEN WWW.MAH.SE 